\title{
From Photoredox Catalysis to the Direct Excitation of EthynylBenziodoXolones: Accessing Alkynylated Quaternary Carbons from Alcohols via Oxalates
}

\author{
Stephanie G. E. Amos, ${ }^{+}{ }^{[a]}$ Diana Cavalli ${ }^{+}{ }^{[a]}$ Franck Le Vaillant, ${ }^{[b]}{ }^{[}$Jerome Waser ${ }^{*[a]}$
}

[a] Laboratory of Catalysis and Organic Synthesis, Institut des Sciences et Ingénierie Chimique, Ecole Polytechnique Fédérale de Lausanne, CH-1015, Lausanne, Switzerland, E-mail: jerome.waser@epfl.ch

[b] Max-Planck-Institut für Kohlenforschung, Mülheim an der Ruhr 45470, Germany

[+] These authors contributed equally to this work.

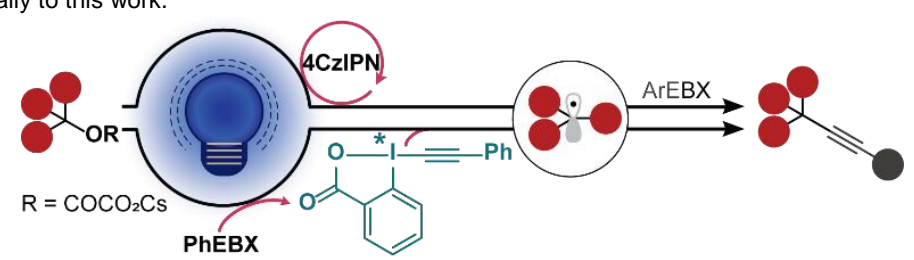

\begin{abstract}
EthynylBenziodoXolones (EBXs) are commonly used as radical traps in photocatalytic alkynylations. Herein, we report their application in two complementary deoxygenation strategies allowing the synthesis of valuable alkynylated all-carbon quaternary centers from tertiary alcohols via stable oxalate salts. Our first approach involves a photocatalytic process using $4 \mathrm{CzIPN}$ as an organic dye to promote oxidative degradation of the oxalate and EBXs to trap the formed radical. In our second approach, we demonstrate the direct photoexcitation of an EBX, which then acts as both oxidant and radical trap, alleviating the need for a photocatalyst in several EBX-mediated alkynylation processes.
\end{abstract}

\section{Introduction}

Alkynes have found broad applications in synthetic and medicinal chemistry, chemical biology, and materials science. ${ }^{[1]}$ Triple $C \equiv$ $\mathrm{C}$ bonds are stable yet offer a versatile panel of reactivity when activated either with transition metal or Lewis acid catalysts, allowing them to be easily converted to complex molecular structures. In medicinal chemistry and chemical biology, they can be used either as an inert and rigid connecting element or as a reactive unit. ${ }^{[2]}$ Alkynes connected to all-carbon quaternary centers constitute an important subset, as they combine the versatility of transformations inherent to triple bonds with the challenge of constructing highly substituted compounds. They have therefore found numerous applications in total synthesis of complex natural products such as (+)-lactacystin, (-)-rhazinilam and $\left( \pm\right.$ )-grandisol. ${ }^{[3]}$ They have also shown unique bioactivity in medicinal chemistry. ${ }^{[4]}$ Unfortunately, accessing such scaffolds remains challenging. Alkyne-transfer reactions are attractive as usually more efficient than multi-step elaboration of the triple bond. The generation of alkynylated quaternary centers has been achieved by the addition of transition metal acetylides onto enones or activated allylic alcohols (Scheme 1A). ${ }^{[5]}$ Metal acetylides have also enabled the alkynylation of diazo compounds, $\alpha$-bromo esters and unactivated alkenes resulting in quaternary centers. ${ }^{[6]}$ In addition to this approach, electrophilic alkynylation strategies with halogenides ${ }^{[7]}$ and hypervalent iodine reagents (HIRs) have been used to functionalize activated carbonyl compounds (Scheme 1B). ${ }^{[8]}$ These strategies rely on the specific reactivity of either electrophilic or nucleophilic functional groups, limiting the structural diversity of the products.

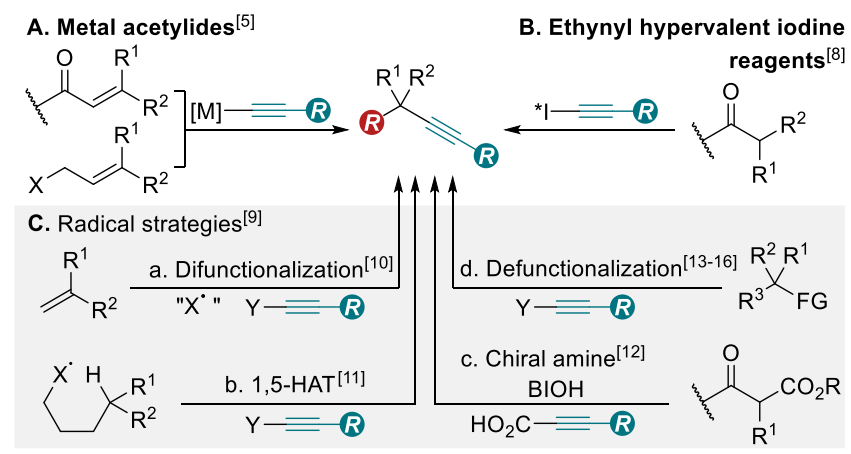

Scheme 1. State of the art: accessing alkynylated quaternary centers. A Transition metal catalyzed approaches. B. Hypervalent iodine for electrophilic alkynylation. C. Generation of tertiary radicals for alkynylation.

Recently, the alkynylation of carbon radicals has emerged as an attractive complementary route for the formation of quaternary alkynylated centers. ${ }^{[9]}$ Indeed, upon the generation of a highly reactive open-shell species, the limitations associated to steric hindrance that often hamper the formation of quaternary centers can be more easily overcome. Nowadays, a variety of mild methods can be used to generate radicals via thermal activation, transition metal catalysis or photomediated approaches (Scheme $1 \mathrm{C})$. In this context, alkynylative alkene difunctionalization (a) ${ }^{[10]}$ and 1,5-HAT $(\mathrm{b})^{[11]}$ strategies have been implemented successfully. Photoorganocatalysis has also enabled the formation of alkynylated quaternary centers from carbonyl compounds (c). ${ }^{[12]}$ Another strategy for the alkynylation of tertiary radicals is to perform radical defunctionalization (d). Decarboxylation has been the most investigated, ${ }^{[13]}$ but it still requires starting materials that themselves contain all-carbon quaternary centers. Alternatively, less abundant organoboron and organosulfur compounds can be used as precursors, ${ }^{[14]}$ whereas $\mathrm{C}-\mathrm{N}$ bond cleavage has been performed using azobis(isobutyronitrile) (AIBN) analogues. ${ }^{[15]}$ In this context, starting from broadly available tertiary alcohols appears highly attractive, yet it has been only rarely reported. The only described photocatalytic deoxy-alkynylation exploited a reductive substrate activation strategy using unstable and non-isolable $N$ phthalimidoyl oxalates as precursors. ${ }^{[16]}$ 
Amidst the various activating groups used for photocatalytic deoxygenation, cesium oxalates appear as attractive starting materials. Not only are they bench stable, easily accessible, and easy to use, their side-products are gaseous $\mathrm{CO}_{2}$ and a second cesium salt, as demonstrated by MacMillan and Overman in a deoxy-alkylation strategy (Scheme 2A). ${ }^{[17]}$ They extended this approach to arylation, ${ }^{[18]}$ and allylation, ${ }^{[19]}$ whereas Reisman and co-workers developed deoxy-halogenation. ${ }^{20]}$ However, this approach has yet to be applied for alkynylation. In this context, we thought that EthynylBenziodoXolone reagents (EBXs) would be suitable radical traps using cesium oxalates as radical precursors. Indeed, EBX reagents have proven themselves to be efficient radical traps when combined with substrate oxidation. ${ }^{[9,11 a, 13 c-d, 21]}$

A. Cesium oxalates as deoxygenation precursors

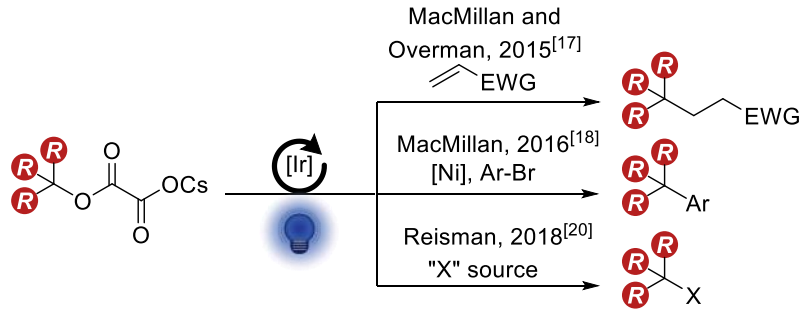

B. Our approaches for deoxy-alkynylation

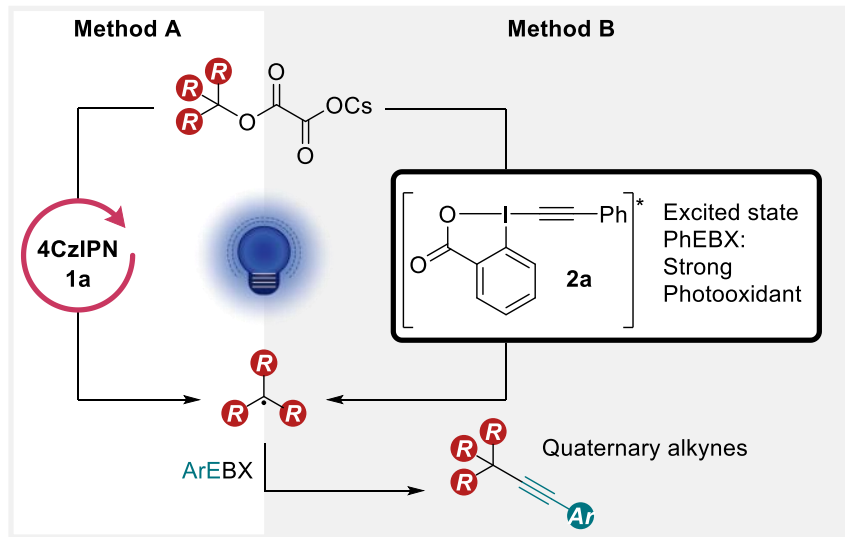

Scheme 2. A. Reported cesium oxalates-based transformations under photoredox catalysis. B. This work: Dual approach for photomediated deoxyalkynylation: metal-free photocatalysis (Method A) vs excited state EBXs as photooxidants (Method B).

Herein, we report the use of the photoorganocatalyst 4CzIPN (2,4,5,6-tetrakis $(9 \mathrm{H}$-carbazol-9-yl) isophthalonitrile, 1a) for the conversion of cesium oxalates to a variety of arylalkynes under visible light irradiation (Scheme 2B, Method A). During our studies, we discovered that the EBX reagent was undergoing photoactivation leading also to deoxy-alkynylation. A variety of HIRs have been reported to undergo excitation through spin forbidden transitions under visible light activation, ${ }^{[22]}$ but, to the best of our knowledge, this activation mode has never been reported for EBXs. We now report that the excited state PhEBX* $\left(\mathbf{2} \mathbf{a}^{\star}\right)$ of PhEBX (2a) can be used as a photooxidant to perform a photocatalyst-free deoxy-alkynylation of cesium oxalates. Preliminary results indicate that the approach can be extended to other photomediated alkynylations (Scheme 2B, Method B).

\section{Results and Discussion}

Photocatalyst mediated deoxy-alkynylation. We started our investigation of the metal-free photocatalytic approach using the cesium oxalate $3 \mathbf{a}$ as model substrate (Table 1 ). In presence of 5 mol\% of 4 CzIPN (1a) $\left(E_{1 / 2}\left(\mathbf{1}^{\star} / \mathbf{1} \mathbf{a}^{-}\right)=+1.35 \mathrm{~V}\right.$ vs SCE $),{ }^{[21 b]}$ under light irradiation at $440 \mathrm{~nm}$ with 1.5 equivalent of PhEBX (2a) in $\mathrm{DCM}$, the desired product (4a) was observed in $75 \%$ NMR yield ( $94 \%$ based on remaining starting material, entry 1$)$. Even if the starting material and reagent were not fully soluble in DCM, using more polar solvents such as $\mathrm{DMSO}, \mathrm{MeCN}$, or a mixture of DME/DMF with 10 equivalents $\mathrm{H}_{2} \mathrm{O},{ }^{[17]}$ led to lower yields (entry 2, see Supporting Information for details).

Table 1. Optimization of the photocatalyzed method (method A).

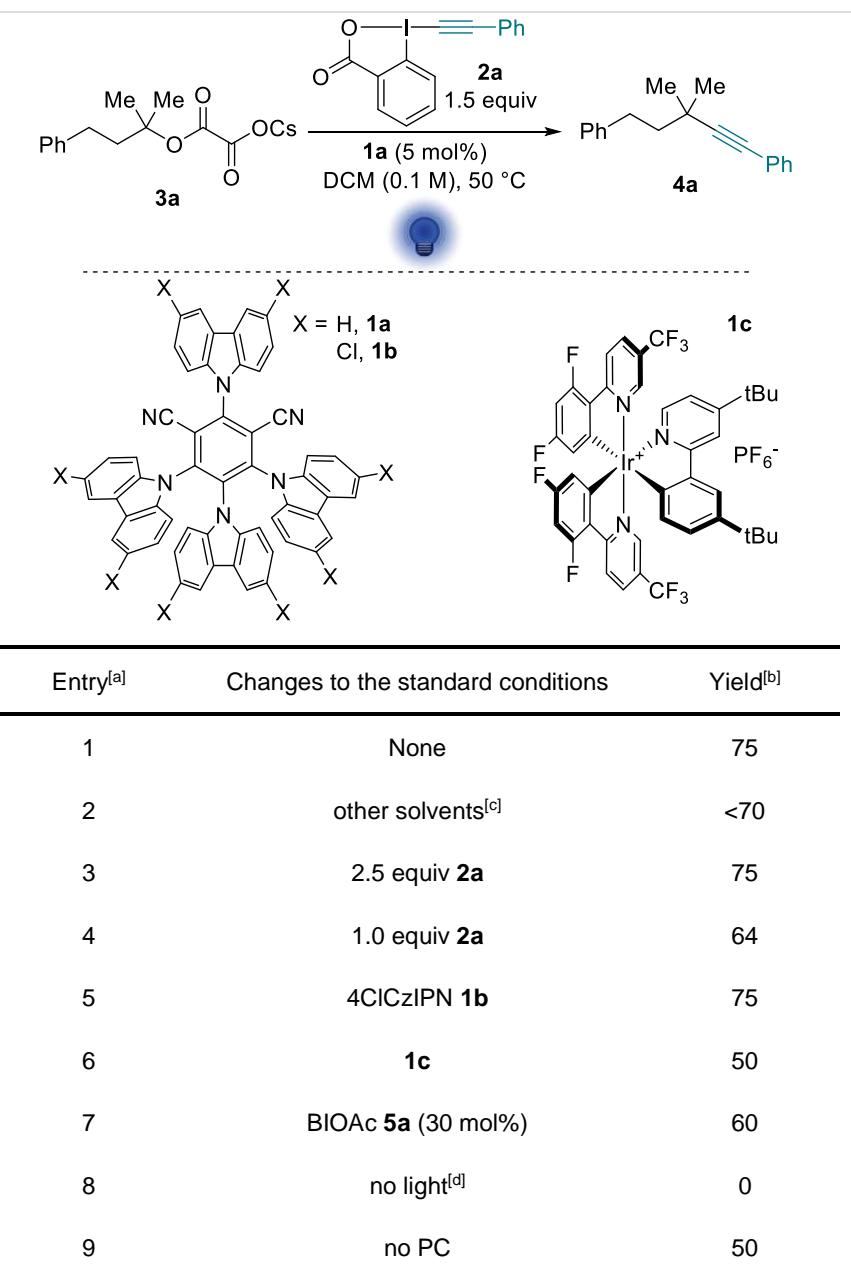

[a] $3 \mathbf{a}(0.1 \mathrm{mmol}), \mathbf{2 a}(1.5$ equiv) and $\mathbf{1 a}(5 \mathrm{~mol} \%)$ were dissolved in solvent [3a] $=0.1 \mathrm{M}$ and irradiated with two lamps $(40 \mathrm{~W}, 440 \mathrm{~nm})$ for $18 \mathrm{~h}$. [b] ${ }^{1} \mathrm{H}$ NMR Yield was determined using 1 equiv of $\mathrm{CH}_{2} \mathrm{Br}_{2}$ as internal standard. [c] DMSO, MeCN $\mathrm{DME} / \mathrm{DMF}+10$ equiv $\mathrm{H}_{2} \mathrm{O}$. [d] Reaction was performed with and without $\mathrm{PC}$

With a larger excess of PhEBX (2a) (2.5 equiv), the same yield was obtained, whereas $4 a$ was formed in only $64 \%$ yield when using one equivalent of $\mathbf{2 a}$ (entries 3 and 4 ). When the reaction was carried out in presence of the more oxidizing photocatalyst 4CICzIPN (1b, $E_{1 / 2}\left(\mathbf{1 b}^{\star} / \mathbf{1 b}^{-}\right)=+1.49 \mathrm{~V}$ vs SCE),[21b] we did not observe any improvement (entry 5 ). In turn, using the less oxidizing iridium photocatalyst $\left[\operatorname{lr}\left(\mathrm{dF}\left(\mathrm{CF}_{3}\right) \mathrm{ppy}\right)_{2}(\mathrm{dtbbpy})\right] \mathrm{PF}_{6}(\mathbf{1 c}$, $\mathrm{E}_{1 / 2}\left(\mathbf{1 c}^{\star} / \mathbf{1} \mathbf{c}^{-}\right)=+1.21 \mathrm{~V}$ vs SCE$)^{[13 \mathrm{~d}]}$ led to less product formation 
(entry 6). A range of other photocatalysts were examined but gave the product in lower yields (see Supporting Information). Furthermore, we investigated the use of benziodoxolone acetate (BIOAc, 5a) as additive, as it was previously shown to improve the yield in photo-mediated alkynylation processes involving EBX reagents. [21d] In our case however, it led to a considerable drop in yield (entry 7 ). When the reaction was run with the exclusion of light, no product was formed, and NMR analysis showed no degradation of the starting material (entry 8). Surprisingly, when the reaction was performed under irradiation without the addition of $4 \mathrm{CzIPN}$ (1a), $50 \%$ yield of 4 a was still obtained (entry 9). Nevertheless, we decided to use the photocatalytic conditions to investigate the scope of the reaction, since they provided the desired product in higher yield.

With the optimized reaction conditions established, we proceeded to explore the scope of cesium oxalates. The model substrate afforded the desired alkyne $\mathbf{4 a}$ in $75 \%$ yield (Scheme 3 ).

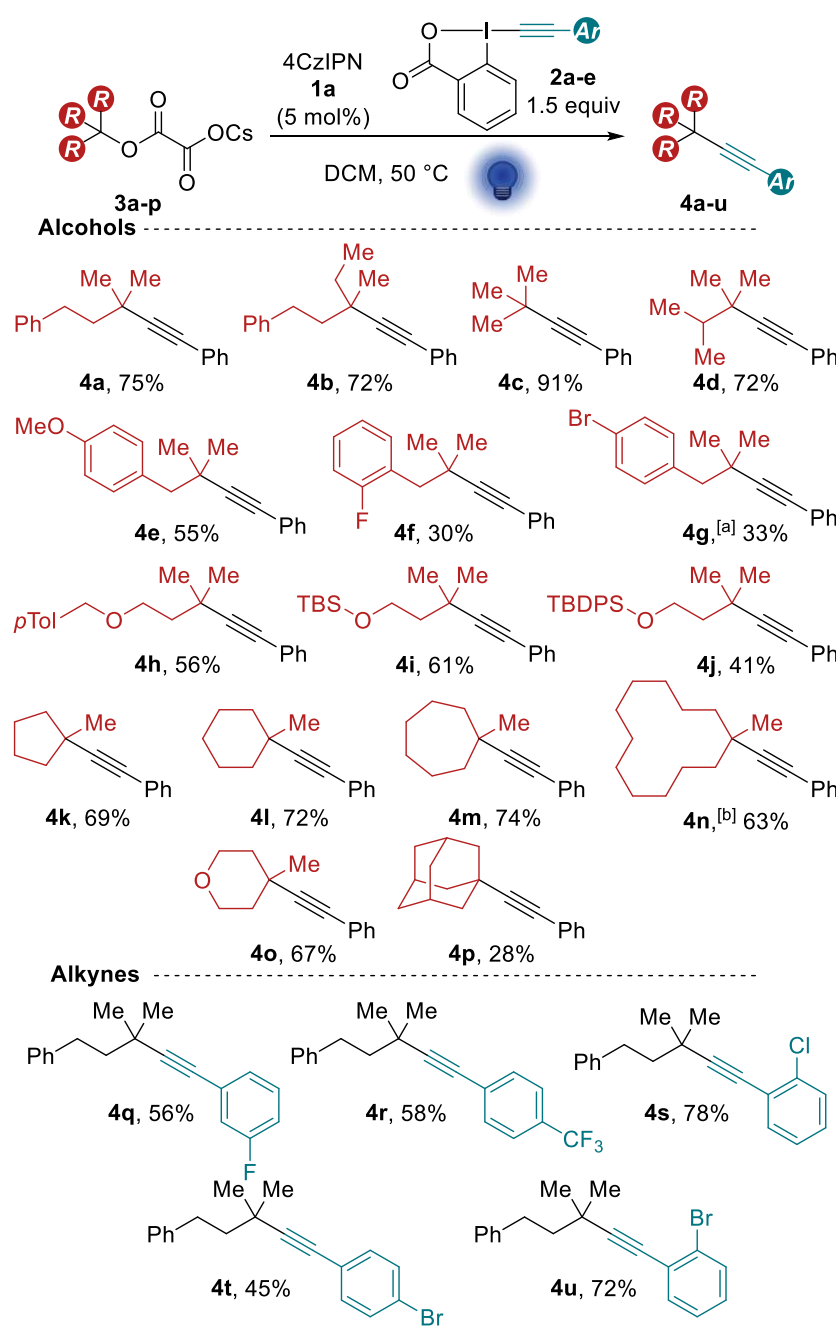

Scheme 3. Scope Method A. Reactions were performed on $0.3 \mathrm{mmol}$ scale using the corresponding cesium oxalate 3a-p (1 equiv) and ArEBX 2a-e (1.5 equiv) with $4 \mathrm{CzIPN}(\mathbf{1 a}, 5 \mathrm{~mol} \%)$ in DCM $(0.1 \mathrm{M})$. [a] Performed on $0.2 \mathrm{mmol}$ scale. [b] Reaction was performed on $0.24 \mathrm{mmol}$ scale with 1.9 equiv of $2 \mathrm{a}$.

When changing one of the methyl groups to an ethyl group the reaction afforded $\mathbf{4 b}$ in $72 \%$ yield. The tert-butyl radical gave $\mathbf{4 c}$ in $91 \%$ yield and bulkier $\mathbf{4 d}$ was obtained in $72 \%$ yield.
Homobenzylic scaffolds yielded compounds $4 \mathrm{e}-\mathrm{g}$ in 30 to $55 \%$ yield. A variety of benzyl and silyl protected alcohols afforded alkynes $\mathbf{4 h}$-j in up to $61 \%$ yield. Cyclic alcohols of variable ring sizes were investigated affording $\mathbf{4 k}-\mathbf{n}$ in $63-78 \%$ yield. A tetrahydropyran substrate was successfully converted to the corresponding alkyne 40 in $67 \%$ yield. The adamantyl alkyne $4 p$ was obtained with an expectable drop in yield when considering the known complication with bridged carbon radicals. ${ }^{[23]}$ Having established the scope of alcohols, we turned to explore different ArEBX reagents. Electron-poor fluorinated reagents afforded the corresponding alkynes $\mathbf{4 q}$ and $\mathbf{4 r}$ in $56 \%$ and $58 \%$ yield. Chlorinated and brominated aryl alkynes $\mathbf{4 s}$, $4 \mathbf{t}$ and $\mathbf{4 u}$ were obtained in 45 to $78 \%$ yield. Silyl- and alkyl- EBX reagents gave the product in only very low yield.

Investigation of the photoactive species in the photocatalystfree transformation. With the photocatalyst promoted transformation established, we attempted to gain a better understanding of the photocatalyst-free alkynylation we had observed with PhEBX (2a) (Table 1, entry 9). We envisaged a variety of mechanistic hypotheses that could account for the oxidation of the starting material even in absence of a photocatalyst (Scheme 4). First, we considered that a residual HIR(III) species BIOX from the PhEBX synthesis could be the oxidant (Scheme 4A, hypothesis 1). Based on literature precedence, the formation of a covalent adduct I between the oxalate and the HIR would be expected. ${ }^{[12,24]}$ Under visible light irradiation, homolytic cleavage of the hypervalent iodine IIII-O would form the O-centered radical, which then undergoes double decarboxylation to give the tertiary radical II.

A. Hypothesis 1: lodine(III) precursors as oxidants

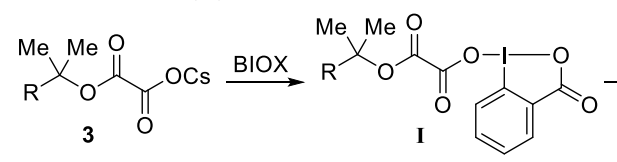

B. Hypothesis 2: Ketone side-products $\mathbf{6 a}$ or $\mathbf{6 b}$ as oxidants<smiles>O=C1OI(C#Cc2ccccc2)c2ccccc21</smiles>

$$
\stackrel{\text { method } \mathrm{A}}{\longrightarrow}
$$

C. Hypothesis 3: Excited-state 2a as an oxidant
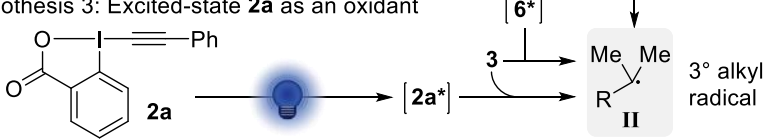

Scheme 4. Alternative mechanisms for the oxidation of cesium oxalate 3 to give tertiary radical II. A. Hypothesis 1: lodine(III) precursors as oxidants. B Hypothesis 2: Ketone side products $\mathbf{6 a}$ and $\mathbf{6 b}$ as oxidants. C. Hypothesis 3 : Excited state of $\mathbf{2 a}$ as an oxidant.

As a second hypothesis, side-products formed during the reaction could act as photooxidants (Scheme 4B, hypothesis 2). Indeed, two aromatic ketones $\mathbf{6 a}$ and $\mathbf{6 b}$ were observed as side products, resulting from degradation PhEBX (2a) with or without addition of the oxalate starting material $3 \mathbf{a}^{\left[{ }^{[25]}\right.}$ Based on the broad use of aromatic ketones as photosensitizers, ${ }^{[26]}$ we hypothesized that $6 \mathbf{a}$ 
or $\mathbf{6 b}$ may be photoactive specie under our reaction conditions and potential oxidants in the excited state to generate II.

Finally, PhEBX (2a) may be undergoing direct photoexcitation (Scheme 4C, hypothesis 3). Surprisingly, we found no reference in the literature for such a process. However, previous studies were performed with less intensive irradiation and/or at different wavelengths, ${ }^{[10 d-e, 11 c, 13 a, c-d, 14 a, 15]}$ which may explain why this activation process has yet to be reported. Here, we speculated that photoexcited $2 \mathbf{a}^{*}$ would be more oxidizing than the ground state $\mathbf{2 a}$ and would generate the O-centered radical through a single electron transfer (SET), leading to II after fragmentation.

In order to gain support for one of the proposed alternatives, further experiments were performed (Table 2). Interestingly, when TIPS-EBX (TIPS = triisopropylsilyl, $2 \mathbf{2 g}$ ) was used as an alkynylation reagent in absence of photocatalyst, no product was observed and both $\mathbf{2} \mathbf{g}$ and the cesium oxalate $\mathbf{3 a}$ remained untouched (Table 2 , entry 1 ). $\mathbf{2 g}$ has been reported to not undergo direct excitation at $400 \mathrm{~nm},{ }^{[27]}$ but is known to work as a radical trap. ${ }^{[13 c-d]}$ When we performed the reaction with $\mathbf{1 a}$ and $\mathbf{2} \mathbf{g}$ based on the conditions for method A, we obtained $25 \%$ of alkynylation product $\mathbf{4} \mathbf{v}$ confirming that it is able to react with the tertiary radical formed from cesium oxalates, even if the overall reaction is not very efficient (entry 2). This result confirmed that the aryl substituent on PhEBX (2a) was required for the reaction to proceed in absence of photocatalyst, but it still did not allow us to distinguish between our three hypotheses. Concerning hypothesis 1 , BIOAc $(\mathbf{5 a})$ and $\mathrm{BIOH}(\mathbf{5 b})$ have been shown as competent reagents to generate such covalent intermediates. ${ }^{[12,}$ ${ }^{24]}$ Especially $\mathbf{5 b}$ could be present in small amounts as impurity in PhEBX (2a), as it is used for its synthesis. However, when the reaction was performed with 0.2 equivalents of either additive, nearly no product formation was obtained (entries 3 and 4). Even with 1.5 equivalents of additive in absence of TIPS-EBX $(\mathbf{2 g})$, very little degradation of the starting material was observed upon irradiation (entries 5 and 6 ). This indicated that, under our reaction conditions, this pathway is not operative for generating the desired tertiary radical. We then turned to hypothesis 2 : the side product ketones $\mathbf{6 a}$ and $\mathbf{6} \mathbf{b}$ as photooxidants. We observed the formation of $\mathbf{6 a}$ and $\mathbf{6} \mathbf{b}$ without the photocatalyst under light irradiation. In addition, when the reaction was performed in the dark at $50{ }^{\circ} \mathrm{C}$, the conversion of $2 \mathbf{a}$ to $6 \mathbf{a}(0.45$ equiv) and $6 \mathbf{b}(0.2$ equiv) could be observed by ${ }^{1} \mathrm{H}$ NMR. This suggests a thermal pathway for the formation of $\mathbf{6 a}$ and $\mathbf{6 b}$. These ketones could result from the formal hydration of $2 \mathbf{a}$ and the incorporation of a nucleophile (iodobenzoate or oxalate). We first explored the possibility of $6 \mathbf{a}$ as a competent photocatalyst and performed the alkynylation with 0.2 equivalents of $6 \mathbf{a}$ and TIPS-EBX $\mathbf{2 g}$ as reagent (entry 7). Only traces of alkynylation product were observed. In presence of one equivalent of $6 \mathbf{a}$, no degradation of the cesium oxalate or 6a was observed under irradiation (entry 8). These results showed that $6 a$ was not able to catalyze the alkynylation process. $\mathbf{6 b}$ was subjected to the same control experiments with no notable alkynylation products detected (See Supporting Information). ${ }^{[28]}$ Finally, we turned to hypothesis 3 and performed the alkynylation with 0.2 equivalents of PhEBX (2a) and 1.5 equivalents of TIPS-EBX (2g). In this case, $20 \%$ conversion of the cesium salt was observed with $8-13 \%$ deoxyalkynylation with phenyl and TIPS alkyne products $\mathbf{4 a}$ and $\mathbf{4 v}$ formed in a $1: 1$ ratio based on ${ }^{1} \mathrm{H}$ NMR analysis (entry 9), giving strong support for $\mathbf{2 a}$ acting as photooxidant.
Table 2. Optimization of the photocatalyzed method (method A).

(2)

[a] 3a $(0.1 \mathrm{mmol}), \mathbf{2 g}$ (1.5 equiv) and the additive were dissolved in DCM [3a] = $0.1 \mathrm{M}$ and irradiated with two lamps $(40 \mathrm{~W}, 440 \mathrm{~nm})$ for $18 \mathrm{~h}$. [b] ${ }^{1} \mathrm{H}$ NMR yield was determined using 1 equiv of $\mathrm{CH}_{2} \mathrm{Br}_{2}$ as internal standard. [c] Overall yield of deoxy-alkynylation, $\mathbf{4 a : 4 v}=1: 1$.

In addition to these results, the irradiation of PhEBX (2a) in the absence of cesium salt led also to non-negligible degradation of 2a with significant formation of diyne 7 (Scheme $5 \mathrm{~A}$ ), ${ }^{[29]}$ whereas the cesium salt $3 a$ did not show any degradation when irradiated separately (Scheme 5B). Interestingly, when we subjected 2 a to irradiation in THF (8) instead of DCM, we obtained $80 \%$ of $\mathrm{C}-\mathrm{H}$ alkynylation product 9 demonstrating that the direct excitation of $\mathbf{2 a}$ can lead to the well-established radical $\mathrm{C}-\mathrm{H}$ functionalization of ethers (Scheme $5 \mathrm{C}$ ). ${ }^{[27,30]}$ We suspect that $2 \mathbf{a}^{*}$ or one of its degradation products can perform a HAT to initiate the a-oxo alkynylation of THF (8). ${ }^{[31]}$ In addition to this HAT transformation, we wondered if $2 \mathbf{a}^{*}$ could also act as a single electron oxidant in other types of EBX-mediated alkynylations beside deoxyalkynylation. We were pleased to see that the enamide difunctionalization previously developed in our group ${ }^{[21 d]}$ gave $35 \%$ yield of the oxy-alkynylated product 11 (Scheme 5D). Furthermore, the deboronative alkynylation reported by Chen and co-workers ${ }^{[14 a]}$ could be also performed using 2.5 equiv of $\mathrm{PhEBX}$ (2a) in absence of photocatalyst and the additive $\mathrm{BIOH}(\mathbf{5 b})$ (Scheme 5E). The alkyne 13 was obtained in 60\% NMR yield. Finally, the decarboxylative fragmentation of oximes previously developed in our group ${ }^{[21 \mathrm{~b}]}$ also worked well with $75 \%$ NMR yield of fragmentation product $\mathbf{1 5}$ (Scheme $5 \mathrm{~F}$ ). It is important to note that the control experiments performed in absence of photocatalyst in the original reports of the reactions presented in Scheme 5D-F showed no product formation. This further 
highlights that the light source used in our work (two $40 \mathrm{~W}$ Kessil lamps with irradiation centered around $440 \mathrm{~nm}$ ) is essential for the observed direct activation of $\operatorname{PhEBX}(\mathbf{2 a})$.

A.

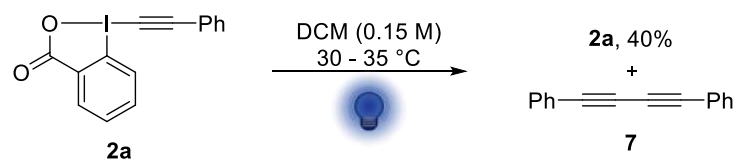<smiles>CC(C)(CCc1ccccc1)OC(=O)C(=O)OC(C)(C)CCc1ccccc1</smiles>

C.

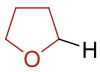

2a, $0.1 \mathrm{mmol}$ $(0.15 \mathrm{M}), 30-35^{\circ} \mathrm{C}$

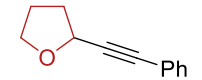

8, solvent

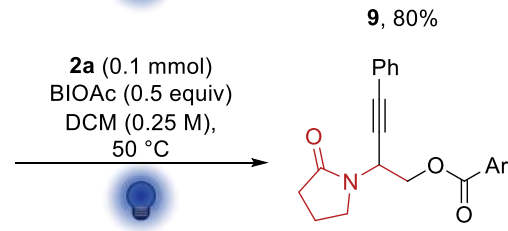

$10,1.5$ equiv

$11,35 \%$

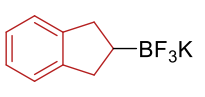

2a (2.5 equiv) DCM: $\mathrm{H}_{2} \mathrm{O}(0.1 \mathrm{M})$, $50^{\circ} \mathrm{C}$

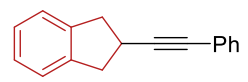

12, 1 equiv

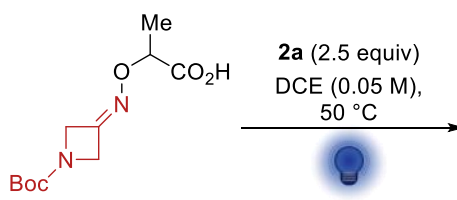

14, 1 equiv
$13,60 \%$

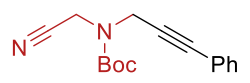

$15,75 \%$

Scheme 5. Control experiments corroborating the photoactivation of PhEBX (2a) and its potential as a photooxidant. The reactions were performed on 0.1 $\mathrm{mmol}$ or $0.2 \mathrm{mmol}$ scale and yields were determined by ${ }^{1} \mathrm{H}$ NMR by addition of 1 equiv of $\mathrm{CH}_{2} \mathrm{Br}_{2}$ as an internal standard. A. Photodegradation of 2a. B. Photostability of 3a. C. Photocatalyst-free THF (8) alkynylation. D. Photocatalyst-free oxyalkynylation of enamide 10 . E. Photocatalyst-free deboronative alkynylation of 12. F. Photocatalyst-free decarboxylative fragmentation ring-opening alkynylation of oxime 14.

With these results in hand, we turned to UV-Vis absorption and fluorescence spectroscopy to have further support for the photoactivity of PhEBX (2a) under our reaction conditions (Figure 1). We observed absorption until $460 \mathrm{~nm}$ (plain blue line) and fluorescence at $485 \mathrm{~nm}$ (dashed red line) (Figure 1A). Fluorescence excitation spectroscopy (dotted grey line) showed that irradiation of 2a from $300 \mathrm{~nm}$ to $440 \mathrm{~nm}$ was responsible for the emission at $485 \mathrm{~nm}$. Specifically, we observed two excitation bands $\left(\lambda_{\max , 1}=380 \mathrm{~nm}, \lambda_{\max , 2}=430 \mathrm{~nm}\right)$ confirming the possibility of the photoexcitation of $\mathbf{2 a}$ with a broad band light source with emission centered around $440 \mathrm{~nm}$. To identify the molar extinction coefficient $\varepsilon$ of $\mathbf{2 a}$, we performed a Beer-Lambert linear regression at $420 \mathrm{~nm}$ and $440 \mathrm{~nm}$ providing $\varepsilon_{420 \mathrm{~nm}}=0.54 \mathrm{~L} . \mathrm{mol}^{-1} . \mathrm{cm}^{-1}$ and $\varepsilon_{440 \mathrm{~nm}}=0.33 \mathrm{~L} \cdot \mathrm{mol}^{-1} . \mathrm{cm}^{-1}$ (Figure 1B). ${ }^{[32]}$ This is coherent with the weak absorption we observe in the $390 \mathrm{~nm}-460 \mathrm{~nm}$ range, even at high concentrations. These low molar extinction coefficients

suggest that the absorption at $420 \mathrm{~nm}$ and $440 \mathrm{~nm}$ could result from a spin forbidden electronic transition. ${ }^{[33]}$
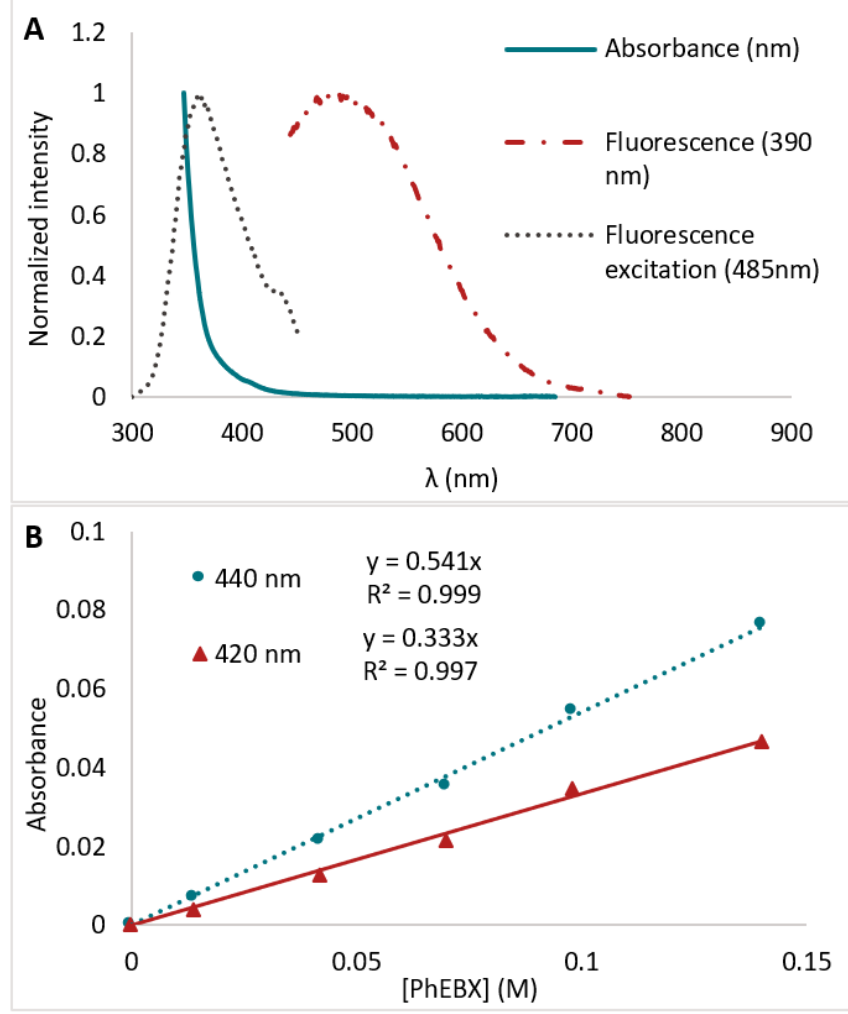

Figure 1. A. Normalized absorption of $2 a$ (blue plain line), emission (red dashed line, excitation at $\lambda=390 \mathrm{~nm}, \lambda_{\max }=485 \mathrm{~nm}$ ) and fluorescence excitation (grey dotted line, for emission at $485 \mathrm{~nm}, \lambda_{\max }=362 \mathrm{~nm}$ ). B. Beer Lambert linear regression for $420 \mathrm{~nm}$ (red plain line) and $440 \mathrm{~nm}$ (blue dotted line). $A=\varepsilon \mid[2 a]$, $\mathrm{I}=1 \mathrm{~cm}), \varepsilon_{420 \mathrm{~nm}}=0.54 \mathrm{~L} \cdot \mathrm{mol}^{-1} \cdot \mathrm{cm}^{-1}$ and $\varepsilon_{440 \mathrm{~nm}}=0.33 \mathrm{~L} \cdot \mathrm{mol}^{-1} \cdot \mathrm{cm}^{-1}$.

Having identified that PhEBX (2a) was photoactive and absorbing under our reaction conditions, it was important to estimate the strength of $\mathbf{2 a}$ as an oxidant in the excited state, in particular when considering the broad scope of substrates that could be oxidized with $2 \mathbf{a}^{\star}$. First, cyclic voltammetry allowed us to determine the redox potential of the ground state $\mathrm{E}_{1 / 2}\left(\mathbf{2} \mathbf{a} / \mathbf{2} \mathrm{a}^{*-}\right)=-0.87 \mathrm{~V}$ vs SCE (Figure 2). We could then calculate an estimate of the excited state $E_{1 / 2}\left(2 \mathbf{a}^{*} / 2 a^{*}\right)=+1.8 \mathrm{~V}$ vs $\operatorname{SCE}_{,}{ }^{[34]}$ thus confirming the thermodynamic feasibility of the SET oxidation of the substrate by the excited state $2 \mathrm{a}^{*}\left(\mathrm{E}_{1 / 2}\right.$ (oxalate $\%$ oxalate $)=1.3 \mathrm{~V}$ vs SCE). ${ }^{[17]} \mathrm{It}$ is also strong enough to oxidize enamide 10 , boronate 12 and oxime $14\left(\mathrm{E}_{1 / 2}\left(\mathbf{1 0}^{*} / \mathbf{1 0}\right) \approx+1.3 \mathrm{~V}\right.$ vs SCE, ${ }^{[21 \mathrm{~d}]} \mathrm{E}_{1 / 2}\left(\mathbf{1 2}^{*+} / \mathbf{1 2}\right) \approx+1.5 \mathrm{~V}$ vs SCE, ${ }^{[35]} \mathrm{E}_{1 / 2}\left(\mathbf{1 4}^{+*} / \mathbf{1 4}\right) \approx+1.5 \mathrm{~V}$ vs SCE for the corresponding potassium salt). ${ }^{[21 b]}$ In addition, the excited state $2 \mathbf{a}^{*}$ is a stronger oxidant than the excited state of $4 \mathrm{Cz} \operatorname{IPN}(\mathbf{1 a})\left(\mathrm{E}_{1 / 2}\left(\mathbf{1}^{*} / \mathbf{1} \mathbf{a}^{*}\right)=1.35\right.$ V vs SCE). 


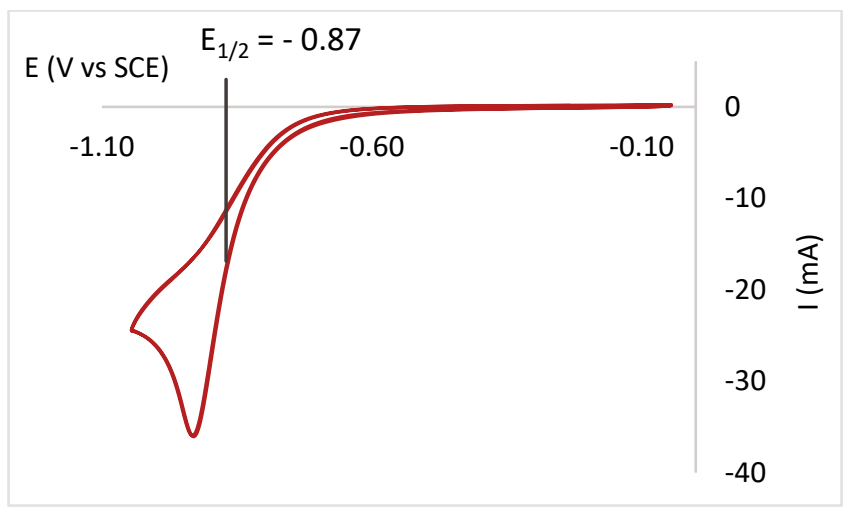

Figure 2. Cyclic voltammogram of $2 \mathbf{a}\left(50 \mathrm{mV} . \mathrm{s}^{-1}, 1.0 \mu \mathrm{M}\right.$ in $\left.\mathrm{MeCN}\right)$.

Excited-state EBX mediated deoxy-alkynylation. With PhEBX (2a) acting both as photooxidant and radical trap, a larger excess would be needed to promote the alkynylation. Furthermore, the formation of ketones $\mathbf{6 a}$ and $\mathbf{6 b}$ proceeds thermally suggesting that cooling the reaction media would be beneficial to avoid side reactions. Indeed, using 2.5 equivalents of PhEBX (2a), cooling the reaction media to $30-35^{\circ} \mathrm{C}$, and extending the reaction time to $24 \mathrm{~h}$ gave alkyne $4 \mathrm{a}$ in $67 \%$ NMR yield on $0.1 \mathrm{mmol}$ scale $(79 \%$ based on remaining starting material, Scheme 6$)$. In addition, the properties of the light source were important, as irradiation at lower wavelengths and intensities gave poorer results. Further fine-tuning of the reaction conditions (time, solvent, concentration, and additives) did not improve the yield (See Supporting Information).

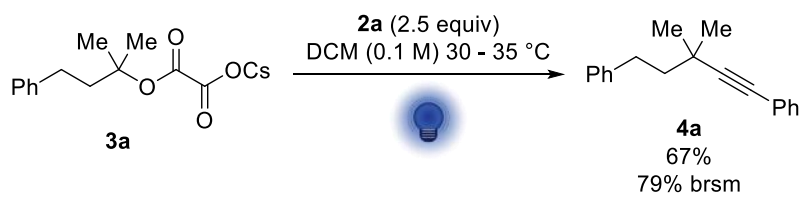

Scheme 6. Optimized reaction conditions for the direct excitation of PhEBX (2a) for deoxy-alkynylation of 3a (Method B).

With these conditions in hand, we briefly examined the generality of the direct photoactivation approach with a few selected substrates on $0.3 \mathrm{mmol}$ scale (Scheme 7). To our delight, we could obtain a variety of alkynylated products in only slightly lower yields when compared to the photocatalytic method A. Acyclic alkynes $4 a$ and $4 c$ could be obtained in 60 and $57 \%$ yield. Cyclic scaffolds $\mathbf{4 k}, \mathbf{4 I}$ and $\mathbf{4 n}$ were isolated in $37-61 \%$ yield. Finally, THP derivative 40 was obtained in $62 \%$ yield.

Alkynylation of Natural Products. Finally, we were delighted to see that both methods A and B could be applied for the diastereoselective deoxy-alkynylation of (-)-cedrol oxalate $3 w$ (Scheme $8 A$ ). Method A afforded alkyne $4 w$ in $58 \%$ yield and method $B$ in $50 \%$. Both methods provided products in over $20: 1$ diastereoselectivity based on NMR analysis. NOESY analysis supported that the isomer obtained is of $(S)$ configuration at $\mathrm{C}_{8}$. Interestingly, when (-)-terpinen-4-ol-derived oxalate $\mathbf{3 x}$ was used, a different outcome was observed: the 5-exo-trig cyclization of the intermediate acyl radical II onto the double bond was observed followed by alkynylation of the formed tertiary carbon radical (Scheme 8B). Both methods resulted in the formation of product $4 x$ in $53 \%$ and $47 \%$ yield, respectively. This indicates that the decarboxylations are stepwise and that the acyl radical II formed from the oxalate radical after the release of $\mathrm{CO}_{2}$ is long-lived enough to undergo cyclization before the second decarboxylation. The remotely alkynylated product $\mathbf{4 x}$ was also obtained in over 20:1 diastereoselectivity.

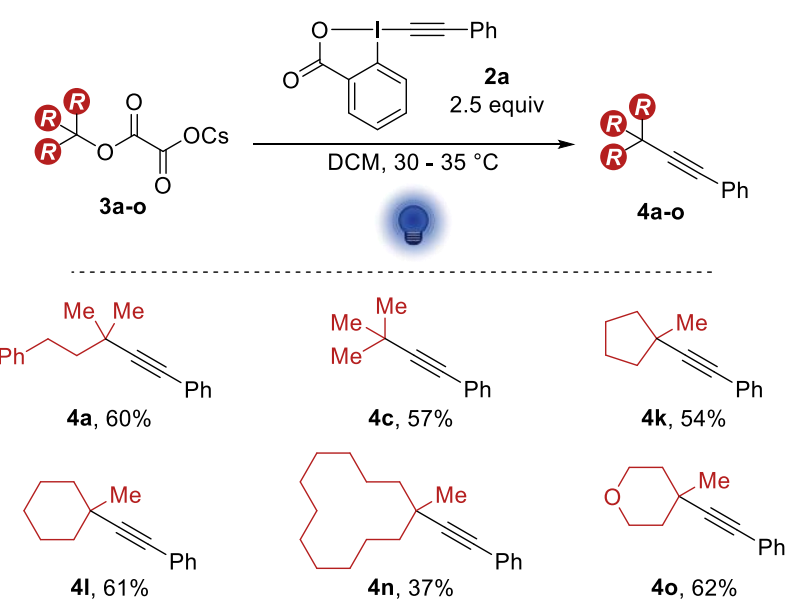

Scheme 7. Scope of Method B. Reactions were performed on $0.3 \mathrm{mmol}$ scale using the corresponding cesium oxalate 3a-o (1 equiv) and PhEBX 2a (2.5 equiv) in DCM (0.1 M).

A.

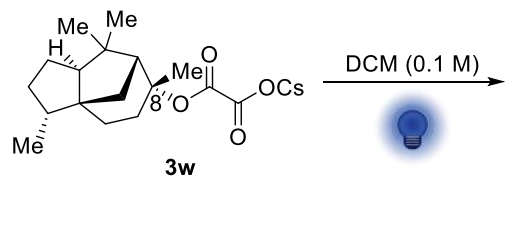<smiles>C[C@H]1CCC2C1(C)C[C@@H](C#Cc1ccccc1)C2(C)C</smiles>

B.<smiles>CC1=CC[C@@](F)(OC(=O)C(=O)OC(C)C)CC1</smiles><smiles>CC1=CC[C@](P)(OC(=O)C(=O)[O-])CC1</smiles>

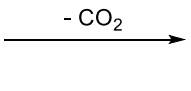<smiles>CC(C)C12CC[C@](C)(C#Cc3ccccc3)[C@H](C1)C(=O)O2</smiles>

4x, Method A $53 \%$ Method B 47\%<smiles>CC1=CC[C@](OC=O)(C(C)(F)I)CC1</smiles>

Scheme 8. Alkynylation of A. (-)-Cedrol oxalate $3 \mathbf{w}$ and B. (-)-Terpinen-4-o oxalate $3 \mathbf{x}$. Reactions were performed on $0.3 \mathrm{mmol}$ scale under blue LED irradiation. Method A: $\mathbf{3}$ w or $\mathbf{3 x}$ (1 equiv), $\mathbf{2 a}$ (1.5 equiv), $\mathbf{1 a}(5 \mathrm{~mol} \%)$ in DCM $(0.1 \mathrm{M}), 50^{\circ} \mathrm{C}$. Method B: $3 \mathbf{w}$ or $3 \mathbf{x}$ (1 equiv), $2 \mathrm{a}(2.5$ equiv) in $\operatorname{DCM}(0.1 \mathrm{M})$.

Overall Mechanistic Proposal. With the results obtained in our work together with literature precedence, ${ }^{[17-20]}$ we propose the following speculative mechanisms for our alkynylation methods (Scheme 9). First, in method A cesium oxalate 3 (for the tert-butyl oxalate $+1.3 \mathrm{~V}$ vs SCE) would undergo oxidation by the excited state 4 CzIPN, $1 \mathbf{a}^{*}(+1.35 \mathrm{~V}$ vs SCE). The resulting O-centered radical I fragments to the acyl radical II and finally the tertiary alkyl 
radical III releasing two molecules of carbon dioxide. The latter can then add to the EBX reagent 2 affording the final product 4 and the iodanyl radical IV. IV $(+0.25 \mathrm{~V} \text { vs SCE })^{[21 \mathrm{a}]}$ can then oxidize 1a*- (-1.2 V vs SCE) back down to the ground state ensuring photocatalytic turnover and affording iodobenzoate (16). ${ }^{[21 b]}$ Second, our experimental data associated to Method B (Figures 1 and 2) suggest that PhEBX (2a) undergoes direct photoexcitation to generate a highly oxidant excited state $2 \mathbf{a}^{*}$ $(+1.8 \mathrm{~V}$ vs SCE), the latter can then perform a SET oxidation of the cesium salt $3(+1.3 \mathrm{~V}$ vs SCE). In the same fashion as method $A$, the resulting O-centered radical I would then fragment twice to the desired radical III before being trapped by a second molecule of $\mathbf{2 a}$ to give the desired alkyne 4 . Following the oxidation of oxalate 3 , we suspect that the reduced $2 a^{*-}$ would be highly unstable and degrade resulting in side products such as the observed 1,4-diphenylbutadiyne (7). Additionally, we cannot exclude the possibility of diyne formation from the excited state $2 a^{*}$. The formation of ketones $\mathbf{6 a}$ and $\mathbf{6} \mathbf{b}$ seems to be $a$ background process occurring under thermal conditions. These ketones impact the yield slightly in the case of the slower direct photoexcitation, due to the consumption of the starting materials. However, they do not seem to play a role in the reaction mechanism. In presence of the photocatalyst, both mechanisms are probably occurring in parallel leading to higher efficiency overall.

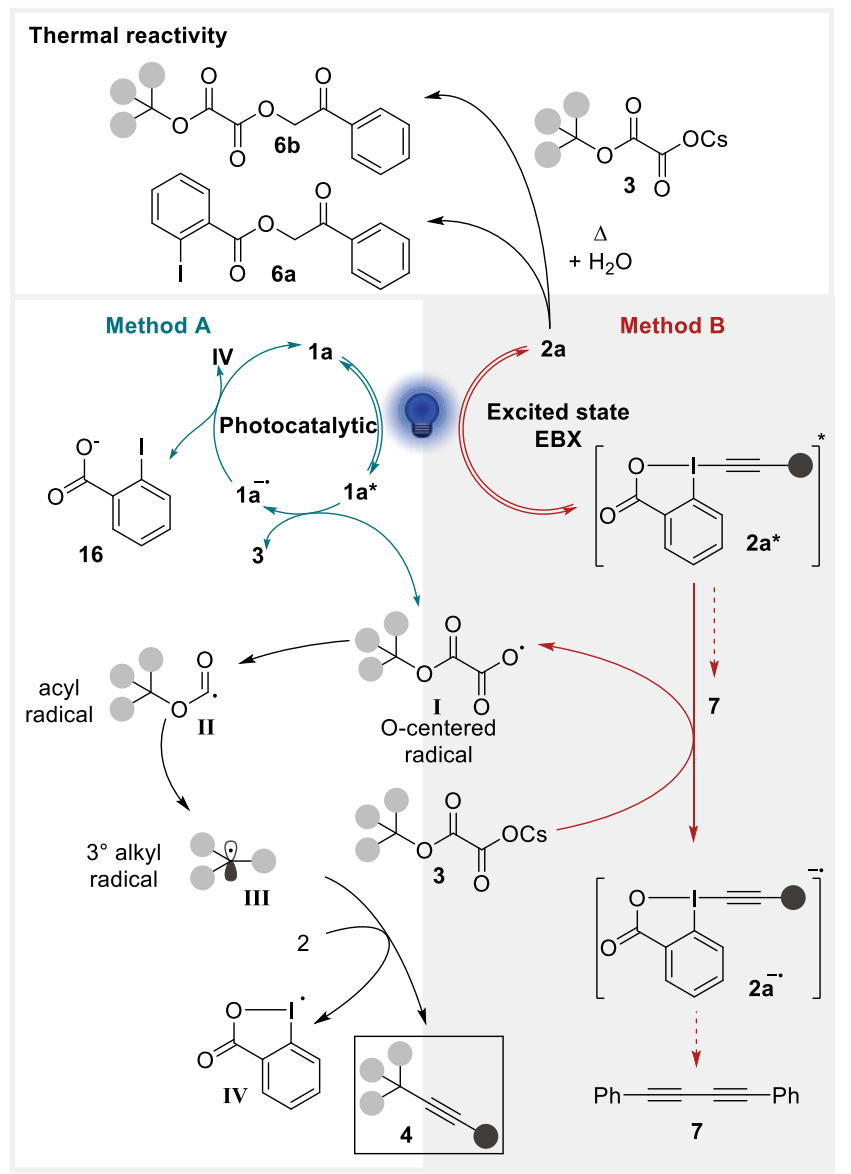

Scheme 9. Speculative mechanism.
In summary, we have developed two methods for the deoxyalkynylation of tertiary alcohols. Both methods use bench stable cesium oxalates easily accessed from the corresponding alcohols and aryl EBX reagents as a somophilic alkyne source. Our first strategy involves a metal-free process using 4CzIPN (1a) as photocatalyst, demonstrating yet again its versatility as an organic dye, and affording a valuable scope of alkynes connected to allcarbon quaternary centers. Our second approach is based on the direct photoexcitation of EBX reagents and their use as strong photooxidants. The relevance of the direct excitation of aryl EBX reagents has been exemplified beyond deoxy-alkynylation by the successful $\mathrm{C}-\mathrm{H}$ alkynylation of THF, the oxyalkynylation of enamides, a deboronative alkynylation and a cascade alkynylation from oximes. From the synthetic point of view, our methods constitute a practical access to alkynylated quaternarycenters and should contribute to further increase the use of these valuable building blocks. From the point of view of designing new photochemical processes, the direct excitation of aryl EBX reagents will open the door for new alkynylation processes without the need for sophisticated catalysts.

\section{Acknowledgements}

We thank the ERC (European Research Council, Starting Grant iTools4MC, number 334840) and EPFL for financial support. This publication was created as part of NCCR Catalysis, a National Centre of Competence in Research funded by the Swiss National Science Foundation.

In addition, we would like to thank members of the Laboratory of Supramolecular Chemistry-Ecole Polytechnique Fédérale de Lausanne LCS-EPFL: Cristian Pezzato and Cesare Berton for enabling us to run our absorption and fluorescence studies and Ophélie Planes who also helped greatly for the cyclic voltammetry studies.

Keywords: Alkynes; Hypervalent lodine; Photochemistry; Quaternary centers; Synthetic methods.

[1] a) Acetylene Chemistry: Chemistry, Biology, and Material Science; F. Diederich, P. J. Stang, R. R. Tykwinski, Eds.; Wiley-VCH: Weinheim 2005. b) I. A. Maretina, J. C. Tebby, Alkynes in Cycloadditions; Wiley: Chichester, West Sussex, 2014. c) Modern Alkyne Chemistry: Catalytic and Atom-Economic Transformations; B. M. Trost, C.-J. Li, Eds.; WileyVCH: Weinheim, 2015. d) Y. Jiang, R. E. McNamee, P. J. Smith, A Sozanschi, Z. Tong, E. A. Anderson, Chem. Soc. Rev. 2021, 50, 58-71.

[2] a) P. Thirumurugan, D. Matosiuk, K. Jozwiak, Chem. Rev. 2013, 113 4905-4979. b) T. T. Talele, J. Med. Chem. 2020, 63, 5625-5663.

[3] Selected examples: a) T. J. A. Graham, E. E. Gray, J. M. Burgess, B. C Goess, J. Org. Chem. 2010, 75, 226-228. b) K. Sugimoto, K. Toyoshima S. Nonaka, K. Kotaki, H. Ueda, H. Tokuyama, Angew. Chem. Int. Ed. 2013, 52, 7168-7171. Angew. Chem. 2013, 125, 7309-7312. c) S Yoshioka, M. Nagatomo, M. Inoue, Org. Lett. 2015, 17, 90-93.

[4] S. Zheng, Y. R. Santosh Laxmi, E. David, A. T. Dinkova-Kostova, K. H. Shiavoni, Y. Ren, Y. Zheng, I. Trevino, R. Bumeister, I. Ojima, W. C. Wigley, J. B. Bliska, D. F. Mierke, T. Honda, J. Med. Chem. 2012, 55, 4837-4846.

[5] Selected examples: a) Y.-S. Kwak, E. J. Corey, Org. Lett. 2004, 6, 33853388. b) J. A. Dabrowski, F. Gao, A. H. Hoveyda, J. Am. Chem. Soc 2011, 133, 4778-4781. c) M. Tissot, D. Poggiali, H. Hénon, D. Müller, L. Guénée, M. Mauduit, A. Alexakis, Chem. - Eur. J. 2012, 18, 8731-8747. d) S. Ahmar, E. Fillion, Org. Lett. 2014, 16, 5748-5751.

\section{Conclusion}


[6] Selected examples: a) Z.-S. Chen, X.-H. Duan, P.-X. Zhou, S. Ali, J.-Y. Luo, Y.-M. Liang, Angew. Chem. Int. Ed. 2012, 51, 1370-1374. Angew. Chem. 2012, 124, 1399-1403. b) C. Wang, F. Ye, C. Wu, Y. Zhang, J. Wang, J. Org. Chem. 2015, 80, 8748-8757. c) Y. Yamane, N. Miwa, T. Nishikata, ACS Catal. 2017, 7, 6872-6876. d) Z.-X. Wang, B.-J. Li, J. Am. Chem. Soc. 2019, 141, 9312-9320

[7] Selected examples: a) J. Sun, M. P. Conley, L. Zhang, S. A. Kozmin, J. Am. Chem. Soc. 2006, 128, 9705-9710. b) T. B. Poulsen, L. Bernardi, J. Alemán, J. Overgaard, K. A. Jørgensen, J. Am. Chem. Soc. 2007, 129 441-449.

[8] Selected examples: a) D. Fernández González, J. P. Brand, J. Waser, Chem. - Eur. J. 2010, 16, 9457-9461. b) H. Shi, L. Fang, C. Tan, L. Shi, W. Zhang, C. Li, T. Luo, Z. Yang, J. Am. Chem. Soc. 2011, 133, 1494414947. c) D. Fernández González, J. P. Brand, R. Mondière, J. Waser, Adv. Synth. Catal. 2013, 355, 1631-1639. d) X. Wu, S. Shirakawa, K. Maruoka, Org. Biomol. Chem. 2014, 12, 5388-5392. e) S. Companys, P. A. Peixoto, C. Bosset, S. Chassaing, K. Miqueu, J.-M. Sotiropoulos, L. Pouységu, S. Quideau, Chem. - Eur. J. 2017, 23, 13309-13313.

[9] F. Le Vaillant, J. Waser, Chem. Sci. 2019, 10, 8909-8923.

[10] Selected examples: a) Y. Shen, B. Huang, J. Zheng, C. Lin, Y. Liu, S. Cui, Org. Lett. 2017, 19, 1744-1747. b) S. Zhou, T. Song, H. Chen, Z. Liu, H. Shen, C. Li, Org. Lett. 2017, 19, 698-701. c) H. Jiang, Y. He, Y. Cheng, S. Yu, Org. Lett. 2017, 19, 1240-1243. d) Y. Li, R. Lu, S. Sun, L. Liu, Org. Lett. 2018, 20,6836-6839. e) H. Jiang, A. Studer, Chem. - Eur. J. 2019, 25, 516-520.

[11] Selected examples: a) T. Hoshikawa, S. Kamijo, M. Inoue, Org. Biomol. Chem. 2012, 11, 164-169. b) L. Wang, Y. Xia, K. Bergander, A. Studer Org. Lett. 2018, 20, 5817-5820. c) S. P. Morcillo, E. M. Dauncey, J. H. Kim, J. J. Douglas, N. S. Sheikh, D. Leonori, Angew. Chem. Int. Ed. 2018 57, 12945-12949. Angew. Chem. 2018, 130, 13127-13131.

[12] D. Wang, L. Zhang, S. Luo, Org. Lett. 2017, 19, 4924-4927.

[13] Selected examples: a) X. Liu, Z. Wang, X. Cheng, C. Li, J. Am. Chem. Soc. 2012, 134, 14330-14333. b) Y.-S. Feng, Z.-Q. Xu, L. Mao, F.-F. Zhang, H.-J. Xu, Org. Lett. 2013, 15, 1472-1475. c) Q. Zhou, W. Guo, W. Ding, X. Wu, X. Chen, L. Lu, W. Xiao, Angew. Chem. Int. Ed. 2015 54, 11196-11199. Angew. Chem. 2015, 127, 11348-11351. d) F. Le Vaillant, T. Courant, J. Waser, Angew. Chem. Int. Ed. 2015, 54, 1120011204. Angew. Chem. 2015, 127, 11352-11356.

[14] Selected examples: a) H. Huang, G. Zhang, L. Gong, S. Zhang, Y. Chen, J. Am. Chem. Soc. 2014, 136, 2280-2283. b) Y. Xia, A. Studer, Angew. Chem. Int. Ed. 2019, 58, 9836-9840. Angew. Chem. 2019, 131, 99419945.

[15] S. Tang, Y. Liu, X. Gao, P. Wang, P. Huang, A. Lei, J. Am. Chem. Soc. 2018, 140, 6006-6013.

[16] C. Gao, J. Li, J. Yu, H. Yang, H. Fu, Chem. Commun. 2016, 52, 7292 7294.

[17] C. C. Nawrat, C. R. Jamison, Y. Slutskyy, D. W. C. MacMillan, L. E. Overman, J. Am. Chem. Soc. 2015, 137, 11270-11273.

[18] X. Zhang, D. W. C. MacMillan, J. Am. Chem. Soc. 2016, 138, 13862 13865.

[19] S. Y. Abbas, P. Zhao, L. E. Overman, Org. Lett. 2018, 20, 868-871.
[20] J. Y. Su, D. C. Grünenfelder, K. Takeuchi, S. E. Reisman, Org. Lett. 2018 20, 4912-4916.

[21] Selected examples: a) F. Le Vaillant, M. D. Wodrich, J. Waser, Chem Sci. 2017, 8, 1790-1800. b) F. Le Vaillant, M. Garreau, S. Nicolai, G. Gryn'ova, C. Corminboeuf, J. Waser, Chem. Sci. 2018, 9, 5883-5889. c) M. Garreau, F. Le Vaillant, J. Waser, Angew. Chem. Int. Ed. 2019, 58 8182-8186. Angew. Chem. 2019, 131, 8266-8270. (d) S. G. E. Amos, S Nicolai, J. Waser, Chem. Sci. 2020, 11, 11274-11279.

[22] M. Nakajima, S. Nagasawa, K. Matsumoto, T. Kuribara, A. Muranaka, M. Uchiyama, T. Nemoto, Angew. Chem. Int. Ed. 2020, 59, 6847 -6852. Angew. Chem. 2020, 132, 6914-6919.

[23] G. L. Lackner, K. W. Quasdorf, L. E. Overman, J. Am. Chem. Soc. 2013, 135, 15342-15345.

[24] K. Jia, Y. Chen, Photochemistry of Hypervalent lodine Compounds. In PATAl'S Chemistry of Functional Groups; American Cancer Society, 2018; pp 1-42.

[25] In absence of the $3 \mathbf{a}$, we observed $60 \%$ degradation of $2 \mathbf{a}$ and $3 \%$ of $6 \mathbf{a}$ In presence of $\mathbf{3 a}$, we observe $90 \%$ degradation of $\mathbf{2 a}, 17 \%$ of $\mathbf{6 a}$ and $12 \% 6 \mathbf{b}$.

[26] J. Pérez-Prieto, R. E. Galian, M. A. Miranda, Diaryl Ketones as Photoactivators. In Mini-Reviews in Organic Chemistry; 2006; Vol. 3, pp 117-135.

[27] K. Matsumoto, M. Nakajima, T. Nemoto, J. Org. Chem. 2020, 85, 1180211811.

[28] Irradiation of $3 \mathbf{a}$ (1 equiv) and $\mathbf{6 b}$ (1 equiv) in DCM led to no observable degradation. Irradiation of $\mathbf{3 a}$ (1 equiv), $\mathbf{6} \mathbf{b}$ ( 0.7 equiv) with $\mathbf{2} \mathbf{g}$ (1.5 equiv) led to less than $5 \%$ product formation. Irradiation of $\mathbf{6 b}$ ( 1 equiv) and $\mathbf{2 a}$ (1.5 equiv) in DCM led to no product formation confirming that $\mathbf{6 b}$ is most likely not a reaction intermediate.

[29] Overlap of signals in the aromatic region of the NMR did not allow us to precisely determine the amount of 1,4-diphenylbutadiyne (7) formed.

[30] a) R.-Y. Zhang, L.-Y. Xi, L. Zhang, S. Liang, S.-Y. Chen, X.-Q. Yu, RSC Adv. 2014, 4, 54349-54353. b) E. Voutyritsa, M. Garreau, M. G. Kokotou I. Triandafillidi, J. Waser, C. G. Kokotos, Chem. - Eur. J. 2020, 26, 14453-14460.

[31] This type of HAT-alkynylation with EBX reagents has been reported to proceed with thermal radical initiators such as $\mathrm{TBHP}^{[30 \mathrm{a}]}$ and in presence of photocatalysts such as phenylglyoxilic acid ${ }^{[30 b]}$ or benzophenone, ${ }^{[27]}$ but its promotion by direct irradiation of the reagent is, to the best of our knowledge, unprecedented.

[32] The reproducibility of the measurement was verified by 3 repetitions of the sample.

[33] Photochemistry of Organic Compounds, 1st ed.; John Wiley \& Sons, Ltd, 2009.

[34] $E_{1 / 2}\left(\mathbf{2}^{\star} / \mathbf{2} \mathbf{a}^{*}\right)=E_{0-0}+E_{1 / 2}\left(\mathbf{2} \mathbf{a} / \mathbf{2} \mathbf{a}^{*}\right) . E_{0-0}$ was determined experimentally from the position of the long wavelength tail of the absorption spectrum at $460 \mathrm{~nm}$, as described in: L. Buzzetti, A. Prieto, S. R. Roy, P. Melchiorre, Angew. Chem. Int. Ed. 2017, 56, 15039-15043. Angew. Chem. 2017, 129, 15235-15239.

[35] Converted from V vs Fc to V vs SCE, taken from: Y. Yasu, T. Koike, M. Akita, Adv. Synth. Catal. 2012, 354, 3414-3420. 


\section{Contents}

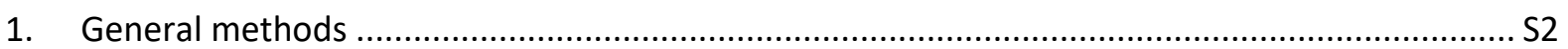

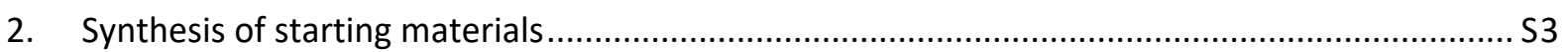

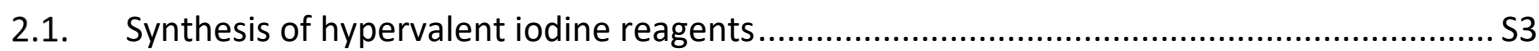

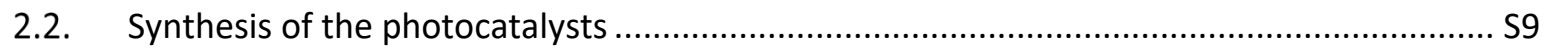

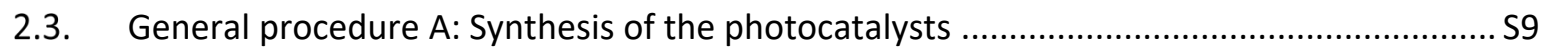

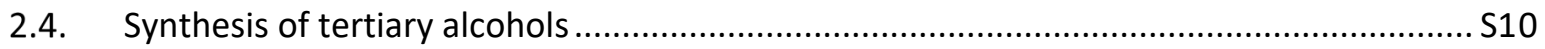

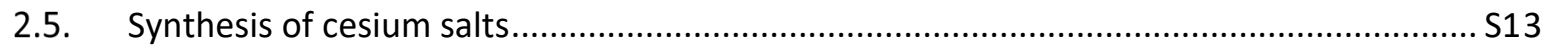

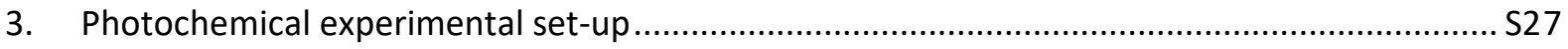

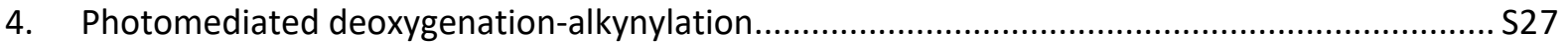

4.1. Optimization studies method A (4CzIPN catalyzed) ..................................................... S27

4.1. Optimization studies method B (Excited state PhEBX 2a) ............................................ S28

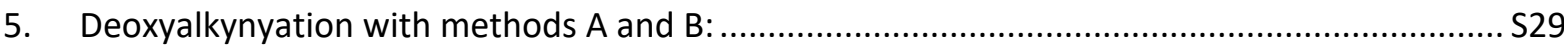

5.1. General procedure E: 4CZIPN catalyzed deoxy-alkynylation ("method A")....................... S29

5.2. General procedure F: Direct excitation of PhEBX for deoxy-alkynylation ("method B").... S29

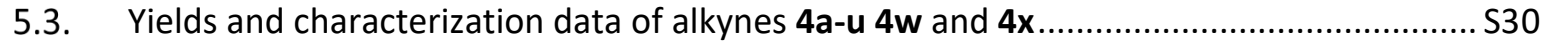

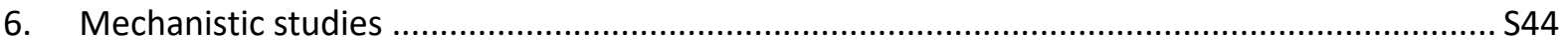

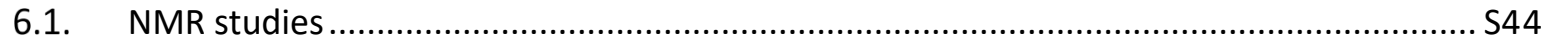

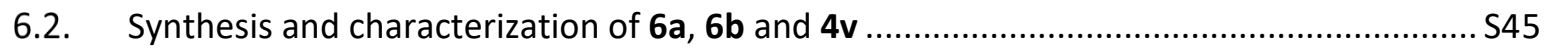

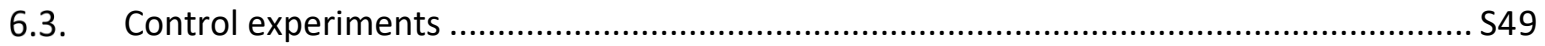

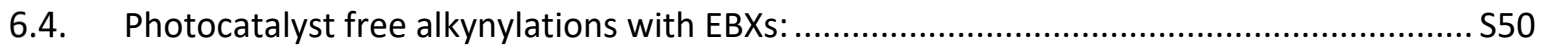

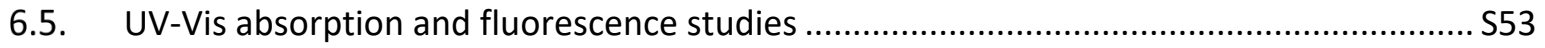

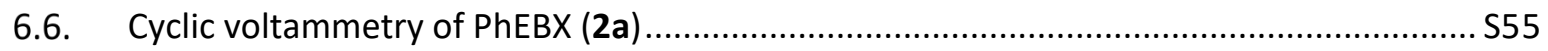

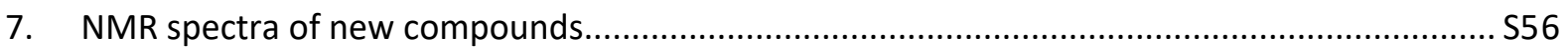




\section{General methods}

All reactions that were carried out in oven dried glassware and under an atmosphere of nitrogen is stated at the start of the reaction conditions. For flash chromatography, distilled technical grade solvents were used. THF, $\mathrm{CH}_{3} \mathrm{CN}$, toluene, $\mathrm{Et}_{2} \mathrm{O}$ and $\mathrm{CH}_{2} \mathrm{Cl}_{2}$ were dried by passage over activated alumina under nitrogen atmosphere $\left(\mathrm{H}_{2} \mathrm{O}\right.$ content $<10 \mathrm{ppm}$, Karl-Fischer titration). The solvents were degassed by Freeze-Pump-Thaw method when mentioned. All chemicals were purchased from Acros, Aldrich, Fluka, VWR, TCl, Merck and used as such unless stated otherwise. Chromatographic purification was performed as flash chromatography using Macherey-Nagel silica 40-63, $60 \AA$, using the solvents indicated as eluent with 0.1-0.5 bar pressure. TLC was performed on Merck silica gel 60 F254 TLC glass plates and visualized with UV light and $p$-anisaldehyde stain (EtOH: $\mathrm{H}_{2} \mathrm{SO}_{4}: \mathrm{AcOH}: p$ anisaldehyde 135:5:1.5:3.7 V:V:V:V).

${ }^{1} \mathrm{H}$-NMR spectra were recorded on a Brucker DPX-400 $400 \mathrm{MHz}$ spectrometer in CDCl3, acetonitrile$d_{3}$, DMSO- $d_{6}$ or acetone- $d_{6}$, all signals Are reported in ppm with the internal chloroform signal at 7.26 $\mathrm{ppm}$, the internal acetonitrile signal at $1.94 \mathrm{ppm}$, the internal methanol signal at $3.30 \mathrm{ppm}$, the internal DMSO signal at $2.50 \mathrm{ppm}$ or the internal acetone signal at $2.05 \mathrm{ppm}$ as standard. The data is reported as $(\mathrm{s}=$ singlet, $\mathrm{d}=$ doublet, $\mathrm{t}=$ triplet, $\mathrm{q}=$ quadruplet, $\mathrm{qi}=$ quintet, $\mathrm{m}=$ multiplet or unresolved, $\mathrm{br}=$ broad signal, app = apparent, coupling constant(s) in $\mathrm{Hz}$, integration, interpretation). ${ }^{13} \mathrm{C}-\mathrm{NMR}$ spectra were recorded with ${ }^{1} \mathrm{H}$-decoupling on a Brucker DPX-400 $100 \mathrm{MHz}$ spectrometer in $\mathrm{CDCl} 3$, acetonitrile$d_{3} \mathrm{CD}_{3} \mathrm{OD}$, DMSO- $d_{6}$ or acetone- $d_{6}$, all signals Are reported in ppm with the internal chloroform signal at $77.0 \mathrm{ppm}$, the internal acetonitrile signal at $1.3 \mathrm{ppm}$ the internal methanol signal at $49.0 \mathrm{ppm}$, the internal DMSO signal at $39.5 \mathrm{ppm}$ or the internal acetone signals at 29.84 and $206.26 \mathrm{ppm}$ as standard. Diastereoiomeric ratios has been determined after purification and stereochemistry has been assigned based on ${ }^{1} \mathrm{H}$ NMR analysis.

Infrared spectra were recorded on a JASCO FT-IR B4100 spectrophotometer with an ATR PRO410-S and $\mathrm{a} \mathrm{ZnSe}$ prisma and is reported in $\mathrm{cm}^{-1}(\mathrm{w}=$ weak, $\mathrm{m}=$ medium, $\mathrm{s}=$ strong).

High resolution mass spectrometric measurements were performed by the mass spectrometry service of ISIC at the EPFL on a MICROMASS (ESI) Q-TOF Ultima API.

All photocatalyzed reactions were carried out in oven dried glassware and under inert atmosphere (freeze pump thaw solvent stored on molecular sieves and under argon for maximum one week) unless specified otherwise. They were performed in screw cap dram vials $(0.5-7,5 \mathrm{~mL})$ which were stuck to a glass plate that was placed on a stirring plate with 2 Kessil lamps ( $440 \mathrm{~nm}, 40 \mathrm{~W}$ ) irradiating from both sides (the hood was free and coated with aluminum foil for personal protection). The distance between the Kessil lamps and the vials was approximatively $10 \mathrm{~cm}$. Long irradiation resulted in temperature increasing up to $50^{\circ} \mathrm{C}$ during overnight reactions unless a fan was used in which case the temperature raised to $30-35^{\circ} \mathrm{C}$. Photos have been provided.

UV/Vis spectroscopy was performed on an Agilent Cary 60 UV-Vis and steady-state luminescence spectroscopy was recorded on a Varian Cary Eclipse spectrophotometer. 


\section{Synthesis of starting materials}

\subsection{Synthesis of hypervalent iodine reagents}

The synthesis of reagents 6 and $\mathbf{2 a - g}$ had already been described before. ${ }^{1,2,3,4,5,6,7}$ Some of the procedures for accessing the ArEBX species have evolved slightly and have been updated with corresponding modifications, the modifications only apply to work-ups and purifications.

1-Hydroxy-1,2-benziodoxol-3-(1H)-one (5b)<smiles>O=C(O)c1ccccc1I</smiles>

16

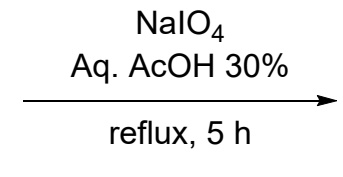<smiles>O=C1OI(O)c2ccccc21</smiles>

5b

Following a reported procedure, ${ }^{1} \mathrm{NalO}_{4}(40.5 \mathrm{~g}, 189 \mathrm{mmol}, 1.05$ equiv) and 2-iodobenzoic acid (16, $44.8 \mathrm{~g}, 180 \mathrm{mmol}, 1.0$ equiv) were suspended in $30 \%$ (v:v) aq. AcOH (350 mL). The mixture was vigorously stirred and refluxed for $5 \mathrm{~h}$. The reaction mixture was then diluted with cold water (250 $\mathrm{mL}$ ) and allowed to cool to rt, protecting it from light. After $1 \mathrm{~h}$, the crude product was collected by filtration, washed on the filter with ice water $(3 \times 150 \mathrm{~mL})$ and acetone $(3 \times 150 \mathrm{~mL})$, and air-dried in the dark overnight to afford 1-Hydroxy-1,2-benziodoxol-3-(1H)-one (5b, $44.3 \mathrm{~g}, 168 \mathrm{mmol}, 93 \%$ yield) as a white solid.

${ }^{1} \mathrm{H}$ NMR (400 MHz, DMSO- $\left.d_{6}\right) \delta 8.02(\mathrm{dd}, J=7.7,1.4 \mathrm{~Hz}, 1 \mathrm{H}, \mathrm{ArH}), 7.97(\mathrm{~m}, 1 \mathrm{H}, \mathrm{ArH}), 7.85$ (dd, $J=8.2$, $0.7 \mathrm{~Hz}, 1 \mathrm{H}, \mathrm{ArH}), 7.71(\mathrm{td}, J=7.6,1.2 \mathrm{~Hz}, 1 \mathrm{H}, \mathrm{ArH})$.

${ }^{13}$ C NMR (100 MHz, DMSO- $\left.d_{6}\right) \delta 167.7,134.5,131.5,131.1,130.4,126.3,120.4$.

Consistent with reported data. ${ }^{1}$

1-Acetoxy-1,2-benziodoxol-3-(1H)-one (5a)<smiles>O=C1OI(O)c2ccccc21</smiles>

$5 \mathbf{b}$

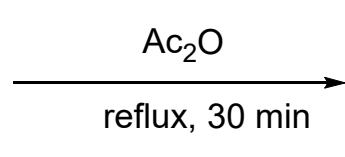

Following a reported procedure, ${ }^{8}$ compound $\mathbf{5 b}$ ( $3.00 \mathrm{~g}, 11.3 \mathrm{mmol}, 1.00$ equiv) was heated in $\mathrm{Ac}_{2} \mathrm{O}$ $(10 \mathrm{~mL})$ to reflux until the solution turned clear (without suspension, ca. $30 \mathrm{~min}$ ). The mixture was then left to cool down and white crystals started to form. The crystallization was continued at $-18{ }^{\circ} \mathrm{C}$.

\footnotetext{
${ }^{1}$ Brand, J. P.; Chevalley, C.; Scopelliti, R.; Waser, J. Chem. - Eur. J. 2012, 18, 5655-5666.

${ }^{2}$ Amos, S. G. E.; Nicolai, S.; Waser, J. Chem. Sci. 2020, 11, 11274-11279.

${ }^{3}$ Lu, B.; Wu, J.; Yoshikai, N. J. Am. Chem. Soc. 2014, 136, 11598.

${ }^{4}$ Jia, K.; Zhang, F.; Huang, H.; Chen, Y. J. Am. Chem. Soc 2016, 138, 1514.

${ }^{5}$ Le Vaillant, F. ; Courant, T. ; Waser, J. Angew. Chem. Int. Ed. 2015, 54, 11200.

${ }^{6}$ Le Vaillant, F. ; Garreau, M. ; Nicolai, S. ; Gryn'Ova, G. ; Corminboeuf, C. ; Waser, J. Chem. Sci. 2018, 9, 5883.

${ }^{7}$ Brand, J. P.; Waser, J. Angew. Chem. Int. Ed. 2010, 49, 7304.

${ }^{8}$ Eisenberger, P.; Gischig, S.; Togni, A. Chem. Eur. J. 2006, 12, 2579
} 
The crystals were then collected and dried overnight under high vacuum to give compound 5a (3.06 g, $10.0 \mathrm{mmol}, 86 \%)$.

${ }^{1}$ H NMR (400 MHz, Chloroform- $d_{3}$ ) $\delta 8.25$ (dd, $\left.1 \mathrm{H}, \mathrm{J}=7.6,1.4 \mathrm{~Hz}, \operatorname{ArH}\right), 8.00$ (dd, $1 \mathrm{H}, \mathrm{J}=8.3,0.5 \mathrm{~Hz}$, $\operatorname{ArH}), 7.92(\mathrm{dt}, 1 \mathrm{H}, \mathrm{J}=7.0,1.7 \mathrm{~Hz}, \mathrm{ArH}), 7.71(\mathrm{td}, 1 \mathrm{H}, \mathrm{J}=7.6,0.9 \mathrm{~Hz}, \mathrm{ArH}), 2.25\left(\mathrm{~s}, 3 \mathrm{H}, \mathrm{COCH}_{3}\right) . \mathrm{NMR}$ data correspond to the reported values. ${ }^{8}$

\section{1-[Phenylethynyl]-1,2-benziodoxol-3(1H)-one (PhEBX, 2a)}<smiles>O=C1OI(O)c2ccccc21</smiles>

$5 b$
TMSOTf

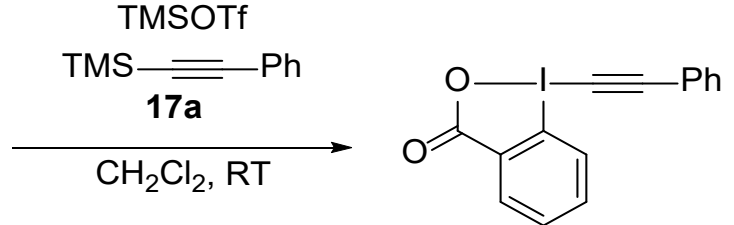

$2 a$

Following a reported procedure, ${ }^{2}$ trimethylsilyltriflate $(9.1 \mathrm{~mL}, 50 \mathrm{mmol}, 1.1$ equiv) was added dropwise to a suspension of 2-iodosylbenzoic acid (5b, $12.1 \mathrm{~g}, 45.8 \mathrm{mmol}, 1.0$ equiv) in $\mathrm{CH}_{2} \mathrm{Cl}_{2}$ (120 $\mathrm{mL}$ ) at $0{ }^{\circ} \mathrm{C}$. The mixture was stirred for $1 \mathrm{~h}$, followed by the dropwise addition of trimethyl(phenylethynyl)silane (17a, $8.8 \mathrm{~mL}, 50 \mathrm{mmol}, 1.1$ equiv) (slightly exothermic). The resulting suspension was stirred for $6 \mathrm{~h}$ at RT, during this time a white solid was formed. A saturated solution of $\mathrm{NaHCO}_{3}(120 \mathrm{~mL})$ was added and the mixture was stirred vigorously for $30 \mathrm{~min}$. The two layers of the mother liquors were separated and the organic layer was washed with sat. $\mathrm{NaHCO}_{3}(2 \times 50 \mathrm{~mL})$, dried over $\mathrm{MgSO}_{4}$, filtered and evaporated under reduced pressure. The resulting solid was recrystalized in EtOAc:MeOH (7:3 v:v) (ca. $20 \mathrm{~mL}$ ). The solution was left to cool to RT then in the freezer ovenight, filtered and dried under high vacuum to afford PhEBX (2a, $6.8 \mathrm{~g}, 25 \mathrm{mmol}, 43 \%$ yield) as colorless crystals.

$\operatorname{Mp}\left(\right.$ Dec.) $155-160^{\circ} \mathrm{C}$.

${ }^{1} \mathrm{H}$ NMR $\left(400 \mathrm{MHz}, \mathrm{CDCl}_{3}\right) \delta 8.46(\mathrm{~m}, 1 \mathrm{H}, \operatorname{ArH}), 8.28(\mathrm{~m}, 1 \mathrm{H}, \operatorname{ArH}), 7.80(\mathrm{~m}, 2 \mathrm{H}, \operatorname{ArH}), 7.63(\mathrm{~m}, 2 \mathrm{H}, \operatorname{ArH})$, $7.48(\mathrm{~m}, 3 \mathrm{H}, \mathrm{ArH})$.

${ }^{13} \mathrm{C} \mathrm{NMR}\left(101 \mathrm{MHz}, \mathrm{CDCl}_{3}\right) \delta 163.9,134.9,132.9,132.5,131.6,131.3 .130 .8,128.8,126.2,120.5,116.2$, 106.6, 50.2.

Consistent with reported data. ${ }^{2}$

1-[3-Fluorophenylethynyl]-1,2-benziodoxol-3(1H)-one (2b)<smiles>CC(C)(C)C#Cc1cccc(F)c1</smiles>

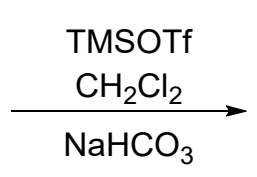

$17 \mathrm{~b}$<smiles>O=C1OI(C#Cc2cccc(F)c2)c2ccccc21</smiles>

2b

Following a slightly modified reported procedure, ${ }^{6}$ trimethylsilyl triflate $(0.44 \mathrm{~mL}, 2.5 \mathrm{mmol}, 1.1$ equiv) was added to a suspension of 2-iodosylbenzoic acid ( $5 \mathbf{b}, 0.589 \mathrm{~g}, 2.23 \mathrm{mmol}, 1.00$ equiv) in $\mathrm{CH}_{2} \mathrm{Cl}_{2}$ (6.8 $\mathrm{mL}$ ) at RT The resulting suspension was stirred for $1 \mathrm{~h}$, followed by the dropwise addition of ((3fluorophenyl)ethynyl)trimethylsilane (17b, $0.50 \mathrm{~mL}, 2.5 \mathrm{mmol}, 1.1$ equiv). The resulting suspension 
was stirred for $6 \mathrm{~h}$ at RT. A saturated solution of $\mathrm{NaHCO}_{3}(10 \mathrm{~mL})$ was then added and the mixture was stirred vigorously for 30 minutes, resulting in a suspension. The mixture was diluted with chloroform $(10 \mathrm{~mL})$, water $(5 \mathrm{~mL})$ and $\mathrm{MeOH}$ (ca. $0.5 \mathrm{~mL}$ ) resulting in two clear layers. The two layers were separated, and the organic layer was washed with sat. $\mathrm{NaHCO}_{3}(7 \mathrm{~mL})$, dried over $\mathrm{Na}_{2} \mathrm{SO}_{4}$, filtered, and evaporated under reduced pressure. The resulting solid was recrystallized in EtOAc:MeOH (7:3 v:v) (ca. $20 \mathrm{~mL}$ ). The solution was left to cool to RT then was placed in the freezer $\left(-20^{\circ} \mathrm{C}\right)$ overnight. The crystals were filtered and washed with $\mathrm{Et}_{2} \mathrm{O}$ to afford $\mathbf{2 b}(787 \mathrm{mg}, 2.15 \mathrm{mmol}, 43 \%$ yield) as colorless crystals.

${ }^{1}{ }_{\mathrm{H}}$ NMR $\left(400 \mathrm{MHz}, \mathrm{DMSO}-d_{6}\right) \delta 8.33(\mathrm{dd}, J=8.2,0.8 \mathrm{~Hz}, 1 \mathrm{H}, \mathrm{ArH}), 8.13(\mathrm{dd}, J=7.4,1.7 \mathrm{~Hz}, 1 \mathrm{H}, \operatorname{ArH})$, 7.91 (ddd, $J=8.2,7.2,1.7 \mathrm{~Hz}, 1 \mathrm{H}, \mathrm{ArH}), 7.81(\mathrm{td}, J=7.3,0.9 \mathrm{~Hz}, 1 \mathrm{H}, \mathrm{ArH}), 7.64-7.59(\mathrm{~m}, 1 \mathrm{H}, \mathrm{ArH}), 7.58$ $-7.53(\mathrm{~m}, 2 \mathrm{H}, \mathrm{ArH}), 7.47-7.37(\mathrm{~m}, 1 \mathrm{H}, \mathrm{ArH})$.

${ }^{13} \mathrm{C}$ NMR $\left(101 \mathrm{MHz}\right.$, DMSO- $\left.d_{6}\right){ }^{9} 166.3,161.8(\mathrm{~d}, J=245.6 \mathrm{~Hz}), 135.3,131.9,131.3,131.2(\mathrm{~d}, J=8.7 \mathrm{~Hz})$, $129.0(\mathrm{~d}, J=2.9 \mathrm{~Hz}), 127.7,122.4(\mathrm{~d}, J=9.6 \mathrm{~Hz}), 119.2(\mathrm{~d}, J=23.4 \mathrm{~Hz}), 118.1(\mathrm{~d}, J=21.1 \mathrm{~Hz}), 116.4$, $102.5(\mathrm{~d}, J=3.3 \mathrm{~Hz}), 53.8$.

${ }^{19}$ F NMR $\left(376 \mathrm{MHz}, \mathrm{DMSO}-d_{6}\right) \delta$-111.7.

Consistent with reported data. ${ }^{5}$

\section{1-[4-Trifluoromethylphenylethynyl]-1,2-benziodoxol-3(1H)-one (2c)}<smiles>O=C1OI(O)c2ccccc21</smiles>

Following a reported procedure, ${ }^{3}$ trimethylsilyl triflate $(1.0 \mathrm{~mL}, 5.5 \mathrm{mmol}, 1.1$ equiv) was added to a suspension of 2-iodosylbenzoic acid ( $5 \mathrm{~b}, 1.3 \mathrm{~g}, 5.0 \mathrm{mmol}, 1.0$ equiv) in $\mathrm{CH}_{2} \mathrm{Cl}_{2}(15 \mathrm{~mL}$ ) at RT The resulting suspension was stirred for $1 \mathrm{~h}$, followed by the dropwise addition of trimethyl((4(trifluoromethyl)phenyl)ethynyl)silane (17c, $1.3 \mathrm{~mL}, 5.5 \mathrm{mmol}, 1.1$ equiv), which was dissolved in $\mathrm{CH}_{2} \mathrm{Cl}_{2}(1 \mathrm{~mL})$. The resulting suspension was stirred for $6 \mathrm{~h}$ at RT A saturated solution of $\mathrm{NaHCO}_{3}(20$ $\mathrm{mL}$ ) was then added and the mixture was stirred vigorously for $30 \mathrm{~min}$, the two layers were separated and the organic layer was washed with sat. $\mathrm{NaHCO}_{3}(20 \mathrm{~mL})$, dried over $\mathrm{MgSO}_{4}$, filtered and evaporated under reduced pressure. The resulting solid was boiled in $\mathrm{CH}_{3} \mathrm{CN}(20 \mathrm{~mL})$. The mixture was cooled down, filtered and dried under high vacuum to afford $2 \mathrm{c}(1.3 \mathrm{~g}, 3.2 \mathrm{mmol}, 64 \%$ yield) as a pale yellow solid.

${ }^{1} \mathrm{H}$ NMR $\left(400 \mathrm{MHz}, \mathrm{CDCl}_{3}\right) \delta 8.46-8.38(\mathrm{~m}, 1 \mathrm{H}, \mathrm{ArH}), 8.28-8.19(\mathrm{~m}, 1 \mathrm{H}, \mathrm{ArH}), 7.84-7.74(\mathrm{~m}, 2 \mathrm{H}$, $\operatorname{ArH}), 7.74-7.65(\mathrm{~m}, 4 \mathrm{H}, \mathrm{ArH})$.

${ }^{13} \mathrm{C}$ NMR $\left(101 \mathrm{MHz}, \mathrm{CDCl}_{3}\right) \delta 166.6,135.0,133.0,132.6,132.2(\mathrm{q}, J=33.0 \mathrm{~Hz}), 131.7,131.2,126.3$, $125.7(q, J=3.6 \mathrm{~Hz}), 124.4,123.4(q, J=272.6 \mathrm{~Hz}), 116.1,104.2$, 53.7 .

Consistent with reported data. ${ }^{3}$

\footnotetext{
${ }^{9}$ One carbon is not resolved.
} 
<smiles>O=C1OI(O)c2ccccc21</smiles>

$5 b$<smiles>C#Cc1ccccc1Cl</smiles>

$17 d$

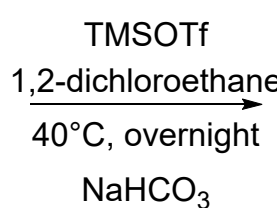

$\mathrm{NaHCO}_{3}$<smiles>O=C1OI(C#Cc2ccccc2Cl)c2ccccc21</smiles>

2d

Following a slightly modified reported procedure, ${ }^{6}$ trimethylsilyl triflate $(0.40 \mathrm{~mL}, 2.2 \mathrm{mmol}, 1.2$ equiv) was added to a suspension of 2-iodosylbenzoic acid ( $5 \mathbf{b}, 0.548 \mathrm{~g}, 2.08 \mathrm{mmol}, 1.1$ equiv) in DCE (5.8 $\mathrm{mL}$ ) at RT The resulting suspension was stirred for $1 \mathrm{~h}$, followed by the drop wise addition of (2chlorophenyl)acetylene (17d, $0.26 \mathrm{~mL}, 0.19 \mathrm{mmol}, 1.0$ equiv). The resulting suspension was stirred for $15 \mathrm{~h}$ at $40{ }^{\circ} \mathrm{C}$ A saturated solution of $\mathrm{NaHCO}_{3}(20 \mathrm{~mL})$ was then added and the mixture was stirred vigorously for 30 minutes resulting in a persistent emulsion/suspension. Water $(5 \mathrm{~mL})$ was added, followed by chloroform ( $15 \mathrm{~mL}$ ) and $\mathrm{MeOH}$ (ca. $0.5 \mathrm{~mL}$ ) resulting in 2 clear layers. The two layers were seperated and the organic layer was washed with sat. $\mathrm{NaHCO}_{3}(5 \mathrm{~mL})$, dried over $\mathrm{Na}_{2} \mathrm{SO}_{4}$, filtered and evaporated under reduced pressure. The resulting solid was recrystallized from EtOAc:MeOH (7:3 v:v, ca. $10 \mathrm{~mL})$. The mixture was cooled down overnight in the freezer $\left(-20^{\circ} \mathrm{C}\right)$, filtered and washed with $\mathrm{Et}_{2} \mathrm{O}$ to afford $2 \mathrm{~d}(0.217 \mathrm{~g}, 0.567 \mathrm{mmol}, 30 \%$ yield) as a white crystalline solid.

${ }^{1} \mathrm{H}$ NMR $\left(400 \mathrm{MHz}, \mathrm{CDCl}_{3}\right) \delta 8.46-8.38(\mathrm{~m}, 2 \mathrm{H}, \mathrm{ArH}), 7.84-7.73(\mathrm{~m}, 2 \mathrm{H}, \mathrm{ArH}), 7.62(\mathrm{dd}, J=7.6,1.7 \mathrm{~Hz}$, $1 \mathrm{H}, \operatorname{ArH}), 7.50(\mathrm{dt}, J=8.2,1.2 \mathrm{~Hz}, 1 \mathrm{H}, \operatorname{Ar} H), 7.46-7.37(\mathrm{~m}, 1 \mathrm{H}, \operatorname{ArH}), 7.33(\mathrm{td}, J=7.6,1.3 \mathrm{~Hz}, 1 \mathrm{H}, \operatorname{ArH})$.

${ }^{13} \mathrm{C} \mathrm{NMR}\left(101 \mathrm{MHz}, \mathrm{CDCl}_{3}\right) \delta 166.6,137.2,135.2,134.5,132.7,131.8,131.7,131.3,129.9,127.0,126.7$, $121.0,116.4,102.7,56.0$.

Consistent with reported data ${ }^{10}$

1-[4-Bromophenylethynyl]-1,2-benziodoxol-3(1H)-one (2e)<smiles>O=C1OI(O)c2ccccc21</smiles>

$5 b$<smiles>CSC#Cc1ccc(Br)cc1</smiles>

$17 e$

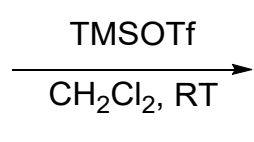<smiles>O=C1OI(C#Cc2ccc(Br)cc2)c2ccccc21</smiles>

$2 \mathrm{e}$

Following a reported procedure, ${ }^{4}$ trimethylsilyl triflate $(1.0 \mathrm{~mL}, 5.5 \mathrm{mmol}, 1.1$ equiv) was added to a suspension of 2-iodosylbenzoic acid (5b, $1.3 \mathrm{~g}, 5.0 \mathrm{mmol}, 1.0$ equiv) in $\mathrm{CH}_{2} \mathrm{Cl}_{2}(15 \mathrm{~mL}$ ) at RT The resulting suspension was stirred for $1 \mathrm{~h}$, followed by the dropwise addition of ((4bromophenyl)ethynyl)trimethylsilane (17e, $1.2 \mathrm{~g}, 5.5 \mathrm{mmol}, 1.1$ equiv), which was dissolved in $\mathrm{CH}_{2} \mathrm{Cl}_{2}$ $(1 \mathrm{~mL})$. The resulting suspension was stirred for $6 \mathrm{~h}$ at RT A saturated solution of $\mathrm{NaHCO}_{3}(20 \mathrm{~mL})$ was then added and the mixture was stirred vigorously for $30 \mathrm{~min}$, the two layers were separated and the organic layer was washed with sat. $\mathrm{NaHCO}_{3}(20 \mathrm{~mL})$, dried over $\mathrm{MgSO}_{4}$, filtered and evaporated under

${ }^{10}$ Li, M.; Li, W.; Lin, C.-D.; Wang, J.-H.; Wen, L.-R. J. Org. Chem. 2019, 84 (11), 6904-6915. 
reduced pressure. The resulting solid was boiled in $\mathrm{CH}_{3} \mathrm{CN}(20 \mathrm{~mL})$. The mixture was cooled down, filtered and dried under high vacuum to afford $2 \mathrm{e}(1.4 \mathrm{~g}, 3.3 \mathrm{mmol}, 66 \%$ yield $)$ as a pale yellow solid.

Mp $158-163{ }^{\circ} \mathrm{C}$ (decomposition).

${ }^{1} \mathbf{H}$ NMR $\left(400 \mathrm{MHz}, \mathrm{CDCl}_{3}\right) \delta 8.51-8.30(\mathrm{~m}, 1 \mathrm{H}, \mathrm{ArH}), 8.30-8.13(\mathrm{~m}, 1 \mathrm{H}, \mathrm{ArH}), 7.84-7.72(\mathrm{~m}, 2 \mathrm{H}$, $\operatorname{ArH}), 7.58(\mathrm{~d}, 2 \mathrm{H}, J=8.5 \mathrm{~Hz}, \operatorname{ArH}), 7.46(\mathrm{~d}, 2 \mathrm{H}, J=8.5 \mathrm{~Hz}, \operatorname{ArH})$.

${ }^{13} \mathrm{C}$ NMR $\left(101 \mathrm{MHz}, \mathrm{CDCl}_{3}\right) \delta$ 166.6, 135.1, 134.3, 132.7, 132.3, 131.9, 131.4, 126.3, 125.7, 119.6, 116.3, 105.4, 52.1.

Consistent with reported data. ${ }^{4}$

1-[2-Bromophenylethynyl]-1,2-benziodoxol-3(1H)-one (2f)<smiles>O=C1OI(O)c2ccccc21</smiles>

$5 b$

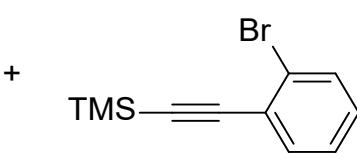

$17 f$

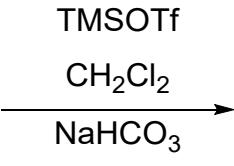<smiles>O=C1OC2(C#Cc3ccccc3)OC1c1ccccc12</smiles>

Following a slightly modified reported procedure, ${ }^{5}$ trimethylsilyl triflate $(0.42 \mathrm{~mL}, 2.4 \mathrm{mmol}, 1.1$ equiv) was added to a suspension of 2-iodosylbenzoic acid ( $5 \mathbf{b}, 0.562 \mathrm{~g}, 2.13 \mathrm{mmol}, 1.00$ equiv) in $\mathrm{CH}_{2} \mathrm{Cl}_{2}$ (6 $\mathrm{mL}$ ) at RT The resulting suspension was stirred for $1 \mathrm{~h}$, followed by the drop wise addition of ((2bromophenyl)ethynyl)trimethylsilane (17f, $0.50 \mathrm{~mL}, 2.4 \mathrm{mmol}, 1.1$ equiv). The resulting suspension was stirred for $6 \mathrm{~h}$ at RT. A saturated solution of $\mathrm{NaHCO}_{3}(10 \mathrm{~mL})$ was then added and the mixture was stirred vigorously for $1 \mathrm{~h}$ resulting in a persistent emulsion/suspension. The mixture was diluted with $\mathrm{CHCl}_{3}(10 \mathrm{~mL})$, water $(5 \mathrm{~mL})$ and $\mathrm{MeOH}$ (ca. $2 \mathrm{~mL}$ ) to afford 2 distinct layers. The two layers were separated, and the organic layer was washed with sat. $\mathrm{NaHCO}_{3}(5 \mathrm{~mL})$, dried over $\mathrm{Na}_{2} \mathrm{SO}_{4}$, filtered, and evaporated under reduced pressure. The resulting solid was recrystallized in EtOAc:MeOH (7:3 v:v) (ca. $20 \mathrm{~mL})$. The solution was left to cool to RT then was placed in the freezer $\left(-20^{\circ} \mathrm{C}\right)$ overnight. The crystals were filtered and washed with $\mathrm{Et}_{2} \mathrm{O}$ afford $2 \mathrm{f}(1.50 \mathrm{~g}, 3.51 \mathrm{mmol}, 70 \%$ yield) as colorless crystals.

${ }^{1} \mathrm{H}$ NMR $\left(400 \mathrm{MHz}, \mathrm{CDCl}_{3}\right) \delta 8.44(\mathrm{td}, J=7.3,2.1 \mathrm{~Hz}, 2 \mathrm{H}, \mathrm{ArH}), 7.84-7.74(\mathrm{~m}, 2 \mathrm{H}, \mathrm{ArH}), 7.68(\mathrm{~d}, J=1.1$ $\mathrm{Hz}, 1 \mathrm{H}, \mathrm{ArH}), 7.61(\mathrm{dd}, J=7.6,1.7 \mathrm{~Hz}, 1 \mathrm{H}, A r H), 7.36(\mathrm{~m}, 2 \mathrm{H}, \mathrm{ArH})$.

${ }^{13} \mathrm{C}$ NMR $\left(101 \mathrm{MHz}, \mathrm{CDCl}_{3}\right)^{7} \delta 166.6,135.2,134.7,133.0,132.7,131.8,131.3,127.6,126.8,126.4$, $123.2,116.5,104.3,55.4$.

Consistent with reported data. ${ }^{5}$

Triisopropylsilyl trimethylsilylacetylene (17g)

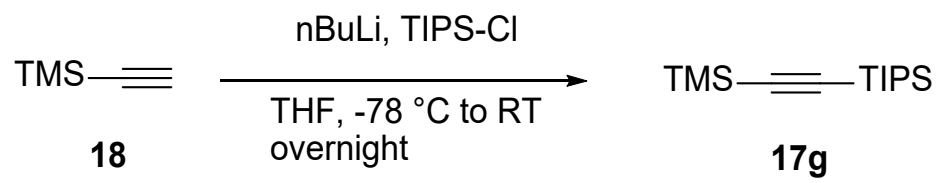


Following a reported procedure, ${ }^{11} \mathrm{n}$-butyllithium ( $2.5 \mathrm{M}$ in hexanes, $28 \mathrm{~mL}, 70 \mathrm{mmol}, 0.98$ equiv) was added dropwise to a stirred solution of ethynyltrimethylsilane $(18,7.0 \mathrm{~g}, 71 \mathrm{mmol}, 1.0$ equiv) in THF $(100 \mathrm{~mL})$ at $-78^{\circ} \mathrm{C}$. The mixture was warmed to $0{ }^{\circ} \mathrm{C}$ and stirred for $5 \mathrm{~min}$. The mixture was then cooled back to $-78{ }^{\circ} \mathrm{C}$ and chlorotriisopropylsilane $(15 \mathrm{~mL}, 71 \mathrm{mmol}, 1.0$ equiv) was added dropwise. The mixture was then allowed to warm to room temperature and stirred overnight. A saturated solution of ammonium chloride $(100 \mathrm{~mL}$ ) was added, and the reaction mixture was extracted with diethyl ether $(2 \times 100 \mathrm{~mL})$. The combined organic layers were washed with water and brine, then dried over MgSO4, filtered and concentrated under reduced pressure to obtain a colorless liquid which was further purified by filtration on silica eluting with pentane $(500 \mathrm{~mL})$ to yield $17 \mathrm{~g}(16 \mathrm{~g}, 64 \mathrm{mmol}, 90 \%$ yield $)$ as a colorless liquid.

${ }^{1} \mathrm{H}$ NMR (400 MHz, Chloroform-d) $\delta 1.08$ (m, 21H, TIPS), 0.18 (s, 9H, TMS).

Consistent with reported data. ${ }^{11}$

\section{1-[(Triiso-propylsilyl)ethynyl]-1,2-benziodoxol-3(1H)-one (TIPS-EBX, 2g)}

This compound can also be accessed in one pot from commercially available $o$-iodobenzoic acid and the free TIPS alkyne, however in the context of this study it was synthesized in the 2 step fashion. ${ }^{12}$<smiles>O=C1OI(O)c2ccccc21</smiles>

$5 b$

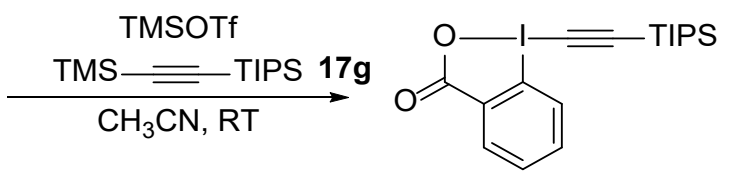

$2 g$

Following a reported procedure, ${ }^{7}$ 2-iodosylbenzoic acid ( $5 \mathrm{~b}, 8.0 \mathrm{~g}, 30 \mathrm{mmol}, 1.0$ equiv) was charged in an oven-dried round-bottomed $250 \mathrm{~mL}$ flask equipped with a magnetic stirrer. The solid was placed under a nitrogen atmosphere and anhydrous acetonitrile $(100 \mathrm{~mL})$ was added. The mixture was cooled to $0{ }^{\circ} \mathrm{C}$. Trimethylsilyltriflate $(6.0 \mathrm{~mL}, 33 \mathrm{mmol}, 1.1$ equiv) was added dropwise. After $15 \mathrm{~min}$, (trimethylsilyl)(triisopropylsilyl)acetylene $(\mathbf{1 7 g}, 8.5 \mathrm{~g}, 33 \mathrm{mmol}, 1.1$ equiv) was added dropwise. After $30 \mathrm{~min}$, the suspension became an orange solution. Pyridine $(2.7 \mathrm{~mL}, 33 \mathrm{mmol}, 1.1$ equiv) was added dropwise. After $15 \mathrm{~min}$, the reaction mixture was transferred in a one-neck $500 \mathrm{~mL}$ flask and concentrated under vacuum to afford a yellow solid. The solid was dissolved in $\mathrm{CH}_{2} \mathrm{Cl}_{2}(100 \mathrm{~mL})$ and transferred in a $500 \mathrm{~mL}$ separatory funnel. The organic layer was washed with a $1 \mathrm{M} \mathrm{HCl}$ solution (50 $\mathrm{mL}$ ) and the aqueous layer was extracted with $\mathrm{CH}_{2} \mathrm{Cl}_{2}(100 \mathrm{~mL})$. The organic layers were combined, washed with a saturated solution of $\mathrm{NaHCO} 3(2 \times 100 \mathrm{~mL})$, dried over MgSO4, filtered and the solvent was evaporated under reduced pressure. Recrystallization from acetonitrile $(40 \mathrm{~mL})$ afforded TIPS-EBX $(2 \mathrm{~g}, 9.2 \mathrm{~g}, 21.5 \mathrm{mmol}, 71 \%$ yield) as colorless crystals.

$\operatorname{Mp}\left(\right.$ Dec.) $170-176^{\circ} \mathrm{C}$.

${ }^{1}$ H NMR (400 MHz, Chloroform-d) $\delta 8.44(\mathrm{~m}, 1 \mathrm{H}, \operatorname{ArH}), 8.29(\mathrm{~m}, 1 \mathrm{H}, \operatorname{ArH}), 7.77(\mathrm{~m}, 2 \mathrm{H}, \operatorname{ArH}), 1.16(\mathrm{~m}$, $21 \mathrm{H}$, TIPS).

\footnotetext{
${ }^{11}$ Helal, C. J.; Magriotis, P. A.; Corey, E. J. J. Am. Chem. Soc. 1996, 118, 10938.

12 Hari, D. P.; Caramenti, P.; Schouwey, L.; Chang, M.; Nicolai, S.; Bachert, D.; Wright, T.; Orella, C.; Waser, J. Org. Process Res. Dev. 2020, 24, 106-110.
} 
${ }^{13}$ C NMR (100 MHz, Chloroform-d) $\delta$ 166.4, 134.6, 132.3, 131.4, 131.4, 126.1, 115.6, 114.1, 64.6, 18.4, 11.1.

IR $\vee 2943(\mathrm{~m}), 2865(\mathrm{~m}), 1716(\mathrm{~m}), 1618(\mathrm{~m}), 1604(\mathrm{~s}), 1584(\mathrm{~m}), 1557(\mathrm{~m}), 1465(\mathrm{~m}), 1439(\mathrm{w}), 1349$ $(\mathrm{m}), 1291(\mathrm{~m}), 1270(\mathrm{w}), 1244(\mathrm{~m}), 1140(\mathrm{~m}), 1016(\mathrm{~m}), 999(\mathrm{~m}), 883(\mathrm{~m}), 833(\mathrm{~m}), 742(\mathrm{~m}), 702(\mathrm{~s})$, $636(\mathrm{~m})$.

Consistent with reported data. ${ }^{7}$

\subsection{Synthesis of the photocatalysts}

\subsection{General procedure A: Synthesis of the photocatalysts}
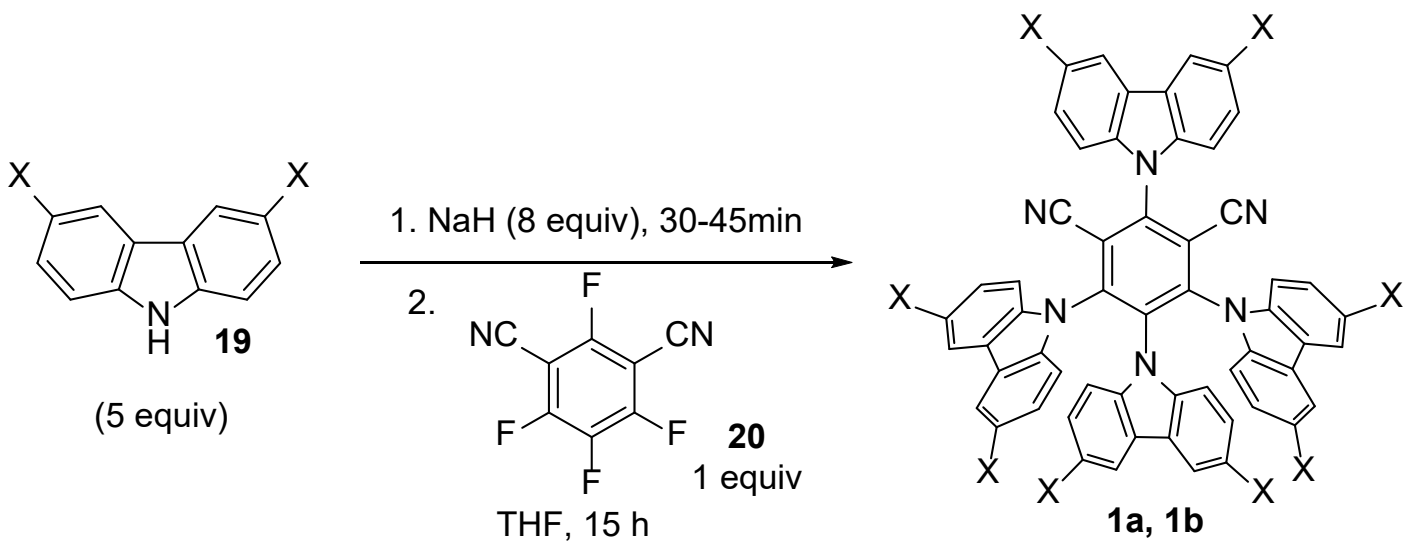

1a, $1 \mathrm{~b}$

Sodium hydride ( $60 \%$ suspension in mineral oil, 8.0 equiv) was added slowly to a stirred solution of substituted-carbazole 19 (5.0 equiv) in dry THF $(0.05 \mathrm{M})$ under a nitrogen atmosphere at RT After 30 min, 2,4,5,6-tetrafluoroisophthalonitrile 20 (1.0 mmol, 1.0 equiv) was added. After stirring at RT for $15 \mathrm{~h}, 2 \mathrm{~mL}$ water was added to the reaction mixture to quench the excess of $\mathrm{NaH}$. The resulting mixture was then concentrated under reduced pressure. The crude product was purified by recrystallization from hexane: $\mathrm{CH}_{2} \mathrm{Cl}_{2}$ then filtered. The brown liquid filtrate was concentrated and recrystallized as before. The combined solids were then purified by column chromatography on silica gel with $\mathrm{CH}_{2} \mathrm{Cl}_{2}$ :Hexane.

\section{2,4,5,6-Tetra(9H-carbazol-9-yl)isophthalonitrile (4CzIPN, 1a)}

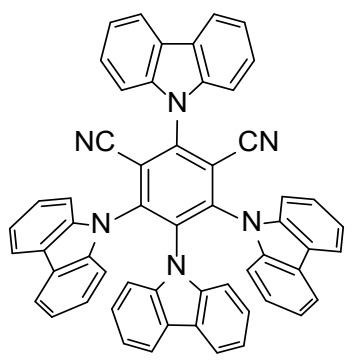

Rf (Hexane; $\left.\mathrm{CH}_{2} \mathrm{Cl}_{2} 1: 1\right)=0.29$. (yellow spot on TLC)

${ }^{1} \mathrm{H}$ NMR $\left(400 \mathrm{MHz}, \mathrm{CDCl}_{3}\right) \delta 8.2(\mathrm{~d}, J=7.7 \mathrm{~Hz}, 2 \mathrm{H}, \mathrm{ArH}), 7.8-7.6(\mathrm{~m}, 8 \mathrm{H}, \mathrm{ArH}), 7.5$ (ddd, $J=8.0,6.6$, $1.6 \mathrm{~Hz}, 2 \mathrm{H}, \operatorname{ArH}), 7.3(\mathrm{~d}, J=7.5 \mathrm{~Hz}, 2 \mathrm{H}, \operatorname{ArH}), 7.2(\mathrm{dd}, J=8.4,1.5 \mathrm{~Hz}, 4 \mathrm{H}, \operatorname{ArH}), 7.2-7.0(\mathrm{~m}, 8 \mathrm{H}, \operatorname{ArH})$, $6.8(\mathrm{t}, J=7.8 \mathrm{~Hz}, 4 \mathrm{H}, \operatorname{ArH}), 6.6(\mathrm{td}, J=7.6,1.2 \mathrm{~Hz}, 2 \mathrm{H}, \operatorname{ArH})$.

${ }^{13} \mathrm{C}$ NMR $\left(101 \mathrm{MHz}, \mathrm{CDCl}_{3}\right) \delta 145.2,144.6,140.0,138.2,136.9,134.7,127.0,125.8,124.9,124.7$, $124.5,123.8,122.4,121.9,121.4,121.0,120.4,119.6,116.3,111.6,109.9,109.5,109.4$. 
${ }^{1} \mathrm{H}$ NMR shift in $\mathrm{CDCl} 3$ are consistent with reported data. ${ }^{13}$

\section{(2r,4s,5r)-2,4,5,6-Tetrakis(3,6-dichloro-9H-carbazol-9-yl)isophthalonitrile (4CICZIPN, 1b)}

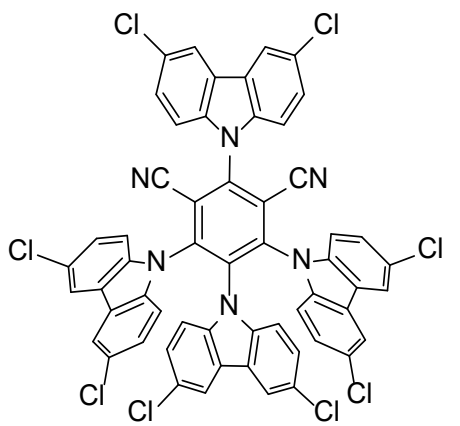

Following general procedure $A$ and starting from 3,6-dichloro-9Hcarbazole 19b (1.96 g, $6.00 \mathrm{mmol}, 6.0$ equiv), sodium hydride $(0.320 \mathrm{~g}, \quad 8.00 \mathrm{mmol}, 8.0$ equiv) and 2,4,5,6tetrafluoroisophthalonitrile 20 (200 mg, $1.00 \mathrm{mmol})$ in $20 \mathrm{~mL}$ of THF. Recrystallization (Hexanes: $\mathrm{CH}_{2} \mathrm{Cl}_{2}(1: 2,80 \mathrm{~mL}$ )) gave $900 \mathrm{mg}$ of yellow powder, then second recrystallization gave $325 \mathrm{mg}$ of brown powder. Column chromatography of the combined solid afforded (2r,4s,5r)-2,4,5,6-tetrakis(3,6-dichloro-9H-carbazol-9$\mathrm{yl}$ )isophthalonitrile (1b) as a bright yellow crystalline solid (830 $\mathrm{mg}, 0.780 \mathrm{mmol}, 87 \%$ yield).

Rf (Hexane: $\mathrm{CH}_{2} \mathrm{Cl}_{2}$ 1:1): 0.25 . (yellow spot on TLC).

${ }^{1} \mathrm{H}$ NMR (400 MHz, DMSO- $\left.d_{6}\right) \delta 8.60(\mathrm{~d}, J=2.1 \mathrm{~Hz}, 2 \mathrm{H}, \operatorname{ArH}), 8.15(\mathrm{~d}, J=2.1 \mathrm{~Hz}, 4 \mathrm{H}, \operatorname{ArH}), 8.08(\mathrm{~d}, J=$ $8.8 \mathrm{~Hz}, 2 \mathrm{H}, \mathrm{ArH}), 7.87(\mathrm{dd}, J=8.8,2.1 \mathrm{~Hz}, 2 \mathrm{H}, \mathrm{ArH}), 7.80(\mathrm{~d}, J=2.2 \mathrm{~Hz}, 2 \mathrm{H}, \operatorname{ArH}), 7.69(\mathrm{~d}, J=8.8 \mathrm{~Hz}$, $4 \mathrm{H}, \mathrm{ArH}), 7.46(\mathrm{~d}, J=8.8 \mathrm{~Hz}, 2 \mathrm{H}, \mathrm{ArH}), 7.32(\mathrm{dd}, J=8.8,2.2 \mathrm{~Hz}, 4 \mathrm{H}, \operatorname{ArH}), 6.93(\mathrm{dd}, J=8.8,2.2 \mathrm{~Hz}, 2 \mathrm{H}$, $\operatorname{ArH})$.

${ }^{13}$ C NMR $\left(101 \mathrm{MHz}\right.$, DMSO- $\left.d_{6}\right) \delta 145.0,144.5,138.5,137.4,136.5,135.8,134.5,127.8,127.0,126.4$, $125.7,125.3,124.2,123.8,123.3,121.6,120.9,120.3,116.8,112.6,112.5,112.3,111.7$.

${ }^{1} \mathrm{H}$ NMR shift in $\mathrm{CDCl} 3$ are consistent with reported data. ${ }^{6}$

\subsection{Synthesis of tertiary alcohols}

Alcohols for substrates $\mathbf{3 a}-\mathbf{d}, \mathbf{3 k}-\mathbf{m}, \mathbf{3 p}, \mathbf{3} \mathbf{w}$ and $\mathbf{3 x}$ were purchased from commercial sources (SigmaAldrich, Acros, $\mathrm{TCl}, \mathrm{abcr}$ ) and used directly without prior purification.

General procedure B: Synthesis of tertiary alcohols from esters

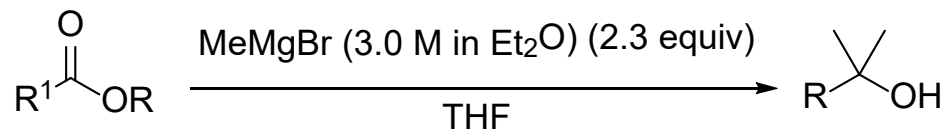

21e-g

22e-g

An oven dried two necked flask, equipped with a magnetic stirrer, was charged with the ester 21e-g (1.0 equiv) and dissolved in anhydrous THF $(1.0 \mathrm{M})$. The reaction was cooled to $0{ }^{\circ} \mathrm{C}$ with an ice bath. The methyl magnesium bromide solution was diluted to $1 \mathrm{M}$ with THF and added dropwise to the cooled solution via syringe. The reaction was left to stir at room temperature overnight (15 to $18 \mathrm{~h}$ ) at this time the reaction was quenched with sat. aq. $\mathrm{NH}_{4} \mathrm{Cl}$. The aqueous layer was extracted 3 times with EtOAc then the combined organic layers were washed with sat. aq. $\mathrm{NaCl}$. The organic layers were then dried on $\mathrm{MgSO}_{4}$, filtered and concentrated under reduced pressure. The compound was purified by column chromatography $\left(\mathrm{SiO}_{2}\right.$, pentane:EtOAc 9:1, 4:1, $p$-Anisaldehyde stain blue to purple and black spots) affording the desired alcohol.

${ }^{13}$ Uoyama, H.; Goushi, K.; Shizu, K.; Nomura, H.; Adachi, C. Nature 2012, 492, 234. 
- 1-(4-Methoxyphenyl)-2-methylpropan-2-ol (22e)<smiles>COc1ccc(CC(C)(C)O)cc1</smiles>

22e was synthesized following the general procedure $B$ : in THF $(60 \mathrm{~mL}, 0.1$ M) using methyl 2-(4-methoxyphenyl)acetate $(21 \mathrm{e}, 1.0 \mathrm{~mL}, 6.3 \mathrm{mmol}, 1.0$

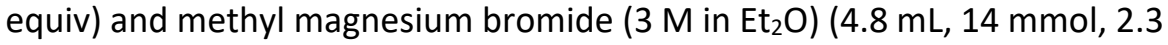
equiv) diluted with $10 \mathrm{~mL}$ of THF.

Column chromatography $\left(\mathrm{SiO}_{2} \mathrm{ca} .40 \mathrm{~g}\right.$, pentane:EtOAc 9:1 to 8:2) afforded 1-(4-methoxyphenyl)2-methylpropan-2-ol 22e (0.898 g, $4.98 \mathrm{mmol}, 79 \%)$.

Rf (pentane:EtOAc 9:1) $=0.3$

${ }^{1} \mathrm{H}$ NMR $\left(400 \mathrm{MHz}, \mathrm{CDCl}_{3}\right) \delta 7.16-7.11(\mathrm{~m}, 2 \mathrm{H}, \mathrm{ArH}), 6.88-6.83(\mathrm{~m}, 2 \mathrm{H}, \mathrm{ArH}), 3.80(\mathrm{~s}, 3 \mathrm{H}, \mathrm{OMe})$, $2.71\left(\mathrm{~s}, 2 \mathrm{H}, \mathrm{ArCH}_{2}\right), 1.21\left(\mathrm{~s}, 6 \mathrm{H}, \mathrm{C}\left(\mathrm{CH}_{3}\right)_{2}\right)$.

${ }^{13} \mathrm{C}$ NMR $\left(101 \mathrm{MHz}, \mathrm{CDCl}_{3}\right) \delta 158.5,131.5,129.9,113.8,70.9,55.4,48.9,29.2$.

The reported NMR data are consitant with the reported data. ${ }^{14}$

- 1-(2-Fluorophenyl)-2-methylpropan-2-ol (22f)<smiles>CC(C)(O)Cc1ccccc1F</smiles>

$22 \mathrm{f}$ was synthesized following the general procedure $B$ : in THF $(60 \mathrm{~mL}, 0.1$ M) using methyl 2-(2-fluorophenyl)acetate $(21 \mathrm{f}, 1.0 \mathrm{~mL}, 6.8 \mathrm{mmol}, 1.0$ equiv) and methyl magnesium bromide ( $3 \mathrm{M}$ in $\left.\mathrm{Et}_{2} \mathrm{O}\right)(5.2 \mathrm{~mL}, 16 \mathrm{mmol}, 2.3$ equiv) diluted with $10 \mathrm{~mL}$ of THF.

Column chromatography $\left(\mathrm{SiO}_{2}\right.$ ca. 40g, pentane:EtOAc 9:1 to 8:2) afforded 1-(2-fluorophenyl)-2methylpropan-2-ol $22 \mathrm{f}(0.723 \mathrm{~g}, 4.30 \mathrm{mmol}, 63 \%)$.

Rf (pentane:EtOAc 9:1) = 0.3.

${ }^{1} \mathbf{H}$ NMR $\left(400 \mathrm{MHz}, \mathrm{CDCl}_{3}\right) \delta 7.29-7.18(\mathrm{~m}, 2 \mathrm{H}, \mathrm{ArH}), 7.13-7.00(\mathrm{~m}, 2 \mathrm{H}, \operatorname{ArH}), 2.83(\mathrm{~d}, \mathrm{~J}=1.5 \mathrm{~Hz}$, $\left.2 \mathrm{H}, \mathrm{CH}_{2}\right), 1.48(\mathrm{~s}, 1 \mathrm{H}, \mathrm{OH}), 1.25\left(\mathrm{~d}, \mathrm{~J}=0.9 \mathrm{~Hz}, 6 \mathrm{H}, \mathrm{C}\left(\mathrm{CH}_{3}\right)_{2}\right)$.

${ }^{13} \mathrm{C}$ NMR $\left(101 \mathrm{MHz}, \mathrm{CDCl}_{3}\right) \delta 161.5(\mathrm{~d}, J=244.7 \mathrm{~Hz}), 132.8(\mathrm{~d}, J=4.7 \mathrm{~Hz}), 128.3(\mathrm{~d}, J=8.2 \mathrm{~Hz}), 124.9$ (d, $J=16.0 \mathrm{~Hz}$ ), $123.8(\mathrm{~d}, J=3.5 \mathrm{~Hz}), 115.4(\mathrm{~d}, J=23.0 \mathrm{~Hz}$ ), 71.3, 42.3, 29.1 .

${ }^{19} \mathrm{~F}$ NMR $\left(376 \mathrm{MHz}, \mathrm{CDCl}_{3}\right) \delta-116.1$.

IR $\left(v_{\max }, \mathrm{cm}^{-1}\right) 3420(\mathrm{~m}), 3061(\mathrm{~m}), 2975(\mathrm{~m}), 2963(\mathrm{~m}), 2936(\mathrm{~m}), 1583(\mathrm{~m}), 1493(\mathrm{~s}), 1455(\mathrm{~s}), 1228$ (s), $1184(\mathrm{~s}), 1134$ (s), 753 (s).

HRMS (APPI/LTQ-Orbitrap) m/z: [M] $]^{+}$Calcd for $\mathrm{C}_{10} \mathrm{H}_{12} \mathrm{~F}^{+}$151.0918; Found 151.0921 .

- 1-(4-Bromophenyl)-2-methylpropan-2-ol (22g)<smiles>CC(C)(O)Cc1ccc(Br)cc1</smiles>

$22 \mathrm{~g}$ was synthesized following the general procedure $B$ : in THF $(40 \mathrm{~mL}, 0.1$ M) using ethyl 2-(4-bromophenyl)acetate $(21 \mathrm{~g}, 1.00 \mathrm{~g}, 4.11 \mathrm{mmol}, 1.0$ equiv) and methyl magnesium bromide ( $\left.3 \mathrm{M}^{\text {in Et}}{ }_{2} \mathrm{O}\right)(3.2 \mathrm{~mL}, 9.5 \mathrm{mmol}, 2.3$ equiv) diluted with $6 \mathrm{~mL}$ of THF.

Column chromatography $\left(\mathrm{SiO}_{2}\right.$ ca. 40 g, pentane:EtOAc 9:1 to 8:2) afforded 1-(4-bromophenyl)-2methylpropan-2-ol 22g (0.781 g, $3.41 \mathrm{mmol}, 83 \%)$.

$\mathbf{R f}$ (pentane:EtOAc 9:1) $=0.3$.

${ }^{1} \mathrm{H}$ NMR $\left(400 \mathrm{MHz}, \mathrm{CDCl}_{3}\right) \delta 7.47-7.39(\mathrm{~m}, 2 \mathrm{H}, \mathrm{ArH}), 7.13-7.06(\mathrm{~m}, 2 \mathrm{H}, \mathrm{ArH}), 2.72\left(\mathrm{~s}, 2 \mathrm{H}, \mathrm{CH}_{2}\right), 1.38$ $(\mathrm{s}, 1 \mathrm{H}, \mathrm{OH}), 1.21\left(\mathrm{~s}, 6 \mathrm{H}, \mathrm{C}\left(\mathrm{CH}_{3}\right)_{2}\right)$.

${ }^{13} \mathrm{C}$ NMR $\left(101 \mathrm{MHz}, \mathrm{CDCl}_{3}\right) \delta 136.8,132.2,131.2,120.5,70.7,49.1,29.2$.

${ }^{14}$ Okamura, T.; Egoshi, S.; Dodo, K.; Sodeoka, M.; Iwabuchi, Y.; Kanoh, N. Chem. - Eur. J. 2019, 25, 16002-16006. 
IR $\left(v_{\max }, \mathrm{cm}^{-1}\right) 3668(\mathrm{~m}), 2972(\mathrm{~s}), 2901$ (s), $1488(\mathrm{~s}), 1406$ (s), 1377 (s), 1229 (s), 1075 (s), 1056 (s), 1012 (s).

HRMS (APPI/LTQ-Orbitrap) m/z: [M] ${ }^{+}$Calcd for $\mathrm{C}_{10} \mathrm{H}_{12}{ }^{79} \mathrm{Br}^{+}$211.0117; Found 211.0122.

General procedure C: Synthesis of tertiary alcohols from ketones

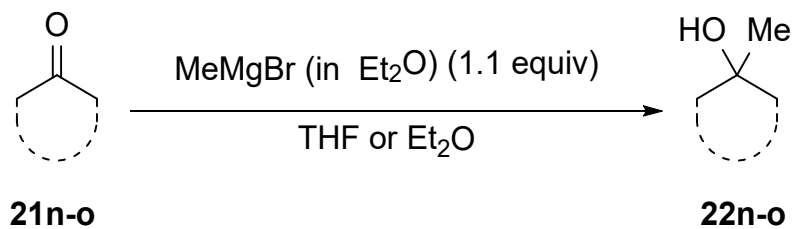

An oven dried two-necked flask, equipped with a magnetic stirrer, was charged with the ketone $\mathbf{2 1 n}$ 0 (1.0 equiv) and dissolved in anhydrous THF or $\mathrm{Et}_{2} \mathrm{O}(0.2 \mathrm{M})$. The reaction was cooled to $0{ }^{\circ} \mathrm{C}$ with an ice bath. The methylmagnesium bromide solution (3.0 M in $\mathrm{Et}_{2} \mathrm{O}$ ) was diluted to $1 \mathrm{M}$ and added dropwise to the cooled solution via a dropping funnel. The reaction was stirred at room temperature overnight (15 to $18 \mathrm{~h}$ ) at this time the reaction was quenched with sat. aq. $\mathrm{NH}_{4} \mathrm{Cl}$, followed by the addition of water and EtOAc. The layers were separated, the aqueous layer was extracted 3 times with EtOAc then the combined organic layers were washed with sat. aq. $\mathrm{NaCl}$. The organic layer was then dried on $\mathrm{MgSO}_{4}$, filtered and concentrated under reduced pressure. The compound was purified by column chromatography $\left(\mathrm{SiO}_{2}\right.$, pentane:EtOAc, $p$-Anisaldehyde stain) affording the desired alcohol.

- Methylcyclododecan-1-ol (22n)

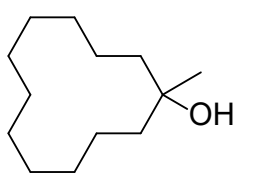

$22 \mathrm{n}$ was synthesized following the general procedure $C$ in $\mathrm{Et}_{2} \mathrm{O}(25 \mathrm{~mL}, 0.2$ M) from cyclododecanone (21n, $1.00 \mathrm{~g}, 5.49 \mathrm{mmol}, 1.0$ equiv) using methylmagnesium bromide ( $3.0 \mathrm{M}$ in $\mathrm{Et}_{2} \mathrm{O}, 2.0 \mathrm{~mL}, 6.00 \mathrm{mmol}, 1.1$ equiv) diluted with THF $(4.0 \mathrm{~mL})$.

Column chromatography $\left(\mathrm{SiO}_{2}, 10 \%\right.$ EtOAC in Pentane) afforded methylcyclododecan-1-ol 22n (609 $\mathrm{mg}, 3.07 \mathrm{mmol}, 56 \%$ ) as a white amorphous solid. The NMR data was collected and the compound was used in the next step without further analysis.

$\mathbf{R f}$ (pentane:EtOAc 9:1) $=0.4$.

${ }^{1} \mathrm{H}$ NMR $\left(400 \mathrm{MHz}, \mathrm{CDCl}_{3}\right) \delta: 1.59-1.52\left(\mathrm{~m}, 2 \mathrm{H}, \mathrm{CH}_{2}\right), 1.45-1.25\left(\mathrm{~m}, 20 \mathrm{H}, \mathrm{CH}_{2}\right), 1.17\left(\mathrm{~s}, 3 \mathrm{H}, \mathrm{CH}_{3}\right)$.

${ }^{13} \mathrm{C}$ NMR $\left(101 \mathrm{MHz}, \mathrm{CDCl}_{3}\right)$ 8: 73.8, 36.3, 29.2, 26.6, 26.2, 22.7, 22.2, 20.1.

- 4-Methyltetrahydro-2H-pyran-4-ol (220)

220 was synthesized following the general procedure $C$ in THF $(50 \mathrm{~mL}, 0.2$ M) from tetrahydro-4H-pyran-4-one $(\mathbf{2 1 0}, 0.94 \mathrm{~mL}, 10 \mathrm{mmol}, 1.0$ equiv)

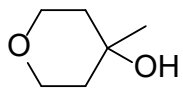
using methylmagnesium bromide $\left(3.0 \mathrm{M}\right.$ in $\mathrm{Et}_{2} \mathrm{O}, 3.7 \mathrm{~mL}, 11 \mathrm{mmol}, 1.1$ equiv) diluted with THF ( $7.3 \mathrm{~mL})$. Column chromatography $\left(\mathrm{SiO}_{2}, 25 \% \mathrm{EtOAC}\right.$ in Pentane) afforded 4-methyltetrahydro-2H-pyran-4-ol 220 (604 mg, 5.20 $\mathrm{mmol}, 52 \%)$ as a colourless oil.

$\mathbf{R f}$ (pentane:EtOAc 3:1) = 0.3.

${ }^{1} \mathrm{H}$ NMR $\left(400 \mathrm{MHz}, \mathrm{CDCl}_{3}\right) \delta: 3.81-3.75\left(\mathrm{~m}, 2 \mathrm{H}, \mathrm{OCH}_{2}\right), 3.72-3.76\left(\mathrm{~m}, 2 \mathrm{H}, \mathrm{OCH}_{2}\right), 1.77-1.62(\mathrm{~m}$, $\left.2 \mathrm{H}, \mathrm{CH}_{2}\right), 1.58-1.48\left(\mathrm{~m}, 2 \mathrm{H}, \mathrm{CH}_{2}\right), 1.28\left(\mathrm{~s}, 3 \mathrm{H}, \mathrm{CH}_{3}\right)$.

${ }^{13} \mathrm{C}$ NMR $\left(101 \mathrm{MHz}, \mathrm{CDCl}_{3}\right) \delta: 67.5,64.4,39.6,30.3$. 


\subsection{Synthesis of cesium salts}

\section{General procedure D: Synthesis of cesium salts from tertiary alcohols}

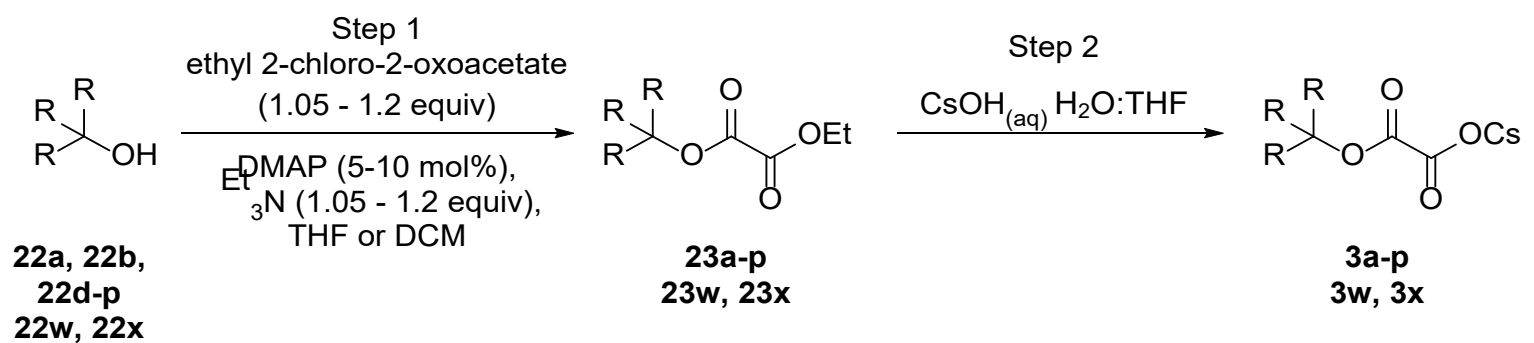

Step 1: Following a modified reported procedure, ${ }^{15}$ a two necked round bottomed flask, equipped with a magnetic stirrer, was charged with THF or $\mathrm{CH}_{2} \mathrm{Cl}_{2}(0.1$ or $0.2 \mathrm{M}),{ }^{16}$ DMAP $(0.15 \mathrm{mmol}, 5 \mathrm{~mol} \%)$, the tertiary alcohol 22a-x ( $3.00 \mathrm{mmol}, 1.00$ equiv) and triethylamine (1.05- 1.2 equiv) were then added. Ethyl 2-chloro-2-oxoacetate (1.05 - 1.2 equiv) was then added dropwise and giving a yellowish solution. The reaction was then stirred for $1 \mathrm{~h}-2 \mathrm{~h}$ at room temperature. Upon full conversion of the alcohol, indicated by TLC analysis, the reactions were quenched with sat. aq. $\mathrm{NH}_{4} \mathrm{Cl}$. The layers were then separated and the organic layer was then washed twice with brine (ca. $10 \mathrm{~mL}$ ). The organic layer was then dried over $\mathrm{Na}_{2} \mathrm{SO}_{4}$ and filtered. A solid deposit for flash chromatography was prepared: (ca. 5-7 $\mathrm{g} \mathrm{SiO}_{2}$ ) concentrated under reduced pressure. The compound was purified by flash chromatography ( $\mathrm{SiO}_{2}$, pentane:EtOAc 9:1, 4:1, p-Anisaldehyde stain blue, green or purple spots) affording the desired alkyl ethyl oxalate 23a-x.

Step 2: Following a modified reported procedure, ${ }^{13}$ a round-bottom flask was charged with ethyl oxoacetate $23 a-x(1.75 \mathrm{mmol}, 1.00$ equiv) followed by the addition of THF (1 M). To this solution, a 1 $\mathrm{M}$ stock solution of aq. $\mathrm{CsOH}$ (1.7 mmol, 1.00 equiv) was added dropwise (ca. $2 \mathrm{~min}$ ). The mixture was stirred vigorously for $5 \mathrm{~min}$ at room temperature, then concentrated immediately under reduced pressure $\left(\mathrm{T}=55^{\circ} \mathrm{C}-60^{\circ} \mathrm{C}: \mathrm{P}=300 \mathrm{mbar}\right.$ to $\left.20 \mathrm{mbar}\right) .{ }^{17}$ The resulting solid was then dried under high vacuum for at least 4 hours affording a dry (rarely hygroscopic, some are soap-like) cesium salt 3a-x.

Synthetic and characterization data for alkyl ethyl oxalate intermediates $23 a-x$ and cesium salts $3 a-x$

Ethyl 2-(2-methyl-4-phenylbutan-2-yl)oxy-2-oxoacetate (23a)<smiles>CCOC(=O)C(=O)OC(C)(C)CCc1ccccc1</smiles>

23a was synthesized following step 1 of general procedure $D$ in THF (90 mL, $0.1 \mathrm{M})$ using 2-methyl-4-phenylbutan-2-ol (22a, $1.6 \mathrm{~mL}, 9.1$ mmol, 1 equiv), DMAP (0.055 g, $0.46 \mathrm{mmol}, 5 \mathrm{~mol} \%)$, triethylamine $(1.3 \mathrm{~mL}, 9.6 \mathrm{mmol}, 1.05$ equiv) and ethyl chloro-oxoacetate $(1.1 \mathrm{~mL}$, $9.6 \mathrm{mmol}, 1.05$ equiv).

Column chromatography $\left(\mathrm{SiO}_{2}\right.$, pentane:EtOAc 85:15) afforded ethyl (2-methyl-4-phenylbutan-2yl) oxalate (23a, $2.00 \mathrm{~g}, 7.57 \mathrm{mmol}, 83 \%)$ as a colorless oil.

\footnotetext{
${ }^{15}$ Nawrat, C. C.; Jamison, C. R.; Slutskyy, Y.; MacMillan, D. W. C.; Overman, L. E. J. Am. Chem. Soc. 2015, 137, 11270-11273.

${ }^{16}$ We have not noticed particular changes of reactivity between THF and $\mathrm{CH}_{2} \mathrm{Cl}_{2}$ or between $0.1 \mathrm{M}$ or $0.2 \mathrm{M}$, use of $\mathrm{CH}_{2} \mathrm{Cl}_{2}$ simplifies extraction.

${ }^{17}$ Other hydrolysis products have been observed when the reaction is left longer or triturated in diethyl ether to attempt purification.
} 
Rf (pentane:EtOAc 9:1) $=0.5$

${ }^{1}$ H NMR $\left(400 \mathrm{MHz}, \mathrm{CDCl}_{3}\right) \delta 7.38-7.29(\mathrm{~m}, 2 \mathrm{H}, \mathrm{ArH}), 7.25(\mathrm{~d}, J=7.1 \mathrm{~Hz}, 3 \mathrm{H}, \operatorname{ArH}), 4.38(\mathrm{q}, J=7.1 \mathrm{~Hz}$, $\left.2 \mathrm{H}, \mathrm{OCH}_{2}-\mathrm{CH}_{3}\right), 2.79-2.71\left(\mathrm{~m}, 2 \mathrm{H}, \mathrm{Ph}-\mathrm{CH}_{2}\right), 2.25-2.16\left(\mathrm{~m}, 2 \mathrm{H}, \mathrm{CH}_{2}\right), 1.66(\mathrm{~s}, 6 \mathrm{H}, \mathrm{d} M e), 1.43(\mathrm{t}, J=$ $\left.7.1 \mathrm{~Hz}, 3 \mathrm{H}, \mathrm{OCH}_{2} \mathrm{CH}_{3}\right)$.

${ }^{13} \mathrm{C}$ NMR $\left(101 \mathrm{MHz}, \mathrm{CDCl}_{3}\right) \delta$ 158.6, 157.1, 141.6, 128.5, 128.4, 126.0, 86.6, 62.8, 42.5, 30.2, 25.7, 14.0.

IR (vmax, cm-1) 3087 (w), 3062 (w), $3029(\mathrm{~m}), 2983(\mathrm{~m}), 2949(\mathrm{~m}), 2872(\mathrm{w}), 1761$ (s), $1737(\mathrm{~s}), 1327$ $(\mathrm{m}), 1188(\mathrm{~s}), 1163(\mathrm{~s}), 1118(\mathrm{~s}), 912(\mathrm{~s})$.

HRMS (ESI/QTOF) m/z: [M + Na] ${ }^{+}$Calcd for $\mathrm{C}_{15} \mathrm{H}_{20} \mathrm{NaO}_{4}{ }^{+}$287.1254; Found 287.1256.

Cesium 2-(2-methyl-4-phenylbutan-2-yl)oxy-2-oxoacetate (3a)

3a was synthesized following step 2 of general procedure $D$ in THF<smiles>CC(C)(C)OC(=O)C(=O)OC(C)(C)CCc1ccccc1</smiles>
(6.5 mL, $0.1 \mathrm{M})$ using ethyl (2-methyl-4-phenylbutan-2-yl) oxalate (23a, $1.70 \mathrm{~g}, 6.43 \mathrm{mmol}, 1.0$ equiv) and $1 \mathrm{M}$ aq. $\mathrm{CsOH}(6.4 \mathrm{~mL}, 6.4$ mmol, 1.0 equiv), affording cesium 2-(2-methyl-4-phenylbutan-2yl)oxy-2-oxoacetate (3a, $2.34 \mathrm{~g}, 6.36 \mathrm{mmol}, 99 \%)$ as an off-white amorphous solid.

${ }^{1} \mathrm{H}$ NMR $\left(400 \mathrm{MHz}, \mathrm{D}_{2} \mathrm{O}\right) \delta 7.31(\mathrm{~m}, 5 \mathrm{H}, \mathrm{ArH}), 2.73-2.64\left(\mathrm{~m}, 2 \mathrm{H}, \mathrm{ArCH}_{2}\right), 2.20-2.11\left(\mathrm{~m}, 2 \mathrm{H}, \mathrm{CH}_{2}\right)$, $1.55\left(\mathrm{~s}, 6 \mathrm{H}, \mathrm{C}\left(\mathrm{CH}_{3}\right)_{2}\right)$.

${ }^{13} \mathrm{C}$ NMR $\left(101 \mathrm{MHz}, \mathrm{D}_{2} \mathrm{O}\right) \delta 165.2,164.1,142.5,128.7,128.5,126.0,86.0,41.3,29.7,25.4$.

HRMS (ESI/QTOF) m/z: [M - Cs] Calcd for $\mathrm{C}_{13} \mathrm{H}_{15} \mathrm{O}_{4}{ }^{-}$235.0976; Found 235.0979.

Ethyl (3-methyl-1-phenylpentan-3-yl) oxalate (23b)<smiles>CCOC(=O)C(=O)OC(C)(CC)CCc1ccccc1</smiles>

23b was synthesized following step 1 of general procedure $D$ in THF (60 mL, $0.1 \mathrm{M}$ ) using 3-methyl-1-phenylpentan-3-ol (22b $1.1 \mathrm{~g}, 6.0$ mmol, 1.0 equiv), DMAP (73 mg, $0.60 \mathrm{mmol}, 10 \mathrm{~mol} \%$ ), triethylamine $(1.0 \mathrm{~mL}, 7.2 \mathrm{mmol}, 1.2$ equiv) and ethyl chloro-oxoacetate $(0.80 \mathrm{~mL}$, $7.2 \mathrm{mmol}, 1.2$ equiv).

Column chromatography $\left(\mathrm{SiO}_{2}, 2 \%\right.$ EtOAC in Pentane) afforded ethyl (3-methyl-1-phenylpentan-3yl) oxalate (23b, $1.61 \mathrm{~g}, 5.78 \mathrm{mmol}, 96 \%)$ as a colourless oil.

Rf (pentane:EtOAc 98:2) $=0.4$.

${ }^{1}$ H NMR $\left(400 \mathrm{MHz}, \mathrm{CDCl}_{3}\right)$ 8: $7.33-7.24(\mathrm{~m}, 2 \mathrm{H}, \mathrm{ArH}), 7.23-7.16(\mathrm{~m}, 3 \mathrm{H}, \mathrm{ArH}), 4.32(\mathrm{q}, J=7.1 \mathrm{~Hz}$, $\left.2 \mathrm{H}, \mathrm{CO}_{2} \mathrm{CH}_{2} \mathrm{CH}_{3}\right), 2.72-2.59\left(\mathrm{~m}, 2 \mathrm{H}, \mathrm{ArCH}_{2}\right), 2.30-2.18\left(\mathrm{~m}, 1 \mathrm{H}, \mathrm{CH}_{2}\right), 2.17-1.99\left(\mathrm{~m}, 2 \mathrm{H}, \mathrm{CH}_{2}\right), 1.97$ $-1.85\left(\mathrm{~m}, 1 \mathrm{H}, \mathrm{CH}_{2}\right), 1.57\left(\mathrm{~s}, 3 \mathrm{H}, \mathrm{CH}_{3}\right), 1.37\left(\mathrm{t}, J=7.1 \mathrm{~Hz}, 3 \mathrm{H}, \mathrm{CO}_{2} \mathrm{CH}_{2} \mathrm{CH}_{3}\right), 0.95(\mathrm{t}, J=7.5 \mathrm{~Hz}, 3 \mathrm{H}$, $\left.\mathrm{CH}_{2} \mathrm{CH}_{3}\right)$.

${ }^{13} \mathrm{C}$ NMR $\left(101 \mathrm{MHz}, \mathrm{CDCl}_{3}\right)$ 8: 158.7, 157.2, 141.8, 128.6, 128.5, 126.1, 89.6, 62.9, 39.7, 30.9, 30.1, 23.1, 14.1, 8.1.

IR $\left(v_{\max }, \mathrm{cm}^{-1}\right)$ : $2979(\mathrm{~m}), 2943(\mathrm{w}), 1739(\mathrm{~s}), 1458(\mathrm{~m}), 1323(\mathrm{~m}), 1185(\mathrm{~s}), 1115(\mathrm{~m}), 1019(\mathrm{~m})$.

HRMS (ESI/QTOF) m/z: [M + Na] ${ }^{+}$Calcd for $\mathrm{C}_{16} \mathrm{H}_{22} \mathrm{NaO}_{4}{ }^{+}$301.1410; Found 301.1412.

Cesium 2-((3-methyl-1-phenylpentan-3-yl)oxy)-2-oxoacetate (3b)<smiles>CCC(C)(CCc1ccccc1)OC(=O)C(=O)O[Na]</smiles>

$3 \mathbf{b}$ was synthesized following step 2 of general procedure $D$ in THF $(3.0 \mathrm{~mL}, 0.1 \mathrm{M})$ using ethyl (3-methyl-1-phenylpentan-3-yl) oxalate (23b, $835 \mathrm{mg}, 3.00 \mathrm{mmol}, 1.0$ equiv) and $1 \mathrm{M}$ aq. $\mathrm{CsOH}(3.0 \mathrm{~mL}, 3.00$ mmol, 1.0 equiv). Cesium 2-((3-methyl-1-phenylpentan-3-yl)oxy)-2oxoacetate (3b, $951 \mathrm{mg}, 2.49 \mathrm{mmol}, 83 \%$ ) was obtained as an offwhite amorphous solid. 
${ }^{1} \mathrm{H}$ NMR $\left(400 \mathrm{MHz}, \mathrm{DMSO}-\mathrm{d}_{6}\right)$ 8: $7.29-7.24(\mathrm{~m}, 2 \mathrm{H}, \mathrm{ArH}), 7.19-7.14(\mathrm{~m}, 3 \mathrm{H}, \mathrm{ArH}), 2.59-2.54(\mathrm{~m}$, $\left.2 \mathrm{H}, \operatorname{Ar}-\mathrm{CH}_{2}\right), 2.11-2.03\left(\mathrm{~m}, 1 \mathrm{H}, \mathrm{CH}_{2}\right), 1.97-1.84\left(\mathrm{~m}, 2 \mathrm{H}, \mathrm{CH}_{2}\right), 1.76-1.67\left(\mathrm{~m}, 1 \mathrm{H}, \mathrm{CH}_{2}\right), 1.36(\mathrm{~s}, 3 \mathrm{H}$, $\left.\mathrm{CH}_{3}\right), 0.84\left(\mathrm{t}, \mathrm{J}=7.53 \mathrm{~Hz}, 3 \mathrm{H}, \mathrm{CH}_{2} \mathrm{CH}_{3}\right)$.

${ }^{13} \mathrm{C}$ NMR $\left(101 \mathrm{MHz}, \mathrm{DMSO}-\mathrm{d}_{6}\right)$ $\delta: 167.7,163.5,142.3,128.3,128.2,125.6,82.3,64.8,30.6,29.3$, 23.2, 7.8.

HRMS (ESI/QTOF) m/z: [M + Na] ${ }^{+}$Calcd for $\mathrm{C}_{14} \mathrm{H}_{17} \mathrm{CsNaO}_{4}{ }^{+}$405.0074; Found 405.0075.

\section{Ethyl (tert-butyl)oxy-2-oxoacetate (23c)}

Ethyl 2-chloro-2-oxoacetate

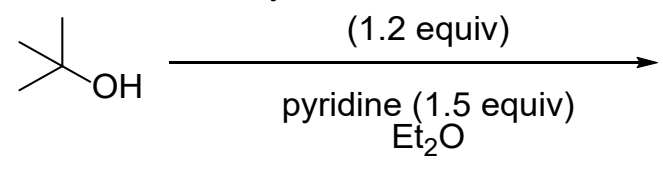

22c<smiles>CCOC(=O)C(=O)OC(C)(C)C</smiles>

23c

Following a reported procedure, ${ }^{18}$ ethyl 2-chloro-2-oxoacetate $(3.6 \mathrm{~mL}, 32 \mathrm{mmol}, 1.2$ equiv) was added to a solution of tert-butanol (22c, $2.0 \mathrm{~g}, 27 \mathrm{mmol}, 1.0$ equiv) and pyridine ( $3.26 \mathrm{~mL}, 40.5 \mathrm{mmol}$ ) in $\mathrm{Et}_{2} \mathrm{O}(100 \mathrm{~mL})$ and the resulting yellow solution was stirred at room temperature for 4 hours. The organic layer was washed with water $(2 \times 50 \mathrm{~mL})$ and sat. aq. $\mathrm{NaHCO}_{3}$ solution $(50 \mathrm{~mL})$, dried over $\mathrm{MgSO}_{4}$ and concentrated under reduced pressure. The crude material was purified by flash column chromatography on a short column of silica gel (1:20 $\mathrm{Et}_{2} \mathrm{O}$ :pentane) to give tert-butyl ethyl oxalate (23c, $4.4 \mathrm{~g}, 25 \mathrm{mmol}, 98 \%$ ) as a colorless oil.<smiles>CCOC(=O)C(=O)OC(C)(C)C</smiles>

${ }^{1} \mathrm{H}$ NMR $\left(400 \mathrm{MHz}, \mathrm{CDCl}_{3}\right) \delta 4.31(\mathrm{q}, J=7.1 \mathrm{~Hz}, 2 \mathrm{H}), 1.55(\mathrm{~s}, 9 \mathrm{H}), 1.36$ $(\mathrm{t}, J=7.1 \mathrm{~Hz}, 3 \mathrm{H})$.

${ }^{13} \mathrm{C}$ NMR $\left(101 \mathrm{MHz}, \mathrm{CDCl}_{3}\right) \delta 158.8,157.3,85.0,62.9,27.9,14.1$. The NMR data obtained are consistent with the reported literature data. ${ }^{16}$

Cesium (tert-butyl)oxy-2-oxoacetate (3c)<smiles>CC(C)(C)OC(=O)C(=O)OC(C)(C)C</smiles>

$3 c$ was synthesized following step 2 of general procedure $D$ in THF $(2.1 \mathrm{~mL}, 0.1 \mathrm{M})$ using tert-butyl ethyl oxalate $(23 \mathrm{c}, 0.366 \mathrm{~g}, 2.10$ $\mathrm{mmol}, 1.0$ equiv) and $1 \mathrm{M}$ aq. $\mathrm{CsOH}(2.1 \mathrm{~mL}, 2.1 \mathrm{mmol}, 1.0$ equiv), affording cesium (tert-butyl)oxy-2-oxoacetate (3c $0.505 \mathrm{~g}, 1.82$ $\mathrm{mmol}, 86 \%)$ as a colorless amorphous solid.

${ }^{1} \mathrm{H}$ NMR $\left(400 \mathrm{MHz}\right.$, DMSO- $\left.d_{6}\right) \delta 1.37\left(\mathrm{~s}, 9 \mathrm{H}, \mathrm{C}\left(\mathrm{CH}_{3}\right)_{3}\right)$.

${ }^{13} \mathrm{C}$ NMR $\left(101 \mathrm{MHz}, \mathrm{DMSO}-d_{6}\right) \delta 167.5,163.5,78.0,27.9$.

HRMS (ESI/QTOF) m/z: [M + Na] ${ }^{+}$Calcd for $\mathrm{C}_{6} \mathrm{H}_{9} \mathrm{CsNaO}_{4}{ }^{+} 300.9448$; Found 300.9451.

\section{Ethyl (2,3-dimethylbutan-2-yl)oxy-2-oxoacetate (23d)}

23d was synthesized following step 1 of general procedure $D$ in<smiles>CCOC(=O)C(=O)OC(C)(C)C(C)C</smiles>
DCM (24 mL, $0.1 \mathrm{M})$ using 2,3-dimethyl-2-butanol (22d, $0.30 \mathrm{~mL}$, $2.4 \mathrm{mmol}, 1.0$ equiv), DMAP (30 $\mathrm{mg}, 0.24 \mathrm{mmol}, 10 \mathrm{~mol} \%$ ), triethylamine ( $0.35 \mathrm{~mL}, 2.5 \mathrm{mmol}, 1.05$ equiv) and ethyl chlorooxoacetate ( $0.3 \mathrm{~mL}, 2.5 \mathrm{mmol}, 1.05$ equiv).

Column chromatography $\left(\mathrm{SiO}_{2}\right.$, pentane:EtOAc 9:1) afforded ethyl (2,3-dimethylbutan-2-yl) oxalate (23d, $0.340 \mathrm{~g}, 1.68 \mathrm{mmol}, 70 \%$ ).

\footnotetext{
${ }^{18}$ Xu, Y.; McLaughlin, M.; Bolton, E. N.; Reamer, R. A. J. Org. Chem. 2010, 75, 8666-8669.
} 
Rf (pentane:EtOAc 9:1) $=0.5$.

${ }^{1} \mathrm{H}$ NMR $\left(400 \mathrm{MHz}, \mathrm{CDCl}_{3}\right) \delta 4.30\left(\mathrm{q}, J=7.2 \mathrm{~Hz}, 2 \mathrm{H}, \mathrm{OCH}_{2} \mathrm{CH}_{3}\right), 2.27$ (hept, $\left.J=6.9 \mathrm{~Hz}, 1 \mathrm{H}, \mathrm{CH}\left(\mathrm{CH}_{3}\right)_{2}\right)$, $1.49\left(\mathrm{~s}, 6 \mathrm{H}, \mathrm{OC}\left(\mathrm{CH}_{3}\right)_{2}\right), 1.35\left(\mathrm{t}, J=7.2 \mathrm{~Hz}, 3 \mathrm{H}, \mathrm{OCH}_{2} \mathrm{CH}_{3}\right), 0.94\left(\mathrm{~d}, J=6.9 \mathrm{~Hz}, 6 \mathrm{H}, \mathrm{CH}\left(\mathrm{CH}_{3}\right)_{2}\right)$.

${ }^{13} \mathrm{C}$ NMR $\left(101 \mathrm{MHz}, \mathrm{CDCl}_{3}\right) \delta 158.9,157.3,90.4,62.8,36.3,22.5,17.4,14.1$.

IR $\left(v_{\max }, \mathrm{cm}^{-1}\right) 2995(\mathrm{~m}), 2983(\mathrm{~m}), 2962(\mathrm{w}), 2946(\mathrm{w}), 2891(\mathrm{w}), 2878(\mathrm{w}), 2840(\mathrm{w}), 1763(\mathrm{~s}), 1737$ (s), $1467(\mathrm{~m}), 1371(\mathrm{~m}), 1324(\mathrm{~s}), 1191(\mathrm{~s}), 1130$ (s), $1094(\mathrm{~s}), 1017(\mathrm{~m})$.

HRMS (ESI/QTOF) m/z: [M + Na] ${ }^{+}$Calcd for $\mathrm{C}_{10} \mathrm{H}_{18} \mathrm{NaO}_{4}{ }^{+}$225.1097; Found 225.1099.

Cesium (2,3-dimethylbutan-2-yl)oxy-2-oxoacetate (3d)

$3 \mathbf{d}$ was synthesized following step 2 of general procedure $D$ in THF<smiles>CCOC(=O)C(=O)OC(C)(C)C(C)C</smiles>
$(1.0 \mathrm{~mL}, 0.1 \mathrm{M})$ using ethyl (2,3-dimethylbutan-2-yl) oxalate (23d, $0.200 \mathrm{~g}, 0.989 \mathrm{mmol}, 1.0$ equiv) and $1 \mathrm{M}$ aq. $\mathrm{CsOH}(0.99 \mathrm{~mL}, 0.99$ mmol, 1.0 equiv), affording cesium (2,3-dimethylbutan-2-yl)oxy-2oxoacetate (3d, $137 \mathrm{mg}, 0.447 \mathrm{mmol}, 45 \%$ ) as a colorless amorphous solid.

${ }^{1} \mathrm{H}$ NMR $\left(400 \mathrm{MHz}, \mathrm{DMSO}-d_{6}\right) \delta 2.22$ (hept, $\left.J=6.9 \mathrm{~Hz}, 1 \mathrm{H}, \mathrm{CH}\left(\mathrm{CH}_{3}\right)_{2}\right), 1.30\left(\mathrm{~s}, 6 \mathrm{H}, \mathrm{OC}\left(\mathrm{CH}_{3}\right)_{2}\right), 0.84(\mathrm{~d}$, $\left.J=6.9 \mathrm{~Hz}, 6 \mathrm{H}, \mathrm{CH}\left(\mathrm{CH}_{3}\right)_{2}\right)$.

${ }^{13} \mathrm{C}$ NMR $\left(101 \mathrm{MHz}, \mathrm{DMSO}-d_{6}\right) \delta 167.6,163.6,83.0,35.3,22.7,17.1$.

HRMS (ESI/QTOF) m/z: [M + Na] ${ }^{+}$Calcd for $\mathrm{C}_{8} \mathrm{H}_{13} \mathrm{CsNaO}_{4}{ }^{+}$328.9761; Found 328.9768.

Ethyl (1-(4-methoxyphenyl)-2-methylpropan-2-yl)oxy-2-oxoacetate (23e)<smiles>CCOC(=O)C(=O)OC(C)(C)Cc1ccc(OC)cc1</smiles>

23e was synthesized following step 1 of general procedure $D$ in $\mathrm{DCM}(30 \mathrm{~mL}, 0.1 \mathrm{M})$ using 1-(4-methoxyphenyl)-2-methylpropan2-ol (22e, $500 \mathrm{mg}, 2.77 \mathrm{mmol}, 1.0$ equiv), DMAP (33 mg, $0.28 \mathrm{mmol}$, $10 \mathrm{~mol} \%)$, triethylamine ( $0.40 \mathrm{~mL}, 2.9 \mathrm{mmol}, 1.05$ equiv) and ethyl chloro-oxoacetate $(0.30 \mathrm{~mL}, 2.9 \mathrm{mmol}, 1.05$ equiv).

Column chromatography ( $\mathrm{SiO}_{2}$, pentane:EtOAc 4:1) afforded ethyl (1-(4-methoxyphenyl)-2methylpropan-2-yl) oxalate (23e, $270 \mathrm{mg}, 0.963 \mathrm{mmol}, 35 \%$ ) as a pale-yellow oil.

$\mathbf{R f}$ (pentane:EtOAc 4:1) $=0.4$.

${ }^{1} \mathrm{H}$ NMR $\left(400 \mathrm{MHz}, \mathrm{CDCl}_{3}\right) \delta 7.20-7.12(\mathrm{~m}, 2 \mathrm{H}, \mathrm{ArH}), 6.87-6.79(\mathrm{~m}, 2 \mathrm{H}, \mathrm{ArH}), 4.32(\mathrm{q}, J=7.2 \mathrm{~Hz}$, $\left.2 \mathrm{H}, \mathrm{OCH}_{2} \mathrm{CH}_{3}\right), 3.79\left(\mathrm{~s}, 3 \mathrm{H}, \mathrm{OCH}_{3}\right), 3.03\left(\mathrm{~s}, 2 \mathrm{H}, \mathrm{ArCH}_{2}\right), 1.53\left(\mathrm{~s}, 6 \mathrm{H}\left(\mathrm{CH}_{3}\right)_{2}\right), 1.38(\mathrm{t}, J=7.1 \mathrm{~Hz}, 3 \mathrm{H}$, $\left.\mathrm{OCH}_{2} \mathrm{CH}_{3}\right)$.

${ }^{13} \mathrm{C}$ NMR $\left(101 \mathrm{MHz}, \mathrm{CDCl}_{3}\right) \delta 158.6,158.6,157.2,131.8,128.5,113.6,86.8,62.9,55.3,46.1,25.4$, 14.1.

IR $\left(v_{\max }, \mathrm{cm}^{-1}\right) 2995(\mathrm{~m}), 2985(\mathrm{~m}), 2953(\mathrm{~m}), 2937(\mathrm{~m}), 2909(\mathrm{~m}), 2837(\mathrm{~m}), 1761(\mathrm{~s}), 1738(\mathrm{~s}), 1612$ (m), $1513(\mathrm{~s}), 1465(\mathrm{~m}), 1370(\mathrm{~m}), 1321$ (s), 1247 (s), 1189 (s), 1177 (s), 1164 (s), 1034 (s), 1019 (s), 851 (s).

HRMS (ESI/QTOF) m/z: [M + Na] $]^{+}$Calcd for $\mathrm{C}_{15} \mathrm{H}_{20} \mathrm{NaO}_{5}{ }^{+}$303.1203; Found 303.1206.

\section{Cesium (1-(4-methoxyphenyl)-2-methylpropan-2-yl)oxy-2-oxoacetate (3e)}<smiles>COc1ccc(CC(C)(C)OC(=O)C(=O)OCc2ccccc2)cc1</smiles>

3e was synthesized following step 2 of general procedure $D$ in THF (0.7 mL, $0.1 \mathrm{M})$ using ethyl (1-(4-methoxyphenyl)-2-methylpropan2-yl) oxalate (23e, $0.20 \mathrm{~g}, 0.71 \mathrm{mmol}, 1.0$ equiv) and $1 \mathrm{M}$ aq. $\mathrm{CsOH}$ (0.7 mL, $0.7 \mathrm{mmol}, 1.0$ equiv), affording cesium (1-(4methoxyphenyl)-2-methylpropan-2-yl)oxy-2-oxoacetate (3e, 251 $\mathrm{mg}, 0.653 \mathrm{mmol}, 92 \%$ ) as a coloroless amorphous solid. 
${ }^{1} \mathrm{H}$ NMR $\left(400 \mathrm{MHz}, \mathrm{DMSO}-d_{6}\right) \delta 7.18-7.11(\mathrm{~m}, 2 \mathrm{H}, \mathrm{ArH}), 6.85-6.78(\mathrm{~m}, 2 \mathrm{H}, \mathrm{ArH}), 3.72\left(\mathrm{~s}, 3 \mathrm{H}, \mathrm{OCH}_{3}\right)$, $2.95\left(\mathrm{~s}, 2 \mathrm{H}, \mathrm{ArCH}_{2}\right), 1.31\left(\mathrm{~s}, 6 \mathrm{H},\left(\mathrm{CH}_{3}\right)_{2}\right)$.

${ }^{13} \mathrm{C}$ NMR $\left(101 \mathrm{MHz}, \mathrm{DMSO}-d_{6}\right) \delta 167.7,163.3,157.7,131.5,129.3,113.2,80.2,54.9,44.5,25.8$.

HRMS (ESI/QTOF) m/z: [M - Cs] $]^{-}$Calcd for $\mathrm{C}_{13} \mathrm{H}_{15} \mathrm{O}_{5}{ }^{-}$251.0925; Found 251.0936.

\section{Ethyl (1-(2-fluorophenyl)-2-methylpropan-2-yl)oxy-2-oxoacetate (23f)}<smiles>CCOC(=O)C(=O)OC(C)(C)Cc1ccccc1F</smiles>

$23 \mathrm{f}$ was synthesized following step 1 of general procedure $D$ in DCM (30 mL, $0.1 \mathrm{M}$ ) using 1-(2-fluorophenyl)-2-methylpropan-2-ol (22f, $500 \mathrm{mg}, 2.97 \mathrm{mmol}, 1.0$ equiv), DMAP (36 mg, $0.30 \mathrm{mmol}, 10$ mol\%), triethylamine $(0.44 \mathrm{~mL}, 3.1 \mathrm{mmol}, 1.05$ equiv) and ethyl chloro-oxoacetate $(0.35 \mathrm{~mL}, 3.1 \mathrm{mmol}, 1.05$ equiv).

Column chromatography ( $\mathrm{SiO}_{2}$, pentane:EtOAc 9:1 to 8:2) afforded ethyl (1-(4-fluorophenyl)-2methylpropan-2-yl) oxalate (23f, $467 \mathrm{mg}, 1.74 \mathrm{mmol}, 59 \%$ ) as a colorless oil.

Rf (pentane:EtOAc 9:1) $=0.35$.

${ }^{1} \mathrm{H}$ NMR $\left(400 \mathrm{MHz}, \mathrm{CDCl}_{3}\right) \delta 7.30(\mathrm{td}, J=7.6,1.8 \mathrm{~Hz}, 1 \mathrm{H}, \mathrm{ArH}), 7.26-7.20(\mathrm{~m}, 1 \mathrm{H}, \operatorname{ArH}), 7.12-6.99$ $(\mathrm{m}, 2 \mathrm{H}, \mathrm{ArH}), 4.32\left(\mathrm{q}, J=7.2 \mathrm{~Hz}, 2 \mathrm{H}, \mathrm{OCH}_{2} \mathrm{CH}_{3}\right), 3.16\left(\mathrm{~d}, J=1.4 \mathrm{~Hz}, 2 \mathrm{H}, \mathrm{ArCH}_{2}\right), 1.57(\mathrm{~d}, J=1.0 \mathrm{~Hz}, 6 \mathrm{H}$, $\left.\left(\mathrm{CH}_{3}\right)_{2}\right), 1.38\left(\mathrm{t}, J=7.2 \mathrm{~Hz}, 3 \mathrm{H}, \mathrm{OCH}_{2} \mathrm{CH}_{3}\right)$.

${ }^{13} \mathrm{C} \mathrm{NMR}\left(101 \mathrm{MHz}, \mathrm{CDCl}_{3}\right) \delta 162.8,160.4,157.8(\mathrm{~d}, J=141.6 \mathrm{~Hz}), 133.2(\mathrm{~d}, J=4.4 \mathrm{~Hz}), 128.8(\mathrm{~d}, J=$ $8.2 \mathrm{~Hz}), 123.9(\mathrm{~d}, J=3.5 \mathrm{~Hz}), 123.5(\mathrm{~d}, J=15.7 \mathrm{~Hz}), 115.4(\mathrm{~d}, J=23.0 \mathrm{~Hz}), 86.6,62.9,39.3,25.4,14.1$. ${ }^{19} \mathrm{~F}$ NMR $\left(376 \mathrm{MHz}, \mathrm{CDCl}_{3}\right) \delta-115.9$.

IR $\left(v_{\max }, \mathrm{cm}^{-1}\right) 3004(\mathrm{~m}), 2989(\mathrm{~m}), 2965(\mathrm{w}), 2938(\mathrm{~m}), 2899(\mathrm{w}), 1764(\mathrm{~s}), 1737(\mathrm{~s}), 1495(\mathrm{~m}), 1456$ $(\mathrm{m}), 1372(\mathrm{~m}), 1319(\mathrm{~m}), 1233(\mathrm{~s}), 1190(\mathrm{~s}), 1172(\mathrm{~s}), 1120(\mathrm{~s}), 759(\mathrm{~s})$.

HRMS (ESI/QTOF) m/z: [M + Na] ${ }^{+}$Calcd for $\mathrm{C}_{14} \mathrm{H}_{17} \mathrm{FNaO}_{4}{ }^{+}$291.1003; Found 291.1002.

\section{Cesium (1-(2-fluorophenyl)-2-methylpropan-2-yl)oxy-2-oxoacetate (3f)}<smiles>CCOC(=O)C(=O)OC(C)(C)Cc1ccccc1F</smiles>

3 f was synthesized following step 2 of general procedure $D$ in THF (1.5 $\mathrm{mL}, 0.1 \mathrm{M})$ using ethyl (1-(2-fluorophenyl)-2-methylpropan-2-yl) oxalate (23f, $0.40 \mathrm{~g}, 1.5 \mathrm{mmol}, 1.0$ equiv) and $1 \mathrm{M}$ aq. $\mathrm{CsOH}(1.5 \mathrm{~mL}$, $1.5 \mathrm{mmol}, 1.0$ equiv), affording cesium (1-(2-fluorophenyl)-2methylpropan-2-yl)oxy-2-oxoacetate (3f, $469 \mathrm{mg}, 1.26 \mathrm{mmol}, 84 \%$ ) as a coloroless amorphous solid.

${ }^{1} \mathrm{H}$ NMR $\left(400 \mathrm{MHz}, \mathrm{DMSO}-d_{6}\right) \delta 7.36(\mathrm{td}, J=7.7,1.9 \mathrm{~Hz}, 1 \mathrm{H}, \mathrm{ArH}), 7.33-7.23(\mathrm{~m}, 1 \mathrm{H}, \mathrm{ArH}), 7.19-$ $7.06(\mathrm{~m}, 2 \mathrm{H}, \mathrm{ArH}), 3.08\left(\mathrm{~s}, 2 \mathrm{H}, \mathrm{ArCH}_{2}\right), 1.34\left(\mathrm{~d}, \mathrm{~J}=1.0 \mathrm{~Hz}, 6 \mathrm{H},\left(\mathrm{CH}_{3}\right)_{2}\right)$.

${ }^{13} \mathrm{C}$ NMR (101 MHz, DMSO- $\left.d_{6}\right) \delta$ 167.6, $163.1,160.9$ (d, $\left.J=243.4 \mathrm{~Hz}\right), 133.1$ (d, $\left.J=4.4 \mathrm{~Hz}\right), 128.5$ (d, $J=8.2 \mathrm{~Hz}$ ), $124.0(\mathrm{~d}, J=4.0 \mathrm{~Hz}), 124.0,114.9(\mathrm{~d}, J=22.9 \mathrm{~Hz}), 80.0,37.5,25.7$.

${ }^{19} \mathrm{~F}$ NMR $\left(376 \mathrm{MHz}, \mathrm{DMSO}-d_{6}\right) \delta$-116.6.

HRMS (ESI/QTOF) m/z: [M - Cs] $]^{-}$Calcd for $\mathrm{C}_{12} \mathrm{H}_{12} \mathrm{FO}_{4}{ }^{-}$239.0725; Found 239.0719.

\section{Ethyl (1-(4-bromophenyl)-2-methylpropan-2-yl)oxy-2-oxoacetate (23g)}

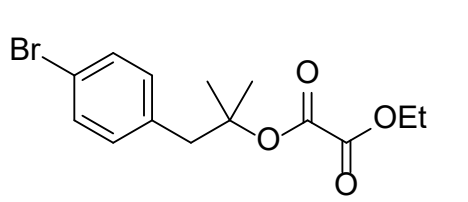

23g was synthesized following step 1 of general procedure $D$ in DCM $(20 \mathrm{~mL}, 0.1 \mathrm{M})$ using 1-(4-bromophenyl)-2-methylpropan-2-ol (22g, $500 \mathrm{mg}, 2.18 \mathrm{mmol}, 1.0$ equiv), DMAP ( $27 \mathrm{mg}, 0.22 \mathrm{mmol}, 10 \mathrm{~mol} \%$ ), triethylamine $(0.30 \mathrm{~mL}, 2.4 \mathrm{mmol}, 1.1$ equiv) and ethyl chlorooxoacetate $(0.30 \mathrm{~mL}, 2.4 \mathrm{mmol}, 1.1$ equiv).

Column chromatography ( $\mathrm{SiO}_{2}$, pentane:EtOAc 9:1 to 8:2) afforded ethyl (1-(4-bromophenyl)-2methylpropan-2-yl) oxalate (3g $461 \mathrm{mg}, 1.40 \mathrm{mmol}, 64 \%$ ). 
$\mathbf{R f}$ (pentane:EtOAc 4:1) $=0.4$.

${ }^{1} \mathrm{H}$ NMR $\left(400 \mathrm{MHz}, \mathrm{CDCl}_{3}\right) \delta 7.46-7.38(\mathrm{~m}, 2 \mathrm{H}, \mathrm{ArH}), 7.16-7.09(\mathrm{~m}, 2 \mathrm{H}, \operatorname{ArH}), 4.32(\mathrm{q}, J=7.1 \mathrm{~Hz}$, $\left.2 \mathrm{H}, \mathrm{OCH}_{2} \mathrm{CH}_{3}\right), 3.03\left(\mathrm{~s}, 2 \mathrm{H}, \mathrm{ArCH}_{2}\right), 1.53\left(\mathrm{~s}, 6 \mathrm{H},\left(\mathrm{CH}_{3}\right)_{2}\right), 1.38\left(\mathrm{t}, J=7.1 \mathrm{~Hz}, 3 \mathrm{H}, \mathrm{OCH}_{2} \mathrm{CH}_{3}\right)$.

${ }^{13} \mathrm{C}$ NMR $\left(101 \mathrm{MHz}, \mathrm{CDCl}_{3}\right) \delta 158.4,157.0,135.4,132.5,131.3,121.0,86.1,63.0,46.5,25.4,14.1$.

IR $\left(v_{\max }, \mathrm{cm}^{-1}\right) 3015(\mathrm{w}), 3002(\mathrm{w}), 2977(\mathrm{w}), 2934(\mathrm{w}), 2844(\mathrm{w}), 1760(\mathrm{~s}), 1738(\mathrm{~s}), 1490(\mathrm{~m}), 1321$ $(\mathrm{m}), 1188(\mathrm{~s}), 1160(\mathrm{~s}), 1115(\mathrm{~s}), 1073(\mathrm{~m}), 1012(\mathrm{~s}), 796(\mathrm{~m})$.

HRMS (ESI/QTOF) m/z: [M + Na] $]^{+}$Calcd for $\mathrm{C}_{14} \mathrm{H}_{17}{ }^{79} \mathrm{BrNaO}_{4}{ }^{+}$351.0202; Found 351.0199.

\section{Cesium (1-(4-bromophenyl)-2-methylpropan-2-yl)oxy-2-oxoacetate (3g)}<smiles>COC(=O)C(=O)OC(C)(C)Cc1ccc(Br)cc1</smiles>

$3 \mathrm{~g}$ was synthesized following step 2 of general procedure $D$ in THF (0.94 mL, $0.1 \mathrm{M})$ using ethyl (1-(4-bromophenyl)-2-methylpropan-2yl) oxalate $(\mathbf{2 3 g}, 310 \mathrm{mg}, 0.941 \mathrm{mmol}, 1.0$ equiv) and $1 \mathrm{M}$ aq. $\mathrm{CsOH}$ (0.94 mL, $0.94 \mathrm{mmol}, 1.0$ equiv), affording cesium (1-(4bromophenyl)-2-methylpropan-2-yl)oxy-2-oxoacetate (3g, $89 \mathrm{mg}$, $0.20 \mathrm{mmol}, 22 \%)$ as a coloroless amorphous solid.

${ }^{1} \mathrm{H}$ NMR $\left(400 \mathrm{MHz}, \mathrm{DMSO}-d_{6}\right) \delta 7.59-7.32(\mathrm{~m}, 2 \mathrm{H}, \operatorname{ArH}), 7.32-7.10(\mathrm{~m}, 2 \mathrm{H}, \operatorname{Ar} H), 3.01(\mathrm{~s}, 2 \mathrm{H}$, $\left.\operatorname{ArCH}_{2}\right), 1.32\left(\mathrm{~s}, 6 \mathrm{H},\left(\mathrm{CH}_{3}\right)_{2}\right)$.

${ }^{13} \mathrm{C}$ NMR $\left(101 \mathrm{MHz}, \mathrm{DMSO}-d_{6}\right) \delta 167.7,163.1,136.9,132.8,130.6,119.5,79.7,44.5,25.9$.

HRMS (ESI/QTOF) m/z: [M - Cs] $]^{-}$Calcd for $\mathrm{C}_{12} \mathrm{H}_{12}{ }^{79} \mathrm{BrO}_{4}^{-}$298.9924; Found 298.9914.

4-Methylbenzylation of 3-methylbutane-1,3-diol (22h)

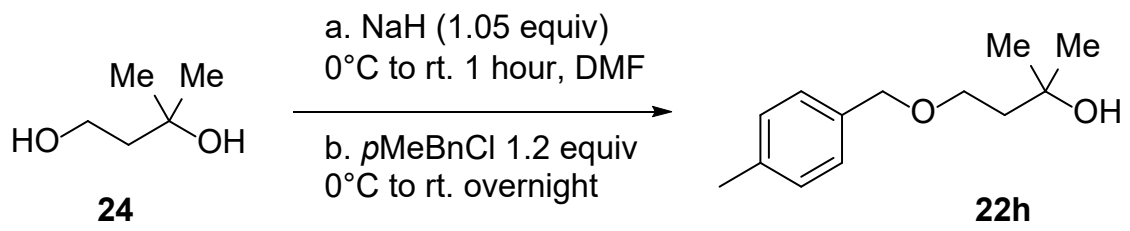

An oven dried $25 \mathrm{~mL}$ flask, equipped with a magnetic stirring bar, was flushed with nitrogen then charged with 3-methylbutane-1,3-diol (24, $0.26 \mathrm{~mL}, 2.4 \mathrm{mmol}, 1.0$ equiv) and anhydrous DMF (12.5 $\mathrm{mL}, 0.2 \mathrm{M}$ ). The solution was cooled to $0{ }^{\circ} \mathrm{C}$ and $\mathrm{NaH}$ ( $60 \%$ oil dispersion, $102 \mathrm{mg}, 2.56 \mathrm{mmol}, 1.05$ equiv) was added portion-wise under nitrogen. The latter solution was stirred for $1 \mathrm{~h}$ at room temperature. The solution was cooled back down to $0{ }^{\circ} \mathrm{C}$ and 1-(chloromethyl)-4-methylbenzene (411 $\mathrm{mg}, 2.92 \mathrm{mmol}, 1.2$ equiv) was added under nitrogen. The reaction was left to warm up to RT slowly and was stirred overnight. The reaction was quenched with sat. aq. $\mathrm{NH}_{4} \mathrm{Cl}(5 \mathrm{~mL})$ then diluted with water $(10 \mathrm{~mL})$ and $\mathrm{CH}_{2} \mathrm{Cl}_{2}(15 \mathrm{~mL})$. The layers were separated, and the aqueous layer was washed with $\mathrm{CH}_{2} \mathrm{Cl}_{2}(15 \mathrm{~mL})$. The organic layers were combined and washed with a (sat. aq. $\mathrm{NaCl}$ ): water (1:1) solution $(15 \mathrm{~mL})$ three times. The organic layers were then dried over $\mathrm{Na}_{2} \mathrm{SO}_{4}$, filtered, and concentrated under reduced pressure. The resulting crude oil was purified by column chromatography ( $\mathrm{SiO}_{2}$, pentane:EtOAc 9:1 to 8:2) affording 2-methyl-4-((4-methylbenzyl)oxy)butan-2-ol (22h, $297 \mathrm{mg}$, $1.43 \mathrm{mmol}, 59 \%)$ as a colorless oil with some trace impurities. After ${ }^{1} \mathrm{H}$ NMR and HRMS confirmation, the compound was used directly in next step with no further purification or analyses.

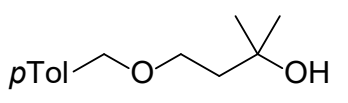

${ }^{1}$ H NMR $\left(400 \mathrm{MHz}, \mathrm{CDCl}_{3}\right) \delta 7.21(\mathrm{~d}, J=8.0 \mathrm{~Hz}, 2 \mathrm{H}, \mathrm{ArH}), 7.15(\mathrm{~d}, J=$ $7.8 \mathrm{~Hz}, 2 \mathrm{H}, \mathrm{ArH}), 4.48\left(\mathrm{~s}, 2 \mathrm{H}, \mathrm{ArCH}_{2}\right), 3.70\left(\mathrm{t}, J=5.9 \mathrm{~Hz}, 2 \mathrm{H}, \mathrm{CH}_{2}\right), 3.14$ (bs, $1 \mathrm{H}, \mathrm{OH}), 2.34\left(\mathrm{~s}, 3 \mathrm{H}, \mathrm{ArCH}_{3}\right), 1.79\left(\mathrm{t}, J=5.9 \mathrm{~Hz}, 2 \mathrm{H}, \mathrm{CH}_{2}\right), 1.23(\mathrm{~s}$, $\left.6 \mathrm{H},\left(\mathrm{CH}_{3}\right)_{2}\right)$.

HRMS (ESI/QTOF) m/z: [M + Na] $]^{+}$Calcd for $\mathrm{C}_{13} \mathrm{H}_{20} \mathrm{NaO}_{2}{ }^{+}$231.1356; Found 231.1358. 
$23 \mathrm{~h}$ was synthesized following step 1 of general procedure $D$ in THF<smiles>CCOCCOCCC(C)(C)OC(=O)C(=O)OCC</smiles>
(11 mL, $0.1 \mathrm{M})$ using 2-methyl-4-((4-methylbenzyl)oxy)butan-2-ol (22h, $220 \mathrm{mg}, 1.06 \mathrm{mmol}, 1.0$ equiv), DMAP (13 mg, $0.11 \mathrm{mmol}, 10$ mol\%), triethylamine $(0.16 \mathrm{~mL}, 1.2 \mathrm{mmol}, 1.1$ equiv) and ethyl chloro-oxoacetate ( $0.16 \mathrm{~mL}, 1.2 \mathrm{mmol}, 1.1$ equiv).

Column chromatography ( $\mathrm{SiO}_{2}$, pentane:EtOAc 9:1 to 8:2) afforded ethyl (2-methyl-4-((4methylbenzyl)oxy)butan-2-yl) oxalate (23h, $224 \mathrm{mg}, 0.726 \mathrm{mmol}, 69 \%)$.

Rf (pentane:EtOAc 9:1) $=0.3$.

${ }^{1} \mathrm{H}$ NMR $\left(400 \mathrm{MHz}, \mathrm{CDCl}_{3}\right) \delta 7.21(\mathrm{~d}, J=8.1 \mathrm{~Hz}, 2 \mathrm{H}, \mathrm{ArH}), 7.18-7.11(\mathrm{~m}, 2 \mathrm{H}, \mathrm{ArH}), 4.44\left(\mathrm{~s}, 2 \mathrm{H}, \mathrm{ArCH}_{2}\right)$, $4.28\left(\mathrm{q}, J=7.1 \mathrm{~Hz}, 2 \mathrm{H}, \mathrm{OCH}_{2} \mathrm{CH}_{3}\right), 3.59\left(\mathrm{t}, J=6.6 \mathrm{~Hz}, 2 \mathrm{H}, \mathrm{CH}_{2}\right), 2.34\left(\mathrm{~s}, 3 \mathrm{H}, \mathrm{ArCH}_{3}\right), 2.19(\mathrm{t}, J=6.6 \mathrm{~Hz}$, $\left.2 \mathrm{H}, \mathrm{CH}_{2}\right), 1.57\left(\mathrm{~s}, 6 \mathrm{H},\left(\mathrm{CH}_{3}\right)_{2}\right), 1.34\left(\mathrm{t}, J=7.1 \mathrm{~Hz}, 3 \mathrm{H}, \mathrm{OCH}_{2} \mathrm{CH}_{3}\right)$.

${ }^{13} \mathrm{C}$ NMR $\left(101 \mathrm{MHz}, \mathrm{CDCl}_{3}\right) \delta 158.6,157.2,137.4,135.3,129.2,127.8,86.1,73.0,66.0,62.9,39.9$, 26.3, 21.3, 14.1 .

IR $\left(v_{\max }, \mathrm{cm}^{-1}\right) 3048(\mathrm{~m}), 3016(\mathrm{~m}), 2991(\mathrm{~m}), 2929(\mathrm{~m}), 2876(\mathrm{~m}), 2860(\mathrm{~m}), 1760(\mathrm{~m}), 1737(\mathrm{~s}), 1370$ $(\mathrm{m}), 1325(\mathrm{~m}), 1187(\mathrm{~s}), 1134(\mathrm{~s}), 1112(\mathrm{~s}), 1096(\mathrm{~s}), 1018(\mathrm{~m}), 802(\mathrm{~s})$.

HRMS (ESI/QTOF) m/z: [M + Na] $]^{+}$Calcd for $\mathrm{C}_{17} \mathrm{H}_{24} \mathrm{NaO}_{5}{ }^{+} 331.1516$; Found 331.1518.

\section{Cesium (2-methyl-4-((4-methylbenzyl)oxy)butan-2-yl)oxy-2-oxoacetate (3h)}

$3 \mathrm{~h}$ was synthesized following step 2 of general procedure $D$ in THF<smiles>COC(=O)C(=O)OC(C)(C)CCOCC=[Zn]</smiles>

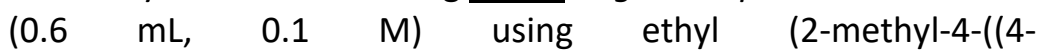
methylbenzyl)oxy)butan-2-yl) oxalate (23h, $0.19 \mathrm{~g}, 0.60 \mathrm{mmol}, 1.0$ equiv) and $1 \mathrm{M}$ aq. $\mathrm{CsOH}(0.6 \mathrm{~mL}, 0.6 \mathrm{mmol}, 1.0$ equiv), affording cesium (2-methyl-4-((4-methylbenzyl)oxy)butan-2-yl)oxy-2oxoacetate (3h, $233 \mathrm{mg}, 0.565 \mathrm{mmol}, 94 \%$ ) as a coloroless amorphous solid.

${ }^{1}$ H NMR $\left(400 \mathrm{MHz}\right.$, DMSO-d $\left.d_{6}\right) \delta 7.23-7.17(\mathrm{~m}, 2 \mathrm{H}, \operatorname{ArH}), 7.14(\mathrm{~d}, J=7.9 \mathrm{~Hz}, 2 \mathrm{H}, \operatorname{ArH}), 4.38(\mathrm{~s}, 2 \mathrm{H}$, $\left.\mathrm{ArCH}_{2}\right), 3.54-3.45\left(\mathrm{~m}, 2 \mathrm{H}, \mathrm{CH}_{2}\right), 2.28\left(\mathrm{~s}, 3 \mathrm{H}, \mathrm{ArCH}_{3}\right), 2.01\left(\mathrm{t}, \mathrm{J}=7.1 \mathrm{~Hz}, 2 \mathrm{H}, \mathrm{CH}_{2}\right), 1.37\left(\mathrm{~s}, 6 \mathrm{H},\left(\mathrm{CH}_{3}\right)_{2}\right)$. ${ }^{13} \mathrm{C}$ NMR $\left(101 \mathrm{MHz}, \mathrm{DMSO}-d_{6}\right) \delta 168.0,163.7,136.9,136.0,129.3,128.0,79.6,72.3,66.3,30.2$ 26.9, 21.2.

HRMS (ESI/QTOF) m/z: [M + Na] ${ }^{+}$Calcd for $\mathrm{C}_{15} \mathrm{H}_{19} \mathrm{CsNaO}_{5}{ }^{+}$435.0179; Found 435.0183 .

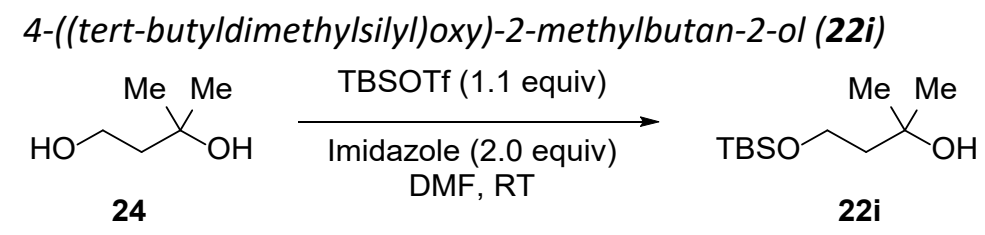

To a solution of 3-methylbutane-1,3-diol (24, $500 \mathrm{mg}, 4.80 \mathrm{mmol}, 1.00$ equiv) and $1 \mathrm{H}$-imidazole (654 $\mathrm{mg}, 9.60 \mathrm{mmol}, 2.00$ equiv) in $\mathrm{N}, \mathrm{N}$-dimethylformamide ( $25 \mathrm{~mL}$ ), TBSOTf (1.4 g, $1.2 \mathrm{~mL}, 5.3 \mathrm{mmol}, 1.1$ equiv) was added dropwise. The reaction mixture was stirred at room temperature until TLC showed full conversion of the starting material. DCM and a 1:1 solution of brine and water were added, the layers were separated and the organic layer was washed with half brine (2x), dried over MgSO4 and solvent removed in vacuo. The crude was purified by flash chromatography $\left(\mathrm{SiO}_{2}, 5 \% \mathrm{EtOAc}\right.$ in pentane) affording 4-((tert-butyldimethylsilyl)oxy)-2-methylbutan-2-ol (22i, $950 \mathrm{mg}, 4.35 \mathrm{mmol}, 91 \%$ yield) as a pale yellow oil. The NMR data was collected and the compound was used in the next step without further analyses. 
Rf (pentane:EtOAc 95:5) $=0.4$.

$\chi_{\mathrm{OH}}$

${ }^{1} \mathbf{H}$ NMR $\left(400 \mathrm{MHz}, \mathrm{CDCl}_{3}\right) \delta: 3.91\left(\mathrm{t}, J=5.8 \mathrm{~Hz}, 2 \mathrm{H}, \mathrm{OCH}_{2}\right), 3.83$ (bs, $1 \mathrm{H}, \mathrm{OH}), 1.70\left(\mathrm{t}, J=5.8 \mathrm{~Hz}, 2 \mathrm{H}, \mathrm{CH}_{2}\right), 1.24\left(\mathrm{~s}, 6 \mathrm{H}, \mathrm{C}\left(\mathrm{CH}_{3}\right)_{2}\right), 0.90(\mathrm{~s}$, $\left.9 \mathrm{H}, \mathrm{C}\left(\mathrm{CH}_{3}\right)_{3}\right), 0.09\left(\mathrm{~s}, 6 \mathrm{H}, \mathrm{Si}\left(\mathrm{CH}_{3}\right)_{2}\right)$.

${ }^{13} \mathrm{C}$ NMR $\left(101 \mathrm{MHz}, \mathrm{CDCl}_{3}\right) \delta 71.0,61.1,43.0,29.4,26.0,18.2,-5.5$.

4-((tert-Butyldimethylsilyl)oxy)-2-methylbutan-2-yl ethyl oxalate (23i)

23i was synthesized following step 1 of general procedure $D$ in THF<smiles>CCOC(=O)C(=O)OC(C)(C)CCOC(C)(C)C</smiles>
$\left(\begin{array}{llll}25 & \mathrm{~mL} & 0.1 & \mathrm{M}\end{array}\right)$ using 4-((tert-butyldimethylsilyl)oxy)-2methylbutan-2-ol (22i, $500 \mathrm{mg}, 2.30 \mathrm{mmol}, 1.0$ equiv), DMAP (28 $\mathrm{mg}, 0.23 \mathrm{mmol}, 10 \mathrm{~mol} \%)$, triethylamine $(0.40 \mathrm{~mL}, 2.8 \mathrm{mmol}, 1.2$ equiv) and ethyl chloro-oxoacetate $(0.30 \mathrm{~mL}, 2.8 \mathrm{mmol}, 1.2$ equiv).

Column chromatography ( $\mathrm{SiO}_{2}, 2 \%$ EtOAc in Pentane) afforded 4-((tert-butyldimethylsilyl)oxy)-2methylbutan-2-yl ethyl oxalate (23i, $517 \mathrm{mg}, 1.62 \mathrm{mmol}, 71 \%$ ) as a yellow oil.

Rf (pentane:EtOAc 98:2) $=0.2$.

${ }^{1} \mathrm{H}$ NMR $\left(400 \mathrm{MHz}, \mathrm{CDCl}_{3}\right) \delta 4.31\left(\mathrm{q}, J=7.2 \mathrm{~Hz}, 2 \mathrm{H}, \mathrm{COOCH}_{2}\right), 3.75\left(\mathrm{t}, J=6.7 \mathrm{~Hz}, 2 \mathrm{H}, \mathrm{OCH}_{2}\right), 2.09(\mathrm{t}, J$ $\left.=6.7 \mathrm{~Hz}, 2 \mathrm{H}, \mathrm{CH}_{2}\right), 1.57\left(\mathrm{~s}, 6 \mathrm{H}, \mathrm{C}\left(\mathrm{CH}_{3}\right)_{2}\right), 1.36\left(\mathrm{t}, J=7.2 \mathrm{~Hz}, 3 \mathrm{H}, \mathrm{COOCH}_{3}\right), 0.88\left(\mathrm{~s}, 9 \mathrm{H}, \mathrm{C}\left(\mathrm{CH}_{3}\right)_{3}\right), 0.05$ $\left(\mathrm{s}, 6 \mathrm{H}, \mathrm{Si}\left(\mathrm{CH}_{3}\right)_{2}\right)$.

${ }^{13} \mathrm{C}$ NMR $\left(101 \mathrm{MHz}, \mathrm{CDCl}_{3}\right) \delta$ 158.7, 157.1, 86.4, 62.9, 59.0, 43.0, 26.3, 26.0, 18.4, 14.1, -5.3.

IR $\left(v_{\max }, \mathrm{cm}^{-1}\right): 2944(\mathrm{~m}), 2891(\mathrm{~m}), 2863(\mathrm{~m}), 1744(\mathrm{~s}), 1468(\mathrm{~m}), 1323(\mathrm{~m}), 1256(\mathrm{~m}), 1190(\mathrm{~s}), 1133$ (s), $1098(\mathrm{~s})$.

HRMS (ESI/QTOF) m/z: [M + Na] ${ }^{+}$Calcd for $\mathrm{C}_{15} \mathrm{H}_{30} \mathrm{NaO}_{5} \mathrm{Si}^{+}$341.1755; Found 341.1752.

Cesium 2-((4-((tert-butyldimethylsilyl)oxy)-2-methylbutan-2-yl)oxy)-2-oxoacetate (3i)

$3 \mathbf{i}$ was synthesized following step 2 of general procedure $D$ in THF<smiles>CCCCOC(=O)C(=O)OC(C)(C)CCOC(C)(C)C</smiles>
$\left(\begin{array}{llll}1.1 & \mathrm{~mL} & 0.1 & \mathrm{M})\end{array}\right)$ using 4-((tert-butyldimethylsilyl)oxy)-2methylbutan-2-yl ethyl oxalate (23i, $350 \mathrm{mg}, 1.10 \mathrm{mmol}, 1.0$ equiv) and $1 \mathrm{M}$ aq. $\mathrm{CsOH}$ (1.1 mL, $1.1 \mathrm{mmol}, 1.0$ equiv). Cesium 2-((4((tert-butyldimethylsilyl)oxy)-2-methylbutan-2-yl)oxy)-2-

oxoacetate (3i, $450 \mathrm{mg}, 1.07 \mathrm{mmol}, 97 \%$ ) was obtained as an offwhite amorphous solid.

${ }^{1} \mathrm{H}$ NMR (400 MHz, DMSO) $\delta 3.65\left(\mathrm{t}, J=7.2 \mathrm{~Hz}, 2 \mathrm{H}, \mathrm{OCH}_{2}\right), 1.94\left(\mathrm{t}, J=7.2 \mathrm{~Hz}, 2 \mathrm{H}, \mathrm{CH}_{2}\right), 1.37(\mathrm{~s}, 6 \mathrm{H}$, $\left.\mathrm{C}\left(\mathrm{CH}_{3}\right)_{2}\right), 0.85\left(\mathrm{~s}, 9 \mathrm{H}, \mathrm{C}\left(\mathrm{CH}_{3}\right)_{3}\right), 0.03\left(\mathrm{~s}, 6 \mathrm{H}, \mathrm{Si}\left(\mathrm{CH}_{3}\right)_{2}\right)$.

${ }^{13} \mathrm{C}$ NMR (101 MHz, DMSO) $\delta 167.5,163.3,79.1,58.7,42.7,26.5,25.8,17.8,-5.3$.

HRMS (ESI/QTOF) m/z: [M + Na] ${ }^{+}$Calcd for $\mathrm{C}_{13} \mathrm{H}_{25} \mathrm{CsNaO}_{5} \mathrm{Si}^{+}$445.0418; Found 445.0418.

4-((tert-butyldiphenylsilyl)oxy)-2-methylbutan-2-ol (22j)

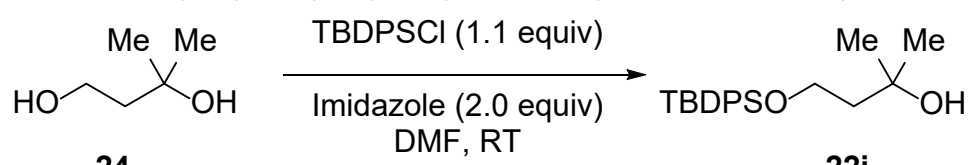

24

22j

To a solution of 3-methylbutane-1,3-diol (24, $500 \mathrm{mg}, 4.80 \mathrm{mmol}, 1.00$ equiv) and $1 \mathrm{H}$-imidazole (654 $\mathrm{mg}, 9.60 \mathrm{mmol}, 2.00$ equiv) in $\mathrm{N}, \mathrm{N}$-dimethylformamide $(25.0 \mathrm{~mL}), \operatorname{TBDPSCl}(1.45 \mathrm{~g}, 1.37 \mathrm{~mL}, 5.28$ mmol, 1.10 equiv) was added dropwise. The reaction mixture was stirred at room temperature until TLC showed full conversion of the starting material. DCM and half brine were added, the layers were separated and the organic layer was washed with half brine (2x), dried over MgSO4 and solvent 
removed under vacuo. The crude product was purified by flash chromatography $\left(\mathrm{SiO}_{2}, 5 \% \mathrm{EtOAc}\right.$ in pentane) affording 4-((tert-butyldiphenylsilyl)oxy)-2-methylbutan-2-ol (22j, $1.64 \mathrm{~g}, 4.80 \mathrm{mmol}, 100 \%$ yield) as a fain yellow oil. The NMR data was collected and the compound was used in the next step without further analyses.

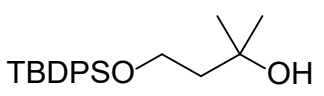

Rf (pentane:EtOAc 95:5) $=0.4$.

${ }^{1} \mathbf{H}$ NMR $\left(400 \mathrm{MHz}, \mathrm{CDCl}_{3}\right) \delta: 7.72-7.65(\mathrm{~m}, 4 \mathrm{H}, \mathrm{ArH}), 7.47-7.36$ $(\mathrm{m}, 6 \mathrm{H}, \mathrm{ArH}), 3.90\left(\mathrm{t}, J=5.8 \mathrm{~Hz}, 2 \mathrm{H}, \mathrm{OCH}_{2}\right), 3.74$ (bs, $\left.1 \mathrm{H}, \mathrm{OH}\right), 1.75$ $\left(\mathrm{t}, J=5.7 \mathrm{~Hz}, 2 \mathrm{H}, \mathrm{CH}_{2}\right), 1.27\left(\mathrm{~s}, 6 \mathrm{H}, \mathrm{C}\left(\mathrm{CH}_{3}\right)_{2} \mathrm{OH}\right), 1.05\left(\mathrm{~s}, 9 \mathrm{H}, \mathrm{C}\left(\mathrm{CH}_{3}\right)_{3}\right)$. ${ }^{13} \mathrm{C}$ NMR $\left(101 \mathrm{MHz}, \mathrm{CDCl}_{3}\right) \delta 135.7,132.9,130.03,128.0,71.1$, $62.2,43.2,29.5,26.9,19.1$.

4-((tert-Butyldiphenylsilyl)oxy)-2-methylbutan-2-yl ethyl oxalate (23j)

23j was synthesized following step 1 of general procedure $D$ in THF<smiles>CCCCOCCC(C)(C)OC(=O)C(=O)OCC</smiles>
$\left(\begin{array}{lll}30 \mathrm{~mL} & 0.1 & \mathrm{M})\end{array}\right)$ using 4-((tert-butyldiphenylsilyl)oxy)-2methylbutan-2-ol (22j, $1.00 \mathrm{~g}, 2.92 \mathrm{mmol}, 1.0$ equiv), DMAP (36 mg, $0.29 \mathrm{mmol}, 10 \mathrm{~mol} \%)$, triethylamine $(0.50 \mathrm{~mL}, 3.5 \mathrm{mmol}, 1.2$ equiv) and ethyl chloro-oxoacetate $(0.40 \mathrm{~mL}, 3.5 \mathrm{mmol}, 1.2$ equiv).

Column chromatography $\left(\mathrm{SiO}_{2}, 2 \%\right.$ EtOAc in Pentane) afforded 4-((tert-butyldiphenylsilyl)oxy)-2methylbutan-2-yl ethyl oxalate (23j, 1.06g, $2.40 \mathrm{mmol}, 82 \%)$ as a pale yellow oil.

Rf (pentane:EtOAc 98:2) $=0.15$.

${ }^{1} \mathrm{H}$ NMR (400 MHz, CDCl $\left.)_{3}\right)$ 8: $7.69-7.64(\mathrm{~m}, 4 \mathrm{H}, \operatorname{ArH}), 7.43-7.35(\mathrm{~m}, 6 \mathrm{H}, \operatorname{ArH}), 4.27(\mathrm{q}, J=7.1 \mathrm{~Hz}$, $\left.2 \mathrm{H}, \mathrm{CO}_{2} \mathrm{CH}_{2} \mathrm{CH}_{3}\right), 3.78\left(\mathrm{t}, J=6.7 \mathrm{~Hz}, 2 \mathrm{H}, \mathrm{OCH}_{2}\right), 2.16\left(\mathrm{t}, J=6.7 \mathrm{~Hz}, 2 \mathrm{H}, \mathrm{CH}_{2}\right), 1.55\left(\mathrm{~s}, 6 \mathrm{H}, \mathrm{C}\left(\mathrm{CH}_{3}\right)_{2}\right), 1.32$ (t, J $\left.=7.1 \mathrm{~Hz}, 3 \mathrm{H}, \mathrm{CO}_{2} \mathrm{CH}_{2} \mathrm{CH}_{3}\right), 1.04\left(\mathrm{~s}, 9 \mathrm{H}, \mathrm{C}\left(\mathrm{CH}_{3}\right)_{3}\right)$.

${ }^{13} \mathrm{C}$ NMR $\left(101 \mathrm{MHz}, \mathrm{CDCl}_{3}\right)$ $\delta: 158.5,157.0,135.6,133.6,129.7,127.7,86.2,62.8,59.8,42.6,26.8$, $26.2,19.1,13.9$.

IR $\left(v_{\max }, \mathrm{cm}^{-1}\right)$ : $3064(\mathrm{w}), 2939(\mathrm{~m}), 2862(\mathrm{~m}), 1743(\mathrm{~s}), 1323(\mathrm{~m}), 1190(\mathrm{~s}), 1104(\mathrm{~s}), 823(\mathrm{~m})$.

HRMS (ESI/QTOF) m/z: [M + Na] ${ }^{+}$Calcd for $\mathrm{C}_{25} \mathrm{H}_{34} \mathrm{NaO}_{5} \mathrm{Si}^{+} 465.2068$; Found 465.2076.

Cesium 2-((4-((tert-butyldiphenylsilyl)oxy)-2-methylbutan-2-yl)oxy)-2-oxoacetate (3j)

$3 \mathbf{j}$ was synthesized following step 2 of general procedure $D$ in THF

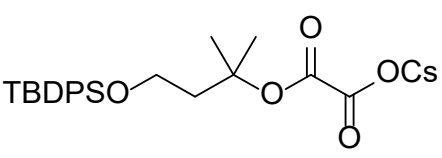
$\left(\begin{array}{llll}1.1 & \mathrm{~mL} & 0.1 & \mathrm{M})\end{array}\right)$ using 4-((tert-butyldiphenylsilyl)oxy)-2methylbutan-2-yl ethyl oxalate (23j, $500 \mathrm{mg}, 1.13 \mathrm{mmol}, 1.0$ equiv) and $1 \mathrm{M}$ aq. $\mathrm{CsOH}$ (1.1 mL, $1.1 \mathrm{mmol}, 1.0$ equiv). Cesium 2-((4-((tertbutyldiphenylsilyl)oxy)-2-methylbutan-2-yl)oxy)-2-oxoacetate (3j, $600 \mathrm{mg}, 1.10 \mathrm{mmol}, 97 \%$ ) was obtained as an off-white amorphous solid.

${ }^{1} \mathrm{H}$ NMR (400 MHz, DMSO) $\delta 7.65-7.58(\mathrm{~m}, 4 \mathrm{H}, \operatorname{ArH}), 7.48-7.41(\mathrm{~m}, 6 \mathrm{H}, \operatorname{ArH}), 3.74(\mathrm{t}, J=7.1 \mathrm{~Hz}$, $\left.2 \mathrm{H}, \mathrm{OCH}_{2}\right), 2.03\left(\mathrm{t}, J=7.1 \mathrm{~Hz}, 2 \mathrm{H}, \mathrm{CH}_{2}\right), 1.34\left(\mathrm{~s}, 6 \mathrm{H}, \mathrm{CH}_{3}\right), 0.99\left(\mathrm{~s}, 9 \mathrm{H}, \mathrm{C}\left(\mathrm{CH}_{3}\right)_{3}\right)$.

${ }^{13} \mathrm{C}$ NMR $(101 \mathrm{MHz}$, DMSO) $\delta 167.5,163.2,135.0,133.2,129.8,127.9,79.1,59.9,42.7,26.7,26.4$, 18.7.

HRMS (ESI/QTOF) m/z: [M + H] $]^{+}$Calcd for $\mathrm{C}_{23} \mathrm{H}_{30} \mathrm{CsO}_{5} \mathrm{Si}^{+}$547.0912; Found 547.0908.

\section{Ethyl 2-(1-methylcyclopent-1-yl)oxy-2-oxoacetate (23k)}<smiles>CCOC(=O)C(=O)OC1(C)CCCC1</smiles>

23k was synthesized following step 1 of general procedure $D$ in THF (16 mL, $0.2 \mathrm{M})$ using 1-methylcyclopentan-1-ol (22k, $337 \mathrm{mg}, 3.36$ mmol, 1.0 equiv), DMAP (21 $\mathrm{mg}, 0.17 \mathrm{mmol}, 5 \mathrm{~mol} \%)$, 
triethylamine ( $0.56 \mathrm{~mL}, 11 \mathrm{mmol}, 1.2$ equiv) and ethyl chlorooxoacetate ( $0.45 \mathrm{~mL}, 11 \mathrm{mmol}, 1.2$ equiv).

Column chromatography ( $\mathrm{SiO}_{2}$, pentane:EtOAc 9:1 to 8:2) afforded ethyl (1-methylcyclopentan-1yl) oxalate (23k, $596 \mathrm{mg}, 2.98 \mathrm{mmol}, 89 \%)$.

Rf (pentane:EtOAc 9:1) $=0.5$.

${ }^{1} \mathrm{H}$ NMR $\left(400 \mathrm{MHz}, \mathrm{CDCl}_{3}\right) \delta 4.31\left(\mathrm{q}, J=7.1 \mathrm{~Hz}, 2 \mathrm{H}, \mathrm{OCH}_{2} \mathrm{CH}_{3}\right), 2.21$ (ttd, $J=10.4,4.8,2.4 \mathrm{~Hz}, 2 \mathrm{H}$, $\left.\mathrm{CH}_{2}\right), 1.83-1.71\left(\mathrm{~m}, 4 \mathrm{H}, \mathrm{CH}_{2}\right), 1.71-1.58\left(\mathrm{~m}, 5 \mathrm{H}, \mathrm{CH}_{2}+\mathrm{CH}_{3}\right), 1.36\left(\mathrm{t}, J=7.1 \mathrm{~Hz}, 3 \mathrm{H}, \mathrm{OCH}_{2} \mathrm{CH}_{3}\right)$.

${ }^{13} \mathrm{C}$ NMR $\left(101 \mathrm{MHz}, \mathrm{CDCl}_{3}\right) \delta 158.8,157.5,94.2,62.9,39.0,24.1,23.8,14.1$.

IR $\left(v_{\max }, \mathrm{cm}^{-1}\right) 2984(\mathrm{~m}), 2942(\mathrm{~m}), 2910(\mathrm{w}), 1762(\mathrm{~s}), 1737(\mathrm{~s}), 1370(\mathrm{~m}), 1324(\mathrm{~m}), 1201(\mathrm{~s}), 1139$ $(\mathrm{s}), 1017(\mathrm{~m}), 846(\mathrm{~m})$.

HRMS (ESI/QTOF) m/z: [M + Na] $]^{+}$Calcd for $\mathrm{C}_{10} \mathrm{H}_{16} \mathrm{NaO}_{4}{ }^{+} 223.0941$; Found 223.0935 .

Cesium 2-(1-methylcyclopent-1-yl)oxy-2-oxoacetate (3k)<smiles>COC(=O)C(=O)OC1(C)CCCC1</smiles>

3k was synthesized following step 2 of general procedure $D$ in THF (1.2 mL, $0.1 \mathrm{M})$ using ethyl (1-methylcyclopent-1-yl) oxalate (23k, $0.37 \mathrm{~g}, 1.8 \mathrm{mmol}, 1.0$ equiv) and $1 \mathrm{M}$ aq. $\mathrm{CsOH}(1.8 \mathrm{~mL}, 1.8 \mathrm{mmol}$, 1.0 equiv), affording cesium 2-(1-methylcyclopent-1-yl)oxy-2oxoacetate (3k, $0.541 \mathrm{~g}, 1.78 \mathrm{mmol}, 97 \%)$.

${ }^{1} \mathrm{H}$ NMR (400 MHz, DMSO) $\delta 1.97$ (dddt, $\left.J=7.1,5.3,3.0,1.8 \mathrm{~Hz}, 2 \mathrm{H}, \mathrm{CH}_{2}\right), 1.72-1.49\left(\mathrm{~m}, 6 \mathrm{H}, \mathrm{CH}_{2}\right)$, $1.46\left(\mathrm{~s}, 3 \mathrm{H}, \mathrm{CH}_{3}\right)$.

${ }^{13} \mathrm{C}$ NMR (101 MHz, DMSO) $\delta 167.5,163.5,87.3,24.3,23.3,14.2$. Consistent with reported data. ${ }^{15}$

Ethyl 2-(1-methylcyclohex-1-yl)oxy-2-oxoacetate (23I)<smiles>CCOC(=O)C(=O)OC1(C)CCCCC1</smiles>

23I was synthesized following step 1 of general procedure $D$ in THF $(90 \mathrm{~mL}, 0.1 \mathrm{M})$ using 1-methylcyclohexan-1-ol $(22 \mathrm{l}, 1.1 \mathrm{~mL}, 8.8$ mmol, 1.0 equiv), DMAP (107 $\mathrm{mg}, 0.876 \mathrm{mmol}, 0.1$ equiv) triethylamine (1.50 mL, $10.5 \mathrm{mmol}, 1.2$ equiv) and ethyl chlorooxoacetate ( $1.20 \mathrm{~mL}, 10.5 \mathrm{mmol}, 1.2$ equiv).

Column chromatography $\left(\mathrm{SiO}_{2}, 2 \%\right.$ EtOAc in Pentane) afforded ethyl (1-methylcyclohexyl) oxalate (23l, $1.18 \mathrm{~g}, 5.51 \mathrm{mmol}, 63 \%$ ) as a pale yellow oil.

Rf (pentane:EtOAc 98:2) $=0.3$.

${ }^{1} \mathrm{H}$ NMR $\left(400 \mathrm{MHz}, \mathrm{CDCl}_{3}\right) \delta: 4.30\left(\mathrm{q}, J=7.12 \mathrm{~Hz}, 2 \mathrm{H}, \mathrm{CO}_{2} \mathrm{CH}_{2}\right), 2.21-2.18\left(\mathrm{~m}, 2 \mathrm{H}, \mathrm{CH}_{2}\right), 1.58-1.44$ (m, $\left.8 \mathrm{H}, \mathrm{CH}_{2}\right), 1.55\left(\mathrm{~s}, 3 \mathrm{H}, \mathrm{CH}_{3}\right), 1.35\left(\mathrm{t}, \mathrm{J}=7.12 \mathrm{~Hz}, 3 \mathrm{H}, \mathrm{CO}_{2} \mathrm{CH}_{2} \mathrm{CH}_{3}\right)$.

${ }^{13} \mathrm{C}$ NMR $\left(101 \mathrm{MHz}, \mathrm{CDCl}_{3}\right)$ 8: 158.8, 157.2, 86.7, 62.8, 36.4, 25.3, 25.1, 22.1, 14.1.

IR $\left(v_{\max }, \mathrm{cm}^{-1}\right)$ : $2979(\mathrm{w}), 2938(\mathrm{~m}), 2864(\mathrm{w}), 1743(\mathrm{~s}), 1454(\mathrm{w}), 1326(\mathrm{~m}), 1192(\mathrm{~s}), 1146(\mathrm{~s})$.

HRMS (ESI/QTOF) $\mathrm{m} / \mathrm{z}$ : [M + Na] $]^{+}$Calcd for $\mathrm{C}_{11} \mathrm{H}_{18} \mathrm{NaO}_{4}{ }^{+} 237.1097$; found 237.1094

\section{Ethyl 2-(1-methylcyclohex-1-yl)oxy-2-oxoacetate (3I)}<smiles>CCOC(=O)C(=O)OC1(C)CCCCC1</smiles>

3 I was synthesized following step 2 of general procedure $D$ in THF $(5.0 \mathrm{~mL}, 0.1 \mathrm{M})$ using ethyl (1-methylcyclohexyl) oxalate (23I, 1.07 g, $5.00 \mathrm{mmol}, 1.0$ equiv) and $1 \mathrm{M}$ aq. $\mathrm{CsOH}(5.0 \mathrm{~mL}, 5.0 \mathrm{mmol}, 1.0$ equiv). Affording cesium 2-((1-methylcyclohexyl)oxy)-2oxoacetate ( $3 \mathrm{l}, 1.4 \mathrm{~g}, 4.4 \mathrm{mmol}, 88 \%)$ as a coloroless amorphous solid. 
${ }^{1} \mathrm{H}$ NMR $\left(400 \mathrm{MHz}\right.$, DMSO-d6) $\delta: 2.08-1.96\left(\mathrm{~m}, 2 \mathrm{H}, \mathrm{CH}_{2}\right), 1.56-1.43\left(\mathrm{~m}, 3 \mathrm{H}, \mathrm{CH}_{2}\right), 1.43-1.29(\mathrm{~m}$, $\left.7 \mathrm{H}, \mathrm{CH}_{2}+\mathrm{CH}_{3}\right), 1.27-1.18\left(\mathrm{~m}, 1 \mathrm{H}, \mathrm{CH}_{2}\right)$.

${ }^{13} \mathrm{C}$ NMR (101 MHz, DMSO) $\delta: 167.7,163.6,79.2,36.2,25.3,25.0,21.5$.

HRMS (ESI/QTOF) m/z: [M - Cs] ${ }^{-}$Calcd for $\mathrm{C}_{9} \mathrm{H}_{13} \mathrm{O}_{4}{ }^{-}$185.0819; Found 185.0819.

\section{Ethyl 2-(1-methylcycloheptan-1-yl)oxy-2-oxoacetate (23m)}<smiles>CCOC(=O)C(=O)OC1(C)CCCCCC1</smiles>

$23 \mathrm{~m}$ was synthesized following step 1 of general procedure $D$ in DCM (35 mL, $0.1 \mathrm{M})$ using 1-methylcycloheptan-1-ol $(22 \mathrm{~m}, 0.30$ $\mathrm{mL}, 3.4 \mathrm{mmol}, 1$ equiv), DMAP (42 $\mathrm{mg}, 0.34 \mathrm{mmol}, 10 \mathrm{~mol} \%$ ), triethylamine $(0.52 \mathrm{~mL}, 3.7 \mathrm{mmol}, 1.1$ equiv) and ethyl chlorooxoacetate $(0.42 \mathrm{~mL}, 3.8 \mathrm{mmol}, 1.1$ equiv).

Column chromatography $\left(\mathrm{SiO}_{2}\right.$, pentane:EtOAc 9:1) afforded ethyl (1-methylcycloheptan-1-yl) oxalate $(23 \mathrm{~m}, 0.373 \mathrm{~g}, 1.84 \mathrm{mmol}, 54 \%)$.

Rf (pentane:EtOAc 9:1) $=0.5$.

${ }^{1} \mathrm{H}$ NMR $\left(400 \mathrm{MHz}, \mathrm{CDCl}_{3}\right) \delta 4.30$ (q, $\left.J=7.1 \mathrm{~Hz}, 2 \mathrm{H}, \mathrm{OCH}_{2} \mathrm{CH}_{3}\right), 2.20$ (ddd, $J=14.9,8.6,1.7 \mathrm{~Hz}, 2 \mathrm{H}$, cyclic- $\left.\mathrm{CH}_{2}\right), 1.82$ (ddd, $J=14.7,9.8,1.8 \mathrm{~Hz}, 2 \mathrm{H}$, cyclic- $\left.\mathrm{CH}_{2}\right), 1.70-1.39\left(\mathrm{~m}, 11 \mathrm{H} \text {, cyclic- }\left(\mathrm{CH}_{2}\right)\right)_{4}+\mathrm{CH}_{3}$, $1.35\left(\mathrm{t}, J=7.2 \mathrm{~Hz}, 3 \mathrm{H}, \mathrm{OCH}_{2} \mathrm{CH}_{3}\right)$.

${ }^{13} \mathrm{C}$ NMR $\left(101 \mathrm{MHz}, \mathrm{CDCl}_{3}\right) \delta 158.9,157.3,91.1,62.8,40.0,29.5,26.6,22.6,14.1$.

IR $\left(v_{\max }, \mathrm{cm}^{-1}\right) 3005(\mathrm{w}), 2929(\mathrm{~m}), 2858(\mathrm{~m}), 1760(\mathrm{~s}), 1736(\mathrm{~s}), 1459(\mathrm{~m}), 1446(\mathrm{~m}), 1371(\mathrm{~m}), 1323$ $(\mathrm{m}), 1205(\mathrm{~s}), 1186(\mathrm{~s}), 1159(\mathrm{~s}), 1128(\mathrm{~s}), 861(\mathrm{~m})$.

HRMS (ESI/QTOF) m/z: [M + Na] $]^{+}$Calcd for $\mathrm{C}_{12} \mathrm{H}_{20} \mathrm{NaO}_{4}{ }^{+}$251.1254; Found 251.1259.

\section{Cesium 2-(1-methylcycloheptan-1-yl)oxy-2-oxoacetate (3m)}

$3 \mathrm{~m}$ was synthesized following step 2 of general procedure $D$ in THF<smiles>CC(C)(O)C(=O)OC(=O)C1(C)CCCCC1</smiles>
$(1.1 \mathrm{~mL}, 0.1 \mathrm{M})$ using ethyl (1-methylcycloheptan-1-yl) oxalate (23m, $0.250 \mathrm{~g}, 1.10 \mathrm{mmol}, 1.0$ equiv) and $1 \mathrm{M}$ aq. $\mathrm{CsOH}(1.1 \mathrm{~mL}$, $1.1 \mathrm{mmol}, 1.0$ equiv), affording cesium 2-(1-methylcycloheptan-1yl)oxy-2-oxoacetate (3m, $0.332 \mathrm{~g}, 1.00 \mathrm{mmol}, 91 \%)$. Amorphous solid.

${ }^{1} \mathrm{H}$ NMR $\left(400 \mathrm{MHz}, \mathrm{DMSO}-d_{6}\right) \delta 2.02$ (ddd, $J=14.3,8.6,1.6 \mathrm{~Hz}, 2 \mathrm{H}$, cyclic- $\mathrm{CH}_{2}$ ), 1.67 (ddd, $J=14.4$, 9.9, $1.9 \mathrm{~Hz}, 2 \mathrm{H}$, cyclic- $\left.\mathrm{CH}_{2}\right), 1.60-1.42\left(\mathrm{~m}, 6 \mathrm{H}\right.$, cyclic- $\left.\mathrm{CH}_{2}\right), 1.41\left(\mathrm{~s}, 3 \mathrm{H}, \mathrm{CH}_{3}\right), 1.40-1.28(\mathrm{~m}, 2 \mathrm{H}$, cyclic- $\left.\mathrm{CH}_{2}\right)$.

${ }^{13} \mathrm{C}$ NMR $\left(101 \mathrm{MHz}, \mathrm{DMSO}-d_{6}\right) \delta 168.2,164.1,83.9,29.3,27.2,22.5 .1$ carbon is unresolved.

\section{Ethyl (1-methylcyclododecyl) oxalate 23n}

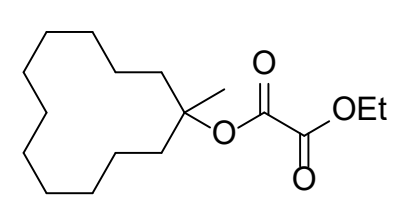

$23 \mathrm{n}$ was synthesized following step 1 of general procedure $D$ in THF (25 mL, 0.1 M) using 1-methylcyclododecan-1-ol (22n, $500 \mathrm{mg}, 2.52$ mmol, 1.0 equiv), DMAP (31 $\mathrm{mg}, 0.25 \mathrm{mmol}, 10 \mathrm{~mol} \%$ ), triethylamine $(0.42 \mathrm{~mL}, 3.0 \mathrm{mmol}, 1.2$ equiv) and ethyl chlorooxoacetate ( $0.34 \mathrm{~mL}, 3.0 \mathrm{mmol}, 1.2$ equiv).

Column chromatography $\left(\mathrm{SiO}_{2}, 20 \%\right.$ EtOAc in Pentane) afforded ethyl (1-methylcyclododecyl) oxalate (23n, $1.08 \mathrm{~g}, 4.25 \mathrm{mmol}, 71 \%$ ) as an off-white amorphous solid.

Rf (pentane:EtOAc 4:1) $=0.5$.

${ }^{1} \mathrm{H}$ NMR $\left(400 \mathrm{MHz}, \mathrm{CDCl}_{3}\right) \delta 4.31\left(\mathrm{q}, J=7.2 \mathrm{~Hz}, 2 \mathrm{H}, \mathrm{CO}_{2} \mathrm{CH}_{2}\right), 2.10-1.98\left(\mathrm{~m}, 2 \mathrm{H}, \mathrm{CH}_{2}\right), 1.74-1.61$ (m, $\left.2 \mathrm{H}, \mathrm{CH}_{2}\right), 1.55\left(\mathrm{~s}, 3 \mathrm{H}, \mathrm{CH}_{3}\right), 1.49-1.23\left(\mathrm{~m}, 21 \mathrm{H}, \mathrm{CH}_{2}+\mathrm{CH}_{2} \mathrm{CH}_{3}\right)$.

${ }^{13} \mathrm{C}$ NMR $\left(101 \mathrm{MHz}, \mathrm{CDCl}_{3}\right)$ ठ 158.9, 157.1, 90.8, 62.9, 32.9, 26.2, 26.2, 24.0, 22.5, 22.0, 19.5, 14.1. 
IR $\left(v_{\max }, \mathrm{cm}^{-1}\right): 2939(\mathrm{~s}), 2861(\mathrm{~m}), 1744(\mathrm{~s}), 1467(\mathrm{~m}), 1375(\mathrm{~m}), 1325(\mathrm{~m}), 1190(\mathrm{~s}), 1152(\mathrm{~s})$

HRMS (ESI/QTOF) m/z: [M + Na] $]^{+}$Calcd for $\mathrm{C}_{17} \mathrm{H}_{30} \mathrm{NaO}_{4}{ }^{+} 321.2036$; Found 321.2037.

Cesium 2-((1-methylcyclododecyl)oxy)-2-oxoacetate (3n)

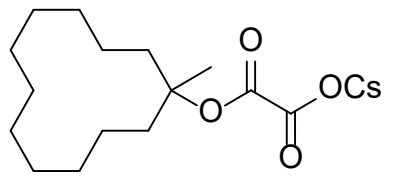

$3 n$ was synthesized following step 2 of general procedure $D$ in THF $(1.0 \mathrm{~mL}, 0.1 \mathrm{M})$ using ethyl (1-methylcyclododecyl) oxalate (23n, $300 \mathrm{mg}, 1.00 \mathrm{mmol}, 1.0$ equiv) and $1 \mathrm{M}$ aq. $\mathrm{CsOH}(1.0 \mathrm{~mL}, 1.00$ mmol, 1.0 equiv). Cesium 2-((1-methylcyclododecyl)oxy)-2oxoacetate (3n, $300 \mathrm{mg}, 0.745 \mathrm{mmol}, 74 \%$ ) was obtained as an offwhite solid.

${ }^{1} \mathrm{H}$ NMR (400 MHz, DMSO) $\delta: 1.90-1.77\left(\mathrm{~m}, 2 \mathrm{H}, \mathrm{CH}_{2}\right), 1.56-1.42\left(\mathrm{~m}, 2 \mathrm{H}, \mathrm{CH}_{2}\right), 1.38\left(\mathrm{~s}, 3 \mathrm{H}, \mathrm{CH}_{3}\right)$, $1.34-1.18\left(\mathrm{~m}, 18 \mathrm{H}, \mathrm{CH}_{2}\right)$.

${ }^{13} \mathrm{C}$ NMR (101 MHz, DMSO) $\delta: 168.1,164.0,83.4,33.21$ 26.3, 26.2, 24.4, 22.3, 22.0, 19.2.

HRMS (ESI/QTOF) m/z: [M + Na] ${ }^{+}$Calcd for $\mathrm{C}_{15} \mathrm{H}_{25} \mathrm{CsNaO}_{4}{ }^{+}$425.0700; Found 425.0695 .

Ethyl (4-methyltetrahydro-2H-pyran-4-yl) oxalate (23o)<smiles>CCOC(=O)C(=O)OC1(C)CCOCC1</smiles>

230 was synthesized following step 1 of general procedure $D$ in THF (45 mL, $0.1 \mathrm{M})$ using 4-methyloxan-4-ol $(\mathbf{2 2 0}, 500 \mathrm{mg}, 4.30 \mathrm{mmol}$, 1.0 equiv), DMAP ( $53 \mathrm{mg}, 0.43 \mathrm{mmol}, 10 \mathrm{~mol} \%)$, triethylamine $(0.72$ $\mathrm{mL}, 5.2 \mathrm{mmol}, 1.2$ equiv) and ethyl chloro-oxoacetate $(0.58 \mathrm{~mL}, 5.2$ mmol, 1.2 equiv).

Column chromatography $\left(\mathrm{SiO}_{2}, 15 \%\right.$ EtOAc in Pentane) afforded ethyl (4-methyltetrahydro- $2 \mathrm{H}$ pyran-4-yl) oxalate (230, $785 \mathrm{mg}, 3.63 \mathrm{mmol}, 84 \%$ ) as a pale yellow oil.

Rf (pentane:EtOAc 85:15) $=0.5$.

${ }^{1} \mathrm{H}$ NMR $\left(400 \mathrm{MHz}, \mathrm{CDCl}_{3}\right) \delta: 4.33\left(\mathrm{q}, J=7.1 \mathrm{~Hz}, 2 \mathrm{H}, \mathrm{CO}_{2} \mathrm{CH}_{2} \mathrm{CH}_{3}\right), 3.83-3.59\left(\mathrm{~m}, 4 \mathrm{H}, \mathrm{OCH}_{2}\right), 2.27-$ $2.17\left(\mathrm{~m}, 2 \mathrm{H}, \mathrm{CH}_{2}\right), 1.78\left(\mathrm{ddd}, J=14.6,10.1,5.0 \mathrm{~Hz}, 2 \mathrm{H}, \mathrm{CH}_{2}\right), 1.62\left(\mathrm{~s}, 3 \mathrm{H}, \mathrm{CH}_{3}\right), 1.37(\mathrm{t}, J=7.1 \mathrm{~Hz}, 3 \mathrm{H}$, $\left.\mathrm{CO}_{2} \mathrm{CH}_{2} \mathrm{CH}_{3}\right)$.

${ }^{13} \mathrm{C}$ NMR $\left(101 \mathrm{MHz}, \mathrm{CDCl}_{3}\right)$ 8: 158.4, 157.1, 83.2, 63.7, 63.1, 36.6, 25.0, 14.1.

IR $\left(v_{\max }, \mathrm{cm}^{-1}\right): 2968(\mathrm{w}), 2864(\mathrm{w}), 1744(\mathrm{~s}), 1462(\mathrm{w}), 1324(\mathrm{~m}), 1192(\mathrm{~s}), 1134(\mathrm{~s}), 1023(\mathrm{~m})$.

HRMS (ESI/QTOF) m/z: [M + Na] ${ }^{+}$Calcd for $\mathrm{C}_{10} \mathrm{H}_{16} \mathrm{NaO}_{5}{ }^{+}$239.0890; Found 239.0894.

cesium (4-methyltetrahydro-2H-pyran-4-yl) oxalate (3o)

3o was synthesized following step 2 of general procedure $D$ in THF<smiles>CCOC(=O)C(=O)OC1(C)CCOCC1</smiles>
(2.5 mL, $0.1 \mathrm{M})$ using ethyl (4-methyltetrahydro-2H-pyran-4-yl) oxalate (23o, $541 \mathrm{mg}, 2.50 \mathrm{mmol}, 1.0$ equiv) and $1 \mathrm{M}$ aq. $\mathrm{CsOH}(2.5$ $\mathrm{mL}, 2.50 \mathrm{mmol}, 1.0$ equiv). Cesium 2-((3-methyl-1-phenylpentan-3yl)oxy)-2-oxoacetate (3o, $725 \mathrm{mg}, 2.27 \mathrm{mmol}, 91 \%$ ) was obtained as an off-white amorphous solid.

${ }^{1} \mathrm{H}$ NMR (400 MHz, DMSO) $\delta: 3.66-3.49\left(\mathrm{~m}, 4 \mathrm{H}, \mathrm{OCH}_{2}\right), 2.04-1.93\left(\mathrm{~m}, 2 \mathrm{H}, \mathrm{CH}_{2}\right), 1.67-1.53(\mathrm{~m}, 2 \mathrm{H}$, $\left.\mathrm{CH}_{2}\right), 1.45\left(\mathrm{~s}, 3 \mathrm{H}, \mathrm{CH}_{3}\right)$.

${ }^{13} \mathrm{C}$ NMR (101 MHz, DMSO-d $\left.\mathrm{d}_{6}\right) \delta: 167.6,163.2,76.5,62.9,36.6,24.9$.

HRMS (ESI/QTOF) m/z: [M + Na] $]^{+}$Calcd for $\mathrm{C}_{8} \mathrm{H}_{11} \mathrm{CsNaO}_{5}{ }^{+}$342.9553; Found 342.9553 . 
23p was synthesized following step 1 of general procedure $D$ in<smiles>CCOC(=O)C(=O)OC12CC3CC(CC(C3)C1)C2</smiles>
DCM (25 mL, $0.1 \mathrm{M})$ using adamant-1-ol (22p, $378 \mathrm{mg}, 2.48 \mathrm{mmol}$, 1.0 equiv), DMAP (30.4 mg, $248 \mathrm{mmol}, 10 \mathrm{~mol} \%$ ), triethylamine (0.41 $\mathrm{mL}, 3.0 \mathrm{mmol}, 1.2$ equiv) and ethyl chloro-oxoacetate $(0.34$ $\mathrm{mL}, 3.0 \mathrm{mmol}, 1.2$ equiv).

Column chromatography $\left(\mathrm{SiO}_{2}, 15 \%\right.$ EtOAc in Pentane) afforded ethyl 2-(((1S,3S)-adamantan-1yl)oxy)-2-oxoacetate (2op, $442 \mathrm{mg}, 1.75 \mathrm{mmol}, 71 \%$ ) as a pale yellow oil.

Rf (pentane:EtOac 9:1) $=0.5$.

${ }^{1} \mathrm{H}$ NMR $\left(400 \mathrm{MHz}, \mathrm{CDCl}_{3}\right) \delta 4.31\left(\mathrm{q}, J=7.1 \mathrm{~Hz}, 2 \mathrm{H}, \mathrm{OCH}_{2} \mathrm{CH}_{3}\right), 2.19\left(\mathrm{~d}, J=2.7 \mathrm{~Hz}, 9 \mathrm{H}\right.$, ad- $\left.\mathrm{CH}_{\mathrm{x}}\right), 1.76-$ $1.55\left(\mathrm{~m}, 6 \mathrm{H}, \mathrm{ad}-\mathrm{CH}_{\mathrm{x}}\right), 1.36\left(\mathrm{t}, J=7.1 \mathrm{~Hz}, 3 \mathrm{H}, \mathrm{OCH}_{2} \mathrm{CH}_{3}\right)$.

${ }^{13} \mathrm{C}$ NMR $\left(101 \mathrm{MHz} \mathrm{CDCl}_{3}\right) \delta 158.8,156.8,85.1,62.9,41.0,36.1,31.1,14.1$.

IR $\left(v_{\max }, \mathrm{cm}^{-1}\right) 2911(\mathrm{~m}), 2854(\mathrm{w}), 1760(\mathrm{~s}), 1733(\mathrm{~s}), 1176$ (s), 1155 (s), $1044(\mathrm{~m})$.

HRMS (APPI/LTQ-Orbitrap) m/z: [M + Na] ${ }^{+}$Calcd for $\mathrm{C}_{14} \mathrm{H}_{20} \mathrm{NaO}_{4}{ }^{+}$275.1254; Found 275.1256.

Cesium 2-(((1S,3S)-adamantan-1-yl)oxy)-2-oxoacetate (3p)

$3 p$ was synthesized following step 2 of general procedure $D$ in THF<smiles>COC(=O)C(=O)OC12CC3CC(CC(C3)C1)C2</smiles>
$(2.5 \mathrm{~mL}, 0.1 \mathrm{M})$ using ethyl 2-(((1S,3S)-adamantan-1-yl)oxy)-2oxalate (23p, $252 \mathrm{mg}, 1.00 \mathrm{mmol}, 1.0$ equiv) and $1 \mathrm{M}$ aq. $\mathrm{CsOH}(2.5$ $\mathrm{mL}, 2.5 \mathrm{mmol}, 1.0$ equiv). cesium 2-(((1S,3S)-adamantan-1-yl)oxy)2-oxoacetate $(3 p, 0.32 \mathrm{~g}, 0.91 \mathrm{mmol}, 91 \%)$ was obtained as an offwhite amorphous solid.

${ }^{1} \mathrm{H}$ NMR (400 MHz, DMSO) $\delta: 2.12-2.07(\mathrm{~m}, 3 \mathrm{H}, \mathrm{CH}), 2.06-1.99\left(\mathrm{~m}, 6 \mathrm{H}, \mathrm{CH}_{2}\right), 1.64-1.59(\mathrm{~m}, 6 \mathrm{H}$, $\mathrm{CH}_{2}$ ).

${ }^{13} \mathrm{C}$ NMR (101 MHz, DMSO) $\delta: 167.3,163.4,78.0,41.0,35.8,30.2$.

HRMS (ESI/QTOF) m/z: [M - Cs] $]^{-}$Calcd for $\mathrm{C}_{12} \mathrm{H}_{15} \mathrm{O}_{4}{ }^{-}$223.0976; Found 223.0974.

\section{Ethyl (2-methyl-4-((4-methylbenzyl)oxy)butan-2-yl) oxalate (23w)}<smiles>CCOC(=O)C(=O)O[C@]1(C)CC[C@H]2C(C)CCC[C@]2(C)C1(C)C</smiles>

Column chromatography (SiO mmol, 1.1 equiv).

23w was synthesized following step 1 of general procedure $D$ in DCM (50 mL, $0.1 \mathrm{M})$ using Cedrol $(22 \mathrm{w}, 1.00 \mathrm{~g}, 4.46 \mathrm{mmol}, 1.0$ equiv), DMAP (0.054 g, $0.45 \mathrm{mmol}, 10 \mathrm{~mol} \%)$, triethylamine $(0.68$ $\mathrm{mL}, 4.9 \mathrm{mmol}, 1.1$ equiv) and ethyl chloro-oxoacetate $(0.55 \mathrm{~mL}, 4.9$

methylbenzyl)oxy)butan-2-yl) oxalate (23w, $0.343 \mathrm{~g}, 0.1 .06 \mathrm{mmol}, 24 \%)$.

Rf (pentane:EtOAc 9:1) $=0.45$.

${ }^{1} \mathrm{H}$ NMR $\left(400 \mathrm{MHz}, \mathrm{CDCl}_{3}\right) \delta 4.30\left(\mathrm{q}, J=7.1 \mathrm{~Hz}, 2 \mathrm{H}, \mathrm{OCH}_{2} \mathrm{CH}_{3}\right), 2.46-2.40(\mathrm{~m}, 1 \mathrm{H}$, aliphatic- $\mathrm{CH}$ or $\left.\mathrm{CH}_{2}\right), 2.17$ (ddt, $\mathrm{J}=13.6,5.8,1.7 \mathrm{~Hz}, 1 \mathrm{H}$, aliphatic- $\mathrm{CH}$ or $\left.\mathrm{CH}_{2}\right), 2.13-2.00\left(\mathrm{~m}, 1 \mathrm{H}\right.$, aliphatic- $\mathrm{CH}$ or $\left.\mathrm{CH}_{2}\right)$, $1.94-1.78\left(\mathrm{~m}, 2 \mathrm{H}\right.$, aliphatic- $\mathrm{CH}$ or $\left.\mathrm{CH}_{2}\right), 1.74-1.64\left(\mathrm{~m}, 2 \mathrm{H}\right.$, aliphatic- $\mathrm{CH}$ or $\left.\mathrm{CH}_{2}\right), 1.62(\mathrm{~d}, \mathrm{~J}=1.0 \mathrm{~Hz}$, $\left.3 \mathrm{H}, \mathrm{CH}_{3}\right), 1.59-1.47\left(\mathrm{~m}, 2 \mathrm{H}\right.$, aliphatic- $\mathrm{CH}$ or $\left.\mathrm{CH}_{2}\right), 1.46-1.33\left(\mathrm{~m}, 6 \mathrm{H}\right.$, aliphatic- $\mathrm{CH}$ or $\left.\mathrm{CH}_{2}+\mathrm{OCH}_{2} \mathrm{CH}_{3}\right)$, $1.32-1.23\left(\mathrm{~m}, 1 \mathrm{H}\right.$, aliphatic- $\mathrm{CH}$ or $\left.\mathrm{CH}_{2}\right), 1.18\left(\mathrm{~s}, 3 \mathrm{H}, \mathrm{CH}_{3}\right), 0.99\left(\mathrm{~s}, 3 \mathrm{H}, \mathrm{CH}_{3}\right), 0.84(\mathrm{~d}, J=7.1 \mathrm{~Hz}, 3 \mathrm{H}$, $\mathrm{CH}_{2} \mathrm{CH}_{3}$ ).

${ }^{13} \mathrm{C}$ NMR $\left(101 \mathrm{MHz}, \mathrm{CDCl}_{3}\right) \delta 158.8,157.1,91.2,62.8,57.0,56.8,54.0,43.7,41.4,41.2,37.1,33.0$, 31.4, 28.5, 27.1, 25.5, 25.4, 15.6, 14.1 .

IR $\left(v_{\max }, \mathrm{cm}^{-1}\right) 2990(\mathrm{w}), 2939(\mathrm{w}), 2876(\mathrm{w}), 1738(\mathrm{~s}), 1373(\mathrm{~s}), 1236(\mathrm{~s}), 1186(\mathrm{~m}), 1044$ (s).

HRMS (ESI/QTOF) m/z: [M + Na] ${ }^{+}$Calcd for $\mathrm{C}_{19} \mathrm{H}_{30} \mathrm{NaO}_{4}{ }^{+}$345.2036; Found 345.2029. 
(-)-Cedrol derived cesium oxalate: cesium (2-methyl-4-((4-methylbenzyl)oxy)butan-2-yl)oxy-2oxoacetate (3w)

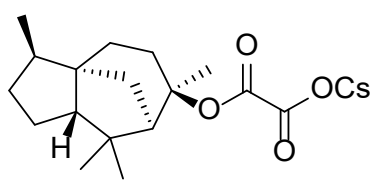

3w was synthesized following step 2 of general procedure $D$ in THF $\left.\begin{array}{llll}0.78 & \mathrm{~mL} & 0.1 & \mathrm{M})\end{array}\right)$ using ethyl (2-methyl-4-((4methylbenzyl)oxy)butan-2-yl) oxalate (23w, $0.250 \mathrm{~g}, 0.775 \mathrm{mmol}$, 1.0 equiv) and $1 \mathrm{M}$ aq. $\mathrm{CsOH}(0.78 \mathrm{~mL}, 0.78 \mathrm{mmol}, 1.0$ equiv), affording cesium (2-methyl-4-((4-methylbenzyl)oxy)butan-2-yl)oxy2-oxoacetate (3w, $0.330 \mathrm{~g}, 0.774 \mathrm{mmol}, 100 \%)$. Amorphous white amorphous solid.

${ }^{1} \mathrm{H}$ NMR $\left(400 \mathrm{MHz}, \mathrm{DMSO}-\mathrm{d}_{6}\right) \delta 2.34(\mathrm{~d}, J=5.1 \mathrm{~Hz}, 1 \mathrm{H}$, aliphatic- $\mathrm{CH}), 1.90-1.71(\mathrm{~m}, 4 \mathrm{H}$, aliphatic$\mathrm{CH}), 1.68-1.54(\mathrm{~m}, 2 \mathrm{H}$, aliphatic- $\mathrm{CH}$ or $\mathrm{CH} 2), 1.45\left(\mathrm{~s}, 4 \mathrm{H}\right.$, aliphatic- $\mathrm{CH}$ or $\left.\mathrm{CH} 2+\mathrm{CH}_{3}\right), 1.42-1.19(\mathrm{~m}$, $5 \mathrm{H}$, aliphatic- $\mathrm{CH}$ or $\mathrm{CH} 2), 1.16\left(\mathrm{~s}, 3 \mathrm{H}, \mathrm{CH}_{3}\right), 0.91\left(\mathrm{~s}, 3 \mathrm{H}, \mathrm{CH}_{3}\right), 0.81\left(\mathrm{~d}, \mathrm{~J}=7.1 \mathrm{~Hz}, 3 \mathrm{H}, \mathrm{CHCH}_{3}\right)$.

${ }^{13} \mathrm{C}$ NMR $\left(101 \mathrm{MHz}\right.$, DMSO- $\left.d_{6}\right) \delta 167.3,163.5,83.8,56.4,56.2,53.6,43.0,40.7,40.3,36.4,33.0,30.6$, 28.4, 27.3, 25.7, 24.9, 15.5 .

HRMS (ESI/QTOF) m/z: [M - Cs] $]^{-}$Calcd for $\mathrm{C}_{17} \mathrm{H}_{25} \mathrm{O}_{4}{ }^{-}$293.1758; Found 293.1751.

(R)-Ethyl (1-isopropyl-4-methylcyclohex-3-en-1-yl) oxalate (23x)<smiles>CCOC(=O)C(=O)OC1(F)CC=C(C)CC1</smiles>

23x was synthesized following step 1 of general procedure $D$ in THF (60 mL, 0.1 M) using (-)-terpinen-4-ol (22x, $1.00 \mathrm{~mL}, 6.00 \mathrm{mmol}, 1.0$ equiv), DMAP (73 $\mathrm{mg}, 0.60 \mathrm{mmol}, 10 \mathrm{~mol} \%)$, triethylamine $(1.00$ $\mathrm{mL}, 7.20 \mathrm{mmol}, 1.2$ equiv) and ethyl chloro-oxoacetate $(0.80 \mathrm{~mL}$, $7.2 \mathrm{mmol}, 1.2$ equiv).

Column chromatography $\left(\mathrm{SiO}_{2}, 2 \%\right.$ EtOAc in Pentane) afforded (R)-ethyl (1-isopropyl-4methylcyclohex-3-en-1-yl) oxalate (23x, $1.08 \mathrm{~g}, 4.25 \mathrm{mmol}, 71 \%)$ as a pale yellow oil.

Rf (pentane:EtOAc 98:2) $=0.4$.

${ }^{1} \mathrm{H}$ NMR $\left(400 \mathrm{MHz}, \mathrm{CDCl}_{3}\right) \delta: 5.29-5.21(\mathrm{~m}, 1 \mathrm{H}, \mathrm{C}=\mathrm{CH}), 4.29\left(\mathrm{q}, J=7.1 \mathrm{~Hz}, 2 \mathrm{H}, \mathrm{CO}_{2} \mathrm{CH}_{2}\right), 2.71$ (hept, $\left.J=6.9 \mathrm{~Hz}, 1 \mathrm{H}, \mathrm{CH}\left(\mathrm{CH}_{3}\right)_{2}\right), 2.54-2.43\left(\mathrm{~m}, 2 \mathrm{H}, \mathrm{CH}_{2}\right), 2.29-2.19\left(\mathrm{~m}, 1 \mathrm{H}, \mathrm{CH}_{2}\right), 2.11-1.98\left(\mathrm{~m}, 1 \mathrm{H}, \mathrm{CH}_{2}\right)$, $1.97-1.87\left(\mathrm{~m}, 1 \mathrm{H}, \mathrm{CH}_{2}\right), 1.78-1.68\left(\mathrm{~m}, 1 \mathrm{H}, \mathrm{CH}_{2}\right), 1.73-1.62\left(\mathrm{~m}, 3 \mathrm{H}, \mathrm{CH}_{3}\right), 1.34(\mathrm{t}, J=7.1 \mathrm{~Hz}, 3 \mathrm{H}$, $\left.\mathrm{CO}_{2} \mathrm{CH}_{2} \mathrm{CH}_{3}\right), 0.95\left(\mathrm{~d}, J=6.9 \mathrm{~Hz}, 3 \mathrm{H}, \mathrm{CH}\left(\mathrm{CH}_{3}\right)_{2}\right), 0.94\left(\mathrm{~d}, J=6.9 \mathrm{~Hz}, 3 \mathrm{H}, \mathrm{CH}\left(\mathrm{CH}_{3}\right)_{2}\right)$.

${ }^{13} \mathrm{C}$ NMR $\left(101 \mathrm{MHz}, \mathrm{CDCl}_{3}\right)$ ठ: 158.9, 157.6, 133.8, 117.2, 91.1, 62.7, 32.7, 29.9, 27.9, 27.3, 23.3, 17.7, 17.2, 14.1 .

IR $\left(v_{\max }, \mathrm{cm}^{-1}\right): 2973(\mathrm{~m}), 2933(\mathrm{~m}), 1738(\mathrm{~s}), 1444(\mathrm{~m}), 1380(\mathrm{~m}), 1324(\mathrm{~m}), 1180(\mathrm{~s}), 1014(\mathrm{~m})$.

HRMS (ESI/QTOF) m/z: [M + Na] $]^{+}$Calcd for $\mathrm{C}_{14} \mathrm{H}_{22} \mathrm{NaO}_{4}{ }^{+}$277.1410; Found 277.1415.

(-)Terpinen-4-ol derived cesium oxalate: cesium (R)-2-((1-isopropyl-4-methylcyclohex-3-en-1-yl)oxy)-2oxoacetate $(3 x)$

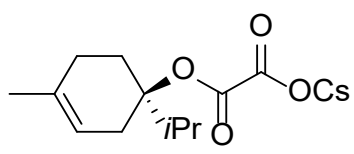

$3 \mathbf{x}$ was synthesized following step 2 of general procedure $D$ in THF $(2.0 \mathrm{~mL}, 0.1 \mathrm{M})$ using $(R)$-ethyl (1-isopropyl-4-methylcyclohex-3-en1 -yl) oxalate (23x, $509 \mathrm{mg}, 2.00 \mathrm{mmol}, 1.0$ equiv) and $1 \mathrm{M}$ aq. $\mathrm{CsOH}$ $(2.0 \mathrm{~mL}, 2.0 \mathrm{mmol}, 1.0$ equiv). Cesium $(R)-2-((1-$-isopropyl-4methylcyclohex-3-en-1-yl)oxy)-2-oxoacetate (3x, $661 \mathrm{mg}, 1.85$ $\mathrm{mmol}, 92 \%)$ was obtained as an off-white amorphous solid.

${ }^{1} \mathrm{H}$ NMR (400 MHz, DMSO) $\delta: 5.20-5.16(\mathrm{~m}, 1 \mathrm{H}, \mathrm{C}=\mathrm{CH}), 2.66\left(\mathrm{p}, \mathrm{J}=7.0 \mathrm{~Hz}, 1 \mathrm{H}, \mathrm{CH}\left(\mathrm{CH}_{3}\right)_{2}\right), 2.40-2.31$ $\left(\mathrm{m}, 1 \mathrm{H}, \mathrm{CH}_{2}\right), 2.23-2.04\left(\mathrm{~m}, 2 \mathrm{H}, \mathrm{CH}_{2}\right), 2.04-1.90\left(\mathrm{~m}, 1 \mathrm{H}, \mathrm{CH}_{2}\right), 1.86-1.71\left(\mathrm{~m}, 1 \mathrm{H}, \mathrm{CH}_{2}\right), 1.60(\mathrm{~s}, 3 \mathrm{H}$, $\left.\left.\mathrm{CH}_{3}\right), 1.59-1.50\left(\mathrm{~m}, 1 \mathrm{H}, \mathrm{CH}_{2}\right), 0.86\left(\mathrm{~d}, J=7.0 \mathrm{~Hz}, 3 \mathrm{H}, \mathrm{CH}\left(\mathrm{CH}_{3}\right)_{2}\right), 0.84(\mathrm{~d}, J=7.0 \mathrm{~Hz}, 3 \mathrm{H}), \mathrm{CH}\left(\mathrm{CH}_{3}\right)_{2}\right)$.

${ }^{13} \mathrm{C}$ NMR (101 MHz, DMSO) $\delta: 168.1,163.5,132.8,117.9,83.3,32.0,29.5,27.7,26.7,23.2,17.4$, 16.7. 


\section{Photochemical experimental set-up}

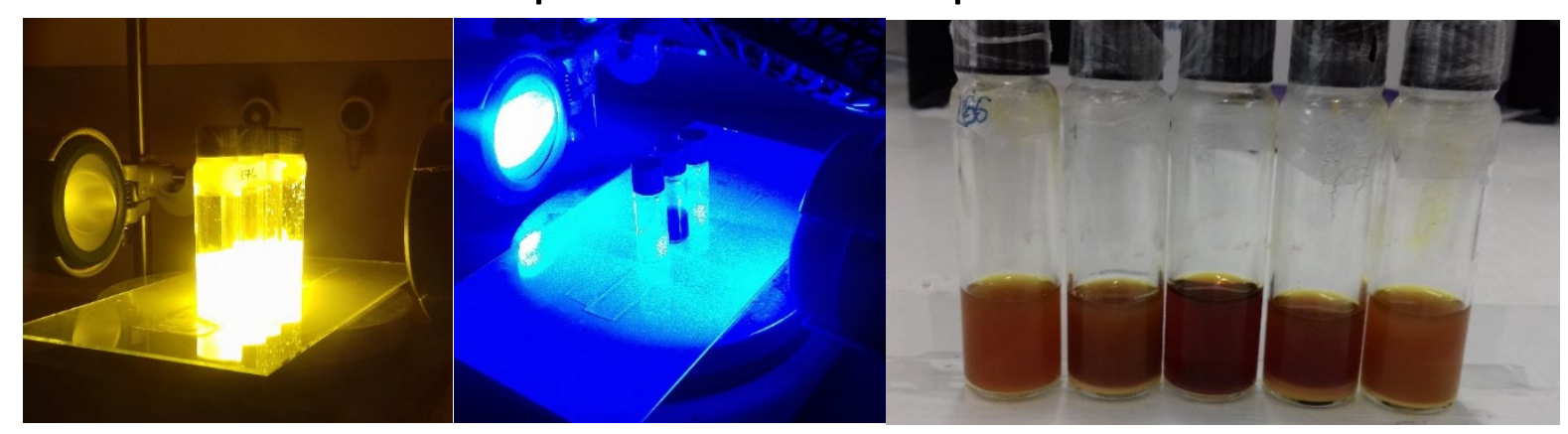

Figure S1. Left: Scope scale reactions (photo taken with a filter applied to it). Middle: optimization scale. Right: Scope scale reactions after irradiation (with PC, same appearance for PC-free reactions without)

\section{Photomediated deoxygenation-alkynylation}

\subsection{Optimization studies method A (4CzIPN catalyzed)}<smiles>CC(C)(C)OC(=O)C(=O)OC(C)(C)CCc1ccccc1</smiles>

3a<smiles>O=C1OI(C#Cc2ccccc2)c2ccccc21</smiles>

2a

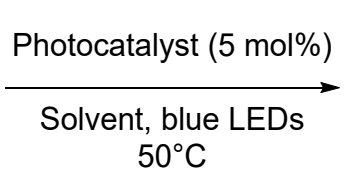

$50^{\circ} \mathrm{C}$

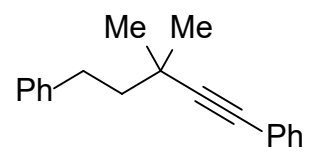

$4 a$

Experimental procedure: an oven dried dram vial $(2 \mathrm{~mL})$, equipped with a magnetic stirrer, was charged with the solid components following table S1: cesium oxalate 3a, PhEBX (2a), photocatalyst, additive (as specified). The reaction vial was sealed with a septum. After 3 vacuum/N2 cycles (backfilling with Ar on the last cycle), dry degassed (freeze pump thaw) solvent was added and the septum was replaced with a screw cap under a flux of $\operatorname{Ar} .{ }^{19}$ The reactions were placed between $2 \mathrm{x}$ $440 \mathrm{~nm}$ Kessil lamps (at ca. $10 \mathrm{~cm}$ distance from both lamps (no ventilation, $\mathrm{T}=\mathrm{ca} .50{ }^{\circ} \mathrm{C}$, with ventilation $\mathrm{T}=\mathrm{ca} \cdot 30-35^{\circ} \mathrm{C}$ as specified) and stirred under irradiation for 18 hours or 24 hours (as specified). The reaction was filtered through a small celite plug which was washed with $\mathrm{CH}_{2} \mathrm{Cl}_{2}$. The reaction crude was concentrated in vacuo, diluted with $\mathrm{CDCl}_{3}$ and 1 equiv of $\mathrm{CH}_{2} \mathrm{Br}_{2}$ was added as internal standard for ${ }^{1} \mathrm{H}$ NMR analysis.

Table S1. Optimization pf method A

\begin{tabular}{lllll}
\hline Entry & Solvent $(\mathbf{M})$ & Photocatalyst & $\begin{array}{l}\text { Stoichiometry } \\
\text { (3a: 2a) }\end{array}$ & ${ }^{\mathbf{1}} \mathbf{H}$ NMR yield (\%) \\
\hline $\mathbf{1}$ & DMSO (0.1 M) & $1 \mathrm{a}$ & $1: 1.5$ & 52 \\
$\mathbf{2}$ & MeCN (0.1 M) & $1 \mathrm{a}$ & $1: 1.5$ & 40 \\
$\mathbf{3}$ & $\mathrm{DME} / \mathrm{DMF}(0.1 \mathrm{M})$ & $1 \mathrm{a}$ & $1: 1.5$ & 70 \\
$\mathbf{4}$ & $\mathrm{DME} / \mathrm{DMF}+10$ eq $\mathrm{H}_{2} \mathrm{O}(0.1 \mathrm{M})$ & $1 \mathrm{a}$ & $1: 1.5$ & 55 \\
\hline
\end{tabular}

\footnotetext{
${ }^{19}$ Use of a screw cap or crimp cap is of great importance to prevent solvent evaporation as the irradiation causes an increase in temperature. When using a test-tube/septum set-up, the latter would fly off within an hour of irradiation. As shown in the optimization section DCE is not as good a solvent as $\mathrm{CH}_{2} \mathrm{Cl}_{2}$.
} 


\begin{tabular}{|c|c|c|c|c|}
\hline 5 & THF (0.1 M) & $1 a$ & $1: 1.5$ & 22 \\
\hline 6 & DCE (0.1 M) & $1 a$ & 1:1.5 & 67 \\
\hline 7 & $\mathrm{DCM}(0.1 \mathrm{M})$ & $1 a$ & 1:1.5 & 75 \\
\hline 8 & DCM (0.1 M) & $1 b$ & $1: 1.5$ & 75 \\
\hline 9 & DCM (0.1 M) & $1 c$ & $1: 1.5$ & 50 \\
\hline 10 & DCM (0.1 M) & DCA & $1: 1.5$ & 55 \\
\hline 11 & DCM (0.1 M) & MesAcr. $\mathrm{BF}_{4}$ & $1: 1.5$ & 53 \\
\hline 12 & DCM (0.1 M) & {$\left[\mathrm{Ru}(\mathrm{bpy})_{3}\right] \mathrm{PF}_{6}$} & $1: 1.5$ & $<10 \%$ decomp \\
\hline 13 & DCM (0.1 M) & {$\left[\mathrm{Ru}(\mathrm{bpz})_{3}\right] \mathrm{PF}_{6}$} & $1: 1.5$ & 20 \\
\hline 14 & $\mathrm{DCM}(0.1 \mathrm{M})$ & $1 a$ & $1.2: 1$ & 45 \\
\hline 15 & DCM (0.1 M) & $1 a$ & $1: 1$ & 64 \\
\hline 16 & DCM (0.1 M) & $1 a$ & $1:: 1.2$ & 56 \\
\hline 17 & DCM (0.1 M) & $1 a$ & $1: 1.8$ & 70 \\
\hline 18 & $\mathrm{DCM}(0.1 \mathrm{M})$ & $1 a$ & $1: 2.5$ & 75 \\
\hline 19 & $\mathrm{DCM}(0.5 \mathrm{M})$ & $1 a$ & $1: 1.5$ & 75 \\
\hline 20 & DCM (0.05 M) & $1 a$ & 1:1.5 & 73 \\
\hline 21 & DCM (0.02 M) & $1 a$ & $1: 1.5$ & 55 \\
\hline $22^{\mathrm{a}}$ & $\mathrm{DCM}(0.1 \mathrm{M})$ & $1 a$ & $1: 1.5$ & 65 \\
\hline
\end{tabular}

aPerformed with 0.3 equiv BIOAc as an additive

\subsection{Optimization studies method B (Excited state PhEBX 2a)}<smiles>CC(C)(CCc1ccccc1)OC(=O)C(=O)O[Na]</smiles>

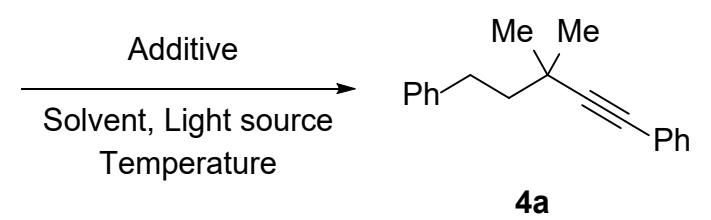

Experimental procedure: an oven dried dram vial $(2 \mathrm{~mL})$, equipped with a magnetic stirrer, was charged with the solid components following table S2: cesium oxalate $3 a, \operatorname{PhEBX}(\mathbf{2 a}), \mathrm{CsOBz}_{2} \mathrm{Cs}_{2} \mathrm{CO}_{3}$. The reaction vial was sealed with a septum. After 3 vacuum/N2 cycles (backfilling with Ar on the last cycle), dry degassed (freeze pump thaw) solvent was added, followed by the liquid additive THF or $\gamma$ terpinene (as specified) and the septum was replaced with a screw cap under a flux of $A r .{ }^{19}$ The reactions were placed between $2 \times 440 \mathrm{~nm}$ Kessil lamps (unless specified otherwise) at ca. $10 \mathrm{~cm}$ distance from both lamps (no ventilation, $\mathrm{T}=\mathrm{ca} .50^{\circ} \mathrm{C}$, with ventilation $\mathrm{T}=\mathrm{ca} .30-35^{\circ} \mathrm{C}$ as specified) and stirred under irradiation for 18 hours or 24 hours (as specified). The reaction was filtered through a small celite plug which was washed with $\mathrm{CH}_{2} \mathrm{Cl}_{2}$. The reaction crude was concentrated in vacuo, diluted with $\mathrm{CDCl}_{3}$ and 1 equiv of $\mathrm{CH}_{2} \mathrm{Br}_{2}$ was added as internal standard for ${ }^{1} \mathrm{H}$ NMR analysis.

Table S2. Optimization of method B

\begin{tabular}{llllllll}
\hline Entry & $\begin{array}{l}\mathbf{2 a} \\
\text { (equiv) }\end{array}$ & $\begin{array}{l}\text { Additive } \\
\text { (equiv) }\end{array}$ & solvent $(\mathbf{M})$ & $\mathbf{T}\left({ }^{\circ} \mathbf{C}\right)$ & $\begin{array}{l}\boldsymbol{\lambda} \\
(\mathbf{n m})\end{array}$ & $\begin{array}{l}\text { residual } \\
\text { 3a (equiv) }\end{array}$ & $\begin{array}{l}{ }^{\mathbf{1}} \mathbf{H} \mathbf{N M R} \\
\text { yield }(\%)\end{array}$ \\
\hline 1 & 1.5 & - & MeCN $(0.1 \mathrm{M})$ & 50 & 440 & nd & 4 \\
2 & 1.5 & - & MeCN $: H 2 O(0.1 \mathrm{M})$ & 50 & 440 & nd & 6 \\
3 & 1.5 & - & DMSO- $d_{6}(0.1 \mathrm{M})$ & 50 & 440 & nd & 4 \\
4 & 1.5 & - & MeOH $(0.1 \mathrm{M})$ & 50 & 440 & nd & 17 \\
5 & 1.5 & - & DCM $(0.1 \mathrm{M})$ & $30-35$ & 440 & 0.10 & 50 \\
$6^{\mathrm{a}}$ & 1.5 & - & $\mathrm{DCM}(0.1 \mathrm{M})$ & $30-35$ & $360^{\mathrm{b}}$ & 0.10 & 50 \\
$7^{\mathrm{b}}$ & 1.5 & - & $\mathrm{DCM}(0.1 \mathrm{M})$ & $30-35$ & $460^{\mathrm{c}}$ & 0.10 & 50 \\
\hline
\end{tabular}




\begin{tabular}{llllllll}
\hline 8 & 2.5 & - & DCM (0.1 M) & $30-35$ & 440 & 0.40 & 57 \\
$9^{c}$ & 2.5 & - & DCM (0.1 M) & $30-35$ & 440 & 0.20 & 67 \\
$10^{c, d}$ & 2.5 & - & DCM (0.1 M) & $30-35$ & 440 & 0.34 & 41 \\
$11^{c, d}$ & 2.5 & - & DCM (0.1 M) & $30-35$ & 427 & 0.36 & 43 \\
$12^{c, d}$ & 2.5 & - & DCM (0.1 M) & $30-35$ & 390 & 0.48 & 34 \\
$13^{c}$ & 2.5 & $\mathrm{Cs}_{2} \mathrm{CO}_{3}(0.5)$ & DCM (0.1 M) & $30-35$ & 440 & nd & 20 \\
$14^{c}$ & 2.5 & $\mathrm{CsOBz}(1)$ & DCM (0.1 M) & $30-35$ & 440 & nd & 10 \\
$15^{c}$ & 2.5 & THF (2) & DCM (0.1 M) & $30-35$ & 440 & nd & nd \\
$16^{c}$ & 2.5 & p-terpinene (2) & DCM (0.1 M) & $30-35$ & 440 & nd & 50 \\
\hline
\end{tabular}

${ }^{\mathrm{a}}$ Reaction was performed in Rayonet reactor, ${ }^{\mathrm{b}}$ Reaction was performed with blue LED strips, ${ }^{\mathrm{C}}$ Reaction was run for 24 hours, ${ }^{\mathrm{d}}$ Reaction was performed with 1 Kessil lamp of the corresponding wavelength

\section{Deoxyalkynyation with methods A and B:}

\subsection{General procedure E: 4CzIPN catalyzed deoxy-alkynylation ("method A")}<smiles>[R]C([R])([R])OC(=O)C(=O)O[Si]</smiles>

$3 w^{3 a-p} 3 x$<smiles>[R]C#CI1COC(=O)c2ccccc21</smiles>

2a-f

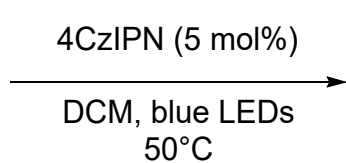

$50^{\circ} \mathrm{C}$<smiles>[R]C#CC([R])([R])[R]</smiles>

$4 w^{4 a \hbar d} 4 x$

An oven dried $(7.5 \mathrm{~mL})$ dram vial equipped with a magnetic stirrer was charged with the cesium salt 3a-x ( $0.30 \mathrm{mmol}, 1.00$ equiv), the EBX reagent (1.5 mmol, 1.5 equiv) and 4CzIPN (1a, $0.015 \mathrm{mmol}, 5$ mol\%). The reaction vial was sealed with a septum. After 3 vacuum/N2 cycles (backfilling with Ar on the last cycle), dichloromethane $(3.00 \mathrm{~mL})$ was added and the septums were replaced with a screw cap under a flux of Ar then the seal was wrapped with parafilm. ${ }^{19}$ The reactions were placed between $2 \times 440 \mathrm{~nm}$ Kessil lamps at ca. $10 \mathrm{~cm}$ distance from both lamps (no ventilation, $\mathrm{T}=\mathrm{ca} .50{ }^{\circ} \mathrm{C}$ ) ${ }^{20}$ and stirred under irradiation for 15-18 hours. The reaction was filtered through a small celite plug which was washed with $\mathrm{CH}_{2} \mathrm{Cl}_{2}$. A solid deposit was prepared (ca. $2 \mathrm{~g} \mathrm{SiO}_{2}$ ). The compound was purified by column chromatography (pentane:EtOAc).

\subsection{General procedure F: Direct excitation of PhEBX for deoxy-alkynylation ("method B")}<smiles>[R]C([R])([R])OC(=O)C(=O)O[AsH3]</smiles>

$3 a$<smiles>O=C1OI(C#Cc2ccccc2)c2ccccc21</smiles>

2a

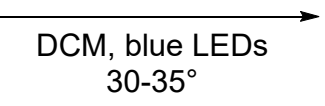
$30-35^{\circ}$<smiles>[R]C#CC([R])([R])[R]</smiles>

4a-x

An oven dried $(7.5 \mathrm{~mL})$ dram vial equipped with a magnetic stirrer was charged with the cesium salt 3a-x ( $0.30 \mathrm{mmol}, 1.00$ equiv) and PhEBX (2a, $2.5 \mathrm{mmol}, 2.5$ equiv). The reaction vial was sealed with a septum. After 3 vacuum/N2 cycles (backfilling with Ar on the last cycle), dichloromethane $(3.00 \mathrm{~mL})$

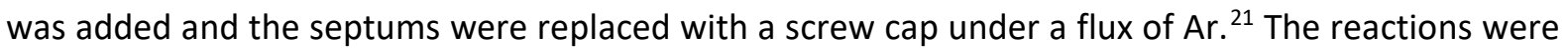

\footnotetext{
20 The reaction temperature was measured with an internal thermometer on a model system using 5 mol\% 4CzIPN in DCM.

${ }^{21}$ Use of a screw cap or crimp cap is of great importance, the irradiation causes an increase in temperature to ca. $50{ }^{\circ} \mathrm{C}$ causing the $\mathrm{CH}_{2} \mathrm{Cl}_{2}$ to evaporate and an overpressure inside the vessel. When using a septum, the latter
} 
placed between $2 \times 440 \mathrm{~nm}$ Kessil lamps at ca. $10 \mathrm{~cm}$ distance from both lamps (with ventilation, $\mathrm{T}=$ $30-35{ }^{\circ} \mathrm{C}$ ) and stirred under irradiation for 24 hours. The reaction was filtered through a small celite plug which was washed with $\mathrm{CH}_{2} \mathrm{Cl}_{2}$. A solid deposit was prepared (ca. $2 \mathrm{~g} \mathrm{SiO}_{2}$ ). The compound was purified by column chromatography (pentane:EtOAc).

\subsection{Yields and characterization data of alkynes $\mathbf{4 a - u} \mathbf{4} \mathbf{w}$ and $\mathbf{4 x}$}

- (3,3-dimethylpent-1-yne-1,5-diyl)dibenzene (4a)<smiles>CC(C)(C#Cc1ccccc1)CCc1ccccc1</smiles>

Method A: 4a was synthesized following general procedure $E$ (Method A) using cesium 2-(methyl-4phenylbutan-2-yl)oxy-2-oxoacetate (3a, $0.110 \mathrm{~g}, 0.300 \mathrm{mmol}, 1.0$ equiv), PhEBX (2a, $0.157 \mathrm{~g}, 0.450$ mmol, 1.50 equiv), 4CzIPN (1a, $0.012 \mathrm{~g}, 1.5 \mu \mathrm{mol}, 5 \mathrm{~mol} \%$ ) in degassed $\mathrm{CH}_{2} \mathrm{Cl}_{2}$ ( $\left.3 \mathrm{~mL}, 0.1 \mathrm{M}\right)$. Column chromatography ( $\mathrm{SiO}_{2}$, pentane) afforded (3,3-dimethylpent-1-yne-1,5-diyl)dibenzene (4a, $0.056 \mathrm{~g}$, $0.23 \mathrm{mmol}, 75 \%)$ as a slightly yellow oil.

Method B: 4a was synthesized following general procedure F (Method B) using cesium 2-(methyl-4phenylbutan-2-yl)oxy-2-oxoacetate (3a, $0.110 \mathrm{~g}, 0.300 \mathrm{mmol}, 1.00$ equiv) and PhEBX (2a, $0.261 \mathrm{~g}$, $0.750 \mathrm{mmol}, 2.50$ equiv) in degassed $\mathrm{CH}_{2} \mathrm{Cl}_{2}(3 \mathrm{~mL}, 0.1 \mathrm{M})$. Column chromatography $\left(\mathrm{SiO}_{2}\right.$, pentane) afforded (3,3-dimethylpent-1-yne-1,5-diyl)dibenzene (4a, $0.045 \mathrm{~g}, 0.18 \mathrm{mmol}, 60 \%)$ as a slightly yellow oil.

$\mathbf{R f}$ (pentane) $=0.4$

${ }^{1} \mathrm{H}$ NMR $\left(400 \mathrm{MHz}, \mathrm{CDCl}_{3}\right) \delta 7.45-7.39(\mathrm{~m}, 2 \mathrm{H}, \mathrm{ArH}), 7.33-7.27(\mathrm{~m}, 5 \mathrm{H}, \mathrm{ArH}), 7.26-7.16(\mathrm{~m}, 3 \mathrm{H}$, $\operatorname{ArH}), 2.95-2.79\left(\mathrm{~m}, 2 \mathrm{H}, \mathrm{ArCH}_{2}\right), 1.86-1.75\left(\mathrm{~m}, 2 \mathrm{H}, \mathrm{ArCH}_{2} \mathrm{CH}_{2}\right), 1.36\left(\mathrm{~s}, 6 \mathrm{H}, \mathrm{C}\left(\mathrm{CH}_{3}\right)_{2}\right)$.

${ }^{13} \mathrm{C}$ NMR $\left(101 \mathrm{MHz}, \mathrm{CDCl}_{3}\right) \delta 142.9,131.7,128.6,128.5,128.3,127.6,125.8,124.1,97.0,81.0,45.7$, 32.3, 32.0, 29.4 .

IR $\left(v_{\max }, \mathrm{cm}^{-1}\right) 3084(\mathrm{~m}), 3060(\mathrm{~m}), 3027(\mathrm{~m}), 2968(\mathrm{~m}), 2945(\mathrm{~m}), 2910(\mathrm{~m}), 2866(\mathrm{~m}), 2224(\mathrm{~m}), 1946$ $(\mathrm{m}), 1878(\mathrm{~m}), 1804(\mathrm{~m}), 1748(\mathrm{~m}), 1491(\mathrm{~m}), 1265(\mathrm{~m}), 1070(\mathrm{~m}), 755(\mathrm{~s}), 740(\mathrm{~s}), 690(\mathrm{~s})$.

HRMS (ESI/QTOF) m/z: [M + Ag] $]^{+}$Calcd for $\mathrm{C}_{19} \mathrm{H}_{20} \mathrm{Ag}^{+}$355.0610; Found 355.0615.

\section{- (3-Ethyl-3-methylpent-1-yne-1,5-diyl)dibenzene (4b)}

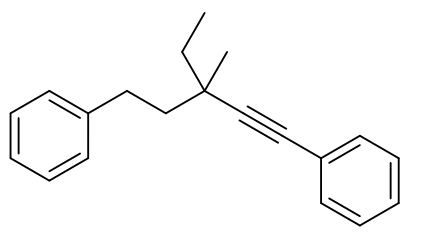

$\mathbf{4 b}$ was synthesized following the general procedure $E$ using cesium 2-((3-methyl-1-phenylpentan-3-yl)oxy)-2-oxoacetate (3b, $115 \mathrm{mg}$, $0.300 \mathrm{mmol}, 1.00$ equiv), PhEBX (2a, $157 \mathrm{mg}, 0.450 \mathrm{mmol}, 1.50$ equiv) and 4CzIPN (1a, $12 \mathrm{mg}, 0.015 \mathrm{mmol}, 5 \mathrm{~mol}$ Column chromatography $\left(\mathrm{SiO}_{2}\right.$, Pentane) affording (3-ethyl-3-methylpent1-yne-1,5-diyl)dibenzene (4b, $57 \mathrm{mg}, 0.22 \mathrm{mmol}, 72 \%$ ) as a pale

$\mathbf{R f}$ (pentane) $=0.3$ yellow oil.

${ }^{1} \mathrm{H}$ NMR $\left(400 \mathrm{MHz}, \mathrm{CDCl}_{3}\right) \delta: 7.44-7.41(\mathrm{~m}, 2 \mathrm{H}, \mathrm{ArH}), 7.32-7.27(\mathrm{~m}, 5 \mathrm{H}, \mathrm{ArH}), 7.25-7.22(\mathrm{~m}, 2 \mathrm{H}$, $\operatorname{ArH}), 7.21-7.17(\mathrm{~m}, 1 \mathrm{H}, \mathrm{ArH}), 2.90-2.77\left(\mathrm{~m}, 2 \mathrm{H}, \mathrm{ArCH}_{2}\right), 1.90-1.82\left(\mathrm{~m}, 1 \mathrm{H}, \mathrm{CH}_{2}\right), 1.76-1.63(\mathrm{~m}$, $\left.2 \mathrm{H}, \mathrm{CH}_{2}\right), 1.59-1.50\left(\mathrm{~m}, 1 \mathrm{H}, \mathrm{CH}_{2}\right), 1.30\left(\mathrm{~s}, 3 \mathrm{H}, \mathrm{CH}_{3}\right), 1.07\left(\mathrm{t}, \mathrm{J}=7.40 \mathrm{~Hz}, 3 \mathrm{H}, \mathrm{CH}_{2} \mathrm{CH}_{3}\right)$.

${ }^{13} \mathrm{C}$ NMR $\left(101 \mathrm{MHz}^{\mathrm{CDCl}} \mathrm{CDC}_{3}\right) \delta: 143.1,131.8,128.6,128.5,128.3,127.6,125.8,124.3,96.1,82.2,43.7$, $36.2,34.5,31.9,26.0,9.5$.

would fly off within an hour of irradiation. As shown in the optimization section DCE is not as good a solvent as $\mathrm{CH}_{2} \mathrm{Cl}_{2}$. 
IR $\left(v_{\max }, \mathrm{Cm}^{-1}\right): 3062(\mathrm{w}), 3031(\mathrm{~m}), 2969(\mathrm{~m}), 2929(\mathrm{~m}), 2858(\mathrm{w}), 1599(\mathrm{~m}), 1493(\mathrm{~m}), 1454(\mathrm{~m})$.

HRMS (APPI/LTQ-Orbitrap) m/z: [M] ${ }^{+}$Calcd for $\mathrm{C}_{20} \mathrm{H}_{22}{ }^{+}$262.1716; Found 262.1716.

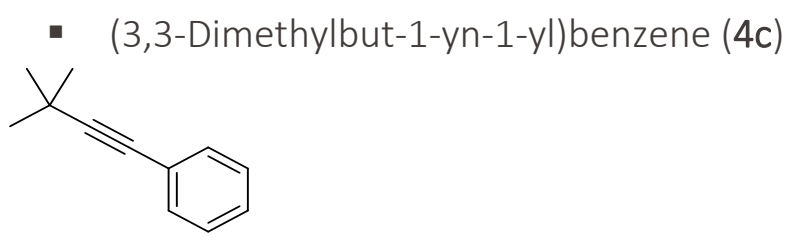

Method A: 4c was synthesized following general procedure $E$ using cesium tert-butoxyl-2oxoacetate (3c, $0.083 \mathrm{~g}, 0.30 \mathrm{mmol}, 1$ equiv), $\operatorname{PhEBX}(2 \mathrm{a}, 0.157 \mathrm{~g}, 0.450 \mathrm{mmol}, 1.50$ equiv), 4CzIPN (1a, $0.012 \mathrm{~g}, 1.5 \mu \mathrm{mol}, 5 \mathrm{~mol} \%)$ in degassed $\mathrm{CH}_{2} \mathrm{Cl}_{2}(3 \mathrm{~mL}, 0.1 \mathrm{M})$. Column chromatography $\left(\mathrm{SiO}_{2}\right.$, pentane) afforded (3,3-dimethylbut-1-yn-1-yl)benzene (4c, $0.051 \mathrm{~g}, 85 \%$ purity, $0.27 \mathrm{mmol}, 91 \%$ ) as a colorless oil. The compound could be partially purified from 1,4-diphenylbuta-1,4-diyne (major impurity) by preparative $\mathrm{TLC}\left(\mathrm{SiO}_{2}\right.$, glass plate, Heptane) allowing full characterization of 4c.

Method B: 4c was synthesized following general procedure $F$ using cesium (tert-butyl)oxy-2oxoacetate (3c, $0.083 \mathrm{~g}, 0.30 \mathrm{mmol}, 1$ equiv) and PhEBX (2a, $0.261 \mathrm{~g}, 0.750 \mathrm{mmol}, 2.50$ equiv) in degassed $\mathrm{CH}_{2} \mathrm{Cl}_{2}$ (3 mL, $\left.0.1 \mathrm{M}\right)$. Column chromatography $\left(\mathrm{SiO}_{2}\right.$, pentane) afforded (3,3-dimethylbut1-yne-1-yl)benzene (4c, $0.067 \mathrm{~g}$, $49 \%$ purity $0.17 \mathrm{mmol}, 57 \%$ ) as a slightly yellow oil.

Rf (pentane) $=0.8$.

${ }^{1}$ H NMR $\left(400 \mathrm{MHz}, \mathrm{CDCl}_{3}\right) \delta 7.43-7.33(\mathrm{~m}, 2 \mathrm{H}, \mathrm{ArH}), 7.32-7.20(\mathrm{~m}, 3 \mathrm{H}, \mathrm{ArH}), 1.32\left(\mathrm{~s}, 9 \mathrm{H}, \mathrm{C}\left(\mathrm{CH}_{3}\right)_{3}\right)$. ${ }^{13} \mathrm{C}$ NMR $\left(101 \mathrm{MHz}, \mathrm{CDCl}_{3}\right) \delta 131.7,128.3,127.5,124.2,98.7,79.1,31.2,28.1$.

IR $\left(v_{\max }, \mathrm{cm}^{-1}\right) 3084(\mathrm{~m}), 3054(\mathrm{~m}), 2971(\mathrm{~m}), 2903(\mathrm{~m}), 2871(\mathrm{~m}), 1780(\mathrm{~m}), 1723(\mathrm{~m}), 909(\mathrm{~s})$.

HRMS (APPI/LTQ-Orbitrap) m/z: [M] ${ }^{+}$Calcd for $\mathrm{C}_{12} \mathrm{H}_{14}{ }^{+}$158.1090; Found 158.1093.

\section{- (3,3,4-trimethylpent-1yn-1-yl)benzene (4d)}

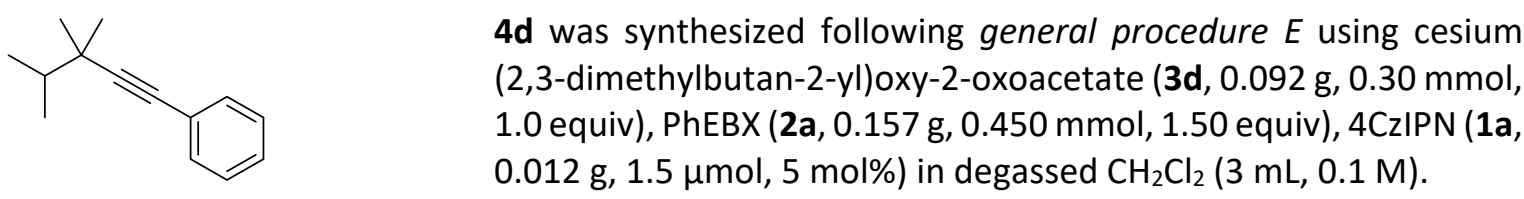

Column chromatography ( $\mathrm{SiO}_{2}$, pentane) afforded (3,3,4-trimethylpent-1-yn-1-yl)benzene (4d, $0.053 \mathrm{~g}, 85 \%$ purity, $0.21 \mathrm{mmol}, 72 \%$ ) as a colorless oil. The compound could be partially purified from 1,4-diphenylbuta-1,4-diyne (major impurity) by preparative $\operatorname{TLC}\left(\mathrm{SiO}_{2}\right.$, glass plate, Heptane) allowing full characterisation of $\mathbf{4 d}$.

$\mathbf{R f}($ pentane $)=0.75$.

${ }^{1} \mathbf{H}$ NMR $\left(400 \mathrm{MHz}, \mathrm{CDCl}_{3}\right) \delta 7.43-7.35(\mathrm{~m}, 2 \mathrm{H}, \mathrm{ArH}), 7.32-7.22(\mathrm{~m}, 3 \mathrm{H}, \mathrm{ArH}), 1.64$ (hept, $\mathrm{J}=6.8$ $\left.\mathrm{Hz}, 1 \mathrm{H}, \mathrm{CH}\left(\mathrm{CH}_{3}\right)_{2}\right), 1.25\left(\mathrm{~s}, 6 \mathrm{H}, \mathrm{C}\left(\mathrm{CH}_{3}\right)_{2}\right), 1.03\left(\mathrm{~d}, J=6.8 \mathrm{~Hz}, 6 \mathrm{H}, \mathrm{CH}\left(\mathrm{CH}_{3}\right)_{2}\right)$.

${ }^{13} \mathrm{C}$ NMR $\left(101 \mathrm{MHz}, \mathrm{CDCl}_{3}\right) \delta 131.7,128.3,127.4,124.4,97.0,81.0,38.0,35.6,27.1,18.5$.

IR $\left(v_{\max }, \mathrm{cm}^{-1}\right) 3083(\mathrm{~m}), 3055(\mathrm{~m}), 2971(\mathrm{~s}), 2939(\mathrm{~m}), 2874(\mathrm{~m}), 2228(\mathrm{~m}), 1599(\mathrm{~m}), 1489(\mathrm{~m}), 1460$ $(\mathrm{m}), 1369(\mathrm{~m}), 1157(\mathrm{~m}), 1061(\mathrm{~m}), 911(\mathrm{~m}), 755(\mathrm{~s}), 691(\mathrm{~s})$.

HRMS (APPI/LTQ-Orbitrap) m/z: [M] ${ }^{+}$Calcd for $\mathrm{C}_{14} \mathrm{H}_{18}{ }^{+}$186.1403; Found 186.1403. 
- 1-(2,2-Dimethyl-4-phenylbut-3-yn-1-yl)-4-methoxybenzene (4e)

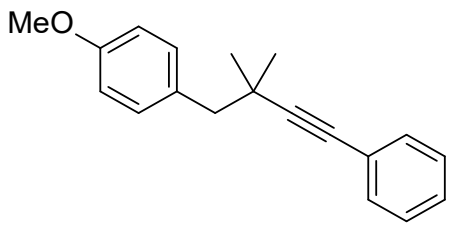

4e was synthesized following general procedure $E$ using cesium (1(4-methoxyphenyl)-2-methylpropan-2-yl)oxy-2-oxoacetate (3e, $0.115 \mathrm{~g}, 0.300 \mathrm{mmol}, 1.00$ equiv), PhEBX ( $2 \mathrm{a}, 0.157 \mathrm{~g}, 0.450 \mathrm{mmol}$, 1.50 equiv), 4CzIPN (1a, $0.012 \mathrm{~g}, 1.5 \mu \mathrm{mol}, 5 \mathrm{~mol} \%)$ in degassed $\mathrm{CH}_{2} \mathrm{Cl}_{2}(3 \mathrm{~mL}, 0.1 \mathrm{M})$.

Column chromatography $\left(\mathrm{SiO}_{2}\right.$, pentane:EtOAc 100:0 to 90:10) afforded 1-(2,2-dimethyl-4phenylbut-3-yn-1-yl)-4-methoxybenzene (4e, $0.044 \mathrm{~g}, 0.17 \mathrm{mmol}, 55 \%)$.

Rf (pentane:EtOAc 9:1) $=0.4$.

${ }^{1} \mathrm{H}$ NMR $\left(400 \mathrm{MHz}, \mathrm{CDCl}_{3}\right) \delta 7.40-7.33(\mathrm{~m}, 2 \mathrm{H}, \mathrm{ArH}), 7.31-7.21(\mathrm{~m}, 5 \mathrm{H}, \mathrm{ArH}), 6.88-6.81(\mathrm{~m}, 2 \mathrm{H}$, $\mathrm{ArH}), 3.80\left(\mathrm{~s}, 3 \mathrm{H}, \mathrm{OCH}_{3}\right), 2.74\left(\mathrm{~s}, 2 \mathrm{H}, \mathrm{ArCH}_{2}\right), 1.28\left(\mathrm{~s}, 6 \mathrm{H},\left(\mathrm{CH}_{3}\right)_{2}\right)$.

${ }^{13} \mathrm{C}$ NMR $\left(101 \mathrm{MHz}, \mathrm{CDCl}_{3}\right) \delta$ 158.4, 131.7, 131.6, 130.7, 128.3, 127.6, 124.2, 113.2, 97.2, 81.7, 55.4, 48.4, 33.1, 29.1.

IR $\left(v_{\max }, \mathrm{cm}^{-1}\right) 3057(\mathrm{~m}), 3034(\mathrm{~m}), 2961(\mathrm{~m}), 2933(\mathrm{~m}), 2835(\mathrm{~m}), 1786(\mathrm{~m}), 1611(\mathrm{~m}), 1512(\mathrm{~s}), 1465$ (m), $1302(\mathrm{~m}), 1246(\mathrm{~s}), 1177(\mathrm{~s}), 1037(\mathrm{~s}), 757(\mathrm{~s}), 739(\mathrm{~s})$.

HRMS (ESI/QTOF) m/z: [M + Ag] $]^{+}$Calcd for $\mathrm{C}_{19} \mathrm{H}_{20} \mathrm{AgO}^{+}$371.0560; Found 371.0552.

- 1-(2,2-Dimethyl-4-phenylbut-3-yn-1-yl)-2-fluorobenzene (4f)

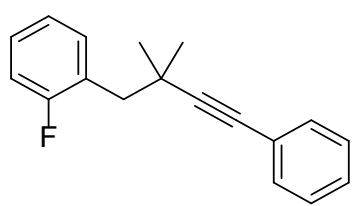

$4 \mathbf{f}$ was synthesized following general procedure $E$ using cesium (1(2-fluorophenyl)-2-methylpropan-2-yl)oxy-2-oxoacetate (3f, 0.112 g, $0.300 \mathrm{mmol}, 1.00$ equiv), PhEBX (2a, $0.157 \mathrm{~g}, 0.450 \mathrm{mmol}, 1.50$ equiv), 4CzIPN (1a, $0.012 \mathrm{~g}, 1.5 \mu \mathrm{mol}, 5 \mathrm{~mol} \%$ ) in degassed $\mathrm{CH}_{2} \mathrm{Cl}_{2}$ (3 mL, $0.1 \mathrm{M}$ ).

Column chromatography $\left(\mathrm{SiO}_{2}\right.$, pentane) afforded 1-(2,2-dimethyl-4-phenylbut-3-yn-1-yl)-2fluorobenzene (4f, $0.023 \mathrm{~g}, 0.091 \mathrm{mmol}, 30 \%)$.

$\mathbf{R f}$ (pentane) $=0.4$.

${ }^{1} \mathrm{H}$ NMR $(400 \mathrm{MHz}, \mathrm{CDCl} 3) \delta 7.43$ (td, $\left.J=7.6,1.9 \mathrm{~Hz}, 1 \mathrm{H}, \operatorname{ArH}\right), 7.38-7.36(\mathrm{~m}, 1 \mathrm{H}, \operatorname{ArH}), 7.35(\mathrm{~d}, J=$ $2.0 \mathrm{~Hz}, 1 \mathrm{H}, \mathrm{ArH}), 7.30-7.25(\mathrm{~m}, 3 \mathrm{H}, \mathrm{ArH}), 7.25-7.17(\mathrm{~m}, 1 \mathrm{H}, \mathrm{ArH}), 7.13-6.99(\mathrm{~m}, 2 \mathrm{H}, \mathrm{ArH}), 2.87$ (d, $\left.J=1.5 \mathrm{~Hz}, 2 \mathrm{H}, \mathrm{ArCH}_{2}\right), 1.33\left(\mathrm{~d}, J=1.0 \mathrm{~Hz}, 6 \mathrm{H}, \mathrm{C}\left(\mathrm{CH}_{3}\right)_{2}\right)$.

${ }^{1} \mathrm{H}$ NMR $\left\{{ }^{19} \mathrm{~F}\right\} \delta 7.42(\mathrm{dd}, J=7.6,1.8 \mathrm{~Hz}, 1 \mathrm{H}, \mathrm{ArH}), 7.39-7.32(\mathrm{~m}, 2 \mathrm{H}, \mathrm{ArH}), 7.31-7.18(\mathrm{~m}, 4 \mathrm{H}, \mathrm{ArH})$, $7.13-7.01(\mathrm{~m}, 2 \mathrm{H}, \mathrm{ArH}), 2.87\left(\mathrm{~s}, 2 \mathrm{H}, \mathrm{ArCH}_{2}\right), 1.33\left(\mathrm{~s}, 6 \mathrm{H}, \mathrm{C}\left(\mathrm{CH}_{3}\right)_{2}\right)$.

${ }^{13} \mathrm{C}$ NMR $\left(101 \mathrm{MHz}, \mathrm{CDCl}_{3}\right) \delta 161.7(\mathrm{~d}, J=245 \mathrm{~Hz}), 133.1(\mathrm{~d}, J=5 \mathrm{~Hz}), 131.6,128.3(\mathrm{~d}, J=8 \mathrm{~Hz})$, $128.3,127.7,125.5$ (d, $J=16 \mathrm{~Hz}), 124.1,123.5(\mathrm{~d}, J=4 \mathrm{~Hz}), 115.2(\mathrm{~d}, J=23 \mathrm{~Hz}$ ), 96.7, 81.5, 41.3, 33.3, 29.1.

${ }^{19} \mathrm{~F}$ NMR $\left(376 \mathrm{MHz}, \mathrm{CDCl}_{3}\right) \delta-116.1$.

IR $\left(v_{\max }, \mathrm{cm}^{-1}\right) 3061(\mathrm{w}), 2969(\mathrm{w}), 2925(\mathrm{w}), 1489(\mathrm{~m}), 1488(\mathrm{~m}), 1467(\mathrm{~m}), 1280(\mathrm{~m}), 1183(\mathrm{~m}), 752$ $(\mathrm{s}), 721(\mathrm{~m})$.

HRMS (APPI/LTQ-Orbitrap) m/z: [M] ${ }^{+}$Calcd for $\mathrm{C}_{18} \mathrm{H}_{17} \mathrm{~F}^{+}$252.1309; Found 252.1308.

- 1-(2,2-Dimethyl-4-phenylbut-3-yn-1-yl)-4-bromobenzene (4g)

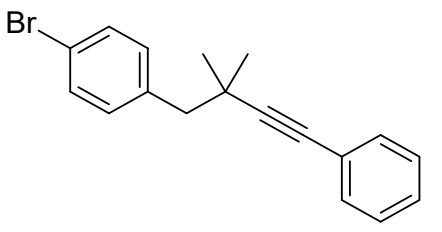

$\mathbf{4 g}$ was synthesized following general procedure $E$ using cesium (1(4-bromophenyl)-2-methylpropan-2-yl)oxy-2-oxoacetate (3g, $0.087 \mathrm{~g}, 0.20 \mathrm{mmol}, 1.0$ equiv), PhEBX (2a, $0.105 \mathrm{~g}, 0.300 \mathrm{mmol}$, 1.5 equiv), 4CzIPN (1a, $0.008 \mathrm{~g}, 1 \mu \mathrm{mol}, 5 \mathrm{~mol} \%$ ) in degassed $\mathrm{CH}_{2} \mathrm{Cl}_{2}$ (3 mL, $0.067 \mathrm{M})$.

Column chromatography $\left(\mathrm{SiO}_{2}\right.$, pentane) afforded 1-(2,2-dimethyl-4-phenylbut-3-yn-1-yl)-4bromobenzene (4g, $0.021 \mathrm{~g}, 0.067 \mathrm{mmol}, 33 \%$ ). 
Rf (pentane) $=0.35$

${ }^{1}$ H NMR $\left(400 \mathrm{MHz}, \mathrm{CDCl}_{3}\right) \delta 7.45-7.39(\mathrm{~m}, 2 \mathrm{H}, \mathrm{ArH}), 7.38-7.31(\mathrm{~m}, 2 \mathrm{H}, \mathrm{ArH}), 7.32-7.25(\mathrm{~m}, 3 \mathrm{H}$, $\operatorname{ArH}), 7.23-7.17(\mathrm{~m}, 2 \mathrm{H}, \mathrm{ArH}), 2.74\left(\mathrm{~s}, 2 \mathrm{H}, \mathrm{ArCH}_{2}\right), 1.29\left(\mathrm{~s}, 6 \mathrm{H}, \mathrm{C}\left(\mathrm{CH}_{3}\right)_{2}\right)$.

${ }^{13} \mathrm{C}$ NMR $\left(101 \mathrm{MHz}, \mathrm{CDCl}_{3}\right) \delta 137.5,132.4,131.6,130.9,128.3,127.8,123.9,120.6,96.5,82.1,48.6$, 32.9, 29.2.

IR $\left(\mathrm{v}_{\max }, \mathrm{cm}^{-1}\right) 3080(\mathrm{~m}), 3060(\mathrm{~m}), 3033(\mathrm{~m}), 2967(\mathrm{~m}), 2921(\mathrm{~m}), 2863(\mathrm{~m}), 1763(\mathrm{~m}), 1737(\mathrm{~m})$, $1598(\mathrm{~m}), 1489(\mathrm{~s}), 1443(\mathrm{~m}), 1384(\mathrm{~m}), 1277(\mathrm{~m}), 1187(\mathrm{~m}), 1072(\mathrm{~s}), 1048(\mathrm{~m}), 1013(\mathrm{~s}), 912(\mathrm{~s})$, $841(\mathrm{~s}), 756(\mathrm{~s}), 741(\mathrm{~s})$.

HRMS (APPI/LTQ-Orbitrap) m/z: [M] ${ }^{+}$Calcd for $\mathrm{C}_{18} \mathrm{H}_{17}{ }^{79} \mathrm{Br}^{+}$312.0508; Found 312.0508.

- 1-(((3,3-Dimethyl-5-phenylpent-4-yn-1-yl)oxy)methyl)-4-methylbenzene (4h)

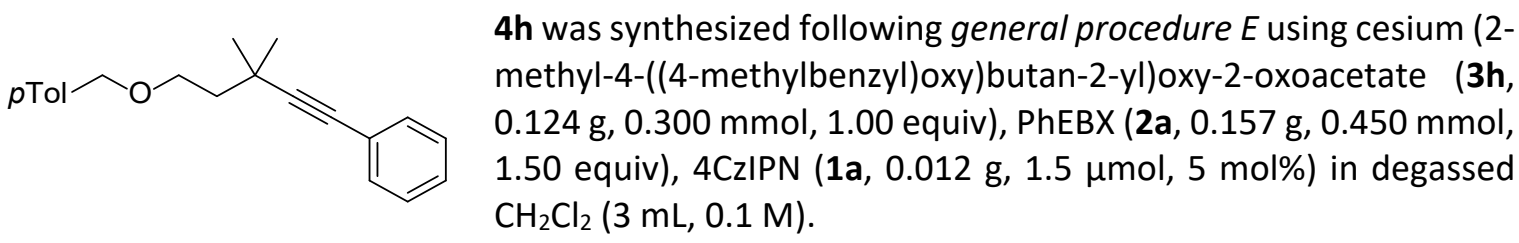

Column chromatography ( $\mathrm{SiO}_{2}$, pentane:EtOAc 100:0 to 80:20) afforded 1-(((3,3-dimethyl-5phenylpent-4-yn-1-yl)oxy)methyl)-4-methylbenzene (4h, $0.049 \mathrm{~g}, 0.17 \mathrm{mmol}, 56 \%)$.

Rf(pentane:EtOAc 8:2) $=0.5$.

${ }^{1}$ H NMR $\left(400 \mathrm{MHz}, \mathrm{CDCl}_{3}\right) \delta 7.37-7.29(\mathrm{~m}, 2 \mathrm{H}, \mathrm{ArH}), 7.30-7.21(\mathrm{~m}, 5 \mathrm{H}, \mathrm{ArH}), 7.17-7.11(\mathrm{~m}, 2 \mathrm{H}$, $\operatorname{ArH}), 4.50\left(\mathrm{~s}, 2 \mathrm{H}, \mathrm{ArCH}_{2}\right), 3.74\left(\mathrm{dd}, J=7.6,6.9 \mathrm{~Hz}, 2 \mathrm{H}, \mathrm{CH}_{2}\right), 2.34\left(\mathrm{~s}, 3 \mathrm{H}, \mathrm{ArCH}_{3}\right), 1.89-1.81(\mathrm{~m}, 2 \mathrm{H}$, $\left.\mathrm{CH}_{2}\right), 1.32\left(\mathrm{~s}, 6 \mathrm{H}, \mathrm{C}\left(\mathrm{CH}_{3}\right)_{2}\right)$.

${ }^{13} \mathrm{C}$ NMR $\left(101 \mathrm{MHz}, \mathrm{CDCl}_{3}\right) \delta 137.3,135.6,131.7,129.2,128.3,127.9,127.6,124.0,96.7,80.8,73.0$, $68.2,42.6,30.6,29.9,21.3$.

IR $\left(v_{\max }, \mathrm{cm}^{-1}\right) 3052(\mathrm{~m}), 3033(\mathrm{~m}), 2969(\mathrm{~m}), 2907(\mathrm{~m}), 2863(\mathrm{~m}), 1960(\mathrm{w}), 1900(\mathrm{w}), 1715(\mathrm{w})$, $1598(\mathrm{~m}), 1490(\mathrm{~m}), 1443(\mathrm{~m}), 1361(\mathrm{~m}), 1096(\mathrm{~s}), 802(\mathrm{~s}), 754(\mathrm{~s})$.

HRMS (APPI/LTQ-Orbitrap) m/z: [M] ${ }^{+}$Calcd for $\mathrm{C}_{21} \mathrm{H}_{24} \mathrm{O}^{+}$292.1822; Found 292.1818.

- tert-Butyl((3,3-dimethyl-5-phenylpent-4-yn-1-yl)oxy)dimethylsilane (4i)

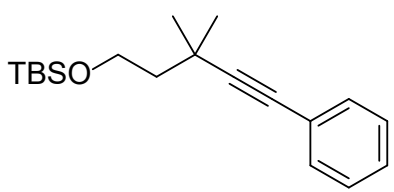

$4 \mathbf{i}$ was synthesized following the general procedure $E$ using cesium 2-((4-((tert-butyldimethylsilyl)oxy)-2-methylbutan-2-yl)oxy)-2oxoacetate (3i, $127 \mathrm{mg}, 0.300 \mathrm{mmol}, 1.00$ equiv), PhEBX (2a, 157 $\mathrm{mg}, 0.450 \mathrm{mmol}, 1.50$ equiv) and 4CzIPN (1a, $12 \mathrm{mg}, 0.015 \mathrm{mmol}, 5$ mol\%).

Column chromatography $\left(\mathrm{SiO}_{2}, 5 \% \mathrm{DCM}\right.$ in Pentane) afforded (3- tert-butyl((3,3-dimethyl-5phenylpent-4-yn-1-yl)oxy)dimethylsilane (4i, $55 \mathrm{mg}, 0.18 \mathrm{mmol}, 61 \%$ ) as a yellow oil.

Rf (pentane:DCM 95:5) = 0.4.

${ }^{1} \mathrm{H}$ NMR $\left(400 \mathrm{MHz}, \mathrm{CDCl}_{3}\right)$ 8: $7.39-7.34(\mathrm{~m}, 2 \mathrm{H}, \mathrm{ArH}), 7.30-7.25(\mathrm{~m}, 3 \mathrm{H}, \mathrm{ArH}), 3.90(\mathrm{t}, J=7.5 \mathrm{~Hz}$, $\left.2 \mathrm{H}, \mathrm{OCH})_{2}\right), 1.76\left(\mathrm{t}, J=7.5 \mathrm{~Hz}, 2 \mathrm{H}, \mathrm{CH}_{2}\right), 1.31\left(\mathrm{~s}, 6 \mathrm{H}, \mathrm{C}\left(\mathrm{CH}_{3}\right)_{2}\right), 0.91\left(\mathrm{~s}, 9 \mathrm{H}, \mathrm{C}\left(\mathrm{CH}_{3}\right)_{3}\right), 0.08\left(\mathrm{~s}, 6 \mathrm{H}, \mathrm{Si}\left(\mathrm{CH}_{3}\right)_{2}\right)$.

${ }^{13} \mathrm{CNMR}\left(101 \mathrm{MHz}, \mathrm{CDCl}_{3}\right) \delta: 131.7,128.3,127.6,124.1,96.8,80.7,61.1,45.8,30.5,29.9,26.1,18.5$, -5.1 .

IR $\left(v_{\max }, \mathrm{cm}^{-1}\right)$ : $3668(\mathrm{w}), 2962(\mathrm{~s}), 2901(\mathrm{~s}), 1467(\mathrm{~m}), 1393(\mathrm{~m}), 1254(\mathrm{~m}), 1092(\mathrm{~s}), 1057(\mathrm{~s})$.

HRMS (nanochip-ESI/LTQ-Orbitrap) m/z: [M + H] ${ }^{+}$Calcd for $\mathrm{C}_{19} \mathrm{H}_{31} \mathrm{OSi}^{+}$303.2139; Found 303.2137. 
- tert-Butyl((3,3-dimethyl-5-phenylpent-4-yn-1-yl)oxy)diphenylsilane (4j)

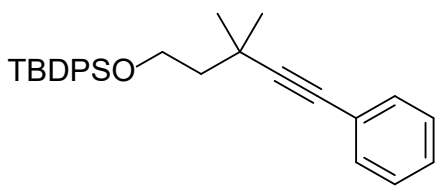

4j was synthesized following the general procedure $E$ using cesium 2-((4-((tert-butyldiphenylsilyl)oxy)-2-methylbutan-2-yl)oxy)-2-

oxoacetate (3j, $164 \mathrm{mg}, 0.300 \mathrm{mmol}, 1.00$ equiv), PhEBX (2a, 157

$\mathrm{mg}, 0.450 \mathrm{mmol}, 1.50$ equiv) and 4CzIPN (1a, $12 \mathrm{mg}, 0.015 \mathrm{mmol}, 5$ mol\%).

Column chromatography $\left(\mathrm{SiO}_{2}, 5 \% \mathrm{DCM}\right.$ in Pentane) afforded tert-butyl((3,3-dimethyl-5phenylpent-4-yn-1-yl)oxy)diphenylsilane (53 mg, $0.12 \mathrm{mmol}, 41 \%$ ) as a yellow oil.

Rf (pentane:DCM, 95:5) $=0.4$.

${ }^{1} \mathrm{H}$ NMR $\left(400 \mathrm{MHz}, \mathrm{CDCl}_{3}\right) \delta: 7.74-7.64(\mathrm{~m}, 4 \mathrm{H}, \mathrm{ArH}), 7.44-7.31(\mathrm{~m}, 6 \mathrm{H}, \mathrm{ArH}), 7.29-7.21(\mathrm{~m}, 5 \mathrm{H}$, $\operatorname{ArH}), 3.96\left(\mathrm{dd}, \mathrm{J}=7.6,6.8 \mathrm{~Hz}, 2 \mathrm{H}, \mathrm{OCH}_{2}\right), 1.82\left(\mathrm{dd}, \mathrm{J}=7.6,6.8 \mathrm{~Hz}, 2 \mathrm{H}, \mathrm{CH}_{2}\right), 1.27\left(\mathrm{~s}, 6 \mathrm{H}, \mathrm{C}\left(\mathrm{CH}_{3}\right)_{2}\right), 1.05$ $\left(\mathrm{s}, 9 \mathrm{H}, \mathrm{C}\left(\mathrm{CH}_{3}\right)_{3}\right)$.

${ }^{13} \mathrm{C}$ NMR $\left(101 \mathrm{MHz}, \mathrm{CDCl}_{3}\right)$ 8: 135.7, 134.1, 131.7, 129.7, 128.2, 127.8, 127.6, 124.0, 96.7, 80.8, 62.0, $45.5,30.5,30.0,27.0,19.3$.

IR $\left(v_{\max }, \mathrm{cm}^{-1}\right)$ : $3668(\mathrm{~m}), 3061(\mathrm{~m}), 2966(\mathrm{~s}), 2935(\mathrm{~s}), 1478(\mathrm{~m}), 1392(\mathrm{~m}), 1258(\mathrm{~m}), 1084(\mathrm{~s})$.

HRMS (nanochip-ESI/LTQ-Orbitrap) $\mathrm{m} / \mathrm{z}$ : $[\mathrm{M}+\mathrm{Na}]^{+}$Calcd for $\mathrm{C}_{29} \mathrm{H}_{34} \mathrm{NaOSi}^{+}$449.2271; Found 449.2269 .

- ((1-Methylcyclopentyl)ethynyl)benzene (4k)<smiles>CC1(C#Cc2ccccc2)CCCC1</smiles>

Method A: 4k was synthesized following the general procedure $E$ using cesium 2-((1methylcyclopentyl)oxy)-2-oxoacetate (3k, $91 \mathrm{mg}, 0.30 \mathrm{mmol}, 1.0$ equiv), PhEBX (2a, $157 \mathrm{mg}, 0.450$ $\mathrm{mmol}, 1.50$ equiv) and 4CzIPN (1a, $12 \mathrm{mg}, 0.015 \mathrm{mmol}, 5 \mathrm{~mol} \%)$. Column chromatography $\left(\mathrm{SiO}_{2}\right.$, Pentane) afforded ((1-methylcyclopentyl)ethynyl)benzene $(\mathbf{4 k}, 39 \mathrm{mg}, 0.20 \mathrm{mmol}, 69 \%)$ as a pale yellow oil.

Method B: 4k was synthesized following general procedure $F$ using cesium 2-((1methylcyclopentyl)oxy)-2-oxoacetate (3k, $91 \mathrm{mg}, 0.30 \mathrm{mmol}, 1.0$ equiv) and PhEBX (2a, $0.261 \mathrm{~g}$, $0.75 \mathrm{mmol}, 2.50$ equiv) in degassed $\mathrm{CH}_{2} \mathrm{Cl}_{2}(3 \mathrm{~mL}, 0.1 \mathrm{M})$. Column chromatography ( $\mathrm{SiO}_{2}$, pentane) afforded ((1-methylcyclopentyl)ethynyl)benzene $(\mathbf{4 k}, 57 \mathrm{mg}, 42 \%$ purity, $0.16 \mathrm{mmol}, 54 \%)$ with major impurity 1,4-diphenylbutadiyne.

$\mathbf{R f}$ (pentane) $=0.6$.

${ }^{1} \mathrm{H}$ NMR $\left(400 \mathrm{MHz}, \mathrm{CDCl}_{3}\right)$ 8: $7.39-7.36(\mathrm{~m}, 2 \mathrm{H}, \mathrm{ArH}), 7.29-7.23(\mathrm{~m}, 3 \mathrm{H}, \mathrm{ArH}), 2.01-1.95(\mathrm{~m}, 2 \mathrm{H}$, $\left.\mathrm{CH}_{2}\right), 1.90-1.80\left(\mathrm{~m}, 2 \mathrm{H}, \mathrm{CH}_{2}\right) 1.75-1.66\left(\mathrm{~m}, 2 \mathrm{H}, \mathrm{CH}_{2}\right), 1.62-1.51\left(\mathrm{~m}, 2 \mathrm{H}, \mathrm{CH}_{2}\right), 1.35\left(\mathrm{~s}, 3 \mathrm{H}, \mathrm{CH}_{3}\right)$

${ }^{13} \mathrm{C}$ NMR $\left(101 \mathrm{MHz}, \mathrm{CDCl}_{3}\right) \delta: 131.7,128.3,127.4,124.4,98.6,79.6,41.8,38.5,27.6,24.5$.

IR $\left(v_{\max }, \mathrm{cm}^{-1}\right): 3060(\mathrm{~m}), 2960(\mathrm{~s}), 2869(\mathrm{~m}), 1742(\mathrm{~m}), 1488(\mathrm{~m}), 1451(\mathrm{~m}), 1322(\mathrm{~m}), 1186(\mathrm{~m})$.

HRMS (APPI/LTQ-Orbitrap) m/z: [M] ${ }^{+}$Calcd for $\mathrm{C}_{14} \mathrm{H}_{16}{ }^{+}$184.1247; Found 184.1248.

- 2-(1-Methylcyclohexyl)ethynylbenzene (4I)

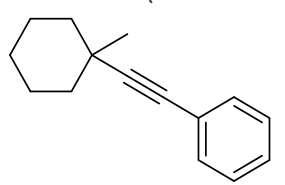

Method A: 4I was synthesized following general procedure $E$ using cesium 2-(1-methylcyclohexan1-yl)oxy-2-oxoacetate ( $3 \mathrm{l}, 0.095 \mathrm{~g}, 0.30 \mathrm{mmol}, 1$ equiv), $\operatorname{PhEBX}(2 \mathrm{a}, 0.157 \mathrm{~g}, 0.450 \mathrm{mmol}, 1.50$ equiv), 4CzIPN (1a, $0.012 \mathrm{~g}, 1.5 \mu \mathrm{mol}, 5 \mathrm{~mol} \%$ ) in degassed $\mathrm{CH}_{2} \mathrm{Cl}_{2}(3 \mathrm{~mL}, 0.1 \mathrm{M})$. Column chromatography 
( $\mathrm{SiO}_{2}$, pentane) afforded (1-methylcyclohexyl)enthynylbenzene (4l, $0.053 \mathrm{~g}$ (80\% purity), 0.22 $\mathrm{mmol}, 72 \%)$ as a colorless oil. The compound could be partially purified from 1,4-diphenylbuta-1,4diyne (major impurity) by preparative $\mathrm{TLC}\left(\mathrm{SiO}_{2}\right.$, glass plate, Heptane) allowing full characterisation of 41 .

Method B: 4 I was synthesized following general procedure $F$ using cesium 2-(1-methylcyclohexan1-yl)oxy-2-oxoacetate (3l, $95 \mathrm{mg}, 0.30 \mathrm{mmol}, 1.0$ equiv) and PhEBX (2a, $0.261 \mathrm{~g}, 0.750 \mathrm{mmol}, 2.50$ equiv) in degassed $\mathrm{CH}_{2} \mathrm{Cl}_{2}(3.0 \mathrm{~mL}, 0.1 \mathrm{M})$. Column chromatography $\left(\mathrm{SiO}_{2}\right.$, pentane) afforded ((1methylcyclohexyl)ethynyl)benzene ( $41,0.063 \mathrm{mg}$ ( $55 \%$ purity), $0.18 \mathrm{mmol}, 61 \%$ ) with major impurity 1,4-diphenylbutadiyne.

Rf (pentane) $=0.7$.

${ }^{1} \mathrm{H}$ NMR $\left(400 \mathrm{MHz}, \mathrm{CDCl}_{3}\right) \delta 7.45-7.37(\mathrm{~m}, 2 \mathrm{H}, \mathrm{ArH}), 7.32-7.22(\mathrm{~m}, 3 \mathrm{H}, \mathrm{ArH}), 1.84-1.55(\mathrm{~m}, 8 \mathrm{H}$, $\left.\mathrm{CH}_{2}\right), 1.28\left(\mathrm{~s}, 3 \mathrm{H}, \mathrm{CH}_{3}\right), 1.27-1.09\left(\mathrm{~m}, 2 \mathrm{H}, \mathrm{CH}_{2}\right)$.

${ }^{13} \mathrm{C}$ NMR $\left(101 \mathrm{MHz}, \mathrm{CDCl}_{3}\right) \delta 131.7,128.3,127.5,124.4,96.9,81.9,39.7,33.3,30.4,26.1,23.6$.

Consistent with the reported NMR data. ${ }^{22}$

- 1-Methyl-1-(phenylethynyl)cycloheptane $(4 \mathrm{~m})$

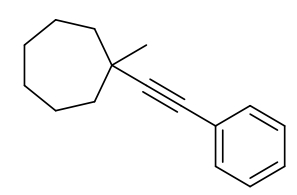

$4 \mathrm{~m}$ was synthesized following general procedure $E$ using cesium 2(1-methylcycloheptan-1-yl)oxy-2-oxoacetate (3m, $0.110 \mathrm{~g}, 0.300$ mmol, 1.00 equiv), PhEBX ( $2 \mathrm{a}, 0.157 \mathrm{~g}, 0.450 \mathrm{mmol}, 1.5$ equiv), 4CZIPN (1a, $0.012 \mathrm{~g}, 1.5 \mu \mathrm{mol}, 5 \mathrm{~mol} \%)$ in degassed $\mathrm{CH}_{2} \mathrm{Cl}_{2}(3 \mathrm{~mL}$, $0.1 \mathrm{M})$.

Column chromatography $\left(\mathrm{SiO}_{2}\right.$, pentane) afforded (3,3-dimethylpent-1-yne-1,5-diyl)dibenzene $(4 \mathrm{~m}, 0.068 \mathrm{~g}, 75 \%$ purity $0.22 \mathrm{mmol}, 74 \%)$ as a colorless oil. The compound could be partially purified from 1,4-diphenylbuta-1,4-diyne (major impurity) by preparative $\mathrm{TLC}$ ( $\mathrm{SiO}_{2}$, glass plate, Heptane) allowing full characterisation of $4 \mathrm{~m}$.

$\mathbf{R f}($ pentane $)=0.7$.

${ }^{1} \mathrm{H}$ NMR $\left(400 \mathrm{MHz}, \mathrm{CDCl}_{3}\right) \delta 7.42-7.39(\mathrm{~m}, 2 \mathrm{H}, \mathrm{ArH}), 7.31-7.24(\mathrm{~m}, 3 \mathrm{H}, \mathrm{ArH}), 1.95-1.84(\mathrm{~m}, 2 \mathrm{H}$, $\left.\mathrm{CH}_{2}\right), 1.82-1.64\left(\mathrm{~m}, 4 \mathrm{H}, \mathrm{CH}_{2}\right), 1.64-1.56\left(\mathrm{~m}, 2 \mathrm{H}, \mathrm{CH}_{2}\right), 1.55-1.44\left(\mathrm{~m}, 4 \mathrm{H}, \mathrm{CH}_{2}\right), 1.29\left(\mathrm{~s}, 3 \mathrm{H}, \mathrm{CH}_{3}\right)$. ${ }^{13} \mathrm{C}$ NMR $\left(101 \mathrm{MHz}, \mathrm{CDCl}_{3}\right) \delta 131.7,128.3,127.4,124.5,98.1,81.1,42.3,36.1,31.5,28.4,24.0$.

$\operatorname{IR}\left(v_{\max }, \mathrm{cm}^{-1}\right) 3081(\mathrm{w}), 3054(\mathrm{w}), 2961(\mathrm{~m}), 2925(\mathrm{~s}), 2855(\mathrm{~m}), 1598(\mathrm{~m}), 1491(\mathrm{~m}), 1460(\mathrm{~m}), 1231$ (m), $912(\mathrm{~m}), 755(\mathrm{~s})$.

HRMS (APPI/LTQ-Orbitrap) m/z: [M] ${ }^{+}$Calcd for $\mathrm{C}_{16} \mathrm{H}_{20}{ }^{+}$212.1560; Found 212.1558.

- 1-Methyl-1-(phenylethynyl)cyclododecane (4n)

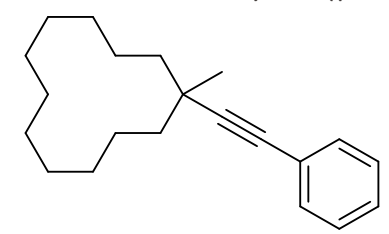

Method A: 4n was synthesized following the general procedure $E$ using cesium 2-((1methylcyclododecyl)oxy)-2-oxoacetate (3n, $121 \mathrm{mg}$ (purity $80 \%), 0.240 \mathrm{mmol}, 1.00$ equiv), PhEBX (2a, $157 \mathrm{mg}, 0.450 \mathrm{mmol}, 1.9$ equiv) and 4CzIPN (1a, $12 \mathrm{mg}, 0.015 \mathrm{mmol}, 6 \mathrm{~mol} \%$ ). Column chromatography $\left(\mathrm{SiO}_{2}\right.$, Pentane) afforded 1-methyl-1-(phenylethynyl)cyclododecane $(4 \mathrm{n}, 43 \mathrm{mg}$, $0.15 \mathrm{mmol}, 63 \%)$ as a pale yellow oil.

${ }^{22}$ Gao, C.; Li, J.; Yu, J.; Yang, H.; Fu, H. Chem. Commun. 2016, 52, 7292-7294. 
Method B: 4n was synthesized following general procedure $F$ using cesium 2-(1methylcyclododecan-1-yl)oxy-2-oxoacetate (3n, $151 \mathrm{mg}$ (purity $80 \%$ ), $0.300 \mathrm{mmol}, 1.00$ equiv) and PhEBX (2a, $0.261 \mathrm{~g}, 0.750 \mathrm{mmol}, 2.50$ equiv) in degassed $\mathrm{CH}_{2} \mathrm{Cl}_{2}$ (3.0 mL, $0.1 \mathrm{M}$ ). Column chromatography $\left(\mathrm{SiO}_{2}\right.$, pentane) afforded ((1-methylcyclododecyl)ethynyl)benzene (4n, $0.062 \mathrm{mg}$ (47\% purity), $0.11 \mathrm{mmol}, 37 \%$ ) with major impurity 1,4-diphenylbutadiyne.

$\mathbf{R f}$ (pentane) $=0.6$.

${ }^{1} \mathrm{H}$ NMR $\left(400 \mathrm{MHz}, \mathrm{CDCl}_{3}\right) \delta: 7.40-7.36(\mathrm{~m}, 3 \mathrm{H}, \mathrm{ArH}), 7.29-7.23(\mathrm{~m}, 2 \mathrm{H}, \mathrm{ArH}), 1.46-1.29(\mathrm{~m}, 22 \mathrm{H}$, $\left.\mathrm{CH}_{2}\right), 1.23\left(\mathrm{~s}, 3 \mathrm{H}, \mathrm{CH}_{3}\right)$.

${ }^{13} \mathrm{C}$ NMR $\left(101 \mathrm{MHz}, \mathrm{CDCl}_{3}\right)$ 8: 131.7, 128.2, 127.4, 124.4, 98.4, 80.6, 35.0, 34.4, 27.5, 26.6, 26.3, 22.7, 22.3, 19.9 .

IR $\left(v_{\text {max }}, \mathrm{cm}^{-1}\right)$ : $3058(w), 2936(\mathrm{~s}), 2859(\mathrm{~m}), 2226(\mathrm{w}), 1597(\mathrm{w}), 1479(\mathrm{~m}), 1449(\mathrm{~m}), 1273(\mathrm{w})$.

HRMS (ESI/QTOF) m/z: [M + Ag] $]^{+}$Calcd for $\mathrm{C}_{21} \mathrm{H}_{30} \mathrm{Ag}^{+} 389.1393$; Found 389.1390.

- 4-Methyl-4-(phenylethynyl)tetrahydro-2H-pyran (40)

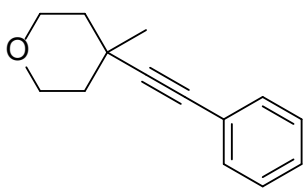

Method A: 40 was synthesized following the general procedure $E$ using ethyl (4-methyltetrahydro2H-pyran-4-yl) oxalate (3o, $96 \mathrm{mg}, 0.30 \mathrm{mmol}, 1.0$ equiv), PhEBX (2a, $157 \mathrm{mg}, 0.450 \mathrm{mmol}, 1.50$ equiv) and 4CzIPN (1a, $12 \mathrm{mg}, 0.015 \mathrm{mmol}, 5 \mathrm{~mol} \%)$. Column chromatography ( $\mathrm{SiO}_{2}, 1 \%$ to $5 \%$ EtOAc in Pentane) afforded, 4-methyl-4-(phenylethynyl)tetrahydro-2H-pyran (4o, 40 mg, purity: 94\%, 0.19 $\mathrm{mmol}, 67 \%)$ as a colorless oil.

Method B: 40 was synthesized following general procedure $F$ using ethyl (4-methyltetrahydro- $2 \mathrm{H}$ pyran-4-yl) oxalate (3o, $0.096 \mathrm{~g}, 0.30 \mathrm{mmol}, 1.0$ equiv) and PhEBX (2a, $0.261 \mathrm{~g}, 0.750 \mathrm{mmol}, 2.50$ equiv) in degassed $\mathrm{CH}_{2} \mathrm{Cl}_{2}(3.0 \mathrm{~mL}, 0.1 \mathrm{M})$. Column chromatography $\left(\mathrm{SiO}_{2}\right.$, pentane) afforded 4methyl-4-(phenylethynyl)tetrahydro-2H-pyran (4o, $0.040 \mathrm{~g}$ (93\% purity), $0.19 \mathrm{mmol}, 62 \%$ ).

Rf (pentane:EtOAc 95:5) $=0.4$.

${ }^{1} \mathrm{H}$ NMR $\left(400 \mathrm{MHz}, \mathrm{CDCl}_{3}\right)$ 8: $7.44-7.39(\mathrm{~m}, 2 \mathrm{H}, \mathrm{ArH}), 7.32-7.27(\mathrm{~m}, 3 \mathrm{H}, \mathrm{ArH}), 3.90-3.77(\mathrm{~m}, 4 \mathrm{H}$, $\left.\mathrm{OCH}_{2}\right), 1.76-1.68\left(\mathrm{~m}, 2 \mathrm{H}, \mathrm{CH}_{2}\right), 1.61$ (ddd, $\left.J=13.2,11.2,5.0 \mathrm{~Hz}, 2 \mathrm{H}, \mathrm{CH}_{2}\right), 1.35\left(\mathrm{~s}, 3 \mathrm{H}, \mathrm{CH}_{3}\right)$.

${ }^{13} \mathrm{C}$ NMR $\left(101 \mathrm{MHz}^{\mathrm{CDCl}} 3\right) \mathrm{\delta}$ : 131.7, 128.4, 127.9, 123.8, 94.7, 83.0, 65.4, 39.4, 31.1, 30.2.

IR $\left(v_{\max }, \mathrm{cm}^{-1}\right): 3058(\mathrm{~m}), 2959(\mathrm{~s}), 2857(\mathrm{~m}), 1746(\mathrm{~m}), 1492(\mathrm{~m}), 1448(\mathrm{~m}), 1174(\mathrm{~s}), 1107(\mathrm{~s})$.

HRMS (APPI/LTQ-Orbitrap) m/z: [M + H] Calcd for $\mathrm{C}_{14} \mathrm{H}_{17} \mathrm{O}^{+}$201.1274; Found 201.1273.

\section{- 1-(Phenylethynyl)adamantane $(4 p)$}

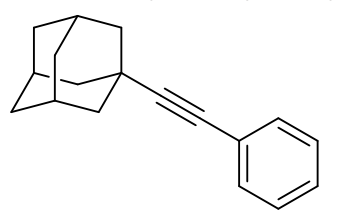

$4 \mathbf{p}$ was synthesized following the general procedure $E$ using cesium 2-(((1S,3S)-adamantan-1-yl)oxy)-2-oxoacetate (3p, $107 \mathrm{mg}, 0.300$ mmol, 1.00 equiv), PhEBX ( $2 \mathrm{a}, 157 \mathrm{mg}, 0.450 \mathrm{mmol}, 1.50$ equiv) and 4CZIPN (1a, $12 \mathrm{mg}, 0.015 \mathrm{mmol}, 5 \mathrm{~mol} \%)$.

Column chromatography $\left(\mathrm{SiO}_{2}\right.$, Pentane) afforded 1-(phenylethynyl)adamantane (4p, $20 \mathrm{mg}, 0.080$ $\mathrm{mmol}, 28 \%$ ) as a pale yellow oil.

$\mathbf{R f}$ (pentane) $=0.5$.

${ }^{1} \mathrm{H}$ NMR $\left(400 \mathrm{MHz}, \mathrm{CDCl}_{3}\right) \delta: 7.44-7.32(\mathrm{~m}, 2 \mathrm{H}, \mathrm{ArH}), 7.32-7.19(\mathrm{~m}, 3 \mathrm{H}, \mathrm{ArH}), 2.07-1.97(\mathrm{~m}, 3 \mathrm{H}$, $\mathrm{CH}), 1.97-1.92\left(\mathrm{~m}, 6 \mathrm{H}, \mathrm{CH}_{2}\right), 1.75-1.69\left(\mathrm{~m}, 6 \mathrm{H}, \mathrm{CH}_{2}\right)$.

${ }^{13} \mathrm{C}$ NMR (101 MHz, CDCl $)$ ): 131.8, 128.2, 127.5, 124.2, 98.6, 79.5, 43.0, 36.6, 30.2, 28.2.

IR $\left(v_{\max }, \mathrm{cm}^{-1}\right): 3060(\mathrm{w}), 2912(\mathrm{~s}), 2853(\mathrm{~m}), 1491(\mathrm{~m}), 1450(\mathrm{~m})$.

HRMS (nanochip-ESI/LTQ-Orbitrap) m/z: [M] ${ }^{+}$Calcd for $\mathrm{C}_{18} \mathrm{H}_{20}{ }^{+}$236.1560; Found 236.1561. 
- 1-(3,3-Dimethyl-5-phenylpent-1-yn-1-yl)-3-fluorobenzene (4q)<smiles>CC(C)(C#Cc1cccc(F)c1)CCc1ccccc1</smiles>

$\mathbf{4 q}$ was synthesized following general procedure $E$ using cesium 2(methyl-4-phenylbutan-2-yl)oxy-2-oxoacetate (3a, $0.110 \mathrm{~g}, 0.300$ mmol, 1 equiv), mFPhEBX ( $2 \mathrm{~b}, 0.164 \mathrm{~g}, 0.450 \mathrm{mmol}, 1.50$ equiv), 4CzIPN (1a, $0.012 \mathrm{~g}, 1.5 \mu \mathrm{mol}, 5 \mathrm{~mol} \%)$ in degassed $\mathrm{CH}_{2} \mathrm{Cl}_{2}(3 \mathrm{~mL}, 0.1$ $\mathrm{M})$.

Column chromatography ( $\mathrm{SiO}_{2}$, pentane) afforded 1-(3,3-dimethyl-5-phenylpent-1-yn-1-yl)-3fluorobenzene (4q, $0.045 \mathrm{~g}, 0.17 \mathrm{mmol}, 56 \%)$.

Rf (pentane) $=0.5$.

${ }^{1} \mathbf{H}$ NMR $\left(400 \mathrm{MHz}, \mathrm{CDCl}_{3}\right) \delta 7.34-7.15(\mathrm{~m}, 7 \mathrm{H}, \mathrm{ArH}), 7.10$ (ddd, $\left.J=9.6,2.7,1.4 \mathrm{~Hz}, 1 \mathrm{H}, \mathrm{ArH}\right), 6.98$ (tdd, $J=8.3,2.7,1.2 \mathrm{~Hz}, 1 \mathrm{H}, \mathrm{ArH}), 2.87-2.79\left(\mathrm{~m}, 2 \mathrm{H}, \operatorname{ArCH}_{2}\right), 1.84-1.75\left(\mathrm{~m}, 2 \mathrm{H}, \mathrm{CH}_{2}\right), 1.35(\mathrm{~s}, 6 \mathrm{H}$, $\left.\mathrm{C}\left(\mathrm{CH}_{3}\right)_{2}\right)$.

${ }^{13}$ C NMR $\left(101 \mathrm{MHz}, \mathrm{CDCl}_{3}\right) \delta 162.4(\mathrm{~d}, J=245.8 \mathrm{~Hz}), 142.6,129.7(\mathrm{~d}, J=8.7 \mathrm{~Hz}), 128.4,127.5(\mathrm{~d}, J=$ $2.9 \mathrm{~Hz}), 126.1-125.3(\mathrm{~m}), 118.4(\mathrm{~d}, J=22.5 \mathrm{~Hz}), 114.8(\mathrm{~d}, J=21.1 \mathrm{~Hz}), 98.0,79.8,45.4,32.1,31.9$, 29.1. 2 carbons are not resolved.

${ }^{19} \mathrm{~F}$ NMR (376 MHz, $\left.\mathrm{CDCl}_{3}\right) \delta-113.5(\mathrm{~d}, J=4.5 \mathrm{~Hz})$.

IR $\left(v_{\max }, \mathrm{cm}^{-1}\right) 3087(\mathrm{~m}), 3062(\mathrm{~m}), 2972(\mathrm{~s}), 2937(\mathrm{~s}), 2911(\mathrm{~s}), 1608(\mathrm{~s}), 1580$ (s), 1075 (s), 1056 (s), $909(\mathrm{~s}), 873(\mathrm{~s}), 784(\mathrm{~s})$.

HRMS (APPI/LTQ-Orbitrap) m/z: [M] ${ }^{+}$Calcd for $\mathrm{C}_{19} \mathrm{H}_{19} \mathrm{~F}^{+}$266.1465; Found 266.1473.

- 1-(3,3-Dimethyl-5-phenylpent-1-yn-1-yl)-4-(trifluoromethyl)benzene (4r)

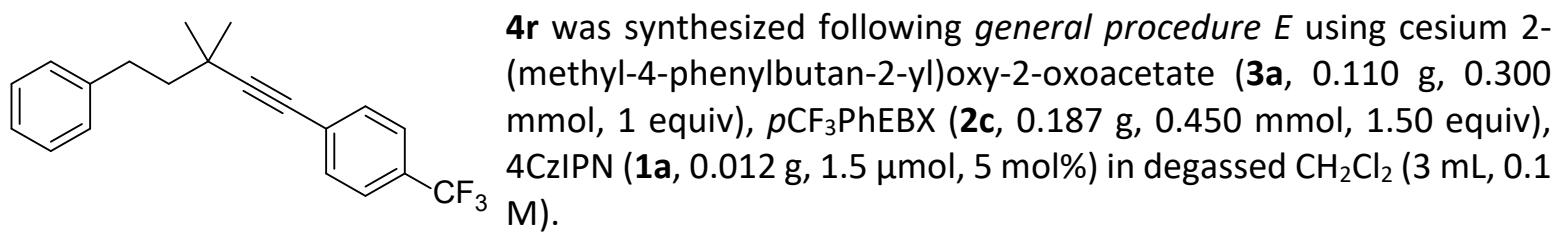

Column chromatography $\left(\mathrm{SiO}_{2}\right.$, pentane) afforded 1-(3,3-dimethyl-5-phenylpent-1-yn-1-yl)-4(trifluoromethyl)benzene (4r, $0.055 \mathrm{~g}, 0.17 \mathrm{mmol}, 58 \%)$.

$\mathbf{R f}($ pentane $)=0.4$.

${ }^{1} \mathrm{H}$ NMR $\left(400 \mathrm{MHz}, \mathrm{CDCl}_{3}\right) \delta 7.58-7.46(\mathrm{~m}, 4 \mathrm{H}, \mathrm{ArH}), 7.34-7.17(\mathrm{~m}, 5 \mathrm{H}, \mathrm{ArH}), 2.88-2.79(\mathrm{~m}, 2 \mathrm{H}$, $\operatorname{ArCH} 2), 1.85-1.77\left(\mathrm{~m}, 2 \mathrm{H}, \mathrm{CH}_{2}\right), 1.36\left(\mathrm{~s}, 6 \mathrm{H}, \mathrm{C}\left(\mathrm{CH}_{3}\right)_{2}\right)$.

${ }^{13} \mathrm{C}$ NMR $\left(101 \mathrm{MHz}, \mathrm{CDCl}_{3}\right) \delta 142.7,132.0,128.5(\mathrm{~m}), 125.9,125.2(\mathrm{~d}, J=3.9 \mathrm{~Hz}), 99.8,80.0,45.5$, 32.3, 32.1, 29.2. 4 carbons not resolved.

${ }^{19} \mathrm{~F}$ NMR $\left(376 \mathrm{MHz}, \mathrm{CDCl}_{3}\right) \delta-62.7$.

IR $\left(v_{\max }, \mathrm{cm}^{-1}\right) 3028(\mathrm{w}), 2975(\mathrm{w}), 2940(\mathrm{~m}), 2859(\mathrm{~m}), 2822(\mathrm{w}), 2239(\mathrm{w}), 1617(\mathrm{~m}), 1505(\mathrm{~m}), 1324$ (s), $1168(\mathrm{~m}), 1130(\mathrm{~s}), 1066(\mathrm{~s}), 910(\mathrm{~s}), 766(\mathrm{~m}), 743(\mathrm{~s})$.

HRMS (ESI/QTOF) m/z: [M + Ag] $]^{+}$Calcd for $\mathrm{C}_{20} \mathrm{H}_{19} \mathrm{AgF}_{3}{ }^{+}$423.0484; Found 423.0479.

- 1-(3,3-Dimethyl-5-phenylpent-1-yn-1-yl)-4-chlorobenzene (4s)<smiles>CC(C)(C#Cc1ccccc1Cl)CCc1ccccc1</smiles>

4s was synthesized following general procedure $E$ using cesium 2(methyl-4-phenylbutan-2-yl)oxy-2-oxoacetate (3a, $0.110 \mathrm{~g}, 0.300$ mmol, 1.00 equiv), oCIPhEBX ( $2 \mathrm{~d}, 0.172 \mathrm{~g}, 0.450 \mathrm{mmol}, 1.50$ equiv), 4CzIPN (1a, $0.012 \mathrm{~g}, 1.5 \mu \mathrm{mol}, 5 \mathrm{~mol} \%)$ in degassed $\mathrm{CH}_{2} \mathrm{Cl}_{2}(3 \mathrm{~mL}, 0.1$ $\mathrm{M})$. 
Column chromatography ( $\mathrm{SiO}_{2}$, pentane) afforded 1-(3,3-dimethyl-5-phenylpent-1-yn-1-yl)-4chlorobenzene (4s, $0.066 \mathrm{~g}, 0.23 \mathrm{mmol}, 78 \%)$.

Rf (pentane) $=0.3$.

${ }^{1} \mathrm{H}$ NMR $\left(400 \mathrm{MHz}, \mathrm{CDCl}_{3}\right) \delta 7.48-7.35(\mathrm{~m}, 2 \mathrm{H}, \mathrm{ArH}), 7.33-7.14(\mathrm{~m}, 7 \mathrm{H}, \mathrm{ArH}), 2.95-2.86(\mathrm{~m}, 2 \mathrm{H}$, $\left.\operatorname{ArCH})_{2}\right), 1.86-1.77\left(\mathrm{~m}, 2 \mathrm{H}, \mathrm{CH}_{2}\right), 1.38\left(\mathrm{~s}, 6 \mathrm{H}, \mathrm{C}\left(\mathrm{CH}_{3}\right)_{2}\right)$.

${ }^{13} \mathrm{C}$ NMR $\left(101 \mathrm{MHz}, \mathrm{CDCl}_{3}\right) \delta 143.0,136.0,133.3,129.3,128.7,128.6,128.5,126.4,125.8,123.9$, 102.7, 78.0, 45.7, 32.4, 32.3, 29.3.

IR $\left(v_{\max }, \mathrm{cm}^{-1}\right) 2972(\mathrm{~m}), 2901(\mathrm{~m}), 1495(\mathrm{w}), 1406(\mathrm{~m}), 1229(\mathrm{~m}), 1075(\mathrm{~s}), 905(\mathrm{~s}), 729(\mathrm{~s})$.

HRMS (APPI/LTQ-Orbitrap) m/z: [M] ${ }^{+}$Calcd for $\mathrm{C}_{19} \mathrm{H}_{19}{ }^{35} \mathrm{Cl}^{+}$282.1170; Found 282.1178 .

- 1-(3,3-Dimethyl-5-phenylpent-1-yn-1-yl)-4-bromobenzene (4t)<smiles>CC(C)(C#Cc1ccc(Br)cc1)CCc1ccccc1</smiles>

4t was synthesized following general procedure $E$ using cesium 2(methyl-4-phenylbutan-2-yl)oxy-2-oxoacetate (3a, $0.110 \mathrm{~g}, 0.300$ mmol, 1.00 equiv), PhEBX (2e, $0.192 \mathrm{~g}, 0.450 \mathrm{mmol}, 1.50$ equiv), 4CZIPN (1a, $0.012 \mathrm{~g}, 1.5 \mu \mathrm{mol}, 5 \mathrm{~mol} \%)$ in degassed $\mathrm{CH}_{2} \mathrm{Cl}_{2}(3 \mathrm{~mL}$, $0.1 \mathrm{M})$.

Column chromatography $\left(\mathrm{SiO}_{2}\right.$, pentane) afforded 1-(3,3-dimethyl-5-phenylpent-1-yn-1-yl)-4bromobenzene (4t, $0.044 \mathrm{~g}, 0.13 \mathrm{mmol}, 45 \%)$.

$\mathbf{R f}$ (pentane) $=0.3$.

${ }^{1} \mathbf{H}$ NMR $\left(400 \mathrm{MHz}, \mathrm{CDCl}_{3}\right) \delta 7.45-7.38(\mathrm{~m}, 2 \mathrm{H}, \mathrm{ArH}), 7.33-7.25(\mathrm{~m}, 2 \mathrm{H}, \mathrm{ArH}), 7.29-7.21(\mathrm{~m}, 3 \mathrm{H}$, $\operatorname{ArH}), 7.25-7.14(\mathrm{~m}, 2 \mathrm{H}, \mathrm{ArH}), 2.87-2.78\left(\mathrm{~m}, 2 \mathrm{H}, \mathrm{ArCH}_{2}\right), 1.83-1.74\left(\mathrm{~m}, 2 \mathrm{H}, \mathrm{CH}_{2}\right), 1.34(\mathrm{~s}, 6 \mathrm{H}$, $\left.\mathrm{C}\left(\mathrm{CH}_{3}\right)_{2}\right)$.

${ }^{13} \mathrm{C}$ NMR $\left(101 \mathrm{MHz}, \mathrm{CDCl}_{3}\right) \delta 142.8,133.2,131.5,128.5,125.9,123.1,121.7,98.3,80.1,45.5,32.3$, 32.1, 29.3. 1 carbon is not resolved.

IR $\left(V_{\max }, \mathrm{cm}^{-1}\right) 3086(\mathrm{~m}), 3062(\mathrm{~m}), 3026(\mathrm{~m}), 2968(\mathrm{~m}), 2920(\mathrm{~m}), 2861(\mathrm{~m}), 1485(\mathrm{~s}), 1469(\mathrm{~m}), 1312$ $(\mathrm{m}), 1265(\mathrm{~m}), 1070(\mathrm{~s}), 1011(\mathrm{~s}), 823(\mathrm{~s}), 745(\mathrm{~s}), 700(\mathrm{~s})$.

HRMS (ESI/QTOF) m/z: [M + Ag] $]^{+}$Calcd for $\mathrm{C}_{19} \mathrm{H}_{19} \mathrm{Ag}^{79} \mathrm{Br}^{+} 432.9716$; Found 432.9707 .

- 1-(3,3-dimethyl-5-phenylpent-1-yn-1-yl)-2-bromobenzene (4u)<smiles>CC(C)(C#Cc1ccccc1Br)CCc1ccccc1</smiles>

4u was synthesized following general procedure $E$ using cesium 2(methyl-4-phenylbutan-2-yl)oxy-2-oxoacetate (3a, $0.110 \mathrm{~g}, 0.300$ mmol, 1.00 equiv), PhEBX (2f, $0.192 \mathrm{~g}, 0.450 \mathrm{mmol}, 1.50$ equiv), 4CzIPN (1a, $0.012 \mathrm{~g}, 1.5 \mu \mathrm{mol}, 5 \mathrm{~mol} \%)$ in degassed $\mathrm{CH}_{2} \mathrm{Cl}_{2}(3 \mathrm{~mL}$, $0.1 \mathrm{M})$.

Column chromatography $\left(\mathrm{SiO}_{2}\right.$, pentane) afforded (3,3-dimethylpent-1-yne-1,5-diyl)dibenzene (4u, $0.071 \mathrm{~g}, 0.22 \mathrm{mmol}, 72 \%$ ).

$\mathbf{R f}$ (pentane) $=0.3$.

${ }^{1} \mathrm{H}$ NMR $\left(400 \mathrm{MHz}, \mathrm{CDCl}_{3}\right) \delta 7.57(\mathrm{dd}, J=8.0,1.2 \mathrm{~Hz}, 1 \mathrm{H}, \mathrm{ArH}), 7.45(\mathrm{dd}, J=7.7,1.7 \mathrm{~Hz}, 1 \mathrm{H}, \operatorname{ArH})$, $7.33-7.18(\mathrm{~m}, 5 \mathrm{H}, \mathrm{ArH}), 7.22-7.14(\mathrm{~m}, 1 \mathrm{H}, \mathrm{ArH}), 7.12(\mathrm{td}, J=7.7,1.7 \mathrm{~Hz}, 1 \mathrm{H}, \mathrm{ArH}), 2.96-2.87(\mathrm{~m}$, $\left.2 \mathrm{H}, \mathrm{ArCH}_{2}\right), 1.87-1.78\left(\mathrm{~m}, 2 \mathrm{H}, \mathrm{CH}_{2}\right), 1.38\left(\mathrm{~s}, 6 \mathrm{H}, \mathrm{C}\left(\mathrm{CH}_{3}\right)_{2}\right)$.

${ }^{13} \mathrm{C}$ NMR $\left(101 \mathrm{MHz}, \mathrm{CDCl}_{3}\right) \delta 142.8,133.2,132.3,128.7,128.5,128.4,126.9,126.0,125.7,101.9$, 79.7, 45.5, 32.3, 32.2, 29.1. 1 carbon is not resolved.

IR $\left(v_{\max }, \mathrm{cm}^{-1}\right) 3062(\mathrm{~m}), 3026(\mathrm{~m}), 2968(\mathrm{~s}), 2925(\mathrm{~m}), 2865(\mathrm{~m}), 2226(\mathrm{~m}), 1466(\mathrm{~s}), 1058(\mathrm{~m}), 1047$ (s), 1027 (s), 753 (s), 700 (s).

HRMS (APPI/LTQ-Orbitrap) m/z: [M] ${ }^{+}$Calcd for $\mathrm{C}_{19} \mathrm{H}_{19}{ }^{79} \mathrm{Br}^{+}$326.0665; Found 326.0676. 
- (3R,3aS,6S,7R,8aS)-3,6,8,8-tetramethyl-6-(phenylethynyl)octahydro-1H-3a,7methanoazulene $(4 \mathrm{w})$

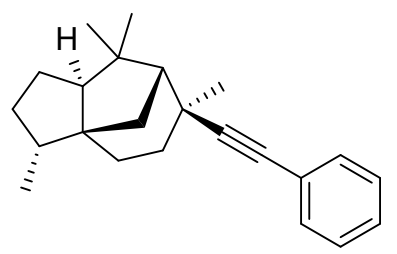

Method A: $\mathbf{4} \mathbf{w}$ was synthesized following general procedure $E$ using cedrol derived cesium oxalate $3 w$ ( $0.128 \mathrm{~g}, 0.300 \mathrm{mmol}, 1$ equiv), PhEBX (2a, $0.157 \mathrm{~g}, 0.450 \mathrm{mmol}, 1.50$ equiv), 4CzIPN (1a, 0.012 g, $1.5 \mu \mathrm{mol}, 5 \mathrm{~mol} \%)$ in degassed $\mathrm{CH}_{2} \mathrm{Cl}_{2}(3 \mathrm{~mL}, 0.1 \mathrm{M})$. Column chromatography ( $\mathrm{SiO}_{2}$, pentane) afforded $(3 R, 3 \mathrm{aS}, 6 \mathrm{~S}, 7 \mathrm{R}, 8 \mathrm{a} S)-3,6,8,8$-tetramethyl-6-(phenylethynyl)octahydro-1H-3a,7methanoazulene ( $4 \mathbf{w}$ ) as a single diasteroisomer (0.075 g (70\% purity), $\mathrm{dr}>20: 1,0.17 \mathrm{mmol}, 58 \%$ ). The compound could be partially purified from 1,4-diphenylbuta-1,4-diyne (major impurity) by preparative $\mathrm{TLC}\left(\mathrm{SiO}_{2}\right.$, glass plate, Heptane) allowing full characterisation of $\mathbf{4} \mathbf{w}$.

Method $B: 4 \mathbf{w}$ was synthesized following general procedure $F$ using cedrol derived cesium oxalate 3w (0.128 g, $0.300 \mathrm{mmol}, 1$ equiv) and PhEBX (2a, $0.261 \mathrm{~g}, 0.750 \mathrm{mmol}, 2.50$ equiv) in degassed $\mathrm{CH}_{2} \mathrm{Cl}_{2}(3 \mathrm{~mL}, 0.1 \mathrm{M})$. Column chromatography $\left(\mathrm{SiO}_{2}\right.$, pentane) afforded (3R,3aS,6S,7R,8aS)-3,6,8,8tetramethyl-6-(phenylethynyl)octahydro-1H-3a,7-methanoazulene (4w) as a single diasteroisomer (0.077 g (48\% purity), dr > 20:1, $0.15 \mathrm{mmol}, 50 \%$ ).

Rf (pentane) $=0.6$.

${ }^{1} \mathrm{H}$ NMR $\left(400 \mathrm{MHz}, \mathrm{CDCl}_{3}\right) \delta 7.42-7.35(\mathrm{~m}, 2 \mathrm{H}, \mathrm{ArH}), 7.32-7.20(\mathrm{~m}, 3 \mathrm{H}, \mathrm{ArH}), 2.18-2.10(\mathrm{~m}, 1 \mathrm{H}$, aliphatic- $\mathrm{CH}$ or $\left.\mathrm{CH}_{2}\right), 1.95-1.83\left(\mathrm{~m}, 2 \mathrm{H}\right.$, aliphatic- $\mathrm{CH}$ or $\left.\mathrm{CH}_{2}\right), 1.85-1.76(\mathrm{~m}, 1 \mathrm{H}$, aliphatic- $\mathrm{CH}$ or $\left.\mathrm{CH}_{2}\right), 1.79-1.65\left(\mathrm{~m}, 5 \mathrm{H}\right.$, aliphatic- $\mathrm{CH}$ or $\left.\mathrm{CH}_{2}\right), 1.60-1.50\left(\mathrm{~m}, 1 \mathrm{H}\right.$, aliphatic- $\mathrm{CH}$ or $\left.\mathrm{CH}_{2}\right), 1.48(\mathrm{~s}, 3 \mathrm{H}$, $\left.\mathrm{CH}_{3}\right), 1.46-1.34\left(\mathrm{~m}, 2 \mathrm{H}\right.$, aliphatic- $\mathrm{CH}$ or $\left.\mathrm{CH}_{2}\right), 1.28(\mathrm{dtd}, J=11.8,7.7,6.0 \mathrm{~Hz}, 1 \mathrm{H}$, aliphatic- $\mathrm{CH}$ or $\left.\mathrm{CH}_{2}\right), 1.22\left(\mathrm{~s}, 3 \mathrm{H}, \mathrm{CH}_{3}\right), 1.03\left(\mathrm{~s}, 3 \mathrm{H}, \mathrm{CH}_{3}\right), 0.87\left(\mathrm{~d}, \mathrm{~J}=7.1 \mathrm{~Hz}, 3 \mathrm{H}, \mathrm{CH}_{3}\right)$.

${ }^{13} \mathrm{C}$ NMR $\left(101 \mathrm{MHz}, \mathrm{CDCl}_{3}\right) \delta 131.6,128.3,127.3,124.6,100.0,80.8,59.0,57.4,54.2,44.4,44.0$, 42.0, 39.1, 37.1, 34.9, 31.9, 29.8, 29.0, 28.5, 25.6, 15.7 .

$\operatorname{IR}\left(v_{\max }, \mathrm{cm}^{-1}\right) 3055(\mathrm{~m}), 3010(\mathrm{~m}), 2950(\mathrm{~m}), 2870(\mathrm{~m}), 2851(\mathrm{~m}), 1648(\mathrm{~m}), 1474(\mathrm{~m}), 1442(\mathrm{~m}), 1246$ (m), $755(\mathrm{~s}), 724(\mathrm{~m}), 690(\mathrm{~s})$.

HRMS (APPI/LTQ-Orbitrap) m/z: [M] ${ }^{+}$Calcd for $\mathrm{C}_{23} \mathrm{H}_{30}{ }^{+}$306.2342; Found 306.2342. 
HMBC

Correlation in $\mathrm{HMBC}$

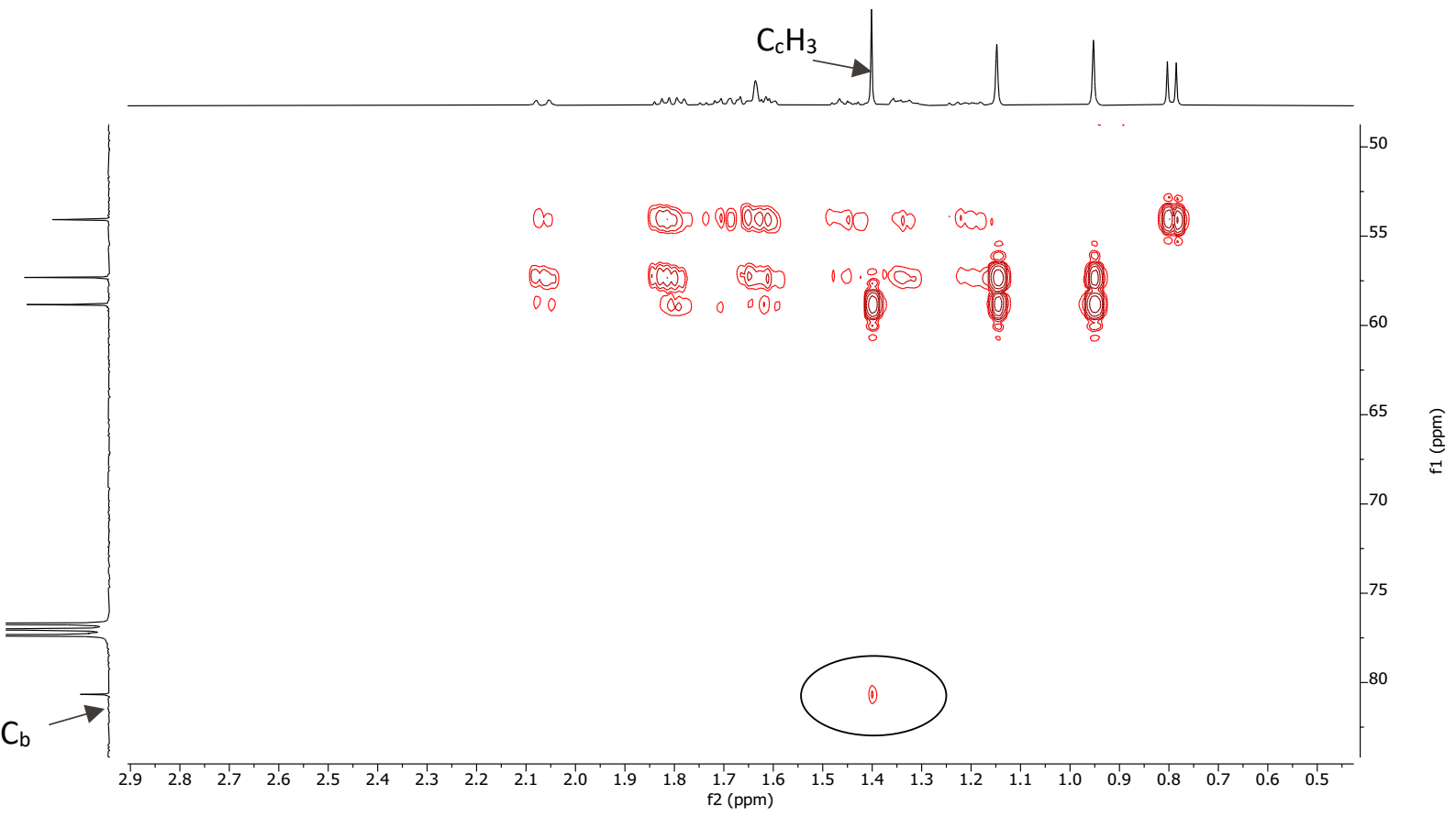


NOESY

Correlation in NOESY
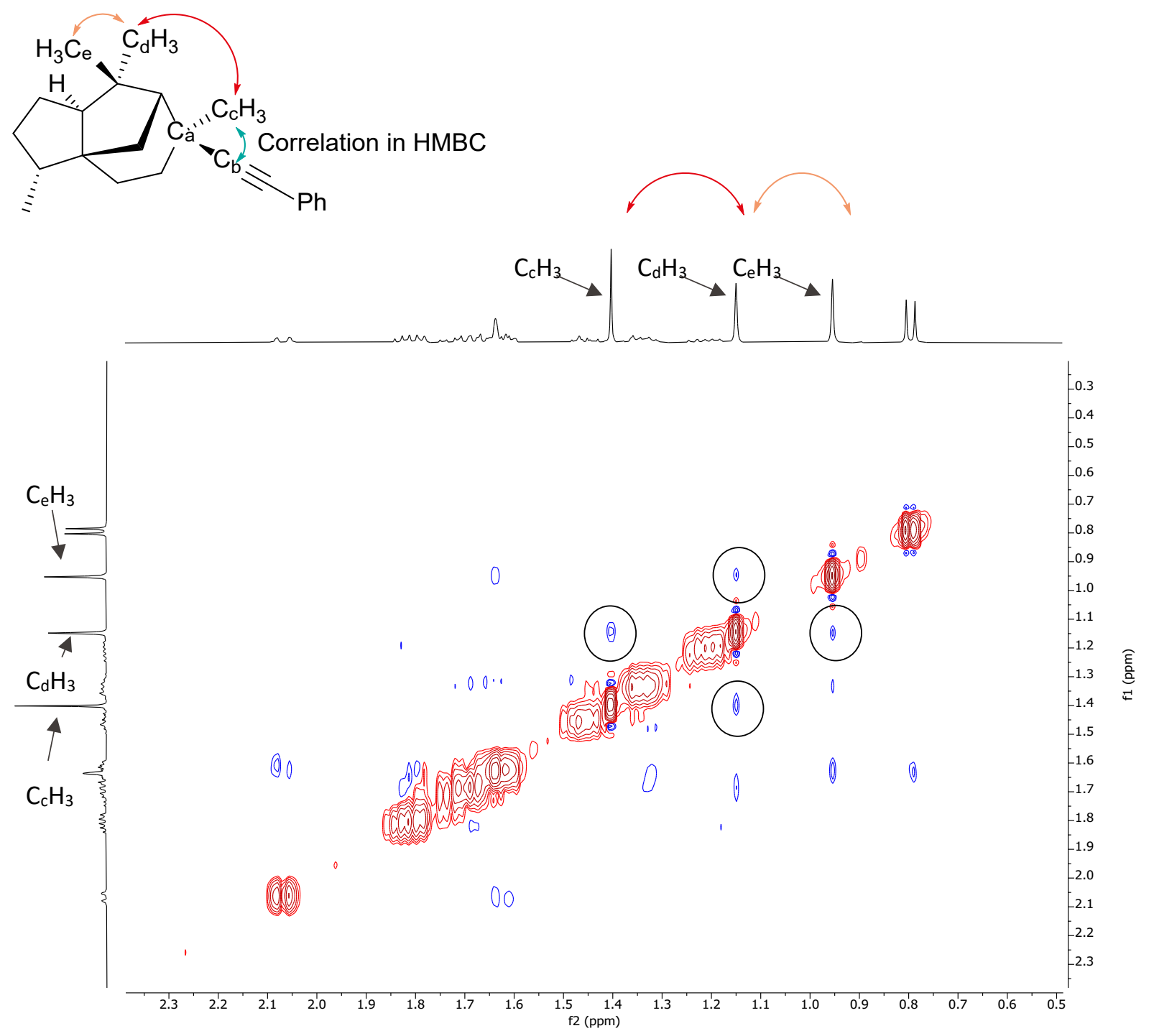
- (1R,2R,5R)-5-isopropyl-2-methyl-2-(phenylethynyl)-6-oxabicyclo[3.2.1]octan-7-one $(4 \mathrm{x})$<smiles>C#CC1(C)CC2(C(C)C)OC(=O)C1C2C#Cc1ccccc1</smiles>

Method A: 4x was synthesized following the general procedure $E$ using Cesium $(R)$-2-((1-isopropyl4-methylcyclohex-3-en-1-yl)oxy)-2-oxoacetate (3x, $107 \mathrm{mg}, 0.300 \mathrm{mmol}, 1.00$ equiv), PhEBX (2a, $157 \mathrm{mg}, 0.450 \mathrm{mmol}, 1.50$ equiv) and $4 \mathrm{CzIPN}$ (1a, $12 \mathrm{mg}, 0.015 \mathrm{mmol}, 5 \mathrm{~mol} \%)$. Column chromatography $\left(\mathrm{SiO}_{2}, 5 \%\right.$ EtOAc in Pentane) afforded $(1 R, 2 R, 5 R)$-5-isopropyl-2-methyl-2(phenylethynyl)-6-oxabicyclo[3.2.1]octan-7-one (4x, $45 \mathrm{mg}, \mathrm{dr}>20: 1,0.16 \mathrm{mmol}, 53 \%$ ) as a colorless oil.

Method B: 4x was synthesized following the general procedure $F$ using Cesium $(R)-2-((1$-isopropyl4-methylcyclohex-3-en-1-yl)oxy)-2-oxoacetate (3x, $107 \mathrm{mg}, 0.300 \mathrm{mmol}, 1.00$ equiv) and PhEBX (2a, $261 \mathrm{mg}, 0.750 \mathrm{mmol}, 2.5$ equiv). Column chromatography $\left(\mathrm{SiO}_{2}, 5 \%\right.$ EtOAc in Pentane) afforded $(1 R, 2 R, 5 R)$-5-isopropyl-2-methyl-2-(phenylethynyl)-6-oxabicyclo[3.2.1]octan-7-one (4x, $40 \mathrm{mg}, \mathrm{dr}>$ 20:1, $0.16 \mathrm{mmol}, 47 \%)$ as a colorless oil.

Rf (pentane:EtOAc 95:5) = 0.4 .

${ }^{1} \mathrm{H}$ NMR (400 MHz, CDCl 3 ) $\delta: 7.44-7.37(\mathrm{~m}, 2 \mathrm{H}, \mathrm{ArH}), 7.35-7.28(\mathrm{~m}, 3 \mathrm{H}, \mathrm{ArH}), 2.65(\mathrm{dd}, J=5.6,1.7$ $\left.\mathrm{Hz}, 1 \mathrm{H}, \mathrm{CHCO}_{2}\right), 2.47\left(\mathrm{~d}, J=11.9 \mathrm{~Hz}, 1 \mathrm{H}, \mathrm{CO}_{2} \mathrm{CHCH}_{a x}\right), 2.15-2.08\left(\mathrm{~m}, 1 \mathrm{H}, \mathrm{CO}_{2} \mathrm{CHCH}_{\text {eq }}\right), 2.07-2.00$ (m, $\left.1 \mathrm{H}, \mathrm{CCH}_{3} \mathrm{CH}_{a x}\right), 2.00-1.91\left(\mathrm{~m}, 1 \mathrm{H}, \mathrm{C}\left(\mathrm{CH}_{3}\right)_{2} \mathrm{H}\right), 1.91-1.85\left(\mathrm{~m}, 1 \mathrm{H}, \mathrm{COCH}_{a x}\right), 1.84-1.77(\mathrm{~m}, 1 \mathrm{H}$, $\left.\mathrm{COCH}_{e q}\right), 1.74-1.63\left(\mathrm{~m}, 1 \mathrm{H}, \mathrm{CCH}_{3} \mathrm{CH}_{e q}\right), 1.48\left(\mathrm{~s}, 3 \mathrm{H}, \mathrm{CH}_{3}\right), 1.01\left(\mathrm{~d}, J=6.8 \mathrm{~Hz}, 3 \mathrm{H}, \mathrm{CH}\left(\mathrm{CH}_{3}\right)_{2}\right), 0.97(\mathrm{~d}$, $\left.J=6.9 \mathrm{~Hz}, 1 \mathrm{H}, \mathrm{CH}\left(\mathrm{CH}_{3}\right)_{2}\right)$.

${ }^{13} \mathrm{C}$ NMR $\left(101 \mathrm{MHz}, \mathrm{CDCl}_{3}\right) \delta: 176.3,131.8,128.5,128.3,123.2,93.1,90.1,82.6,51.0,37.4,35.3$, $34.7,34.3,27.3,26.8,17.2,16.8$.

IR $\left(v_{\max }, \mathrm{cm}^{-1}\right)$ : $3059(\mathrm{w}), 2967(\mathrm{~m}), 2881(\mathrm{~m}), 1773(\mathrm{~s}), 1593(\mathrm{w}), 1461(\mathrm{~m}), 1171(\mathrm{~m}), 930(\mathrm{~m})$. HRMS (APPI/LTQ-Orbitrap) m/z: [M + Na] ${ }^{+}$Calcd for $\mathrm{C}_{19} \mathrm{H}_{22} \mathrm{NaO}_{2}{ }^{+}$305.1512; Found 305.1512. 

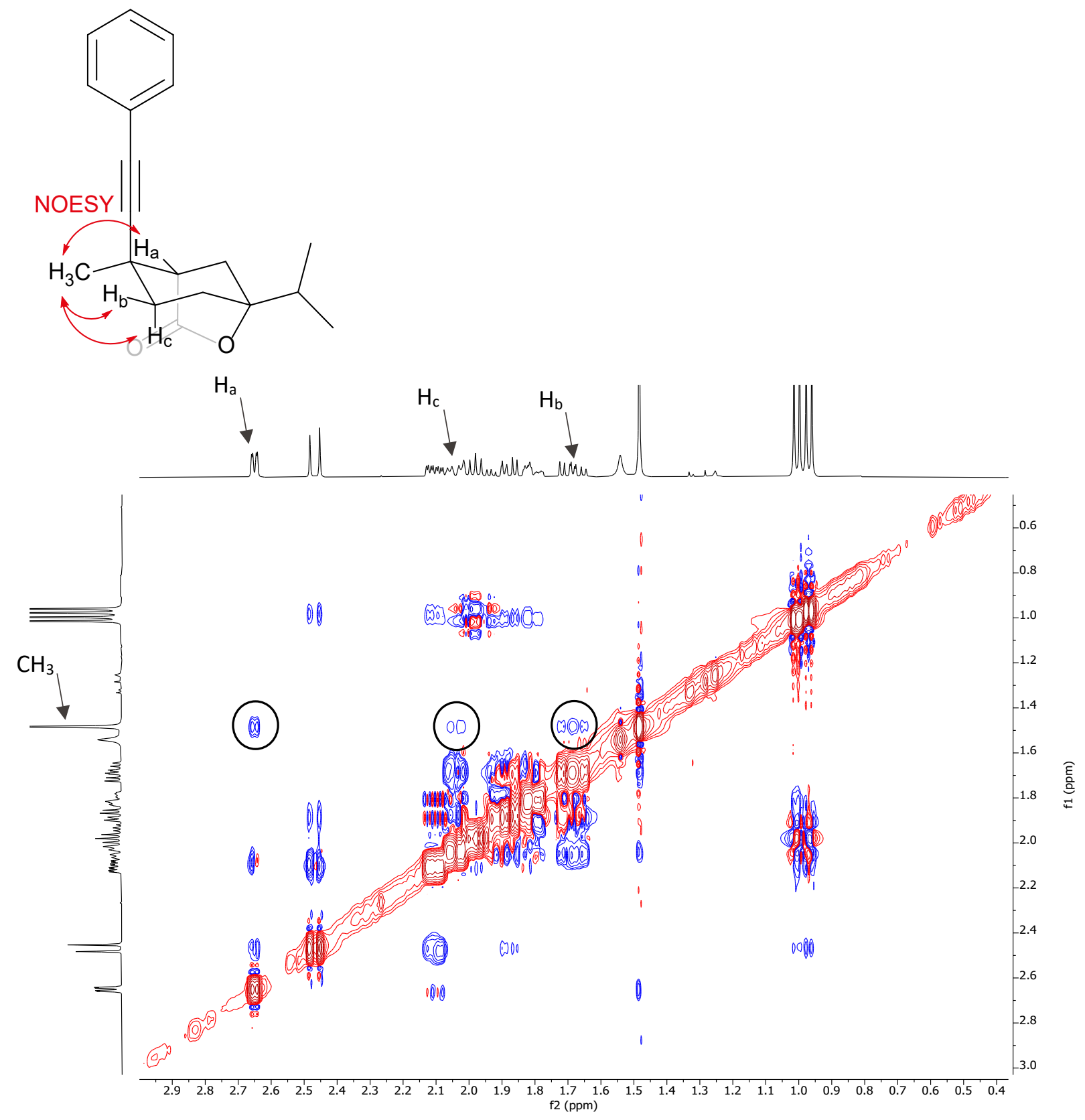


\section{Mechanistic studies}

\subsection{NMR studies}

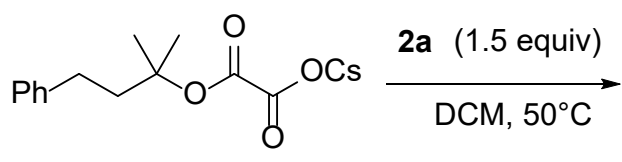

$3 a$

PhEB

X $2 a$<smiles>Cc1ccccc1C(=O)OCC(=O)c1ccccc1</smiles>

$6 a$

0.45 equiv<smiles>CC(C)(CC[PH+]c1ccccc1)OC(=O)C(=O)OCC(=O)c1ccccc1</smiles>

6b

0.2 equiv

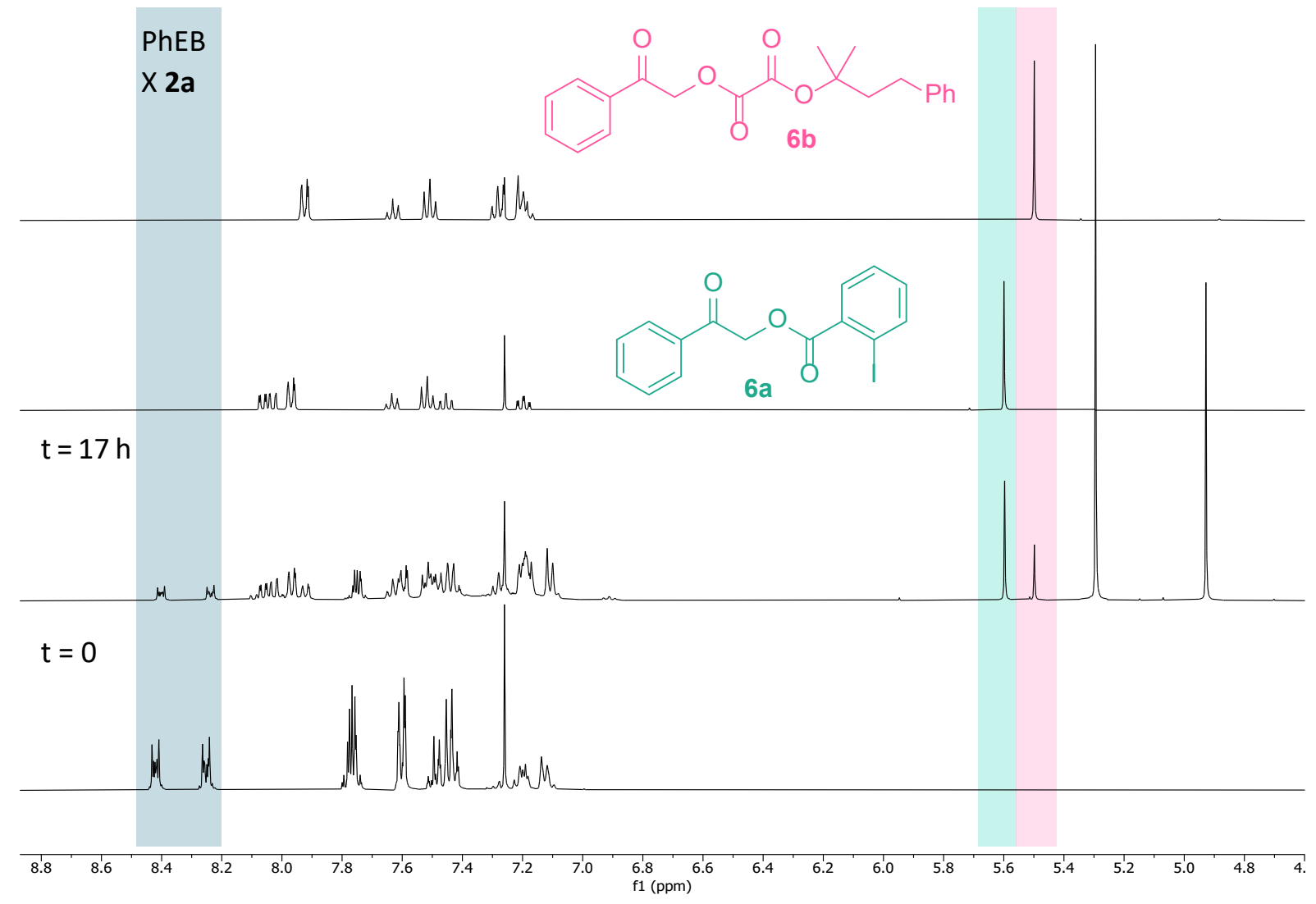

Figure S2. Thermal degradation of PhEBX in presence of $\mathbf{3 a}$ 
<smiles>CC(C)(CCc1ccccc1)OC(=O)C(=O)O[Na]</smiles>

$3 a$

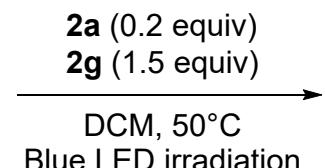

Blue LED irradiation<smiles>CC(C)(C#Cc1ccccc1)CCc1ccccc1</smiles>

$4 a$<smiles>CC(C)(C#C[In])CCc1ccccc1</smiles>

$4 v$

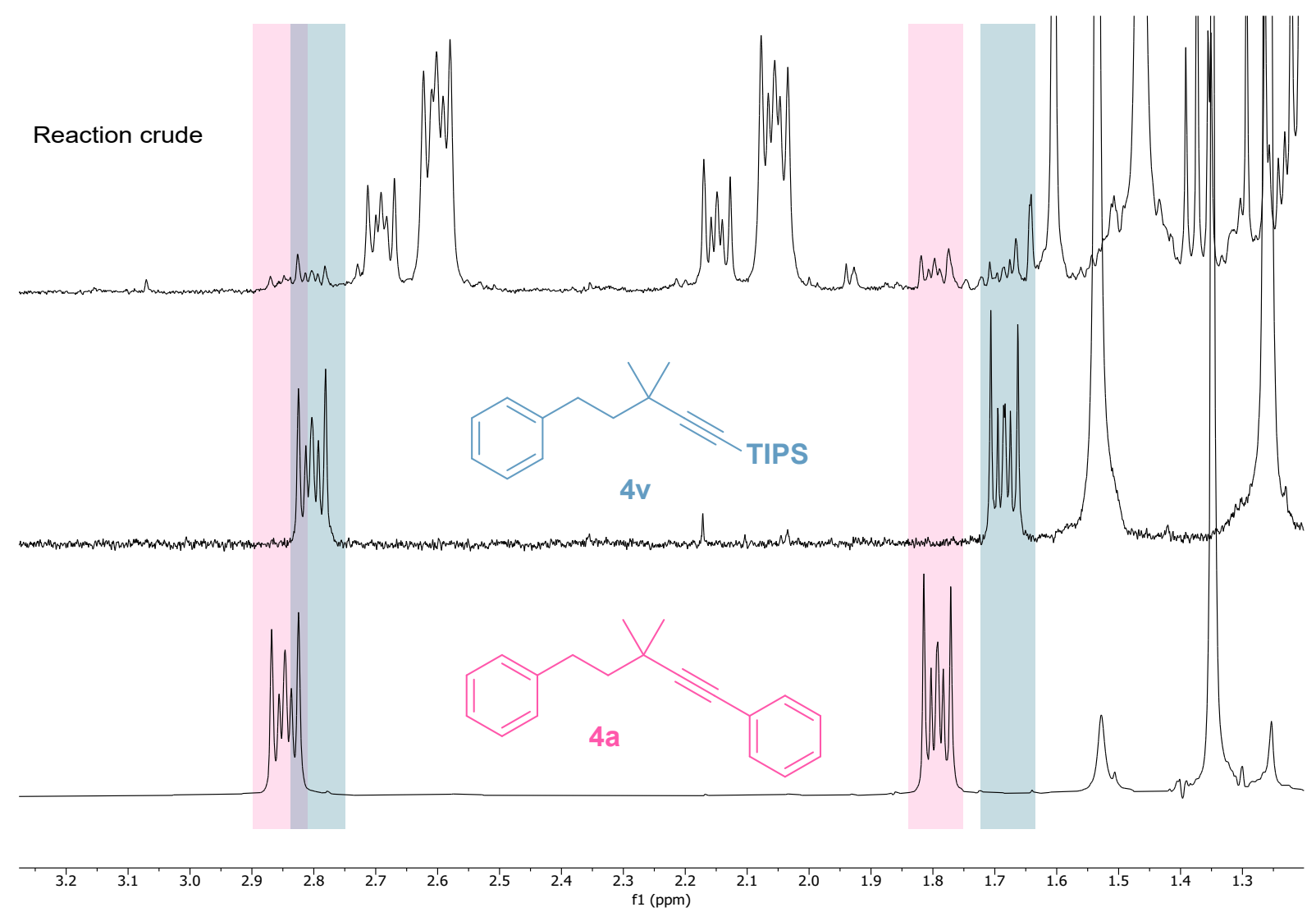

Figure S3. TIPS-alkynylation with PhEBX as a photooxidant

\subsection{Synthesis and characterization of $6 a, 6 b$ and $\mathbf{4 v}$}

2-Oxo-2-phenylethyl 2-iodobenzoate (6a)<smiles>O=C(O)c1ccccc1I</smiles>

16<smiles>O=C(CBr)c1ccccc1</smiles>

25

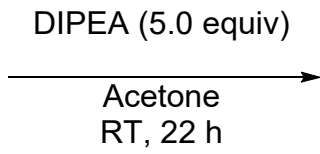

$\mathrm{RT}, 22 \mathrm{~h}$<smiles>O=C(COC(=O)c1ccccc1I)c1ccccc1</smiles>

$6 a$

Following a reported procedure, ${ }^{23} 2$-iodobenzoic acid $(16,744 \mathrm{mg}, 3.00 \mathrm{mmol}, 1.00$ equiv) and 2bromo-1-phenylethanone $(25,657 \mathrm{mg}, 3.30 \mathrm{mmol}, 1.10$ equiv) were dissolved in acetone $(12.0 \mathrm{~mL})$. DIPEA ( $2.6 \mathrm{~mL}, 15 \mathrm{mmol}, 5.0$ equiv) was then added and the reaction mixture was stirred overnight. The mixture was then diluted with EtOAc and washed with water. The organic layer was dried over

${ }^{23}$ Speckmeier, E.; Zeitler, K. ACS Catal. 2017, 7, 6821-6826. 
$\mathrm{MgSO}_{4}$, filtered and the solvent was removed under reduced pressure. The crude product was purified by flash chromatography $\left(\mathrm{SiO}_{2}, 10 \%\right.$ EtOAc in pentane, $\left.\mathrm{Rf}=0.4\right)$ obtaining 2-oxo-2-phenylethyl 2iodobenzoate (6a, $660 \mathrm{mg}, 1.80 \mathrm{mmol}, 60 \%$ yield) as an off-white solid.<smiles>O=C(COC(=O)c1ccccc1I)c1ccccc1</smiles>

${ }^{1} \mathbf{H}$ NMR $\left(400 \mathrm{MHz}, \mathrm{CDCl}_{3}\right) \delta: 8.06(\mathrm{dd}, J=7.8,1.7 \mathrm{~Hz}, 1 \mathrm{H}, \mathrm{ArH}), 8.03$ $(\mathrm{dd}, J=8.0,1.2 \mathrm{~Hz}, 1 \mathrm{H}, \mathrm{ArH}), 8.00-7.94(\mathrm{~m}, 2 \mathrm{H}, \mathrm{ArH}), 7.68-7.59(\mathrm{~m}$, $1 \mathrm{H}, \operatorname{ArH}), 7.56-7.49(\mathrm{~m}, 2 \mathrm{H}, \operatorname{ArH}), 7.45(\mathrm{td}, J=7.7,1.2 \mathrm{~Hz}, 1 \mathrm{H}, \operatorname{ArH})$, $7.20(\mathrm{td}, \mathrm{J}=7.7,1.7 \mathrm{~Hz}, 1 \mathrm{H}, \mathrm{ArH}), 5.60\left(\mathrm{~s}, 2 \mathrm{H}, \mathrm{CH}_{2}\right)$.

${ }^{13} \mathrm{C}$ NMR $(101 \mathrm{MHz}, \mathrm{CDCl} 3)$ ) $: 191.8,165.9,141.6,134.3,134.2,133.2$, $131.8,129.1,128.2,128.0,94.6,66.9 .1$ Carbon atom is unresolved. Constitent with reported literature data. ${ }^{23}$

\section{2-methyl-4-phenylbutan-2-yl (2-oxo-2-phenylethyl) oxalate (6b)}<smiles>CC(C)(O)CCc1ccccc1</smiles><smiles>CC(C)(CCc1ccccc1)OC(=O)C(=O)O</smiles>

26 22a

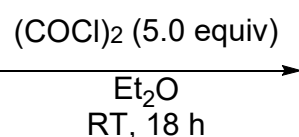

$\mathrm{RT}, 18 \mathrm{~h}$

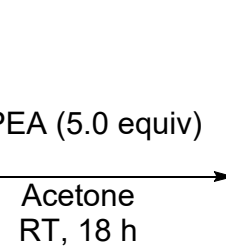

25<smiles>CC(C)(CCc1ccccc1)OC(=O)C(=O)O</smiles>

26<smiles>CC(C)(CCc1ccccc1)OC(=O)C(=O)OCC(=O)c1ccccc1</smiles>

$6 b$

Following a modified reported procedure, ${ }^{24}$ a solution of 2-methyl-4-phenylbutan-2-ol (22a, $0.85 \mathrm{~mL}$, $5.0 \mathrm{mmol}, 1.0$ equiv) in $\mathrm{Et}_{2} \mathrm{O}(40 \mathrm{~mL})$ was cooled to $0{ }^{\circ} \mathrm{C}$. Oxalyl dichloride $(0.90 \mathrm{~mL}, 10 \mathrm{mmol}, 2.0$ equiv) was then added dropwise. The mixture was warmed to room temperature after $10 \mathrm{~min}$, and after an additional $1.5 \mathrm{~h}$, oxalyl dichloride $(0.44 \mathrm{~mL}, 5.0 \mathrm{mmol}, 1.0$ equiv) were added. After an additional $1 \mathrm{~h}$ oxalyl dichloride ( $0.90 \mathrm{~mL}, 10 \mathrm{mmol}, 2.0$ equiv) was added and the reaction was stirred for another hour. The reaction was carefully quenched at $0{ }^{\circ} \mathrm{C}$ by the dropwise addition of $\mathrm{H} 2 \mathrm{O}(30 \mathrm{~mL})$ after addition of a vent needle. The mixture was stirred vigorously and warmed to room temperature. The layers were separated, and the aqueous layer extracted with $\mathrm{Et}_{2} \mathrm{O}(3 \times 15 \mathrm{~mL})$, and the combined organic layers dried with $\mathrm{Na}_{2} \mathrm{SO}_{4}$, filtered, and concentrated under reduced pressure affording 2-(2methyl-4-phenylbutan-2-yl)oxy-2-oxoacetic acid as a clear oil (767 mg, $3.25 \mathrm{mmol}, 65 \%$ yield), which was used directly in the next step. Following a modified reported procedure, ${ }^{23}$ the crude oil of 2-(2methyl-4-phenylbutan-2-yl)oxy-2-oxoacetic acid (26, $767 \mathrm{mg}, 3.25 \mathrm{mmol}, 1.0$ equiv) was dissolved in acetone (12 mL). DIPEA ( $2.4 \mathrm{~mL}, 15 \mathrm{mmol}, 5$ equiv) and phenacyl bromide (25, $597 \mathrm{mg}, 3.00 \mathrm{mmol}, 0.9$ equiv) were then added. The reaction was stirred overnight. The reaction was quenched with water $(5 \mathrm{~mL})$, diluted with EtOAc $(20 \mathrm{~mL})$. The organic layer was washed with sat. aq. $\mathrm{NH} 4 \mathrm{Cl}(3 \times 10 \mathrm{~mL})$, then brine $(10 \mathrm{~mL})$. The organic layer was dried over $\mathrm{Na}_{2} \mathrm{SO}_{4}$, filtered, and concentrated in vacuo to afford 2-methyl-4-phenylbutan-2-yl (2-oxo-2-phenylethyl) oxalate as a crude yellow oil (6b, $930 \mathrm{mg}, 2.62$ $\mathrm{mmol}, 87 \%$ yield, $52 \%$ yield over both steps).

\footnotetext{
${ }^{24}$ Su, J. Y.; Grünenfelder, D. C.; Takeuchi, K.; Reisman, S. E. Org. Lett. 2018, 20, 4912-4916.
} 
<smiles>CC(C)(CCc1ccccc1)OC(=O)C(=O)OCC(=O)c1ccccc1</smiles>

${ }^{1} \mathbf{H}$ NMR $\left(400 \mathrm{MHz}, \mathrm{CDCl}_{3}\right) \delta 7.96-7.89(\mathrm{~m}, 2 \mathrm{H}, \mathrm{ArH}), 7.67-7.58$ $(\mathrm{m}, 1 \mathrm{H}, \mathrm{ArH}), 7.50(\mathrm{t}, J=7.7 \mathrm{~Hz}, 2 \mathrm{H}, \mathrm{ArH}), 7.29(\mathrm{t}, J=7.5 \mathrm{~Hz}, 2 \mathrm{H}, \mathrm{ArH})$, $7.25-7.17(\mathrm{~m}, 3 \mathrm{H}, \mathrm{ArH}), 5.50\left(\mathrm{~s}, 2 \mathrm{H}, \mathrm{C}(\mathrm{O}) \mathrm{CH}_{2} \mathrm{O}\right), 2.77-2.69(\mathrm{~m}, 2 \mathrm{H}$, $\left.\mathrm{PhCH}_{2}\right), 2.23-2.14\left(\mathrm{~m}, 2 \mathrm{H}, \mathrm{CH}_{2}\right), 1.65\left(\mathrm{~s}, 6 \mathrm{H}, \mathrm{C}\left(\mathrm{CH}_{3}\right)_{2}\right.$.

${ }^{13} \mathrm{C}$ NMR $\left(101 \mathrm{MHz}, \mathrm{CDCl}_{3}\right) \delta 190.1,157.7,156.2,141.6,134.2$, $133.9,129.0,128.5,128.5,127.9,126.0,87.2,67.6,42.6,30.3,25.8$. IR $\left(V_{\max }, \mathrm{cm}^{-1}\right) 2978(\mathrm{~s}), 2904(\mathrm{~s}), 1739(\mathrm{~m}), 1705(\mathrm{~m}), 1381(\mathrm{~m}), 1242(\mathrm{~m}), 1165(\mathrm{~s}), 1111(\mathrm{~s}), 1065$ (s).

HRMS (ESI/QTOF) m/z: [M + Na] $]^{+}$Calcd for $\mathrm{C}_{21} \mathrm{H}_{22} \mathrm{NaO}_{5}{ }^{+}$377.1359; Found 377.1363.

\section{(3,3-Dimethyl-5-phenylpent-1-yn-1-yl)triisopropylsilane (4v)}<smiles>CC(C)(C)OC(=O)C(=O)OCc1ccccc1</smiles>

$3 a$

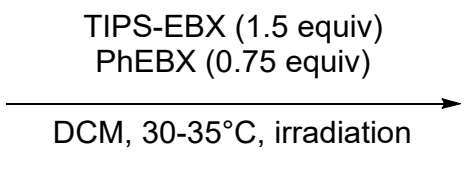

$\mathrm{DCM}, 30-35^{\circ} \mathrm{C}$, irradiation

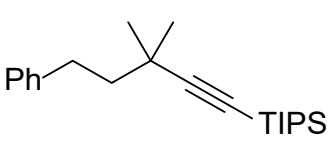

$4 v$

An oven dried dram vial ( $2 \mathrm{~mL}$ ), equipped with a magnetic stirrer, was charged with cesium oxalate (3a, $0.036 \mathrm{~g}, 0.10 \mathrm{mmol}, 1$ equiv), TIPS-EBX ( $\mathbf{2 g}, 0.064 \mathrm{~g}, 0.15 \mathrm{mmol}, 1.5$ equiv) and PhEBX (2a, 0.026 g, $0.075 \mathrm{mmol}, 0.75$ equiv). The reaction vial was sealed with a septum. After 3 vacuum/N2 cycles (backfilling with $\mathrm{Ar}$ on the last cycle), dry degassed (freeze pump thaw) $\mathrm{CH}_{2} \mathrm{Cl}_{2}$ was added and the septum was replaced with a screw cap under a flux of Ar. The reactions were placed between $2 \times 440$ $\mathrm{nm}$ Kessil lamps at ca. $10 \mathrm{~cm}$ distance from both lamps (with ventilation $\mathrm{T}=\mathrm{ca} .30-35^{\circ} \mathrm{C}$ as specified) and stirred under irradiation for 18 hours. The reaction was filtered through a small celite plug which was washed with $\mathrm{CH}_{2} \mathrm{Cl}_{2}$. The reaction crude was concentrated in vacuo, and purified by preparative TLC (SiO2, heptane), affording (3,3-dimethyl-5-phenylpent-1-yn-1-yl)triisopropylsilane (4v, $2 \mathrm{mg}$, $0.006 \mathrm{mmol}, 6 \%$ yield)

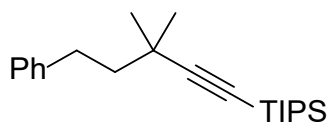

Rf (pentane) $=0.55$

${ }^{1} \mathrm{H}$ NMR $\left(400 \mathrm{MHz}, \mathrm{CDCl}_{3}\right) \delta 7.29(\mathrm{~d}, J=7.6 \mathrm{~Hz}, 2 \mathrm{H}, \mathrm{ArH}), 7.23-7.14(\mathrm{~m}$, $3 \mathrm{H}, \mathrm{ArH}), 2.85-2.75(\mathrm{~m}, 2 \mathrm{H}, \mathrm{PhCH}), 1.72-1.65\left(\mathrm{~m}, 2 \mathrm{H}, \mathrm{CH}_{2}\right), 1.26(\mathrm{~s}, 6 \mathrm{H}$, $\left.\mathrm{C}\left(\mathrm{CH}_{3}\right)_{2}\right), 1.13-0.98(\mathrm{~m}, 22 \mathrm{H}, \mathrm{TIPS})$.

${ }^{13} \mathrm{C}$ NMR $(101 \mathrm{MHz}, \mathrm{CDCl} 3) \delta 143.0,128.4,128.4,125.6,116.2,79.4,45.8,32.2,29.7,29.4,18.7$, 11.3.

HRMS (APPI/LTQ-Orbitrap) m/z: [M + Na] $]^{+}$Calcd for $\mathrm{C}_{22} \mathrm{H}_{36} \mathrm{NaSi}^{+}$351.2478; Found 351.2485.

\subsection{Control experiments}<smiles>CCOC(=O)C(=O)OC(C)(C)CCc1ccccc1</smiles>

3a

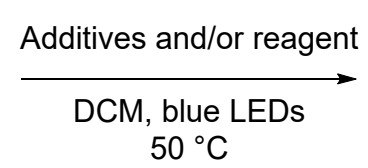
$50^{\circ} \mathrm{C}$

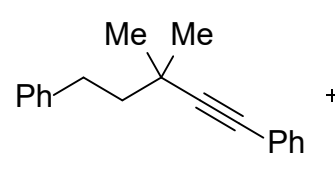

$4 a$<smiles>CC(C)(C[In-])CCc1ccccc1</smiles>

$4 \mathrm{v}$

An oven dried dram vial $(2 \mathrm{~mL})$, equipped with a magnetic stirrer, was charged with the solid components following table S3: cesium oxalate 3a, TIPSEBX (2g) , PhEBX (2a), 4CzIPN (1a), BIOAc (5a), $\mathrm{BIOH}$ (5b), 6a, 6b. The reaction vial was sealed with a septum. After 3 vacuum/N2 cycles (backfilling with $\mathrm{Ar}$ on the last cycle), $\mathrm{CH}_{2} \mathrm{Cl}_{2}(3.0 \mathrm{~mL})$ was added and the septum was replaced with a screw cap 
under a flux of $\mathrm{Ar}^{25}$ The reactions were placed between $2 \times 440 \mathrm{~nm}$ Kessil lamps (unless specified otherwise) at ca. $10 \mathrm{~cm}$ distance from both lamps (no ventilation, $\mathrm{T}=\mathrm{ca} .50^{\circ} \mathrm{C}$ ) and stirred under irradiation for 18 hours. The reaction was filtered through a small celite plug which was washed with $\mathrm{CH}_{2} \mathrm{Cl}_{2}$. The reaction crude was concentrated in vacuo, diluted with $\mathrm{CDCl}_{3}$ and 1 equiv of $\mathrm{CH}_{2} \mathrm{Br}_{2}$ was added as internal standard for ${ }^{1} \mathrm{H}$ NMR analysis.

Table S3. Control reactions for the indetification of the photoactive species without photocatalyst

\begin{tabular}{lllll}
\hline entry $^{\mathbf{a}}$ & Reagent (1.5 equiv) & additive (equiv) & residual 3a (\%) & ${ }^{\mathbf{1}} \mathbf{H}$ NMR yield $\left.\mathbf{~}^{\mathbf{b}} \mathbf{\%}\right)$ \\
\hline $\mathbf{1}$ & $\mathbf{2 g}$ & - & 100 & $\mathrm{nd}$ \\
$\mathbf{2}$ & $\mathbf{2 g}$ & $\mathbf{1 a}(0.05)$ & 30 & 25 \\
$\mathbf{3}$ & $\mathbf{2 g}$ & $\mathbf{5 a}(0.2)$ & 92 & 5 \\
$\mathbf{4}$ & $\mathbf{2 g}$ & $\mathbf{5 b}(0.2)$ & 92 & 5 \\
$\mathbf{5}$ & - & $\mathbf{5 a}(1.5)$ & 90 & - \\
$\mathbf{6}$ & - & $\mathbf{5 b}(1.5)$ & 100 & - \\
$\mathbf{7}$ & $2 \mathrm{~g}$ & $\mathbf{6 a}(0.2)$ & 98 & 2 \\
$\mathbf{8}$ & - & $\mathbf{6 a}(1.0)$ & 100 & - \\
$\mathbf{9}^{\mathbf{c}}$ & $\mathrm{Ph}$ & $\mathbf{6 b}(1.0)$ & - & $\mathrm{nd}^{\mathrm{d}}$ \\
$\mathbf{1 0}$ & $2 \mathrm{~g}$ & $\mathbf{6 b}(0.7)$ & $>90$ & $<5$ \\
$\mathbf{1 7}$ & - & $\mathbf{6 b}(1.0)$ & $>95$ & - \\
$\mathbf{1 8}$ & $\mathbf{2 g}$ & $\mathbf{2 a}(0.2)$ & 80 & 16 \\
\hline
\end{tabular}

${ }^{c}$ No cesium salt was used. ${ }^{d}$ No degradation of $\mathbf{6 b}$ was observed, full decomposition of PhEBX. This suggests that $\mathbf{6} \mathbf{b}$ is not a reaction intermediate.

\subsection{Photocatalyst free alkynylations with EBXs:}

HAT

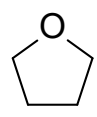

8

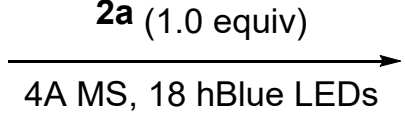

4A MS, 18 hBlue LEDs

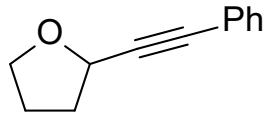

9

Following a modified reported procedure, ${ }^{26}$ an oven dried $(7.5 \mathrm{~mL})$ dram vial equipped with a magnetic stirrer was charged with MS $4 \AA \AA$ ( $20 \mathrm{mg}$ ) and PhEBX (2a, $70 \mathrm{mg}, 0.20 \mathrm{mmol}, 1.0$ equiv). The reaction vial was sealed with a septum. After 3 vacuum/N2 cycles (backfilling with Ar on the last cycle), THF ( $4.00 \mathrm{~mL}$ ) was added and the septum is replaced with a screw cap under a flux of Ar. The reactions were placed between $2 \times 460 \mathrm{~nm}$ Kessil lamps at ca. $10 \mathrm{~cm}$ distance from both lamps (no ventilation, $\mathrm{T}=\mathrm{ca} .50^{\circ} \mathrm{C}$ ) and stirred under irradiation for 18 hours. The reaction was filtered through a small celite plug which was washed with $\mathrm{CH}_{2} \mathrm{Cl}_{2}$. The reaction crude was concentrated in vacuo. An NMR sample of the crude was prepared with 1 equiv of $\mathrm{CH}_{2} \mathrm{Br}_{2}\left(14.0 \mu \mathrm{L}, 0.200 \mathrm{mmol}, 1\right.$ equiv) in $\mathrm{CDCl}_{3}$. The ${ }^{1} \mathrm{H} \mathrm{NMR}$ yield was determined using the signal at $4.81\left(\mathrm{dd}, J=7.2,5.2 \mathrm{~Hz}, 1 \mathrm{H}, \mathrm{CH}_{x} \mathrm{O}\right): 80 \%$.

\footnotetext{
${ }^{25}$ Use of a screw cap or crimp cap is of great importance, the irradiation causes an increase in temperature causing the $\mathrm{CH}_{2} \mathrm{Cl}_{2}$ to evaporate and an overpressure inside the vessel. When using a septum, the latter would fly off within an hour of irradiation. As shown in the optimization section DCE is not as good a solvent as $\mathrm{CH}_{2} \mathrm{Cl}_{2}$. ${ }^{26}$ Matsumoto, K.; Nakajima, M.; Nemoto, T. J. Org. Chem. 2020, 85 (18), 11802-11811.
} 


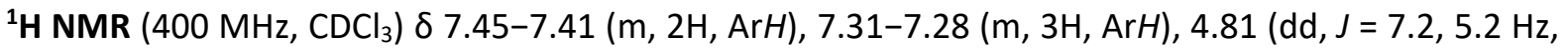
$\left.1 \mathrm{H}, \mathrm{CH}_{x} \mathrm{O}\right), 4.04-3.99\left(\mathrm{~m}, 1 \mathrm{H}, \mathrm{CH}_{x} \mathrm{O}\right), 3.89-3.83\left(\mathrm{~m}, 1 \mathrm{H}, \mathrm{CH}_{x} \mathrm{O}\right), 2.29-2.19\left(\mathrm{~m}, 1 \mathrm{H}, \mathrm{CH}_{x}\right), 2.15-2.04(\mathrm{~m}$, $\left.2 \mathrm{H}, \mathrm{CH}_{\mathrm{x}}\right), 1.99-1.90\left(\mathrm{~m}, 1 \mathrm{H}, \mathrm{CH}_{\mathrm{x}}\right)$. Corresponds to the reported literature data. ${ }^{26}$

\section{Difunctionalisation}<smiles>C=CN1CCCC1=O</smiles>

1.5 equiv 10

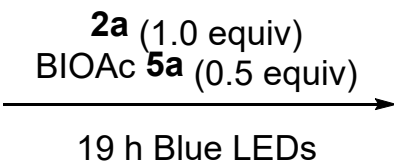

$19 \mathrm{~h}$ Blue LEDs<smiles>Cc1ccccc1C(=O)OCC(C#Cc1ccccc1)N1CCCC1=O</smiles>

11

Following a modified reported procedure, ${ }^{27}$ an oven dried dram vial, equipped with a magnetic stir bar was charged with $2 \mathrm{a}$ ( $35 \mathrm{mg}, 0.10 \mathrm{mmol}, 1.0$ equiv) and 5 a (15 mg, $0.050 \mathrm{mmol}, 0.50$ equiv). After 3 vacuum/nitrogen cycles refilling with $A r$ on the last cycle, degassed $\mathrm{CH}_{2} \mathrm{Cl}_{2}(0.40 \mathrm{~mL})$ was added followed by $N$-vinylpyrolidinone (10) $(16.7 \mathrm{mg}, 16.0 \mu \mathrm{L}, 150 \mu \mathrm{mol}, 1.50$ equiv). The reaction was irradiated for 19 hours with $2 \times 440 \mathrm{~nm}$ Kessil lamps. The reaction was concentrated in vacuo. An NMR sample of the crude was prepared with 1 equiv of $\mathrm{CH}_{2} \mathrm{Br}_{2}(7.0 \mu \mathrm{L}, 0.10 \mathrm{mmol}, 1$ equiv) in $\mathrm{CD}_{3} \mathrm{CN}$. The ${ }^{1} \mathrm{H}$ NMR yield was determined using the signal at $5.53 \mathrm{ppm}(\mathrm{dd}, J=8.6,4.8 \mathrm{~Hz}, 1 \mathrm{H}$, $\left.\mathrm{NCHCH}_{2} \mathrm{O}\right): 35 \%$

${ }^{1} \mathrm{H}$ NMR $(400 \mathrm{MHz}$, Acetonitrile-d3) $\delta 8.06(\mathrm{dd}, J=7.9,1.2 \mathrm{~Hz}, 1 \mathrm{H}, \mathrm{ArH}), 7.81(\mathrm{dd}, J=7.8,1.7 \mathrm{~Hz}, 1 \mathrm{H}$, $\mathrm{ArH}), 7.58-7.47(\mathrm{~m}, 3 \mathrm{H}, \mathrm{ArH}$ and $\mathrm{PhH}), 7.47-7.33(\mathrm{~m}, 3 \mathrm{H}, \mathrm{PhH}), 7.27(\mathrm{td}, J=7.7,1.8 \mathrm{~Hz}, 1 \mathrm{H}, \mathrm{ArH})$, $5.53(\mathrm{dd}, J=8.6,4.8 \mathrm{~Hz}, 1 \mathrm{H}, \mathrm{NCHCH} 2 \mathrm{O}), 4.64\left(\mathrm{dd}, J=11.3,8.6 \mathrm{~Hz}, 1 \mathrm{H}, \mathrm{NCHCH}_{2} \mathrm{O}\right.$ ), 4.50 (dd, $J=11.2$, $\left.4.8 \mathrm{~Hz}, 1 \mathrm{H}, \mathrm{NCHCH}_{2} \mathrm{O}\right), 3.75-3.52\left(\mathrm{~m}, 2 \mathrm{H}, \mathrm{CH}_{2}\right), 2.39-2.30\left(\mathrm{~m}, 2 \mathrm{H}, \mathrm{CH}_{2}\right), 2.12-2.02\left(\mathrm{~m}, 2 \mathrm{H}, \mathrm{CH}_{2}\right)$. Corresponds to the reported literature data. ${ }^{27}$

\section{Deboronative alkynylation}

potassium 2,3-dihydro-1H-inden-2-yl-tris(fluoranyl)borate (12)

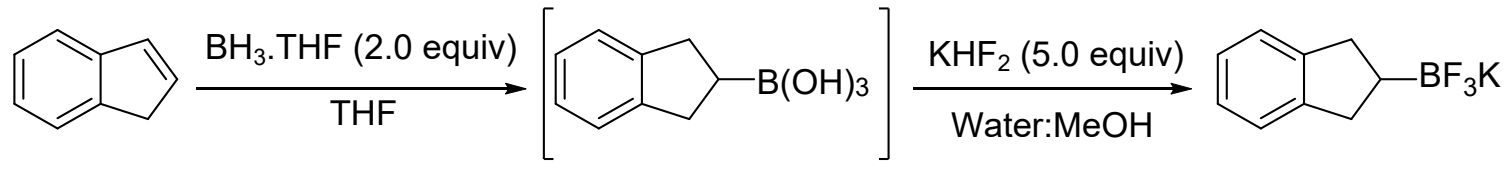

1.0 equiv 28 12 27

Following a reported procedure, ${ }^{28}$ a flame dried round bottom flask containing a solution of $\mathrm{BH}_{3}$. THF (34.0 mL, $34.0 \mathrm{mmol}, 1.00 \mathrm{M}, 2.00$ equiv) in THF was cooled to $0{ }^{\circ} \mathrm{C}$. A solution of $1 \mathrm{H}$-indene (27) $(1.98 \mathrm{~mL}, 17.0 \mathrm{mmol}, 1.00$ equiv) in tetrahydrofuran $(3.40 \mathrm{~mL})$ was added and the mixture was warm to rt and stirred for $2 \mathrm{~h}$. Water $(3.40 \mathrm{~mL})$ was added dropwise and the mixture was stirred for 3 $h$ at $r$. The mixture was concentrated in vacuo to remove the solvents except water. Ethyl acetate

\footnotetext{
${ }^{27}$ Amos, S. G. E.; Nicolai, S.; Waser, J. Chem. Sci. 2020, 11, 11274-11279

${ }^{28}$ Weng, W.-Z.; Liang, H.; Zhang, B. Org. Lett. 2018, 20 (16), 4979-4983.
} 
$\left(50 \mathrm{~mL}\right.$ ) was added to the suspension and the mixture was washed with a sat. sol. of $\mathrm{NaHCO}_{3}(50 \mathrm{~mL})$ and brine $(50 \mathrm{~mL})$. The organic layers were combined, dried over $\mathrm{MgSO}_{4} \cdot\left(\mathrm{H}_{2} \mathrm{O}\right)_{2}$ and concentrated in vacuo. The crude oil was used directly in next step. To a round bottom flask (PFA) containing a solution of potassium hydrogen fluoride $(6.64 \mathrm{~g}, 85.0 \mathrm{mmol}, 5.00$ equiv) in water $(25.0 \mathrm{~mL})$ were added the crude boronic acid $(\mathbf{2 8})$ and methanol $(34.0 \mathrm{~mL})$. The mixture was stirred at $\mathrm{rt}$ open to air for $2 \mathrm{~h}$. The mixture was concentrated in vacuo, the wet solid obtained was further dried by coevaporation with acetone ( 3 times). The resulting solid was diluted with acetone $(30 \mathrm{~mL})$ and was put on the rotavap at $\mathrm{P}_{\text {atm }}$ with the bath at $45{ }^{\circ} \mathrm{C}$ for 10 minutes. The solution was filtered with care to leave the remaining insoluble solid in the flask. This process was repeated 2 more times, the solution of acetone was concentrated in vacuo to $1 / 3$ of the initial volume. The solution was left to cool to $\mathrm{rt}$ then $\mathrm{Et}_{2} \mathrm{O}$ was added to induce precipitation $(\sim 40 \mathrm{~mL})$. The solution was cooled to $0{ }^{\circ} \mathrm{C}$ and left for $15 \mathrm{~min}$ standing at this temperature. The solid was filtered, washed with $\mathrm{Et}_{2} \mathrm{O}$ and dried in vacuo to afford potassium 2,3-dihydro- $1 \mathrm{H}$-inden-2-yl-tris(fluoranyl)borate (12) (1.38 g, $6.14 \mathrm{mmol}, 36 \%$ yield) as a white solid.

${ }^{1}$ H NMR (400 MHz, Acetone) $\delta 7.07$ (dd, $\left.J=5.3,3.3 \mathrm{~Hz}, 2 \mathrm{H}, \operatorname{ArH}\right), 6.95(\mathrm{dd}, J=5.5,3.1 \mathrm{~Hz}, 2 \mathrm{H}, \operatorname{ArH})$, $2.75\left(\mathrm{dd}, J=9.9,3.6 \mathrm{~Hz}, 4 \mathrm{H}, \mathrm{CH}_{2}\right), 1.29(\mathrm{~m}, 1 \mathrm{H}, \mathrm{CHB})$.

${ }^{13} \mathrm{C}$ NMR (101 MHz, Acetone) $\delta 148.3,125.6,124.6,36.8$. One carbon is not resolved.

${ }^{19} \mathrm{~F}$ NMR (376 MHz, Acetone) $\delta-146.34\left(\mathrm{~d}, J=95.0 \mathrm{~Hz}\right.$ ). Corresponds to the reported literature data. ${ }^{29}$

Deboronative alkynylation: synthesis of 13

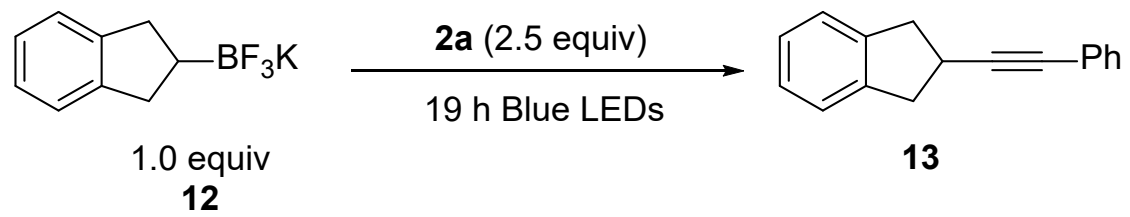

Following a modified reported procedure, ${ }^{29}$ a dram vial, equipped with a magnetic stirring bar, was charged with potassium 2,3-dihydro-1H-inden-2-yl(trifluoro)borate (12, $22 \mathrm{mg}, 0.10 \mathrm{mmol}, 1.0$ equiv), 2a ( $87.0 \mathrm{mg}, 250 \mu \mathrm{mol}, 2.50$ equiv) and sodium carbonate $(21 \mathrm{mg}, 0.20 \mathrm{mmol}, 2.0$ equiv). Degassed $\mathrm{H}_{2} \mathrm{O}(0.5 \mathrm{~mL}$ ) (water was bubbled with nitrogen gas for 30 minutes to remove oxygen before the experiment) and $\mathrm{CH}_{2} \mathrm{Cl}_{2}(0.5 \mathrm{~mL})$ were added, the vial was sealed and the reaction was irradiated with $2 \times 440 \mathrm{~nm}$ Kessil lamps under strong agitation for 19 hours. The reaction mixture was extracted with $\mathrm{CH}_{2} \mathrm{Cl}_{2}(3 \times 7 \mathrm{~mL})$. The organic layers were combined, washed with sat. aq. $\mathrm{NaCl}(10 \mathrm{~mL})$ then dried over $\mathrm{Na}_{2} \mathrm{SO}_{4}$, filtered and concentrated in vacuo. An NMR sample of the crude was prepared with 1 equiv of $\mathrm{CH}_{2} \mathrm{Br}_{2}\left(7.0 \mu \mathrm{L}, 0.10 \mathrm{mmol}, 1\right.$ equiv) in $\mathrm{CDCl} 3$. The ${ }^{1} \mathrm{H}$ NMR yield was determined using the signal at $3.30 \mathrm{ppm}(\mathrm{p}, J=8.5 \mathrm{~Hz}, 1 \mathrm{H}, \mathrm{CH}$-alkyne): $60 \%$.

${ }^{1}{ }_{\text {H NMR }}\left(400 \mathrm{MHz}, \mathrm{CDCl}_{3}\right)$ 8 7.44-7.37 (m, 2H, $\left.\mathrm{ArH}\right), 7.31-7.24(\mathrm{~m}, 3 \mathrm{H}, \mathrm{ArH}), 7.24-7.19(\mathrm{~m}, 2 \mathrm{H}, \mathrm{ArH})$, 7.19-7.13 (m, 2H, ArH), 3.43 (p, J=8.5 Hz, 1H, CH-alkyne), 3.30 (dd, J=15.4, 8.0 Hz, 2H, CH2), 3.12 (dd, $\left.J=15.4,8.0 \mathrm{~Hz}, 2 \mathrm{H}, \mathrm{CH}_{2}\right)$. Corresponds to reported literature data. ${ }^{29}$

\footnotetext{
${ }^{29}$ Huang, H.; Zhang, G.; Gong, L.; Zhang, S.; Chen, Y. J. Am. Chem. Soc. 2014, 136 (6), 2280-2283.
} 


\section{Oxime fragmentation-alkynylation}

\section{2-((Cyclobutylideneamino)oxy)propanoic acid (14)}<smiles>O=C(O)c1ccccc1</smiles>

29

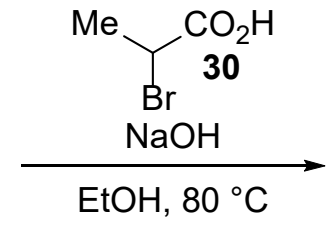<smiles>CC(ONC(=O)c1ccccc1)C(=O)O</smiles>

31

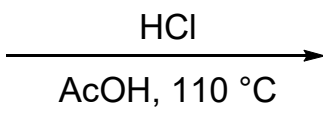

${ }_{\mathrm{H}_{2} \mathrm{~N}^{-}}^{\mathrm{Me}} \mathrm{HCl}$

32

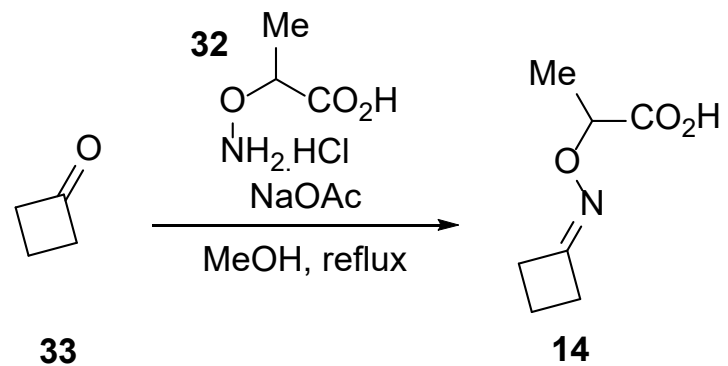

Compound 14 was taken directly from the batch synthesised for a previous project within our group. ${ }^{30}$

Following the reported procedure, ${ }^{30} \mathrm{~N}$-hydroxybenzamide (29) $(6.08 \mathrm{~g}, 44.3 \mathrm{mmol}, 1.0$ equiv) and finely ground $\mathrm{NaOH}(5.32 \mathrm{~g}, 133 \mathrm{mmol}, 3.0$ equiv) were suspended in absolute EtOH (66 mL). To the resulting thick, off-white suspension, 2-bromopropanoic acid (30) $(4.1 \mathrm{~mL}, 44 \mathrm{mmol}, 1.0$ equiv) was added slowly via syringe under stirring. This resulted in the conversion of the homogeneous suspension into a pale brown solution, which was then heated to $80{ }^{\circ} \mathrm{C}$. Once this temperature was reached, the mixture looked again as a homogeneous, off-white suspension, which was stirred overnight. The mixture was then concentrated under reduced pressure to provide a solid residue, which was dissolved in water $(90 \mathrm{~mL})$. The resulting aqueous solution was washed once with diethyl ether $(100 \mathrm{~mL})$ and then acidified by careful addition of aq. $\mathrm{HCl}$ $(37 \% \mathrm{w} / \mathrm{w})$ until $\mathrm{pH}=1$. It was then extracted with EtOAc $(3 \times 100 \mathrm{~mL})$ and the combined organic layers were dried over $\mathrm{MgSO}_{4}$, filtered and concentrated under vacuum to provide an off-white solid. Recrystallization from hexane $(50 \mathrm{~mL})$ and EtOAc $(100 \mathrm{~mL})$ afforded 2(benzamidooxy)propanoic acid (31) $(7.08 \mathrm{~g}, 33.9 \mathrm{mmol}, 76 \%$ yield) as a colorless solid. The compound was used directly in next step with no further analyses.

2-(Benzamidooxy)propanoic acid (31) (7.08 g, $33.8 \mathrm{mmol}, 1.0$ equiv) was suspended in acetic acid (20.5 $\mathrm{mL})$. Aq. $\mathrm{HCl}(5.0 \mathrm{M} ; 68 \mathrm{~mL}, 34 \mathrm{mmol}, 10$ equiv) was then added and the mixture was heated to reflux $\left(110{ }^{\circ} \mathrm{C}\right)$, which resulted in the formation of a pale yellow, clear solution. The latter was refluxed for 18 hours. It was then allowed to cool down to room temperature. This led to the precipitation of a crystalline solid (benzoic acid), which was filtered off. The resulting solution was stored at $4{ }^{\circ} \mathrm{C}$ overnight, which permitted the precipitation of a further amount of benzoic acid. Upon removal of the latter $(4.13 \mathrm{~g}, 33.8 \mathrm{mmol}, 100 \%$ yield $)$ through filtration, the so-obtained clear solution was concentrated under vacuum. The resulting wet solid was further dried under vacuum at $60{ }^{\circ} \mathrm{C}$ for 3 hours. It was then refluxed in a mixture of EtOAc $(30 \mathrm{~mL})$ and EtOH $(1.5 \mathrm{~mL})$ for 20 minutes, filtered,

${ }^{30}$ H. Jiang, A. Studer, Angew. Chem. Int. Ed. 2017, 56, 12273-12276. 
washed with pentane, and dried in the air. 2-(Aminooxy)propanoic acid hydrochloride (32) was obtained as a colorless solid ( $4.15 \mathrm{~g}, 29.3 \mathrm{mmol}, 87 \%$ yield). The compound was used directly in next step with no further analyses.

Following a reported procedure, ${ }^{31}$ a solution of cyclobutanone (32) (1.0 equiv) in $\mathrm{MeOH}(0.20 \mathrm{M})$ was treated with 2-(aminooxy)- propanoic acid hydrochloride (31) $(226 \mathrm{mg}, 1.60 \mathrm{mmol}, 1.2$ equiv), sodium acetate (2.4 equiv) and heated to reflux until complete by TLC analysis ( $4.5-6.0$ hours). The mixture was then allowed to cool to room temperature and aq. $\mathrm{Na}_{2} \mathrm{CO}_{3}(2.0 \mathrm{M})$ was added. In some cases, the addition of a small volume of water was necessary to achieve the complete dissolution of the solids. The resulting aqueous solution was extracted once with $\mathrm{Et}_{2} \mathrm{O}$ and the organic layer was washed with aq. $\mathrm{Na}_{2} \mathrm{CO}_{3}(2.0 \mathrm{M} ; 2 \mathrm{x})$. The combined aqueous extracts were then acidified by careful addition of aq. $\mathrm{HCl}$ solution $(30 \% \mathrm{v} / \mathrm{v})$ until $\mathrm{pH}<2$, and extracted with DCM $(3 \mathrm{x})$. The combined organic layers were dried over $\mathrm{MgSO}_{4}$, filtered, and concentrated under vacuum to provide the pure product 2-methyl-2(((cyclobutylidene)amino)oxy)propanoic acid (14) was obtained as a pale yellow solid $(0.150 \mathrm{~g}, 0.954$ $\mathrm{mmol}, 72 \%$ yield).

${ }^{1} \mathrm{H}$ NMR $\left(400 \mathrm{MHz}, \mathrm{CDCl}_{3}\right) \delta 11.51\left(\mathrm{~s}, 1 \mathrm{H}, \mathrm{CO}_{2} \mathrm{H}\right), 4.63(\mathrm{q}, J=7.1 \mathrm{~Hz}, 1 \mathrm{H}, \mathrm{OCH}), 3.09-2.77(\mathrm{~m}, 4 \mathrm{H}$, $\mathrm{CH}_{2} \mathrm{CH}_{2} \mathrm{CH}_{2} \mathrm{C}=\mathrm{N}$ ), $2.00\left(\mathrm{p}, \mathrm{J}=8.4 \mathrm{~Hz}, 2 \mathrm{H}, \mathrm{CH}_{2} \mathrm{CH}_{2} \mathrm{CH}_{2}\right), 1.47(\mathrm{~d}, J=7.1 \mathrm{~Hz}, 3 \mathrm{H}, \mathrm{Me}) .{ }^{13} \mathrm{C}$ NMR $(101$ $\left.\mathrm{MHz}, \mathrm{CDCl}_{3}\right) \delta 178.7,161.4,76.5,31.6,31.3,16.8,14.5$.

Oxime fragmentation-alkynylation: synthesis of $\mathbf{1 5}$

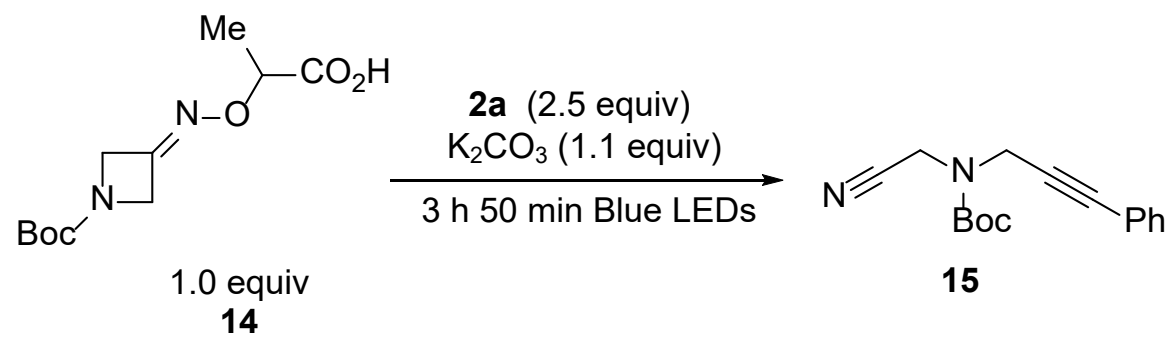

Following a modified reported procedure, ${ }^{32}$ a dram vial, equipped with a magnetic stirring bar, was charged with $2 \mathrm{a}(87.0 \mathrm{mg}, 250 \mu \mathrm{mol}, 2.50$ equiv), 14 ( $25.8 \mathrm{mg}, 100 \mu \mathrm{mol}, 1.00$ equiv), potassium carbonate (15.2 mg, $110 \mu \mathrm{mol}, 1.10$ equiv). After 3 vacuum/nitrogen cycles, refilling with argon upon the last cycle, 1,2-dichloroethane $(2.00 \mathrm{~mL}$, degassed by bubbling $\mathrm{Ar})$ was then added and the reaction was irradiated with $2 \times 440 \mathrm{~nm}$ Kessil lamps for $3 \mathrm{~h} 50 \mathrm{~min}$. An NMR sample of the crude was prepared with 1 equiv of $\mathrm{CH}_{2} \mathrm{Br}_{2}\left(7.0 \mu \mathrm{L}, 0.10 \mathrm{mmol}, 1\right.$ equiv) in $\mathrm{CDCl}_{3}$. The ${ }^{1} \mathrm{H}$ NMR yield was determined using the signal at $4.34 \mathrm{ppm}\left(\mathrm{s}, 2 \mathrm{H}, \mathrm{CH}_{2}\right): 75 \%$.

${ }^{1} \mathrm{H}$ NMR H NMR $\left(400 \mathrm{MHz}\right.$, Acetonitrile- $\left.d_{3}\right) \delta 7.46(\mathrm{~m}, 2 \mathrm{H}, \mathrm{PhH}), 7.37(\mathrm{~m}, 3 \mathrm{H}, \mathrm{PhH}), 4.34\left(\mathrm{~s}, 2 \mathrm{H}, \mathrm{CH}_{2}\right)$, $4.27\left(\mathrm{~s}, 2 \mathrm{H}, \mathrm{CH}_{2}\right), 1.49(\mathrm{~s}, 9 \mathrm{H}, \mathrm{Boc})$. Corresponds to reported literature data. ${ }^{32}$

\footnotetext{
${ }^{31}$ E. M. Dauncey, S. P. Morcillo, J. J. Douglas, N. S. Sheikh, D. Leonori, Angew. Chem. Int. Ed. 2018, 57, 744-748.

${ }^{32}$ Le Vaillant, F.; Garreau, M.; Nicolai, S.; Gryn'ova, G.; Corminboeuf, C.; Waser, J. Chem. Sci. 2018, 9, 5883-5889.
} 


\subsection{UV-Vis absorption and fluorescence studies}

\section{Absorption and fluorescence studies of PhEBX $2 \mathrm{a}$ and the cesium oxalate $3 \mathrm{a}$}

A $5 \mathrm{~mL} 0.2 \mathrm{M}$ stock solution of PhEBX ( $348 \mathrm{mg}, 1.00 \mathrm{mmol}$ ) and a $2 \mathrm{~mL} 0.2 \mathrm{M}$ stock solution of 3a (147 $\mathrm{mg}, 0.4 \mathrm{mmol}$ ) were prepared in DMSO (from fresh ampoules, degassed and deuterated) were prepared in a $5 \mathrm{~mL}$ and $2 \mathrm{~mL}$ volumetric flask. The samples were prepared by dissolving $0.50 \mathrm{~mL}$ of stock solution with $0.5 \mathrm{~mL}$ of fresh DMSO, final concentration: $0.1 \mathrm{M}$. The samples were then submitted to UV-Vis, fluorescence and fluorescence excitation spectroscopy.

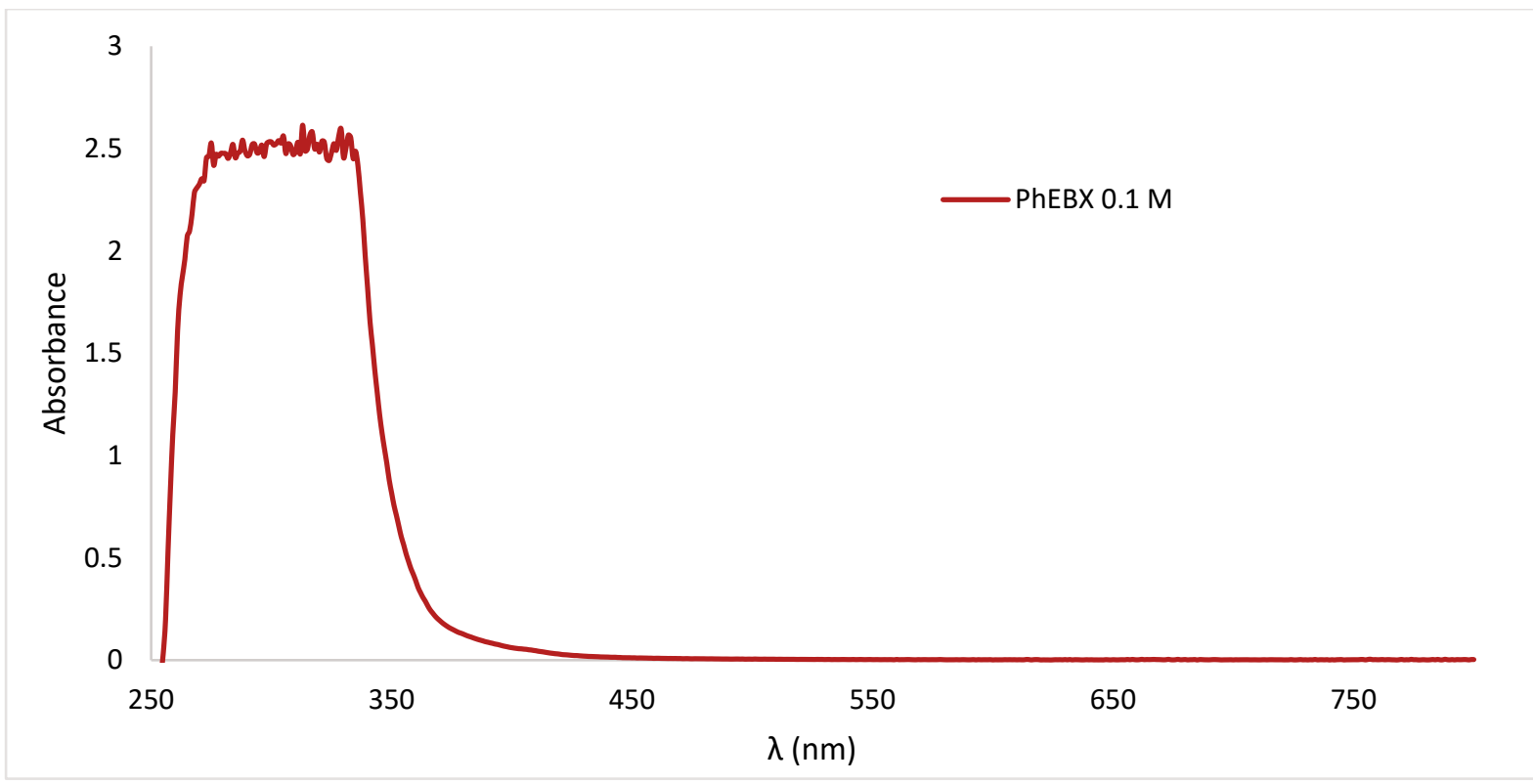

Figure S4. Absorption of PhEBX 2a, 0.1 M in DMSO

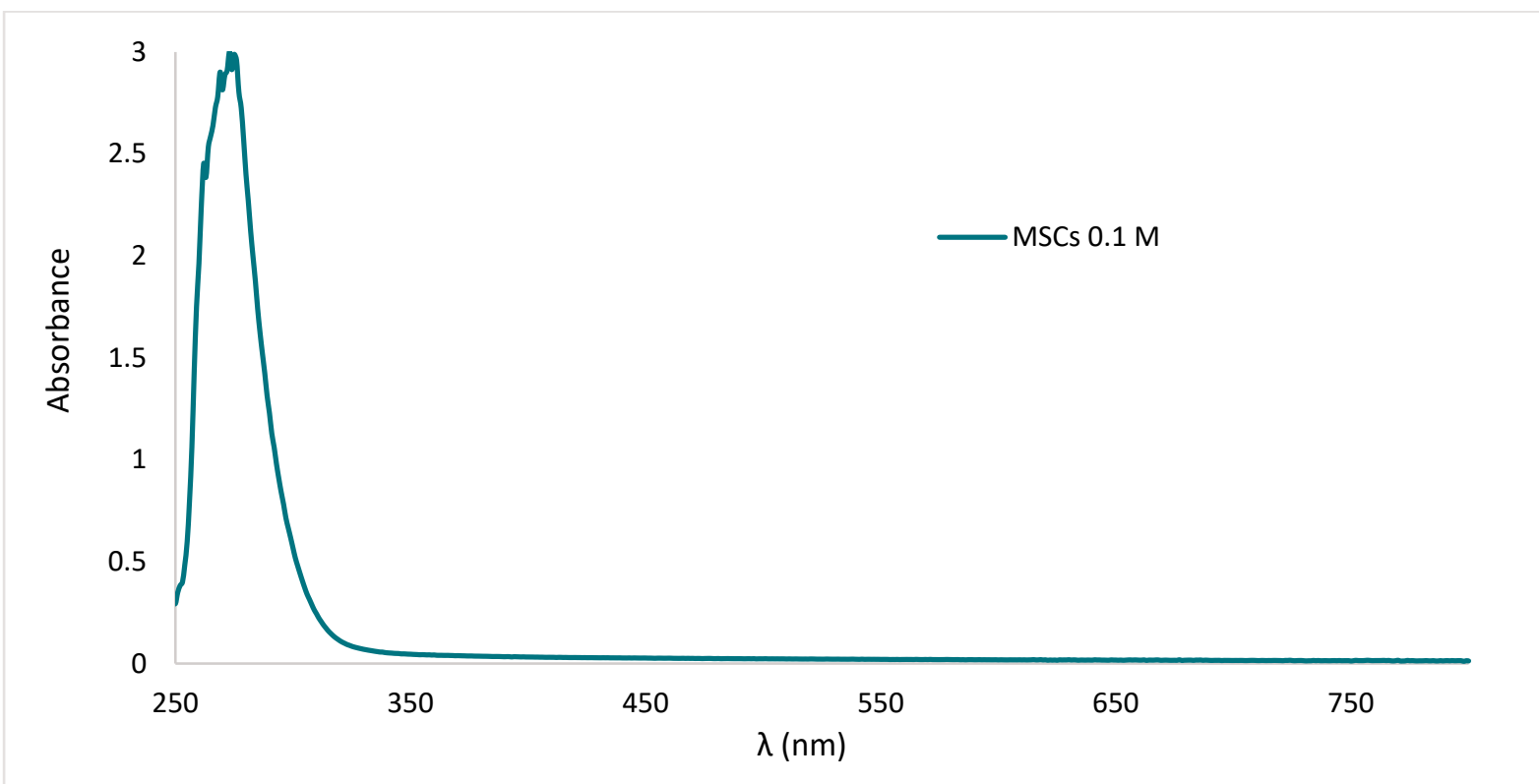

Figure S5. Absorption of 3a $0.1 \mathrm{M}$ in DMSO

We checked for the presence of an EDA complex by combining $0.50 \mathrm{~mL}$ of both stock solutions of 2a and 3a and measuring the UV-Vis spectrum, no new band can be observed (Figure S6) 


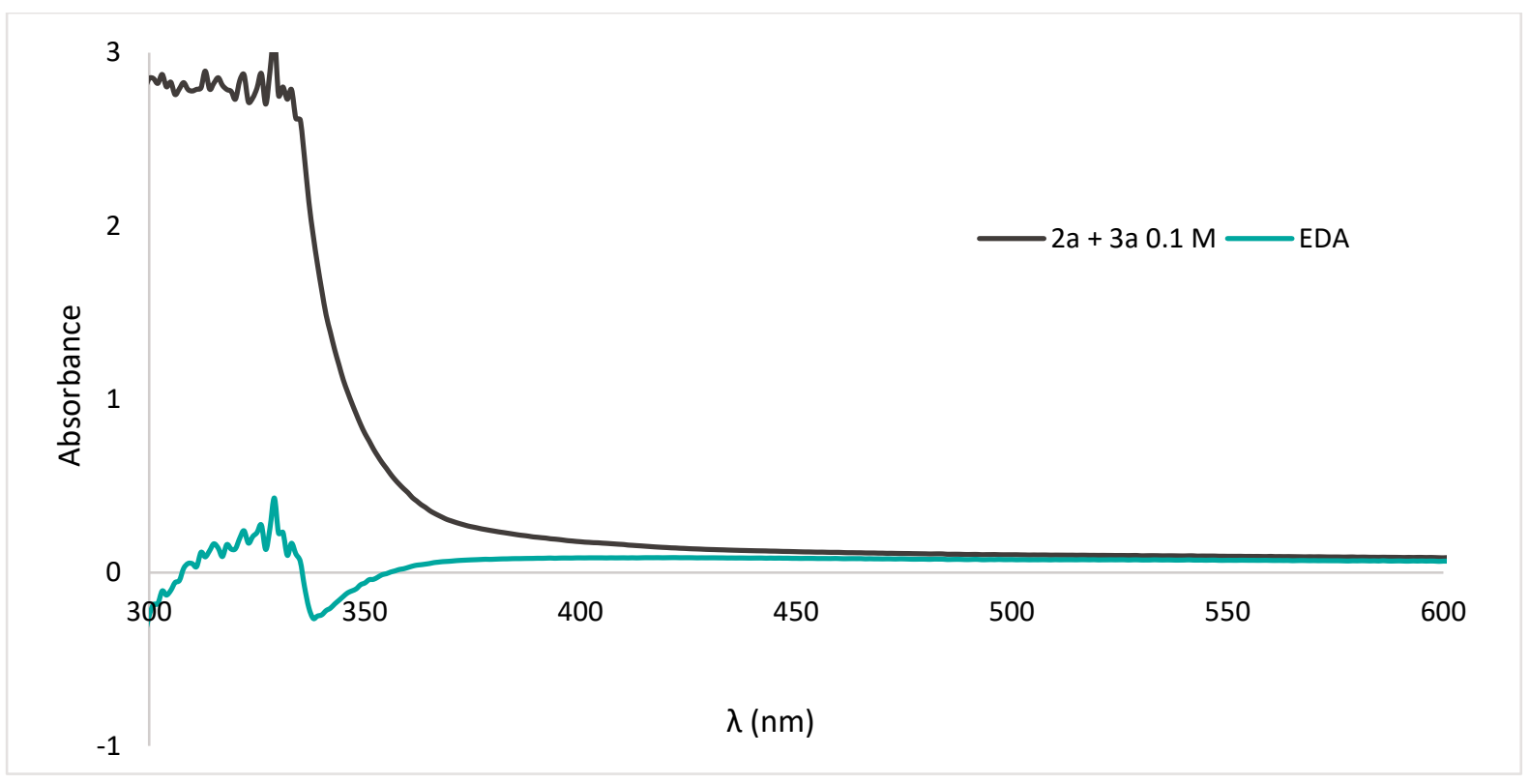

Figure S6. Absorption of a 1:1 mixture 2a:3a.

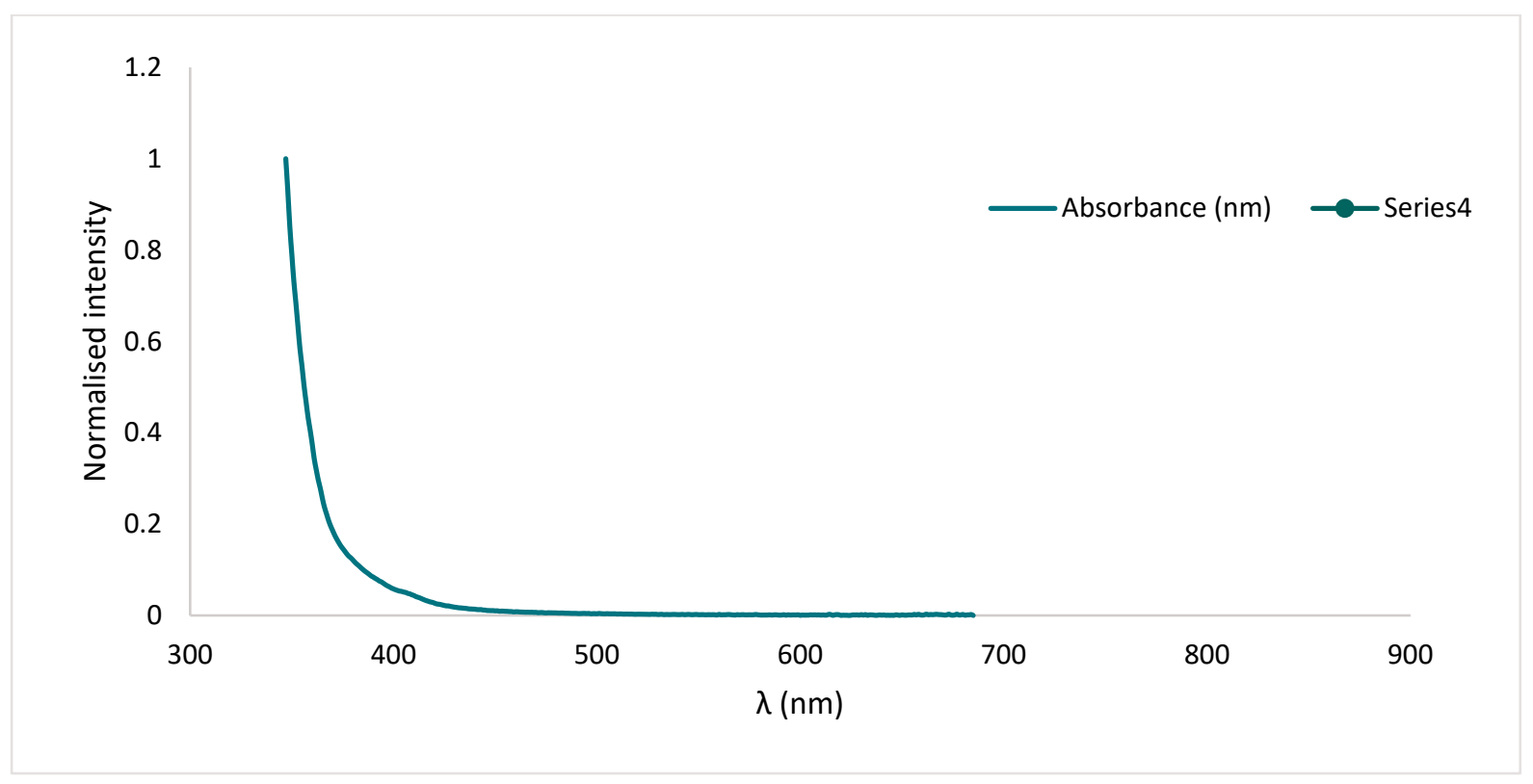

Figure S7. Normalized absorption, fluorescence $(390 \mathrm{~nm})$ and fluorescence excitation (485 nm) of 2a $(0.1 \mathrm{M})$ in DMSO

Absorption and Beer-Lambert linear regression at $420 \mathrm{~nm}$ and $440 \mathrm{~nm}$ of PhEBX (2a)

A $5 \mathrm{~mL} 0.14 \mathrm{M}$ stock solution of PhEBX (2a, $243 \mathrm{mg}, 0.700 \mathrm{mmol}$ ) in DMSO (from fresh ampoules, degassed and deuterated) was prepared in a $5 \mathrm{~mL}$ volumetric flask. Then $1 \mathrm{~mL}$ solutions were prepared following table $\mathrm{S} 4$, where $\mathrm{C}(\mathbf{2 a})$ is the concentration of the stock solution, $\mathrm{V}(\mathbf{2 a})$ is the volume of the stock solution used for the sample, $\mathrm{V}(\mathrm{DMSO})$ the volume of DMSO added for the dilution $\mathrm{C}_{\mathrm{f}}(\mathbf{2 a})$ the final concentration of the sample. UV-Vis spectra of each sample were then measured. Reproducibility of the measure was verified by repetition of the analyses. 
Table S4. Sample preparation table for UV-Vis analyses for the Beer-Lambert linear regression

\begin{tabular}{llll}
\hline $\mathbf{C}(\mathbf{2 a})(\mathbf{M})$ & $\mathbf{V}(\mathbf{2 a})(\mathbf{m L})$ & $\mathbf{V}(\mathbf{D M S O})(\mathbf{m L})$ & $\mathbf{C}_{\mathrm{f}}(\mathbf{2 a})(\mathbf{M})$ \\
\hline 0.14 & 0 & 1.00 & 0 \\
0.14 & 0.10 & 0.90 & 0.014 \\
0.14 & 0.30 & 0.70 & 0.042 \\
0.14 & 0.50 & 0.50 & 0.07 \\
0.14 & 0.70 & 0.30 & 0.098 \\
0.14 & 1.00 & 0 & 0.14 \\
\hline
\end{tabular}

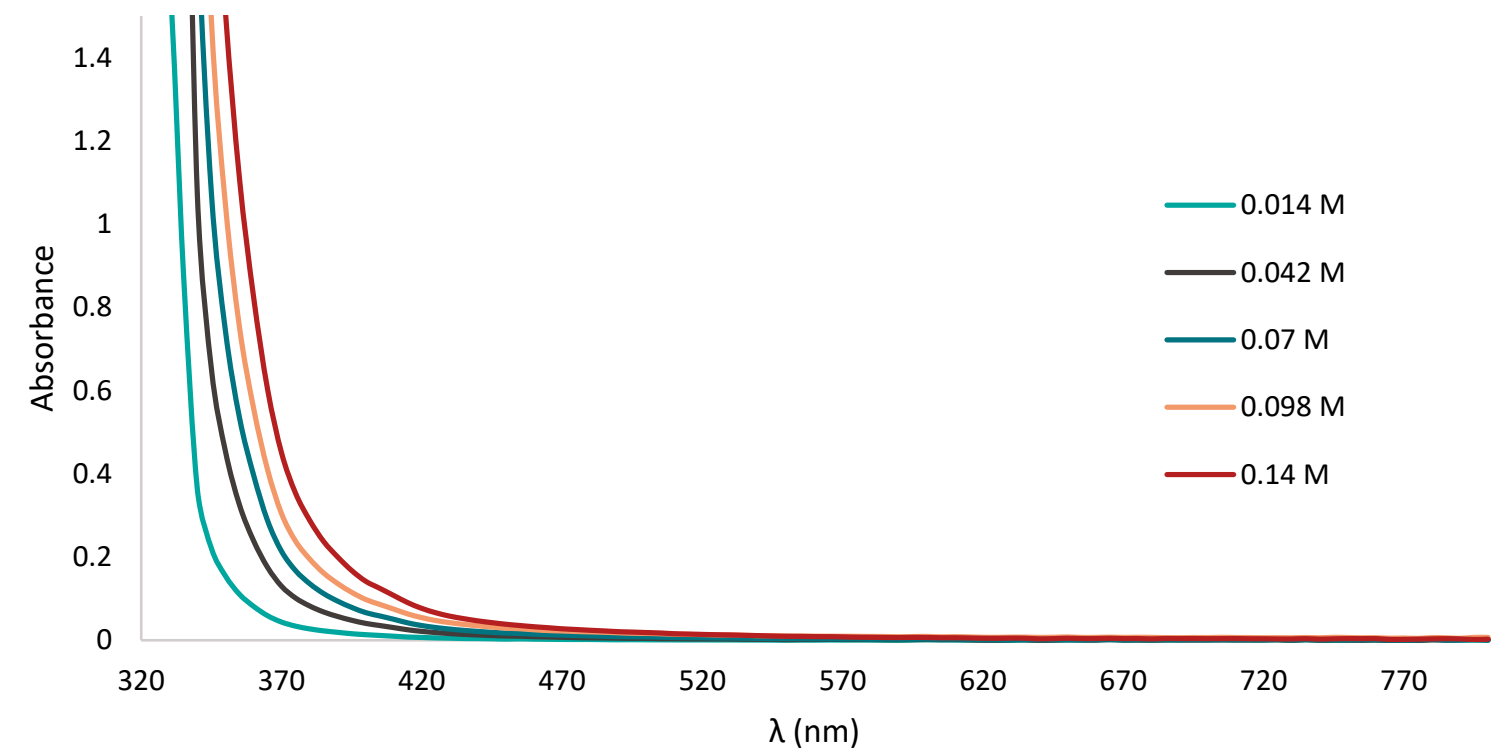

Figure S8. Absorption spectra of $2 \mathrm{a}$ at concentrations from $0.014 \mathrm{M}$ to $0.14 \mathrm{M}$ in DMSO

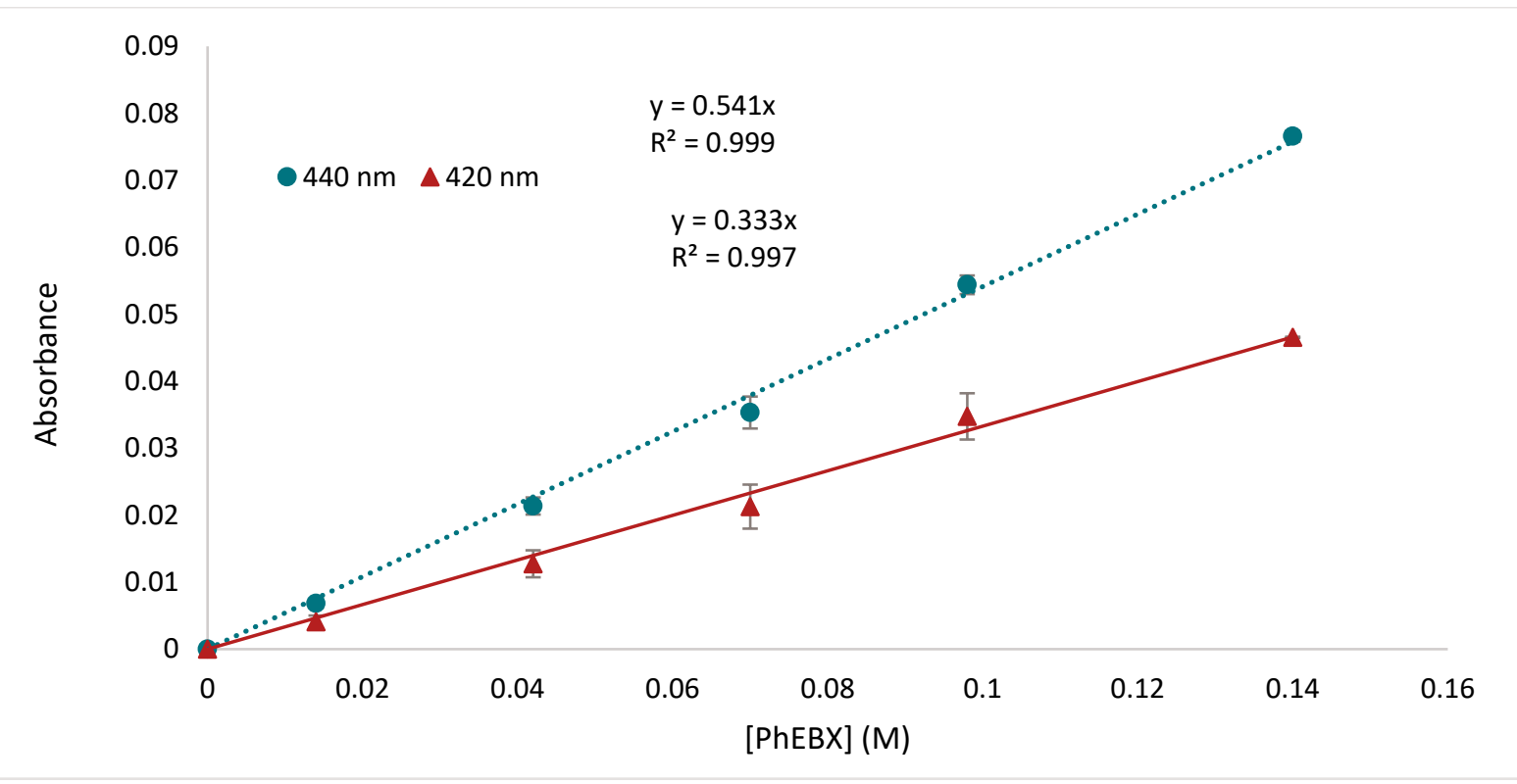

Figure S9. Beer-Lambert linear regression for $420 \mathrm{~nm}$ and $440 \mathrm{~nm}$

\subsection{Cyclic voltammetry of PhEBX (2a)}


An Autolab potentiostat with a 3 electrode cell configuration: glassy carbon (working electrode), $\mathrm{Pt}$ wire as (control electrode), and $\mathrm{Ag} / \mathrm{AgCl}(\mathrm{KCl}, 3 \mathrm{M}$ aq.) as (reference electrode) was used for the measures. Tetrabutyl ammonium hexafluorophosphhate (TBAP, $0.1 \mathrm{M}$ in $\mathrm{MeCN}$ ) was used as an electrolyte. PhEBX (2a, $3.5 \mathrm{mg}, 0.01 \mathrm{mmol})$ was dissolved in a stock solution of TBAP $(0.1 \mathrm{M}, 10 \mathrm{~mL}$ in $\mathrm{MeCN})$ and was degassed by bubbling Argon directly before measure. The redox couple $\mathrm{E}\left(\mathbf{2 a} / \mathbf{2} \mathrm{a}^{\circ}\right)$ is defined as the potential $E$ measured for $\frac{I_{\max }}{2}$.

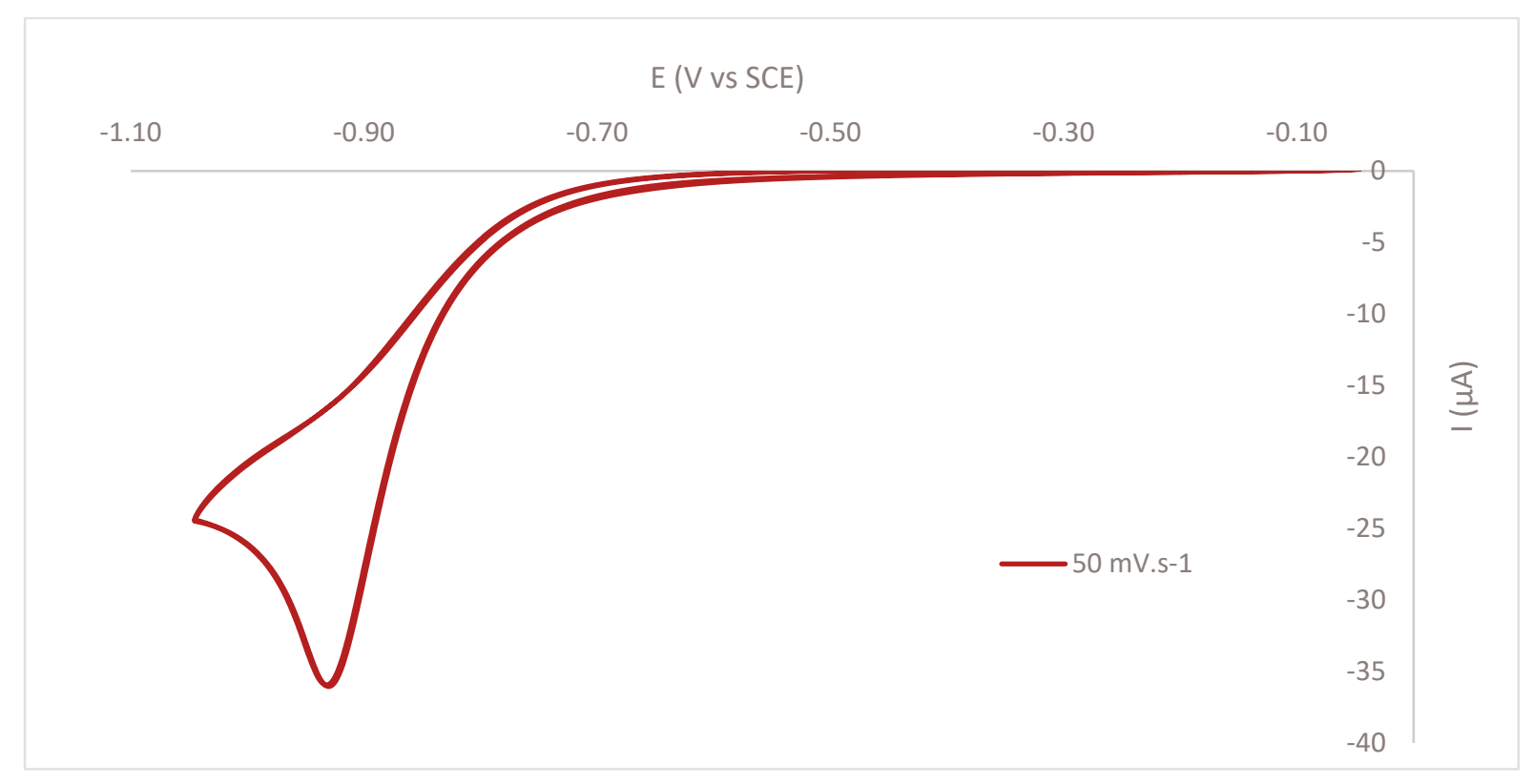

Figure S10. Cyclic voltammogram of $\mathbf{2 a}$

$$
\begin{gathered}
I_{\max }=36 \mu \mathrm{A} ; \frac{I_{\max }}{2}=18 \mu \mathrm{A} E=-0.87 \mathrm{~V} \text { vs SCE for } \mathrm{I}=18 \mu \mathrm{A} \\
\mathrm{E}_{1 / 2}\left(\mathbf{2 a} / \mathbf{2 \mathbf { a } ^ { - - }}\right)=-0.87 \mathrm{~V} \text { vs SCE }
\end{gathered}
$$

$E_{1 / 2}\left(2 a^{*} / \mathbf{2} a^{*}\right)=E_{0-0}+E_{1 / 2}\left(2 a / 2 a^{*}\right)$. $E_{0-0}$ was determined experimentally by position of the long wavelength tail of the absorption spectrum at $460 \mathrm{~nm}$ (Figure S8). ${ }^{33}$

$$
\begin{aligned}
& \mathrm{E}=\frac{\mathrm{hc}}{\lambda} \\
& \mathrm{E}_{0-0}=\frac{1240}{460}=2.7 \mathrm{eV}
\end{aligned}
$$

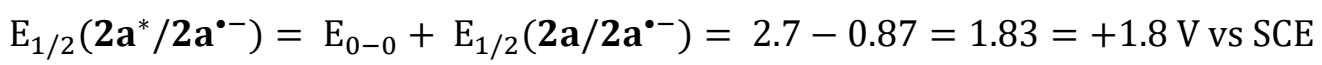

\section{NMR spectra of new compounds}

\footnotetext{
${ }^{33}$ Buzzetti, L.; Prieto, A.; Roy, S. R.; Melchiorre, P. Angew. Chem. Int. Ed. 2017, 56 (47), 15039-15043.
} 
Compound 2d

${ }^{1} \mathrm{H} \mathrm{NMR}, \mathrm{CDCl}_{3}, 400 \mathrm{MHz}$

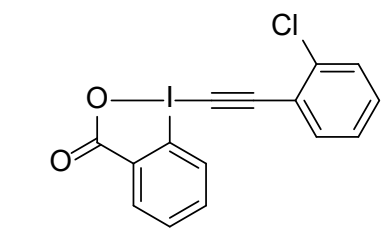

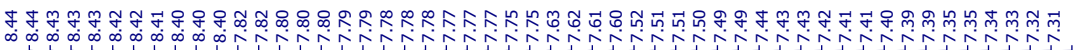

$\underbrace{\infty}_{0.000}$
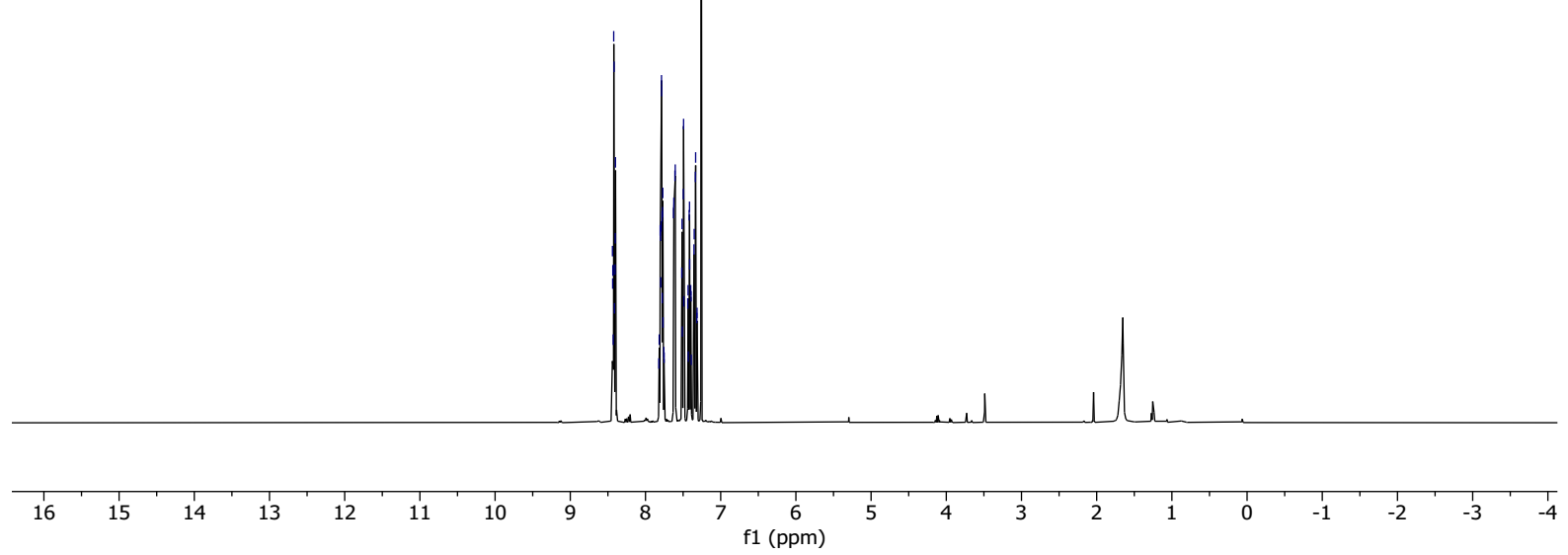

${ }^{13} \mathrm{CNMR}, \mathrm{CDCl}_{3}, 101 \mathrm{MHz}$

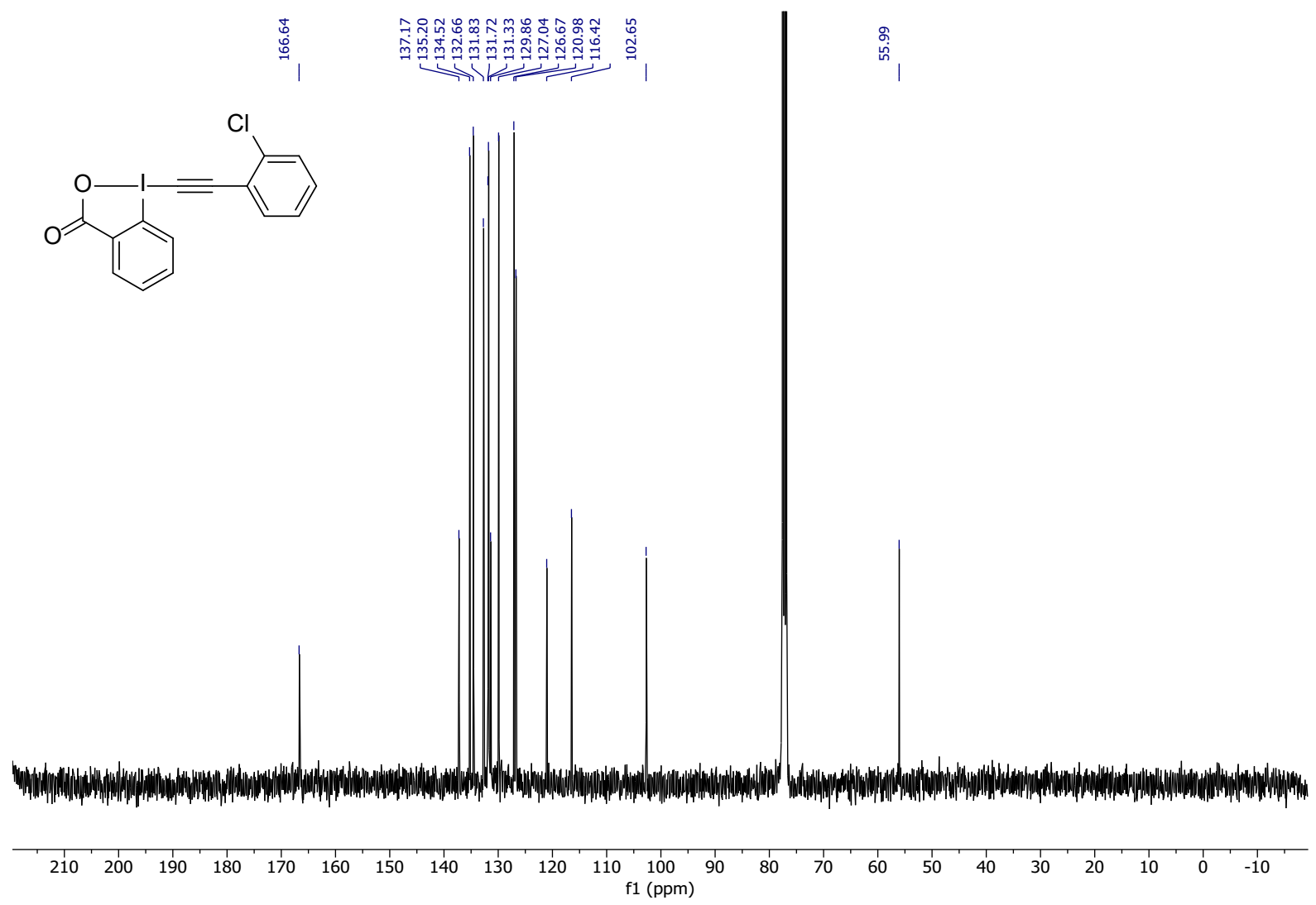

S57 
Compound 22e

${ }^{1} \mathrm{H} \mathrm{NMR}, \mathrm{CDCl}_{3}, 400 \mathrm{MHz}$

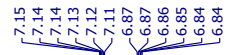

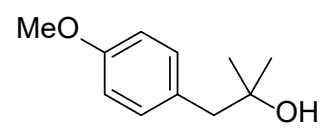

$\stackrel{\infty}{\infty}$

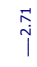

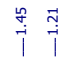
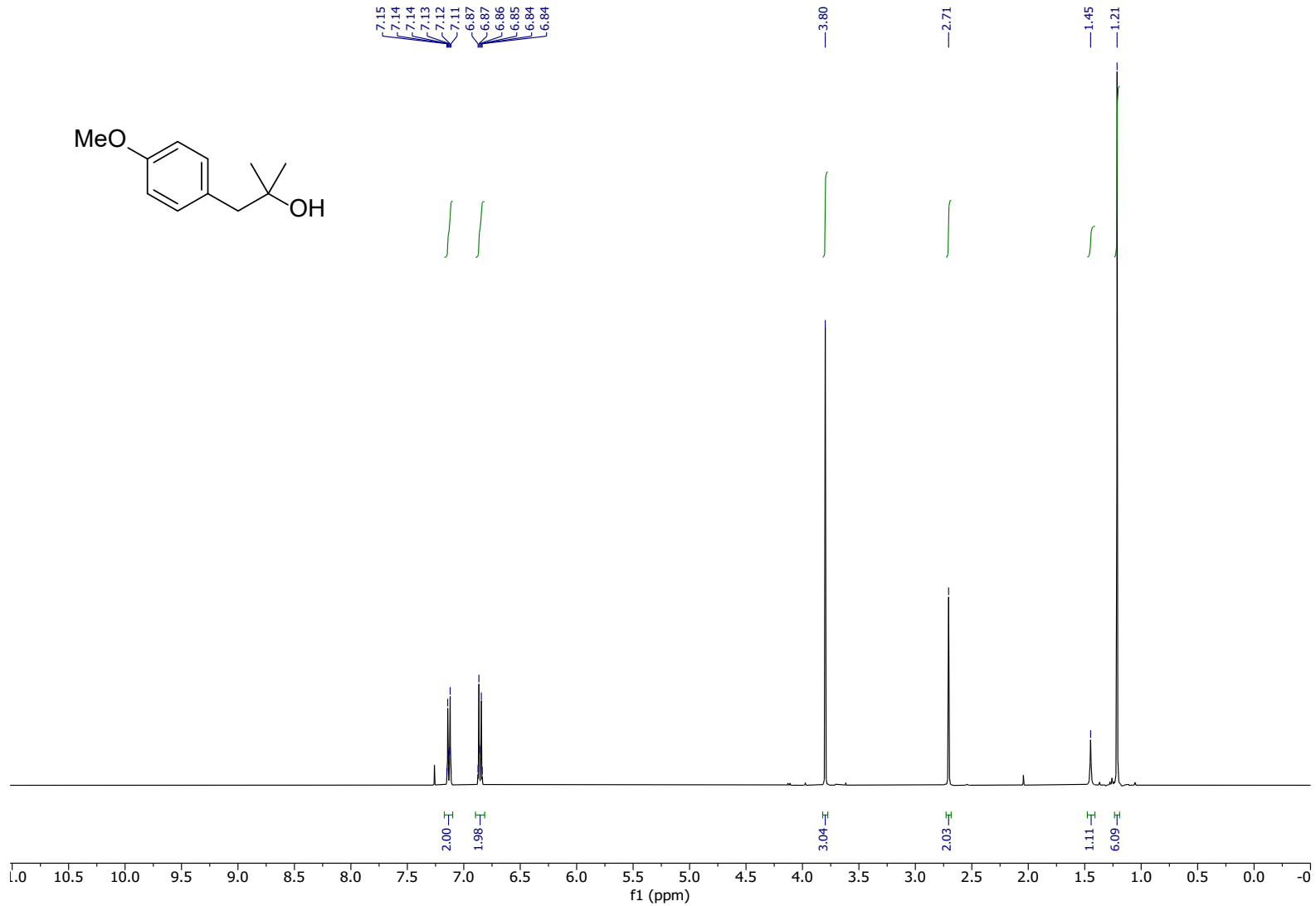

${ }^{13} \mathrm{CNMR}_{\mathrm{N}} \mathrm{CDCl}_{3}, 101 \mathrm{MHz}$
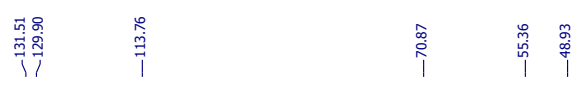

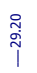
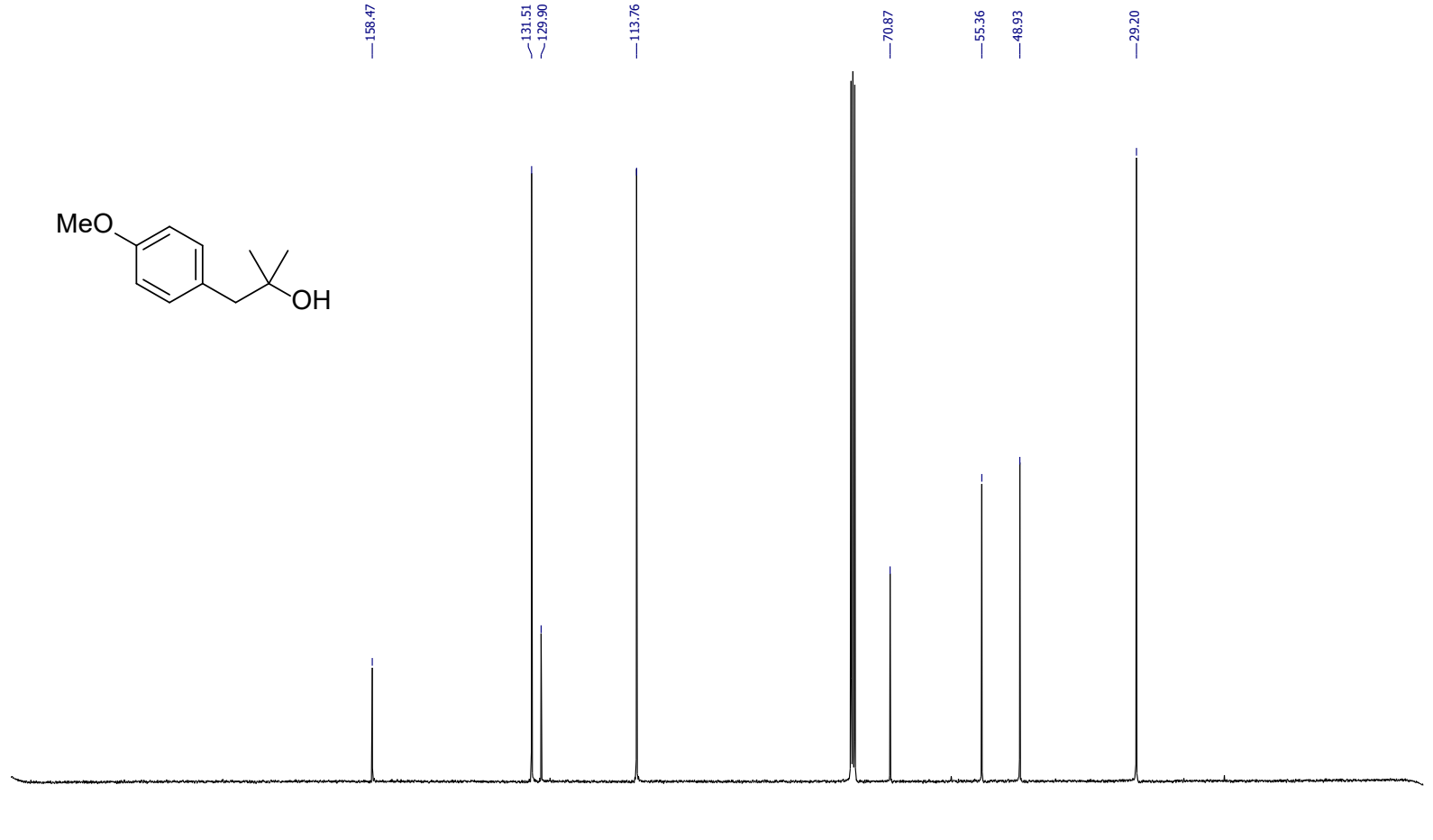

$\begin{array}{llllll}210 & 200 & 190 & 180 & 170 & 160\end{array}$

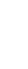

S58 
Compound $\mathbf{2 2 f}$

${ }^{1} \mathrm{H} \mathrm{NMR}, \mathrm{CDCl}_{3}, 400 \mathrm{MHz}$

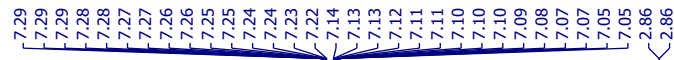
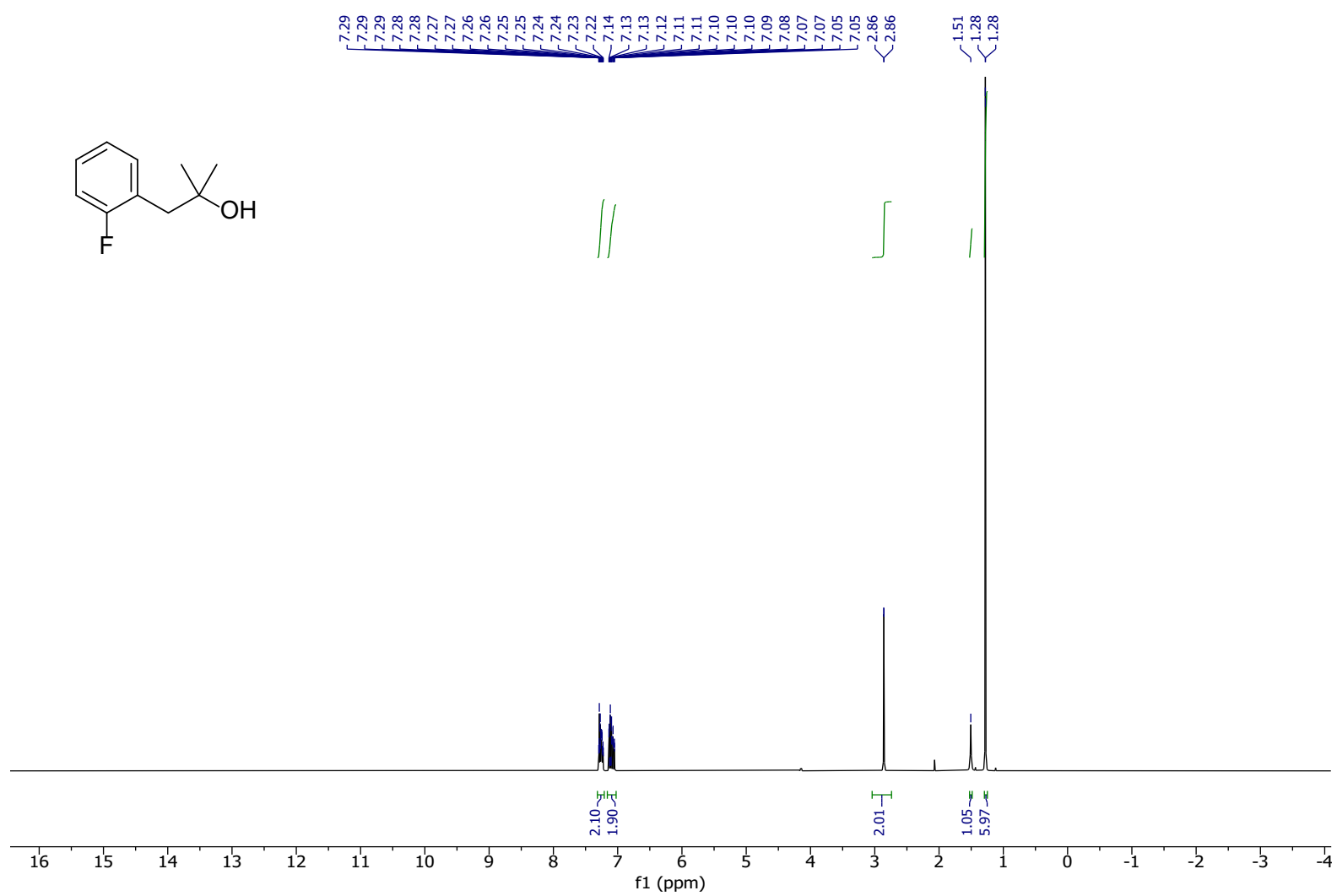

${ }^{13} \mathrm{C} \mathrm{NMR}, \mathrm{CDCl}_{3}, 101 \mathrm{MHz}$

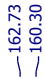

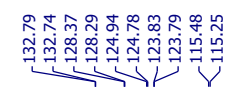
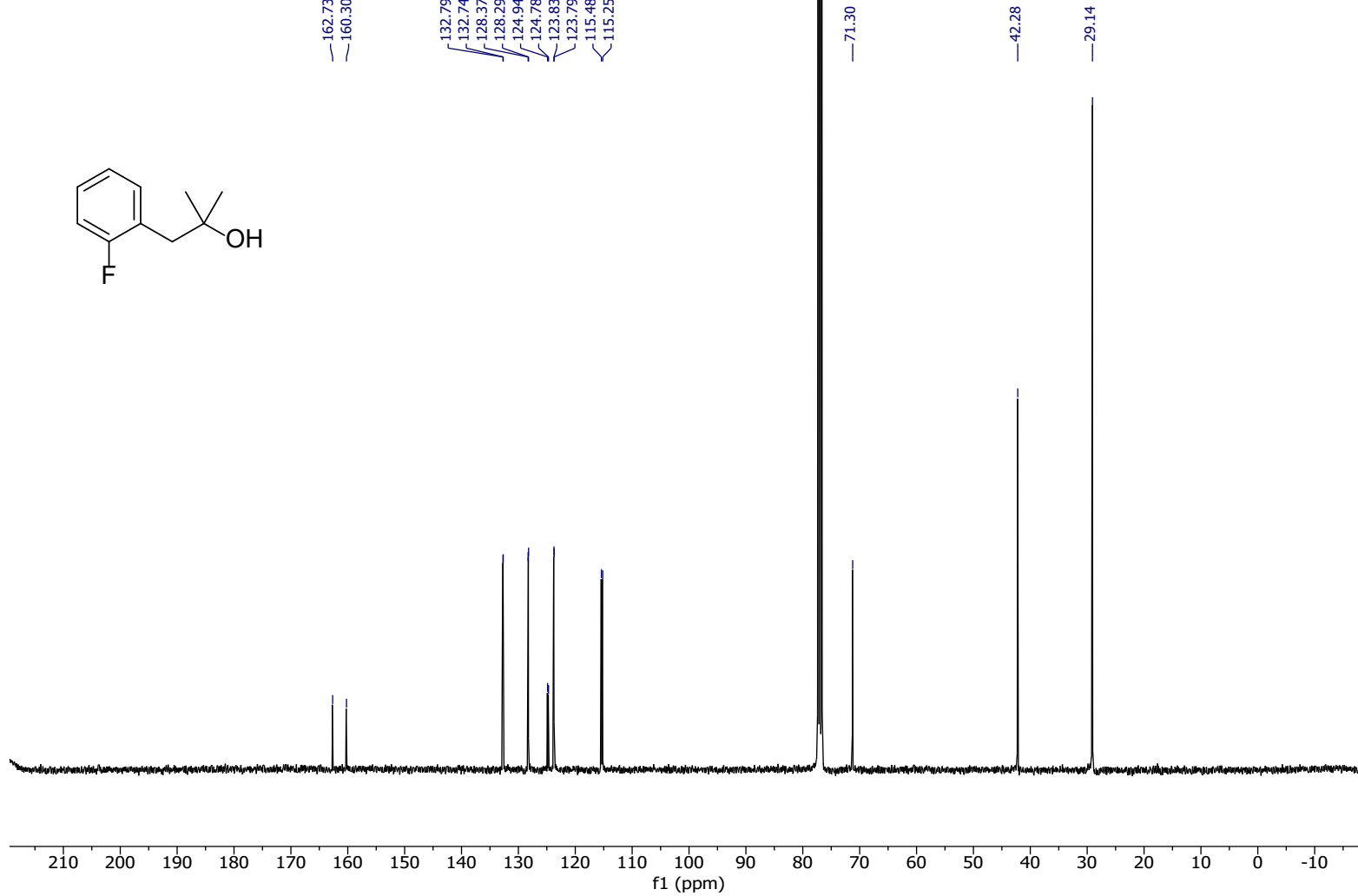

S59 
${ }^{19} \mathrm{~F} \mathrm{NMR}, \mathrm{CDCl}_{3}, 376 \mathrm{MHz}$

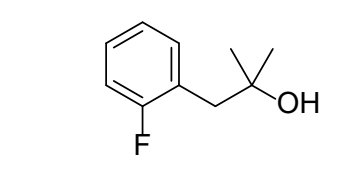

$\infty$
$\stackrel{0}{0}$
0

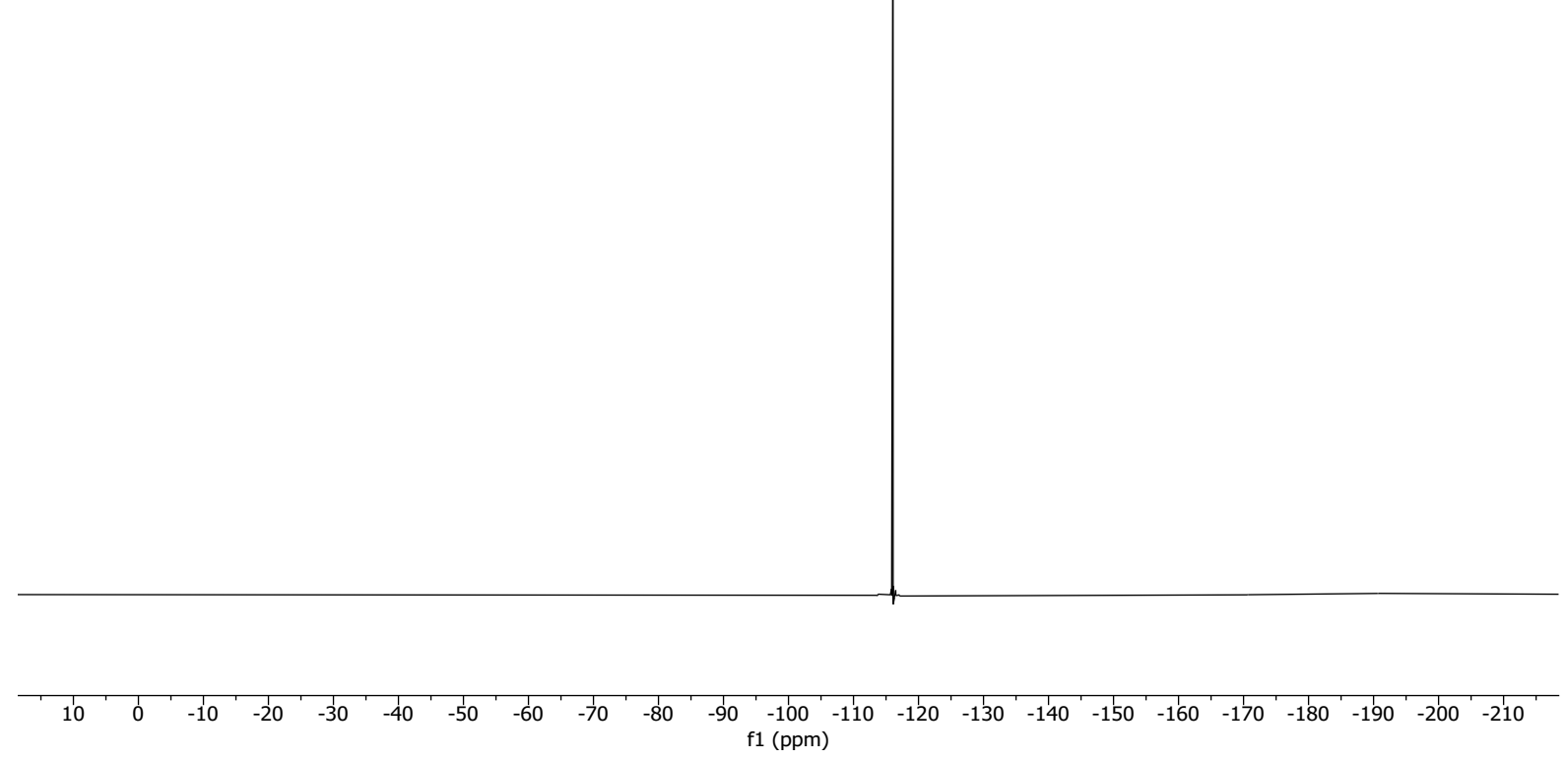

S60 
Compound 22g

${ }^{1} \mathrm{H} \mathrm{NMR}, \mathrm{CDCl}_{3}, 400 \mathrm{MHz}$

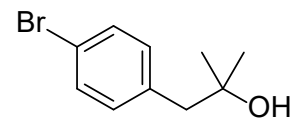

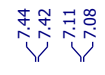

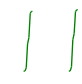

$\stackrel{\text { ก }}{1}$

|

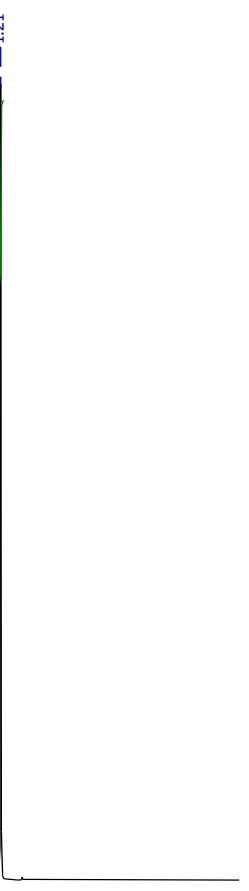

管落

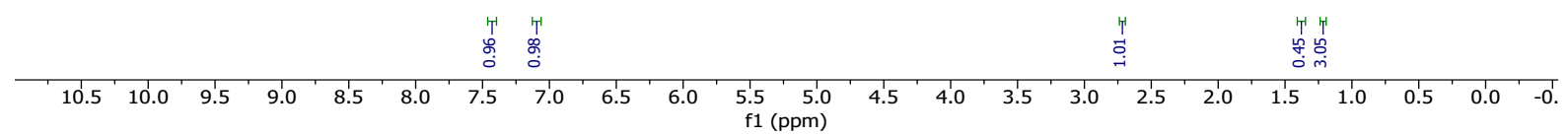

${ }^{13} \mathrm{CNMR}, \mathrm{CDCl}_{3}, 101 \mathrm{MHz}$

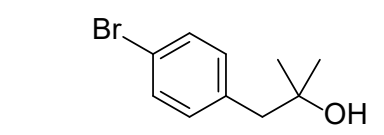

חָ̃
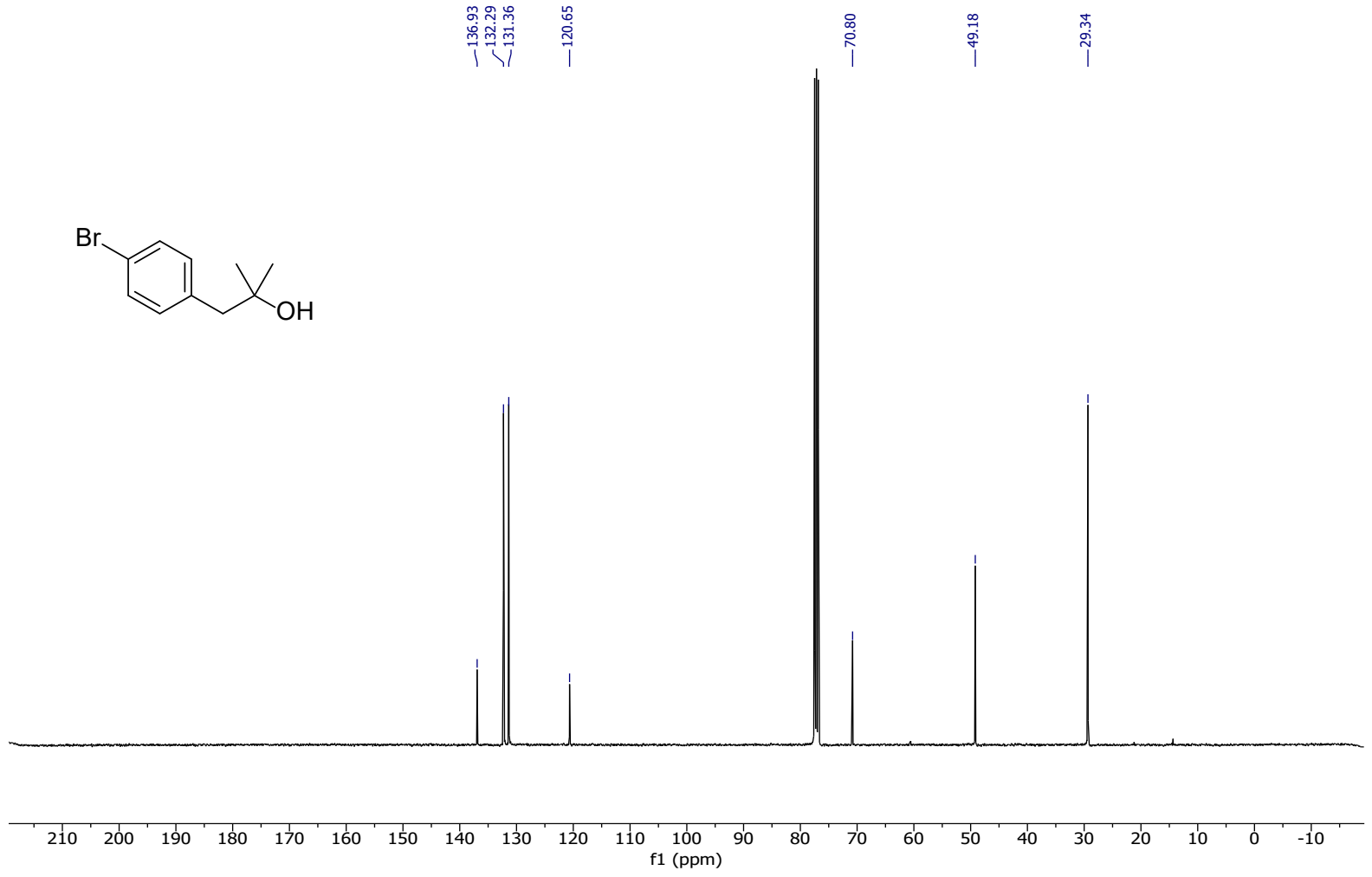

S61 


\section{Compound 22n}

${ }^{1} \mathrm{H} \mathrm{NMR}, \mathrm{CDCl}_{3}, 400 \mathrm{MHz}$

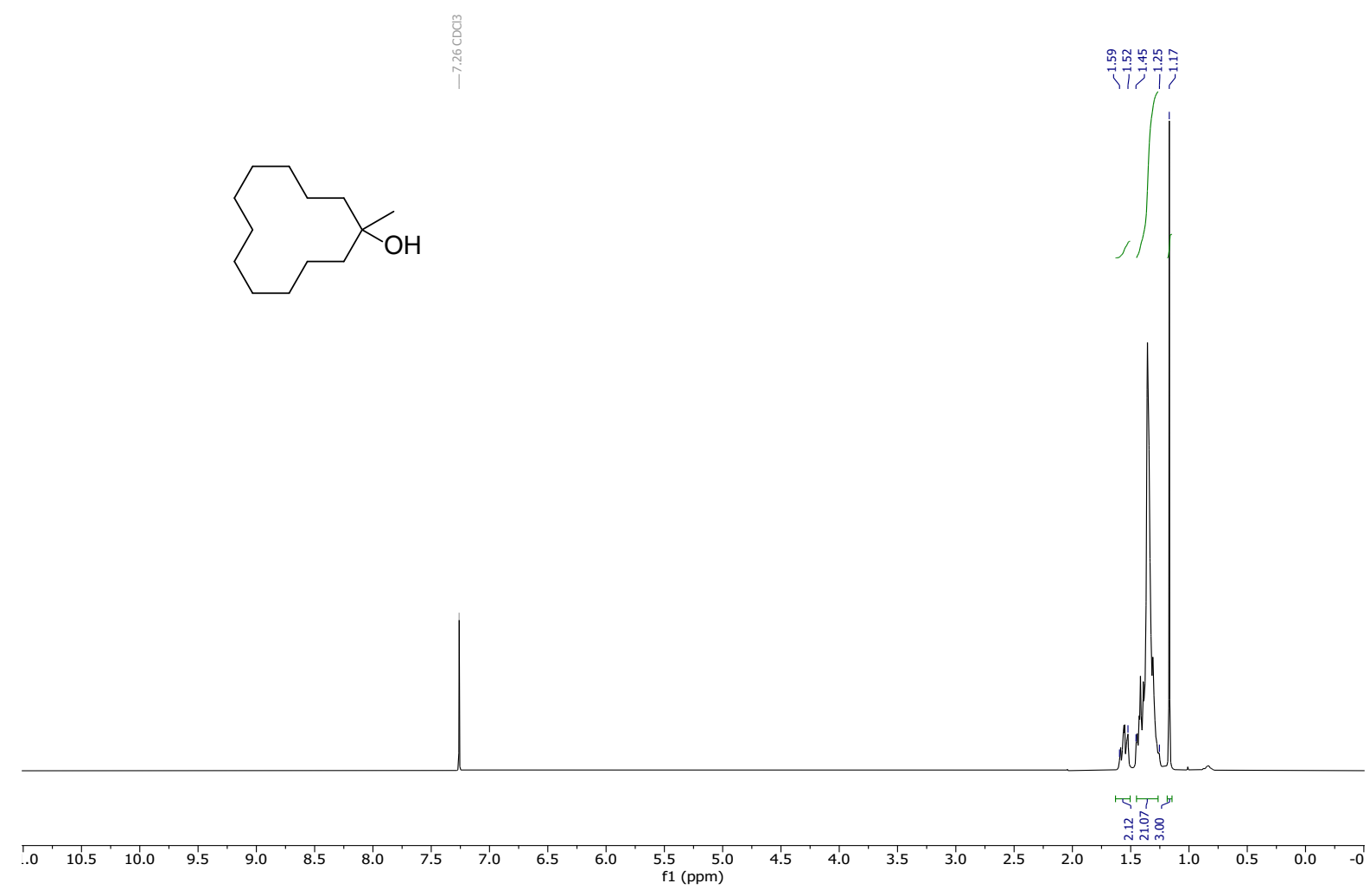

${ }^{13} \mathrm{C}$ NMR, $\mathrm{CDCl}_{3}, 101 \mathrm{MHz}$

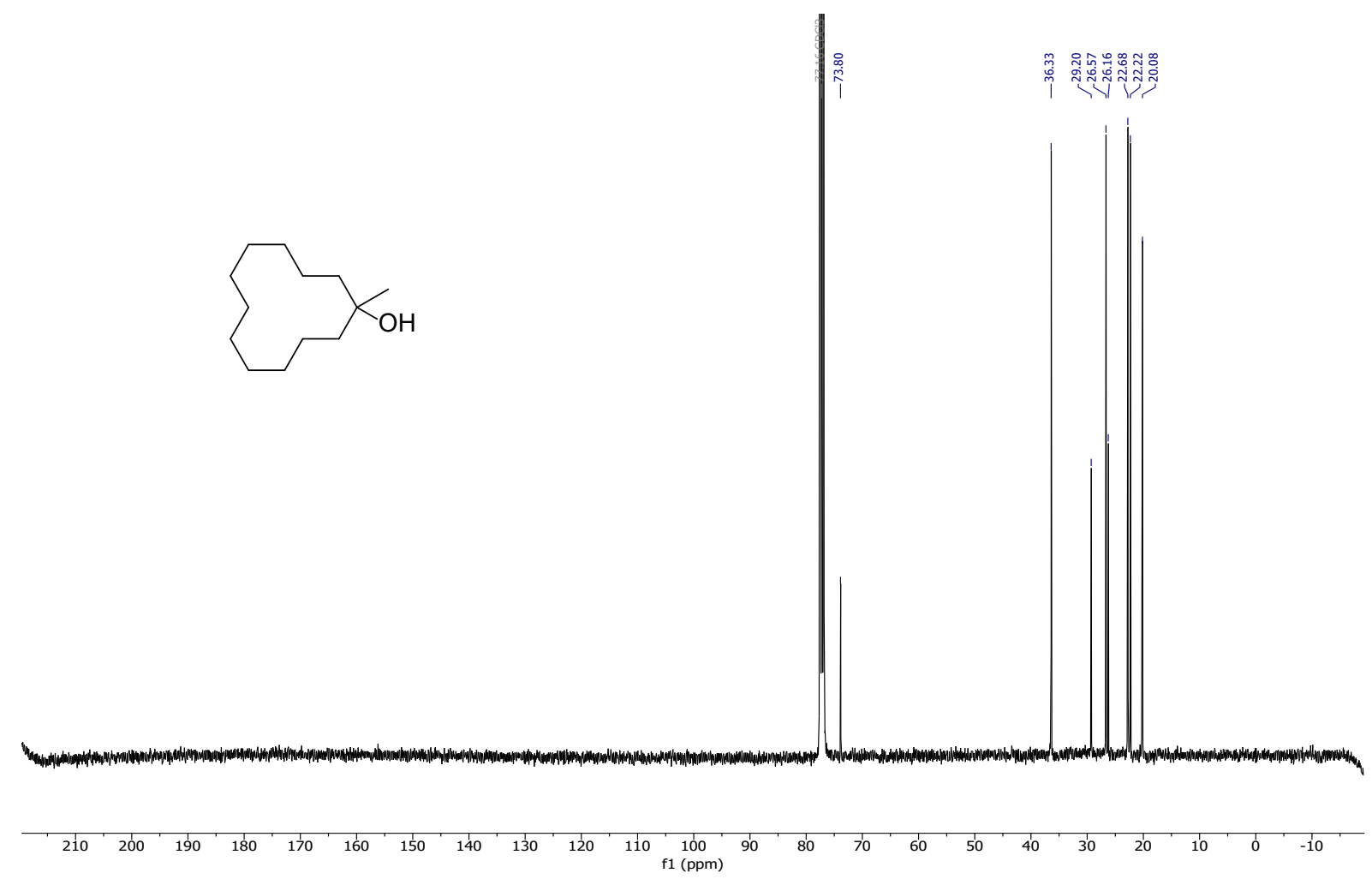




\section{Compound $\mathbf{2 2 0}$}

${ }^{1} \mathrm{H} \mathrm{NMR}, \mathrm{CDCl}_{3}, 400 \mathrm{MHz}$

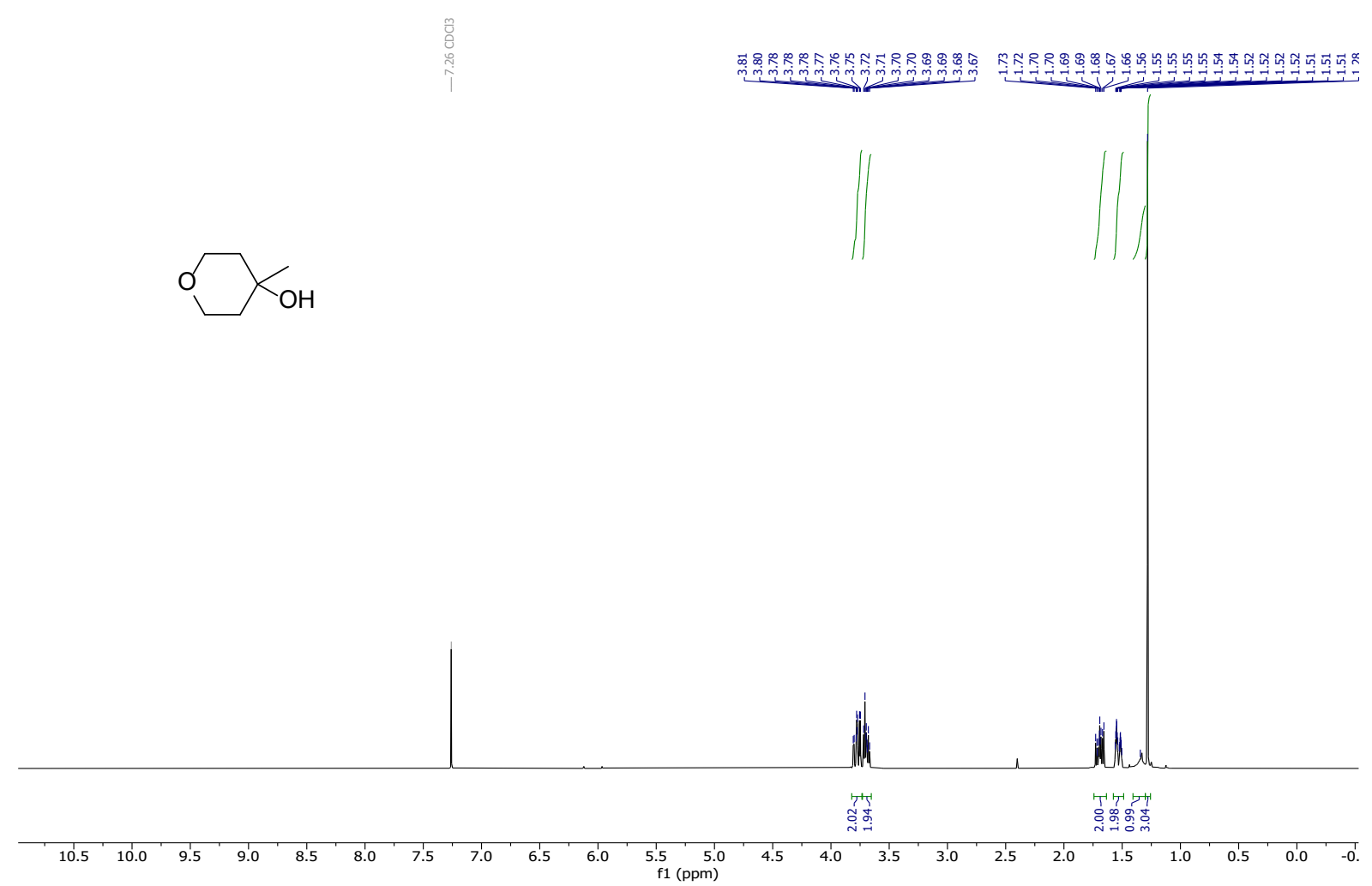

${ }^{13} \mathrm{C} \mathrm{NMR}, \mathrm{CDCl}_{3}, 101 \mathrm{MHz}$

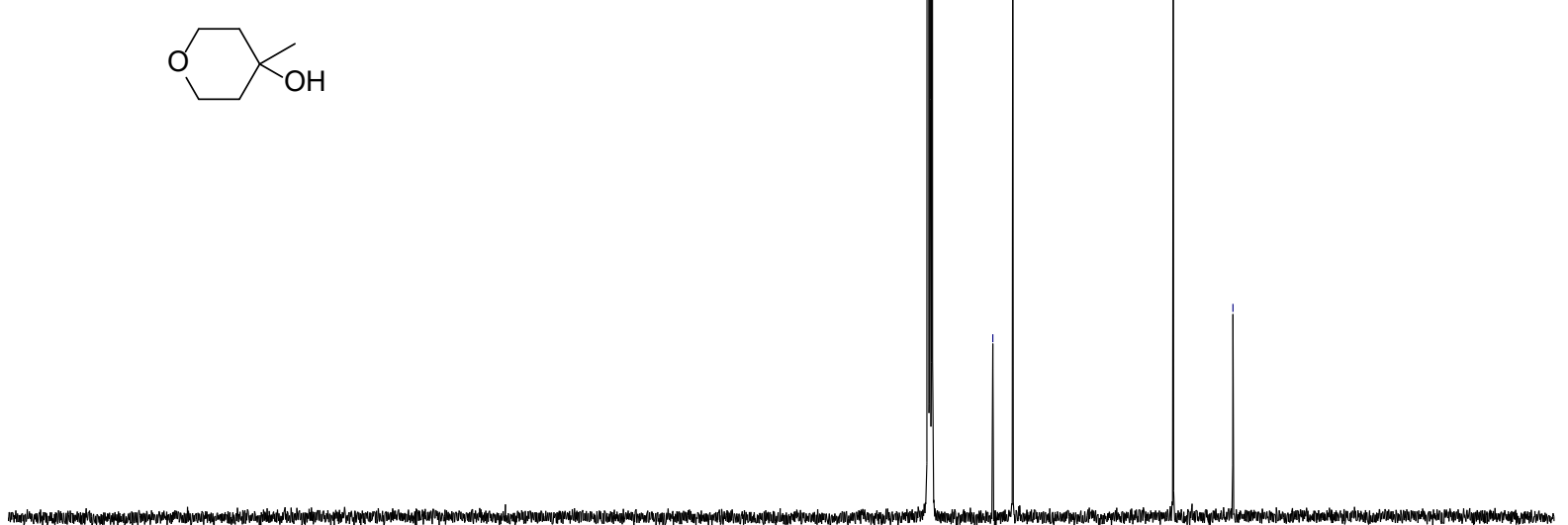

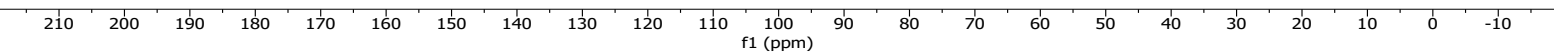




\section{Compound 23a}

${ }^{1} \mathrm{H} \mathrm{NMR}, \mathrm{CDCl}_{3}, 400 \mathrm{MHz}$

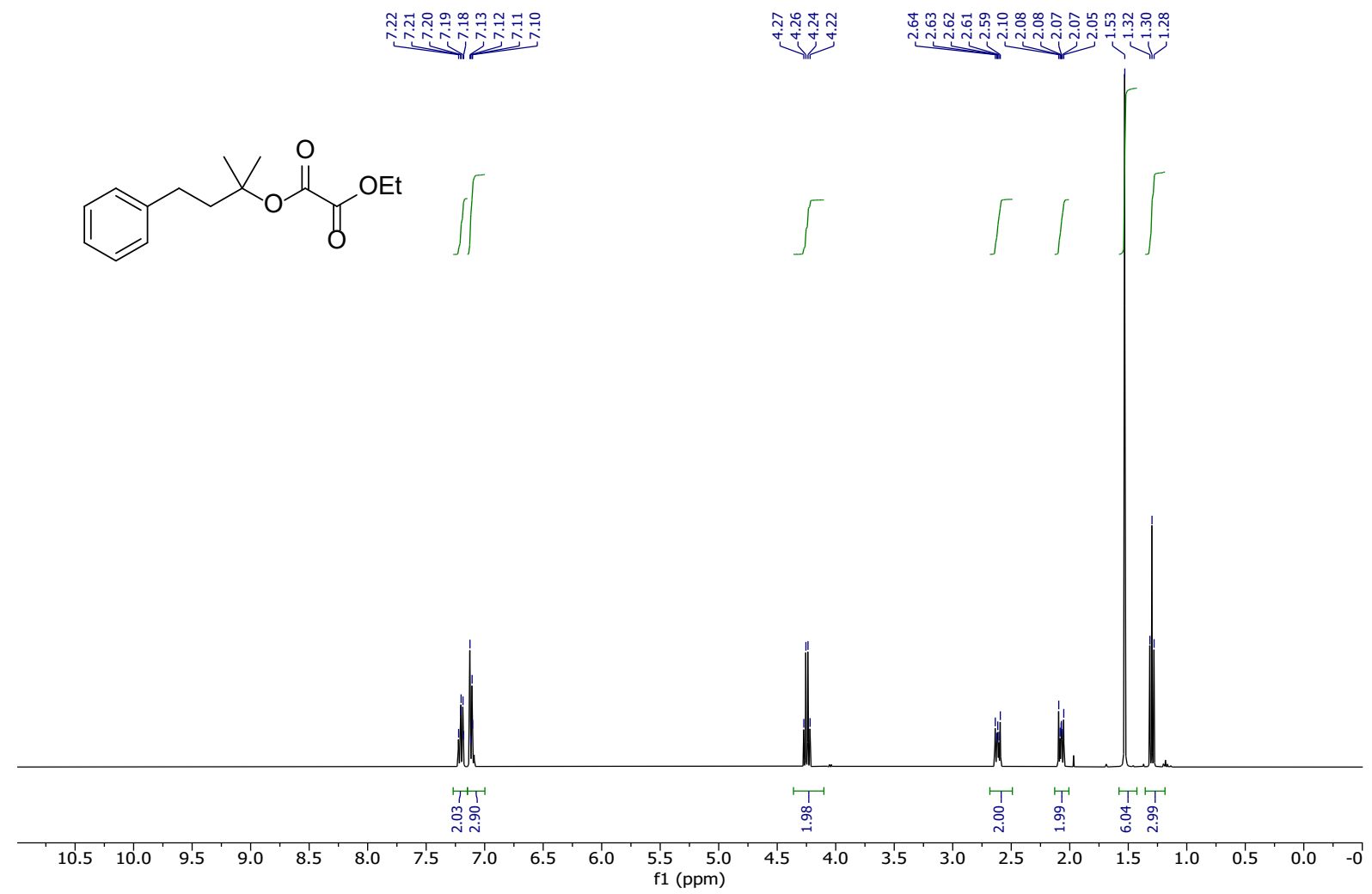

${ }^{13} \mathrm{CNMR}_{\mathrm{NDCl}}, 400 \mathrm{MHz}$

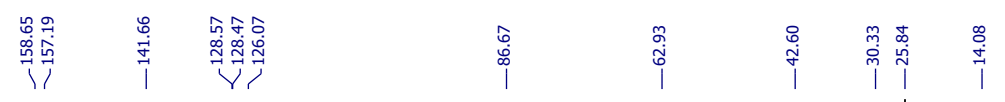<smiles>CCOC(=O)C(=O)OC(C)(C)CCc1ccccc1</smiles>

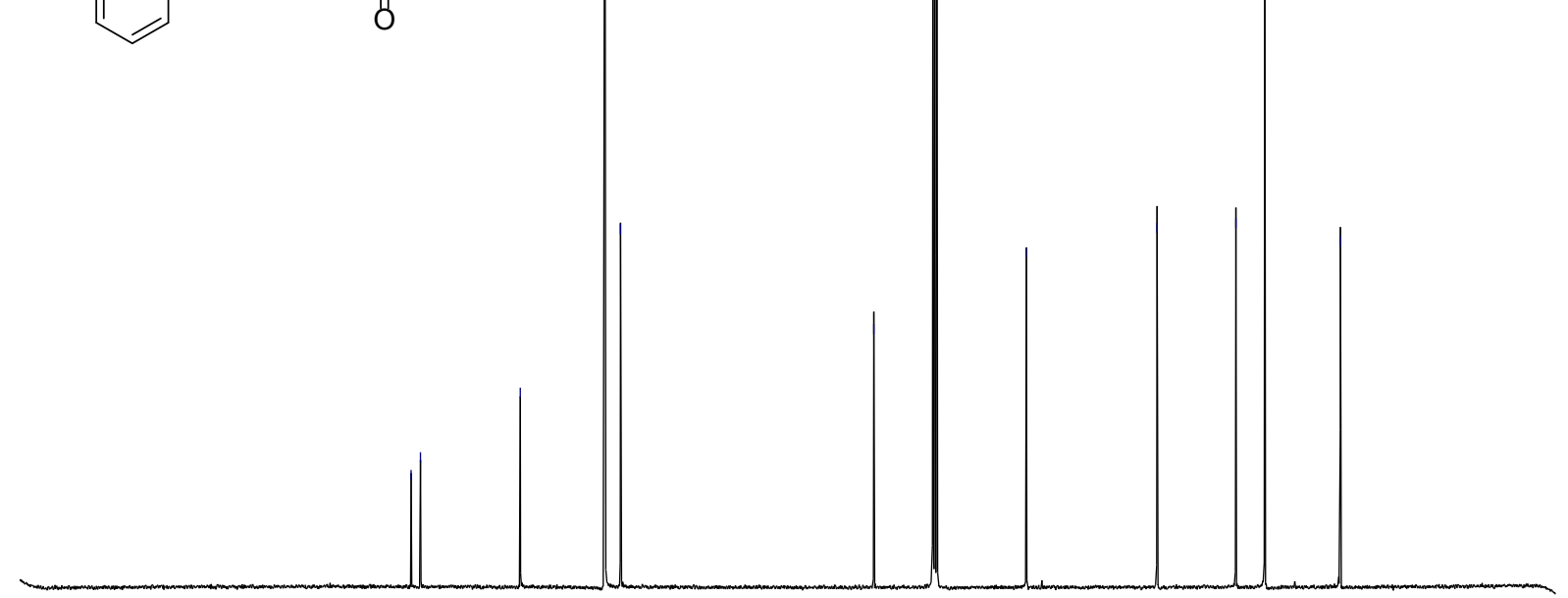

$\begin{array}{llllllllllllllllllllllll}210 & 200 & 190 & 180 & 170 & 160 & 150 & 140 & 130 & 120 & 110 & 100 & 90 & 80 & 70 & 60 & 50 & 40 & 30 & 20 & 10 & 0 & -10 & 10\end{array}$ 


\section{Compound 3a}

${ }^{1} \mathrm{H} N M R, \mathrm{D}_{2} \mathrm{O}, 400 \mathrm{MHz}$

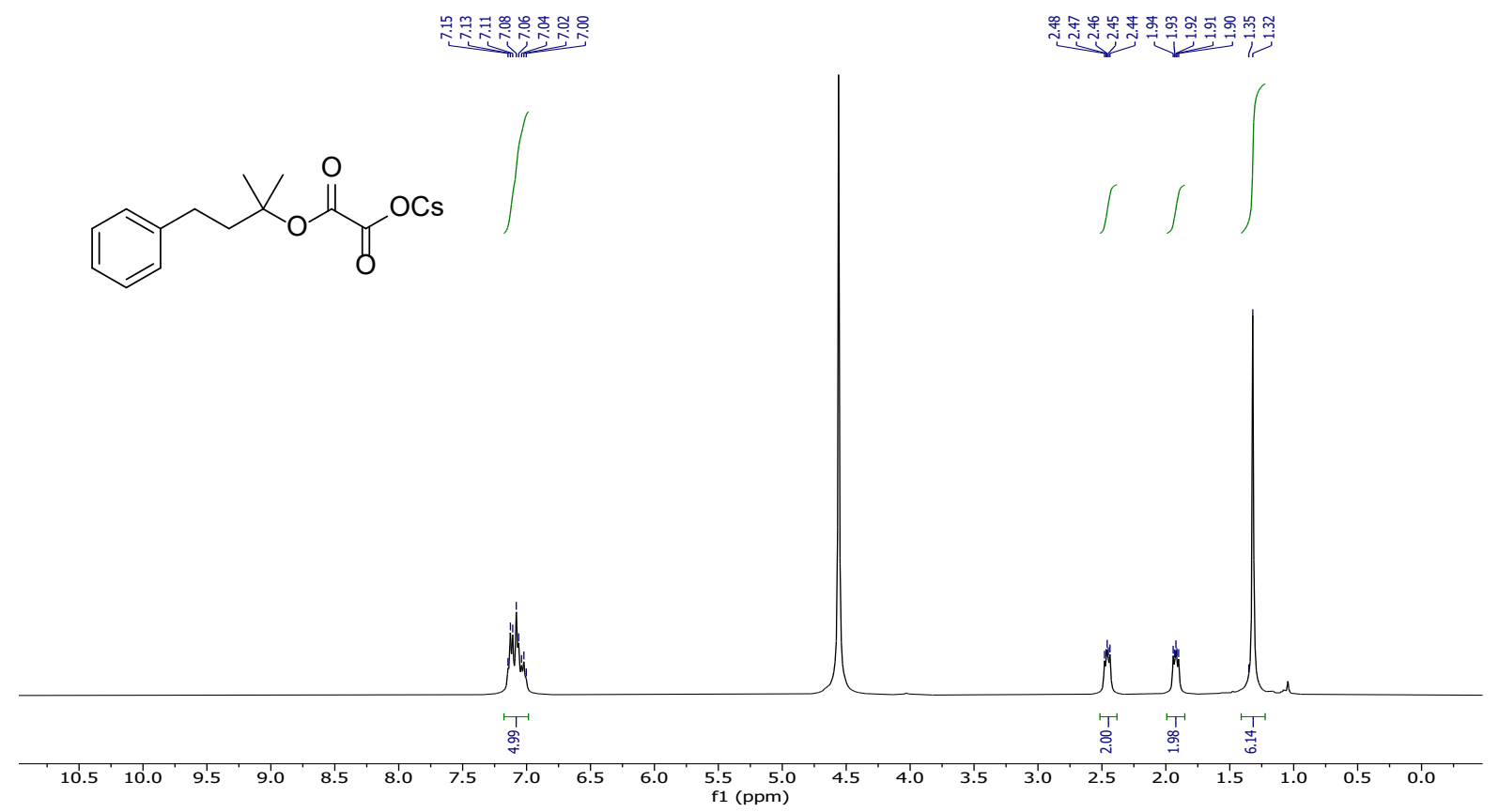

${ }^{13} \mathrm{C}$ NMR, $\mathrm{D}_{2} \mathrm{O}, 101 \mathrm{MHz}$

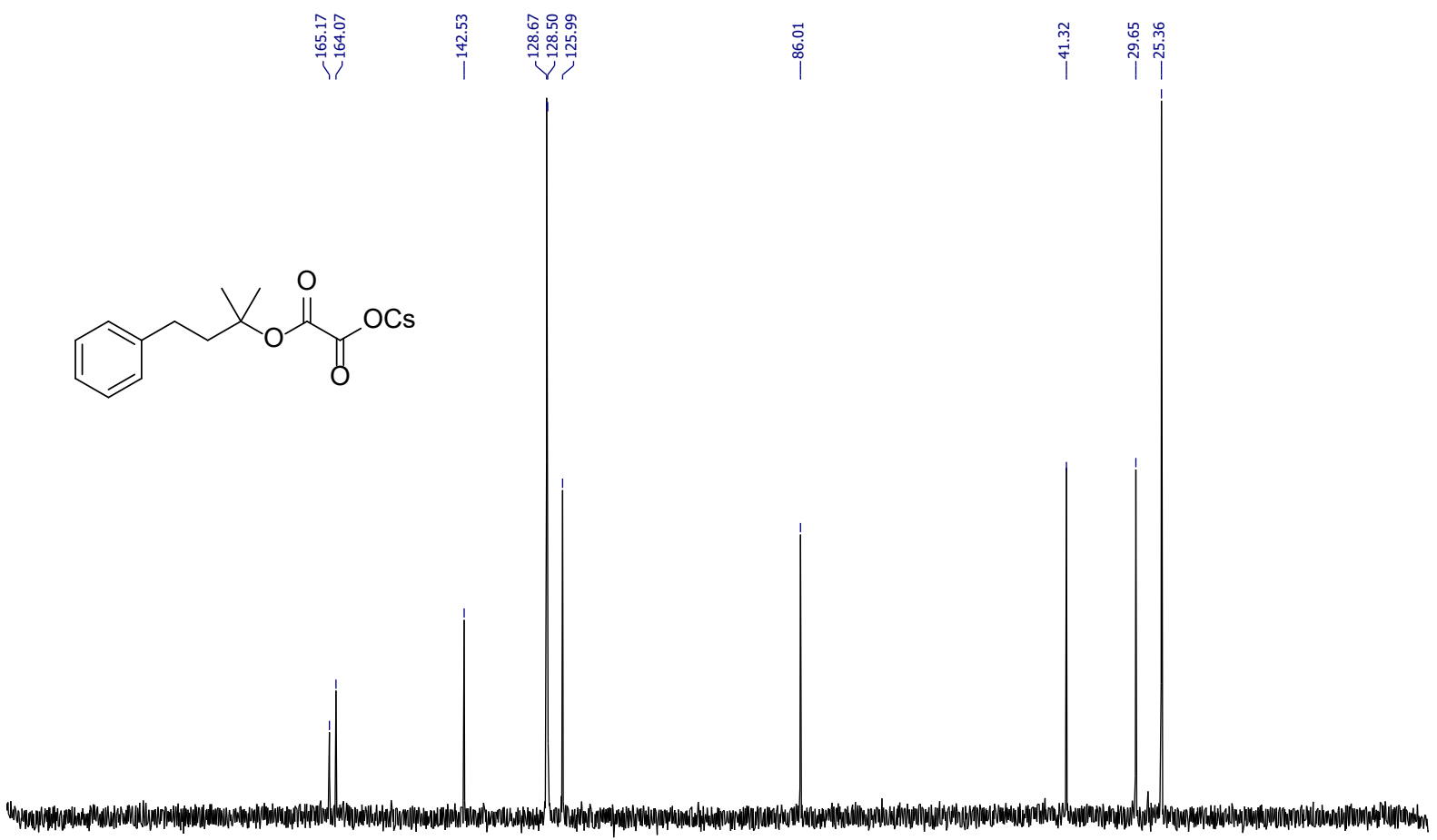

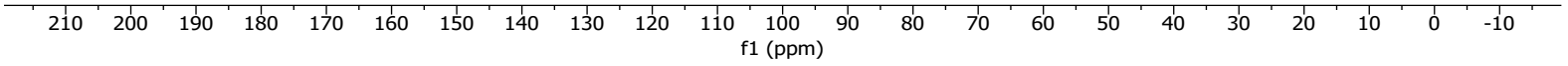




\section{Compound 23b}

${ }^{1} \mathrm{H} \mathrm{NMR}, \mathrm{CDCl}_{3}, 400 \mathrm{MHz}$

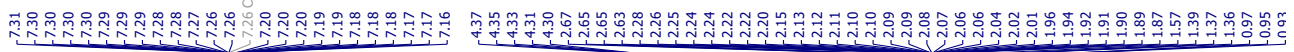<smiles>CCOC(=O)C(=O)OC(C)(CC)CCc1ccccc1</smiles>
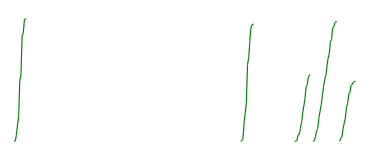

ind

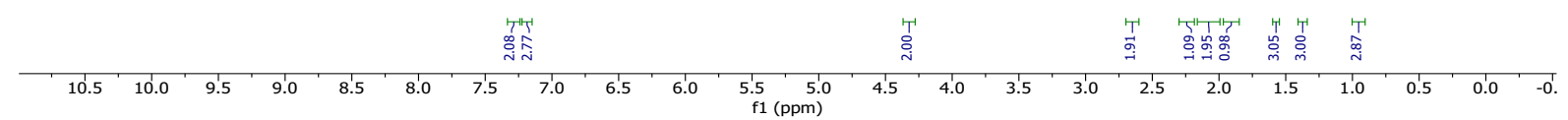

${ }^{13} \mathrm{C} \mathrm{NMR}, \mathrm{CDCl}_{3}, 101 \mathrm{MHz}$

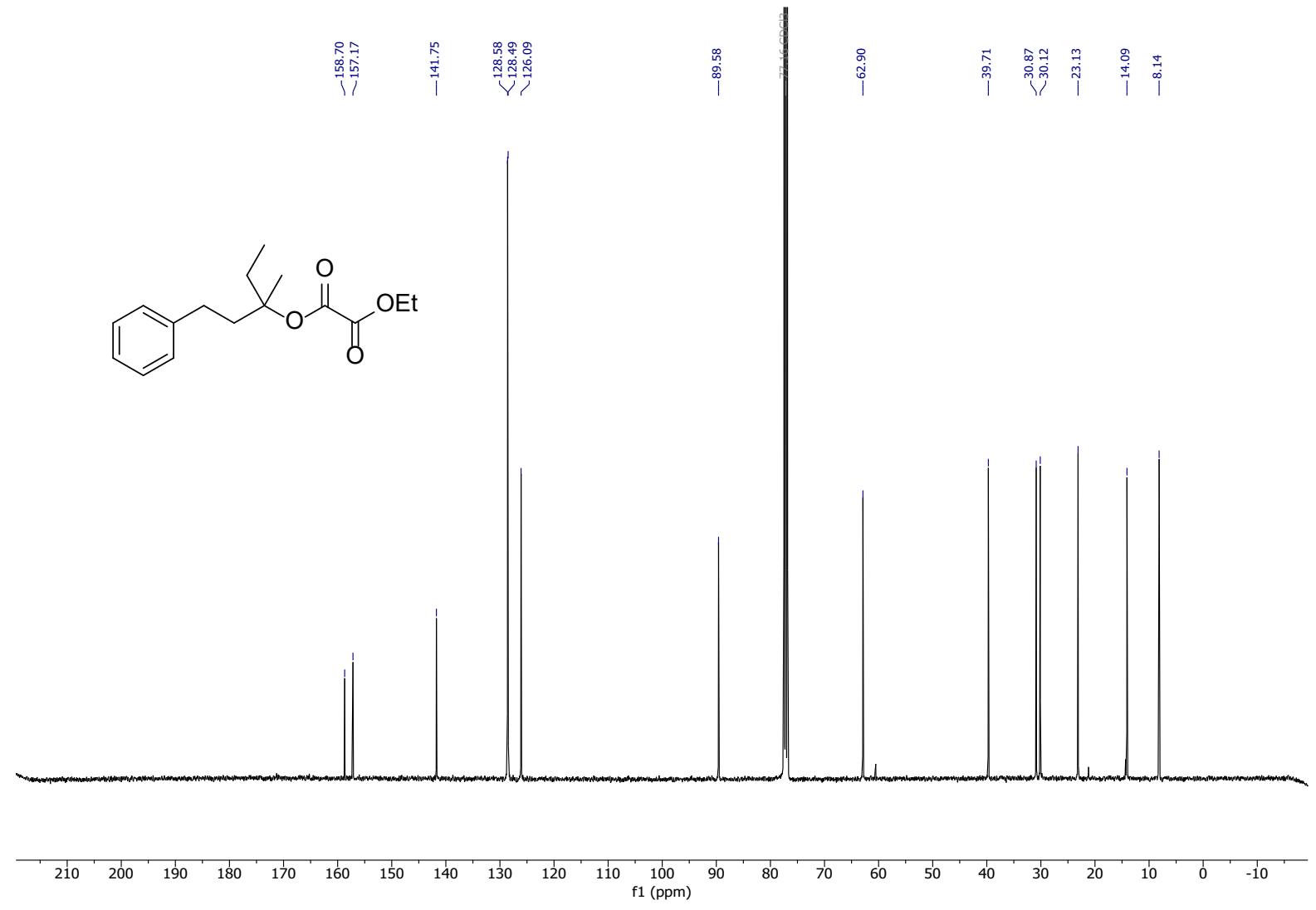




\section{Compound $\mathbf{3 b}$}

${ }^{1} \mathrm{H}$ NMR, DMSO, $400 \mathrm{MHz}$

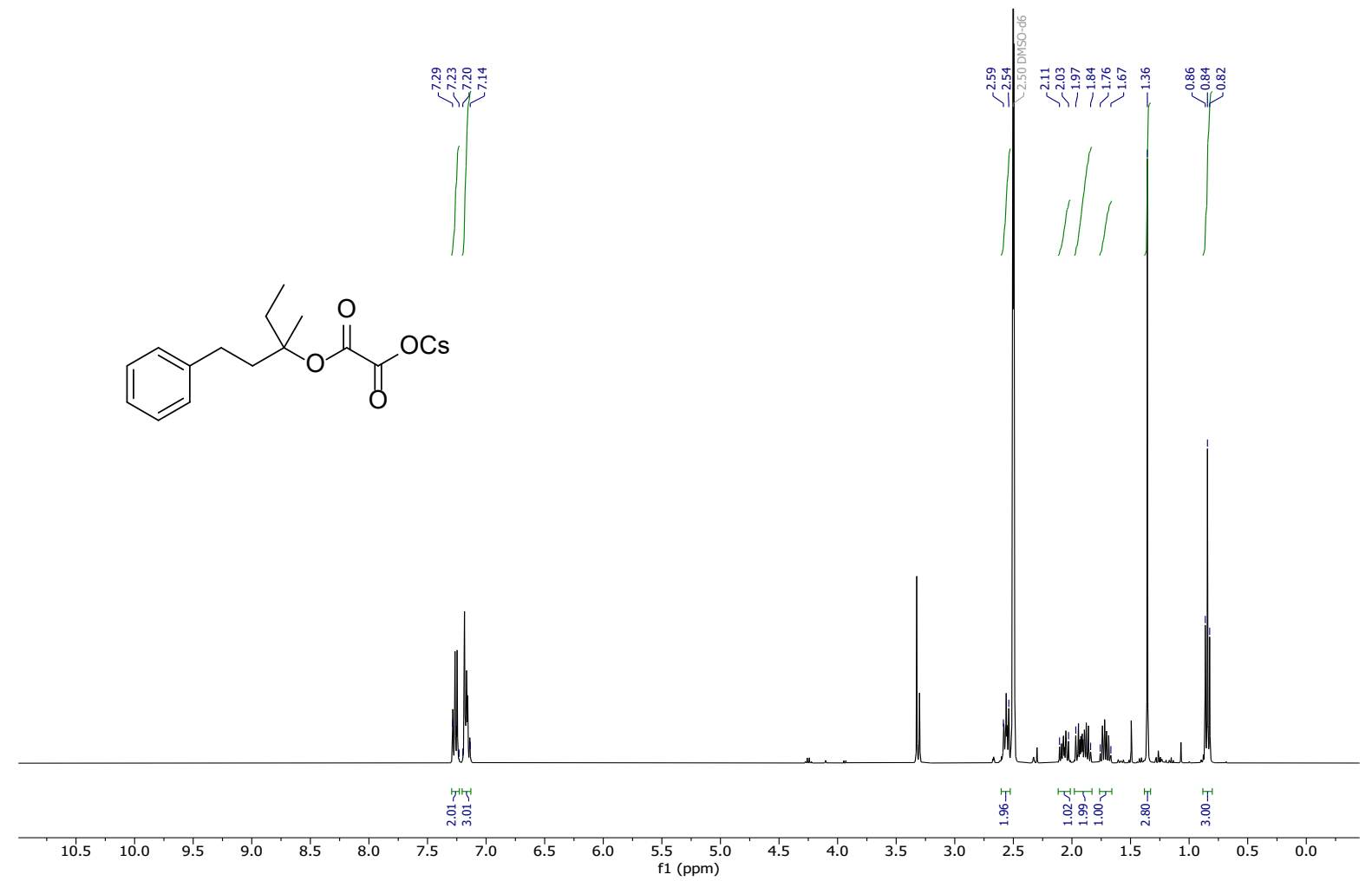

${ }^{13} \mathrm{C}$ NMR, DMSO, $101 \mathrm{MHz}$

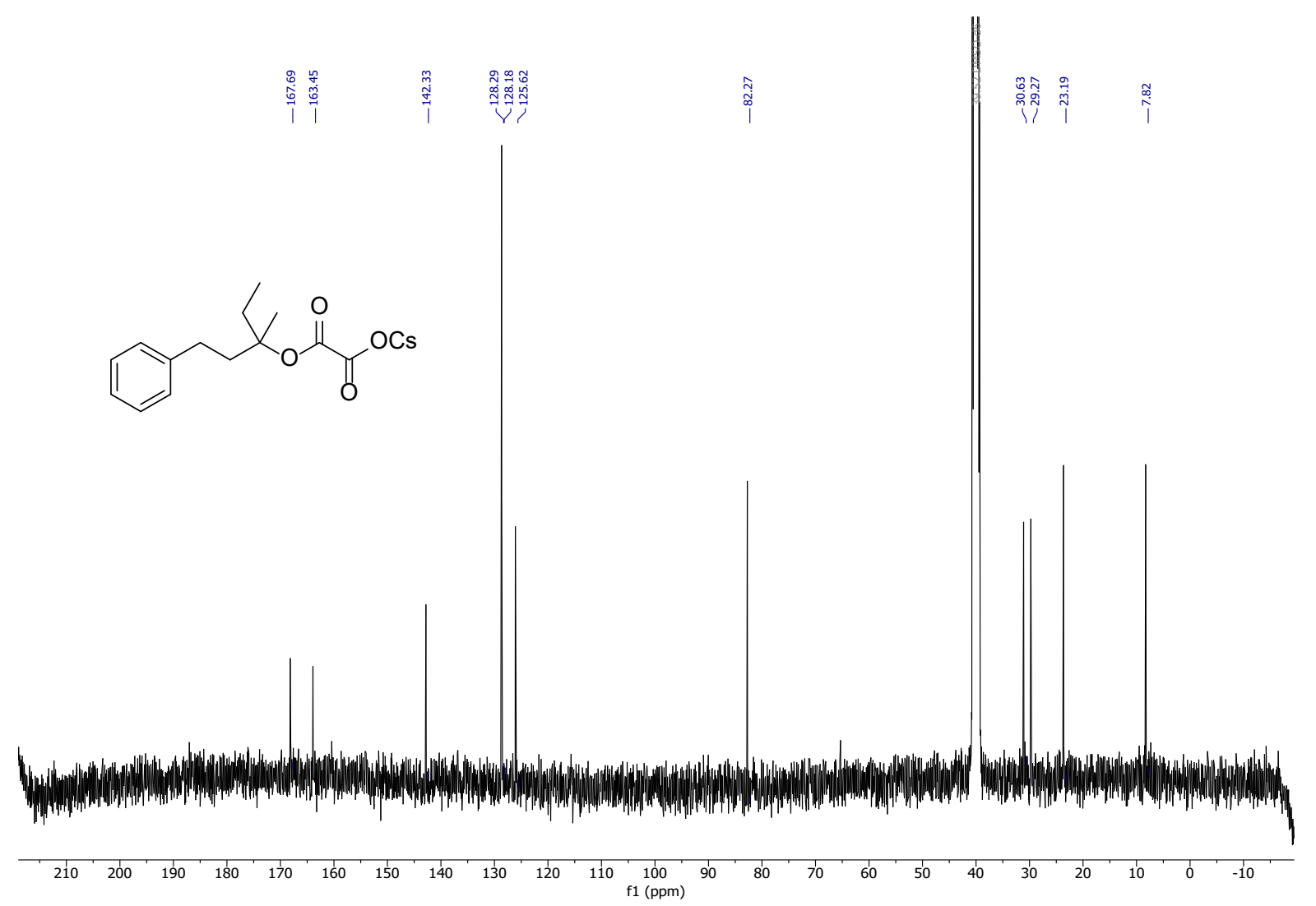


Compound 23c

${ }^{1} \mathrm{H} \mathrm{NMR}, \mathrm{CDCl}_{3}, 400 \mathrm{MHz}$

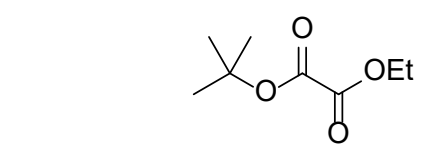

趋是

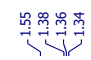

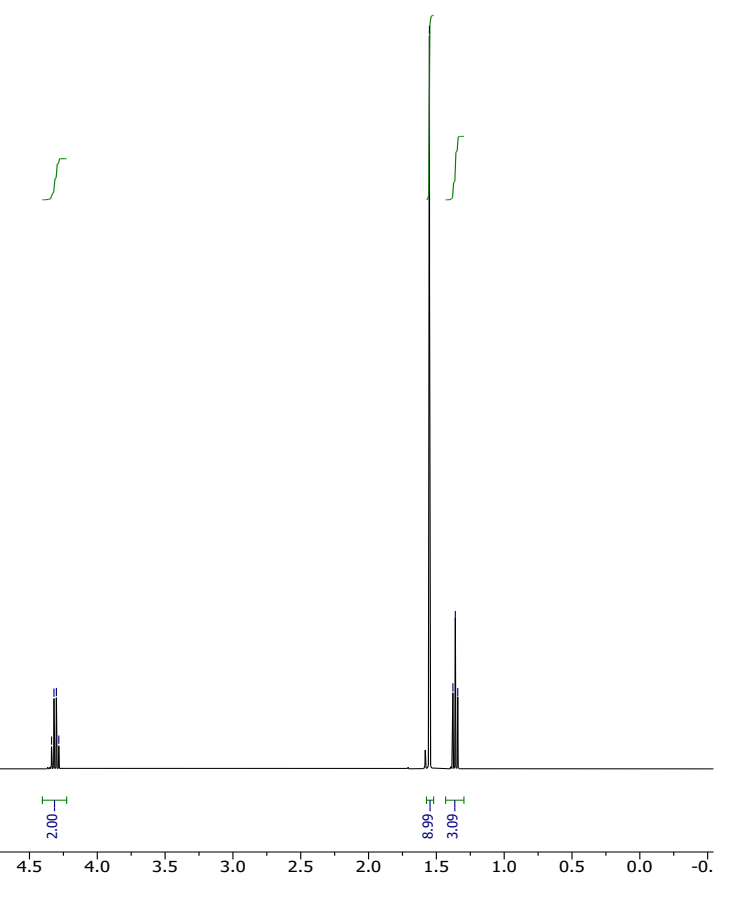

${ }^{13} \mathrm{CNMR}, \mathrm{CDCl}_{3}, 101 \mathrm{MHz}$

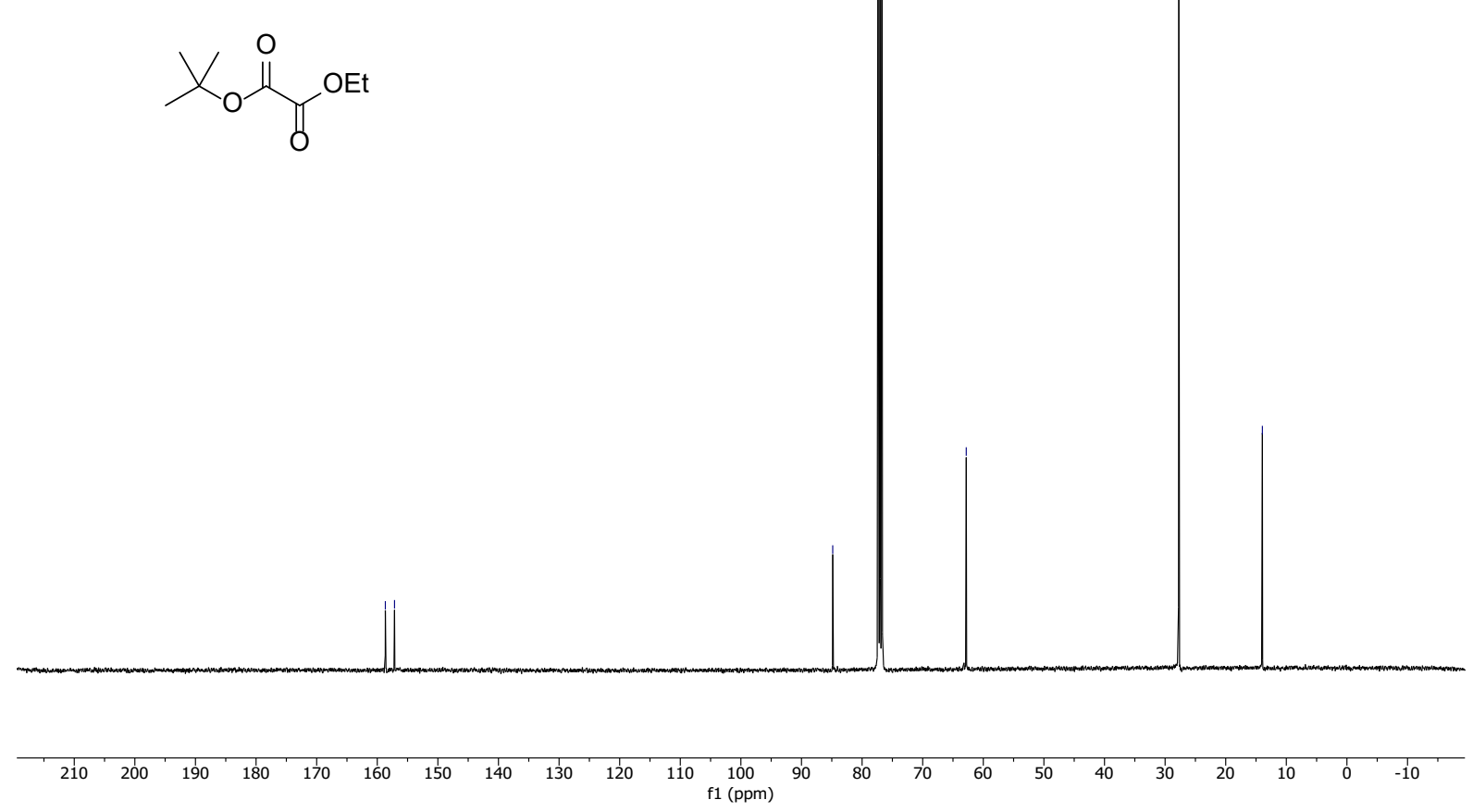

S68 


\section{Compound 3c}

${ }^{1} \mathrm{H}$ NMR, DMSO, $400 \mathrm{MHz}$<smiles>CC(C)(C)OC(=O)C(=O)OCc1ccccc1</smiles>

$$
\text { s }
$$

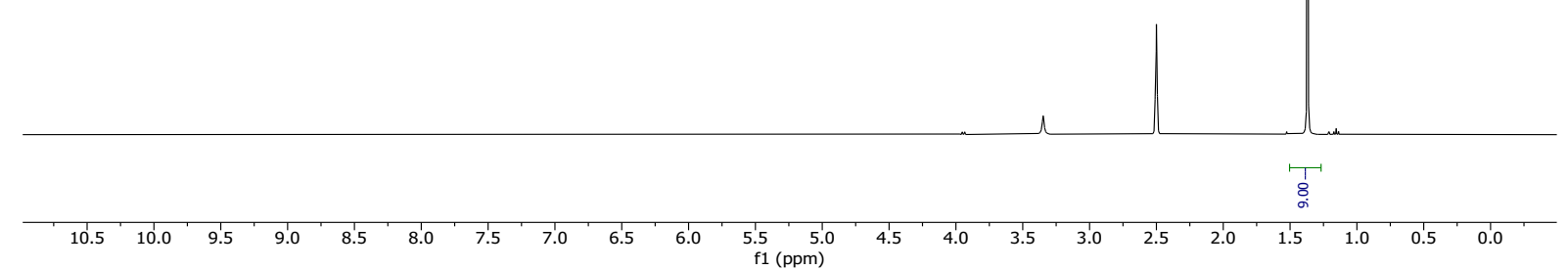

${ }^{13} \mathrm{C} N M R$, DMSO, $101 \mathrm{MHz}$

$$
\overbrace{0}^{0}
$$

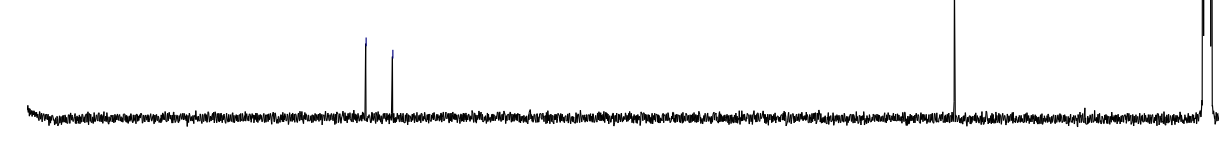




\section{Compound 23d}

${ }^{1} \mathrm{H} \mathrm{NMR}, \mathrm{CDCl}_{3}, 400 \mathrm{MHz}$

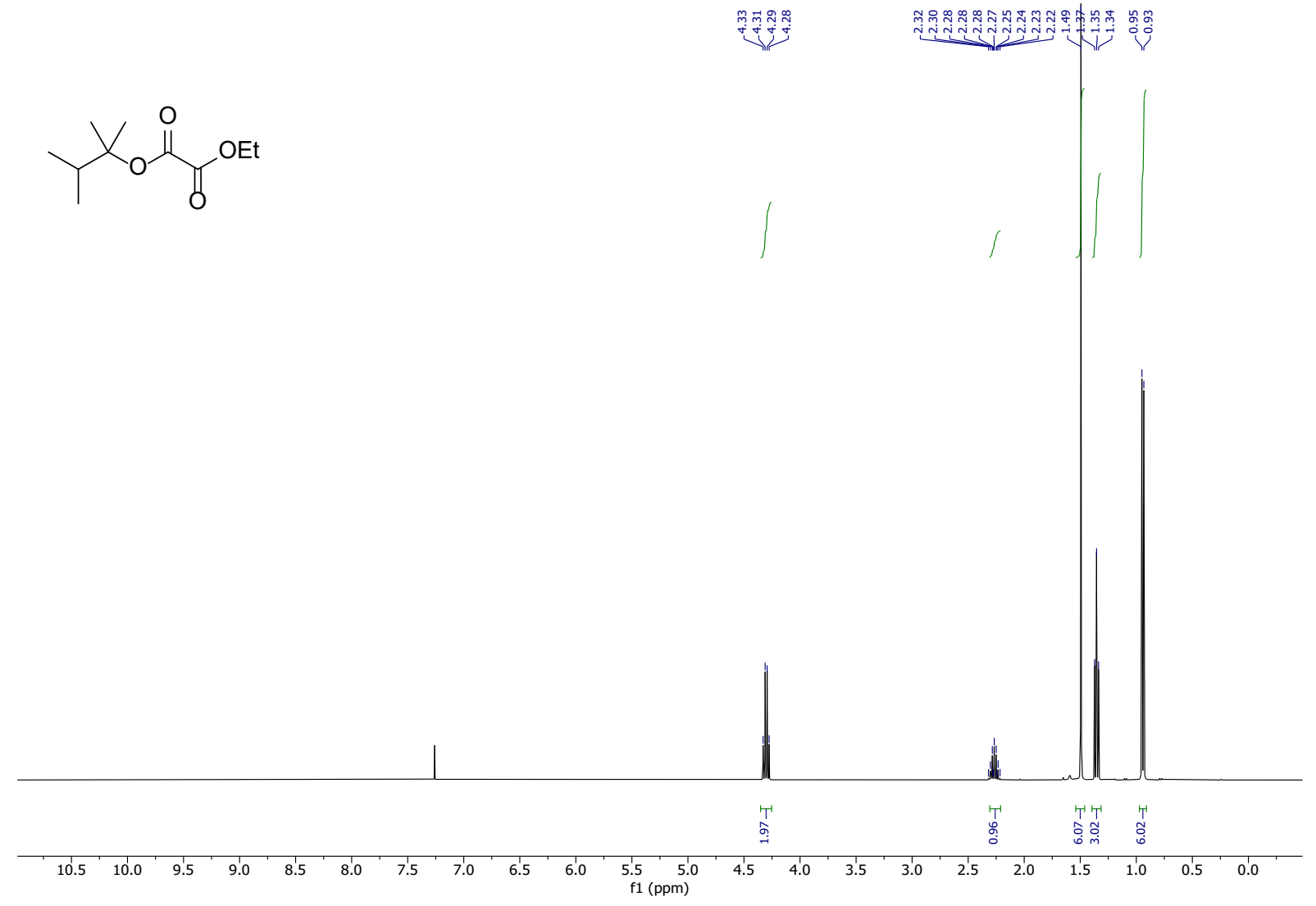

${ }^{13} \mathrm{C} \mathrm{NMR}, \mathrm{CDCl}_{3}, 101 \mathrm{MHz}$

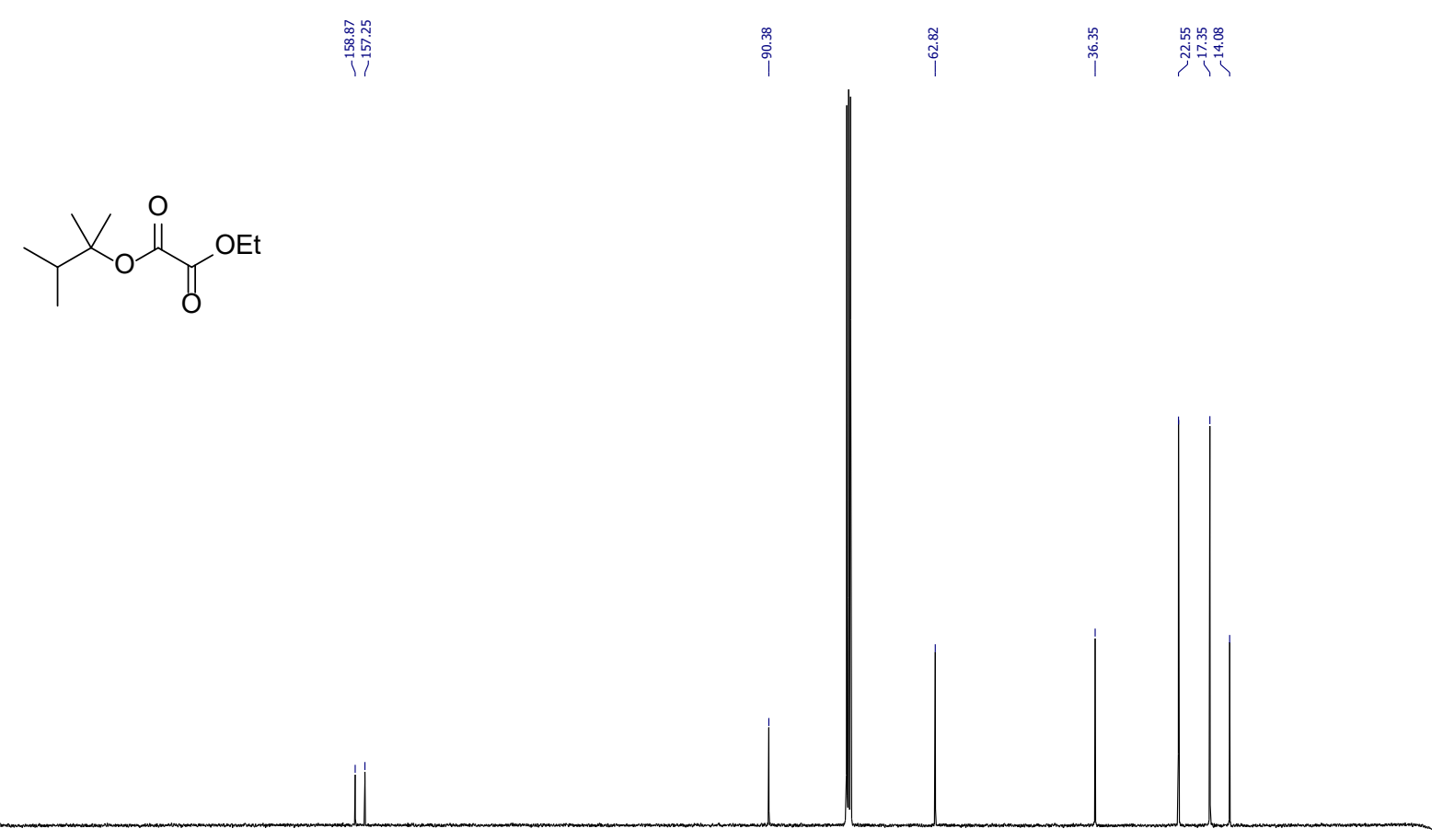

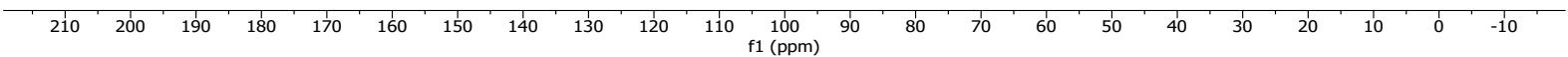




\section{Compound 3d}

${ }^{1} \mathrm{H}$ NMR, DMSO, $400 \mathrm{MHz}$<smiles>CCOC(=O)C(=O)OC(C)(C)C(C)C</smiles>

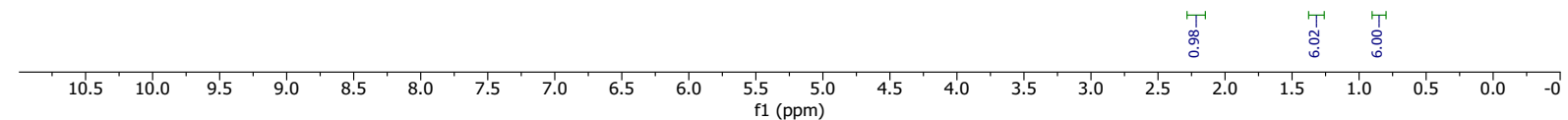

${ }^{13} \mathrm{C}$ NMR, DMSO, $101 \mathrm{MHz}$
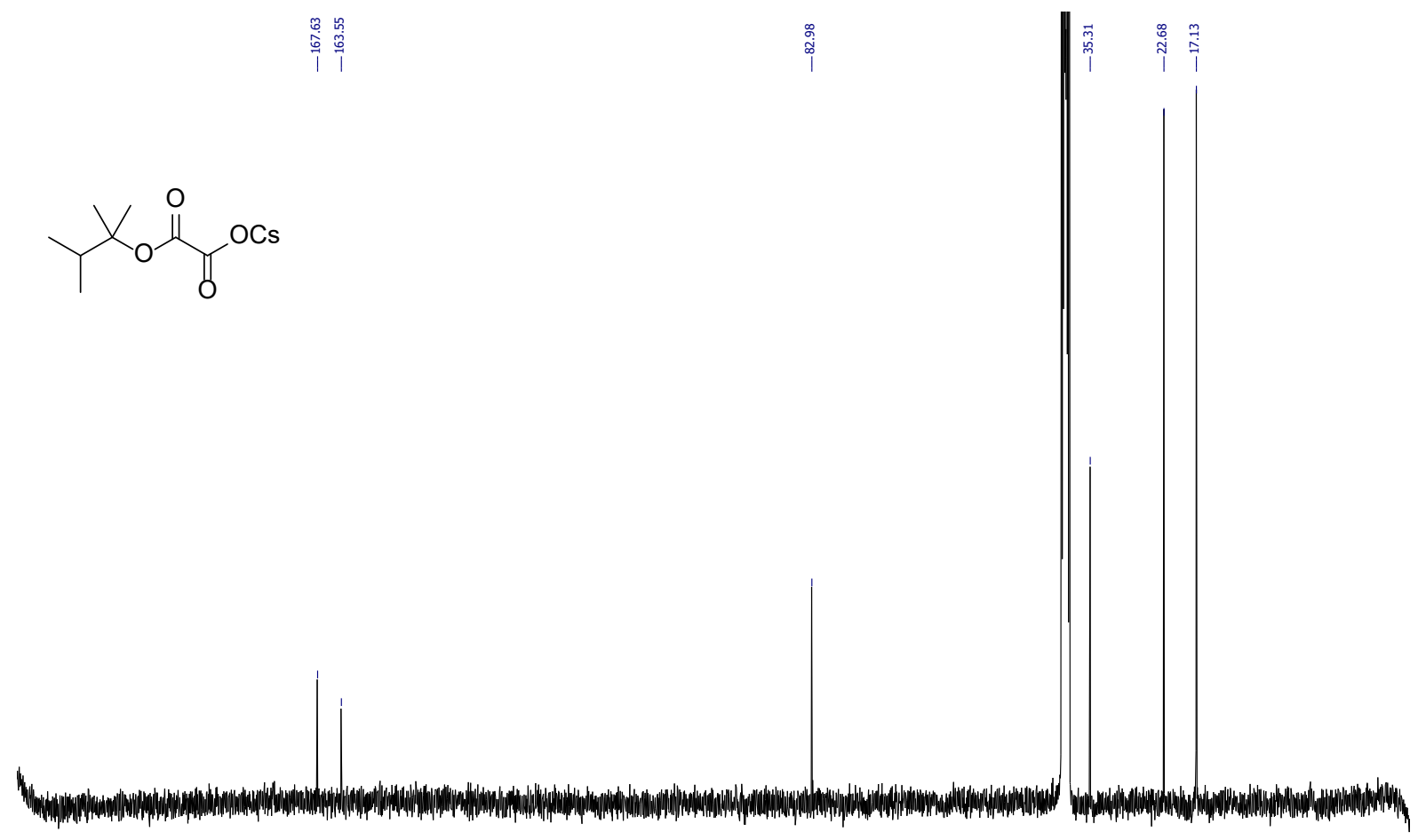

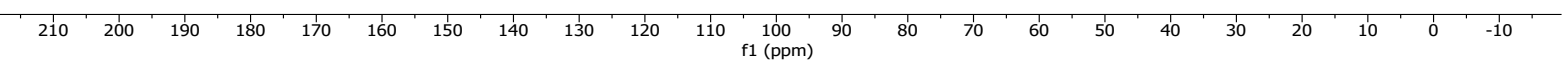


Compound 23e

${ }^{1} \mathrm{H}$ NMR, $\mathrm{CDCl}_{3}, 400 \mathrm{MHz}$

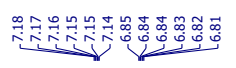
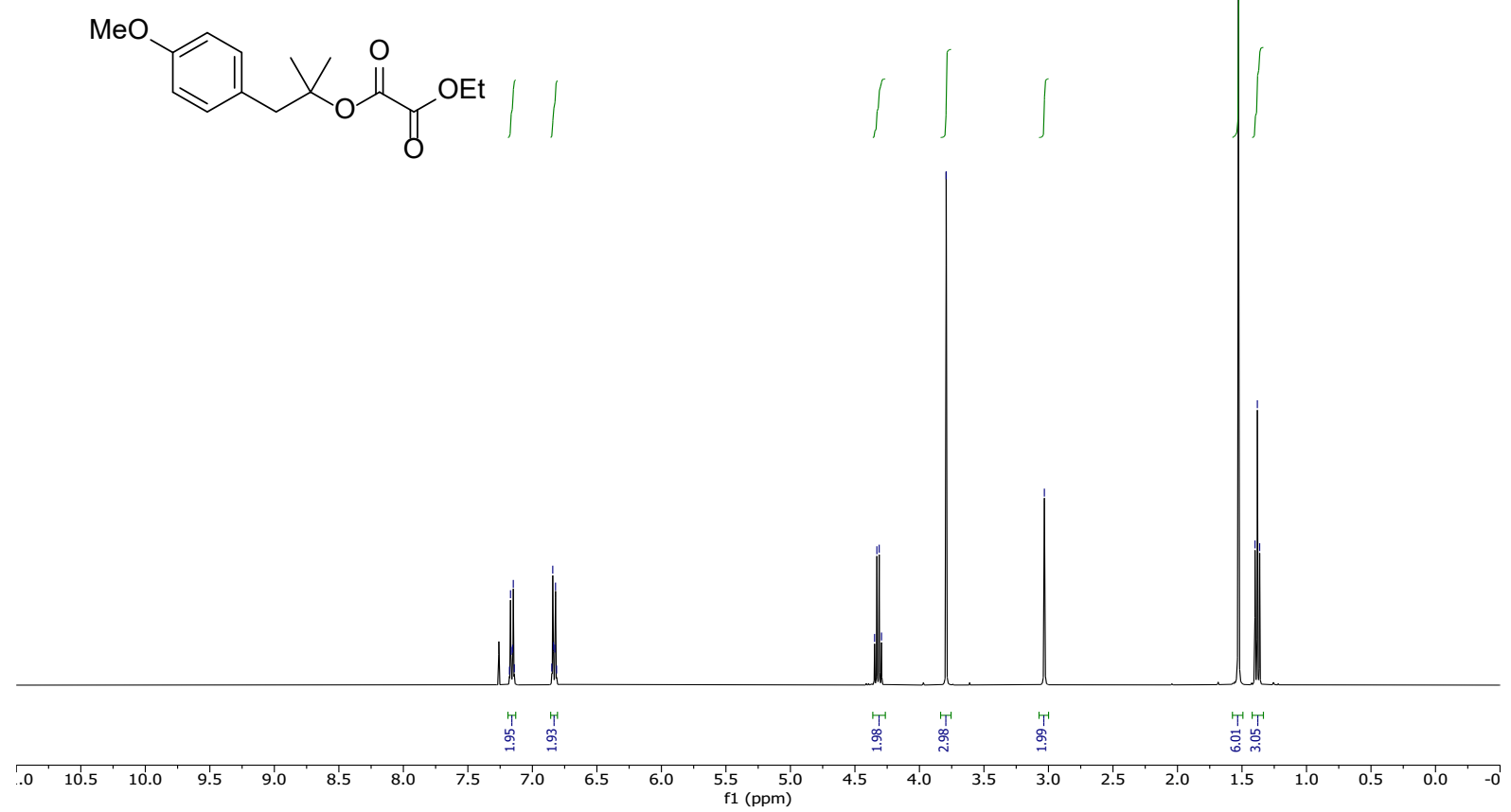

${ }^{13} \mathrm{C} \mathrm{NMR}, \mathrm{CDCl}_{3}, 101 \mathrm{MHz}$

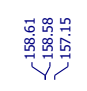

篙

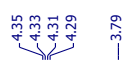

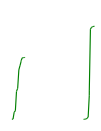


Compound Be

${ }^{1} \mathrm{H}$ NMR, DMSO, $400 \mathrm{MHz}$

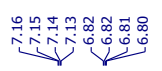

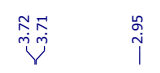

$\stackrel{\vec{p}}{\vec{p}}$
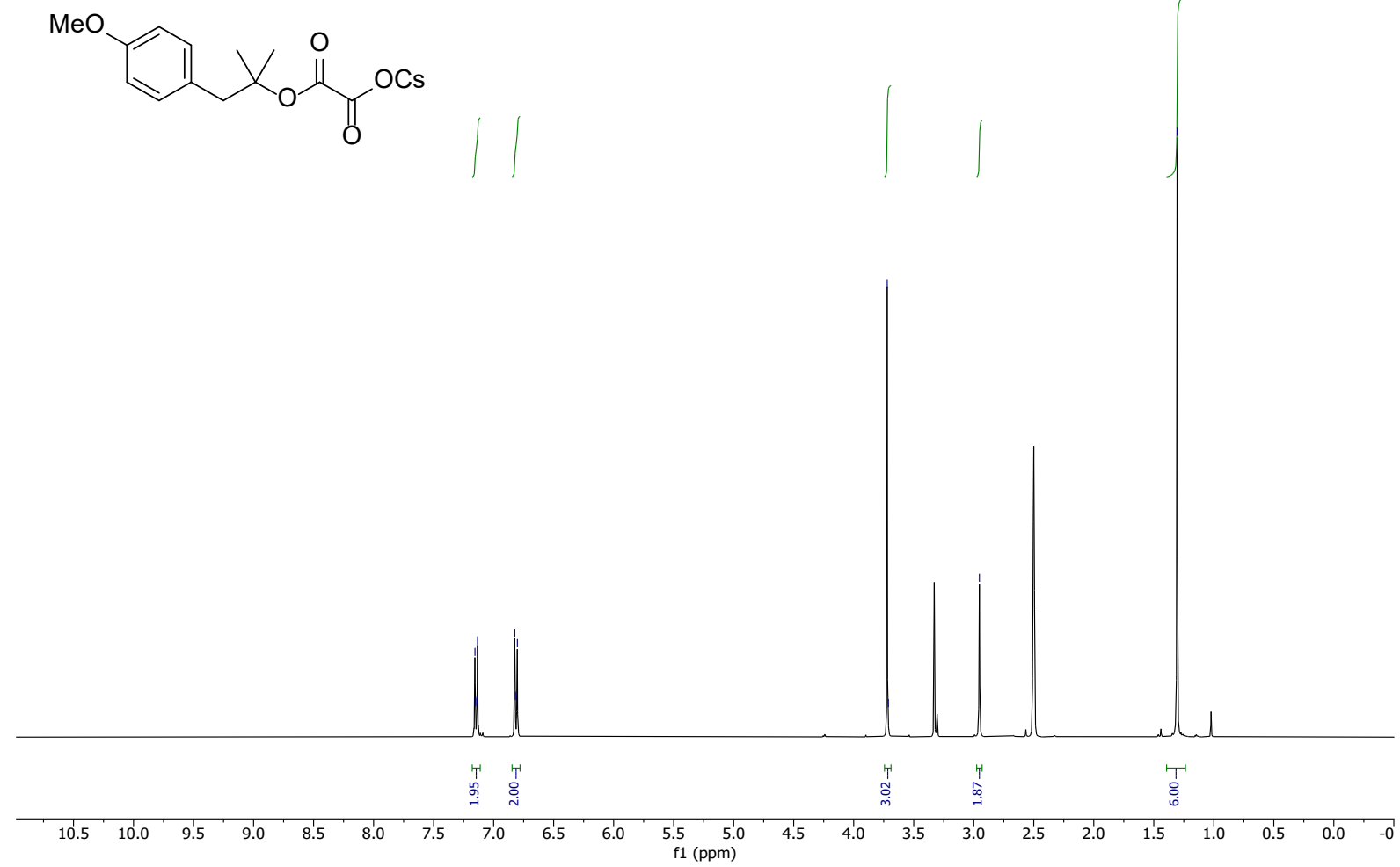

${ }^{13} \mathrm{C}$ NMR, DMSO, $101 \mathrm{MHz}$

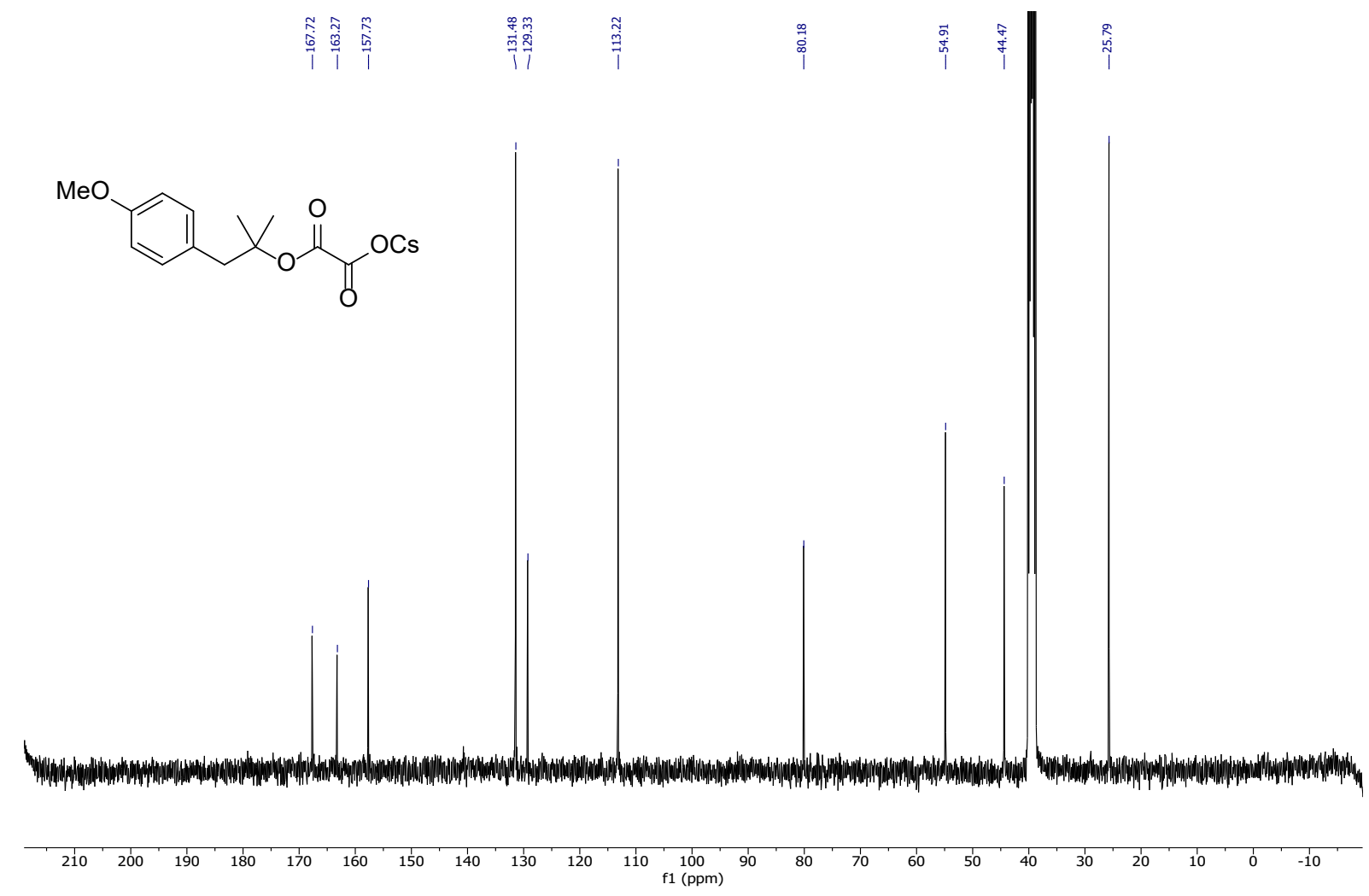

S73 
Compound 23f

${ }^{1} \mathrm{H} \mathrm{NMR}, \mathrm{CDCl}_{3}, 400 \mathrm{MHz}$

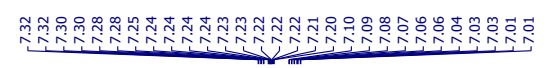
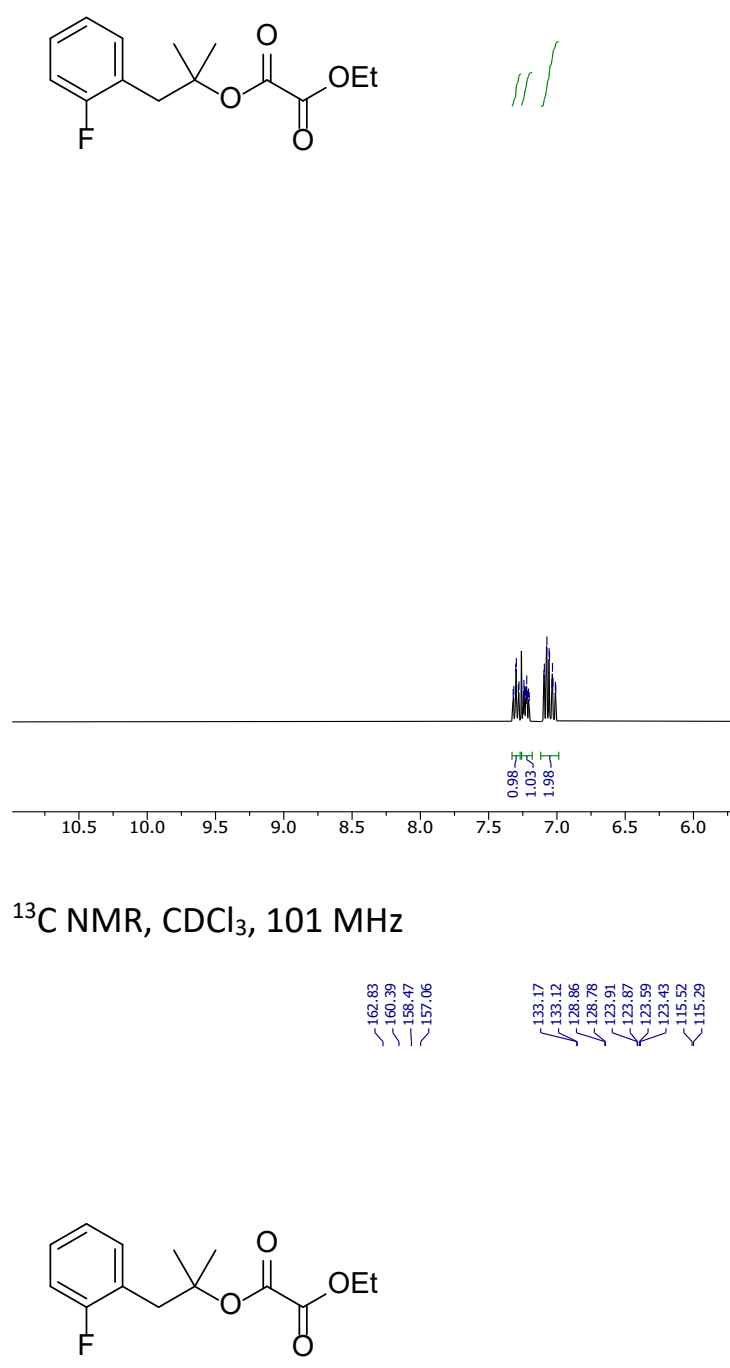

${ }^{13} \mathrm{CNMR}, \mathrm{CDCl}_{3}, 101 \mathrm{MHz}$
踶是是

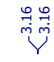
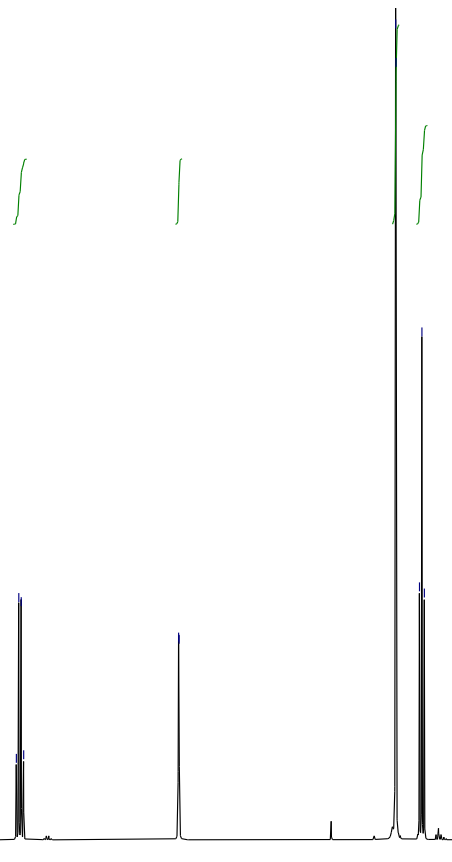

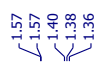
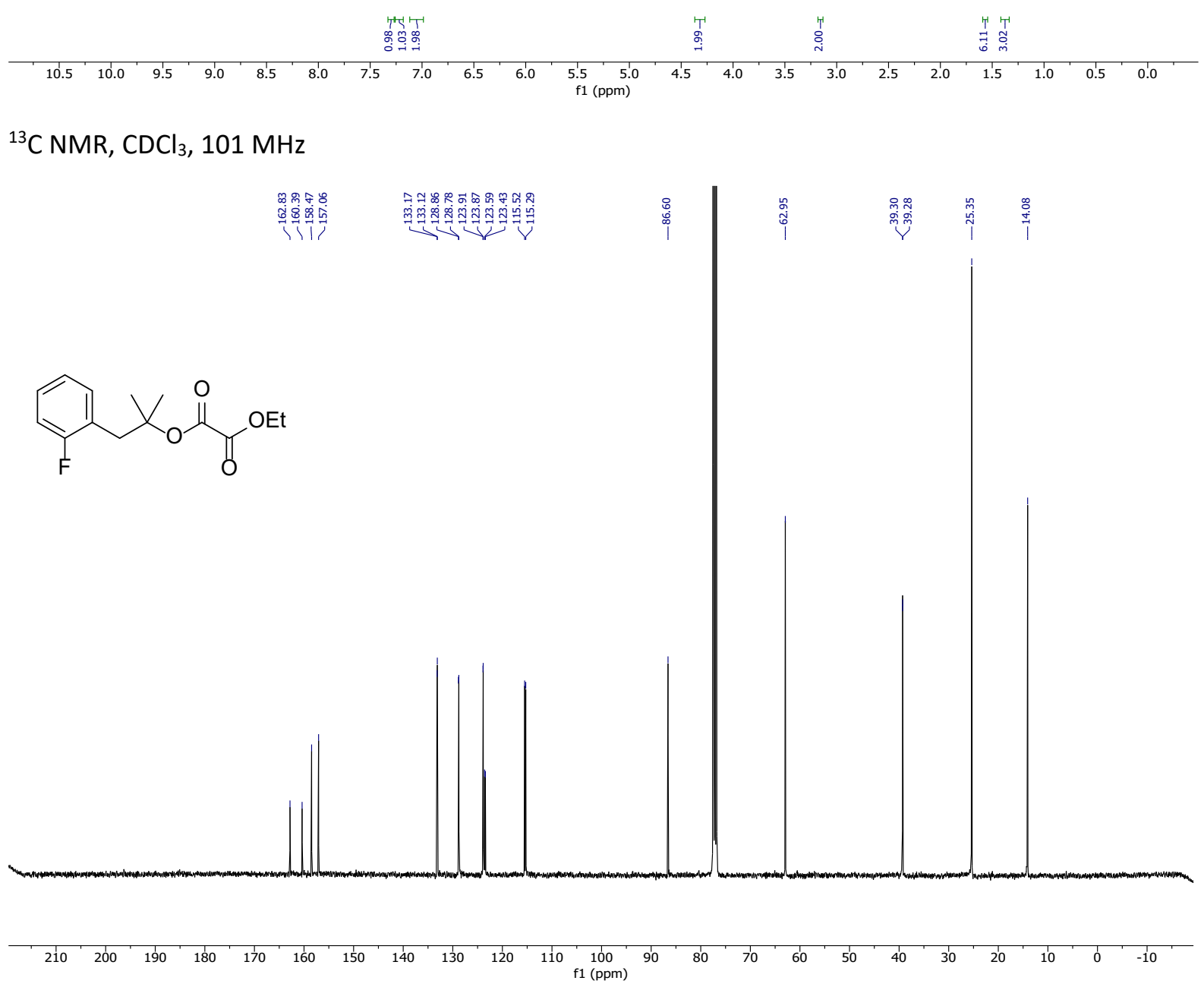

S74 
${ }^{19} \mathrm{~F} \mathrm{NMR}, \mathrm{CDCl}_{3}, 376 \mathrm{MHz}$

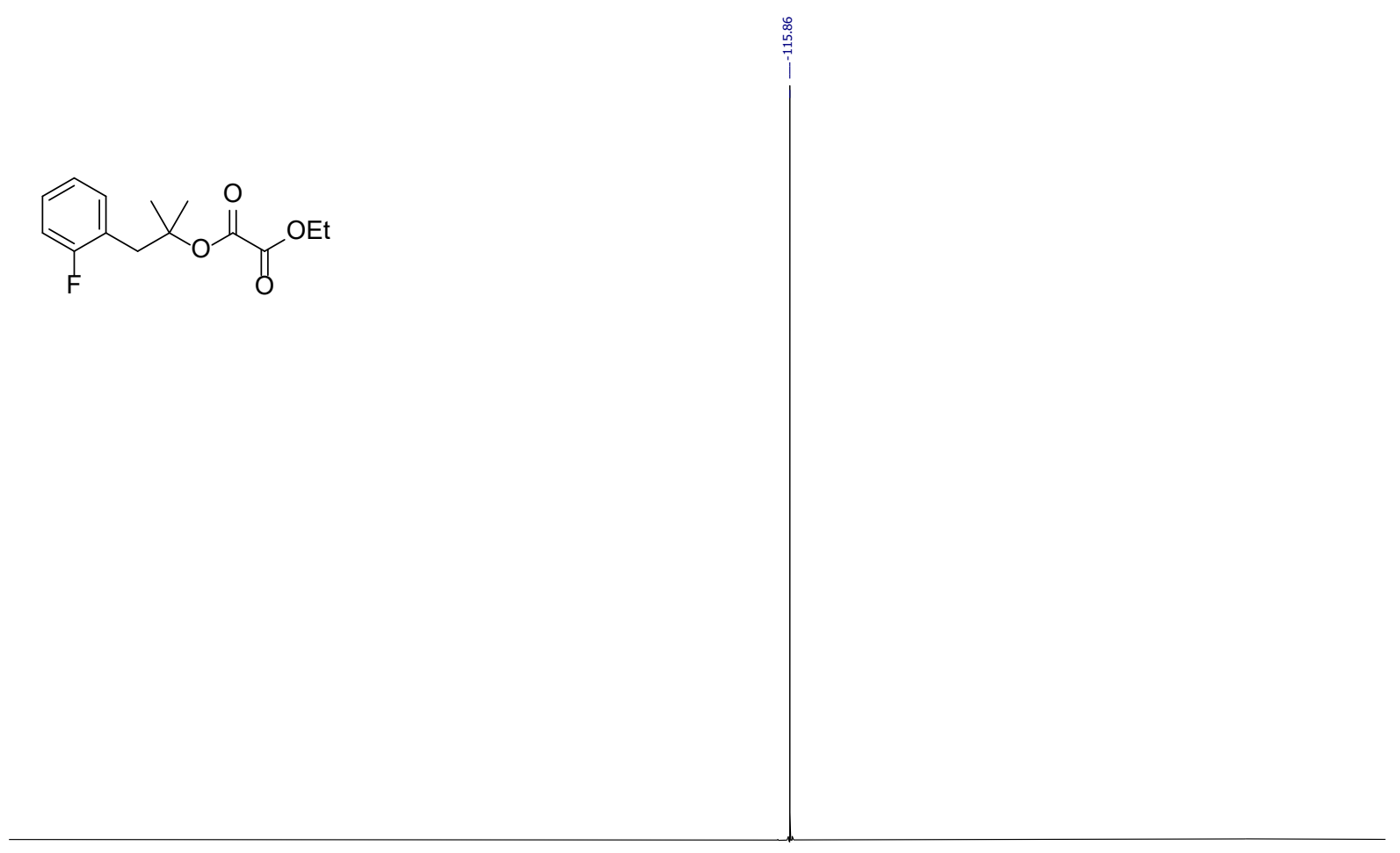

\begin{tabular}{llllllllllllllllllllllll}
\hline 10 & 0 & -10 & -20 & -30 & -40 & -50 & -60 & -70 & -80 & -90 & -100 & -110 & -120 & -130 & -140 & -150 & -160 & -170 & -180 & -190 & -200 & -210 & 1
\end{tabular} 


\section{Compound $\mathbf{3 f}$}

${ }^{1} \mathrm{H}$ NMR, DMSO, $400 \mathrm{MHz}$

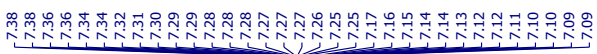

$\overbrace{\mathrm{F}} \mathrm{X}_{\mathrm{O}}^{\mathrm{O}} \mathrm{OCs}$

H.

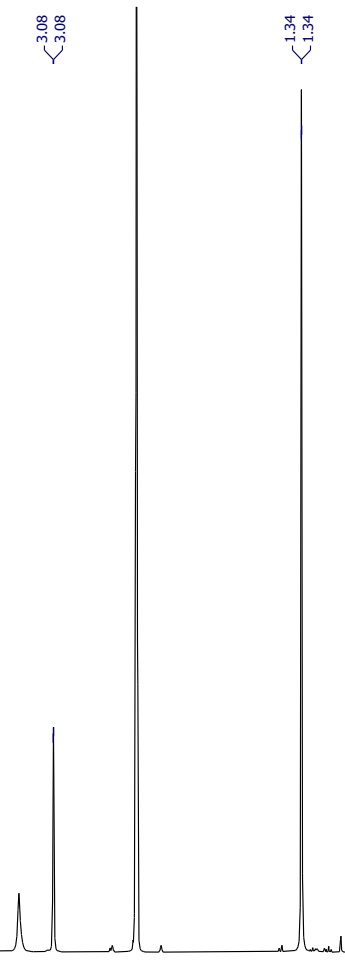

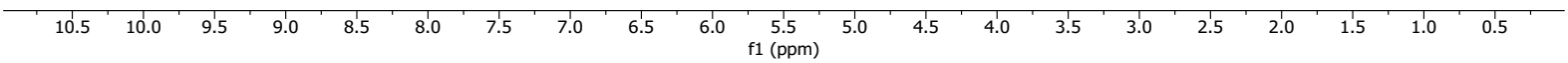

${ }^{13} \mathrm{C}$ NMR, DMSO, $101 \mathrm{MHz}$

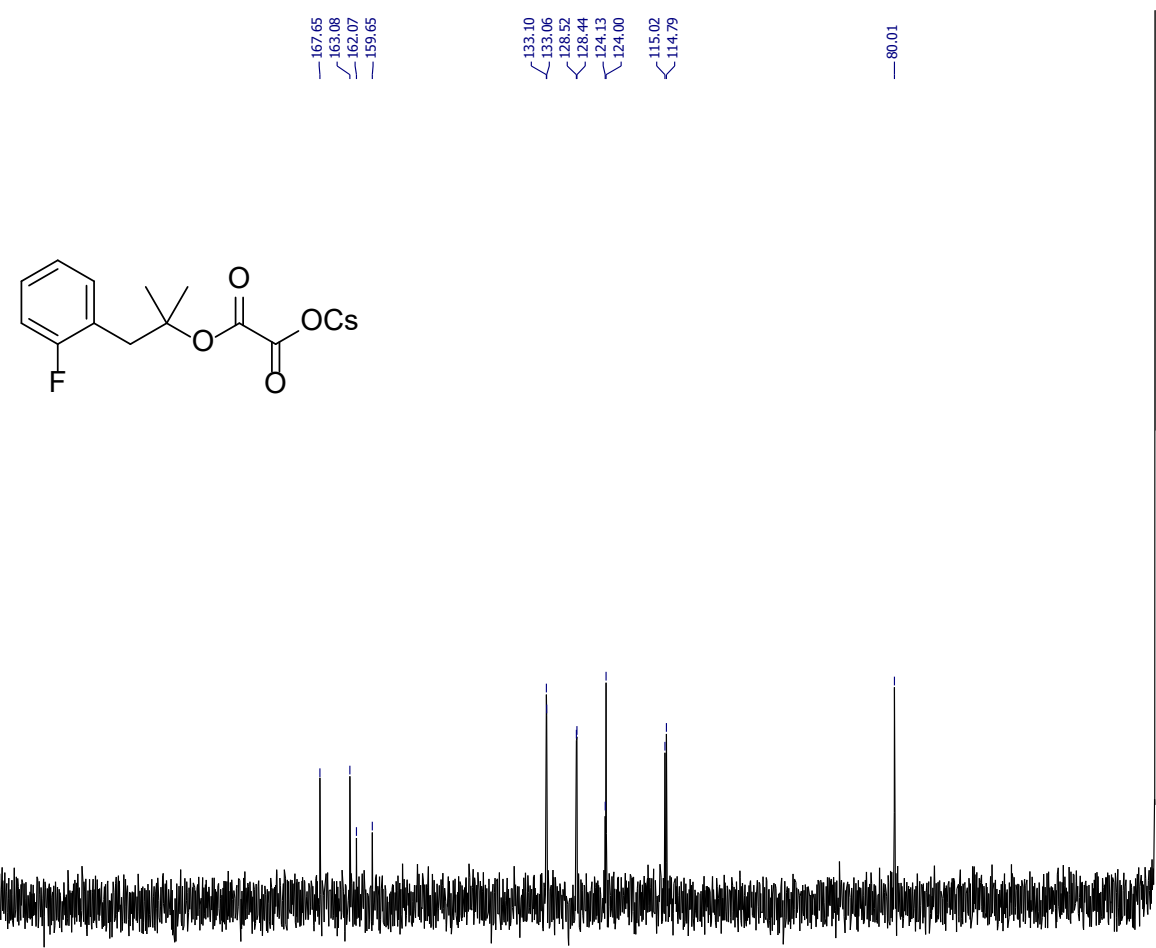


${ }^{19} \mathrm{~F}$ NMR, DMSO, $376 \mathrm{MHz}$<smiles>CCOC(=O)C(=O)OC(C)(C)Cc1ccccc1F</smiles>

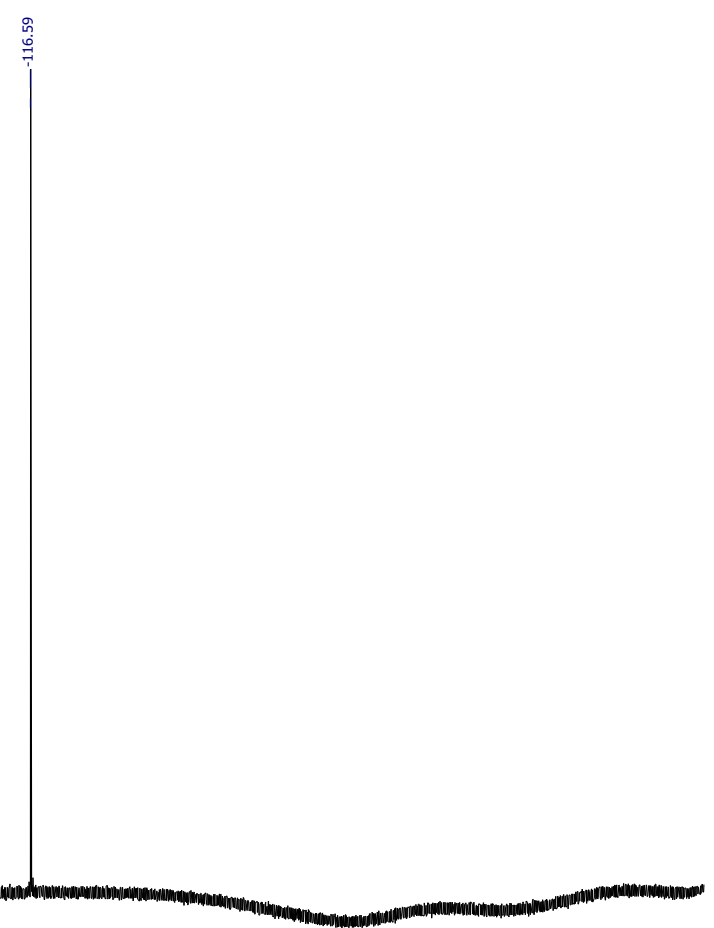

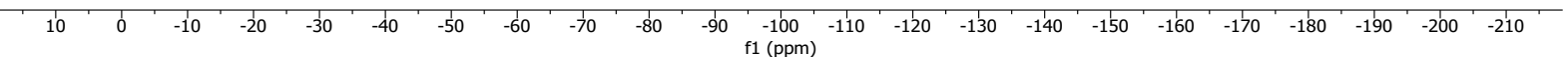


Compound 23g

${ }^{1} \mathrm{H}$ NMR, $\mathrm{CDCl}_{3}, 400 \mathrm{MHz}$

影影责

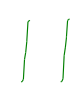

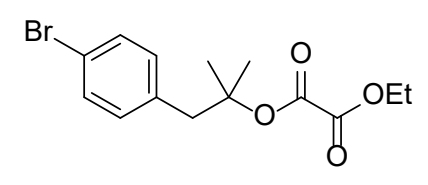

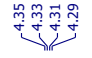

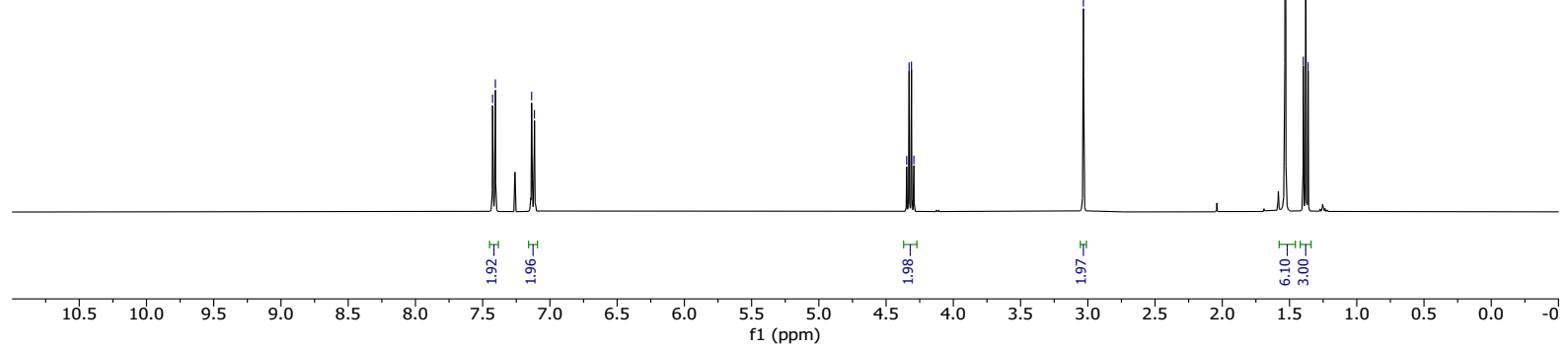

${ }^{13} \mathrm{C} \mathrm{NMR}, \mathrm{CDCl}_{3}, 101 \mathrm{MHz}$

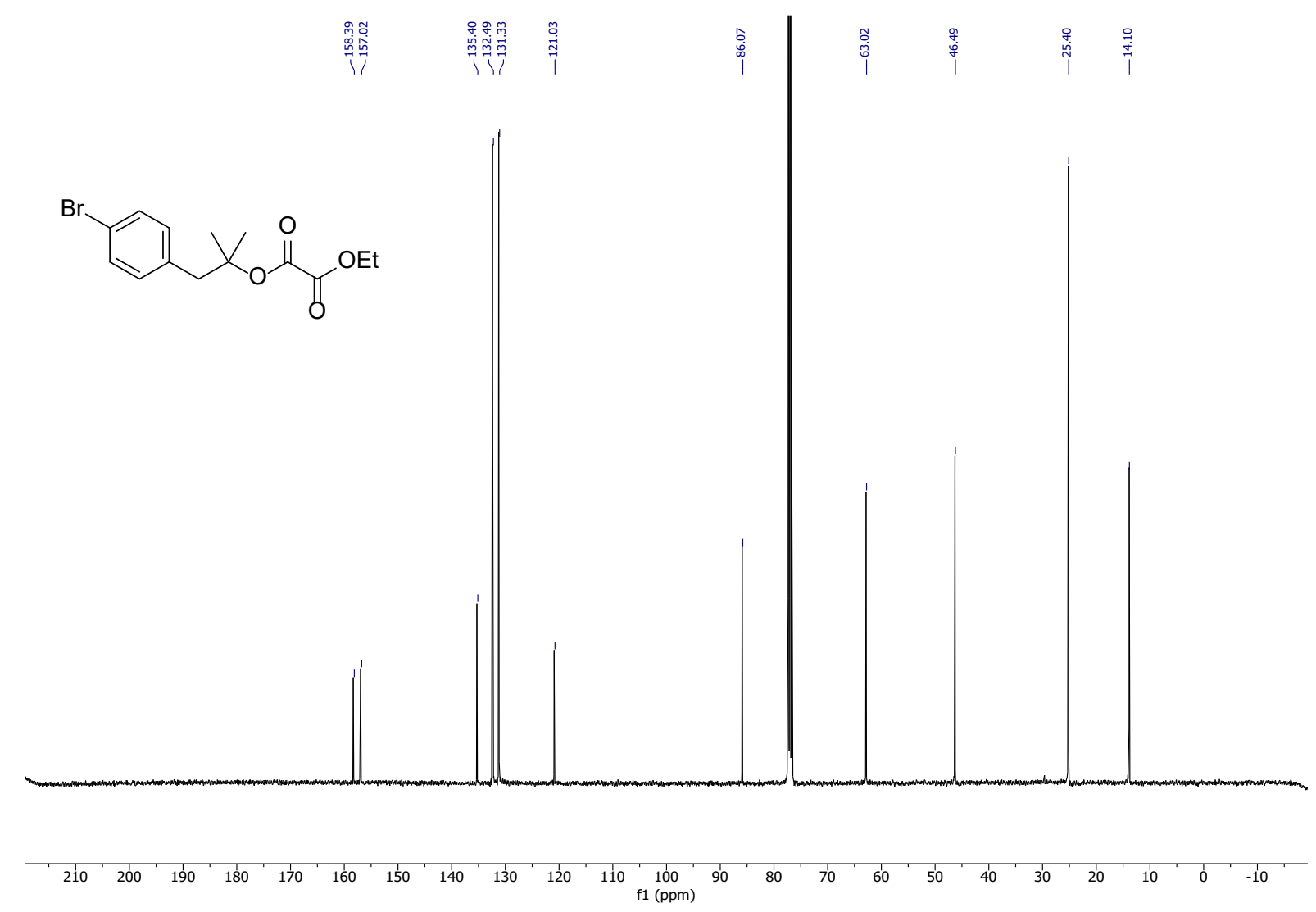

S78 
Compound Jg

${ }^{1} \mathrm{H}$ NMR, DMSO, $400 \mathrm{MHz}$

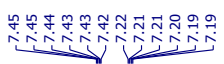

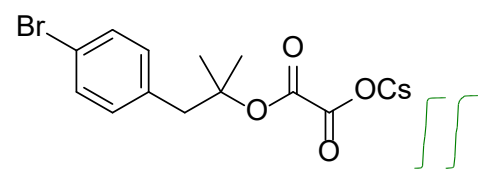

$\stackrel{\overrightarrow{0}}{i}$

羿
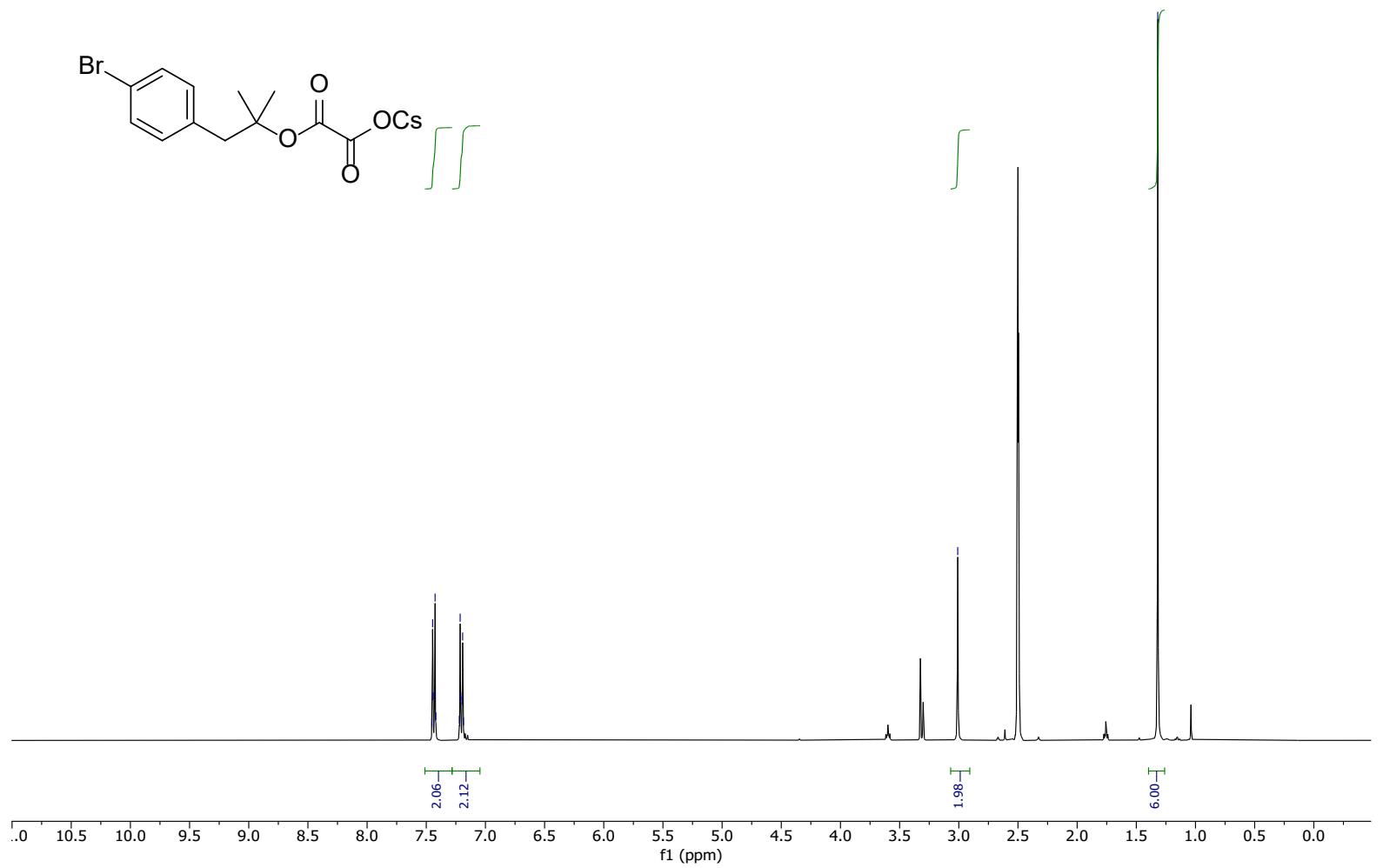

${ }^{13} \mathrm{C}$ NMR, DMSO, $101 \mathrm{MHz}$

部

斟
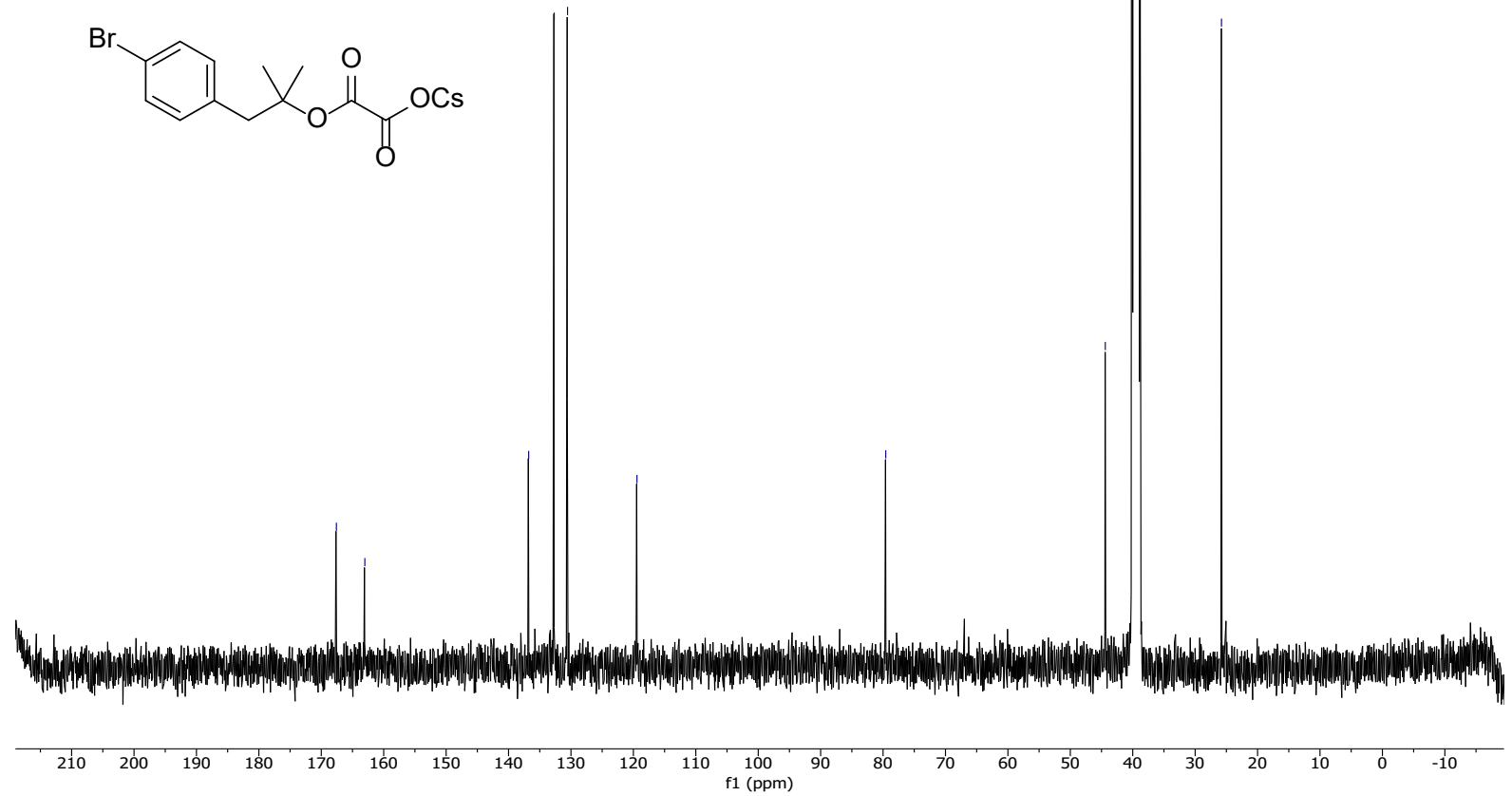

S79 


\section{Compound 23h}

${ }^{1} \mathrm{H} \mathrm{NMR}, \mathrm{CDCl}_{3}, 400 \mathrm{MHz}$

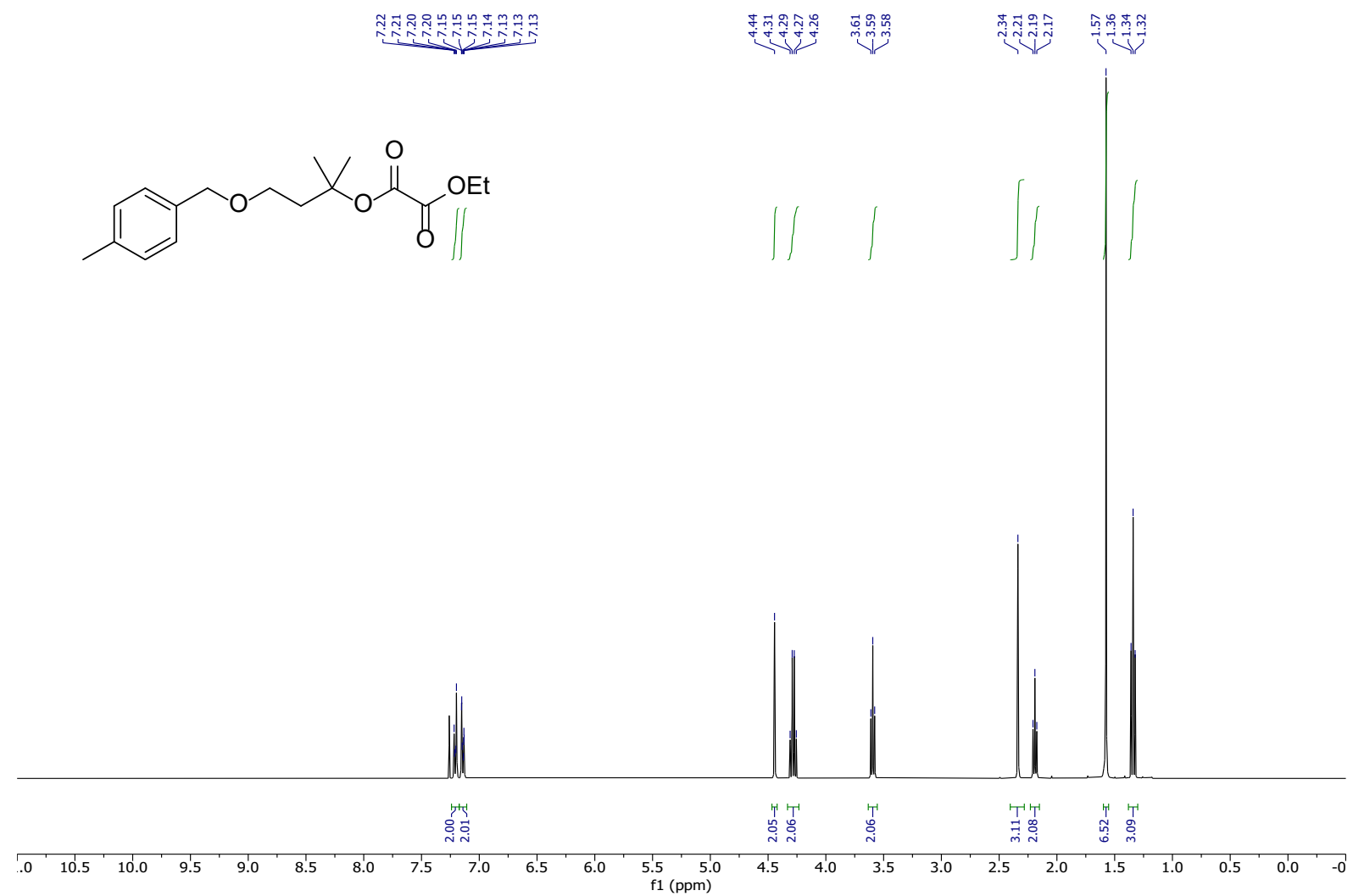

${ }^{13} \mathrm{CNMR}, \mathrm{CDCl}_{3}, 101 \mathrm{MHz}$

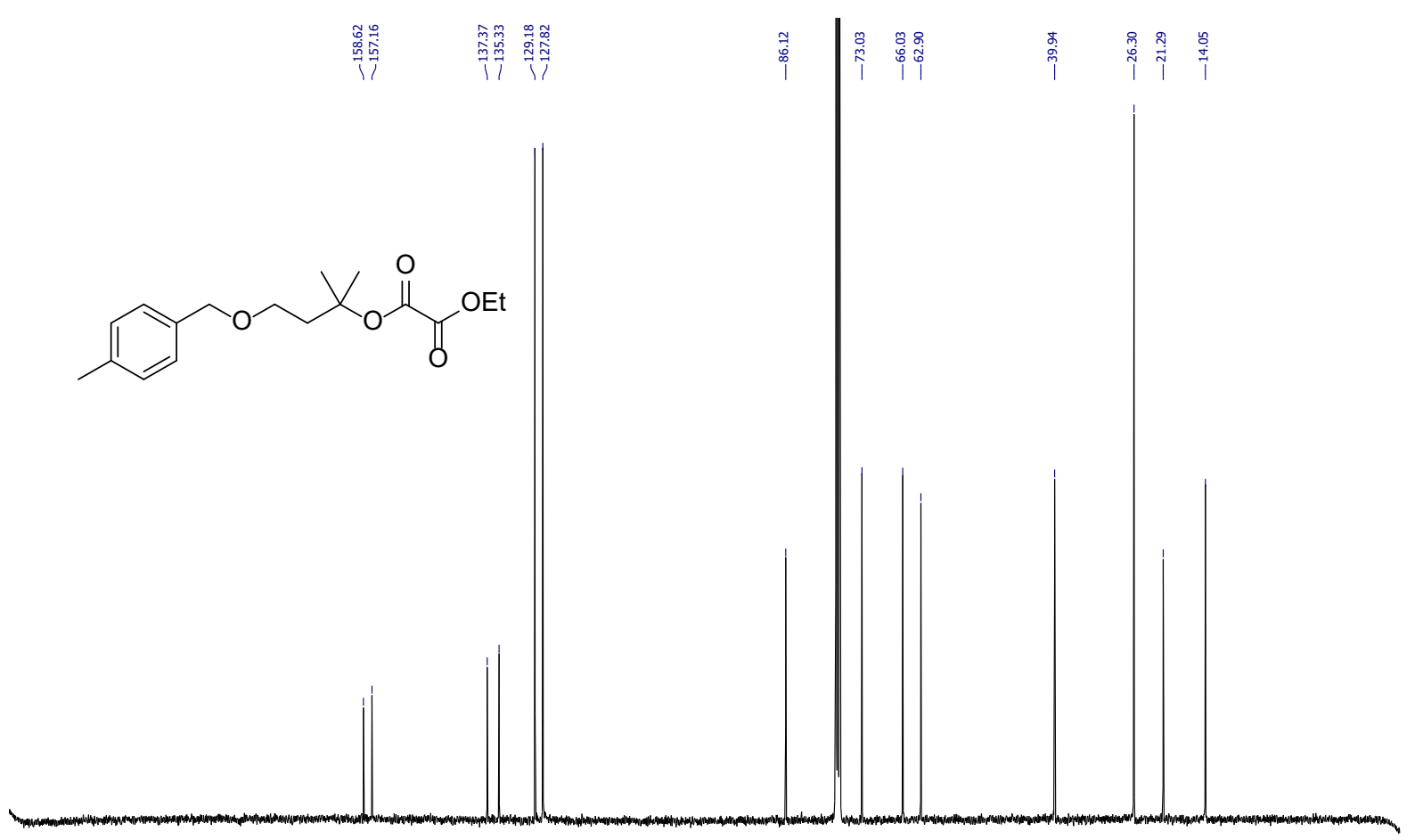

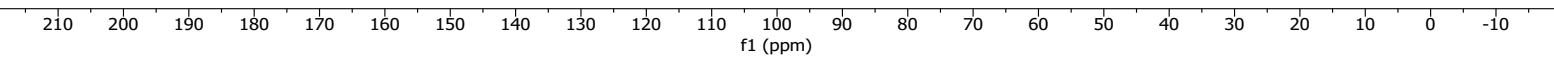




\section{Compound $3 \mathrm{~h}$}

${ }^{1} \mathrm{H}$ NMR, DMSO, $400 \mathrm{MHz}$

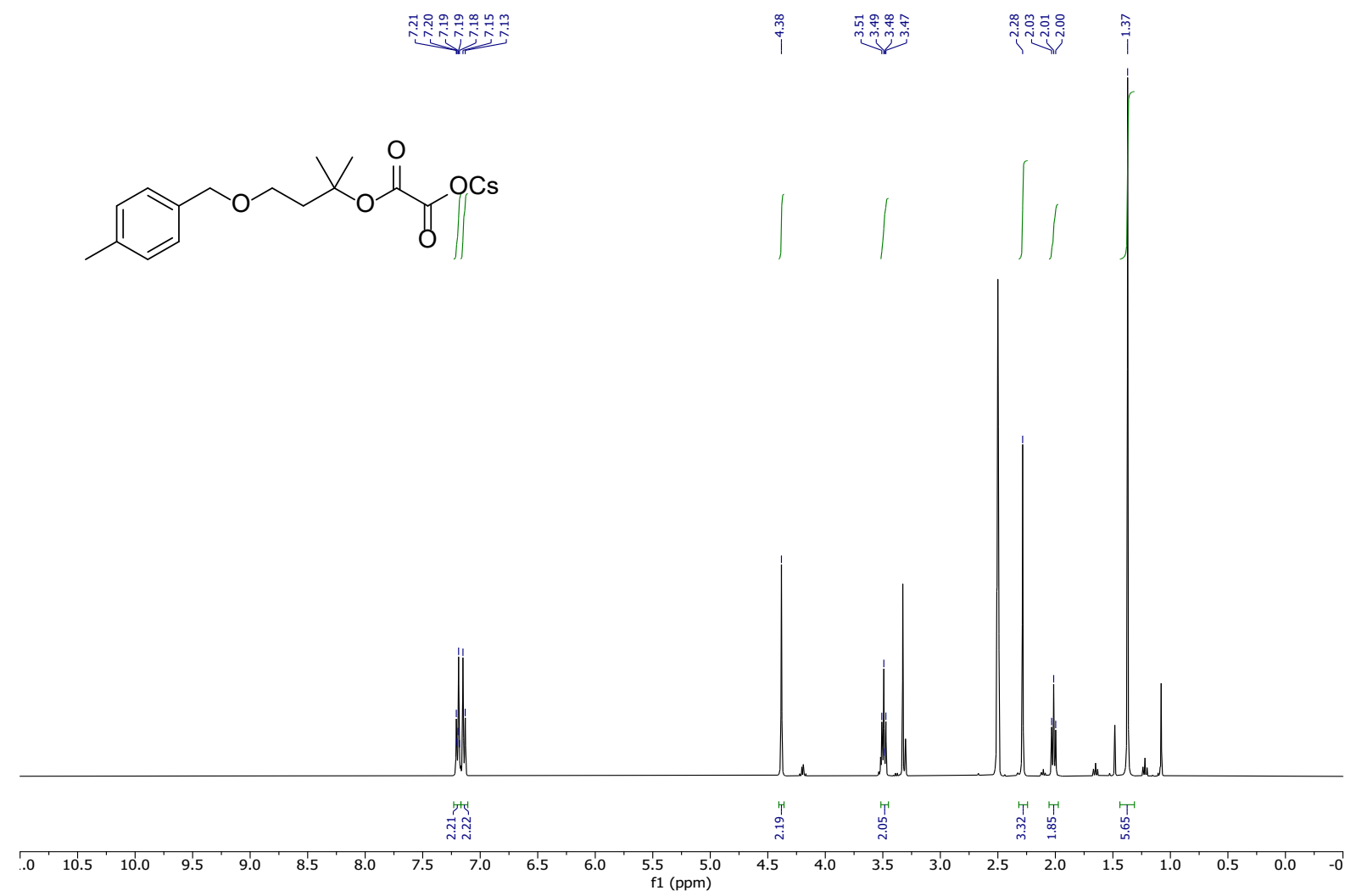

${ }^{13} \mathrm{C}$ NMR, DMSO, $101 \mathrm{MHz}$

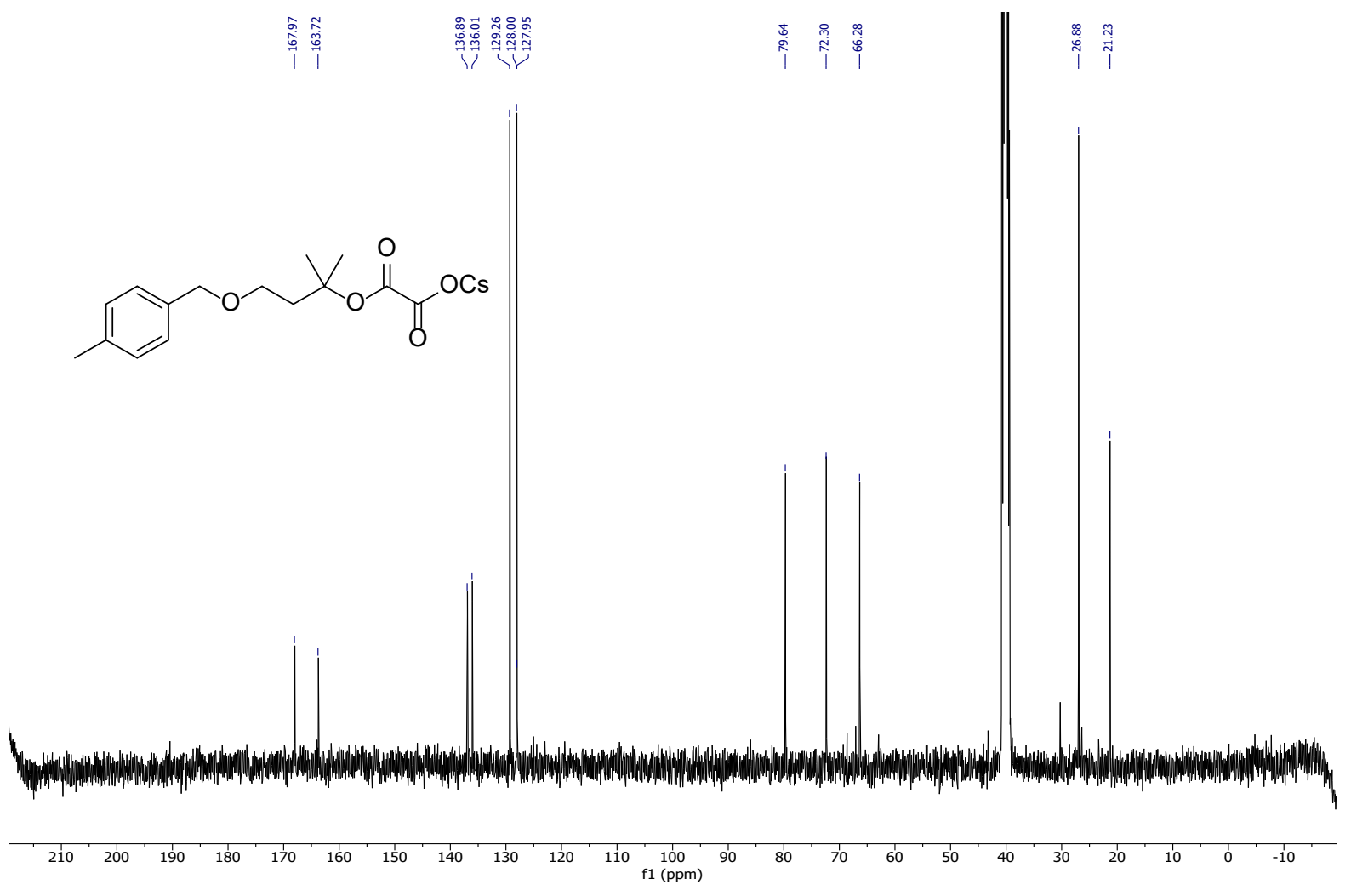




\section{Compound 23i}

${ }^{1} \mathrm{H} \mathrm{NMR}, \mathrm{CDCl}_{3}, 400 \mathrm{MHz}$

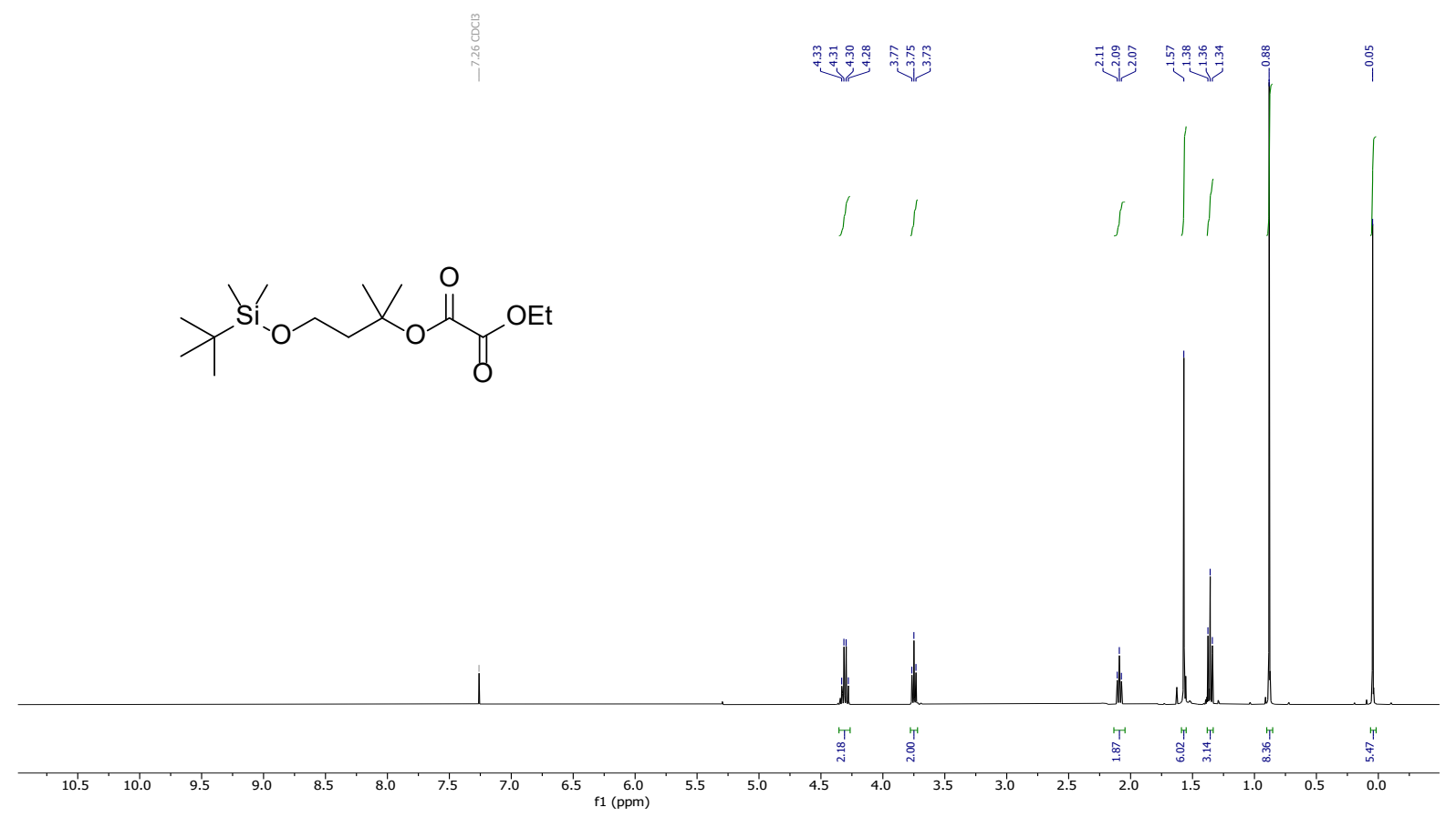

${ }^{13} \mathrm{C} \mathrm{NMR}, \mathrm{CDCl}_{3}, 101 \mathrm{MHz}$
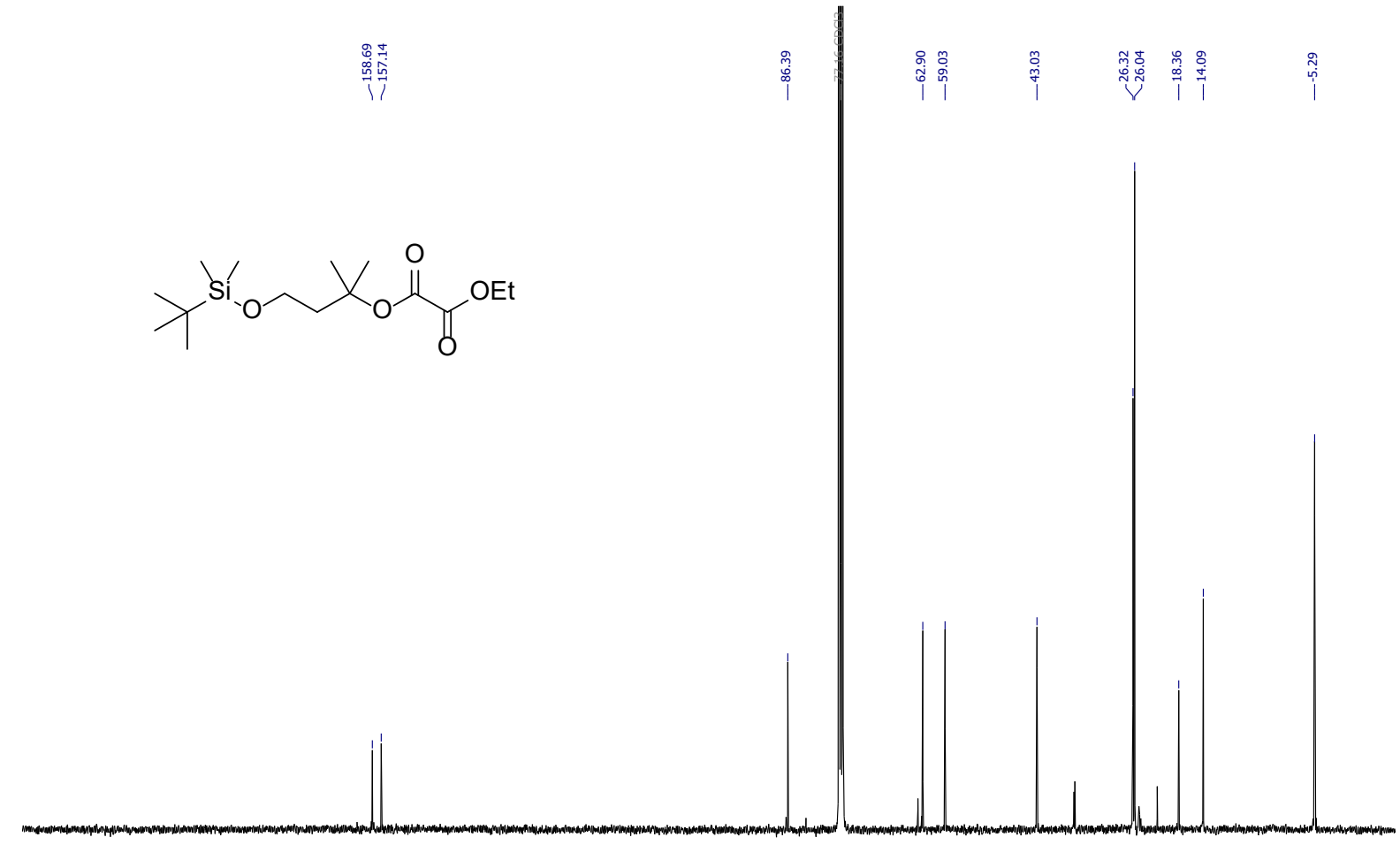

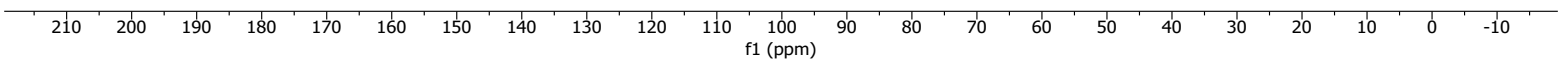




\section{Compound $\mathbf{3 i}$}

${ }^{1} \mathrm{H}$ NMR, DMSO, $400 \mathrm{MHz}$<smiles>CC(C)(CCO[Si](C)(C)C(C)(C)C)OC(=O)C(=O)O[Na]</smiles>

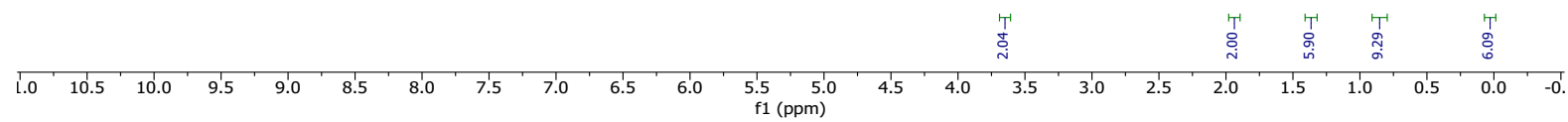

${ }^{13} \mathrm{C}$ NMR, DMSO, $101 \mathrm{MHz}$

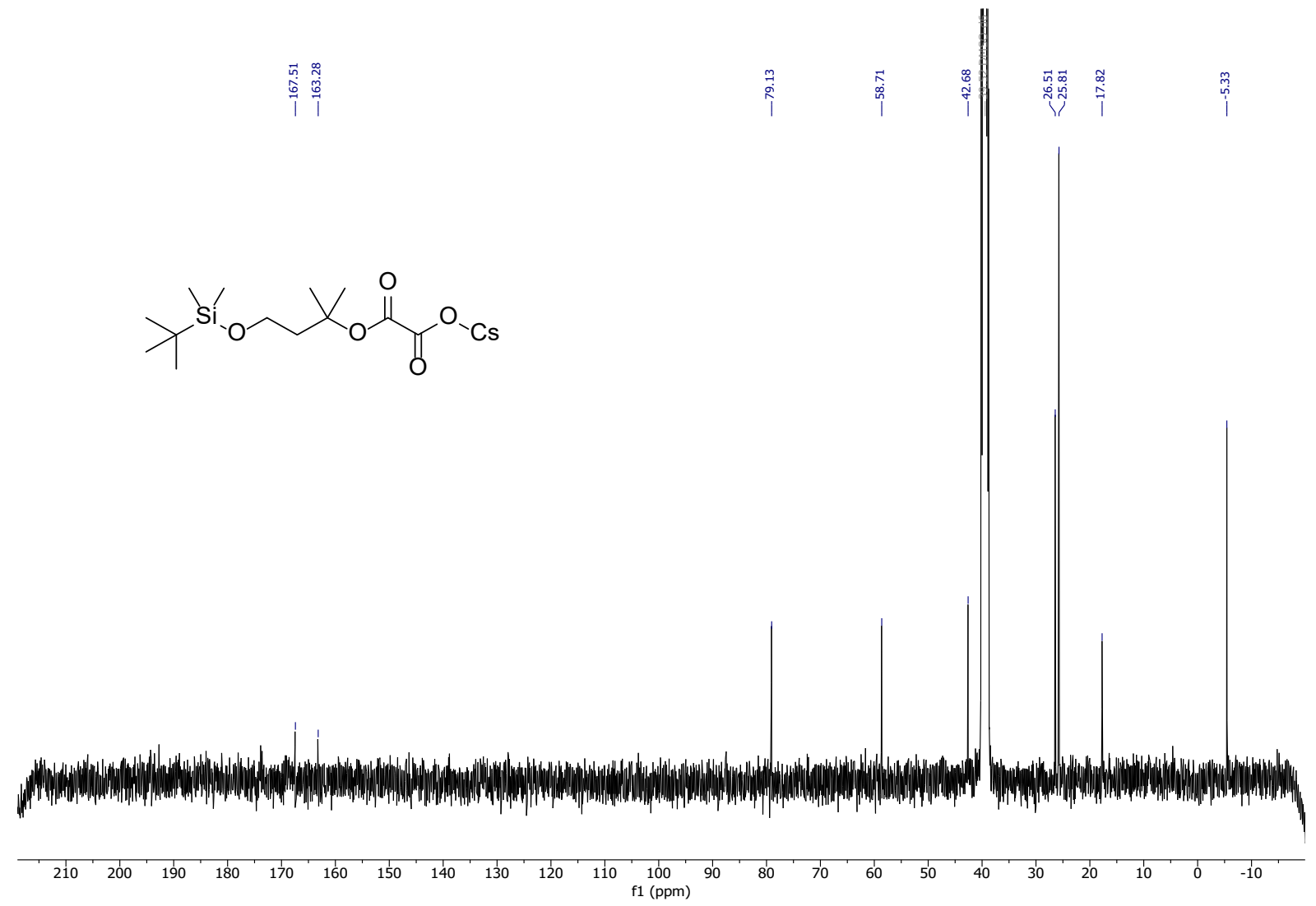




\section{Compound 23j}

${ }^{1} \mathrm{H} \mathrm{NMR}, \mathrm{CDCl}_{3}, 400 \mathrm{MHz}$

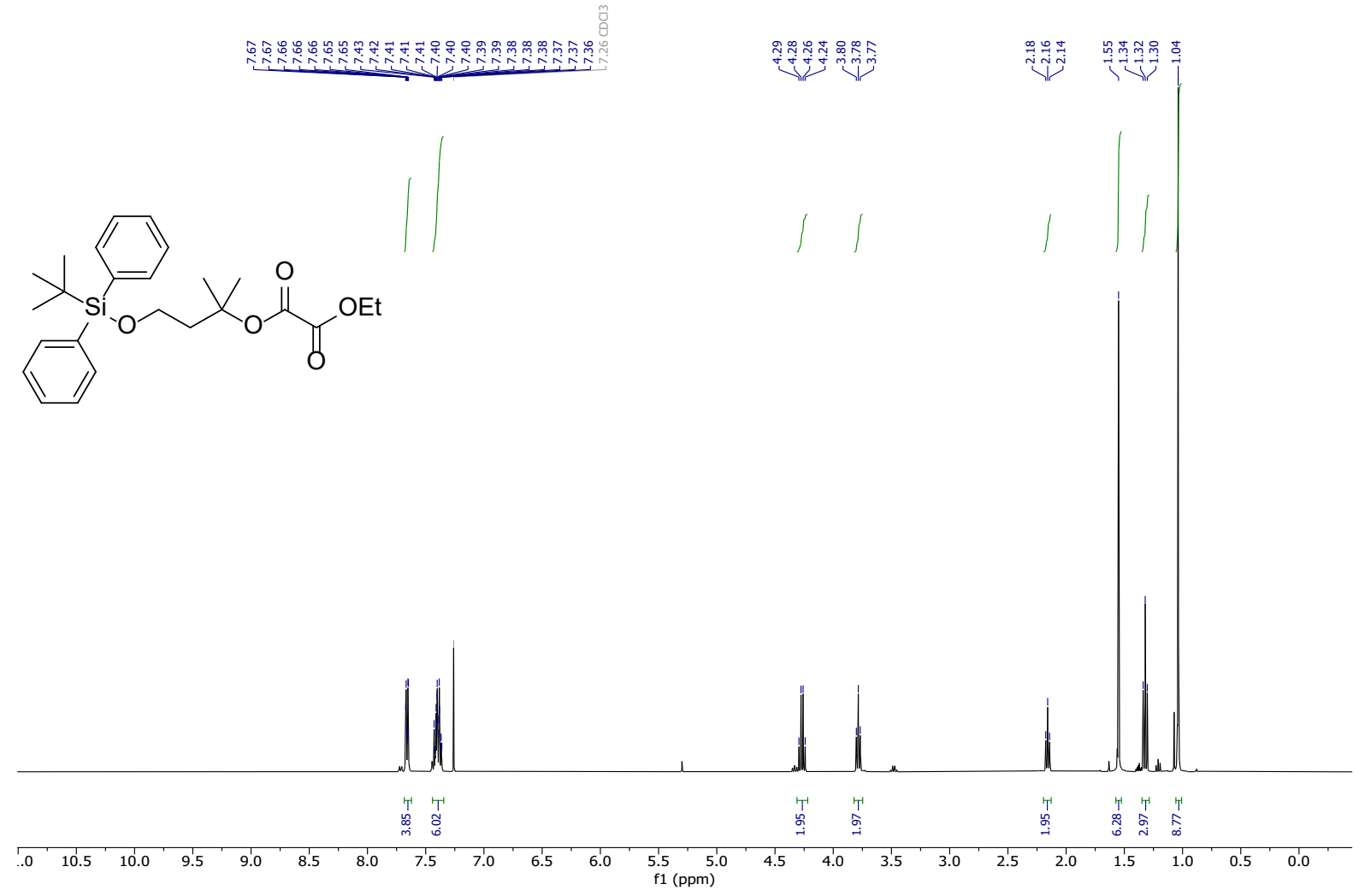

${ }^{13} \mathrm{C} \mathrm{NMR}, \mathrm{CDCl}_{3}, 101 \mathrm{MHz}$

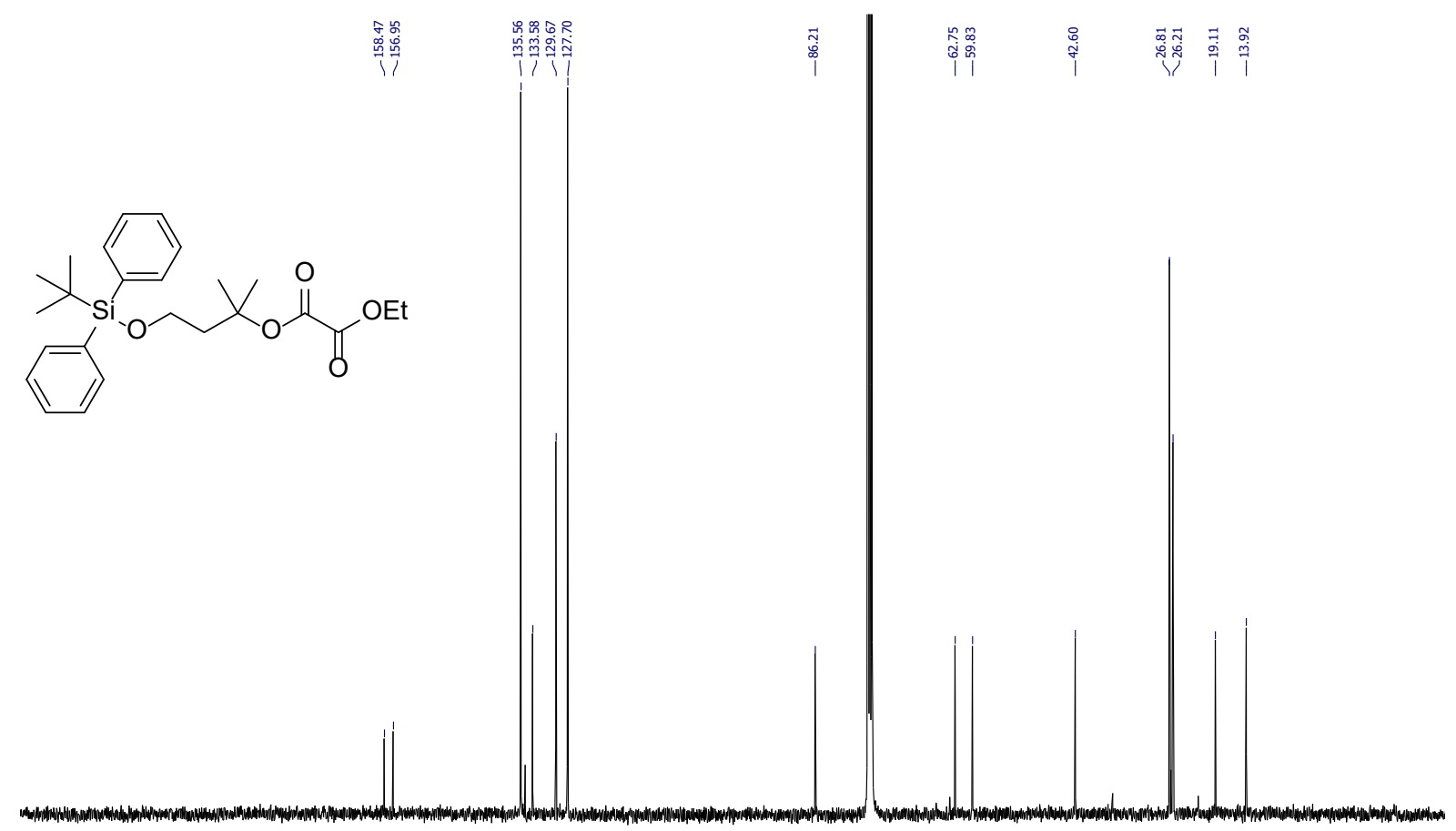

$\begin{array}{llllllllllllllllllllllllllllllll}210 & 200 & 190 & 180 & 170 & 160 & 150 & 140 & 130 & 120 & 110 & 100 & 90 & 80 & 70 & 60 & 50 & 40 & 30 & 20 & 10 & 0 & -10\end{array}$ 


\section{Compound 3j}

${ }^{1} \mathrm{H}$ NMR, DMSO, $400 \mathrm{MHz}$

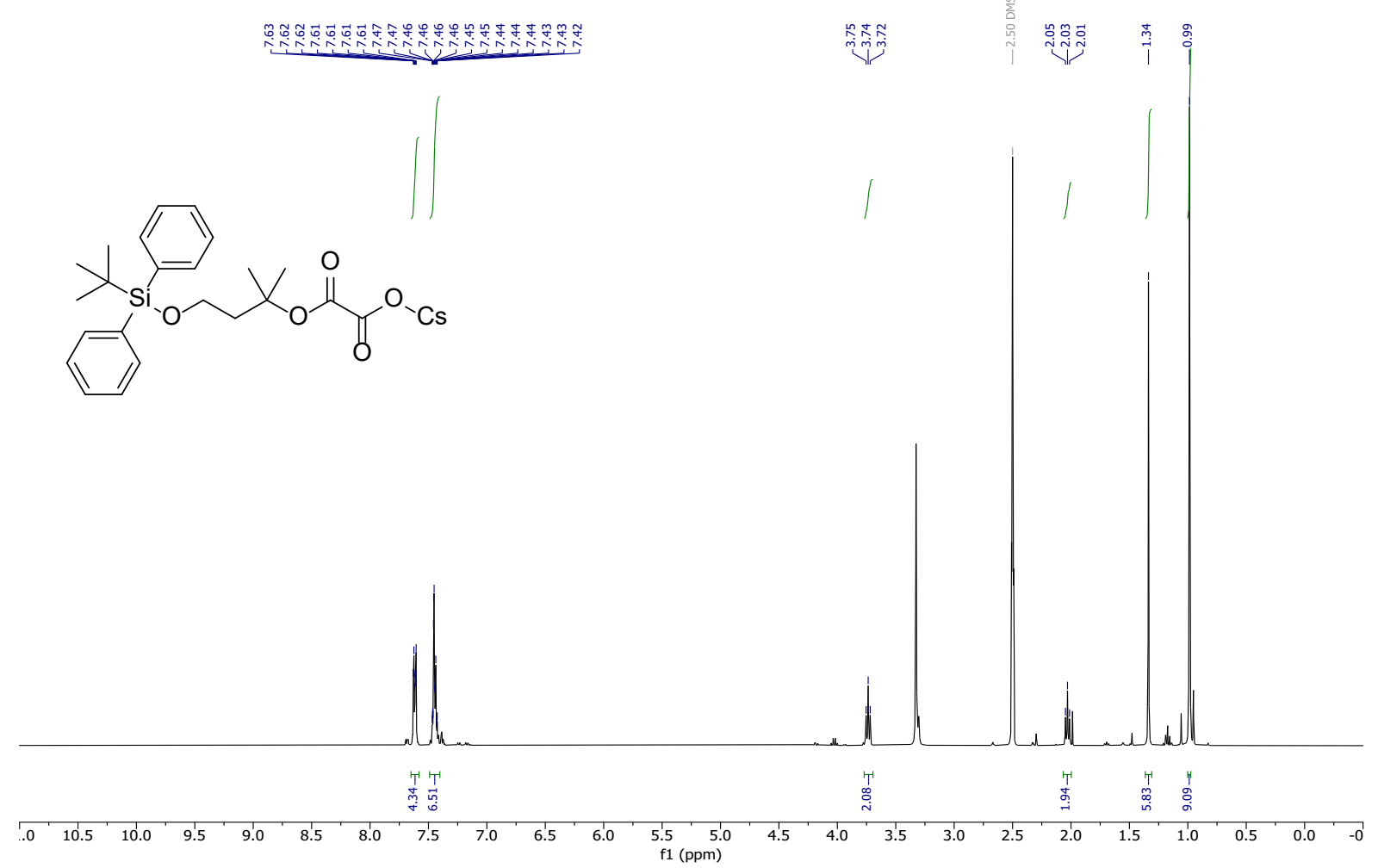

${ }^{13} \mathrm{C}$ NMR, DMSO, $101 \mathrm{MHz}$

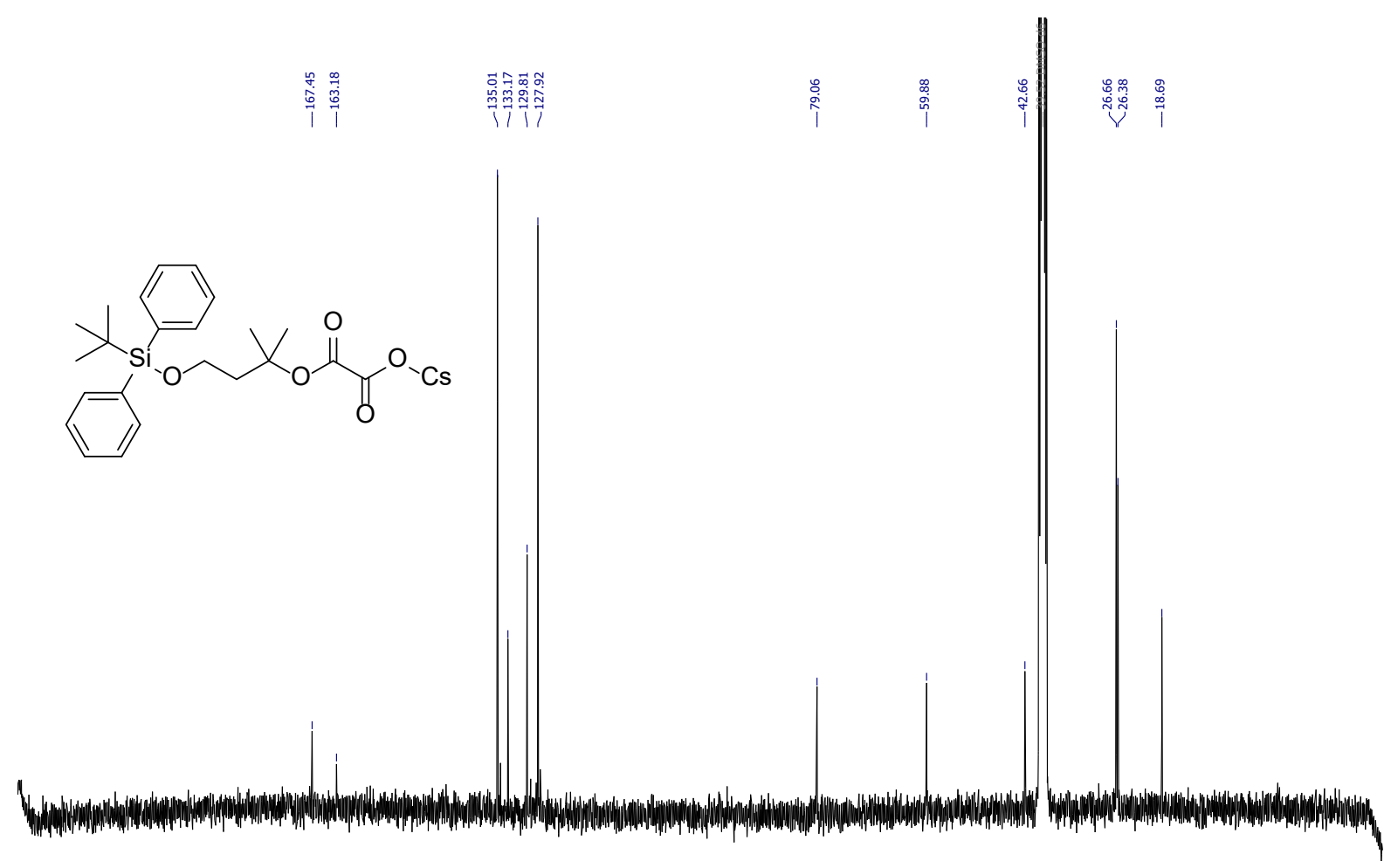

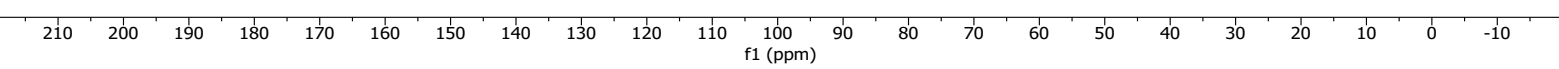


Compound 23k

${ }^{1} \mathrm{H} \mathrm{NMR}, \mathrm{CDCl}_{3}, 400 \mathrm{MHz}$

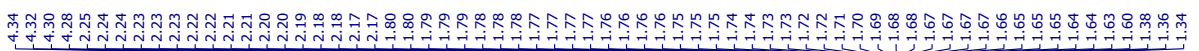
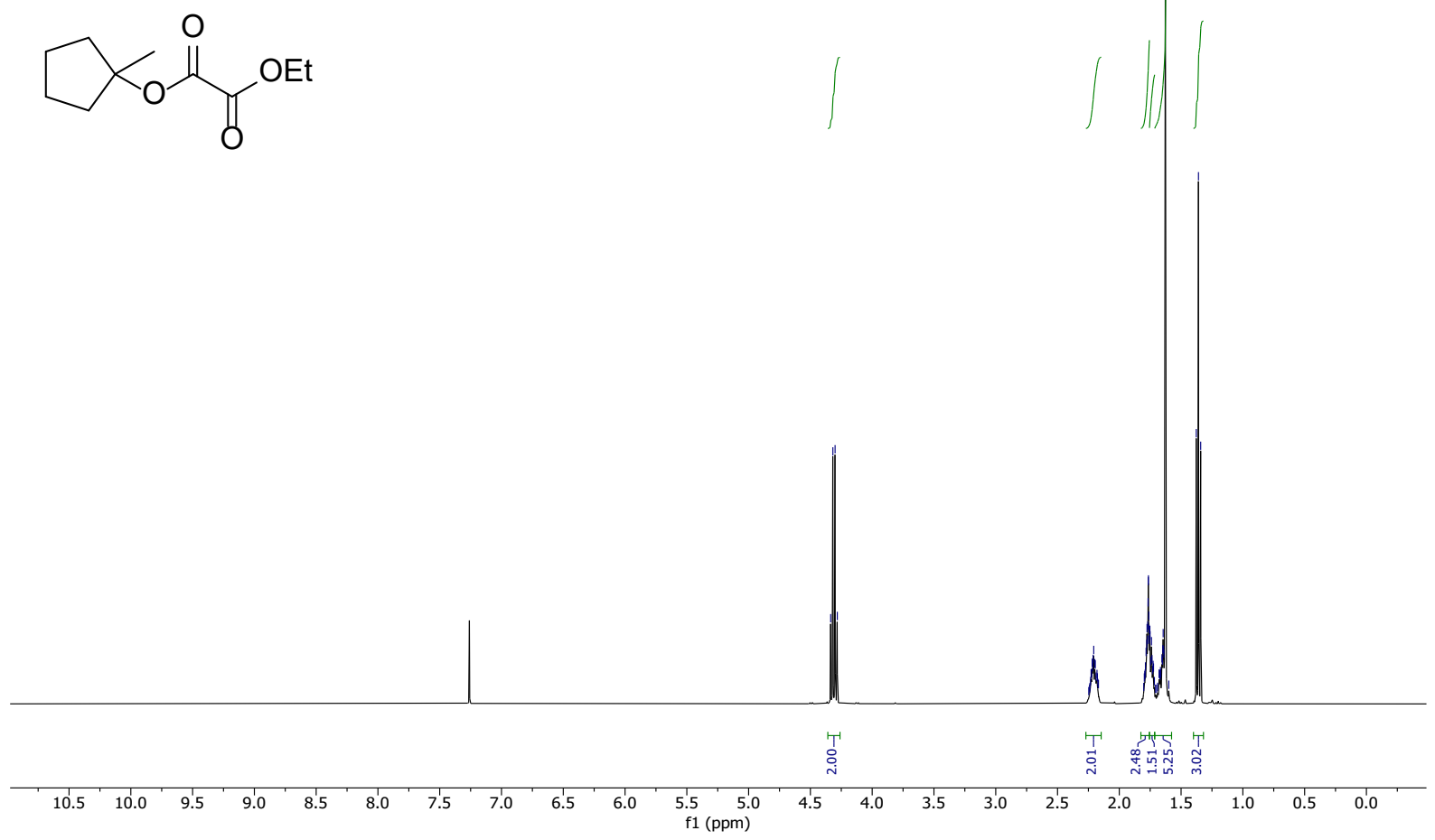

${ }^{13} \mathrm{CNMR}, \mathrm{CDCl}_{3}, 101 \mathrm{MHz}$

籍
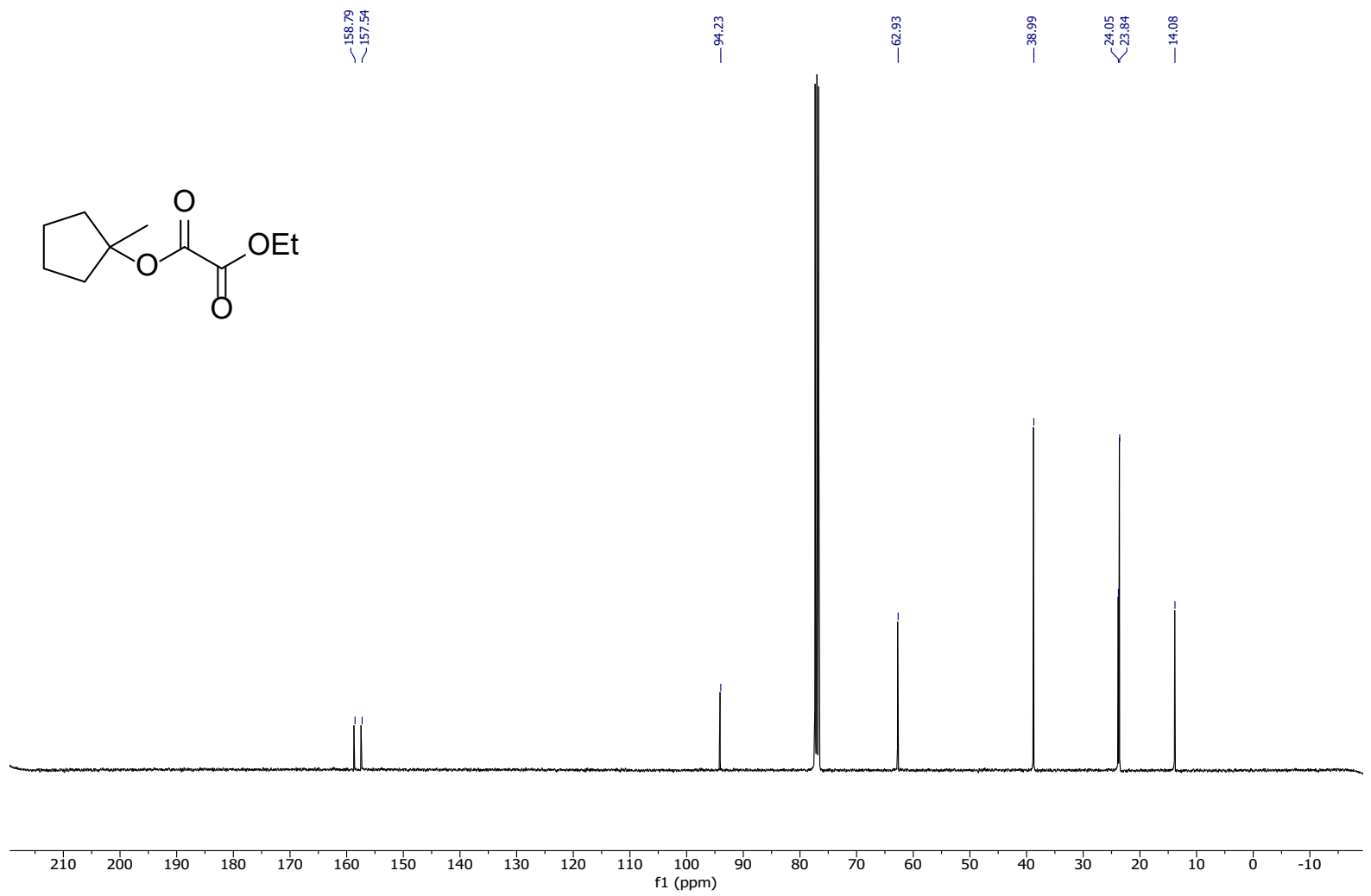

S86 


\section{Compound 3k}

${ }^{1} \mathrm{H}$ NMR, DMSO, $400 \mathrm{MHz}$

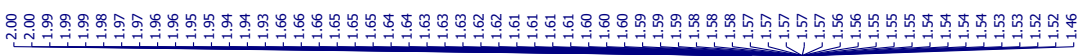

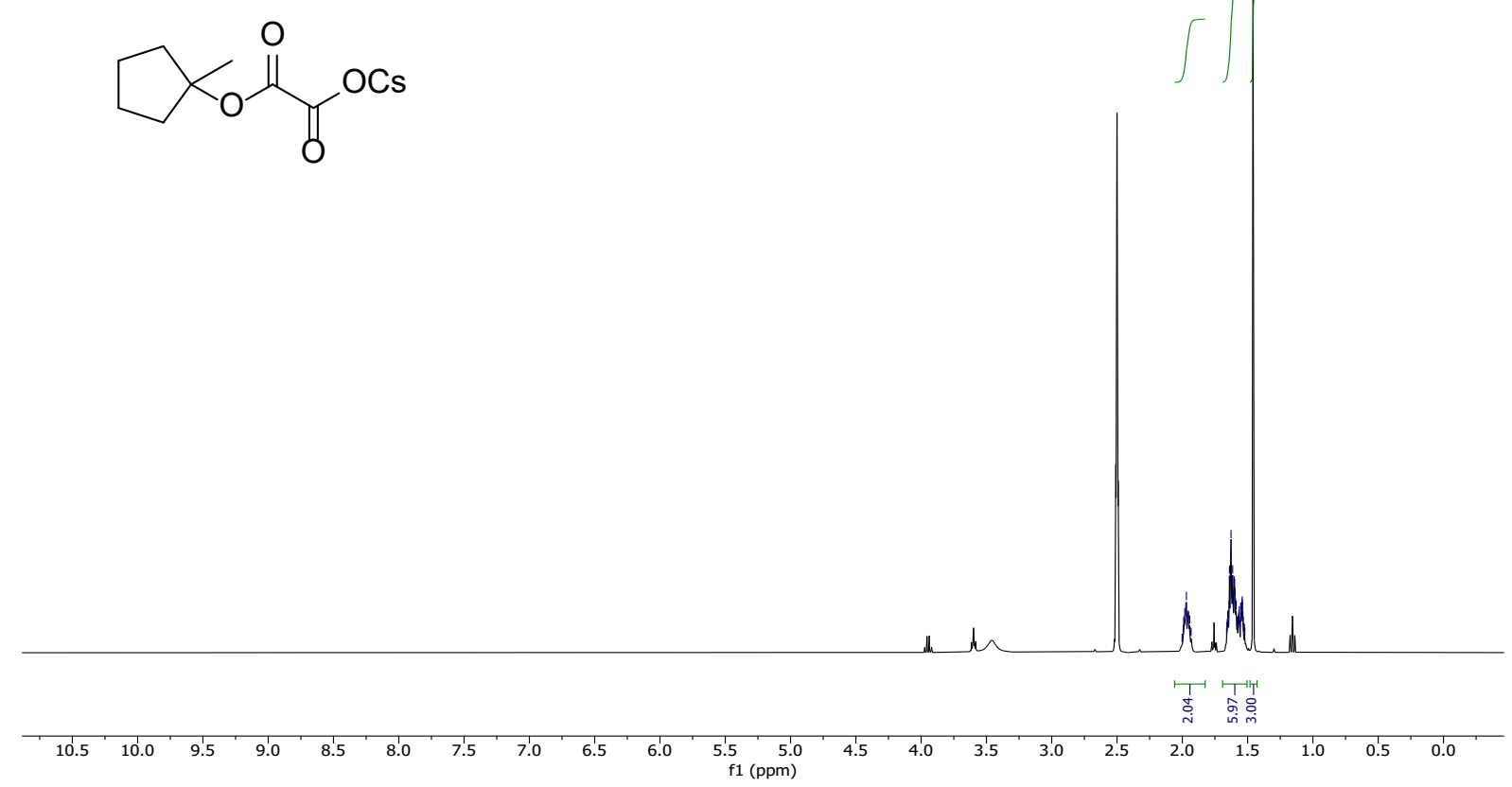

${ }^{13} \mathrm{C}$ NMR, DMSO, $101 \mathrm{MHz}$<smiles>CC1(OC(=O)C(=O)OCc2ccccc2)CCCC1</smiles>

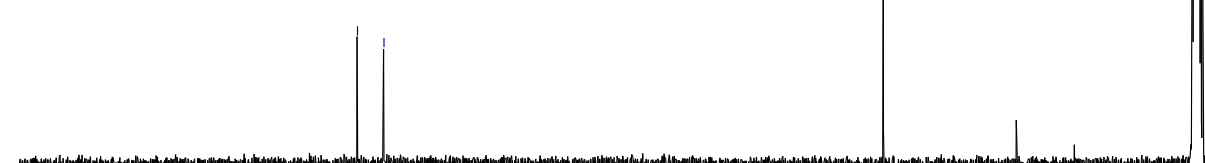




\section{Compound 23I}

${ }^{1} \mathrm{H} \mathrm{NMR}, \mathrm{CDCl}_{3}, 400 \mathrm{MHz}$

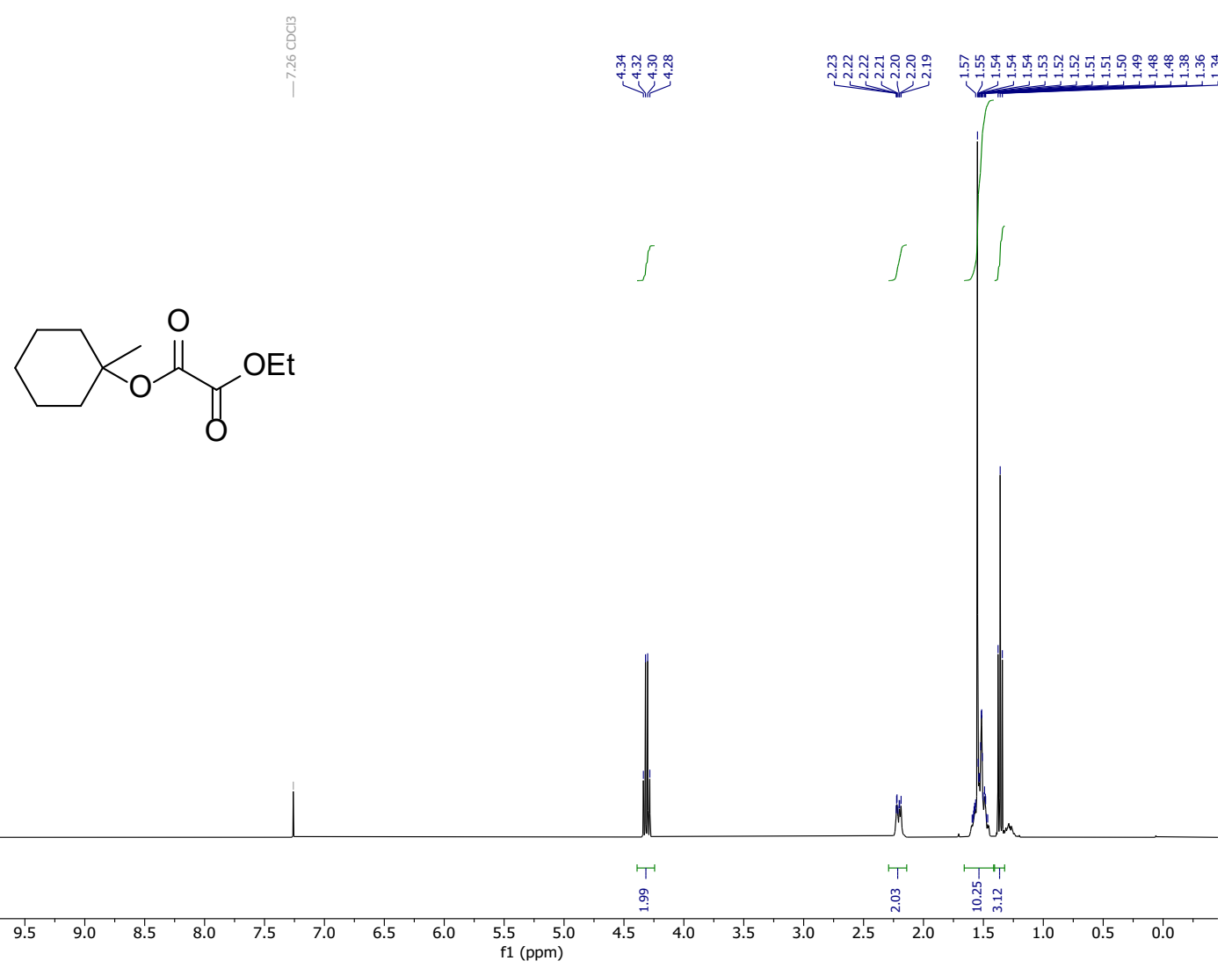

${ }^{13} \mathrm{CNMR}_{\mathrm{N}} \mathrm{CDCl}_{3}, 101 \mathrm{MHz}$

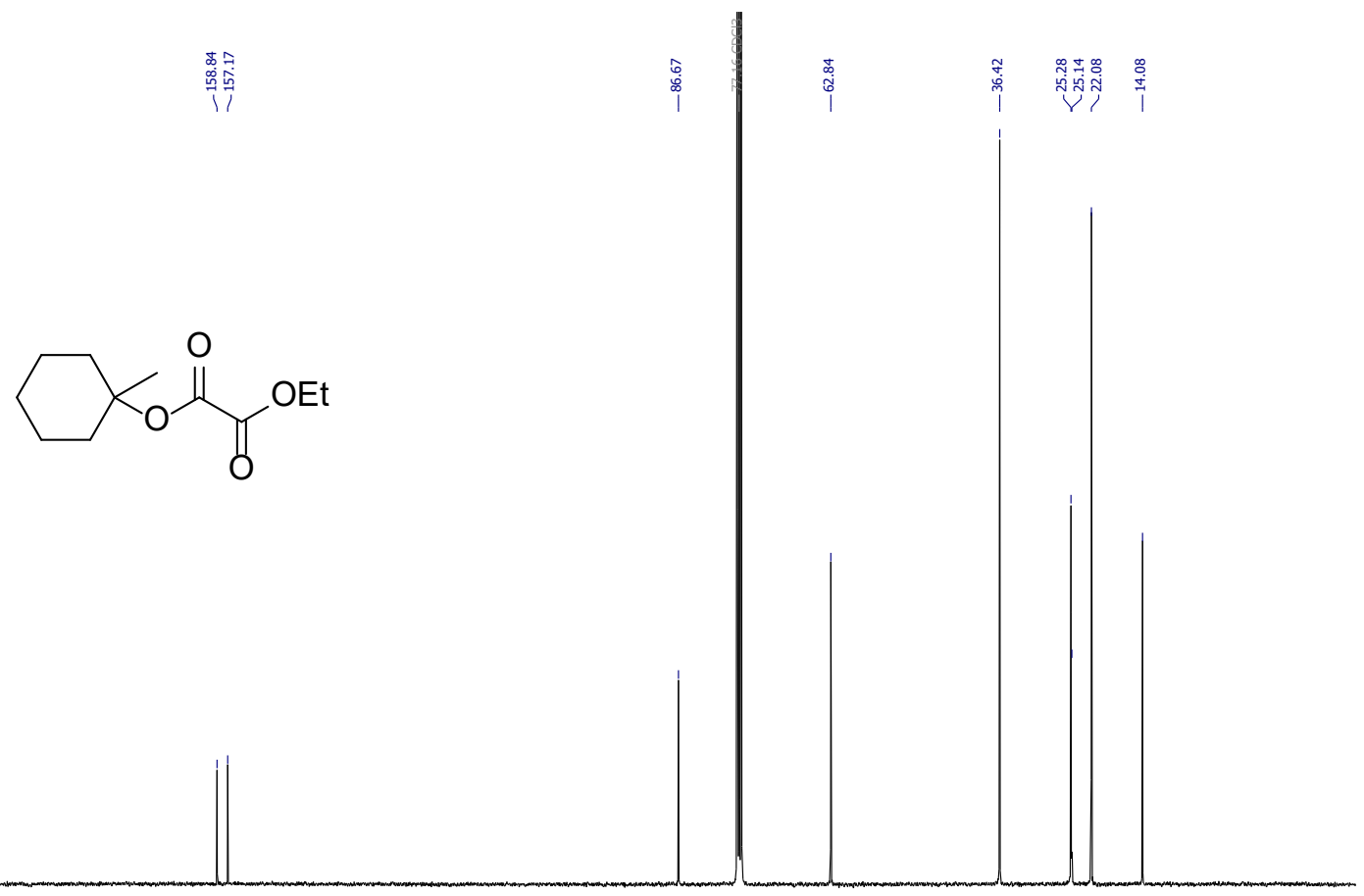

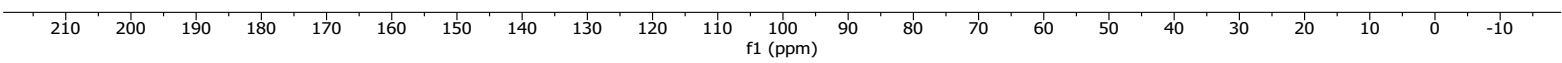




\section{Compound $\mathbf{3 l}$}

${ }^{1} \mathrm{H}$ NMR, DMSO, $400 \mathrm{MHz}$<smiles>CCOC(=O)C(=O)OC1(C)CCCCC1</smiles>
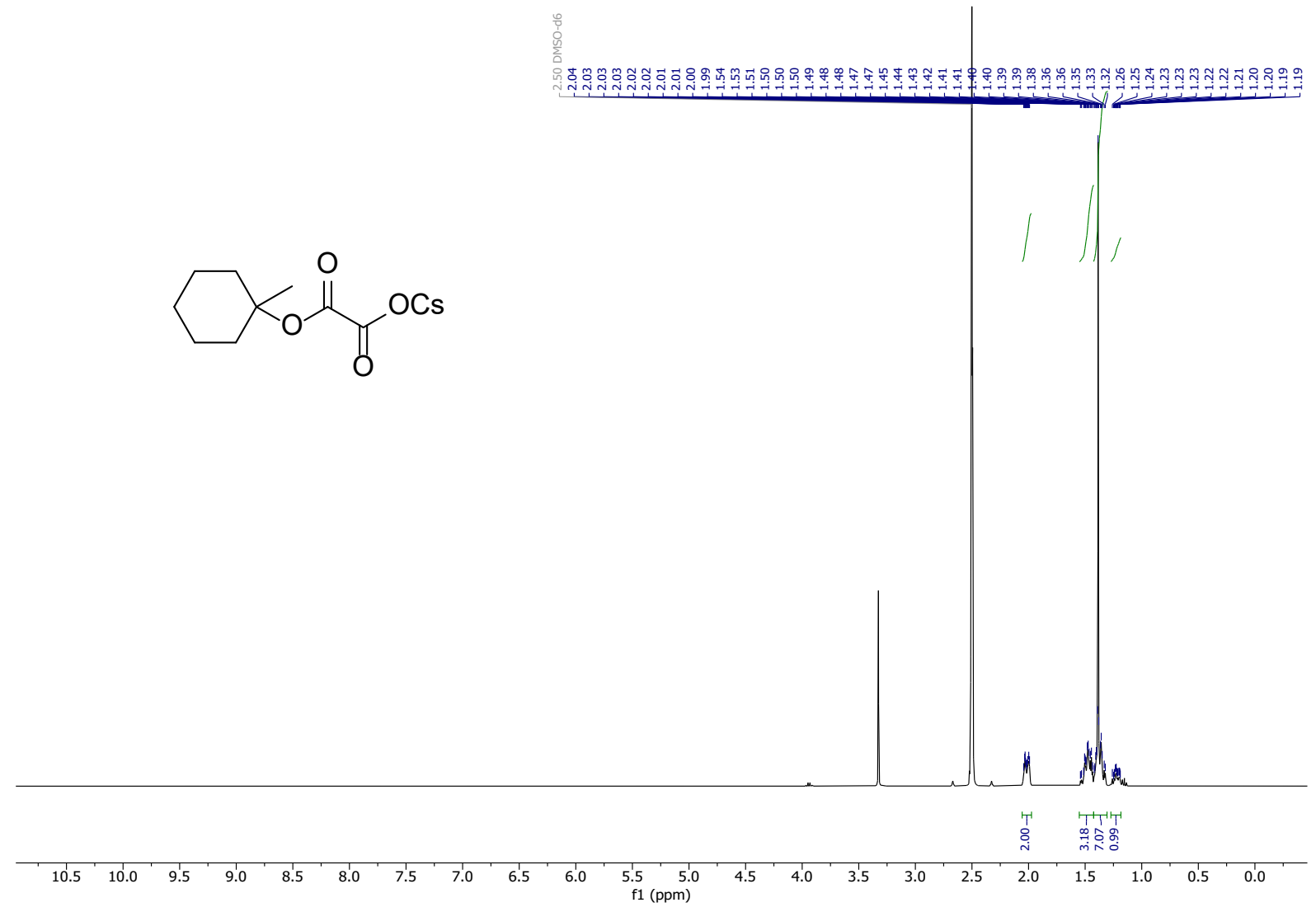

${ }^{13} \mathrm{C}$ NMR, DMSO, $101 \mathrm{MHz}$
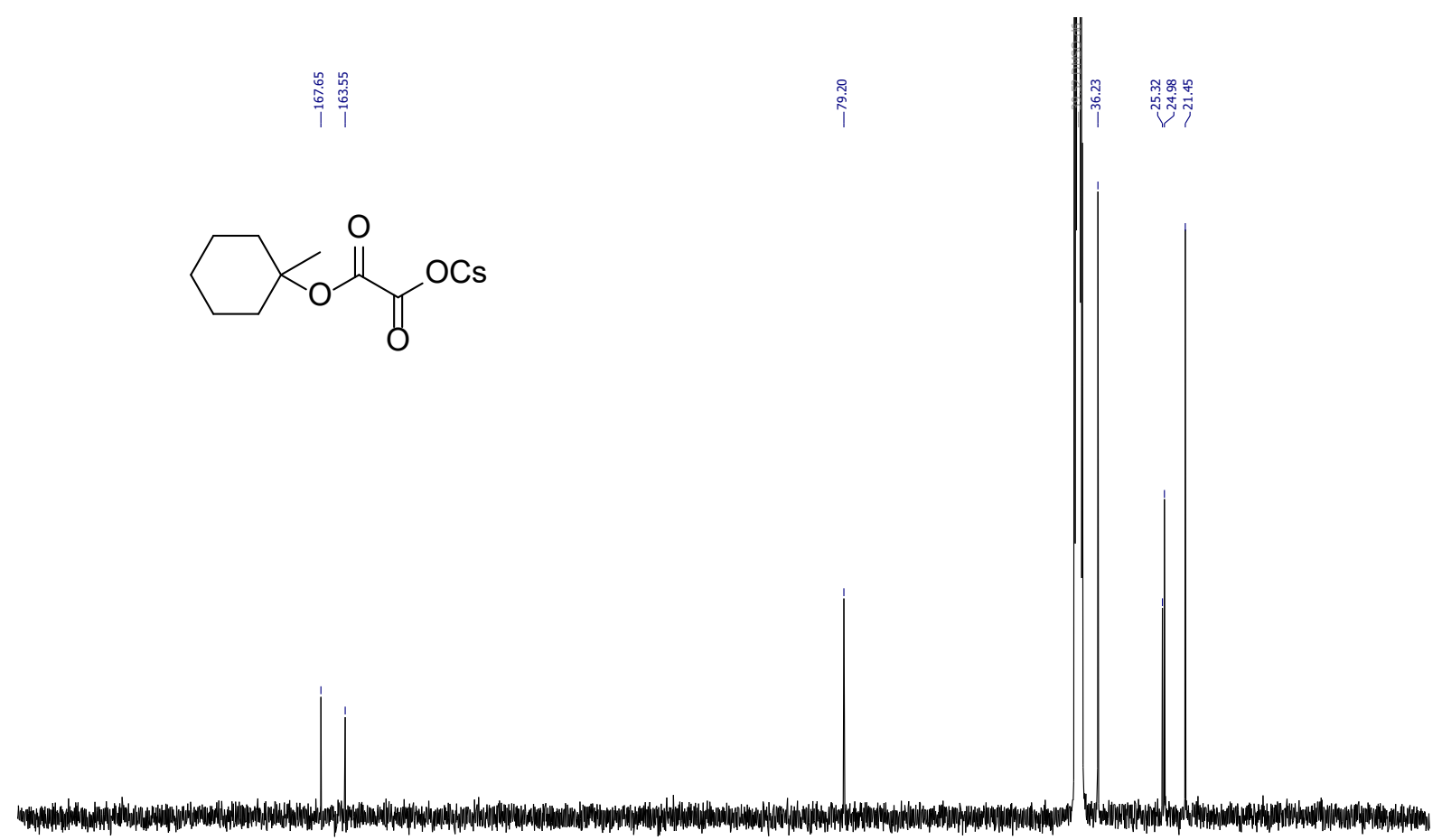

$\begin{array}{lllllllllllllllllllllll}210 & 200 & 190 & 180 & 170 & 160 & 150 & 140 & 130 & 120 & 110 & \begin{array}{c}100 \\ \mathrm{f} 1(\mathrm{ppm})\end{array} & 90 & 80 & 70 & 60 & 50 & 40 & 30 & 20 & 10 & 0 & -10\end{array}$ 


\section{Compound 23m}

${ }^{1} \mathrm{H} \mathrm{NMR}, \mathrm{CDCl}_{3}, 400 \mathrm{MHz}$

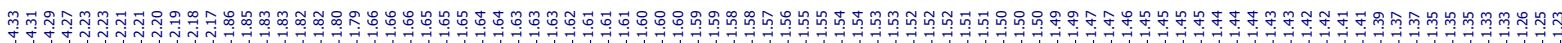<smiles>CCOC(=O)C(=O)OC1(C)CCCCCC1</smiles>
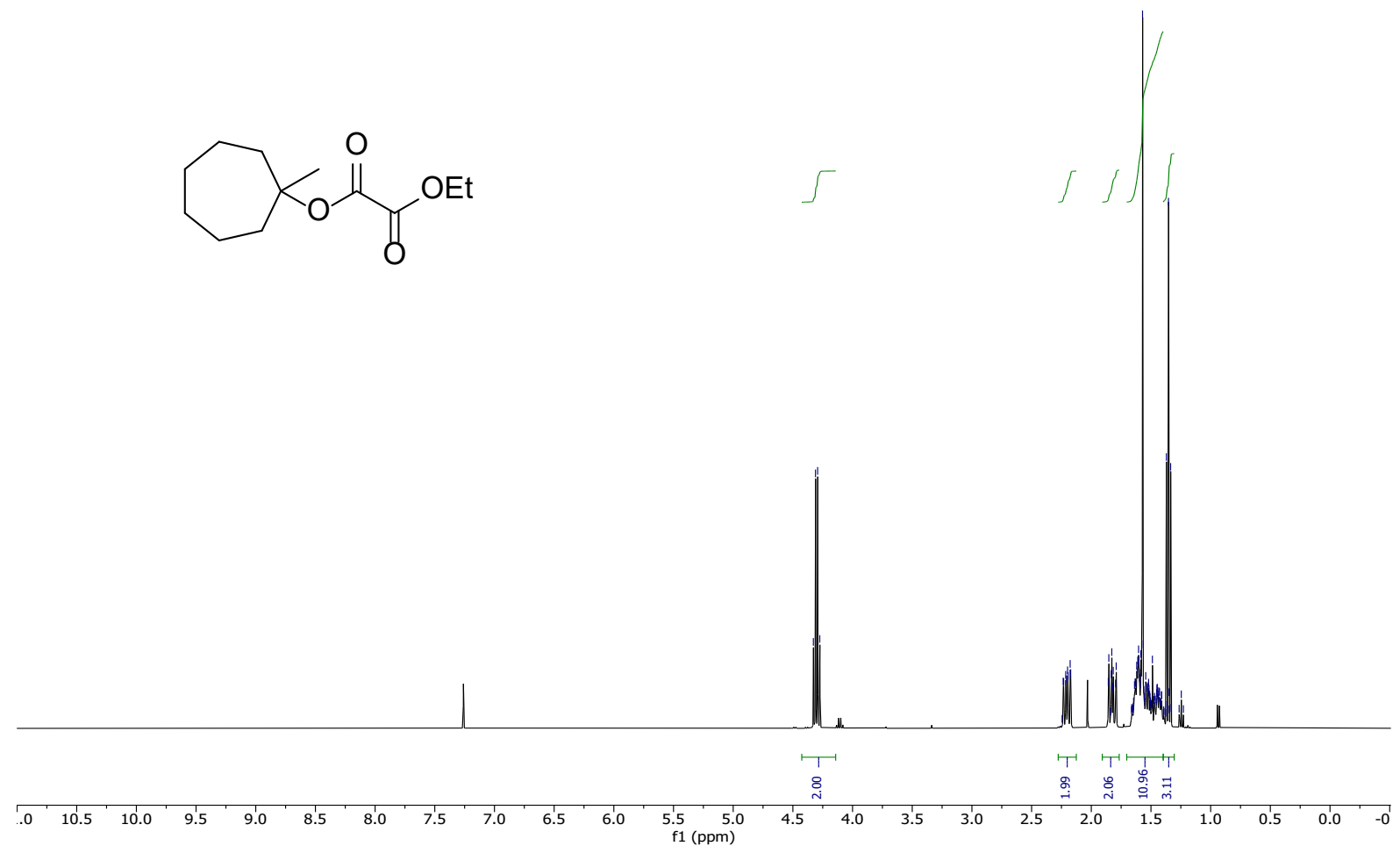

${ }^{13} \mathrm{C} \mathrm{NMR,} \mathrm{CDCl}_{3}, 101 \mathrm{MHz}$

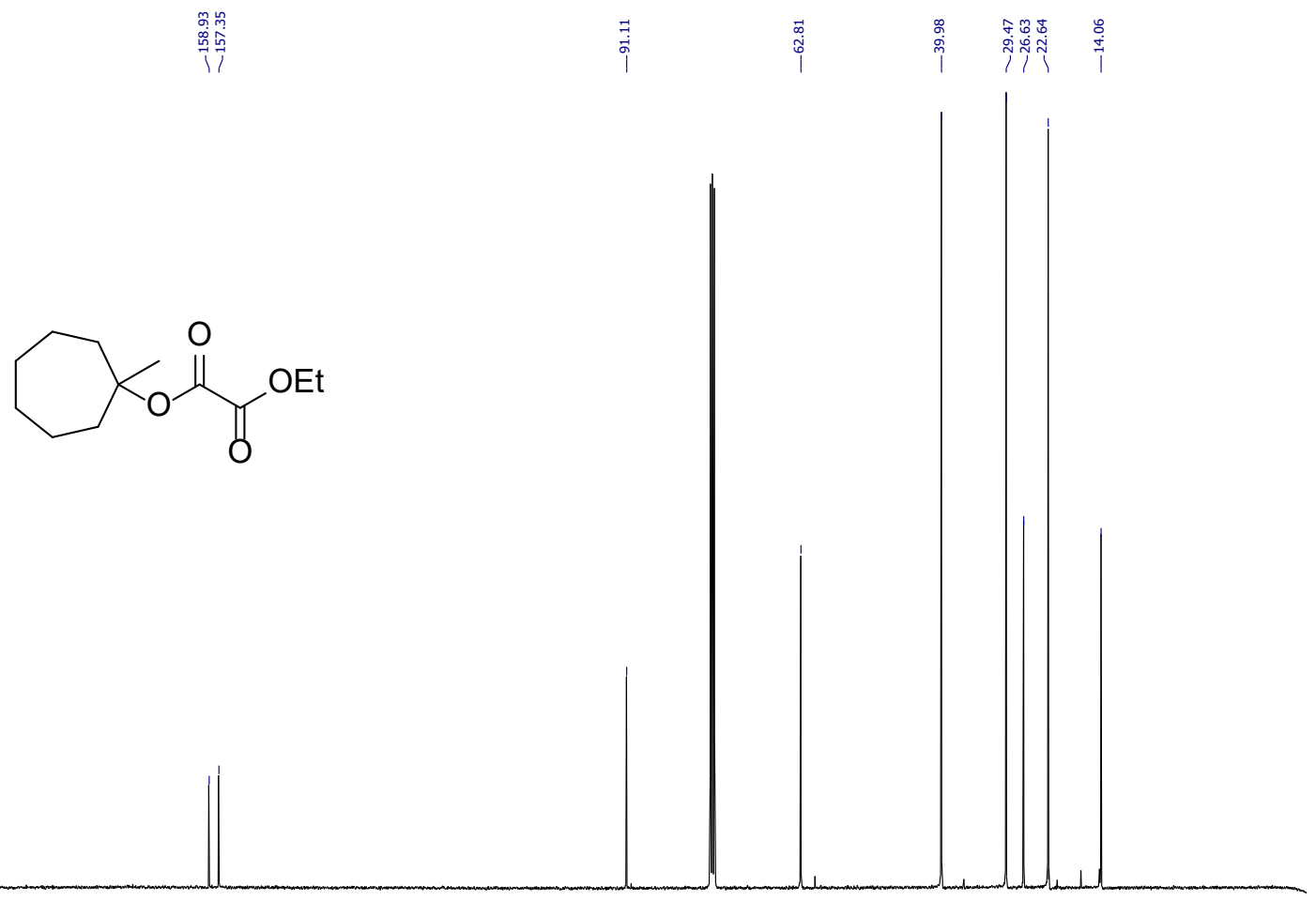

\begin{tabular}{|llllllllllllllllllllllll}
\hline 210 & 200 & 190 & 180 & 170 & 160 & 150 & 140 & 130 & 120 & 110 & $\begin{array}{c}100 \\
\mathrm{f} 1(\mathrm{ppm})\end{array}$ & 90 & 80 & 70 & 60 & 50 & 40 & 30 & 20 & 10 & 0 & -10 & 10
\end{tabular} 


\section{Compound 3m}

${ }^{1} \mathrm{H}$ NMR, DMSO, $400 \mathrm{MHz}$

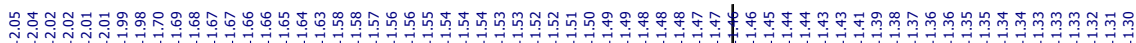<smiles>CCOC(=O)C(=O)OC1(C)CCCCCC1</smiles>

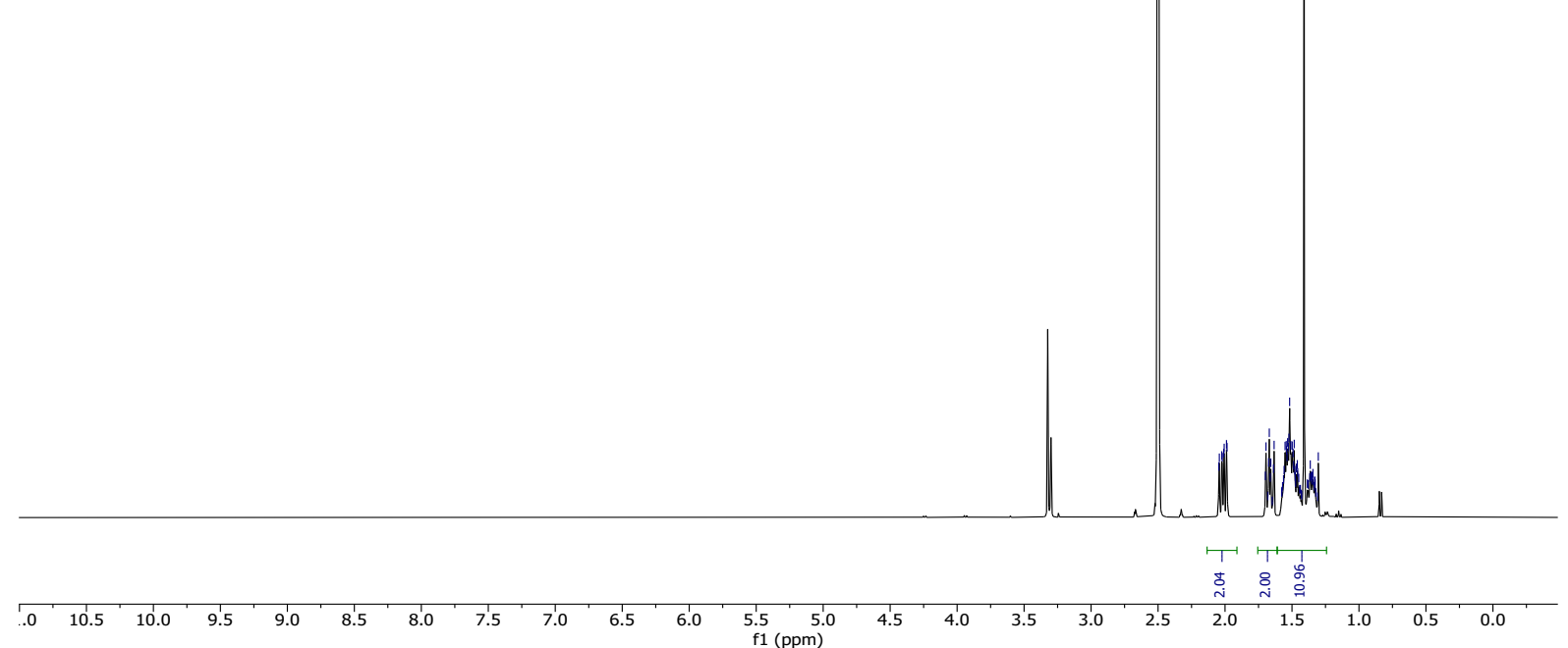

${ }^{13} \mathrm{C}$ NMR, DMSO, $101 \mathrm{MHz}$
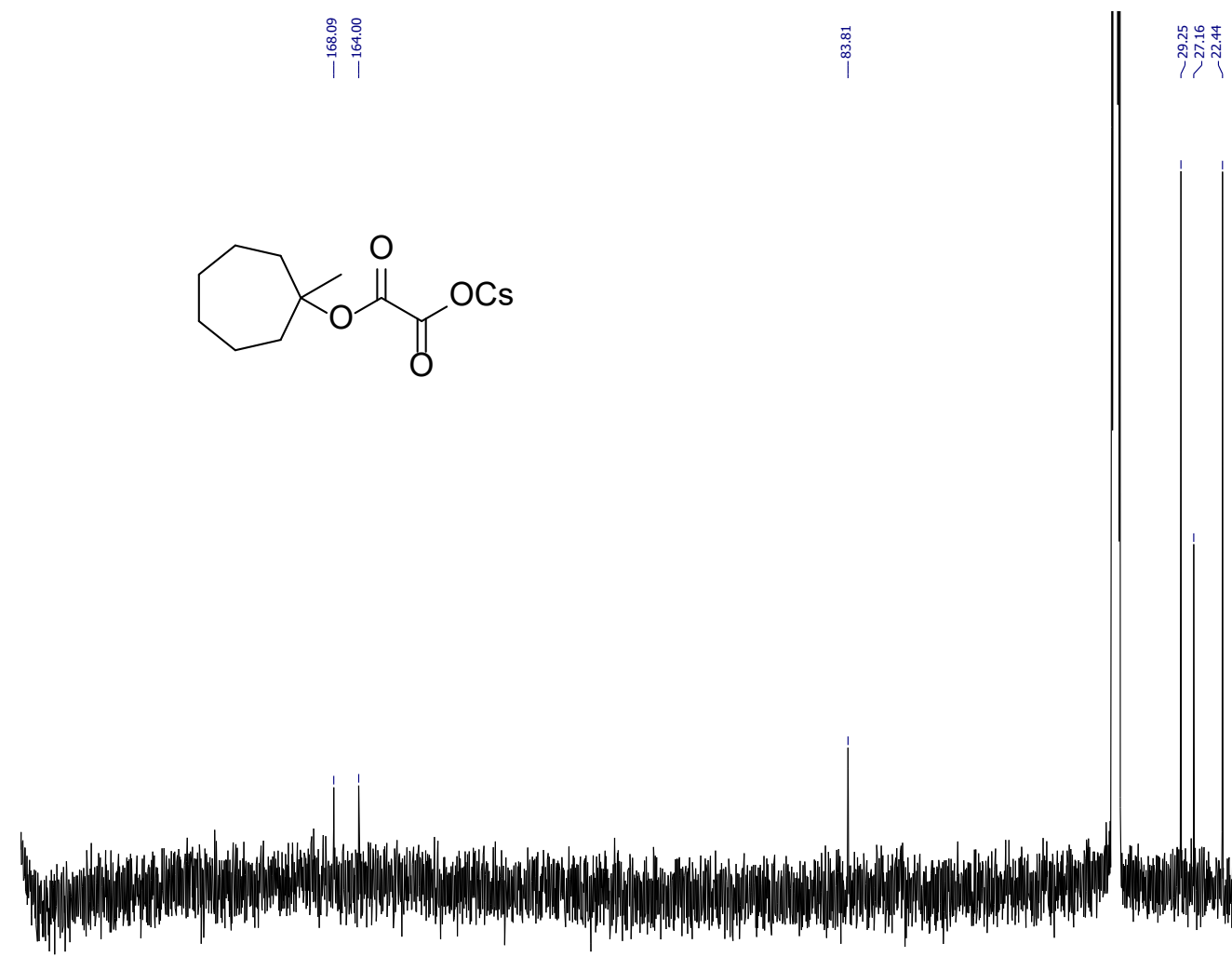

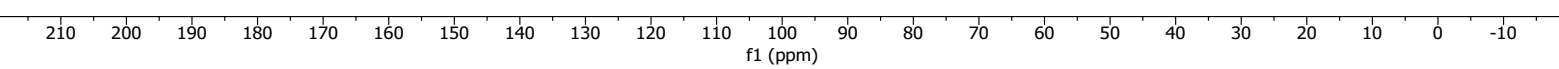




\section{Compound 23n}

${ }^{1} \mathrm{H} \mathrm{NMR}, \mathrm{CDCl}_{3}, 400 \mathrm{MHz}$

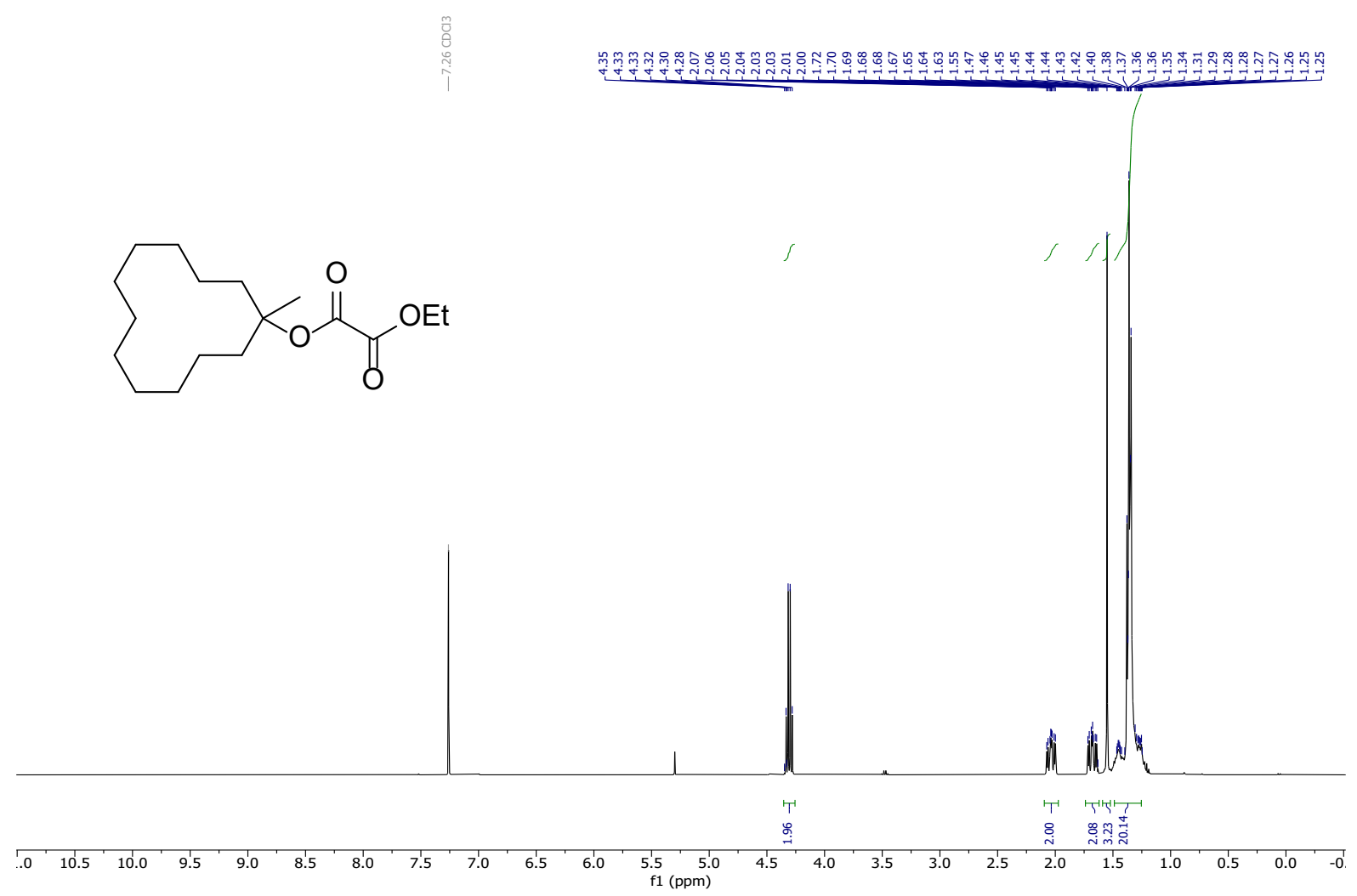

${ }^{13} \mathrm{C} \mathrm{NMR}, \mathrm{CDCl}_{3}, 101 \mathrm{MHz}$
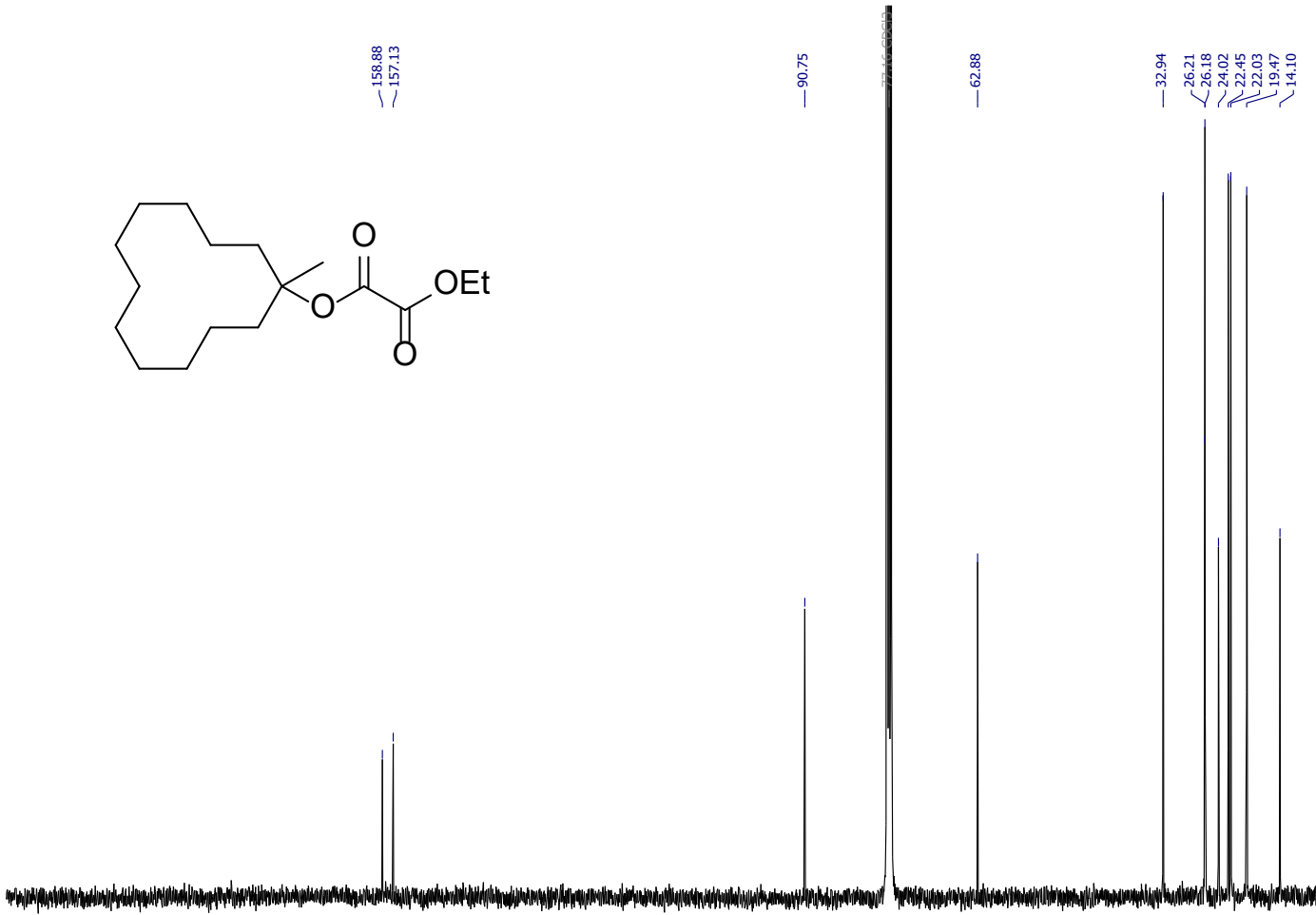

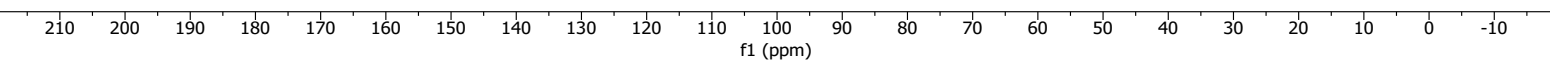




\section{Compound 3n}

${ }^{1} \mathrm{H}$ NMR, DMSO, $400 \mathrm{MHz}$

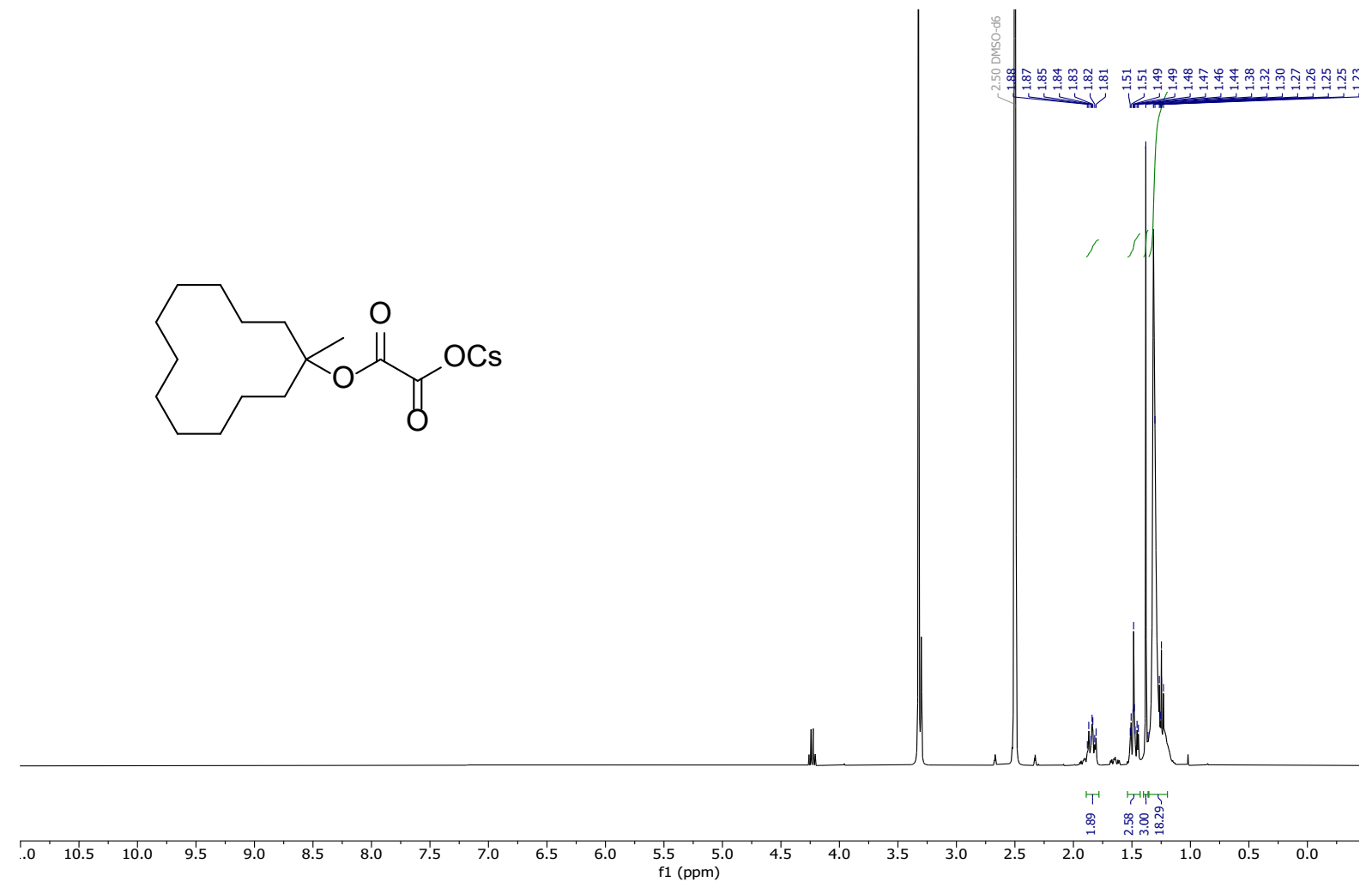

${ }^{13} \mathrm{C}$ NMR, DMSO, $101 \mathrm{MHz}$

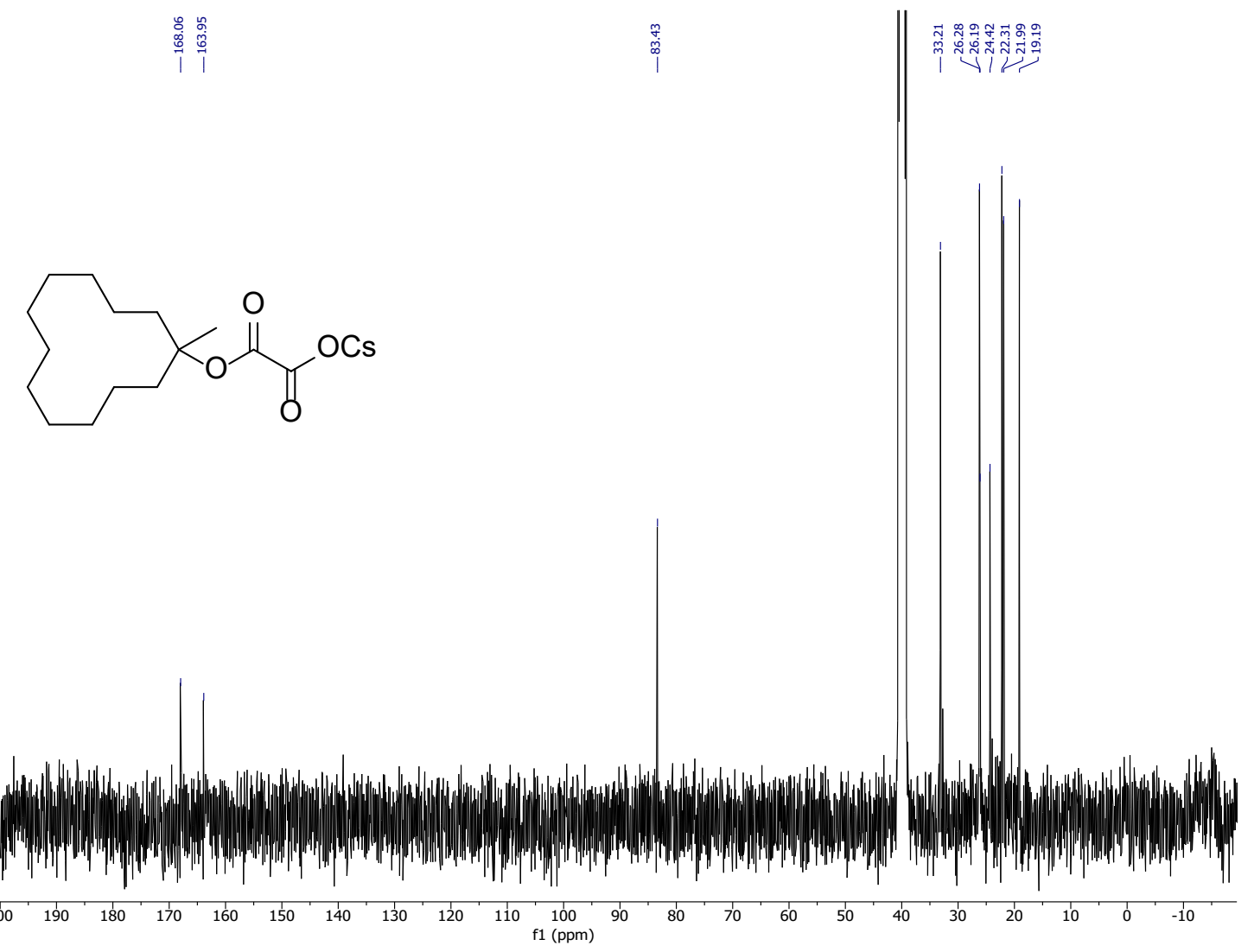




\section{Compound 230}

${ }^{1} \mathrm{H} \mathrm{NMR}, \mathrm{CDCl}_{3}, 400 \mathrm{MHz}$

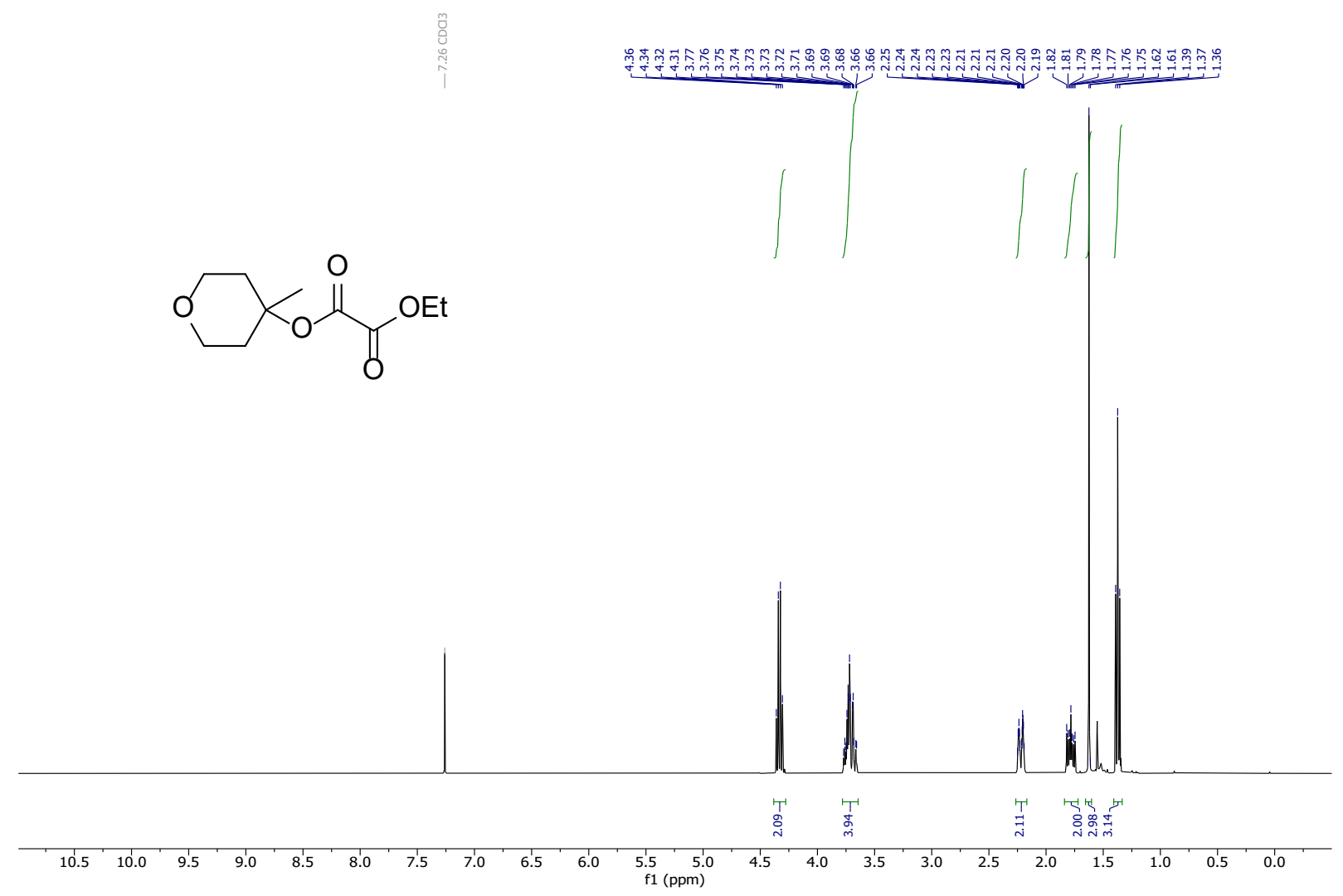

${ }^{13} \mathrm{C} \mathrm{NMR}, \mathrm{CDCl}_{3}, 101 \mathrm{MHz}$

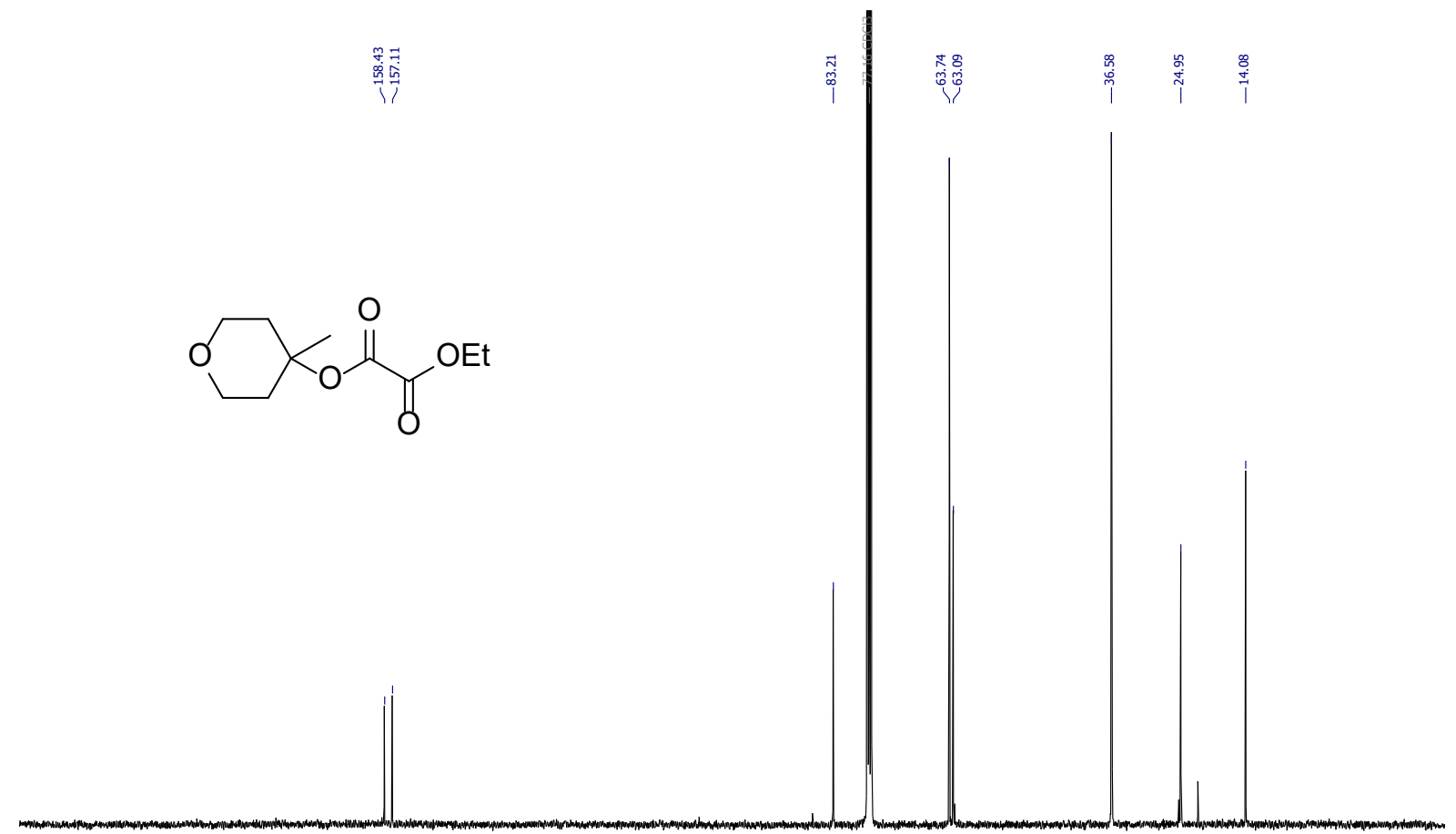

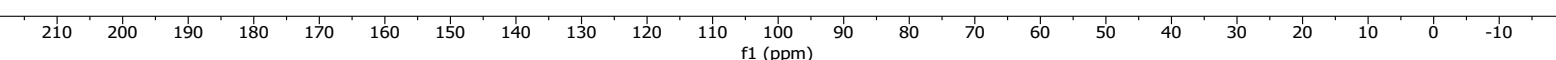




\section{Compound 30}

${ }^{1} \mathrm{H}$ NMR, DMSO, $400 \mathrm{MHz}$
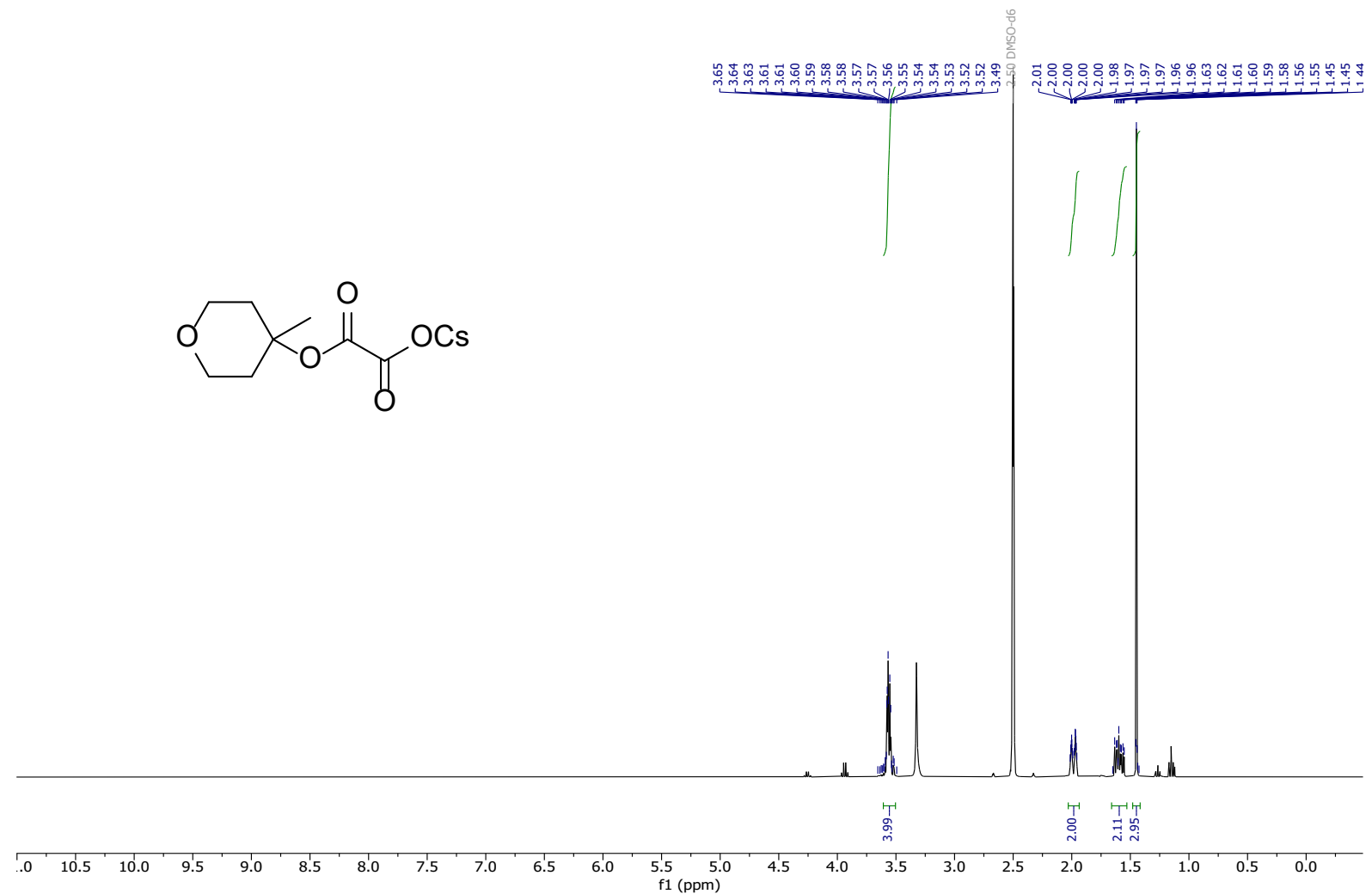

${ }^{13} \mathrm{C}$ NMR, DMSO, $101 \mathrm{MHz}$

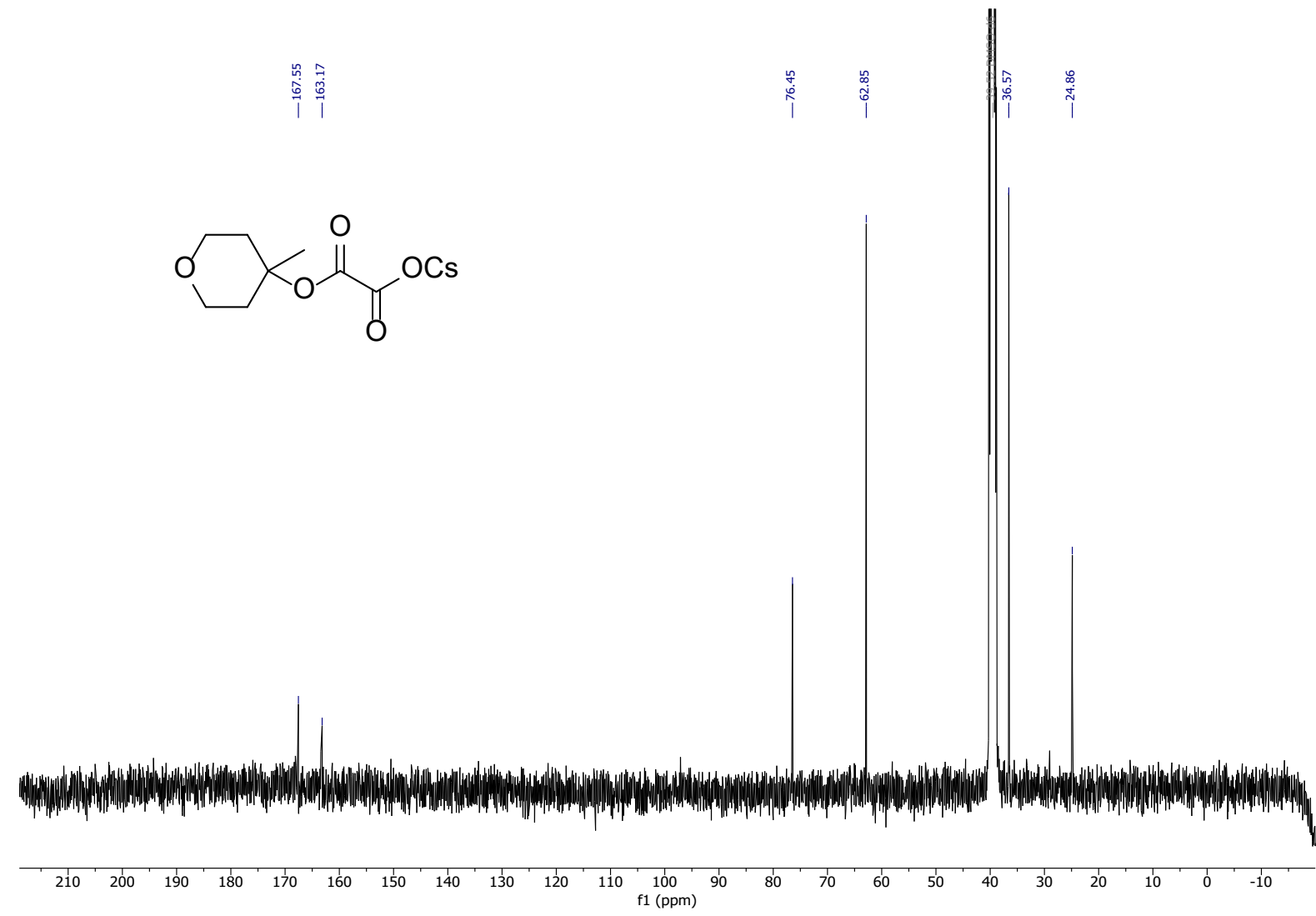




\section{Compound 23p}

${ }^{1} \mathrm{H} \mathrm{NMR}, \mathrm{CDCl}_{3}, 400 \mathrm{MHz}$

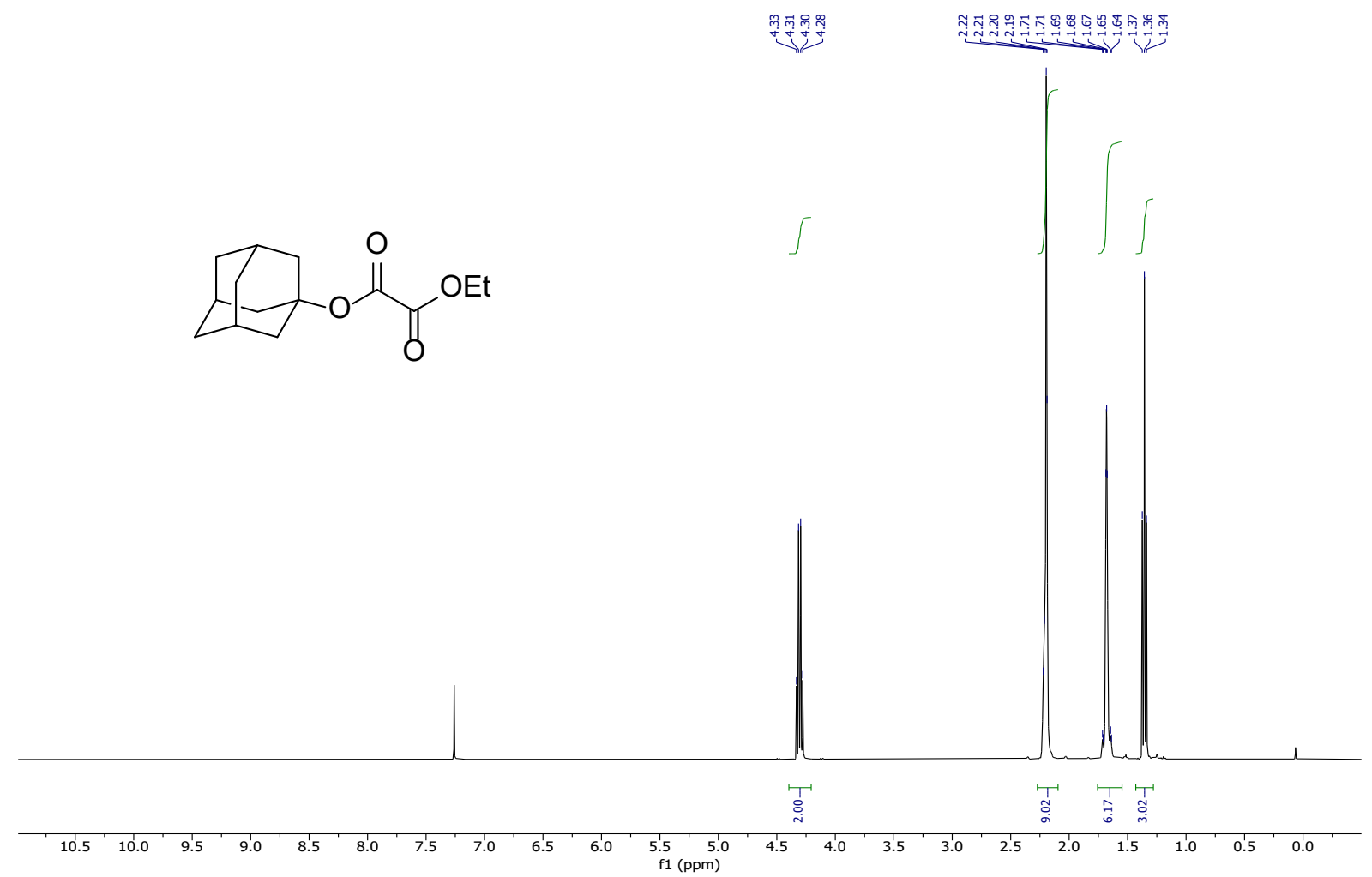

${ }^{13} \mathrm{CNMR}_{\mathrm{N}} \mathrm{CDCl}_{3}, 101 \mathrm{MHz}$<smiles>CCOC(=O)C(=O)OC12CC3CC(CC(C3)C1)C2</smiles>

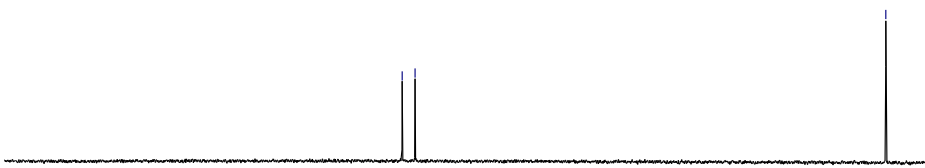


Compound 3p (with 25\% 23p)

${ }^{1} \mathrm{H} N M R, \mathrm{DMSO}, 400 \mathrm{MHz}$<smiles>O=C(OC(F)(F)F)C(=O)OC12CC3CC(CC(C3)C1)C2</smiles>

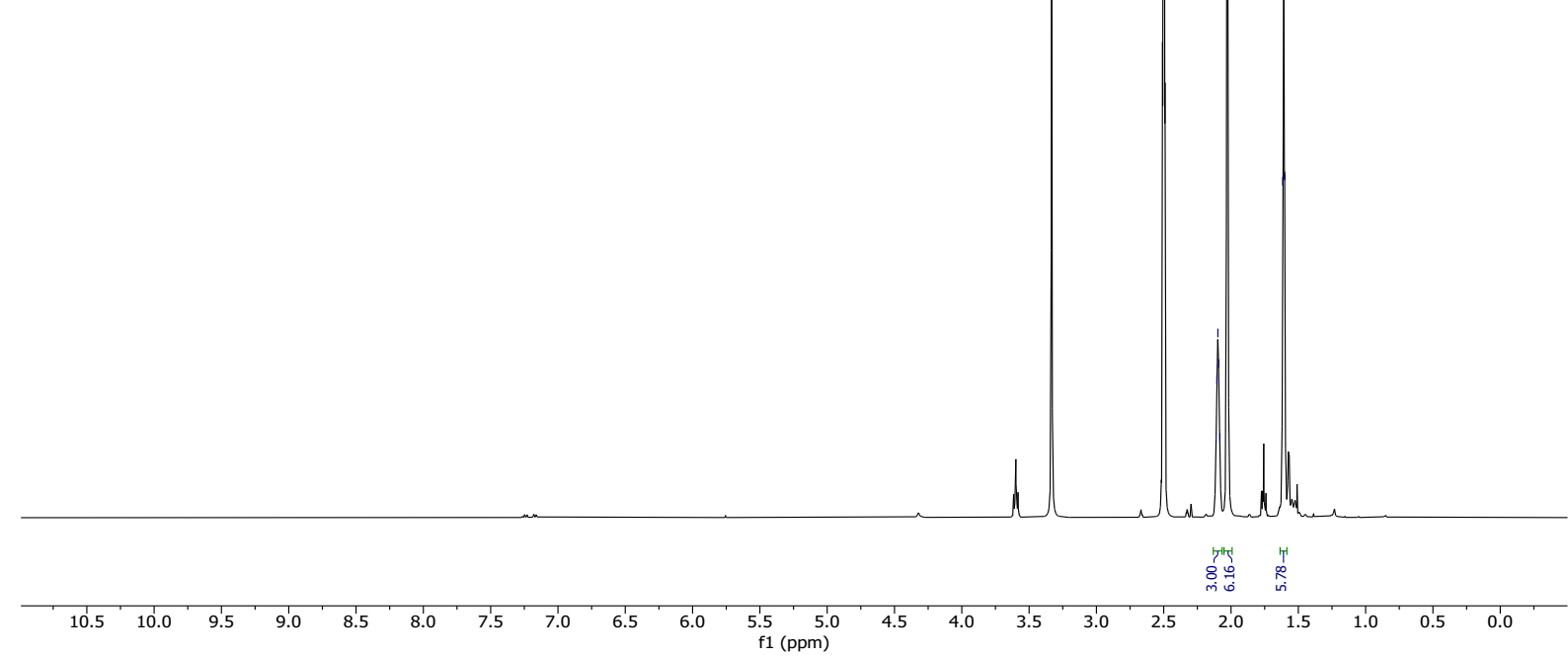

${ }^{13} \mathrm{C}$ NMR, DMSO, $101 \mathrm{MHz}$

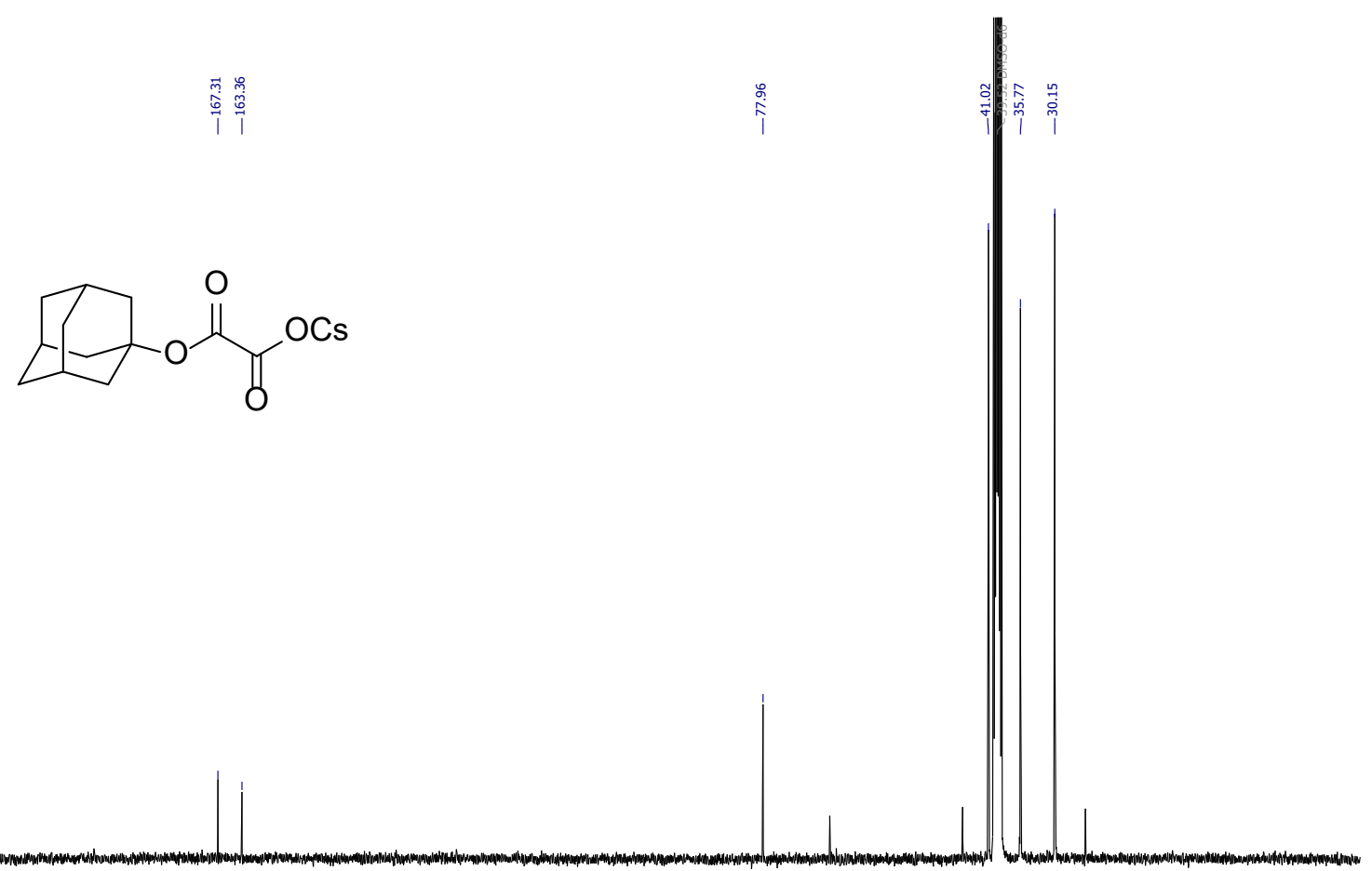

$\begin{array}{llllllllllllllllllllllllllll}210 & 200 & 190 & 180 & 170 & 160 & 150 & 140 & 130 & 120 & 110 & 100 & 90 & 80 & 70 & 60 & 50 & 40 & 30 & 20 & 10 & 0 & -10\end{array}$ 


\section{Compound 23w}

${ }^{1} \mathrm{H} \mathrm{NMR}, \mathrm{CDCl}_{3}, 400 \mathrm{MHz}$

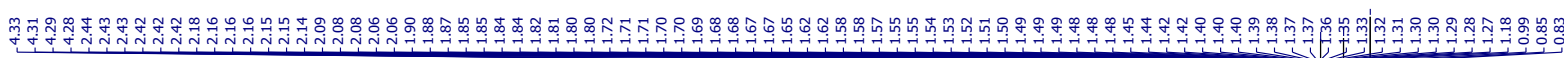<smiles>CCOC(=O)C(=O)O[C@]1(C)CC[C@]23C[C@H](C(C)(C)[C@H]2CC[C@H]3C)C1(C)C</smiles><smiles>[C]1CCCC1</smiles>

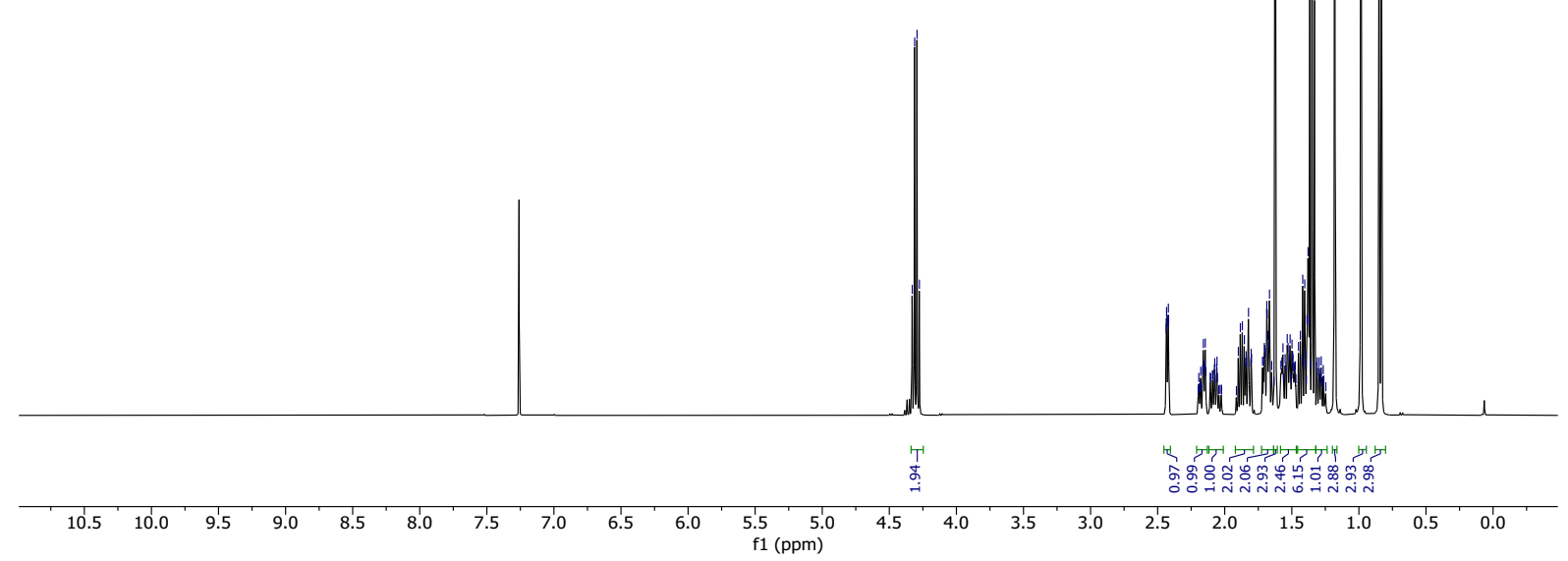

${ }^{13} \mathrm{C} \mathrm{NMR}, \mathrm{CDCl}_{3}, 101 \mathrm{MHz}$

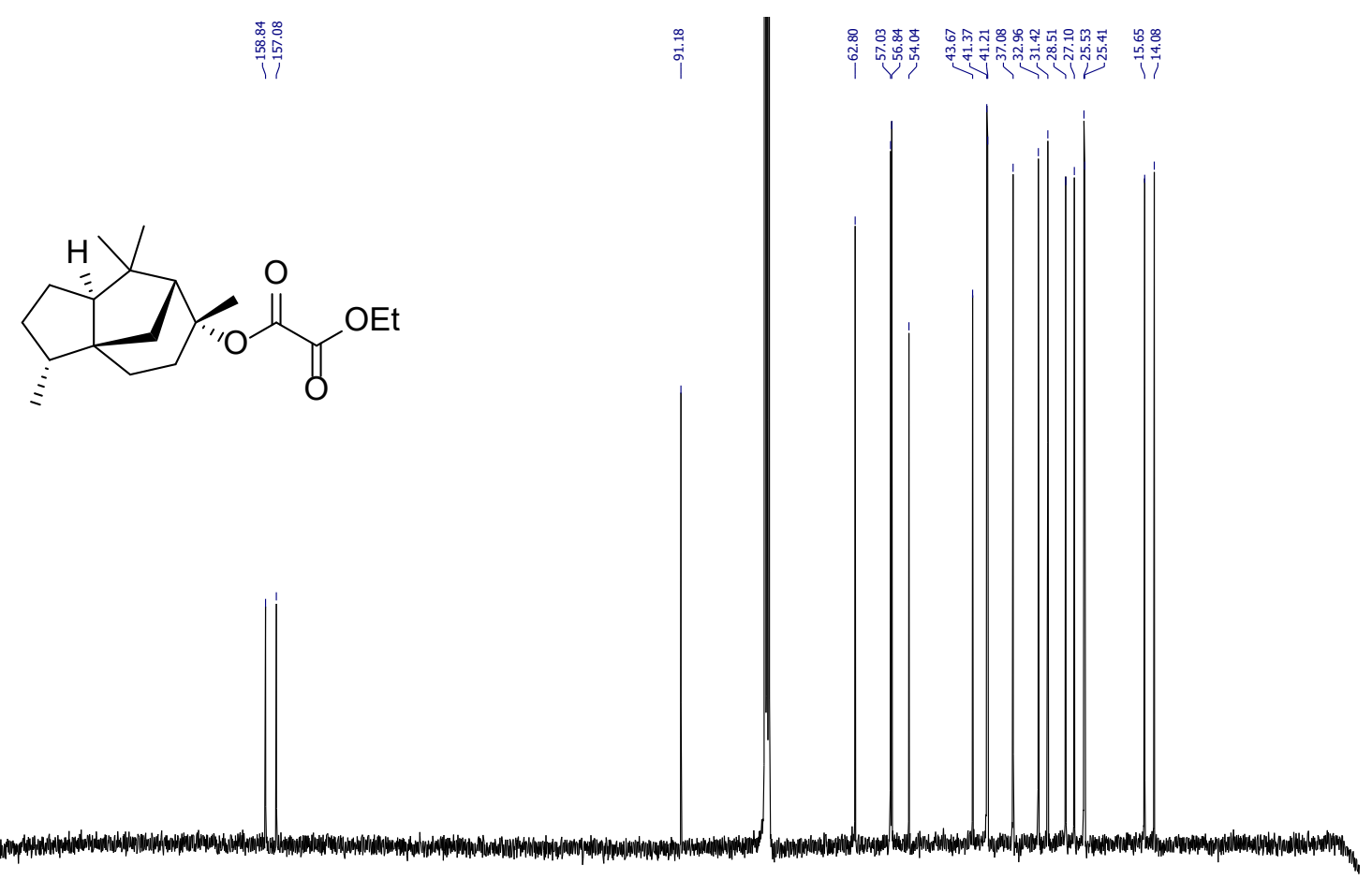

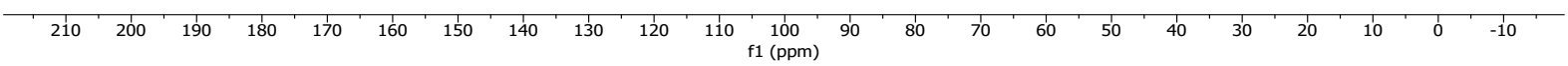




\section{Compound 3w}

${ }^{1} \mathrm{H}$ NMR, DMSO, $400 \mathrm{MHz}$

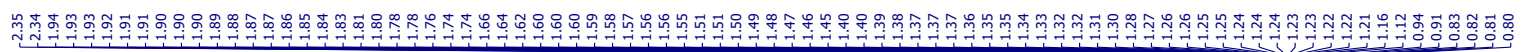

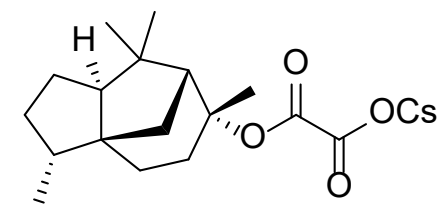

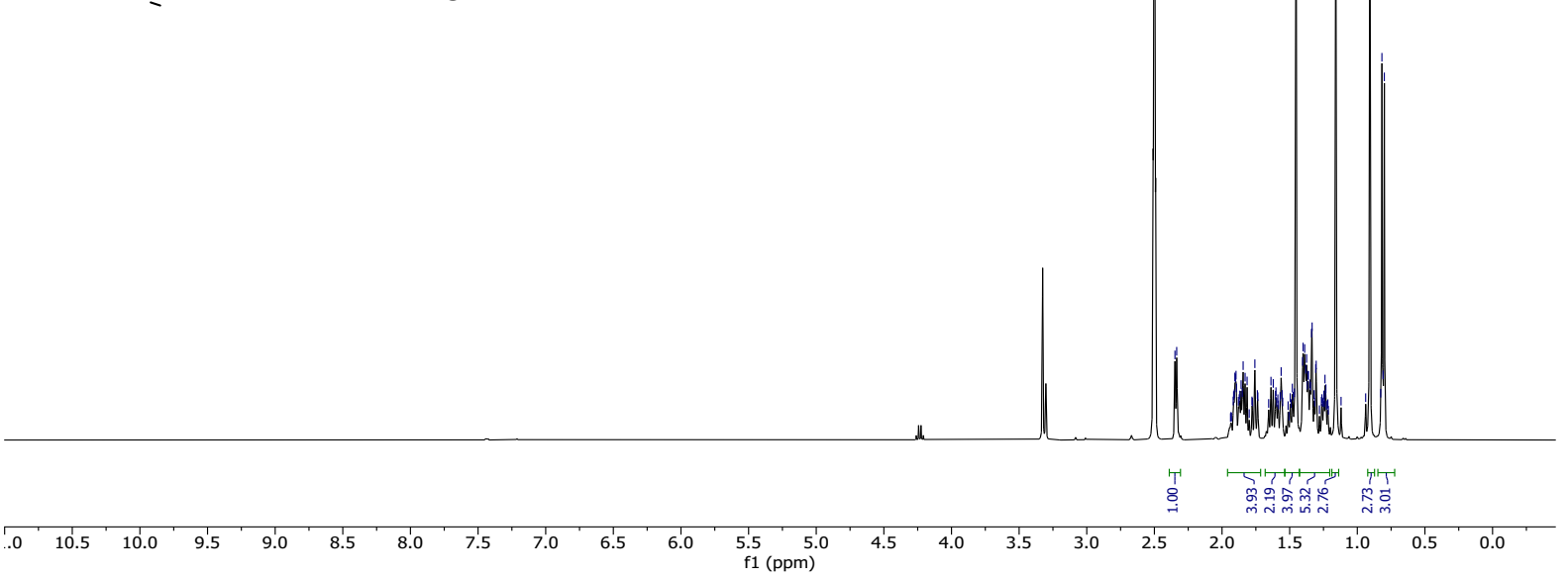

${ }^{13} \mathrm{C}$ NMR, DMSO, $101 \mathrm{MHz}$
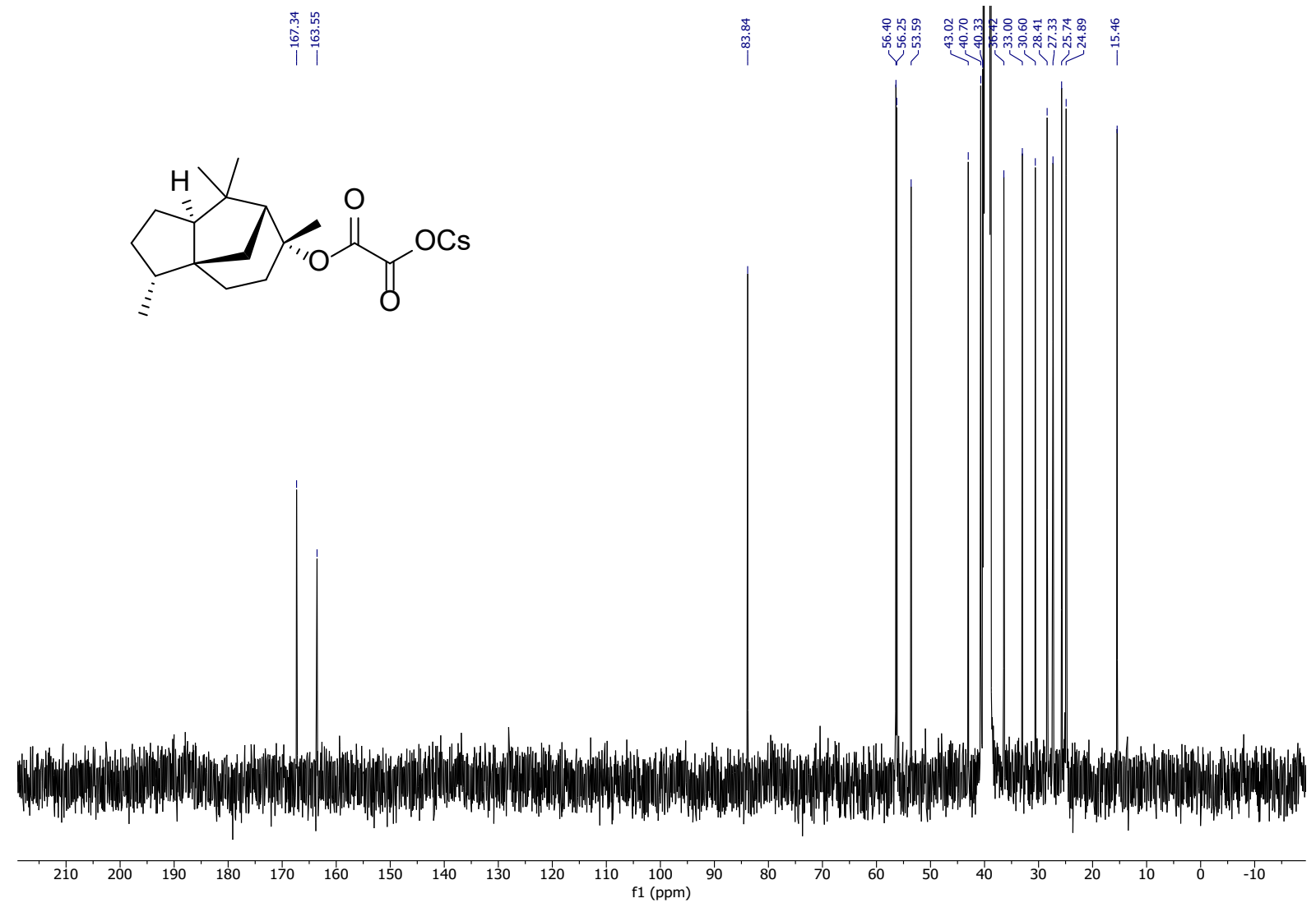


\section{Compound 23x}

${ }^{1} \mathrm{H} \mathrm{NMR}, \mathrm{CDCl}_{3}, 400 \mathrm{MHz}$

8

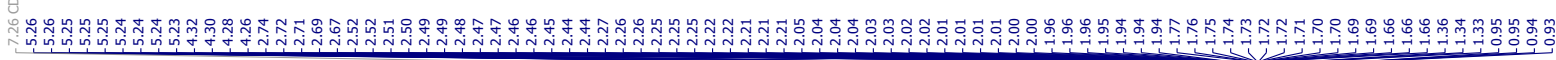<smiles>CCOC(=O)C(=O)OC1(I(F)F)CC=C(C)CC1</smiles>

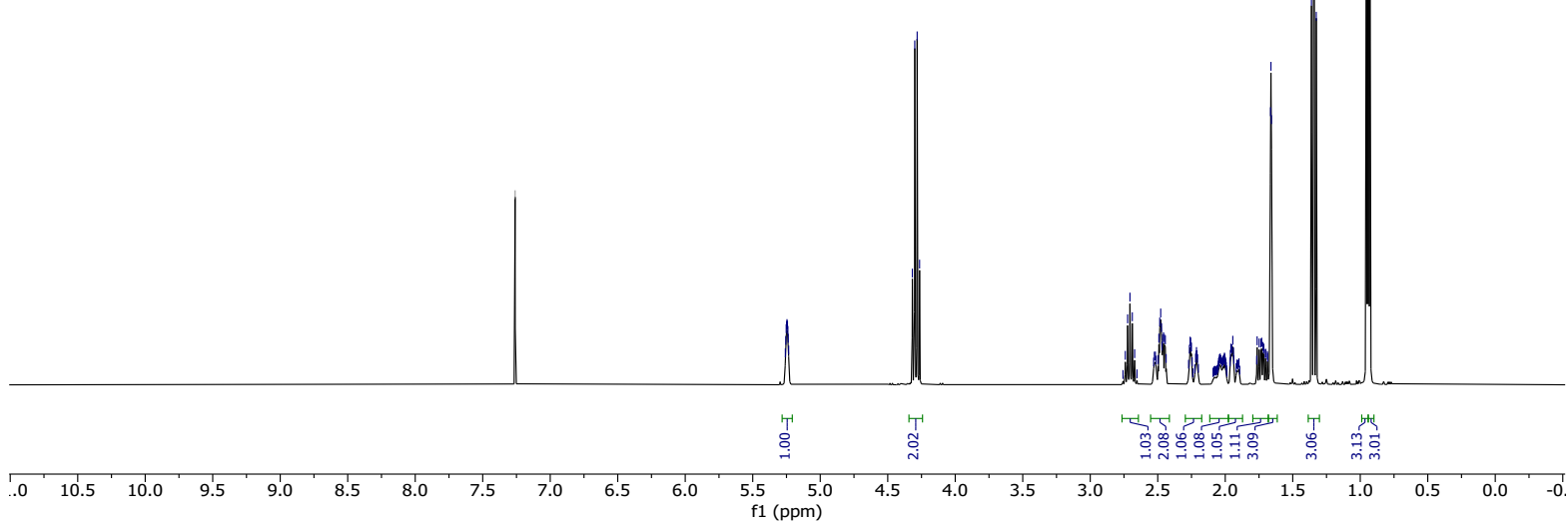

${ }^{13} \mathrm{CNMR}, \mathrm{CDCl}_{3}, 101 \mathrm{MHz}$

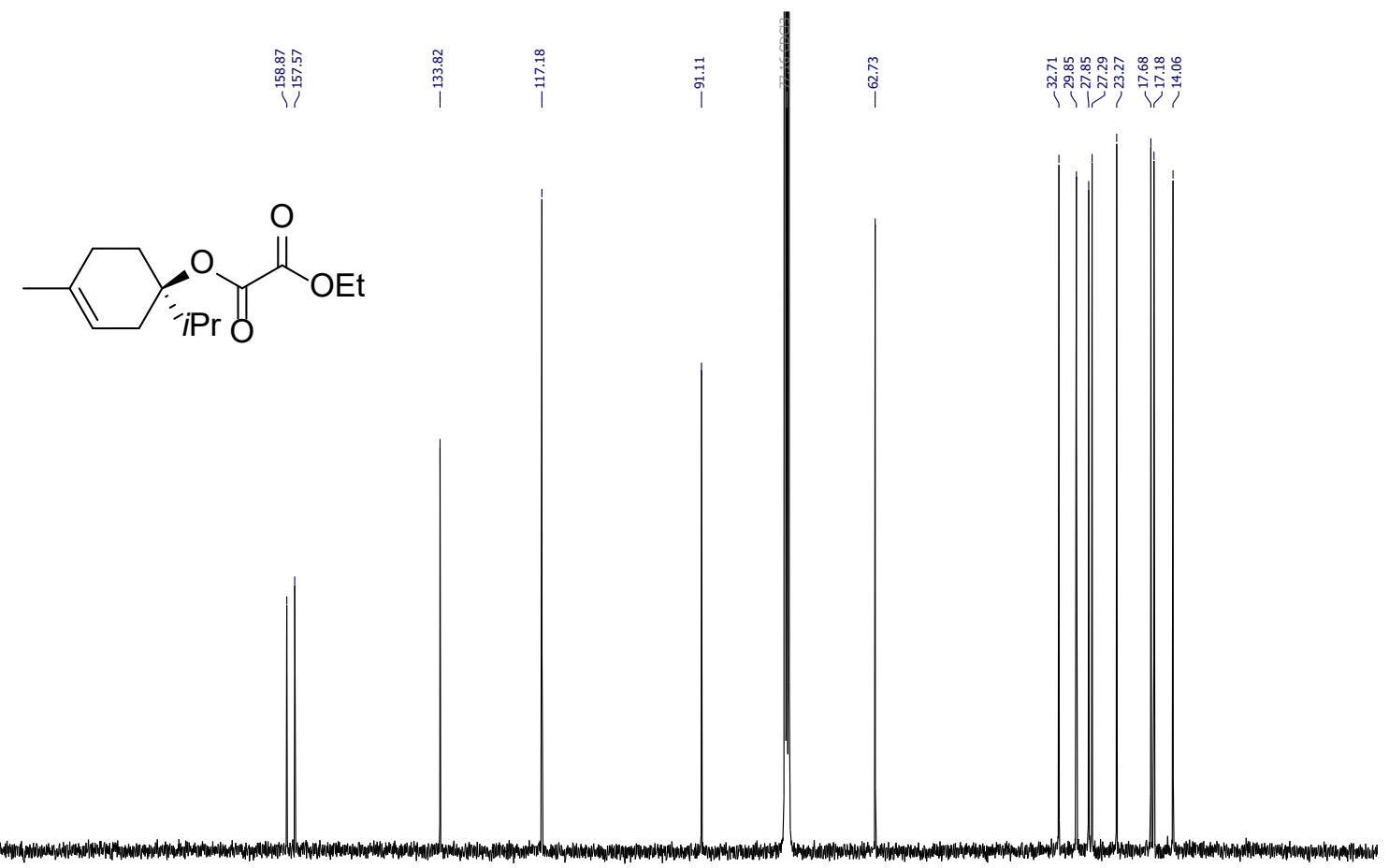

\begin{tabular}{|lllllllllllllllllllllllllll}
210 & 200 & 190 & 180 & 170 & 160 & 150 & 140 & 130 & 120 & 110 & $\begin{array}{c}100 \\
\mathrm{f} 1(\mathrm{ppm})\end{array}$ & 90 & 80 & 70 & 60 & 50 & 40 & 30 & 20 & 10 & 0 & -10
\end{tabular} 


\section{Compound $\mathbf{3 x}$}

${ }^{1} \mathrm{H}$ NMR, DMSO, $400 \mathrm{MHz}$

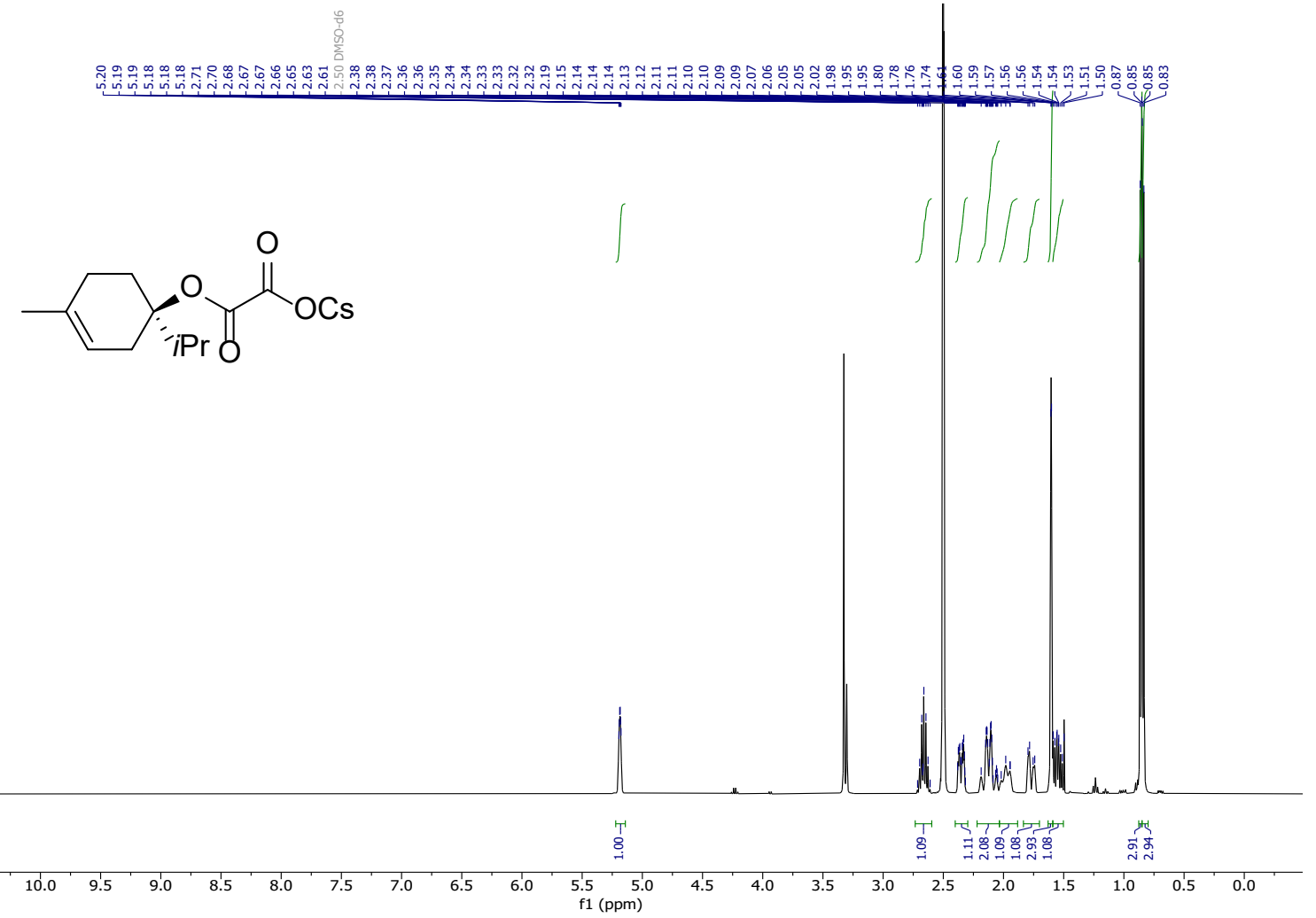

${ }^{13} \mathrm{C}$ NMR, DMSO, $101 \mathrm{MHz}$

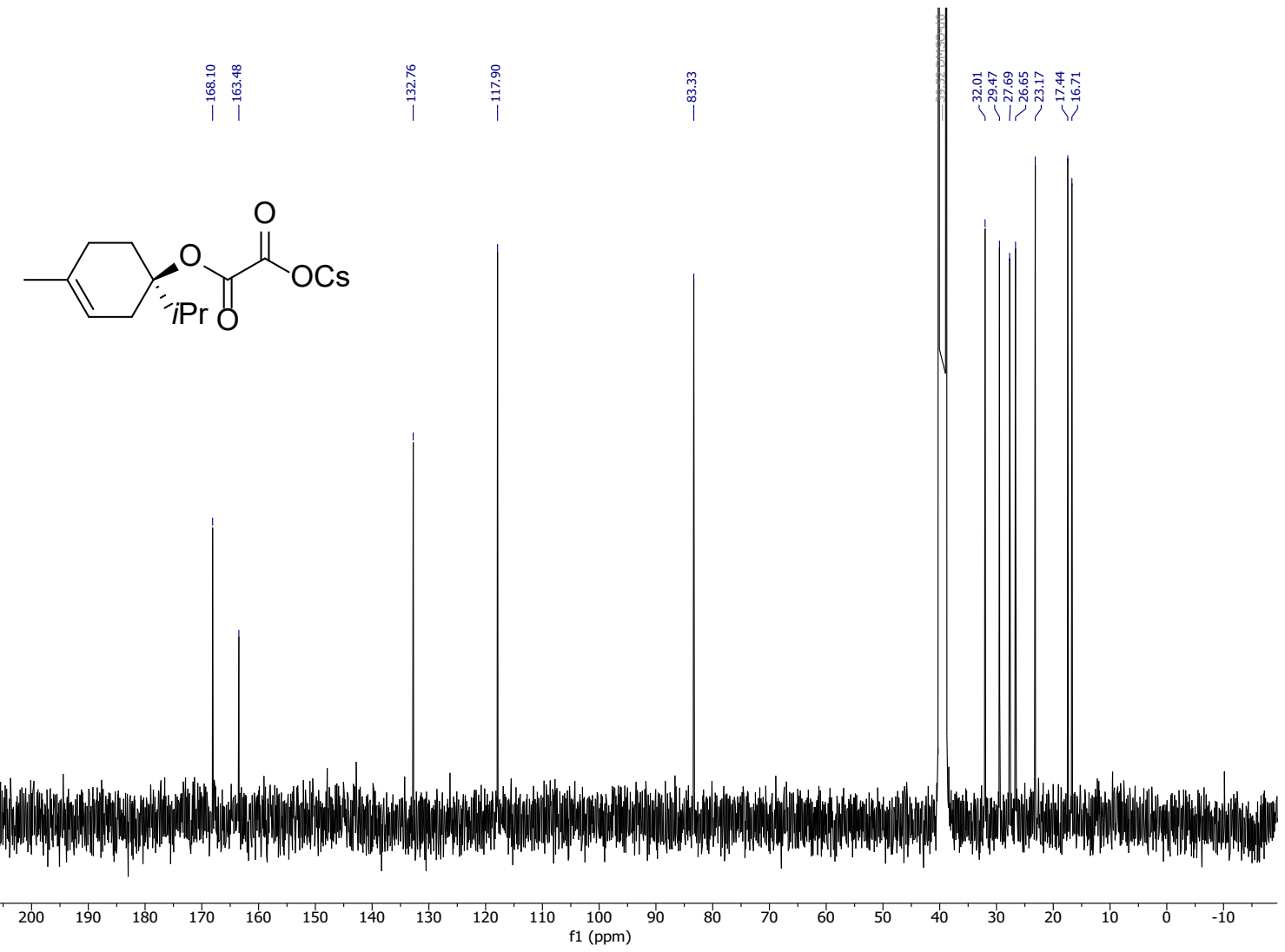




\section{Compound $4 a$}

${ }^{1} \mathrm{H} \mathrm{NMR}, \mathrm{CDCl}_{3}, 400 \mathrm{MHz}$

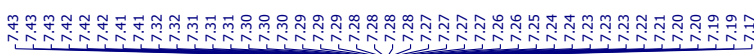

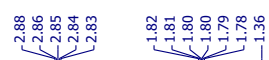<smiles>CC(C)(C#CPc1ccccc1)CCc1ccccc1</smiles>
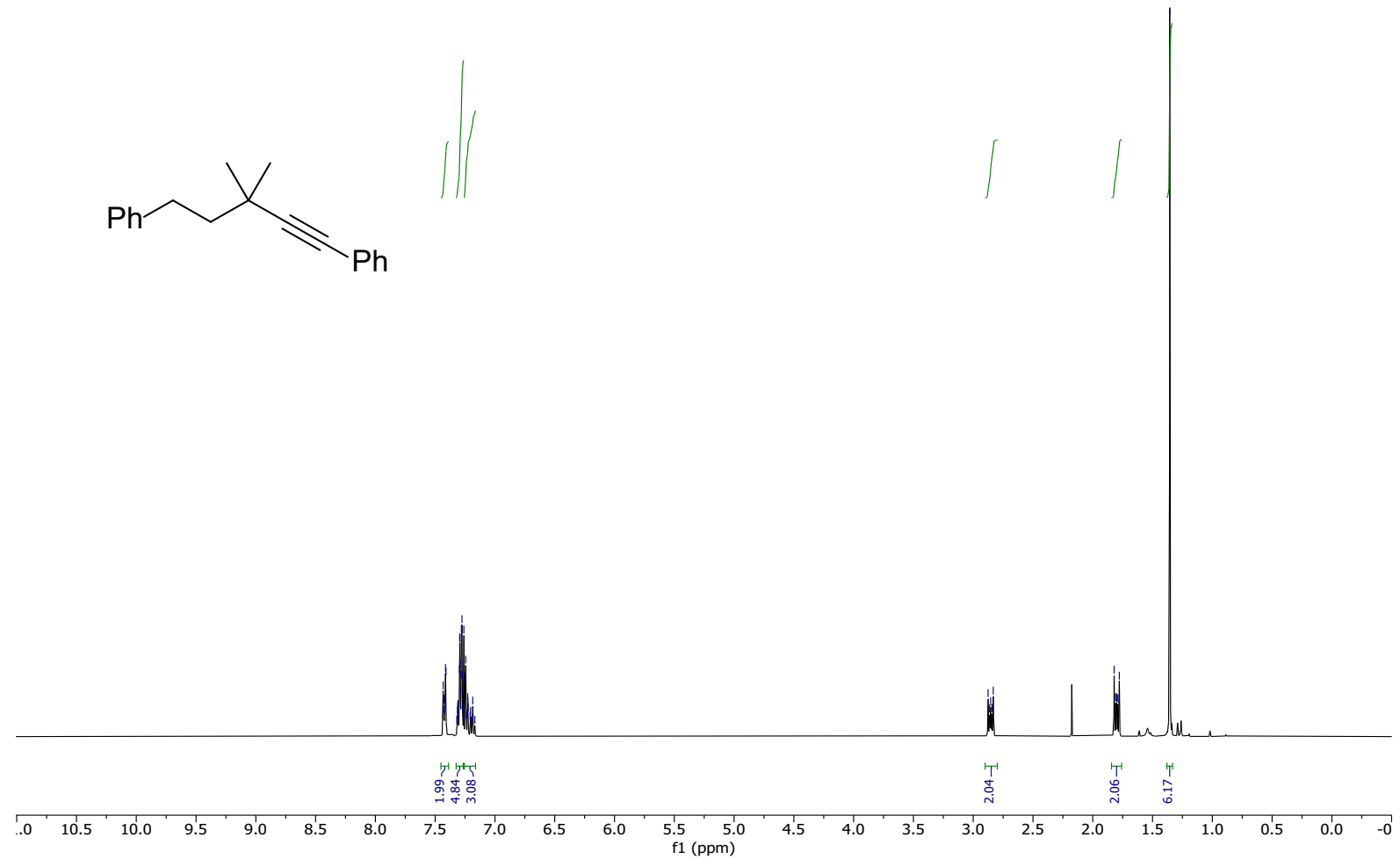

${ }^{13} \mathrm{C} \mathrm{NMR}, \mathrm{CDCl}_{3}, 101 \mathrm{MHz}$

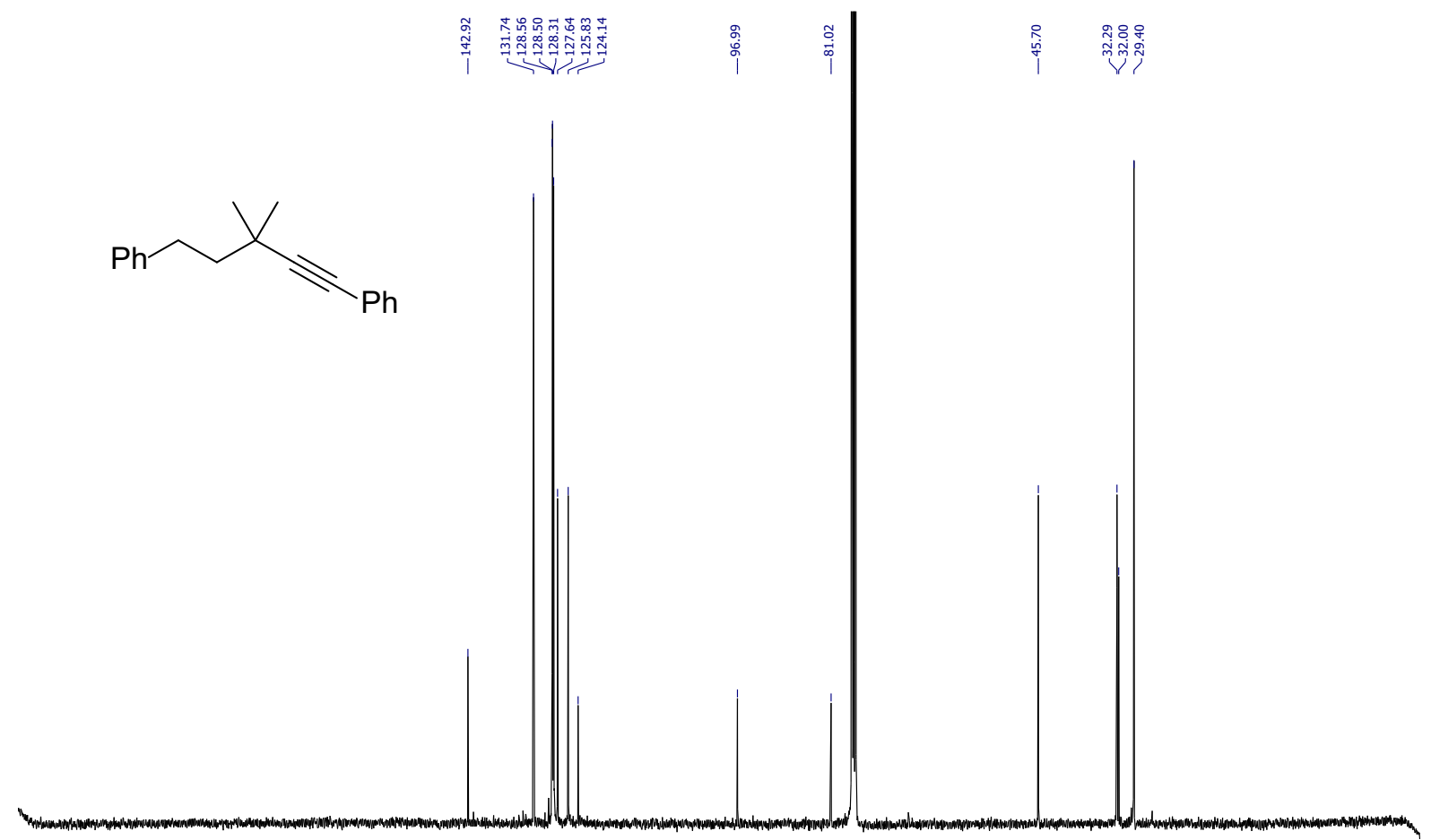

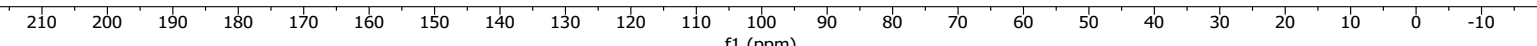




\section{Compound $\mathbf{4 b}$}

${ }^{1} \mathrm{H} \mathrm{NMR}, \mathrm{CDCl}_{3}, 400 \mathrm{MHz}$

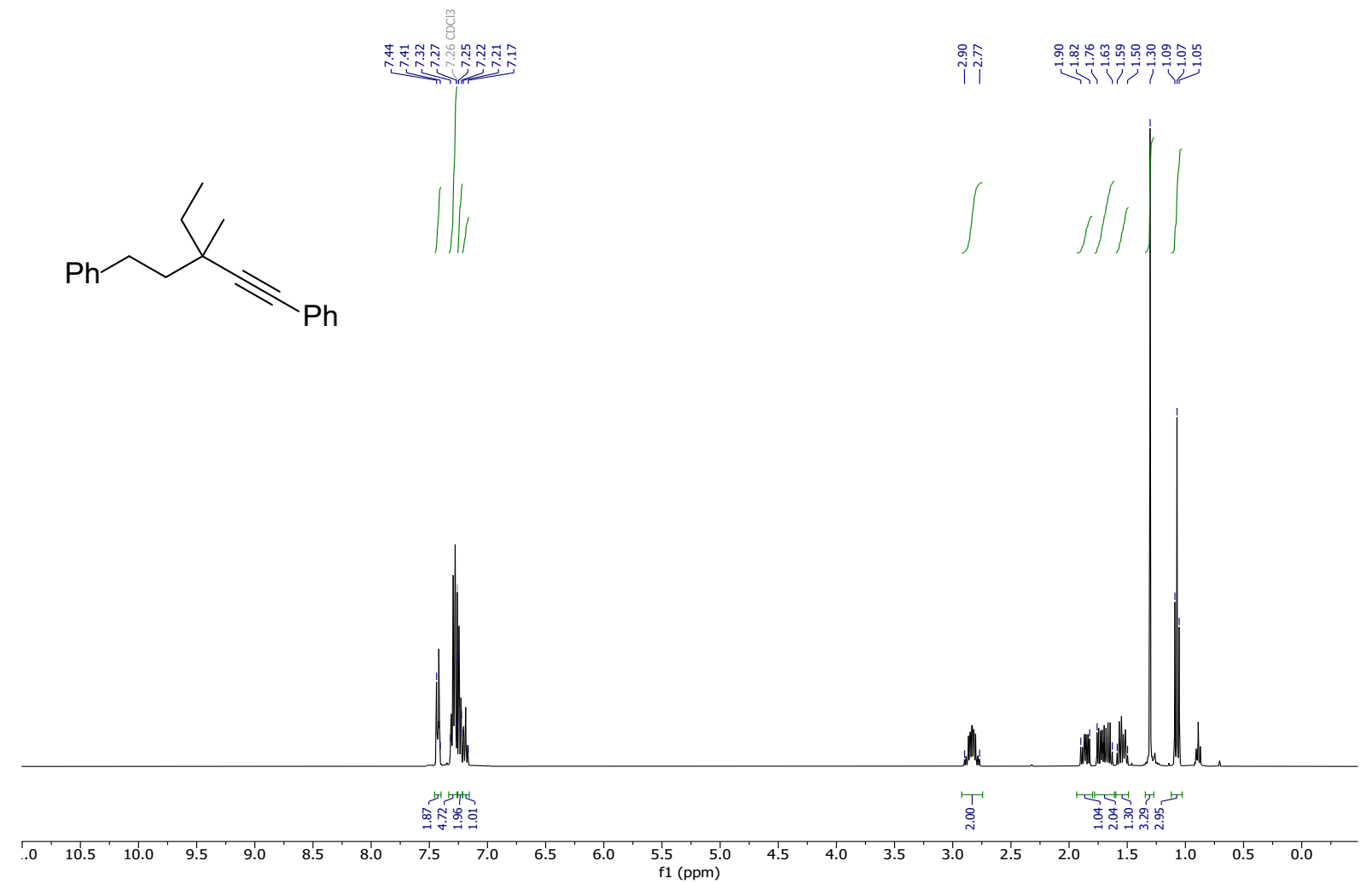

${ }^{13} \mathrm{CNMR}, \mathrm{CDCl}_{3}, 101 \mathrm{MHz}$

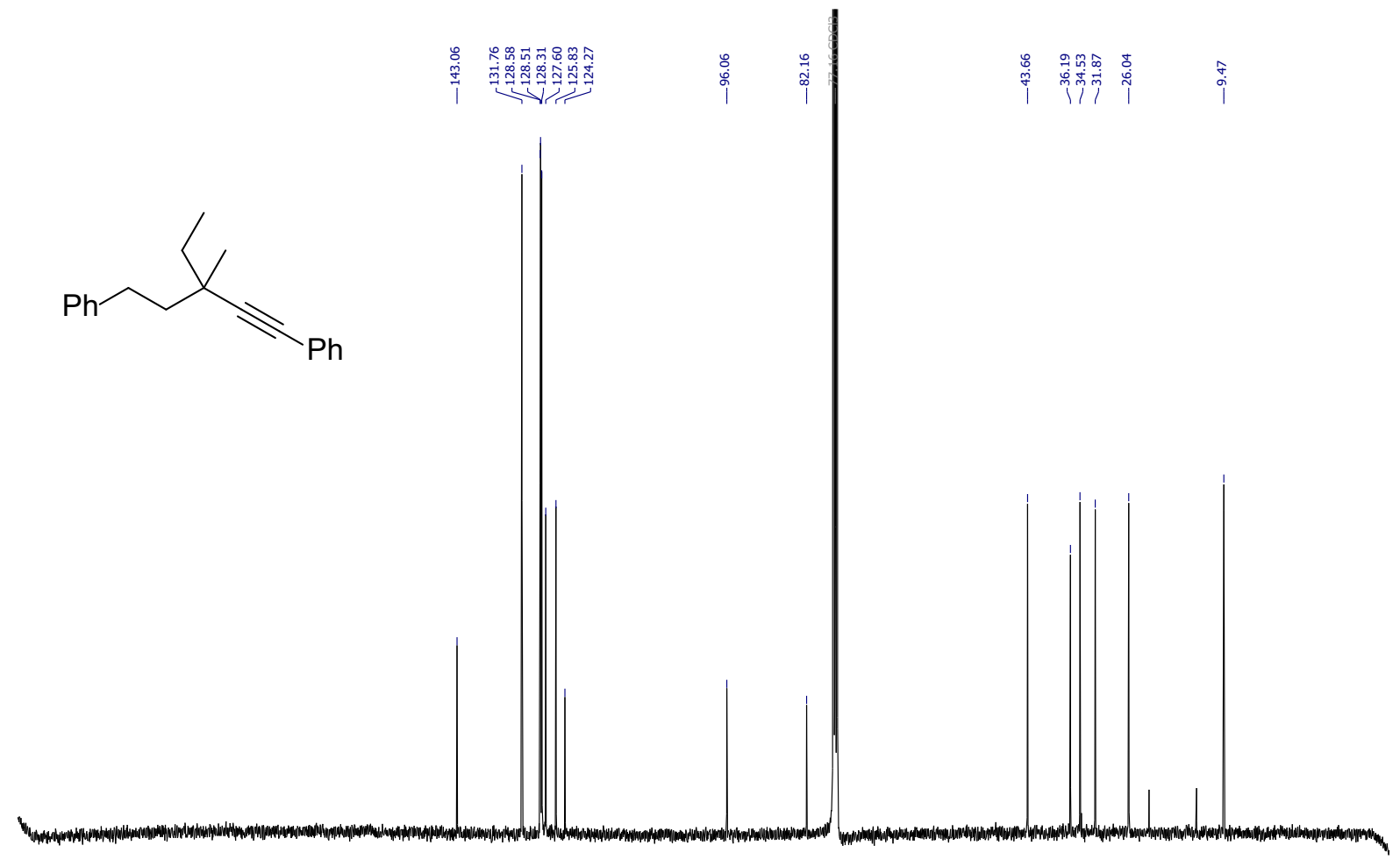

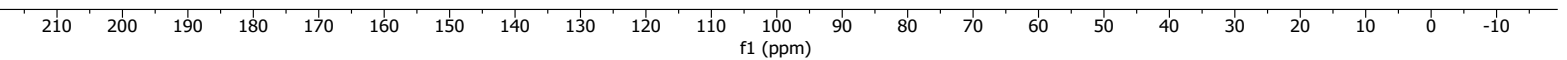




\section{Compound $4 \mathrm{c}$}

${ }^{1} \mathrm{H} \mathrm{NMR}, \mathrm{CDCl}_{3}, 400 \mathrm{MHz}$

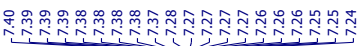<smiles>CC(C)(C)C#Cc1ccccc1</smiles><smiles>C1=CC=C1</smiles>

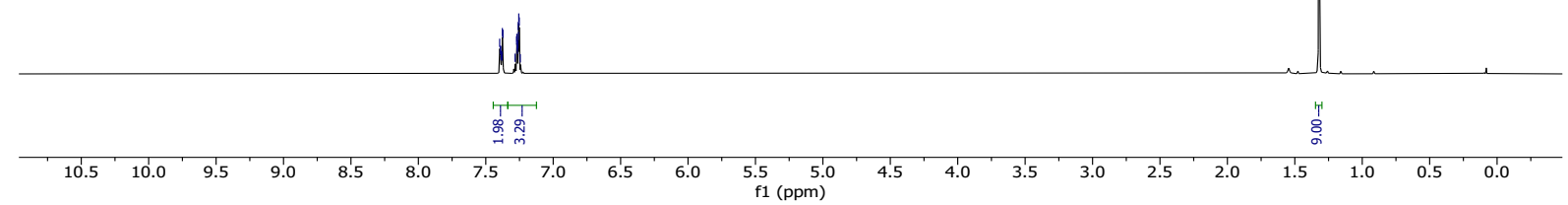

${ }^{13} \mathrm{C} \mathrm{NMR}, \mathrm{CDCl}_{3}, 101 \mathrm{MHz}$

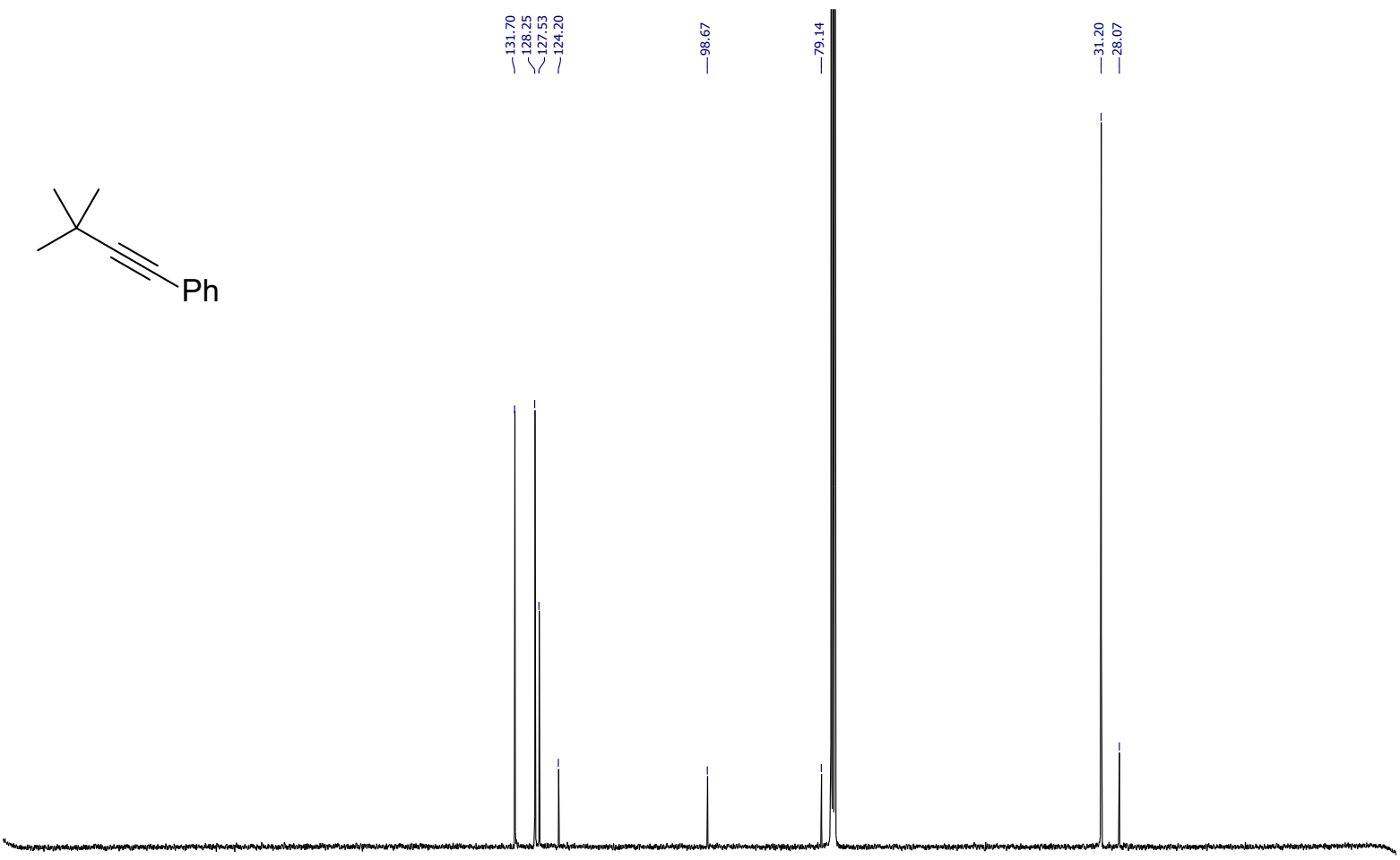

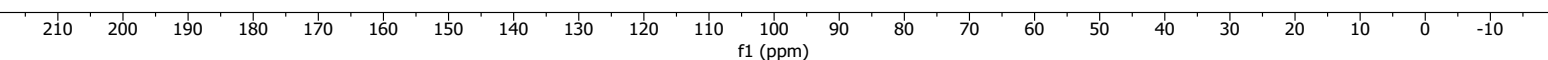




\section{Compound 4d}

${ }^{1} \mathrm{H} \mathrm{NMR}, \mathrm{CDCl}_{3}, 400 \mathrm{MHz}$

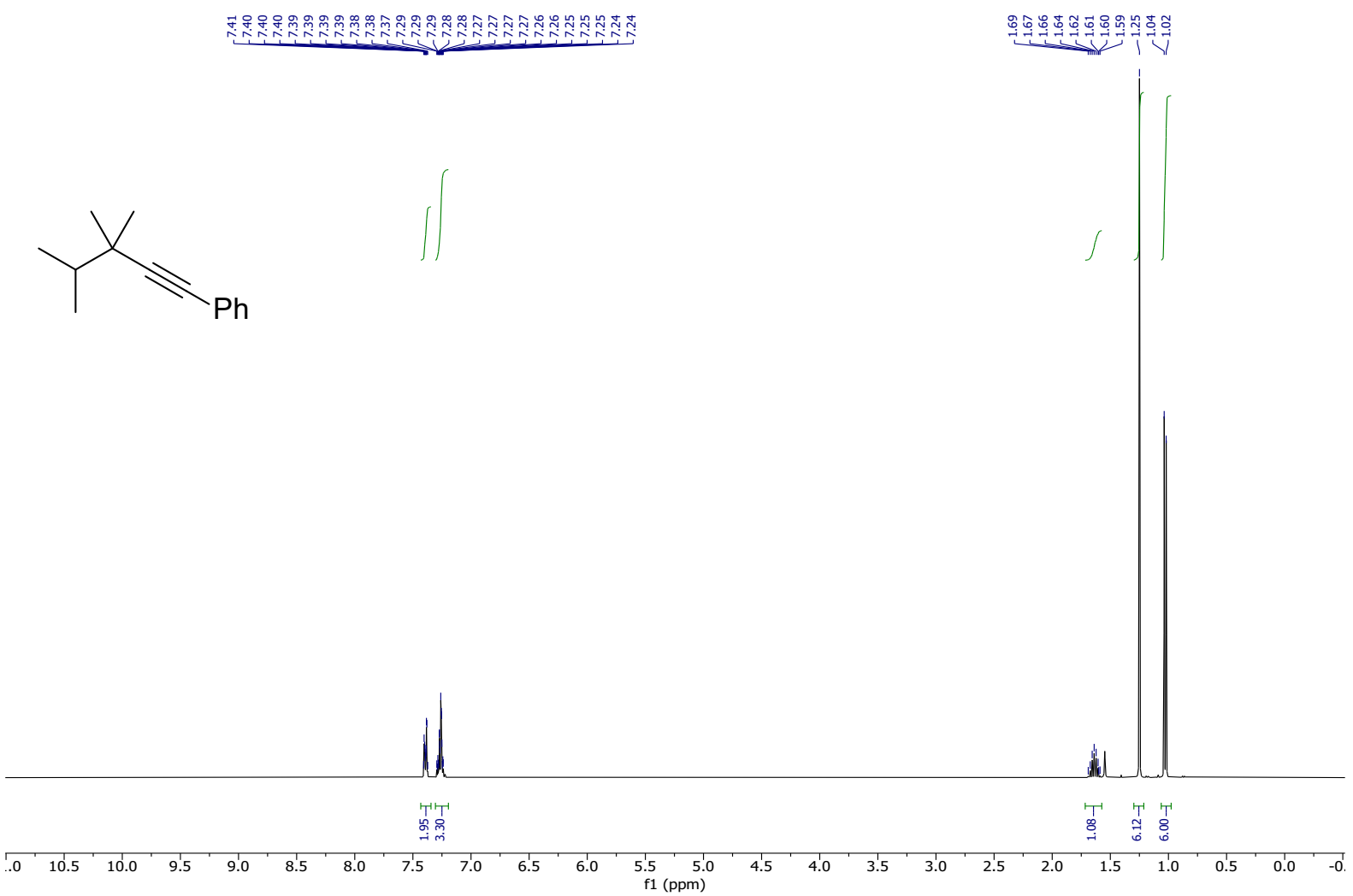

${ }^{13} \mathrm{C} \mathrm{NMR}, \mathrm{CDCl}_{3}, 101 \mathrm{MHz}$

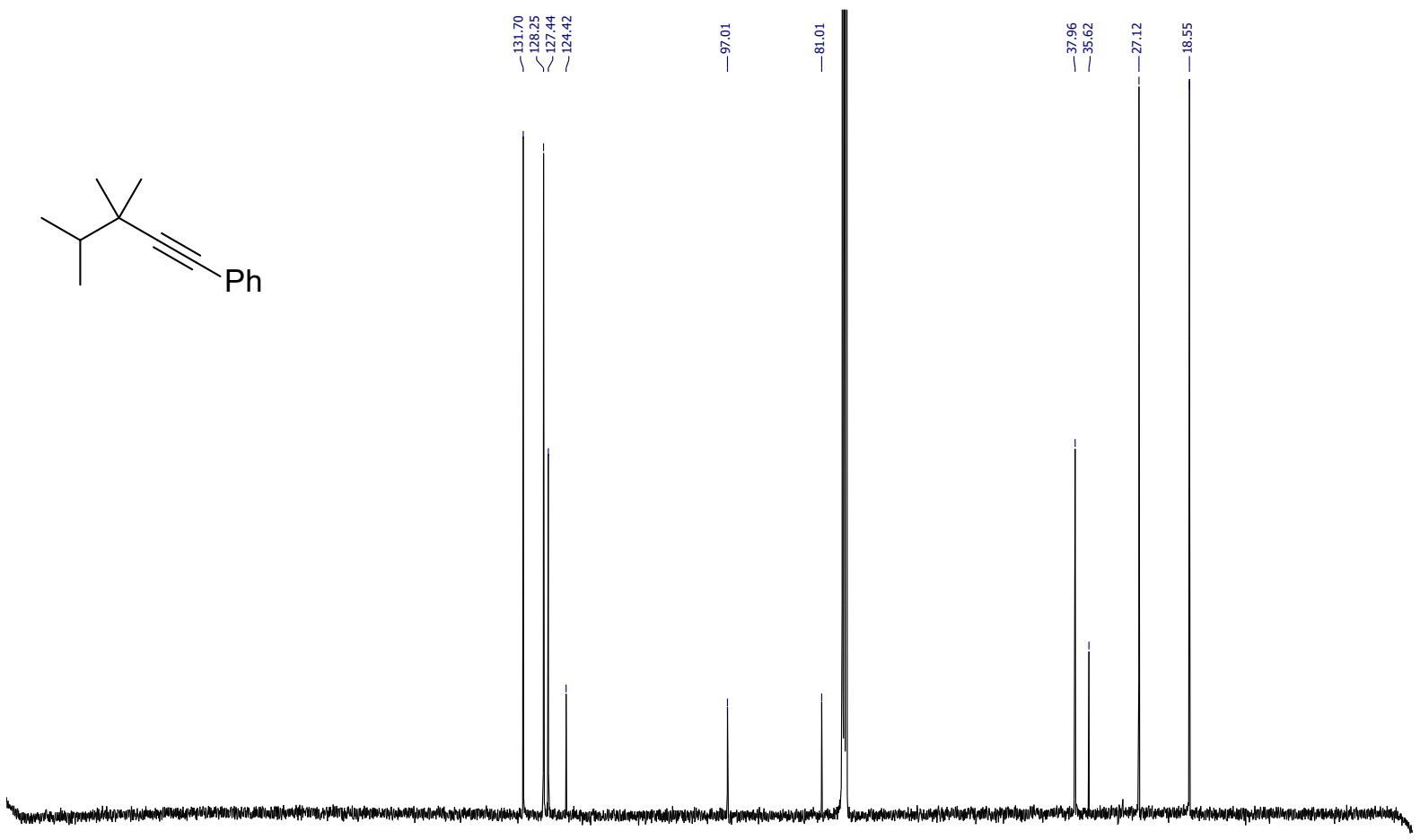

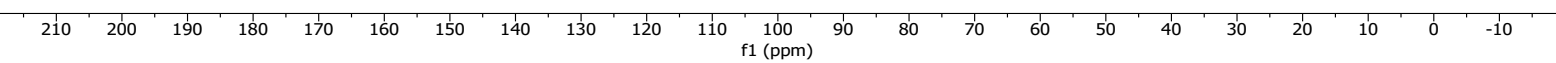


Compound $4 \mathrm{e}$

${ }^{1} \mathrm{H} \mathrm{NMR}, \mathrm{CDCl}_{3}, 400 \mathrm{MHz}$

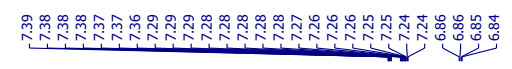

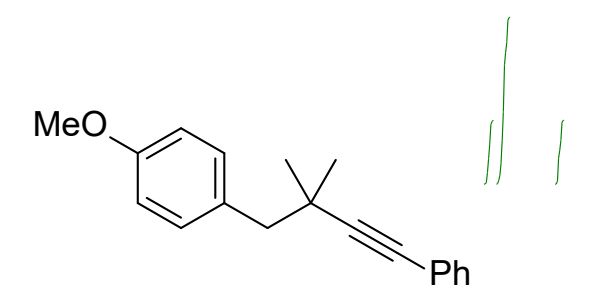

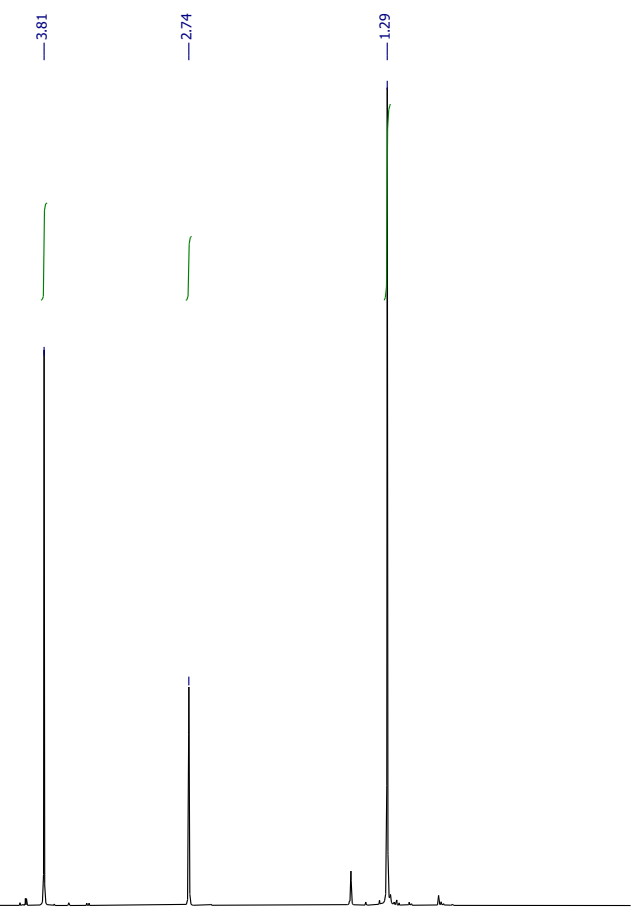

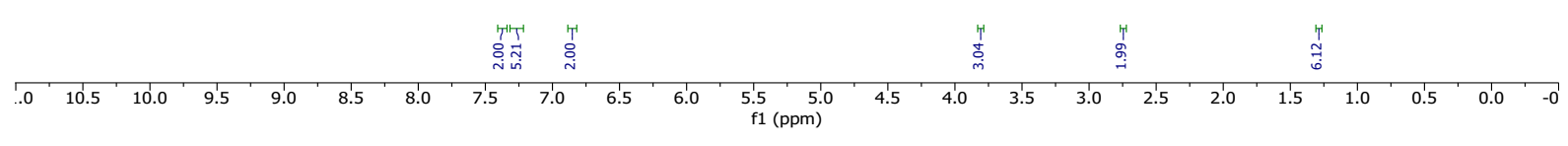

${ }^{13} \mathrm{C} \mathrm{NMR}, \mathrm{CDCl}_{3}, 101 \mathrm{MHz}$

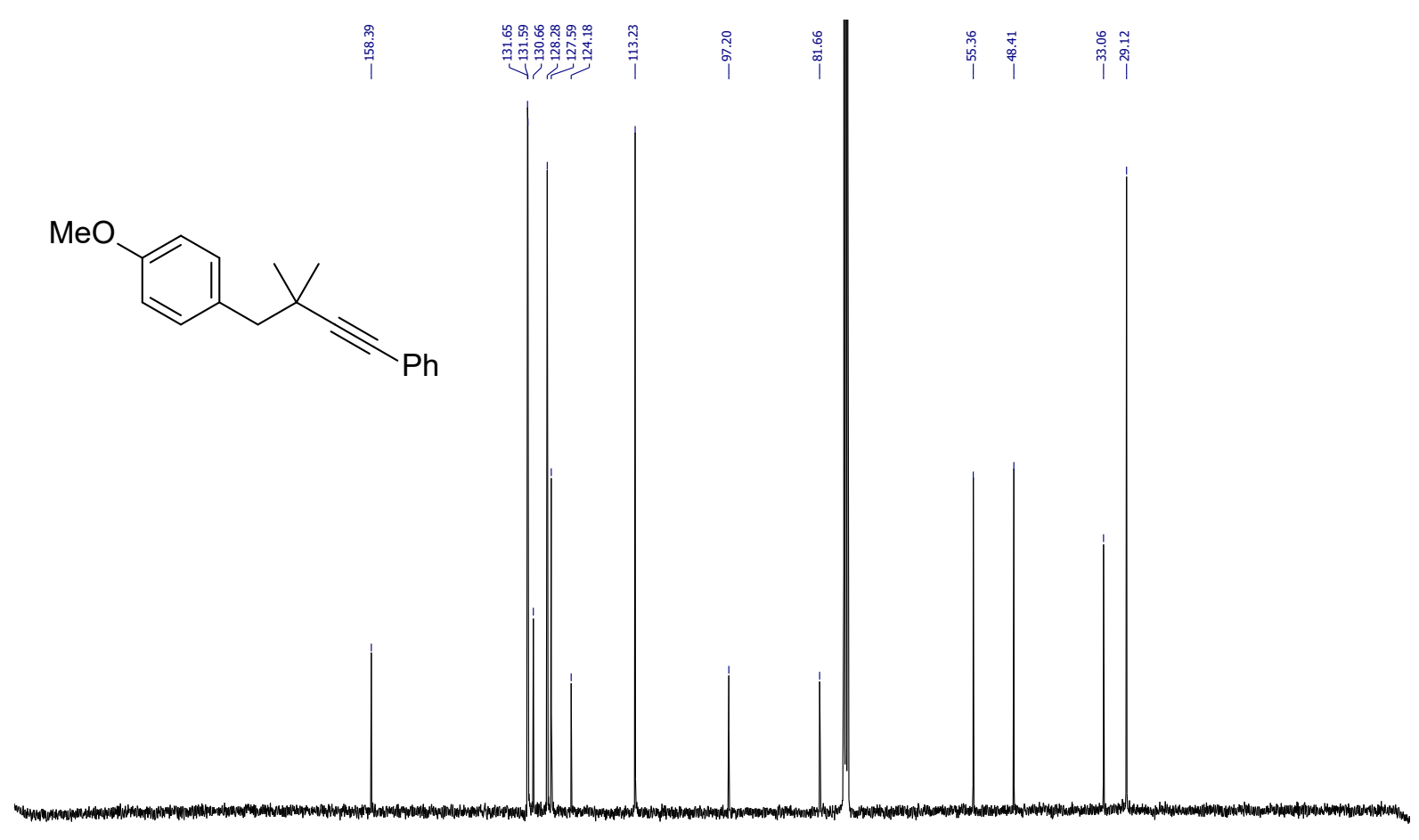

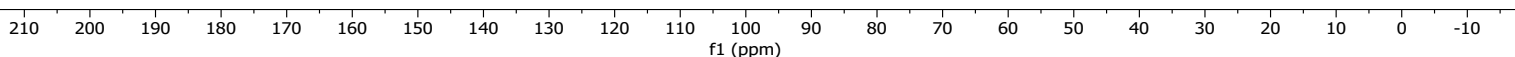

S106 
Compound $\mathbf{4 f}$

${ }^{1} \mathrm{H} \mathrm{NMR}, \mathrm{CDCl}_{3}, 400 \mathrm{MHz}$

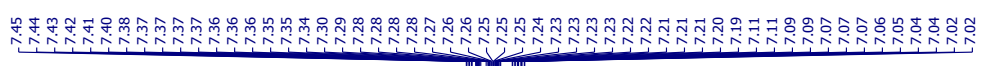

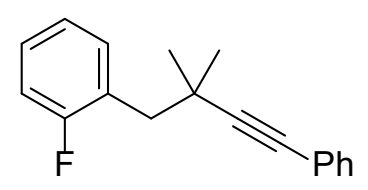

कุल

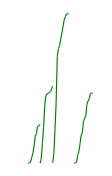

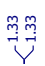
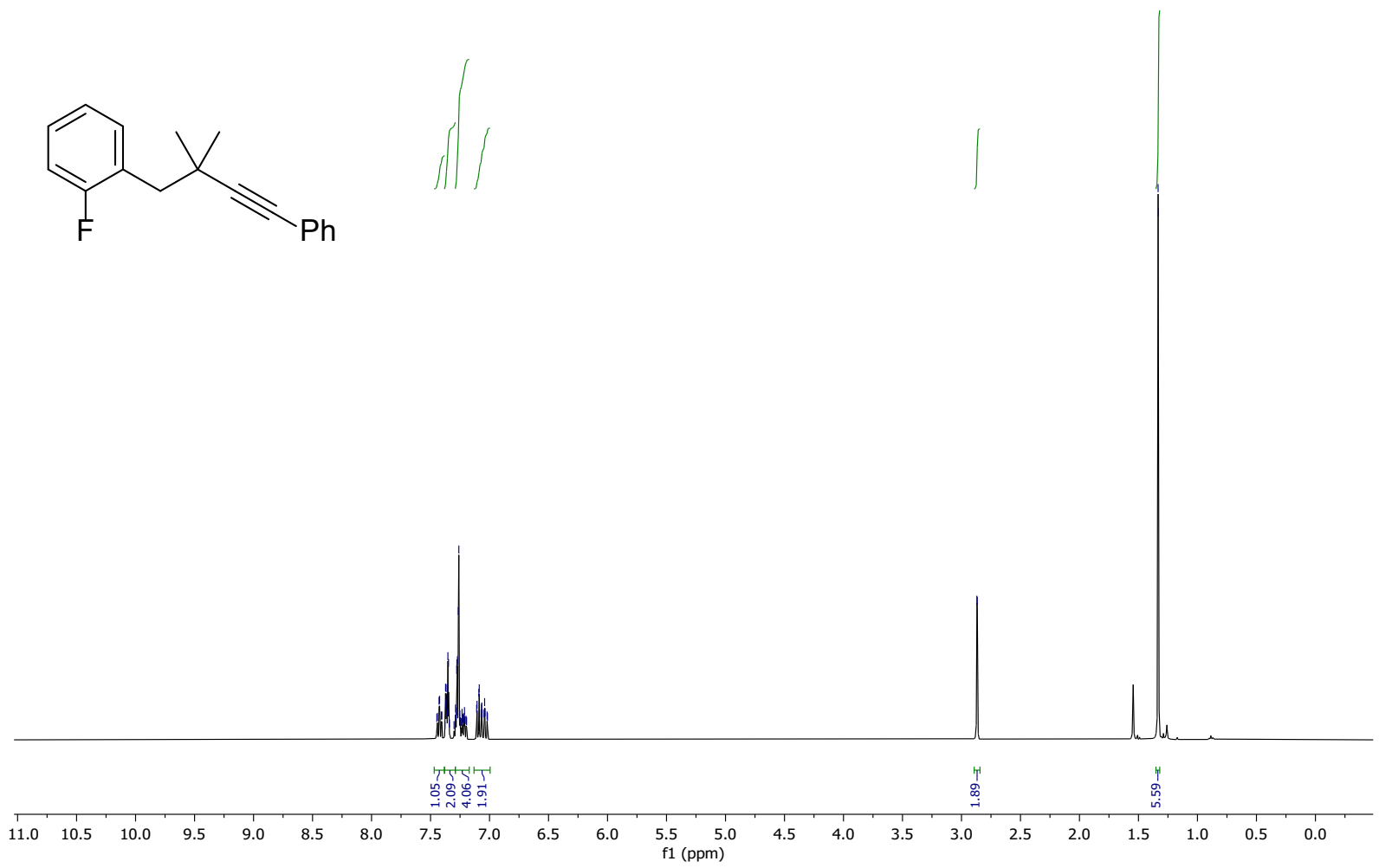

${ }^{13} \mathrm{C} \mathrm{NMR}, \mathrm{CDCl}_{3}, 101 \mathrm{MHz}$

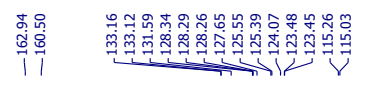

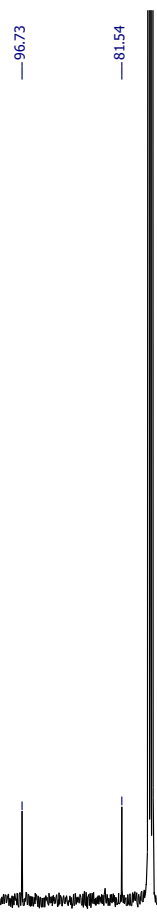

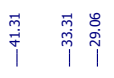
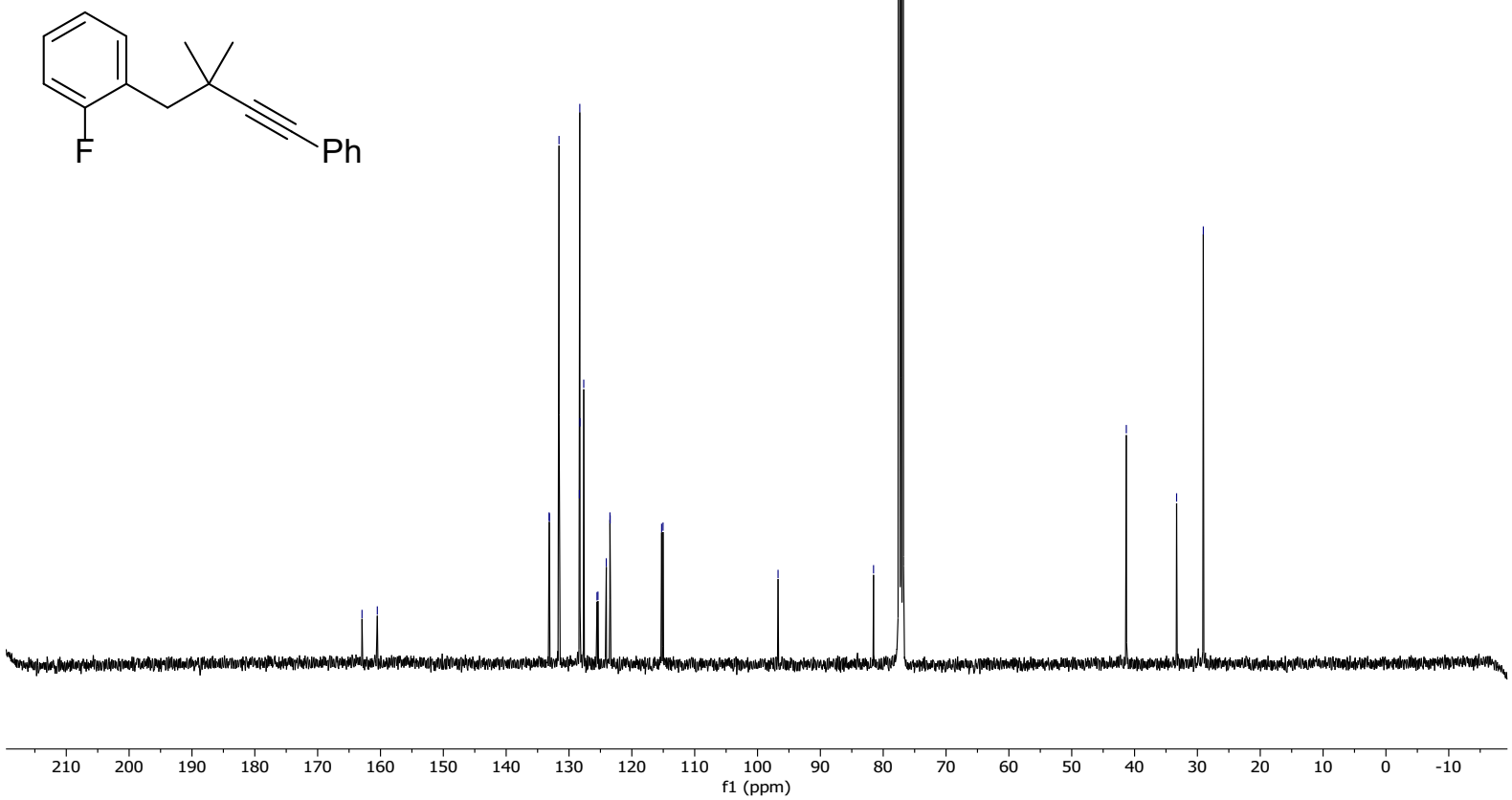

S107 
${ }^{19} \mathrm{~F} \mathrm{NMR,} \mathrm{CDCl}_{3}, 376 \mathrm{MHz}$

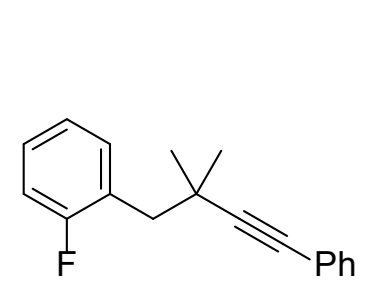

$\begin{array}{lllllllllllllllllllllllll}10 & 1 & -10 & -20 & -30 & -40 & -50 & -60 & -70 & -80 & -90 & -100 & -110 & -120 & -130 & -140 & -150 & -160 & -170 & -180 & -190 & -200 & -210 & 1\end{array}$ 


\section{Compound 4g}

${ }^{1} \mathrm{H} \mathrm{NMR}, \mathrm{CDCl}_{3}, 400 \mathrm{MHz}$

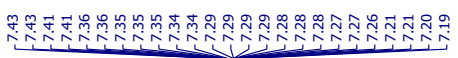

$\mathrm{Br}$<smiles>Cc1ccc(CC(C)(C)C#CP)cc1</smiles>
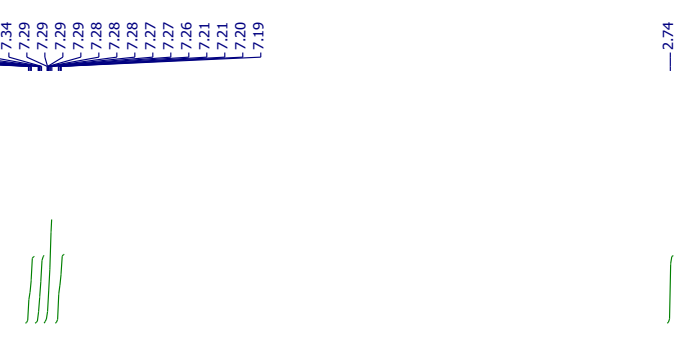

$\mathrm{Ph}$

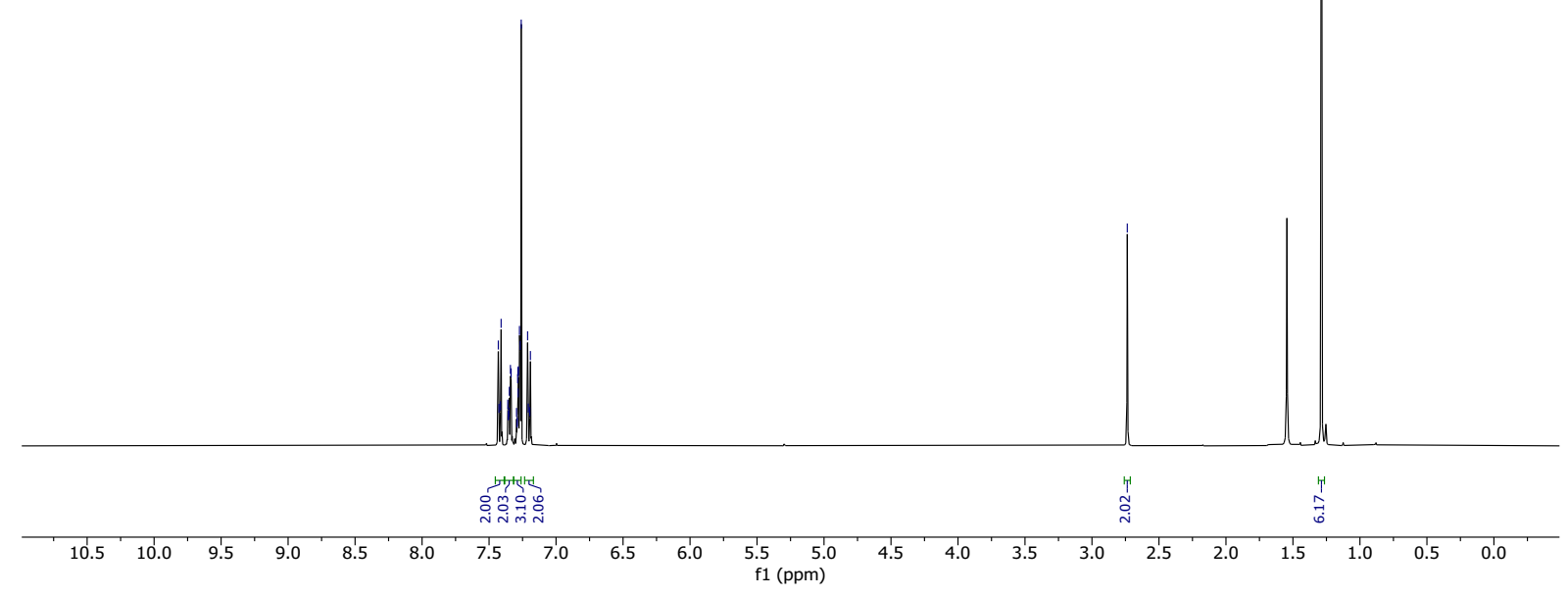

${ }^{13} \mathrm{C} \mathrm{NMR}, \mathrm{CDCl}_{3}, 101 \mathrm{MHz}$

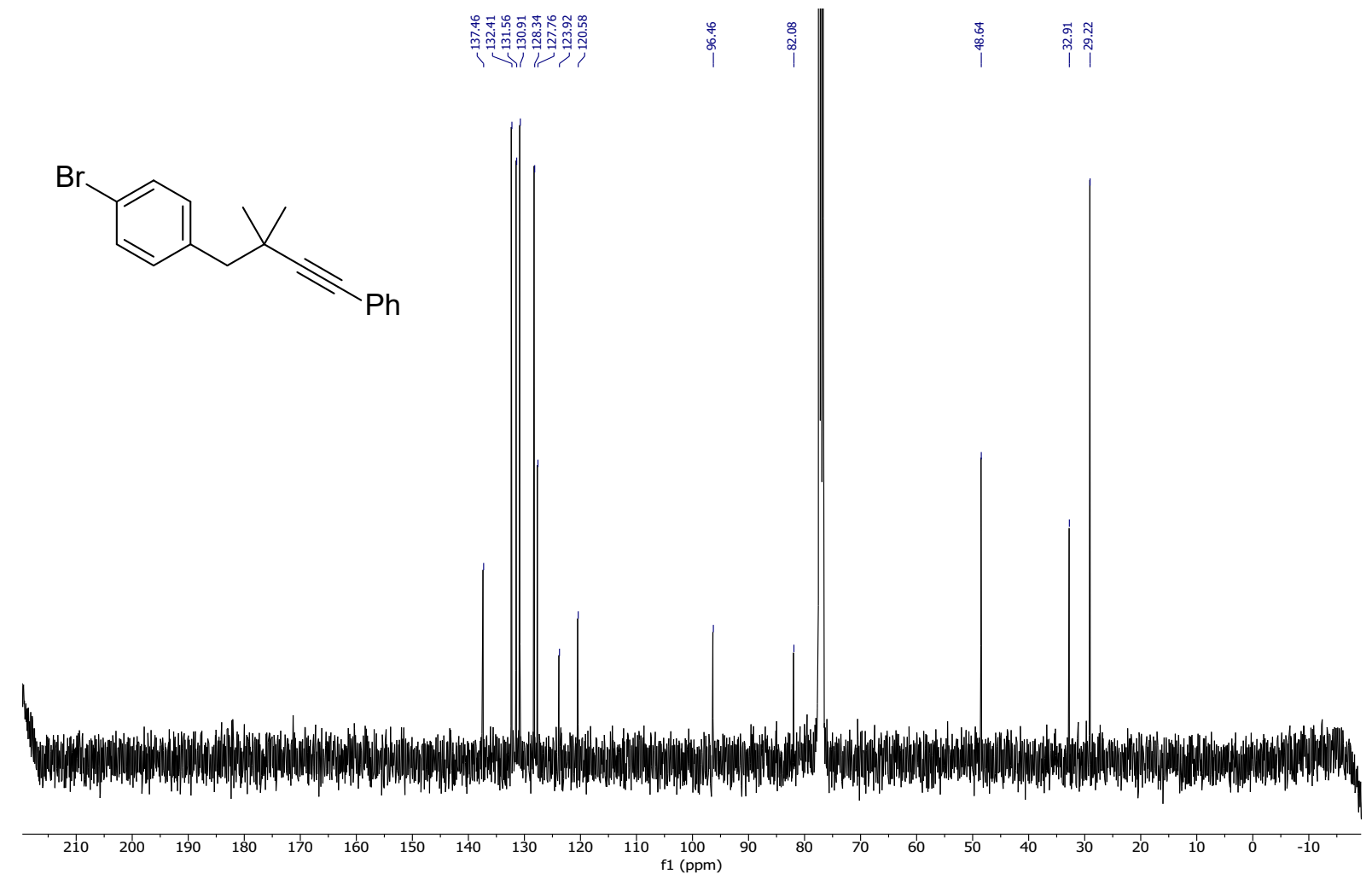




\section{Compound $\mathbf{4 h}$}

${ }^{1} \mathrm{H} \mathrm{NMR}, \mathrm{CDCl}_{3}, 400 \mathrm{MHz}$

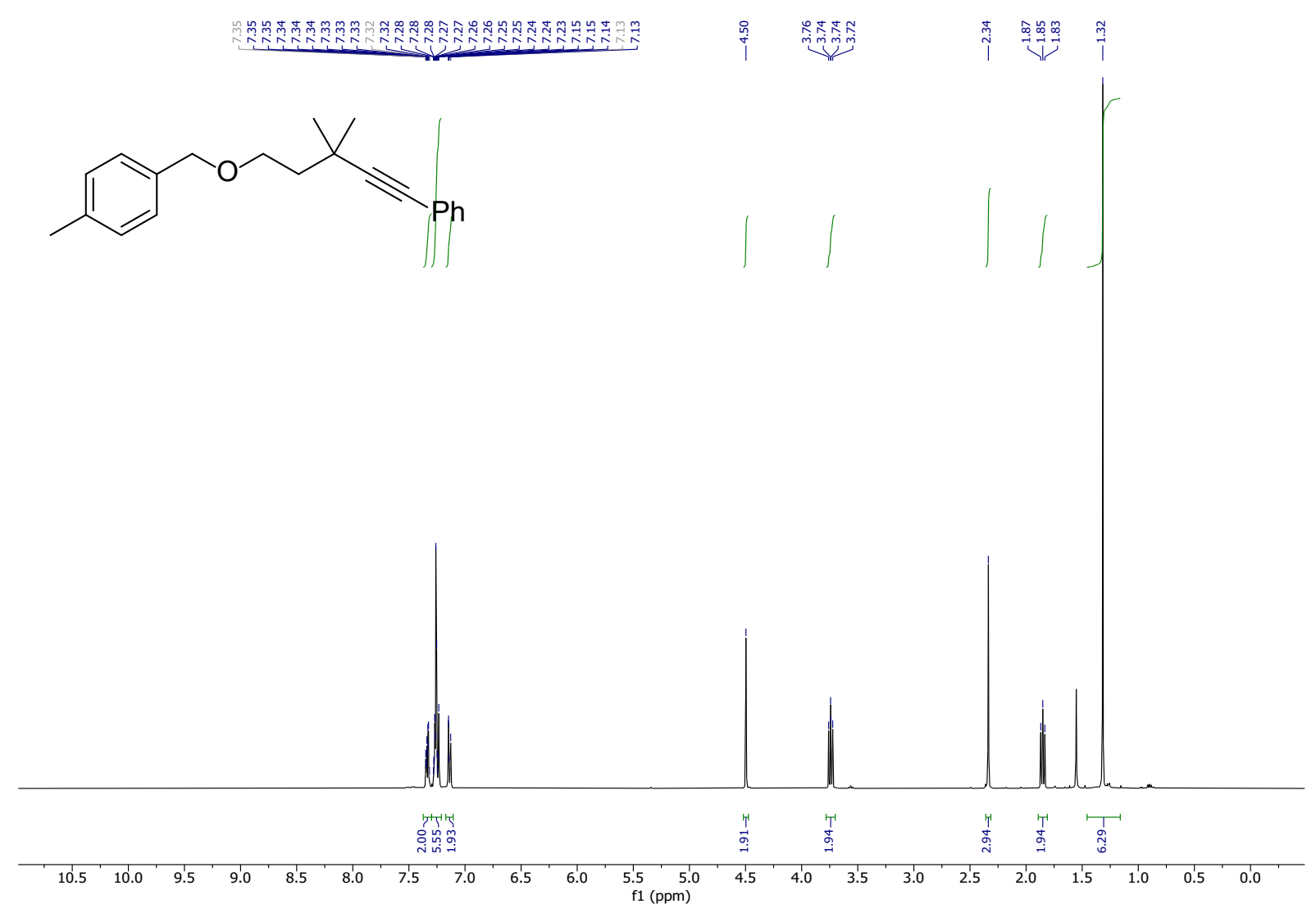

${ }^{13} \mathrm{CNMR}_{\mathrm{N}} \mathrm{CDCl}_{3}, 101 \mathrm{MHz}$

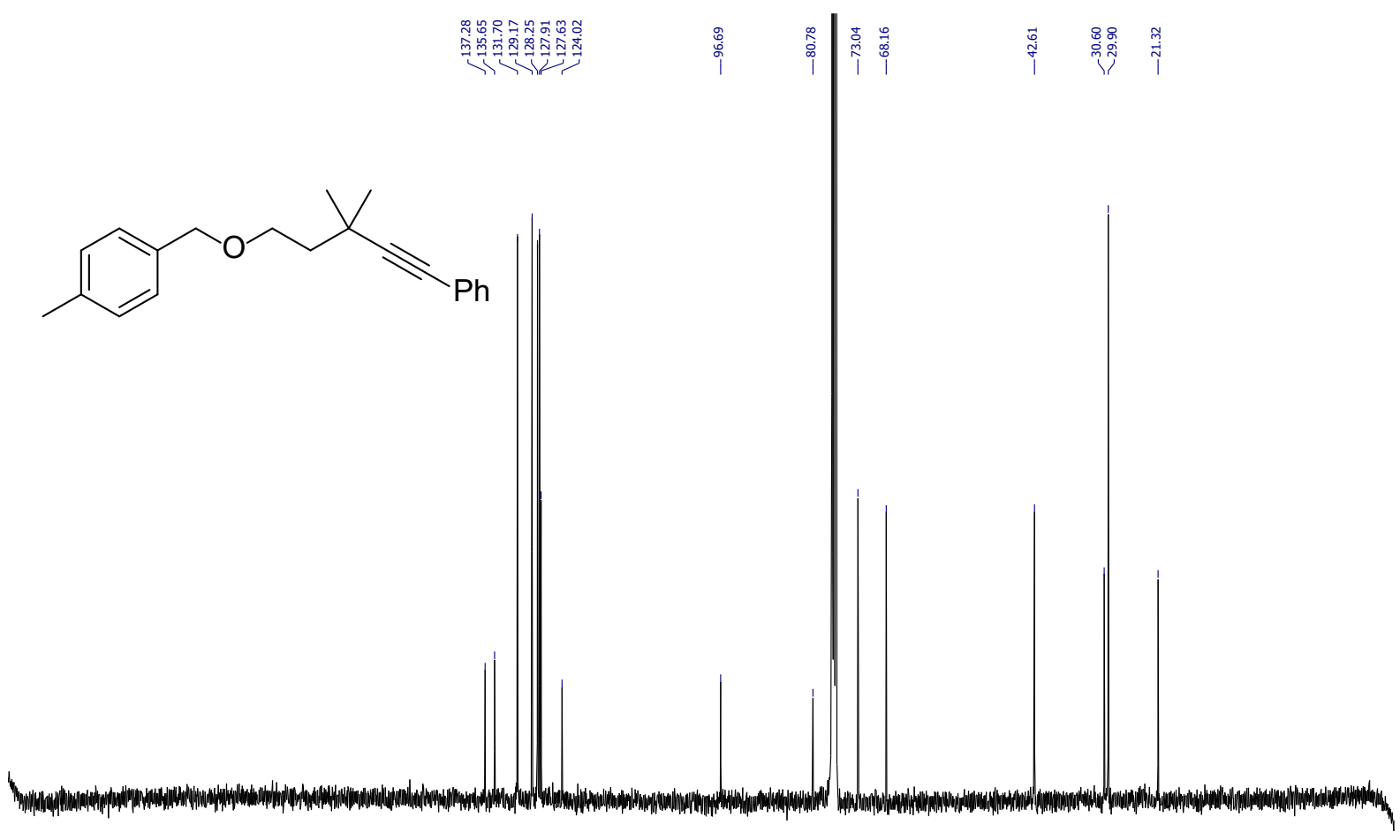

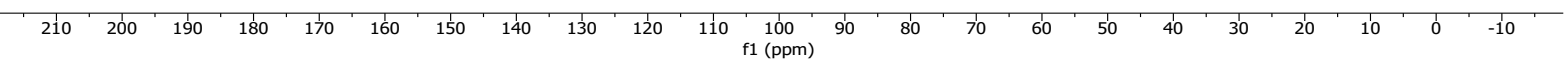




\section{Compound 4i}

${ }^{1} \mathrm{H} \mathrm{NMR}, \mathrm{CDCl}_{3}, 400 \mathrm{MHz}$

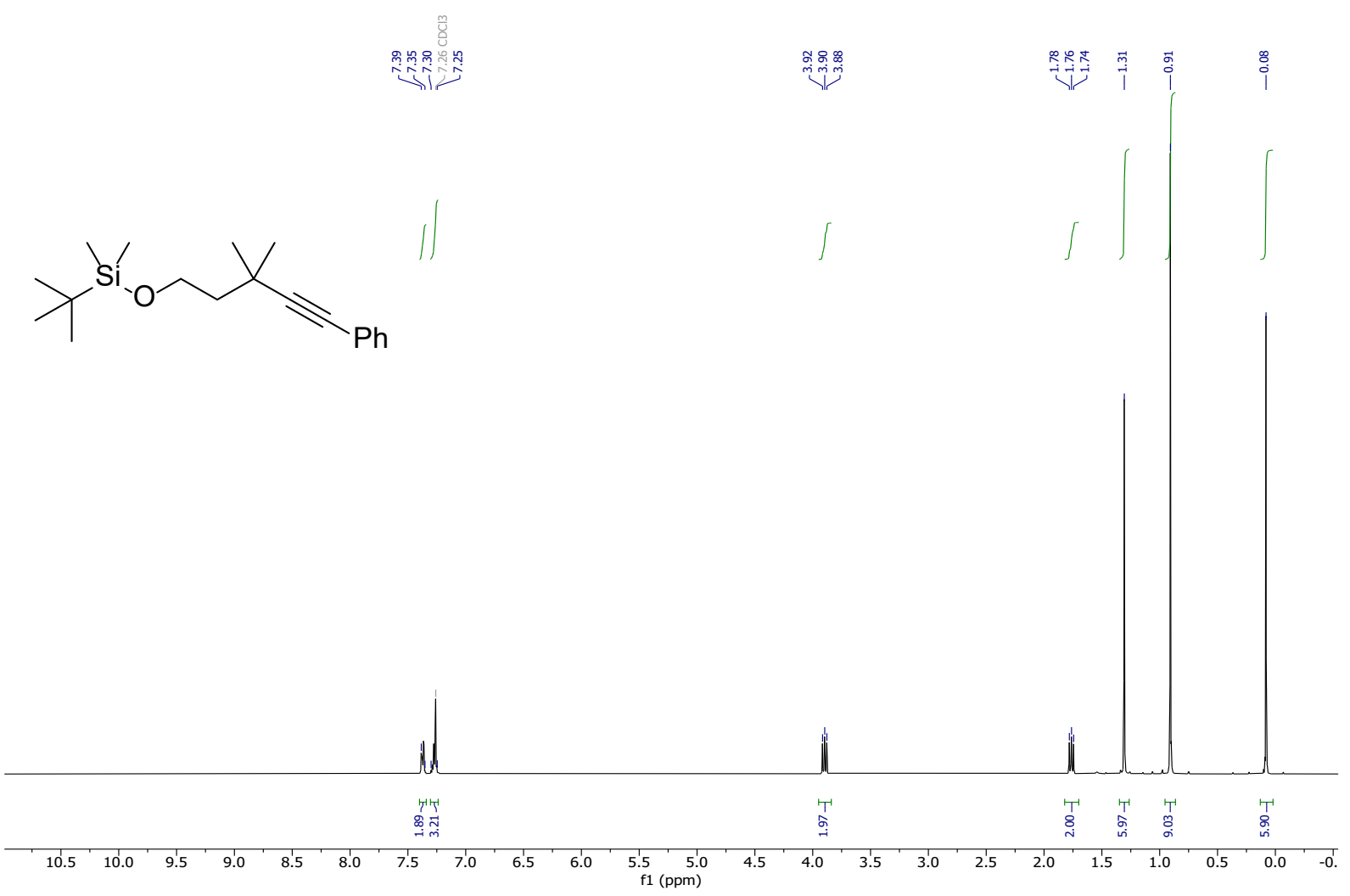

${ }^{13} \mathrm{C} \mathrm{NMR}, \mathrm{CDCl}_{3}, 101 \mathrm{MHz}$

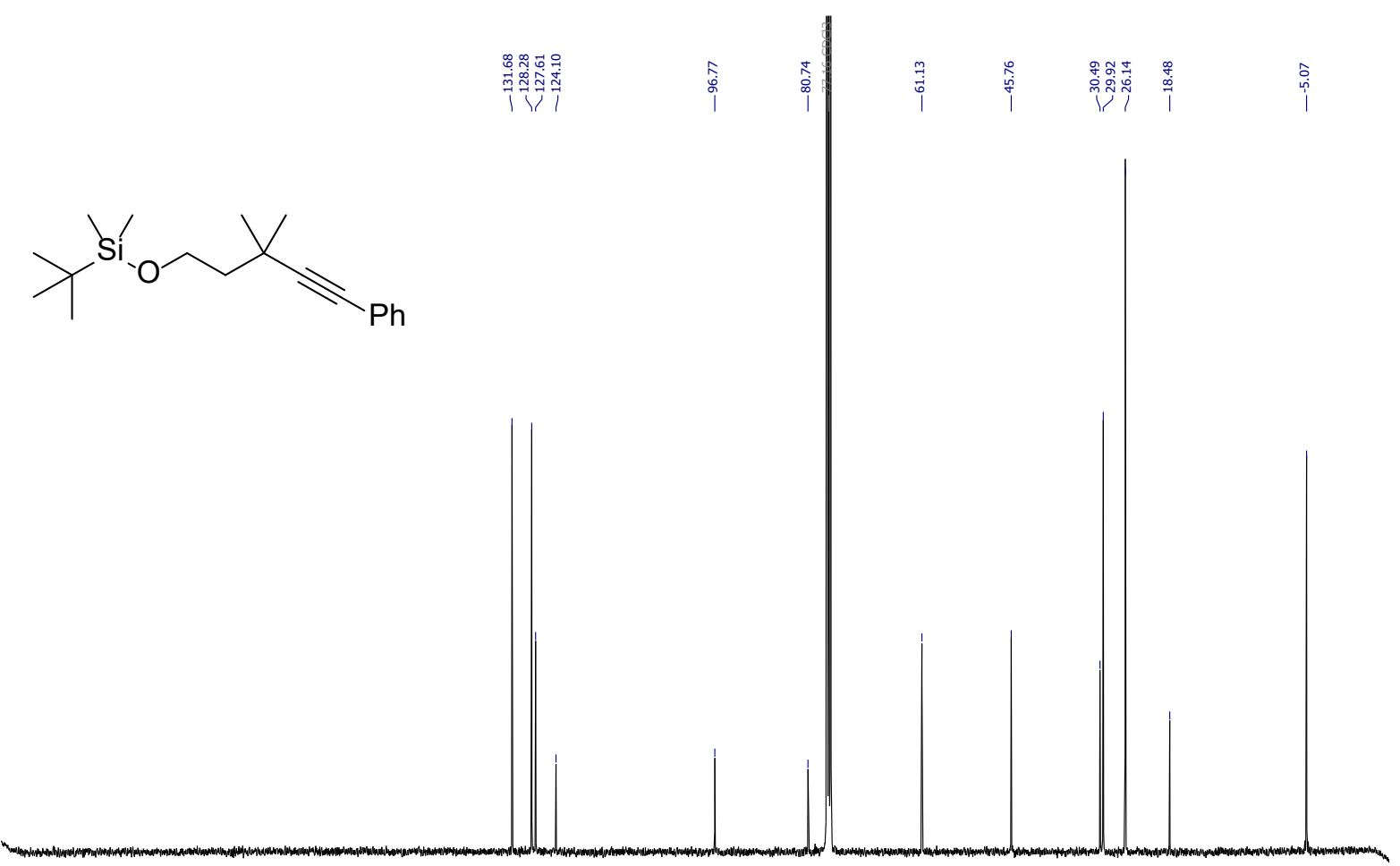

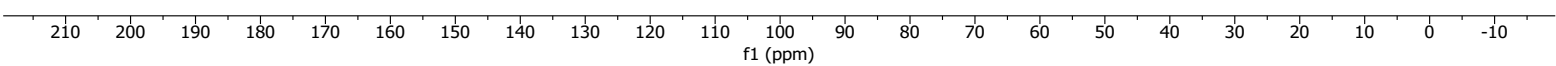




\section{Compound 4j}

${ }^{1} \mathrm{H} \mathrm{NMR}, \mathrm{CDCl}_{3}, 400 \mathrm{MHz}$

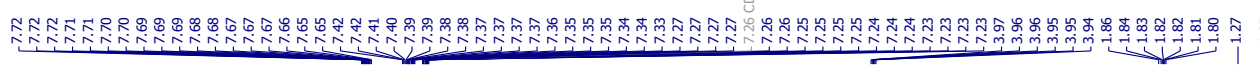

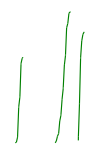<smiles>CC(C)(C#Cc1ccccc1)CCO[Si](c1ccccc1)(c1ccccc1)C(C)(C)C</smiles>

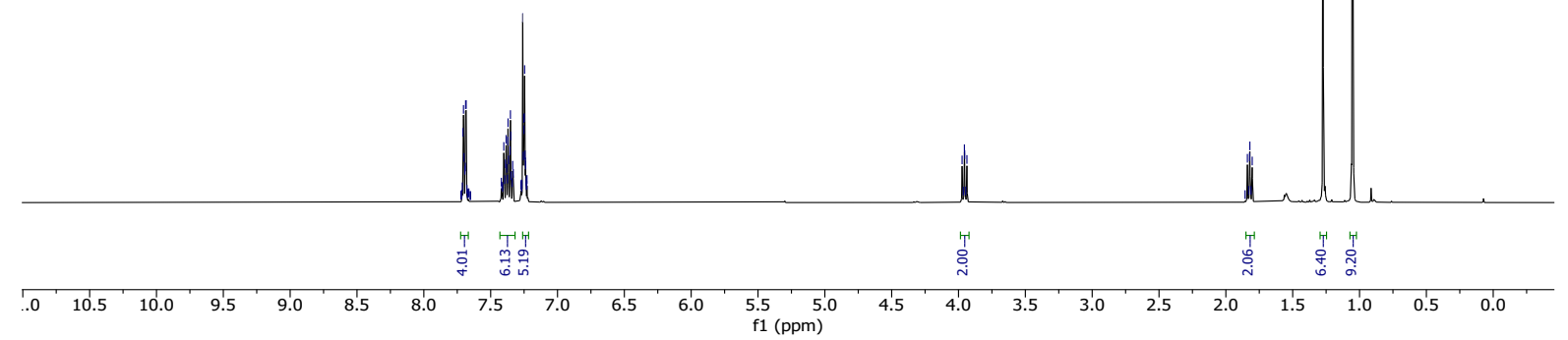

${ }^{13} \mathrm{C} \mathrm{NMR}, \mathrm{CDCl}_{3}, 101 \mathrm{MHz}$

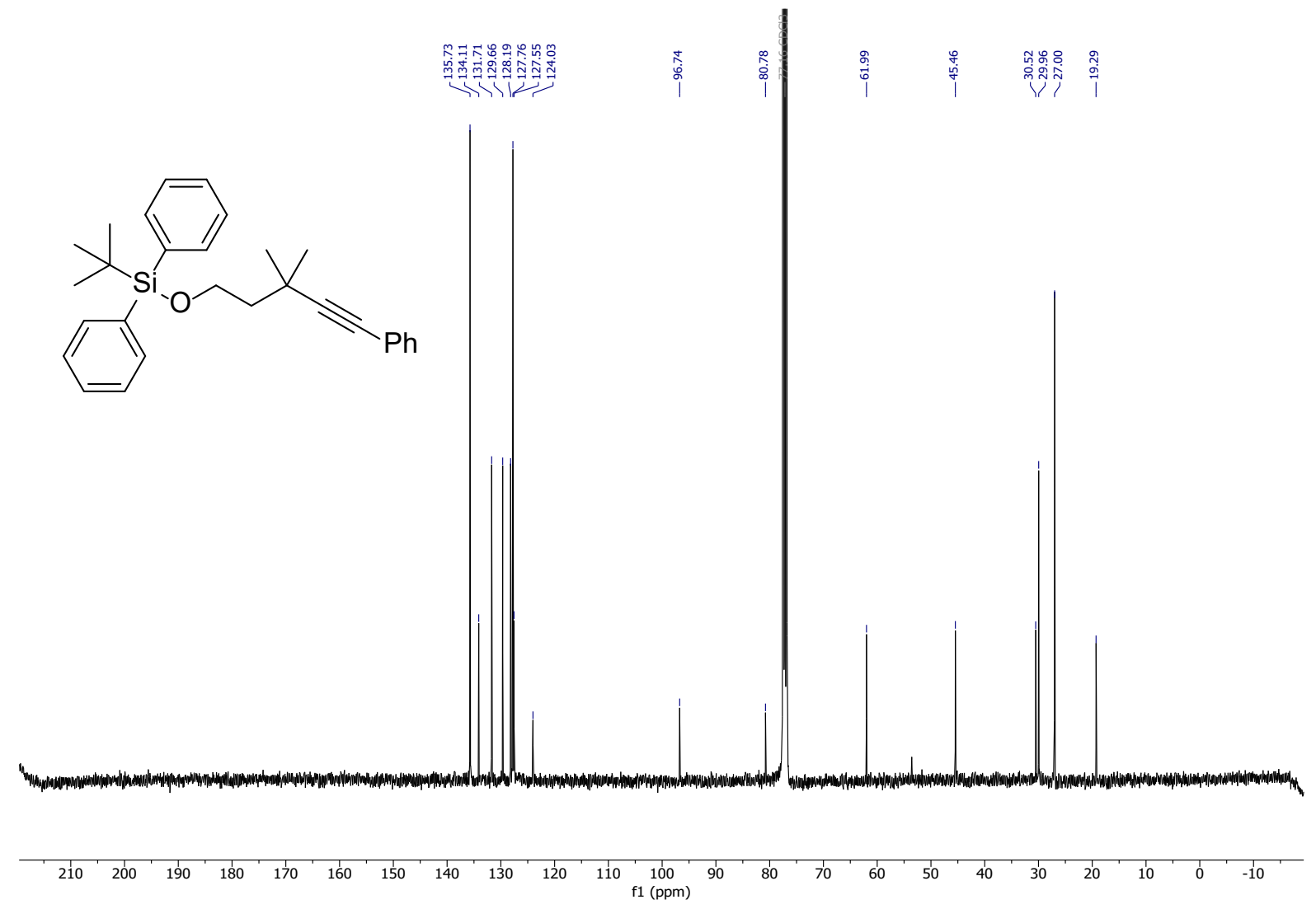




\section{Compound $\mathbf{4 k}$}

${ }^{1} \mathrm{H} \mathrm{NMR}, \mathrm{CDCl}_{3}, 400 \mathrm{MHz}$
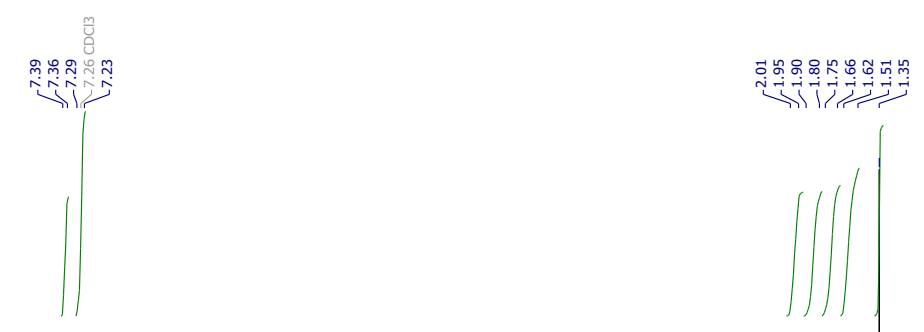

$\gamma_{P h}$

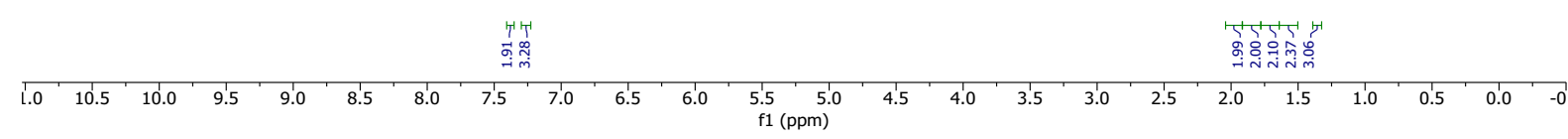

${ }^{13} \mathrm{C} \mathrm{NMR}, \mathrm{CDCl}_{3}, 101 \mathrm{MHz}$
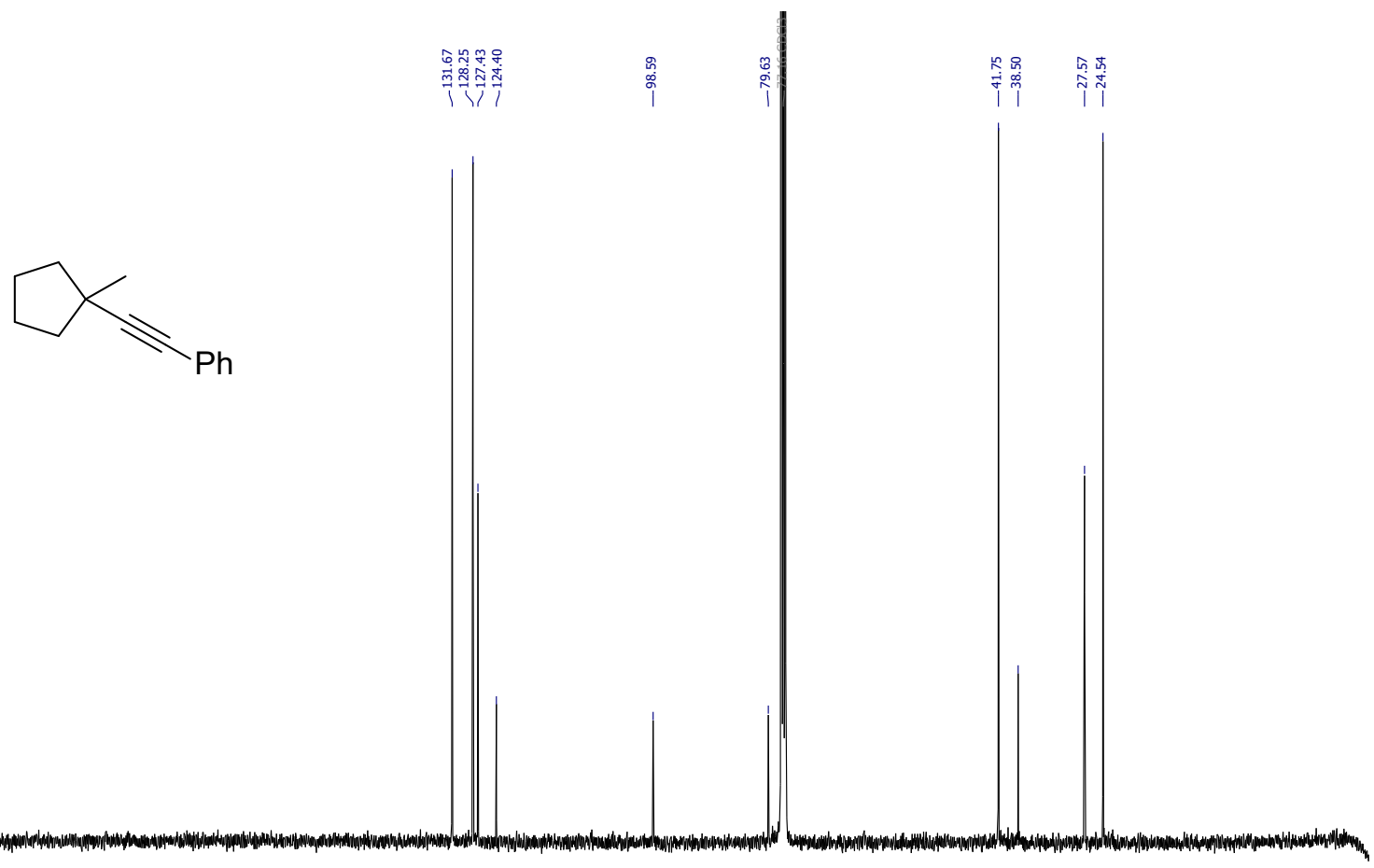

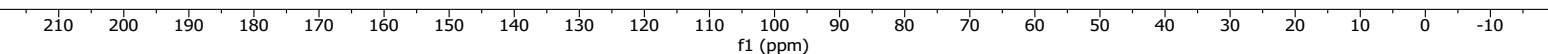




\section{Compound $4 \mathbf{I}$}

${ }^{1} \mathrm{H} \mathrm{NMR}, \mathrm{CDCl}_{3}, 400 \mathrm{MHz}$

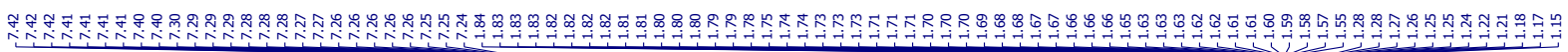
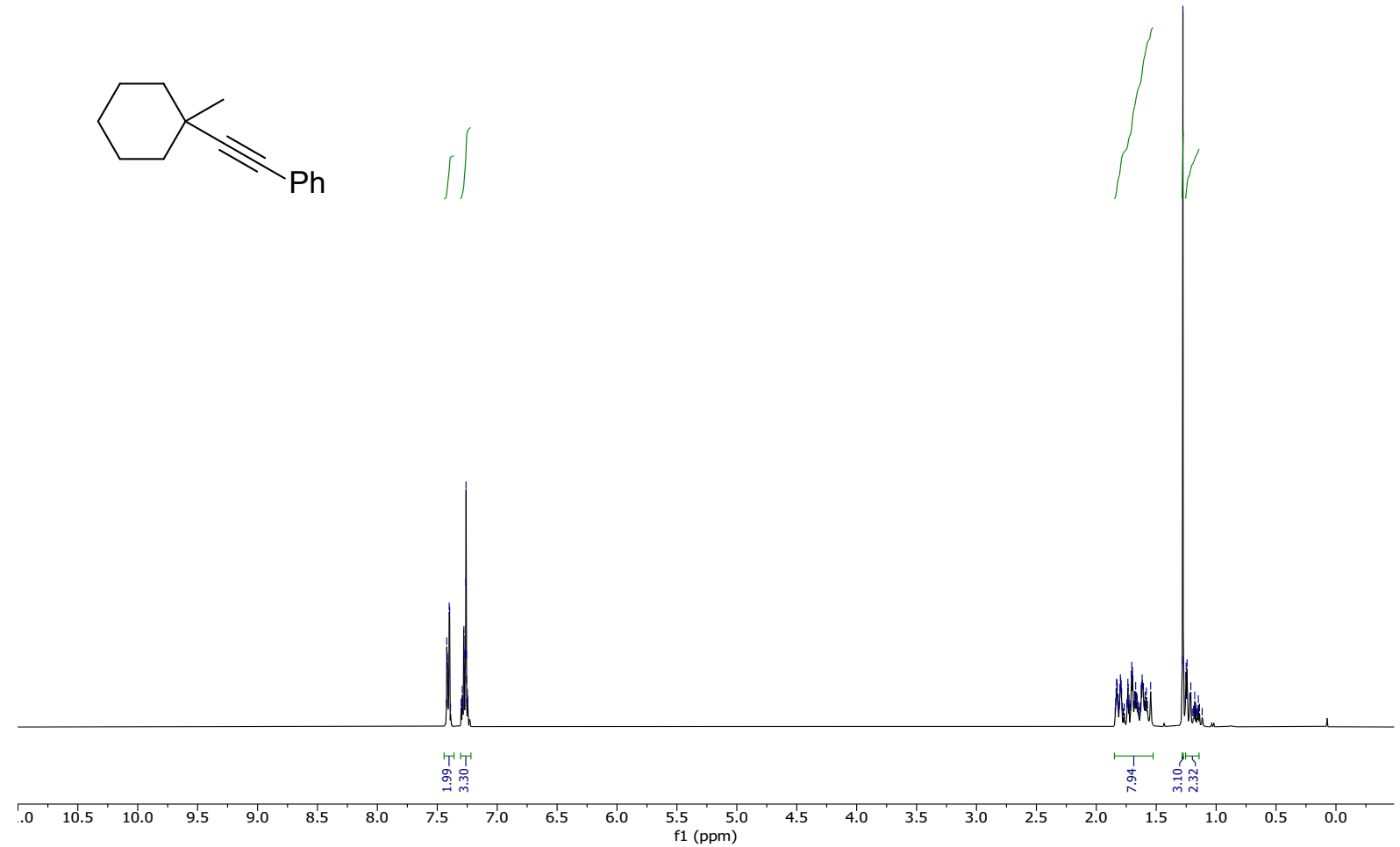

${ }^{13} \mathrm{CNMR}, \mathrm{CDCl}_{3}, 400 \mathrm{MHz}$

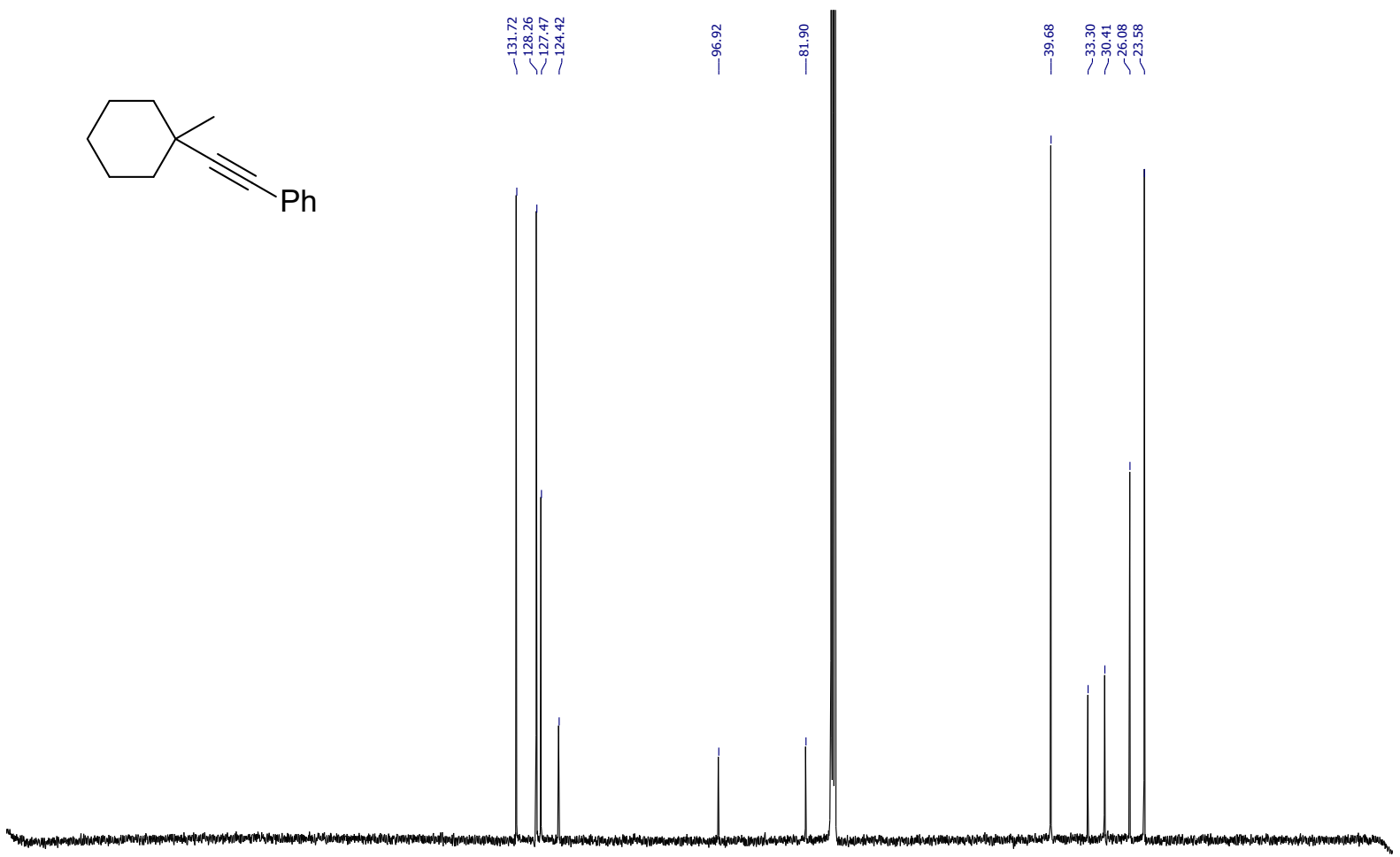

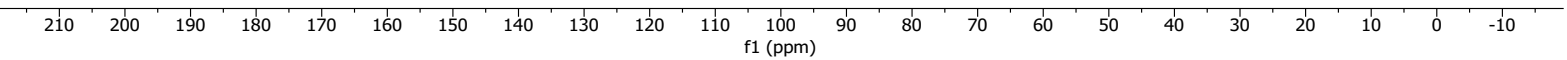




\section{Compound $4 m$}

${ }^{1} \mathrm{H} \mathrm{NMR}, \mathrm{CDCl}_{3}, 400 \mathrm{MHz}$

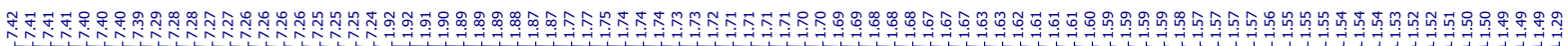<smiles>CC1(C#Cc2ccccc2)CCCCCC1</smiles>
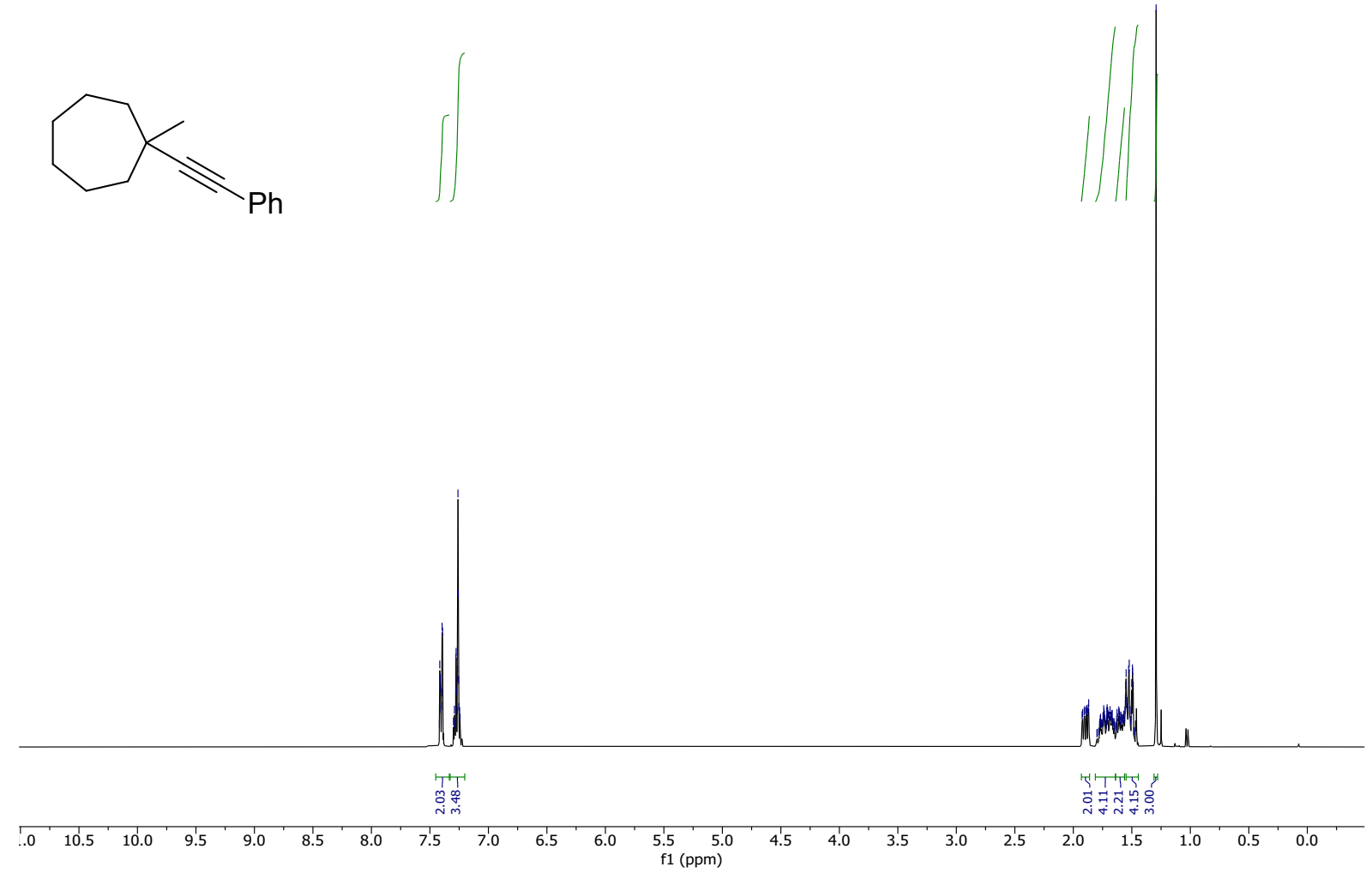

${ }^{13} \mathrm{CNMR}, \mathrm{CDCl}_{3}, 400 \mathrm{MHz}$

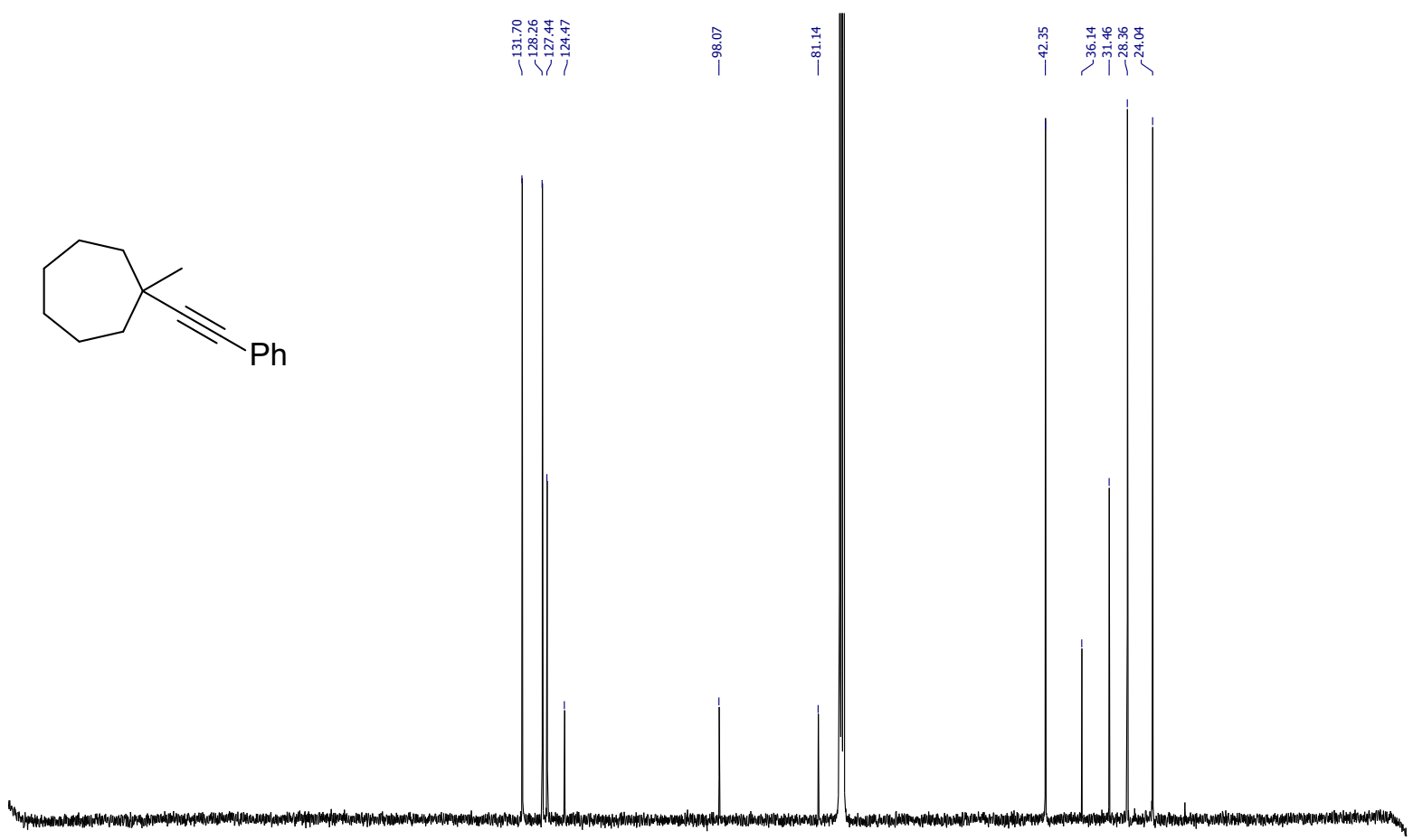

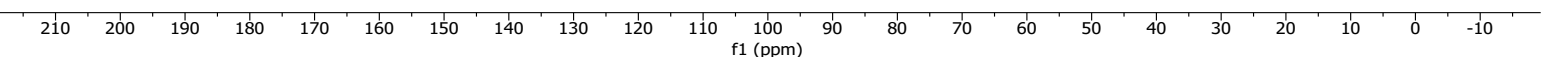




\section{Compound $4 n$}

${ }^{1} \mathrm{H} \mathrm{NMR}, \mathrm{CDCl}_{3}, 400 \mathrm{MHz}$

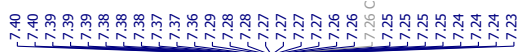

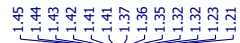
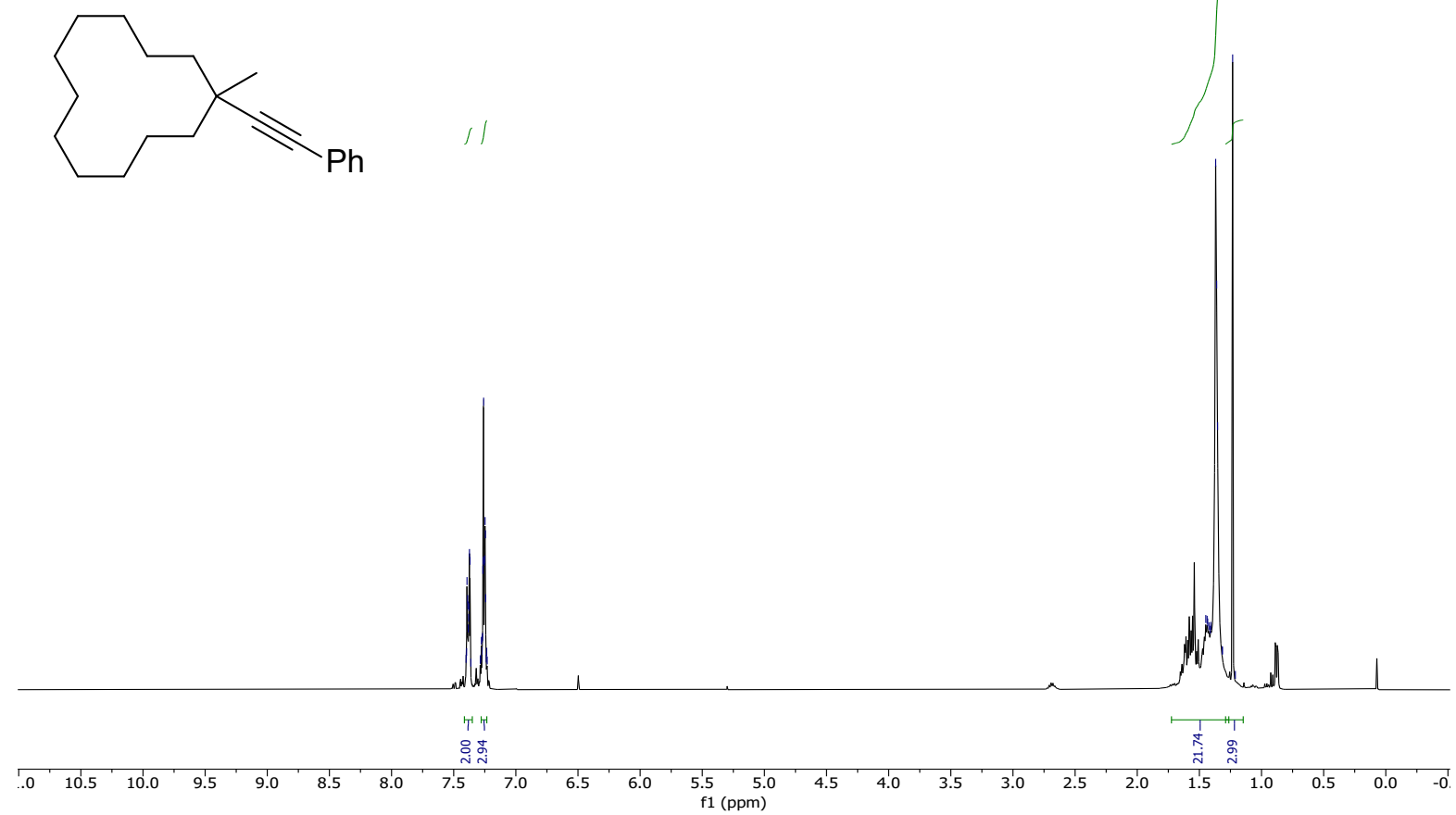

${ }^{13} \mathrm{C} \mathrm{NMR}, \mathrm{CDCl}_{3}, 101 \mathrm{MHz}$
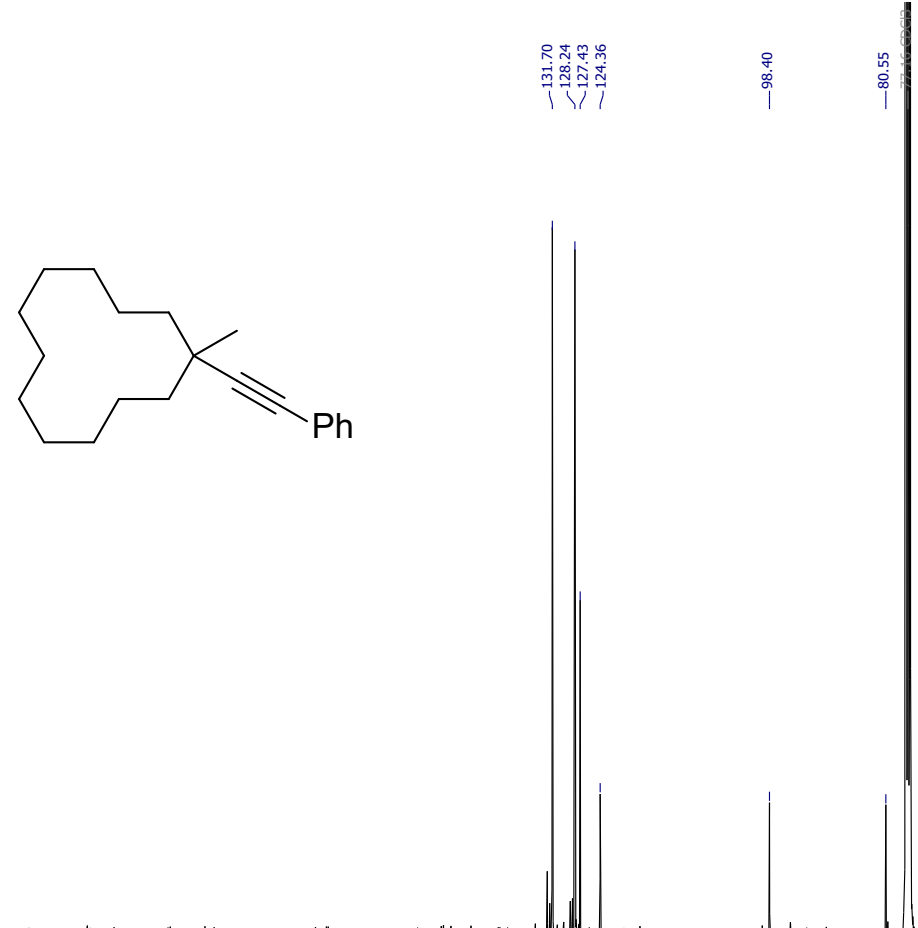

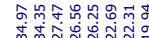

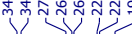




\section{Compound 40}

${ }^{1} \mathrm{H} \mathrm{NMR}, \mathrm{CDCl}_{3}, 400 \mathrm{MHz}$

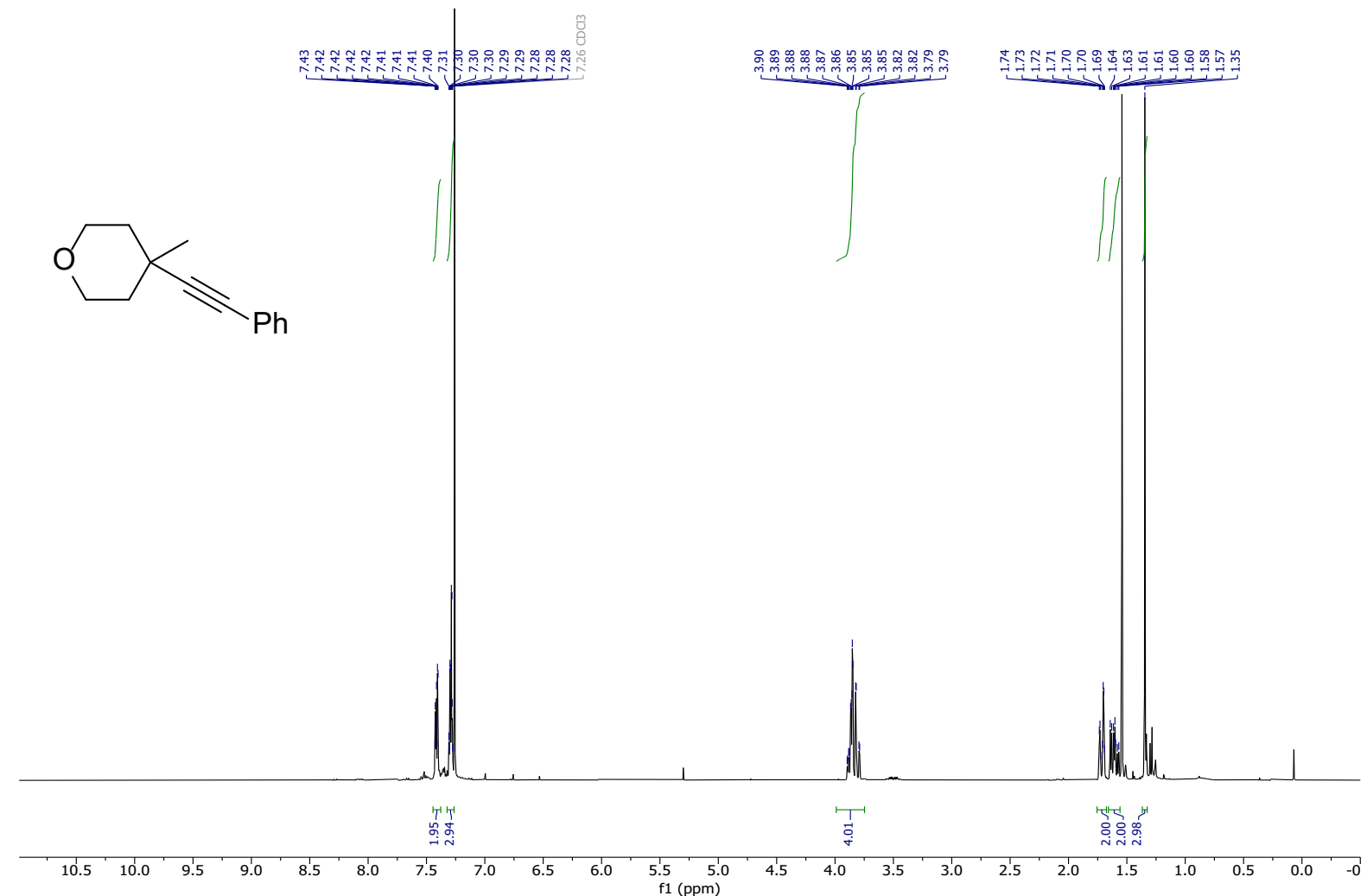

${ }^{13} \mathrm{C} \mathrm{NMR}, \mathrm{CDCl}_{3}, 101 \mathrm{MHz}$

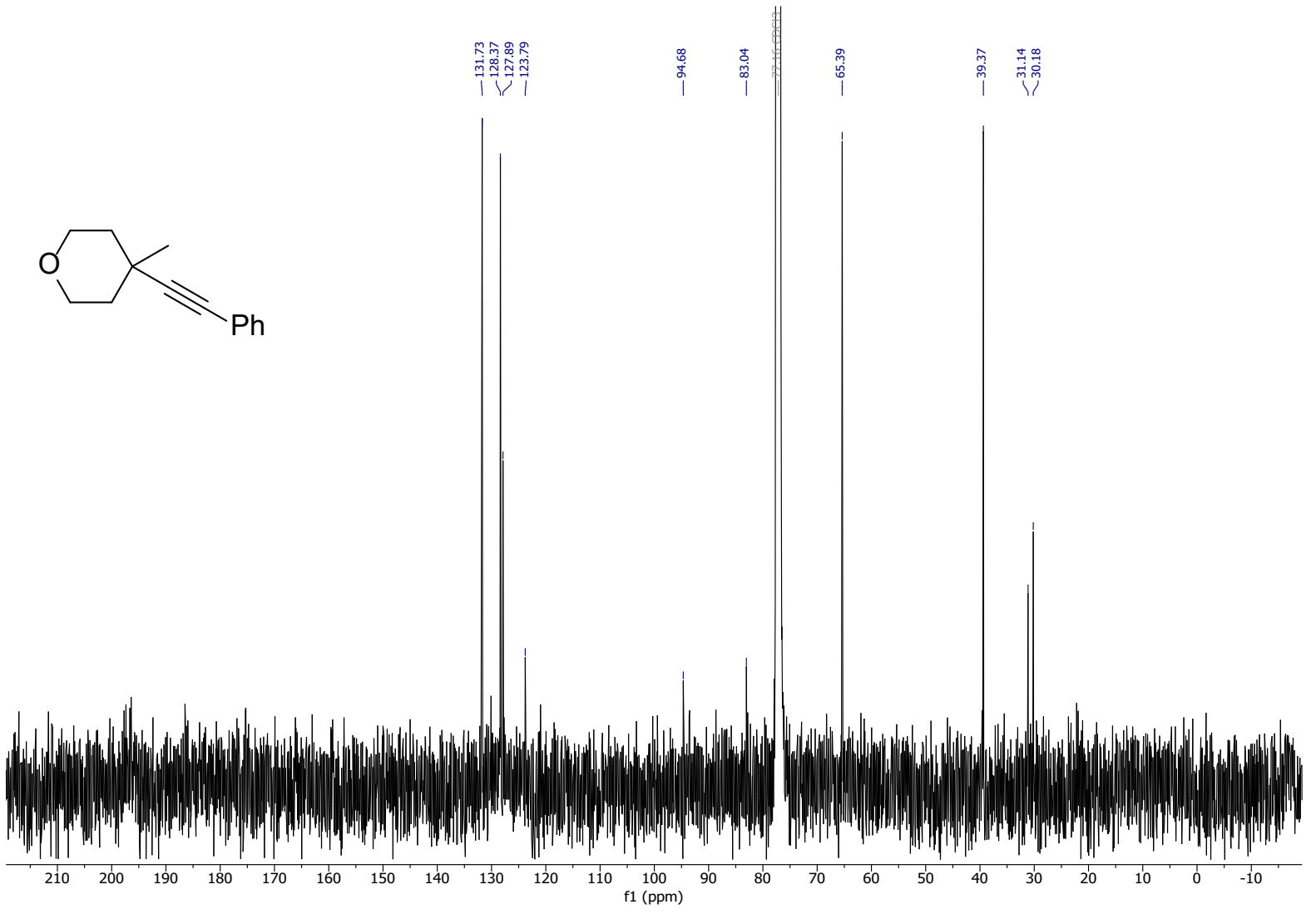




\section{Compound $4 p$}

${ }^{1} \mathrm{H} \mathrm{NMR}, \mathrm{CDCl}_{3}, 400 \mathrm{MHz}$

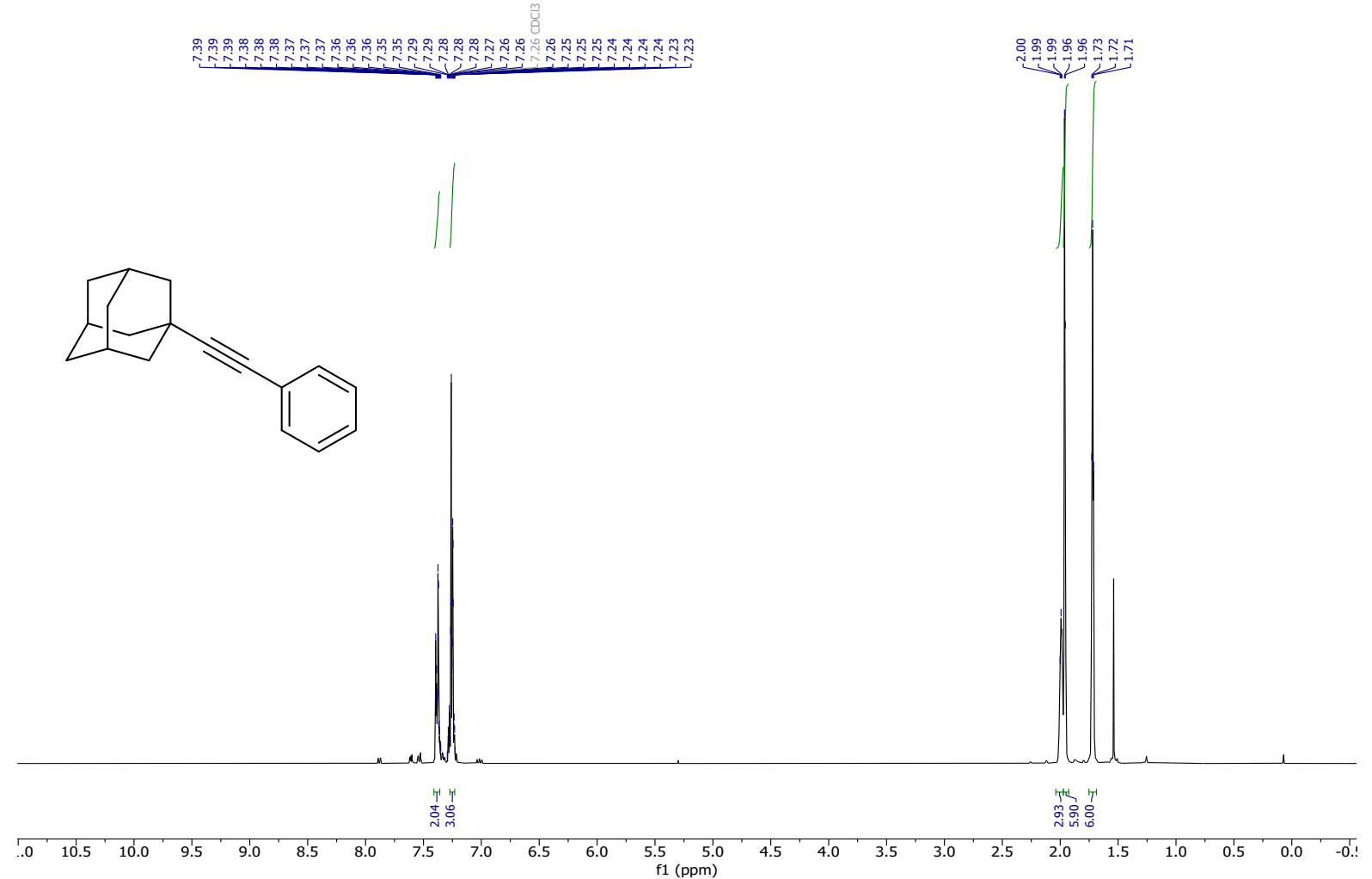

${ }^{13} \mathrm{C} \mathrm{NMR}, \mathrm{CDCl}_{3}$ (with 1V\% TMS), $101 \mathrm{MHz}$

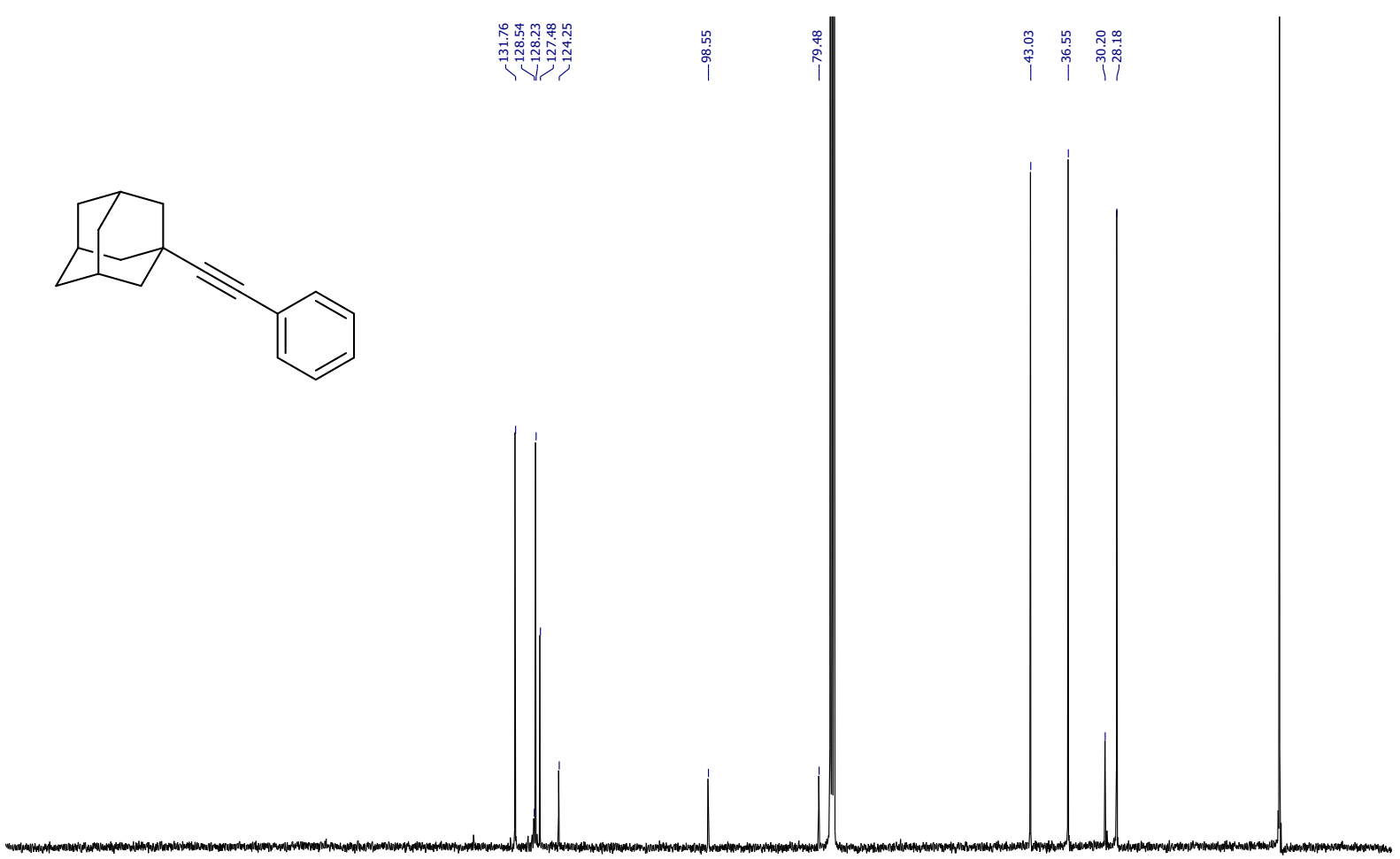

\begin{tabular}{lllllllllllllllllllllll}
\hline 210 & 200 & 190 & 180 & 170 & 160 & 150 & 140 & 130 & 120 & 110 & 100 & 90 & 80 & 70 & 60 & 50 & 40 & 30 & 20 & 10 & 0 & -10
\end{tabular} 


\section{Compound $\mathbf{4 q}$}

${ }^{1} \mathrm{H} \mathrm{NMR}, \mathrm{CDCl}_{3}, 400 \mathrm{MHz}$

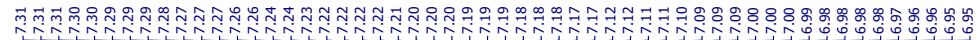

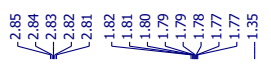

Y

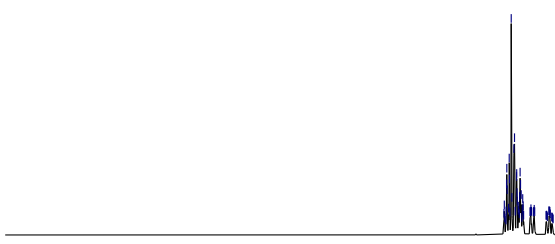

1

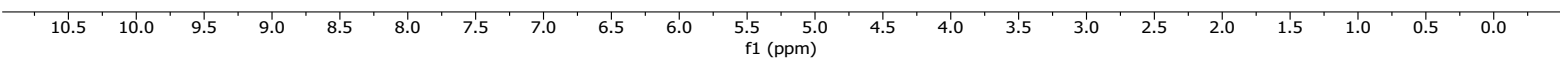

${ }^{13} \mathrm{CNMR}, \mathrm{CDCl}_{3}, 101 \mathrm{MHz}$

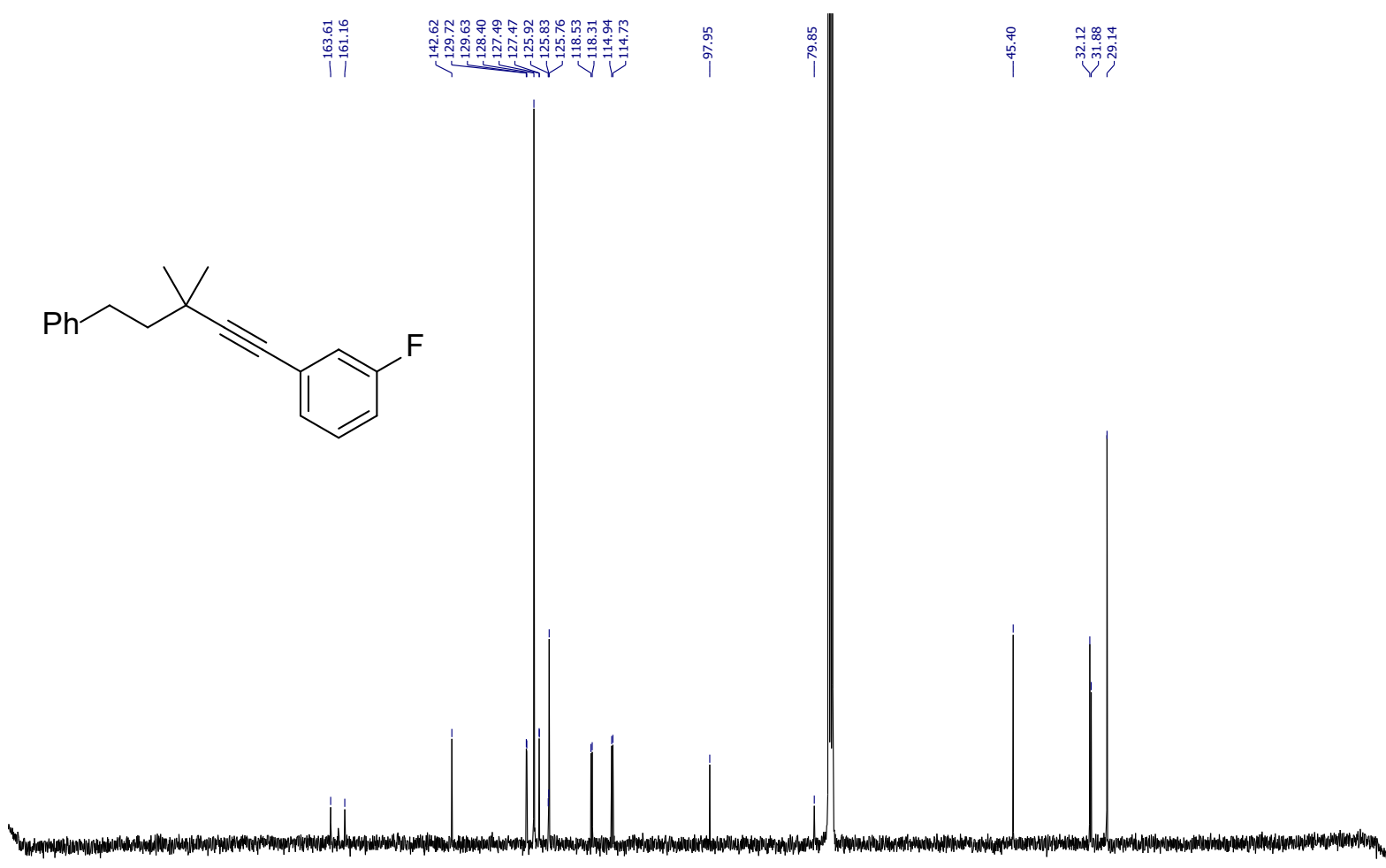

$\begin{array}{lllllllllll}210 & 200 & 190 & 180 & 170 & 160 & 150 & 140 & 130 & 120 & 110 \\ \mathrm{f} 1(\mathrm{ppm})\end{array}$ 
${ }^{19} \mathrm{~F} \mathrm{NMR}, \mathrm{CDCl}_{3}, 376 \mathrm{MHz}$

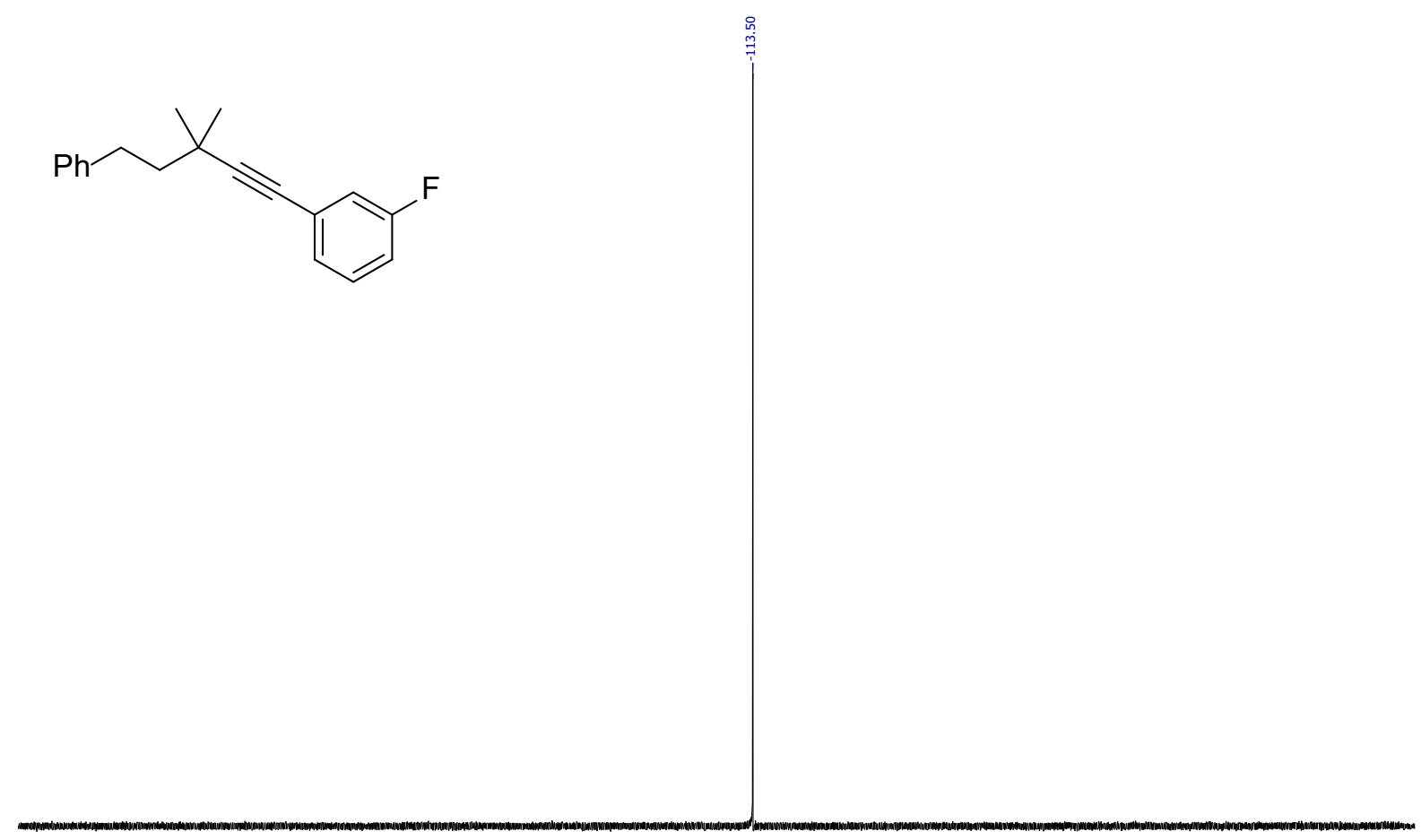

\begin{tabular}{ccccccccccccccccccccccc}
\hline 0 & -10 & -20 & -30 & -40 & -50 & -60 & -70 & -80 & -90 & -100 & -110 & -120 & -130 & -140 & -150 & -160 & -170 & -180 & -190 & -200 & -210 & 1
\end{tabular} 


\section{Compound $\mathbf{4 r}$}

${ }^{1} \mathrm{H} \mathrm{NMR}, \mathrm{CDCl}_{3}, 400 \mathrm{MHz}$

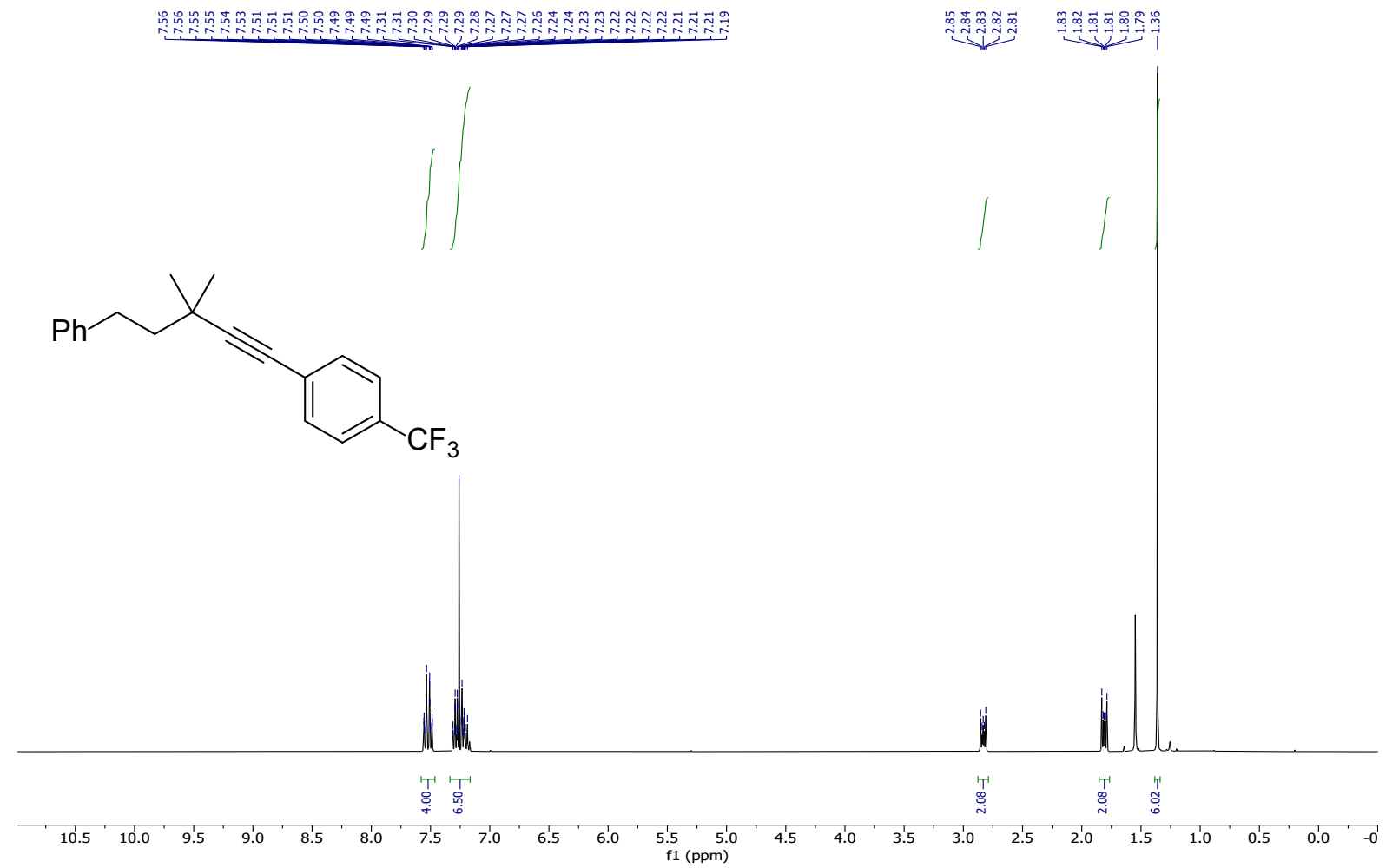

${ }^{13} \mathrm{C} \mathrm{NMR}, \mathrm{CDCl}_{3}, 101 \mathrm{MHz}$

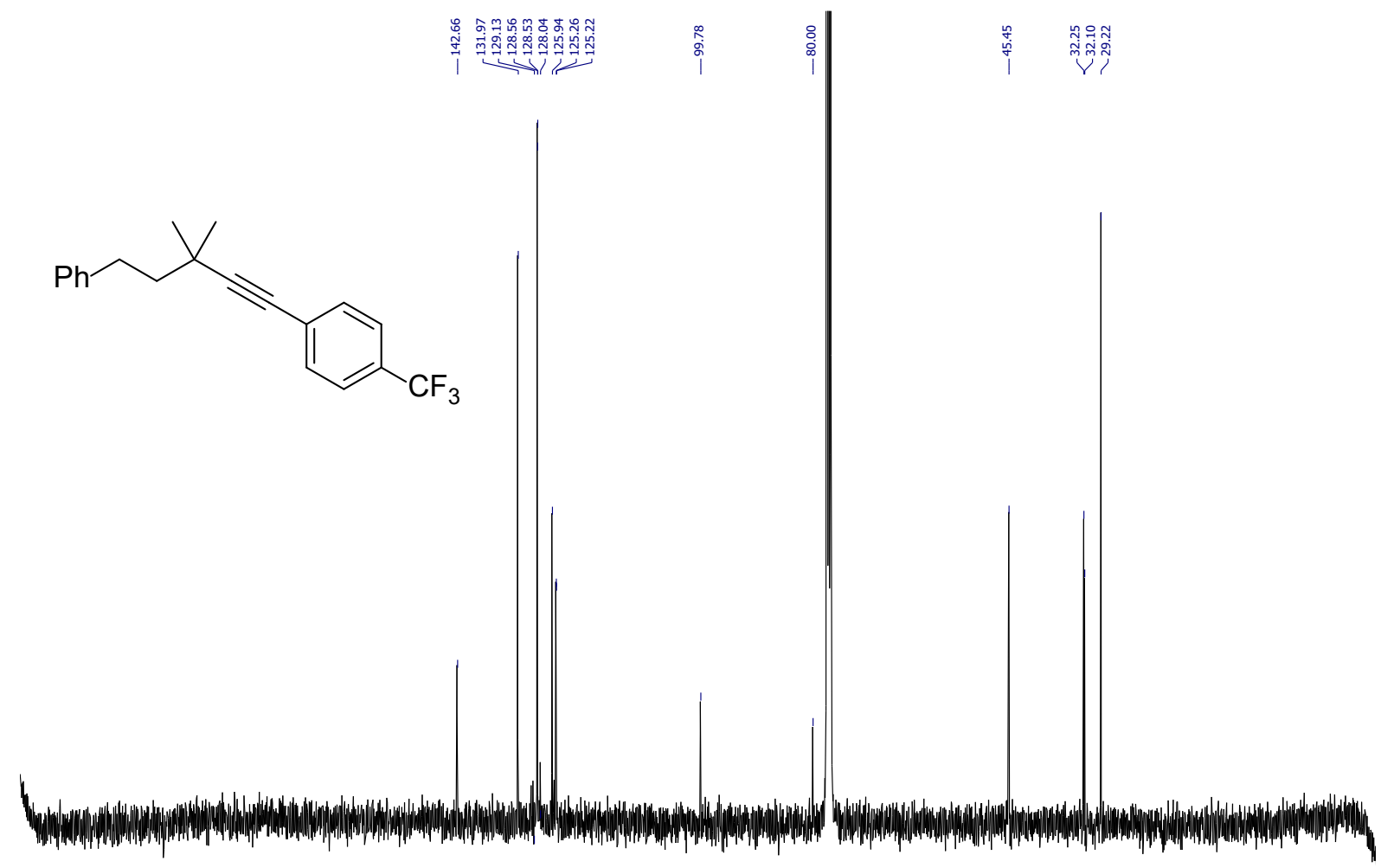

\begin{tabular}{llllllllllllllllllllllll}
\hline & 110 & 200 & 190 & 180 & 170 & 160 & 150 & 140 & 130 & 120 & 110 & 100 & 90 & 80 & 70 & 60 & 50 & 40 & 30 & 20 & 10 & 0 & -10
\end{tabular} 
${ }^{19} \mathrm{~F} \mathrm{NMR}^{\mathrm{CDCl}}{ }_{3}, 376 \mathrm{MHz}$,

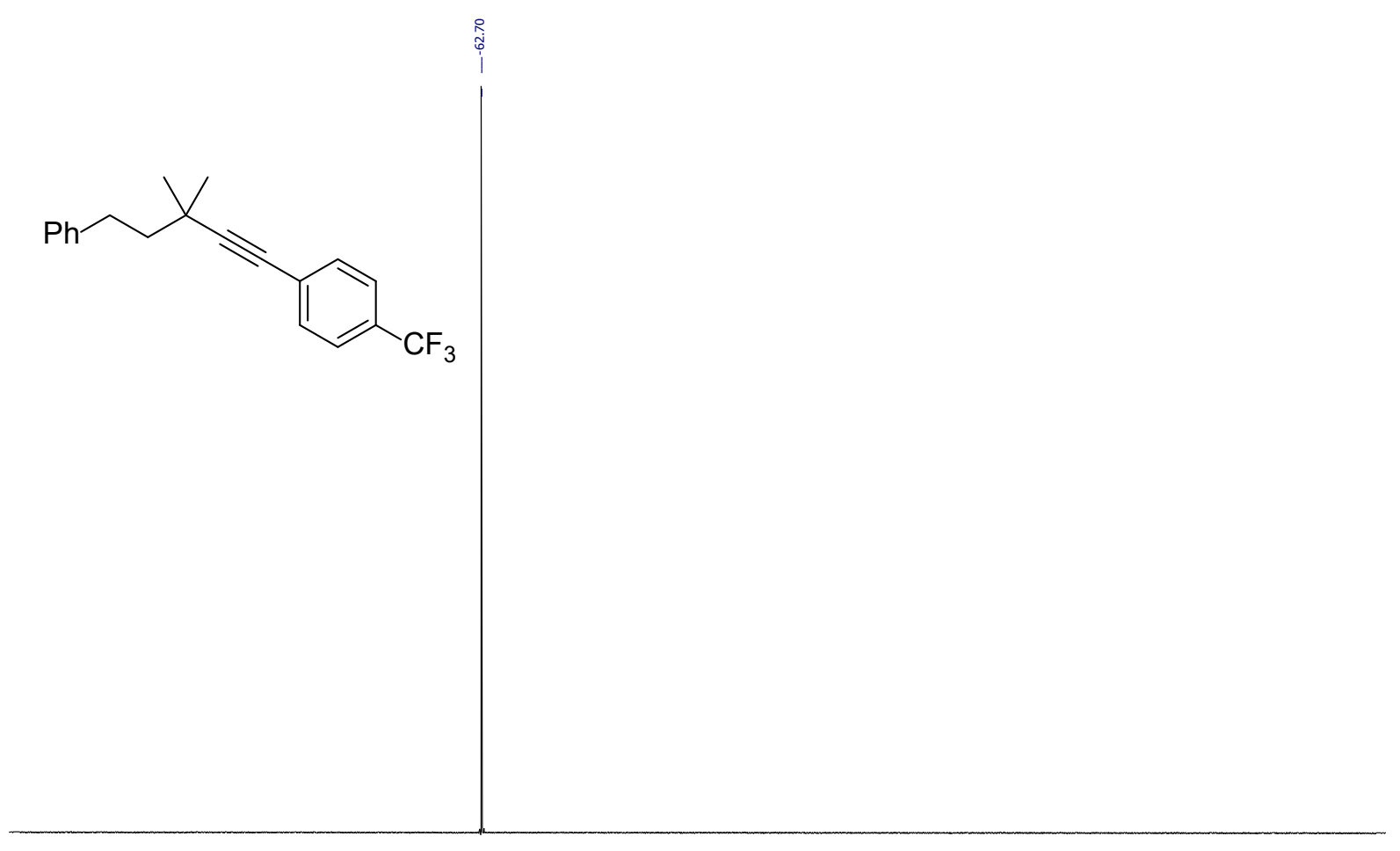

\begin{tabular}{lllllllllllllllllllllllllll}
\hline 10 & 0 & -10 & -20 & -30 & -40 & -50 & -60 & -70 & -80 & -90 & -100 & -110 & -120 & -130 & -140 & -150 & -160 & -170 & -180 & -190 & -200 & -210 &
\end{tabular} 


\section{Compound $4 \mathrm{~s}$}

${ }^{1} \mathrm{H} \mathrm{NMR}, \mathrm{CDCl}_{3}, 400 \mathrm{MHz}$

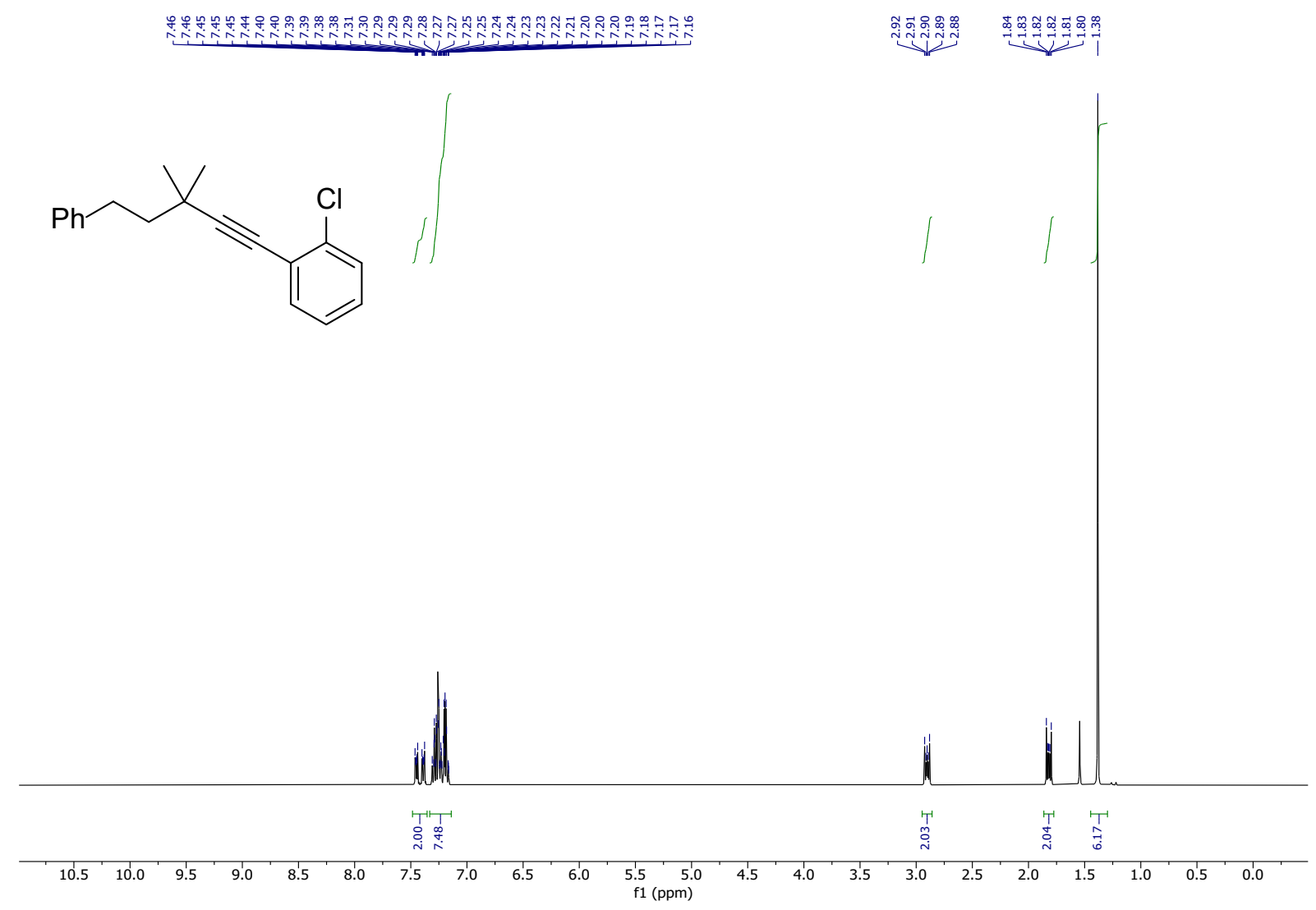

${ }^{13} \mathrm{C} \mathrm{NMR}, \mathrm{CDCl}_{3}, 400 \mathrm{MHz}$

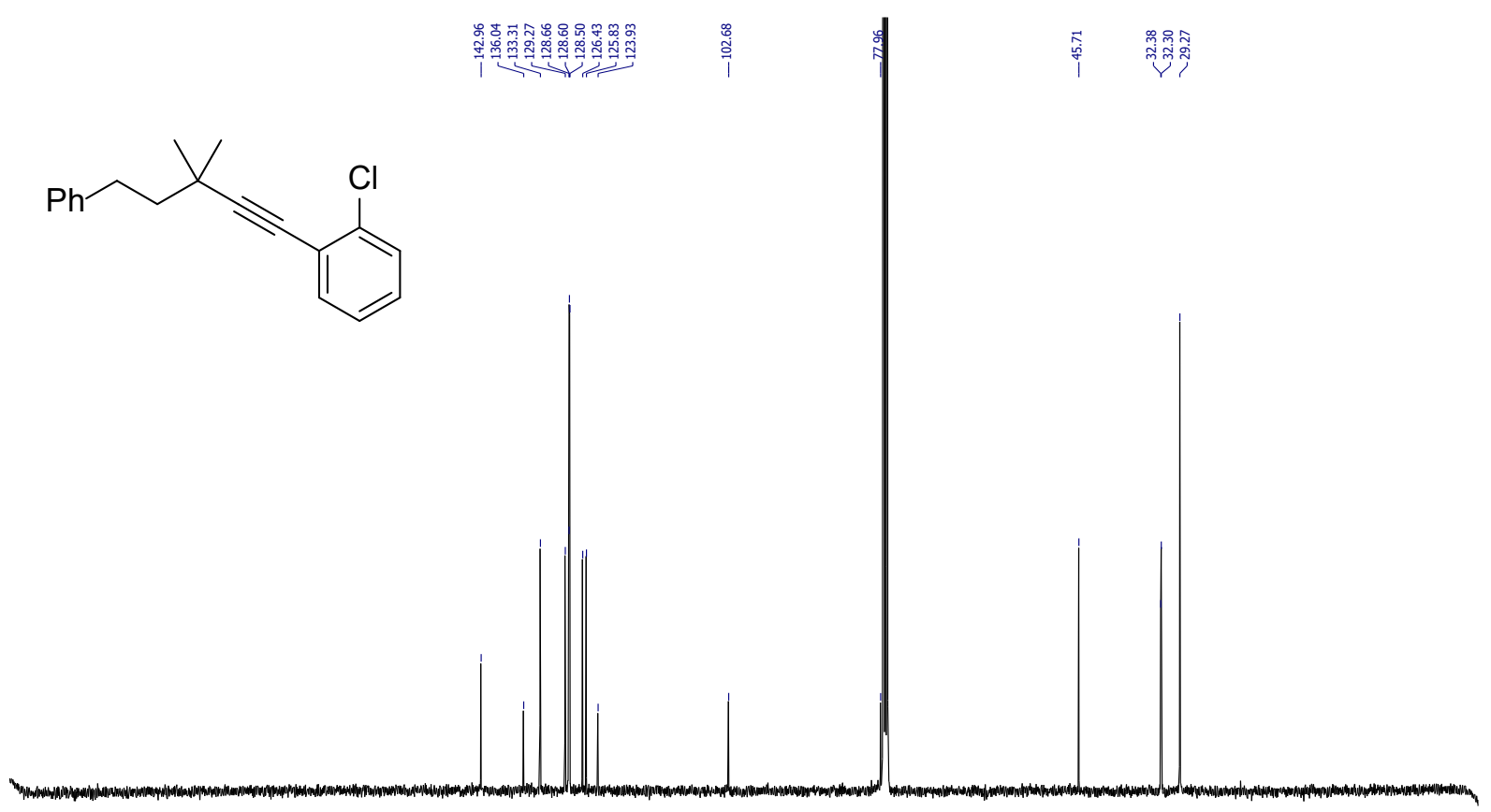

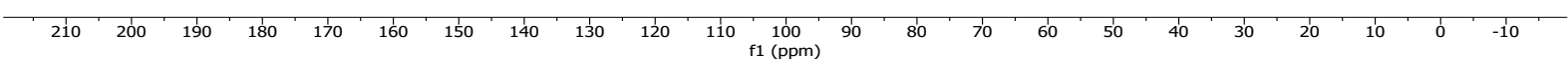




\section{Compound 4t}

${ }^{1} \mathrm{H} \mathrm{NMR}, \mathrm{CDCl}_{3}, 400 \mathrm{MHz}$

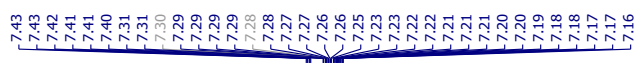

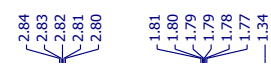

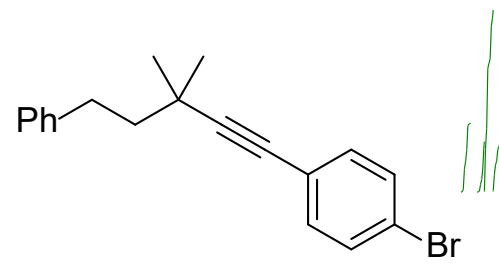

$$
\text { ( }
$$

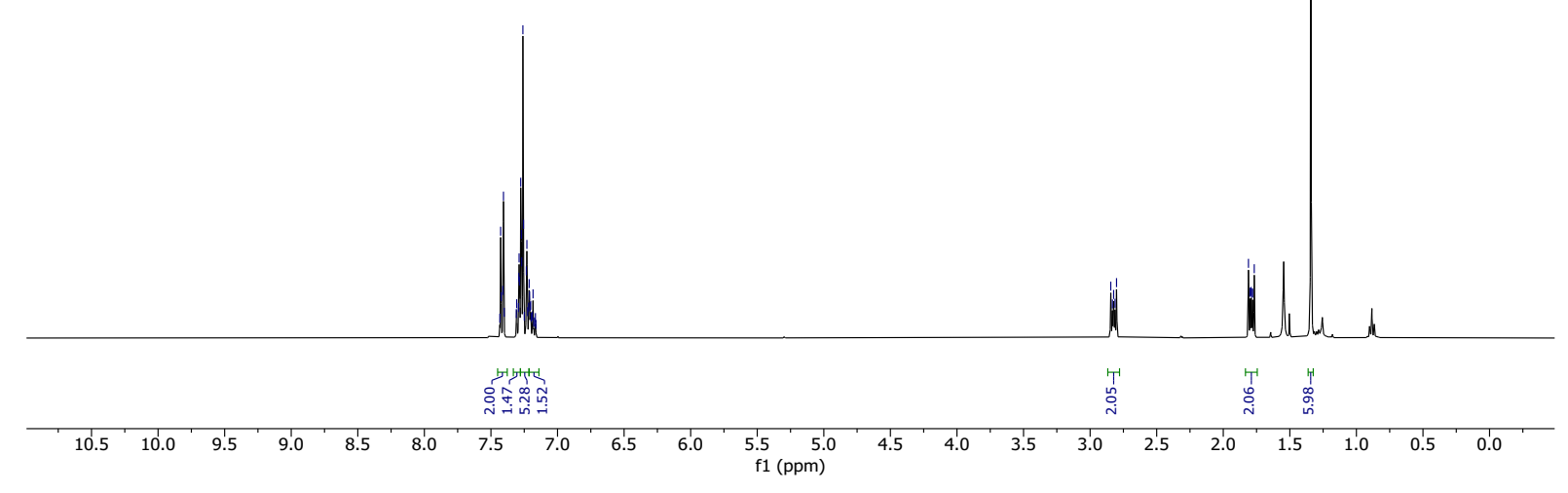

${ }^{13} \mathrm{C} \mathrm{NMR}, \mathrm{CDCl}_{3}, 101 \mathrm{MHz}$

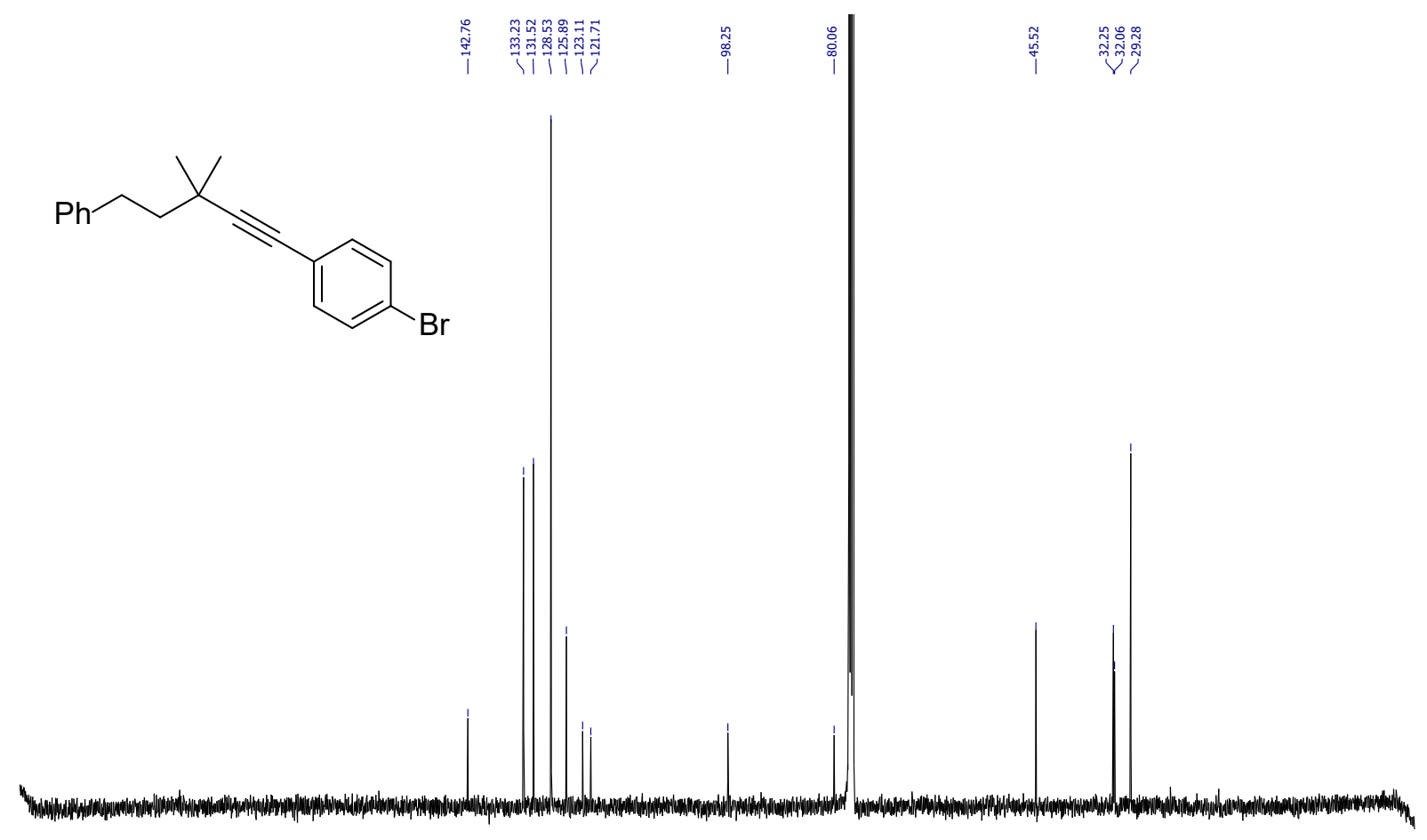

\begin{tabular}{llllllllllllllllllllllllll}
\hline 210 & 200 & 190 & 180 & 170 & 160 & 150 & 140 & 130 & 120 & 110 & 100 & 90 & 80 & 70 & 60 & 50 & 40 & 30 & 20 & 10 & 0 & -10 \\
$\mathrm{f} 1(\mathrm{ppm})$
\end{tabular} 


\section{Compound $\mathbf{4 u}$}

${ }^{1} \mathrm{H} \mathrm{NMR}, \mathrm{CDCl}_{3}, 400 \mathrm{MHz}$

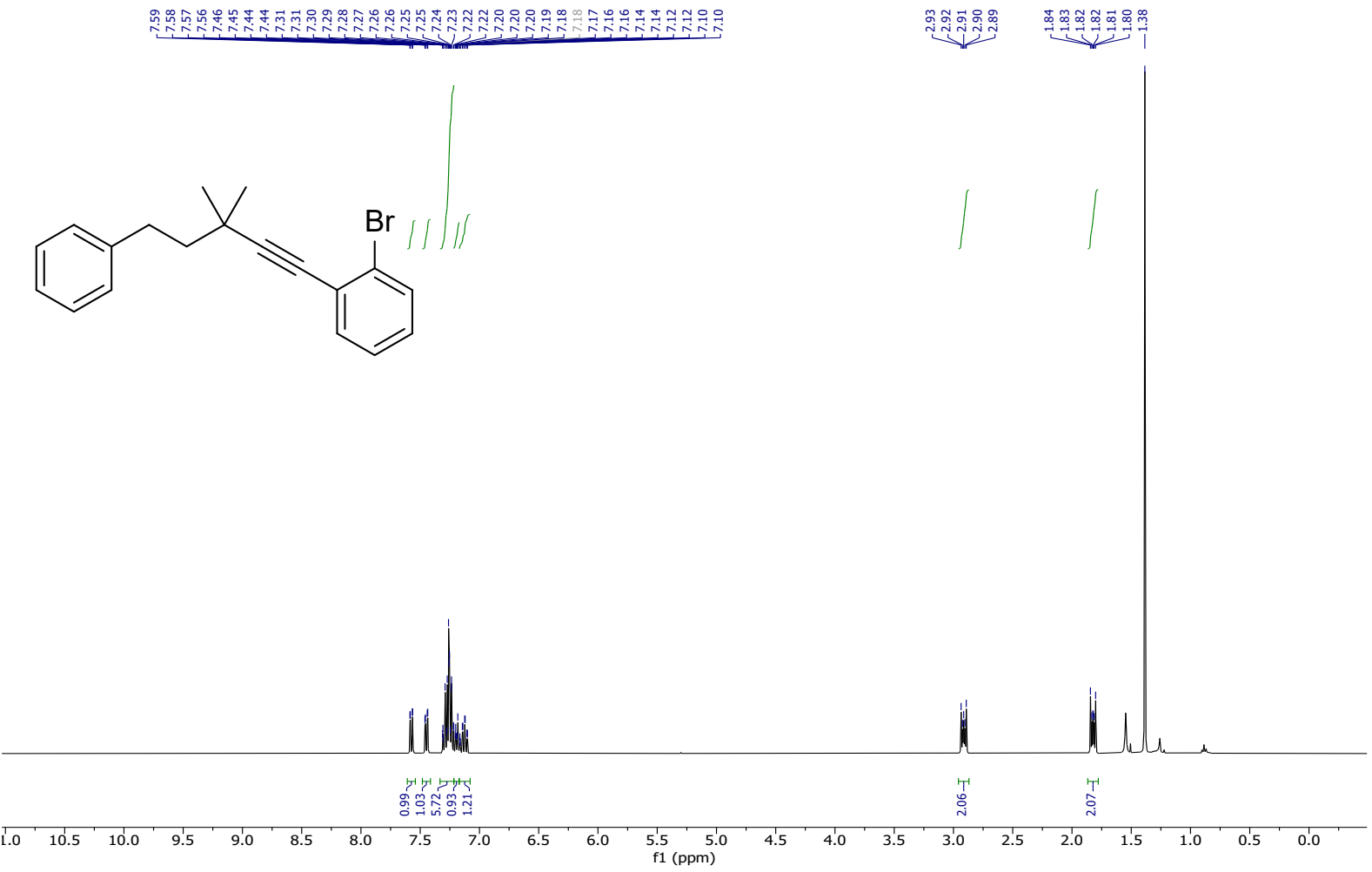

${ }^{13} \mathrm{C} \mathrm{NMR}, \mathrm{CDCl}_{3}, 101 \mathrm{MHz}$

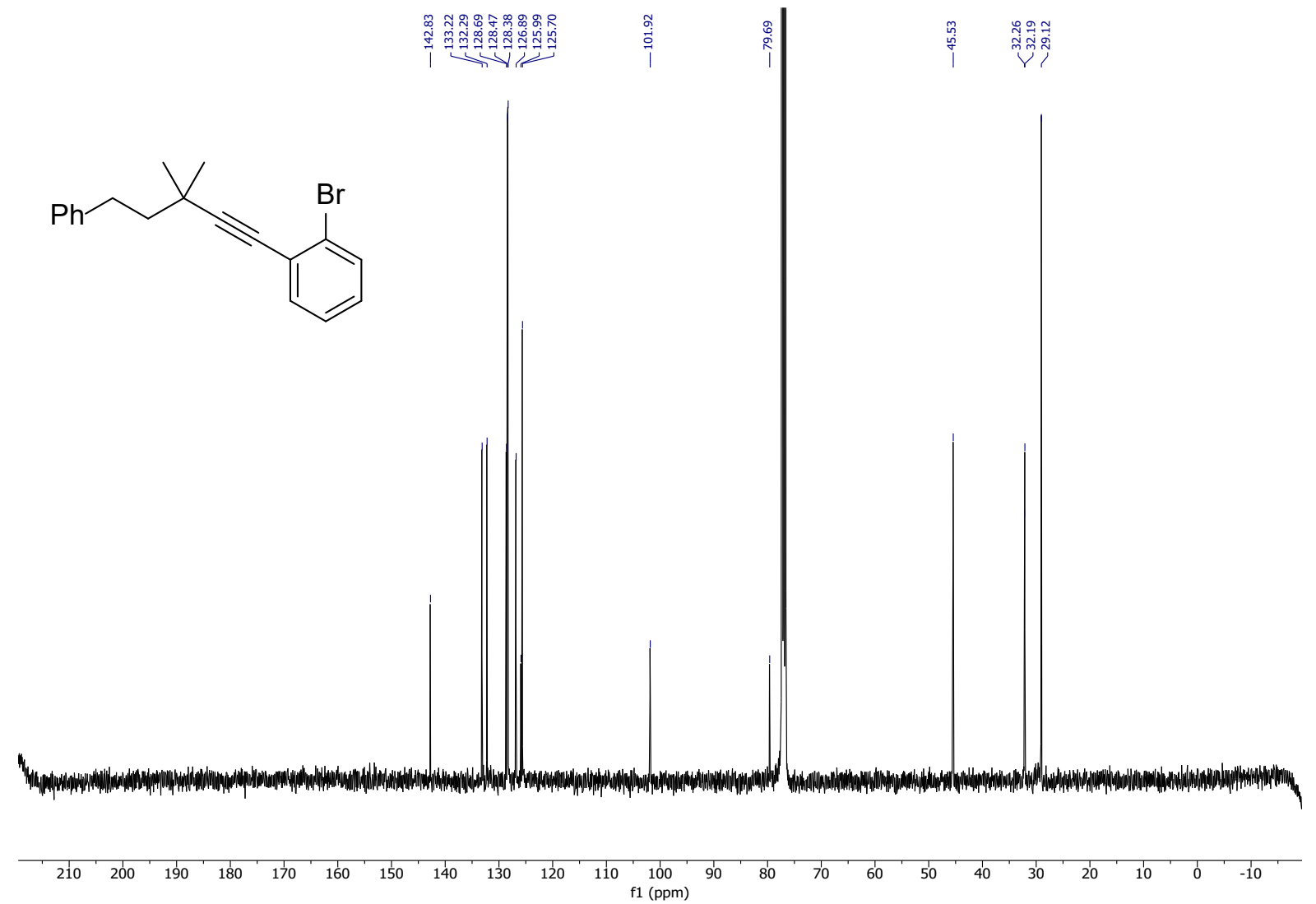




\section{Compound $\mathbf{4 v}$}

${ }^{1} \mathrm{H} \mathrm{NMR}, \mathrm{CDCl}_{3}, 400 \mathrm{MHz}$

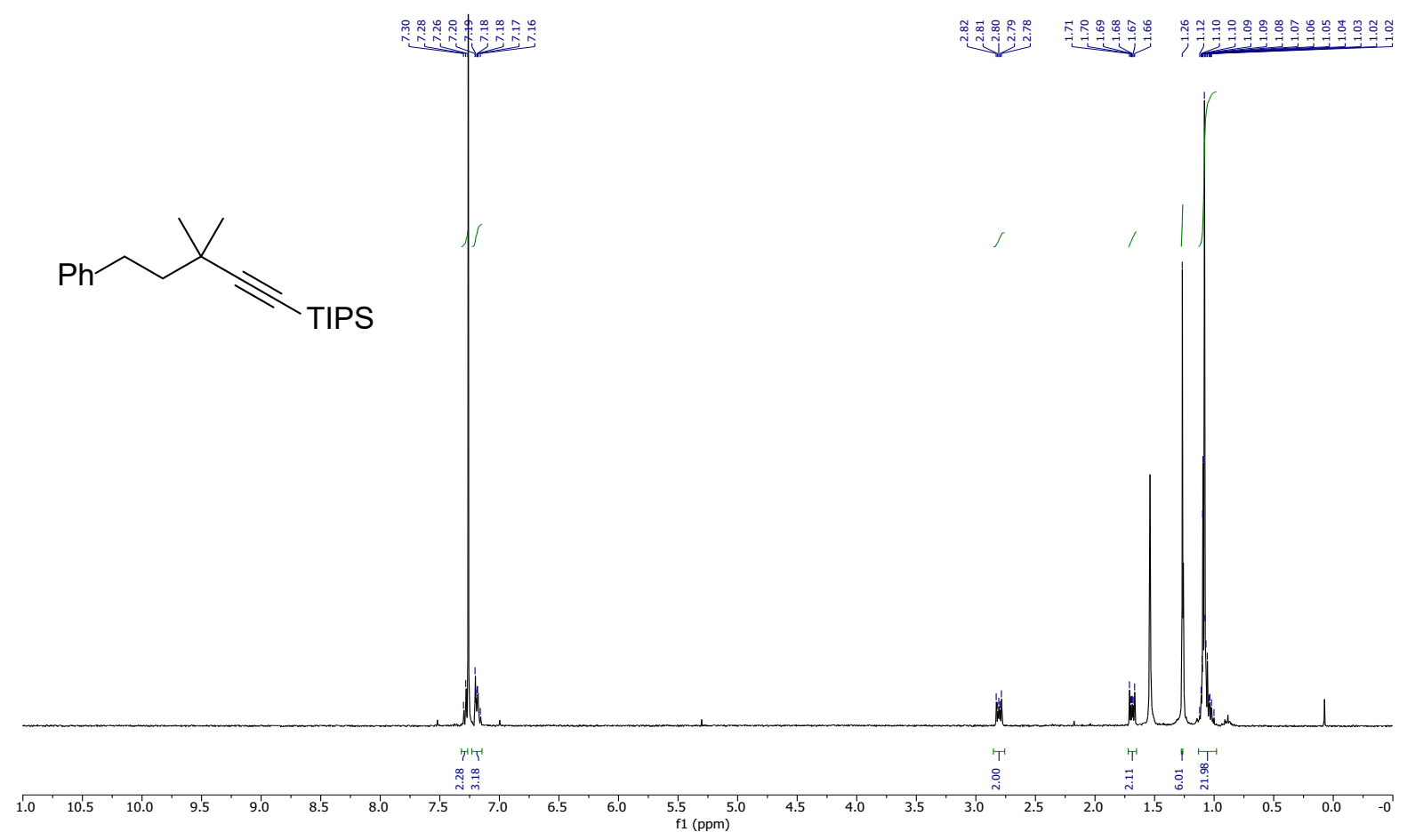

${ }^{13} \mathrm{CNMR}_{\mathrm{N}} \mathrm{CDCl}_{3}, 101 \mathrm{MHz}$

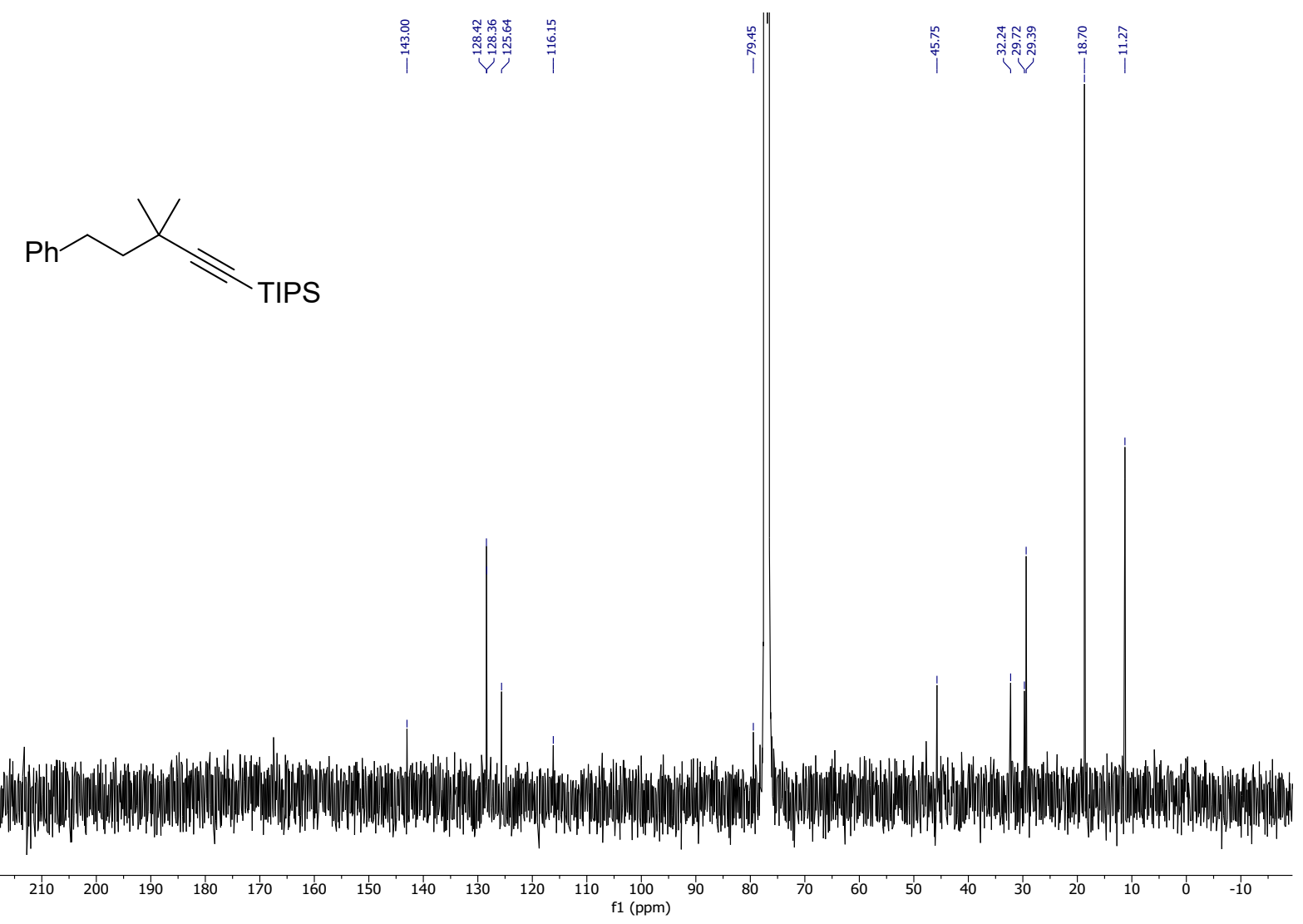




\section{Compound $4 w$}

${ }^{1} \mathrm{H} \mathrm{NMR}, \mathrm{CDCl}_{3}, 400 \mathrm{MHz}$

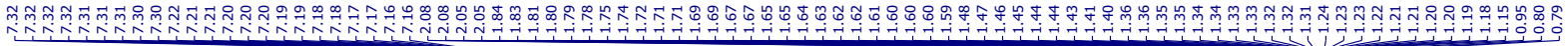<smiles>C[C@H]1CCC2[C@@H]1CC[C@]2(C)C#Cc1ccccc1</smiles>
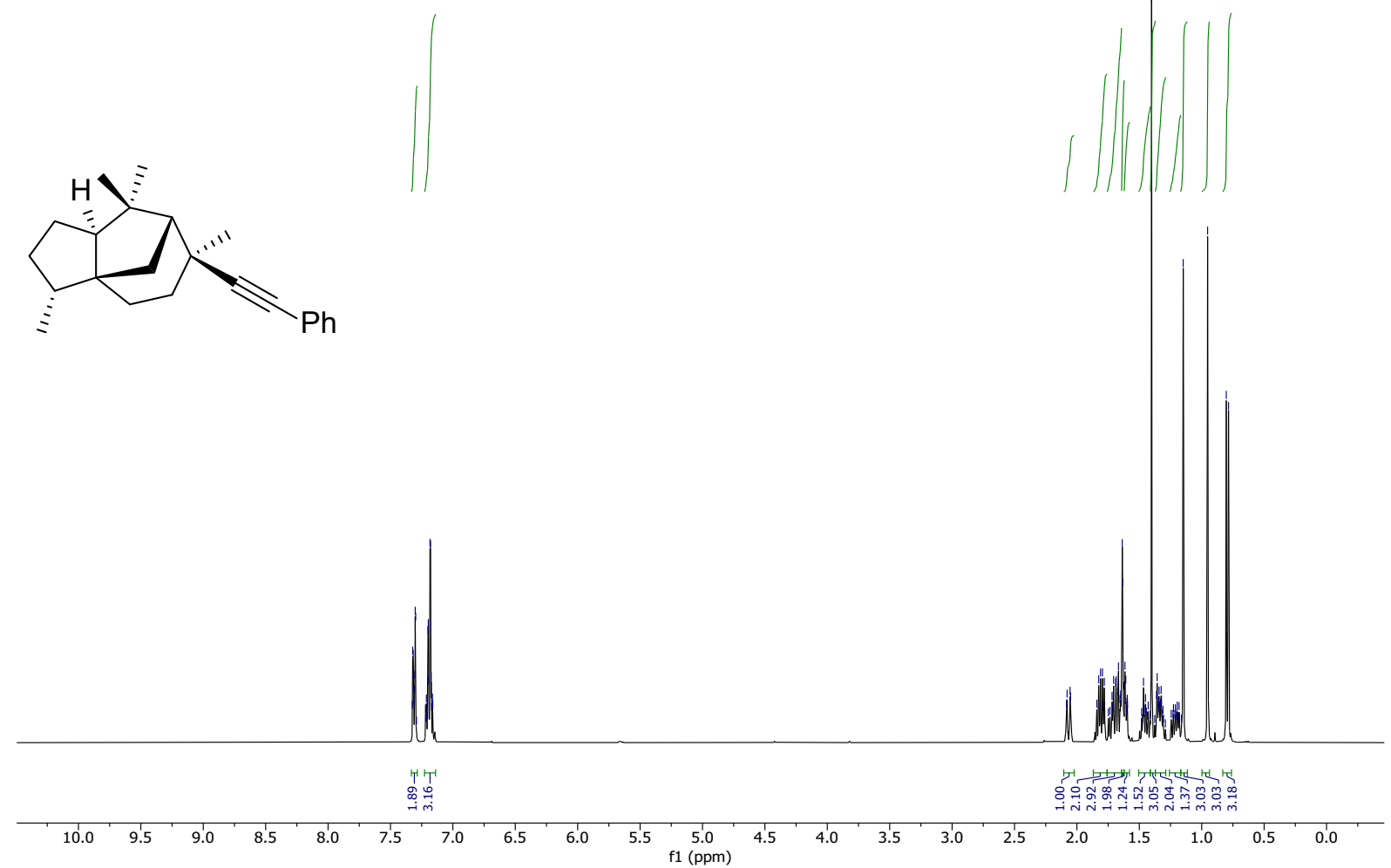

${ }^{13} \mathrm{C} \mathrm{NMR}, \mathrm{CDCl}_{3}, 101 \mathrm{MHz}$
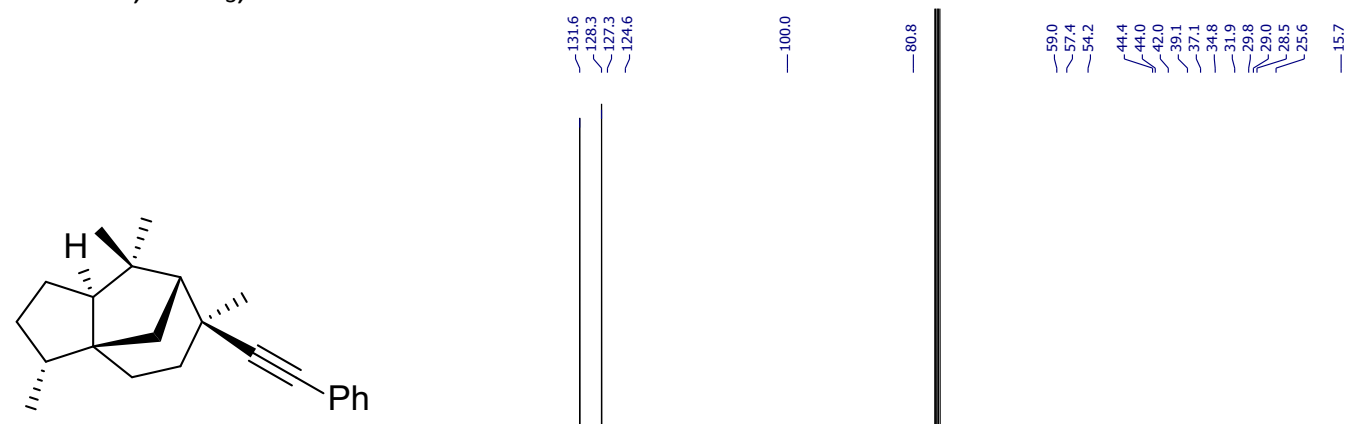

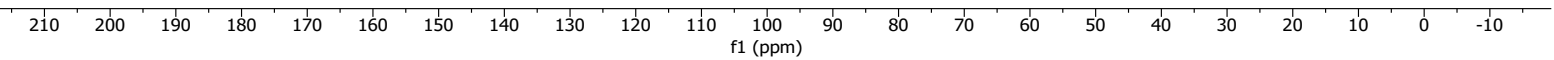




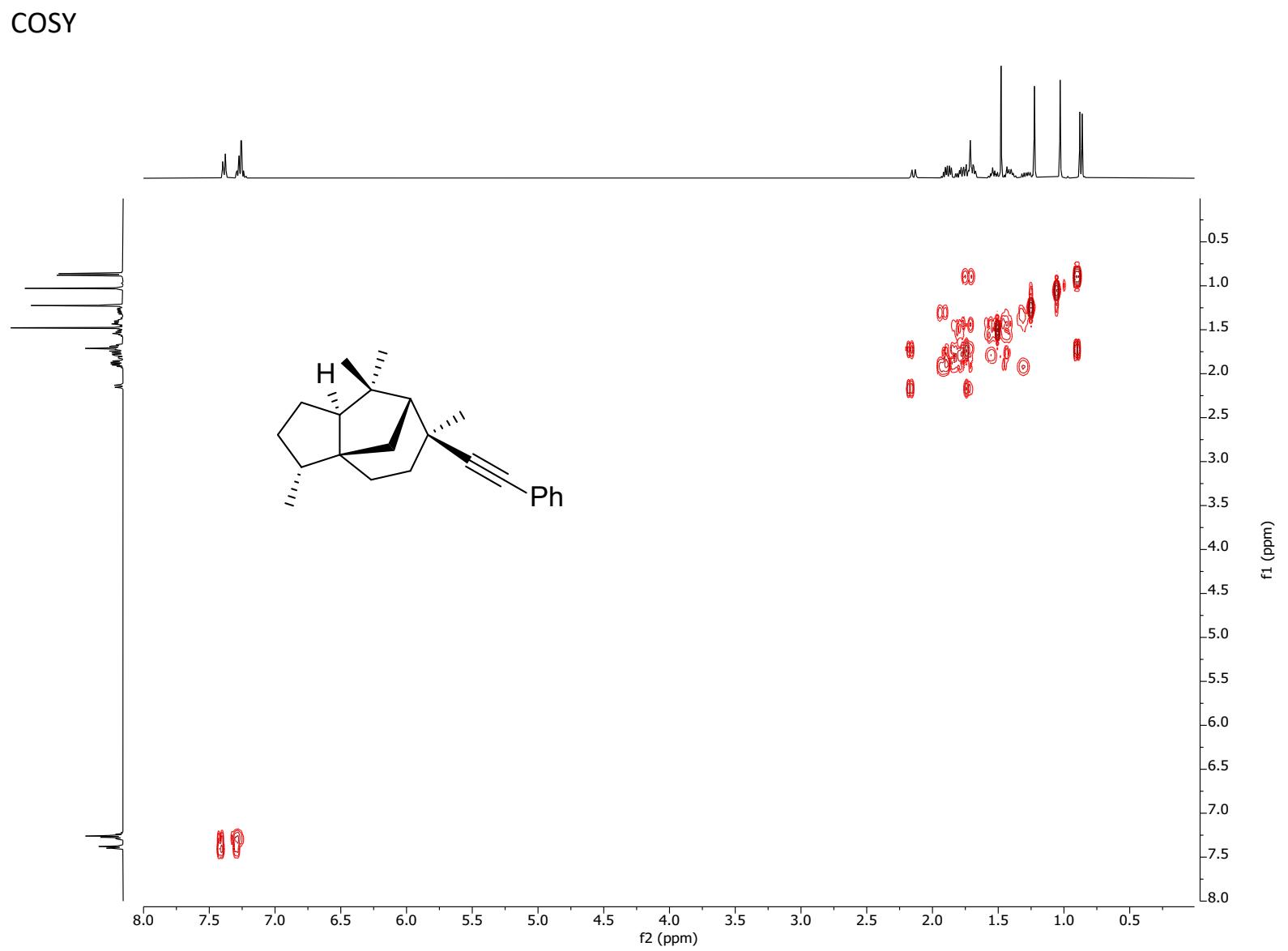

HSQC (O1P $=55$ ppm, SW =80 ppm)

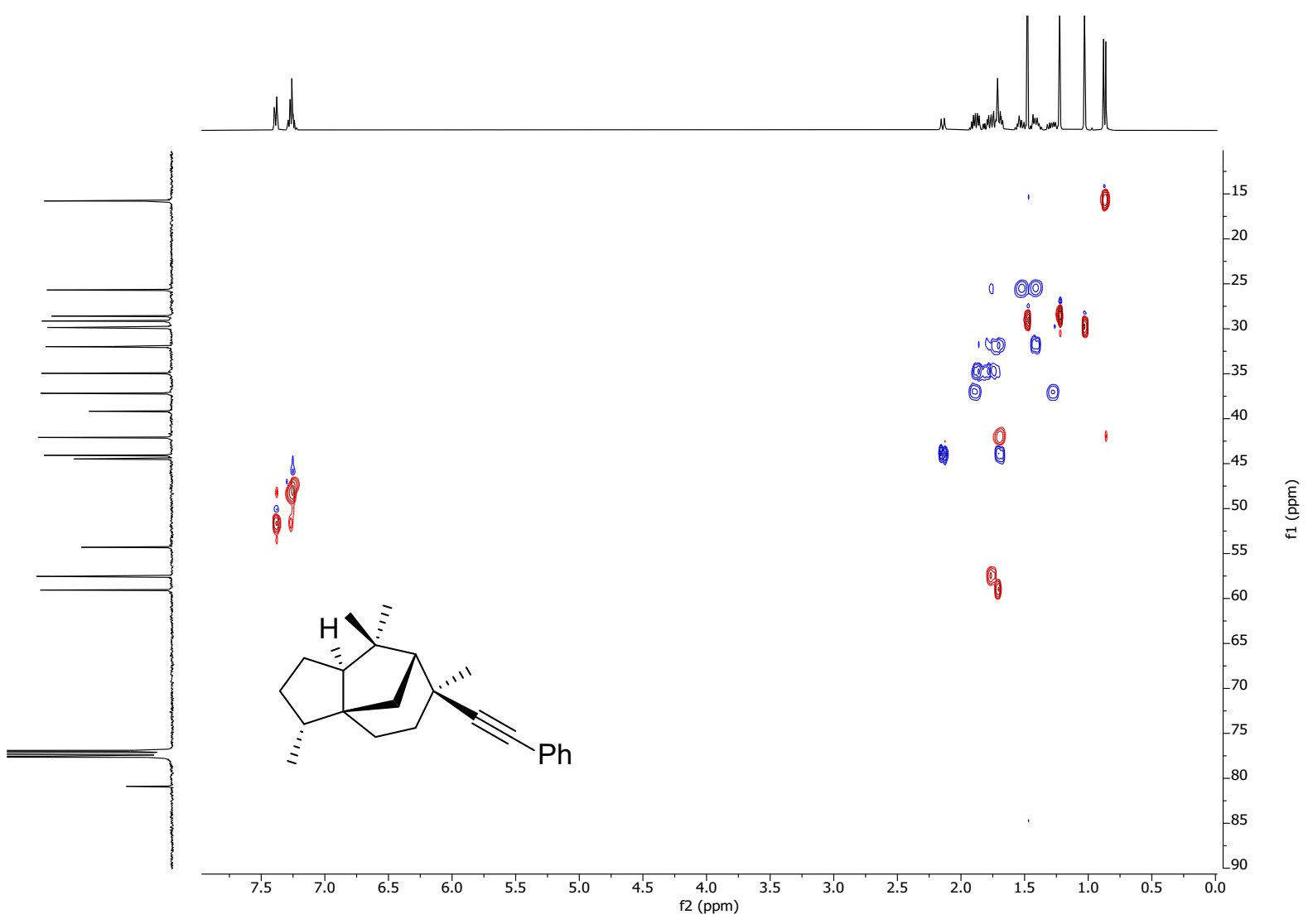


$\mathrm{HMBC}(\mathrm{O} 1 \mathrm{P}=55 \mathrm{ppm} ; \mathrm{SW}=80 \mathrm{ppm})$

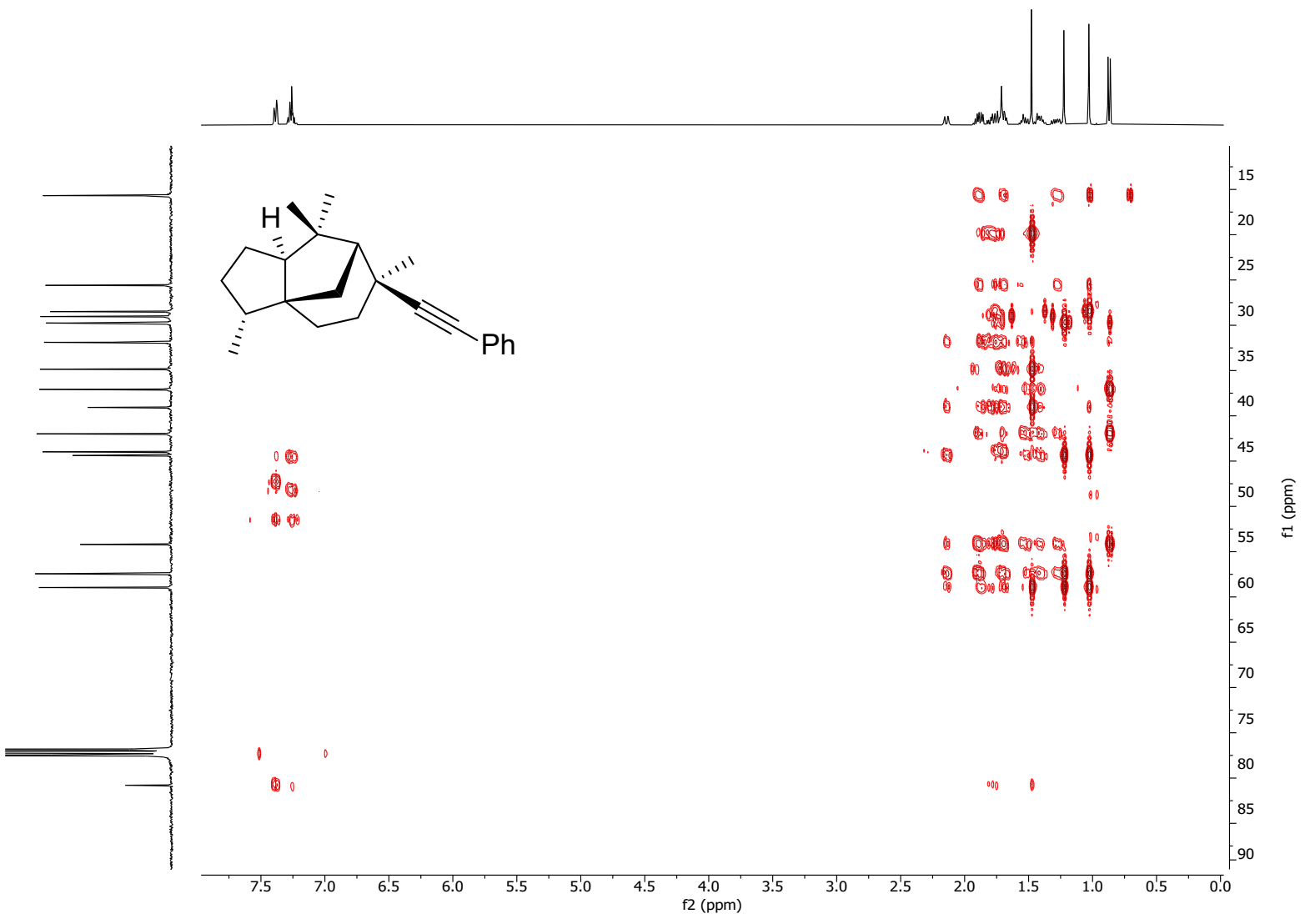

$\operatorname{NOESY}(\mathrm{O} 1 \mathrm{P}=4 \mathrm{ppm}, \mathrm{SW}=8 \mathrm{ppm})$

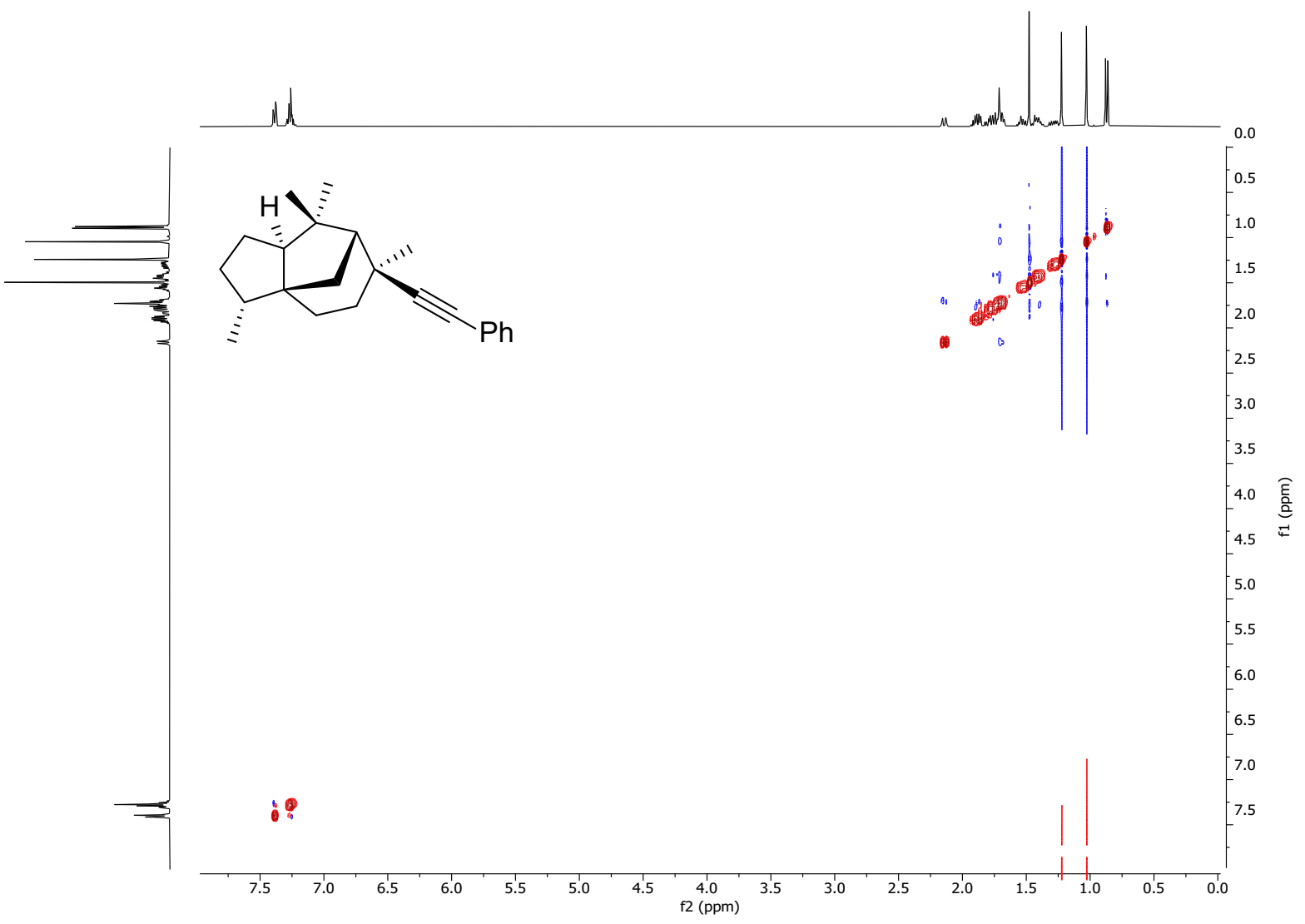




\section{Compound $\mathbf{4 x}$}

${ }^{1} \mathrm{H} \mathrm{NMR}, \mathrm{CDCl}_{3}, 400 \mathrm{MHz}$

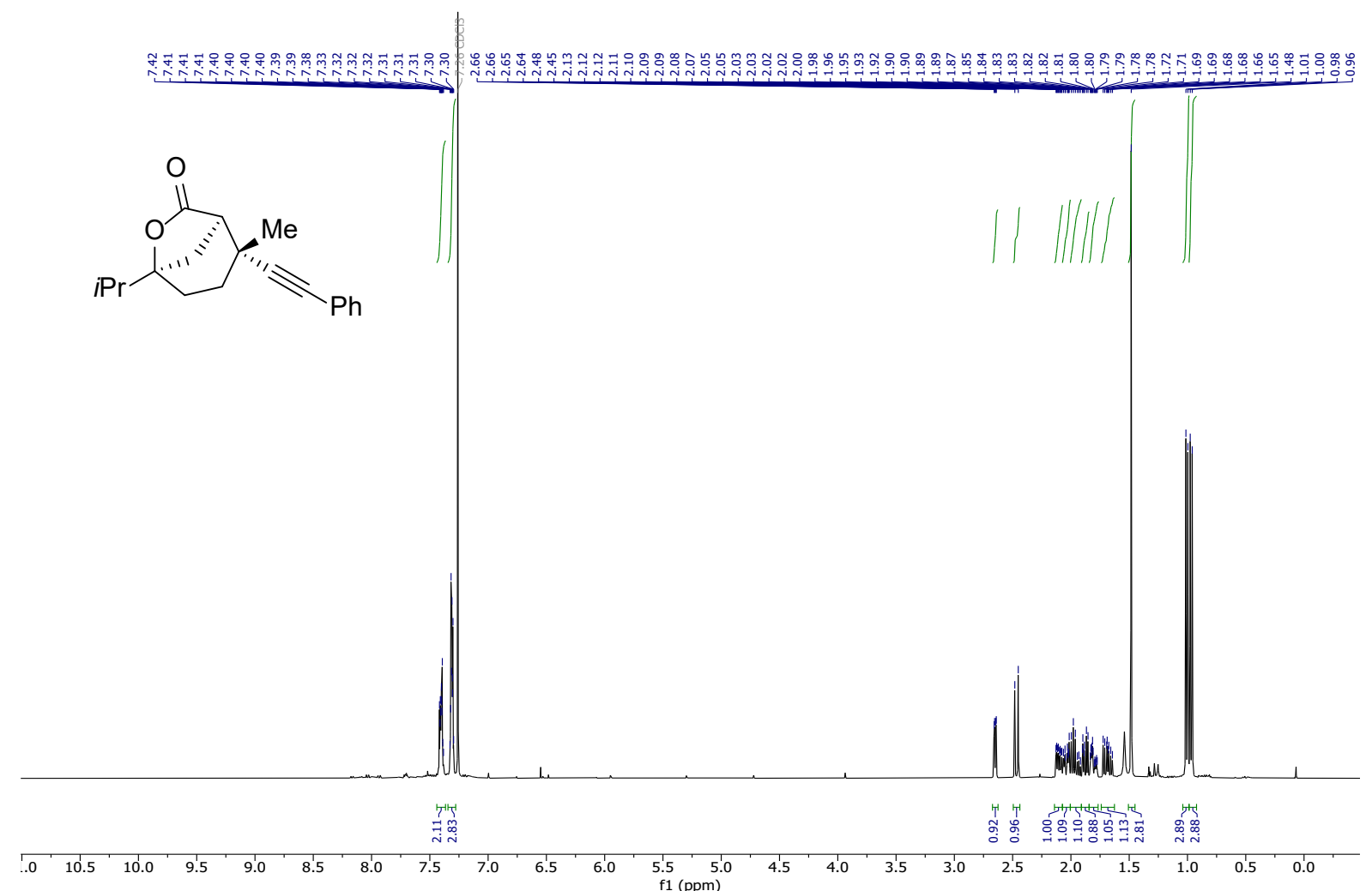

${ }^{13} \mathrm{C} \mathrm{NMR}, \mathrm{CDCl}_{3}, 101 \mathrm{MHz}$

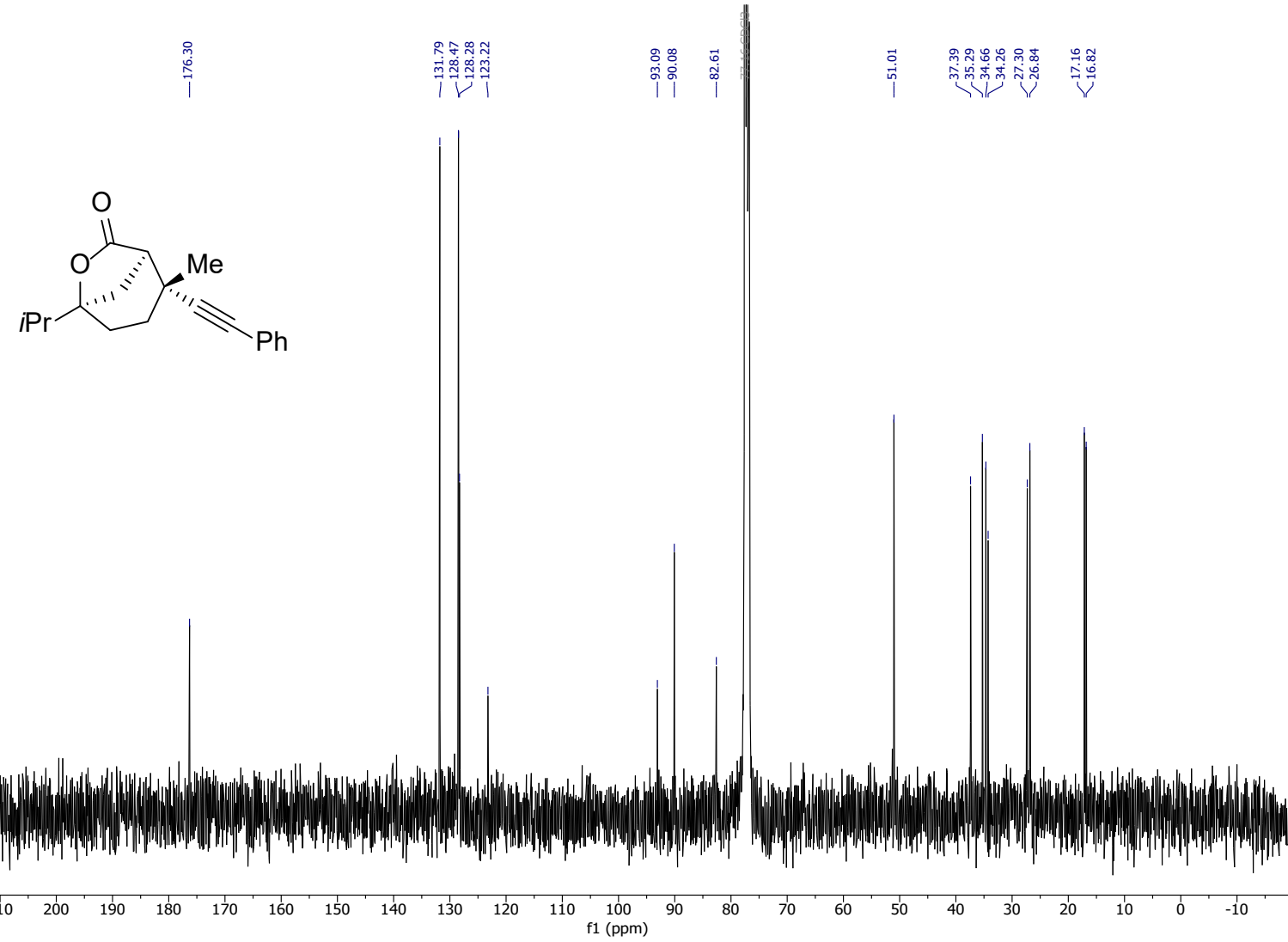


$\operatorname{COSY}(\mathrm{O} 1 \mathrm{P}=3 \mathrm{ppm} ; \mathrm{SW}=6 \mathrm{ppm})$

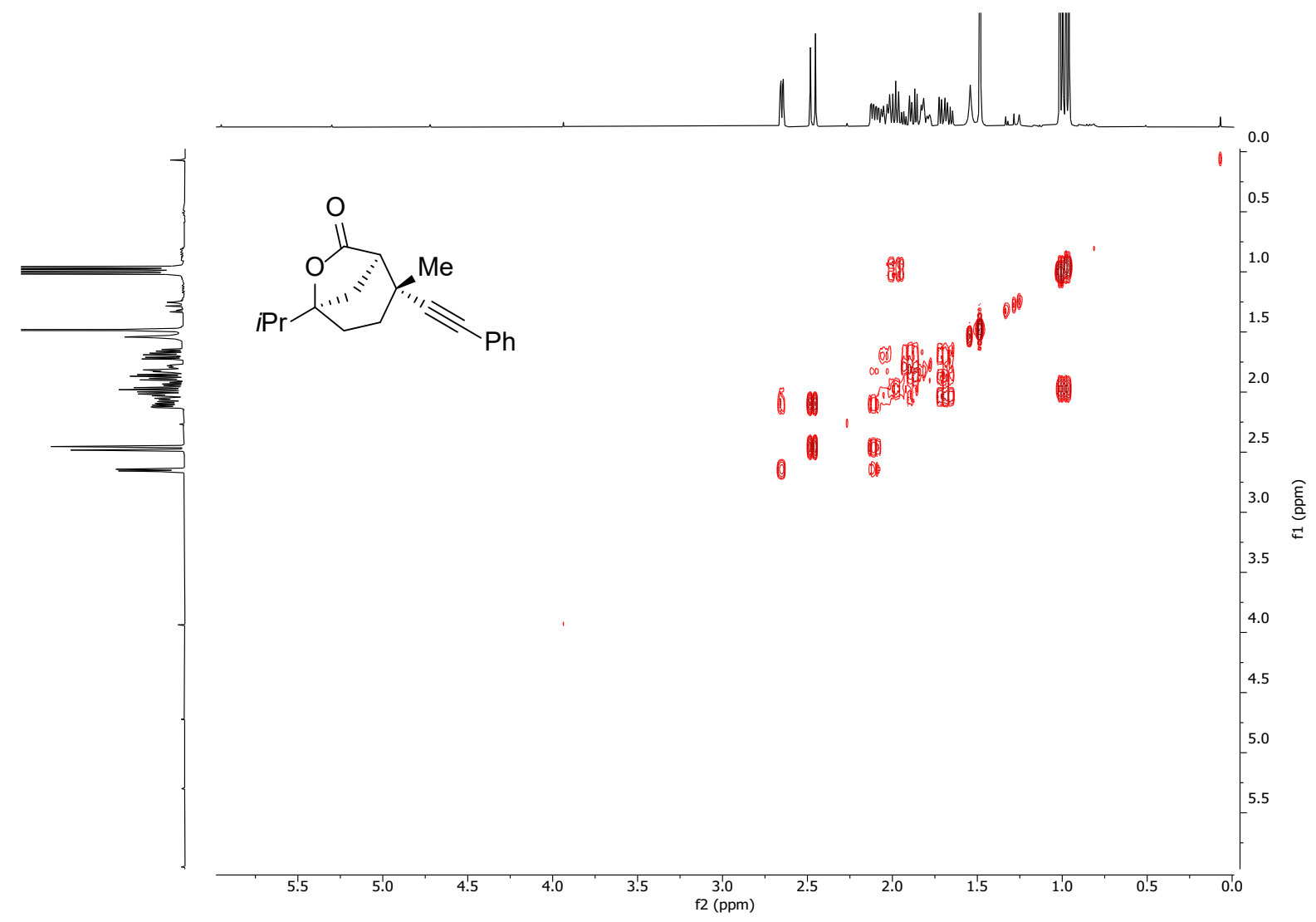

HSQC

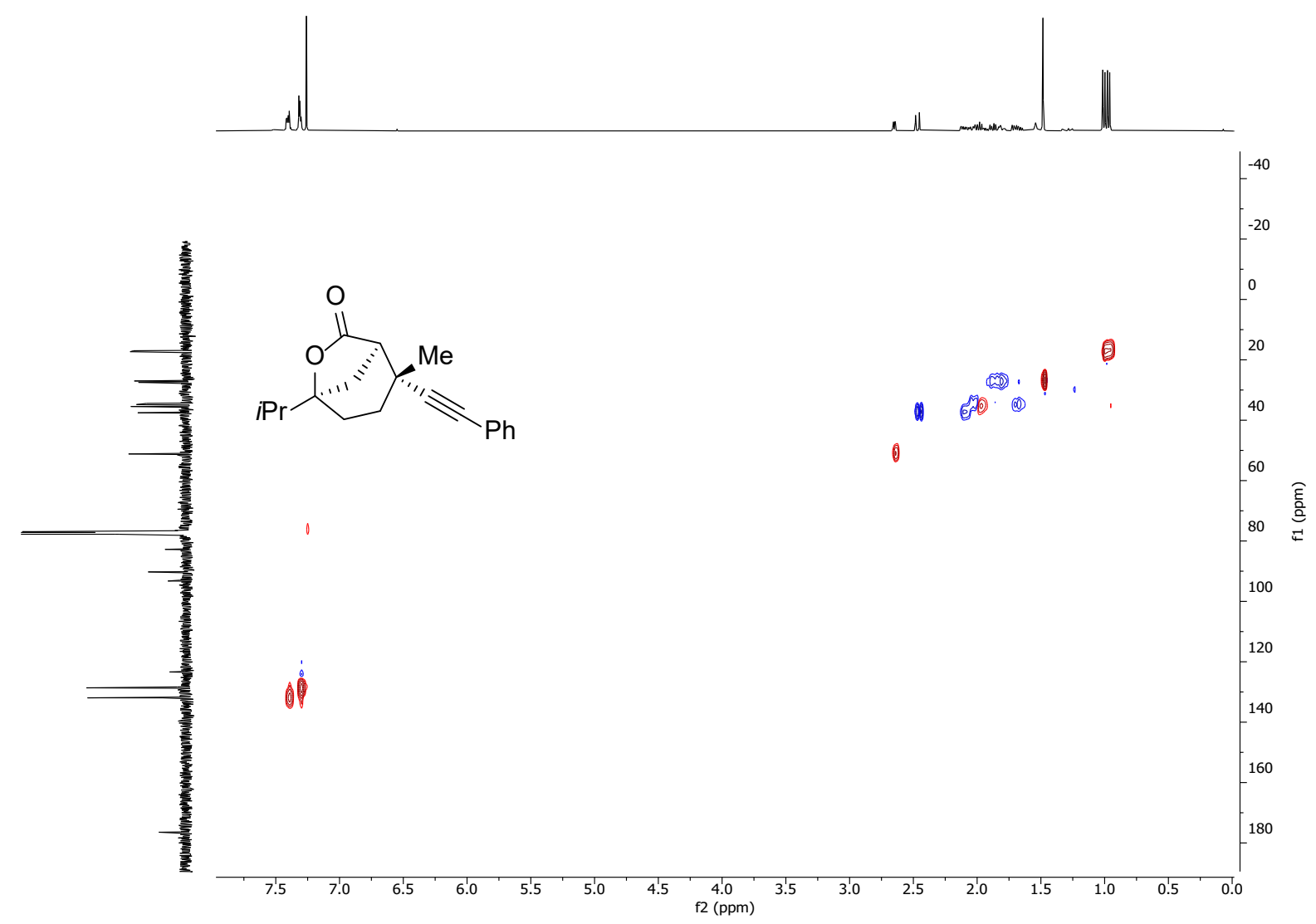


HMBC

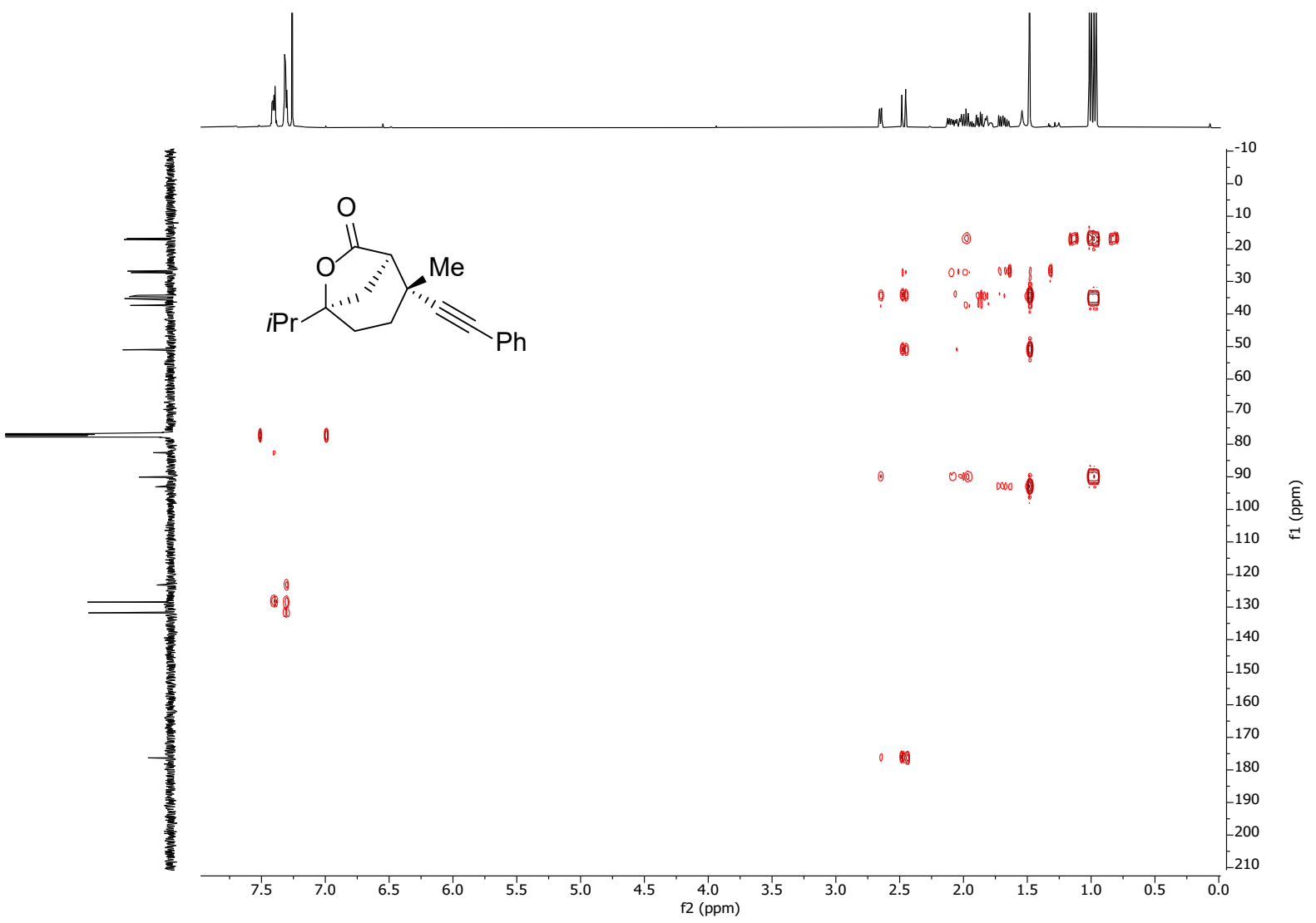

NOESY

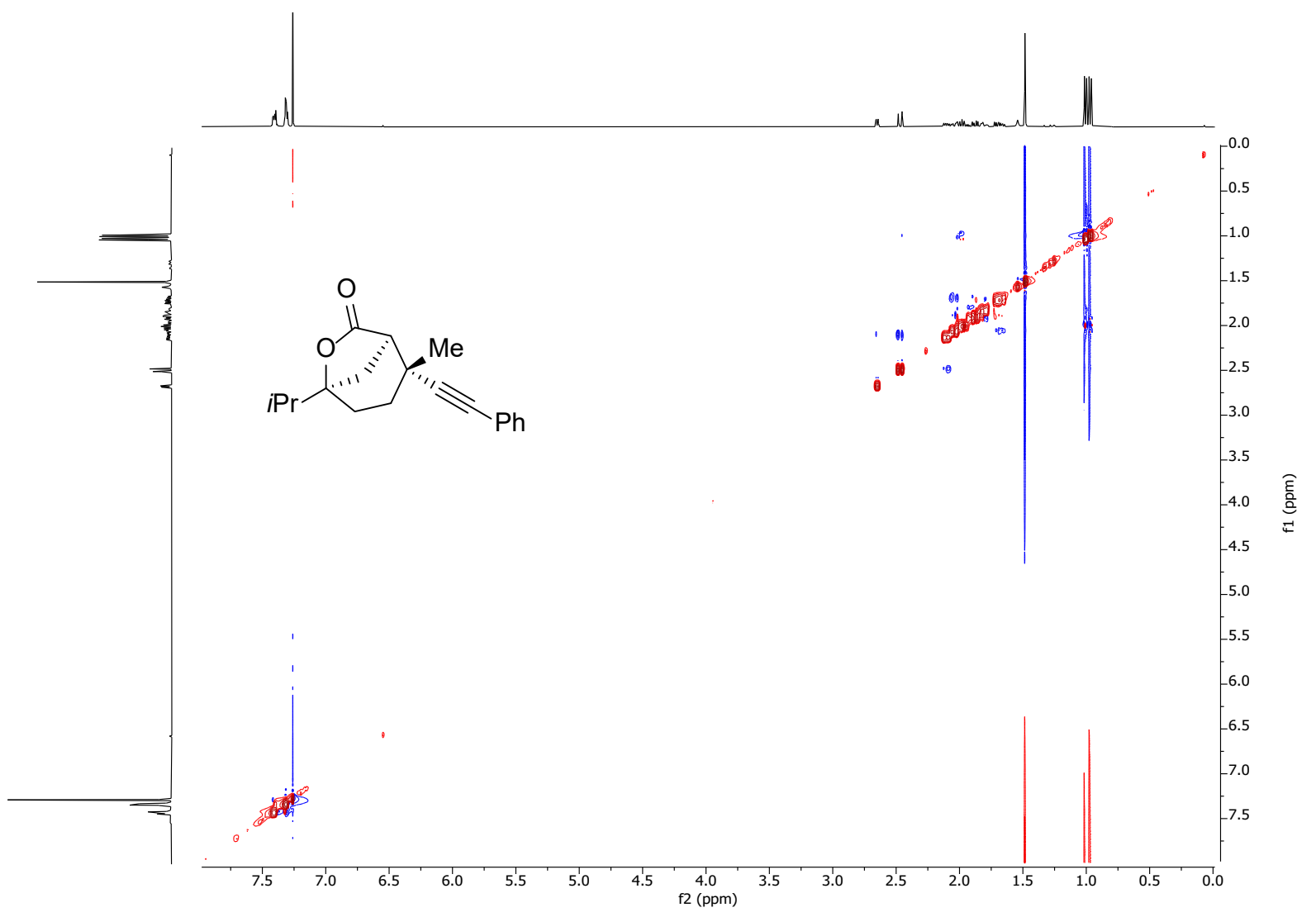




\section{Compound $\mathbf{6 b}$}

${ }^{1} \mathrm{H} \mathrm{NMR}, \mathrm{CDCl}_{3}, 400 \mathrm{MHz}$

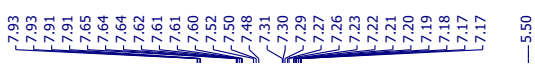
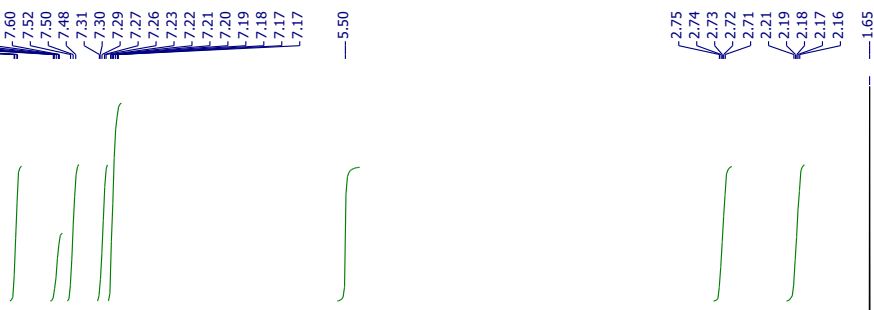

$\chi_{\mathrm{O}}^{\mathrm{O}} \overbrace{\mathrm{O}}^{\mathrm{O}} \stackrel{\mathrm{H}}{\mathrm{Ph}}^{\mathrm{O}}$

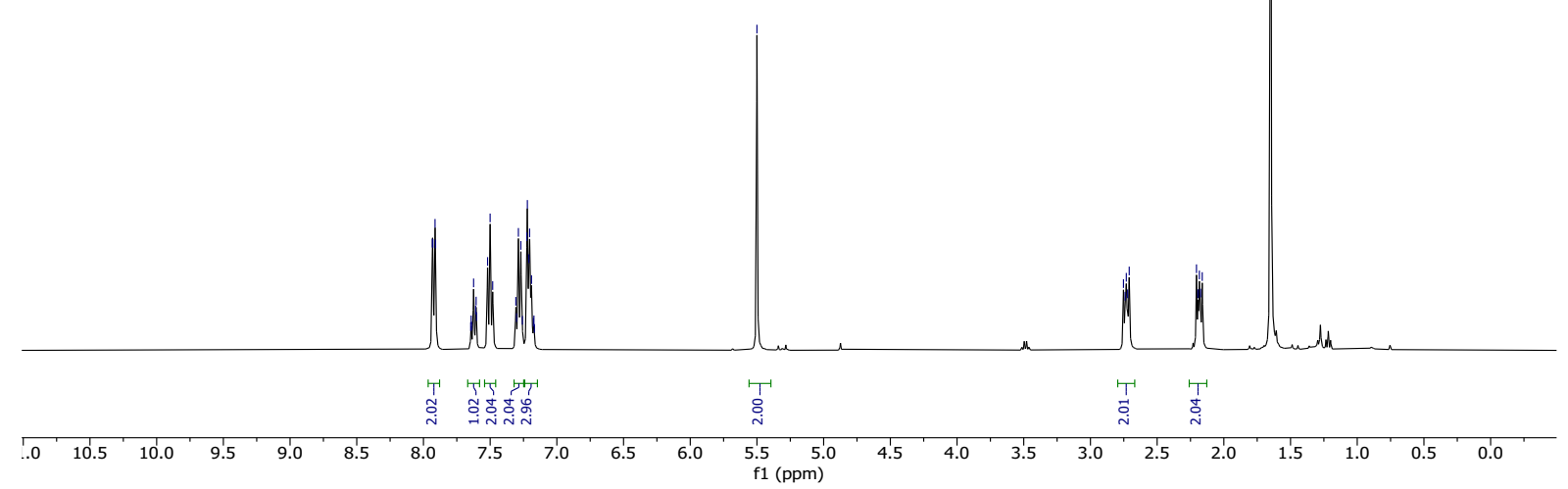

${ }^{13} \mathrm{C} \mathrm{NMR,} \mathrm{CDCl}_{3}, 101 \mathrm{MHz}$

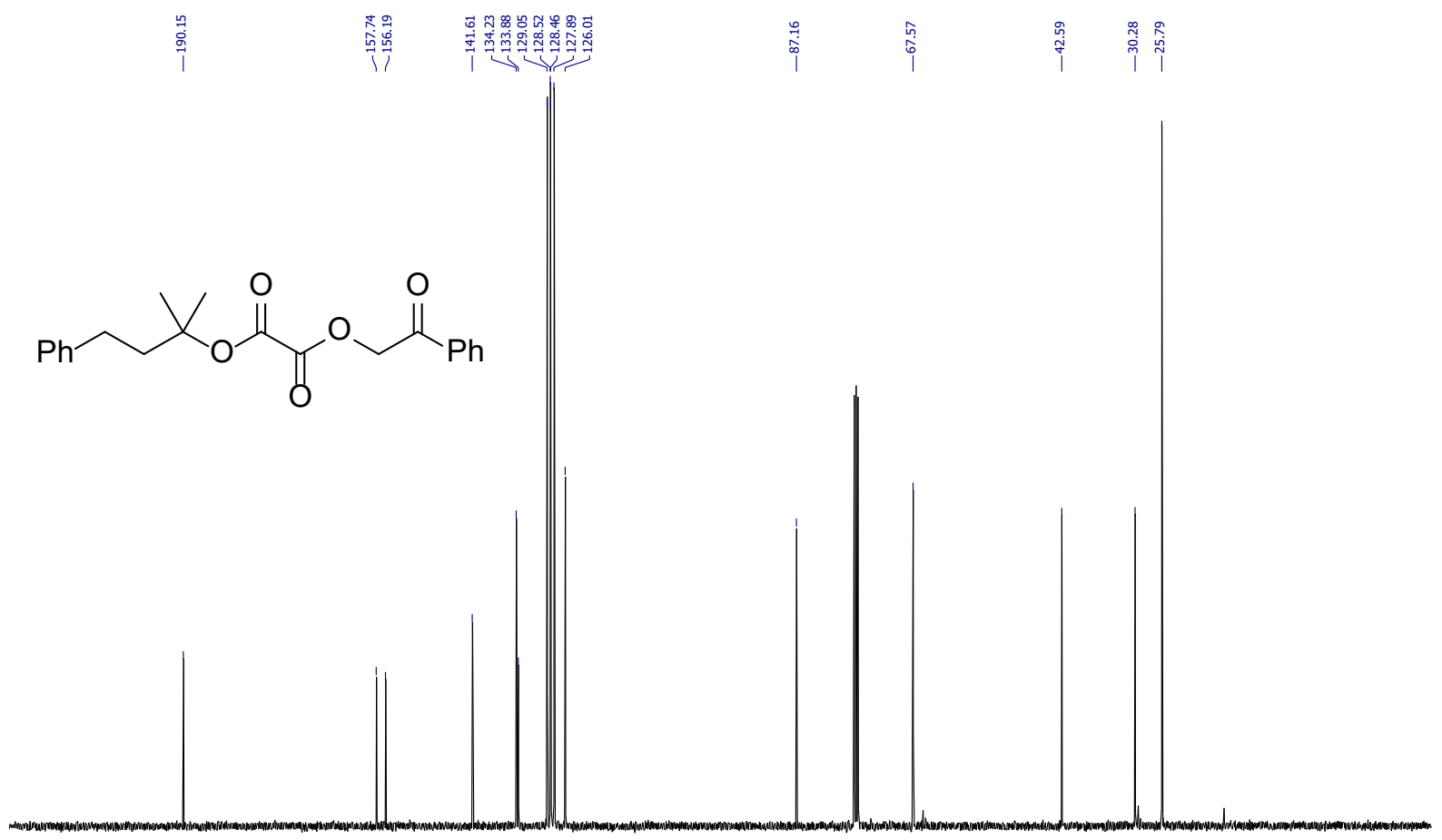

